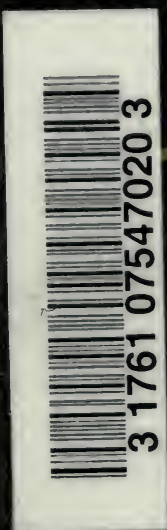

CORRUGATED BAR COMPANY, INC. BUFFALO 


A.E.MOUrSE
Jor.1920 

A.E.MOURSE

\section{USEFUL DATA ON}

Reinforced Concrete Buildings FOR THE

Designer and Estimator o BY THE

ENGINEERING STAFF

OF THE

CORRUgated BAR COMPANY, INC.

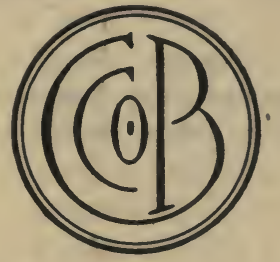

PRICE, $\$ 2.50$

CORRUGATED BAR COMPANY, INC.

1) BUFFALO, N.Y.

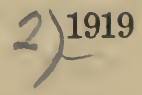


Copyright, 1919, by

Corrugated Bar Company, Inc.

Buffalo, N. Y.

IBRAR

AUE 31967 


\section{PREFACE}

$\mathrm{H}$

por more than 25 years we have been engaged in the design and development of reinforced concrete construction. During this period we have had occasion to publish technical data from time to time of interest to the engineer, the architect and the contractor. As a result of these publications we find there is a growing demand for a compilation of data relating to the design of reinforced concrete buildings. A number of comprehensive treatises have been published to meet this demand, but they deal generally with method and theory of design rather than with quantitative results.

It is not intended, nor is it expected, that this handbook can take the place of any of the excellent textbooks on concrete design or replace the services of the designing engineer. It is hoped that it will supplement the works of reference and as far as possible eliminate the manual labor involved in the repeated application of formulas and diagrams to the determination of the dimensions of a structure. It is the further aim of the book to give under one cover all of the data needed by the busy engineer or estimator in meeting the everyday problems in concrete building design, or briefly to place in his hands designing information that in a measure parallels the familiar handbook of the structural steel manufacturer.

Until such time as a national building code may be adopted, there will be recognized the impossibility of preparing a thoroughly satisfactory set of reinforced concrete standards so that we have of necessity confined ourselves to stress combinations most widely accepted and within these limits to give a satisfactory range of working values,- -values that give the "answer," without further resort to calculation, when the conditions of the problem are known.

Several of the more comprehensive publications on reinforced concrete contain some excellent diagrams that greatly facilitate the designer's work. A few of these diagrams have been incorporated in the present volume and due acknowledgment for their use is hereby made to the work of Messrs. Turneaure \& Maurer entitled "Principles of Reinforced Concrete Construction" and to "Reinforced Concrete Construction," by George A. Hool, S. B., Professor of Structural Engineering, University of Wisconsin. Further acknowledgment is also made to those members of our organization who so ably assisted in the compilation of the data and to their efforts is due what measure of success may attend the publication of this volume.

Corrugated Bar Company, Inc.

Buffalo, N. Y., March 15, 1919. 


\section{A 4 misolyty}

ir:

41

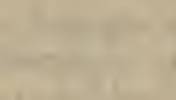
(1)

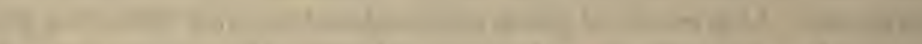

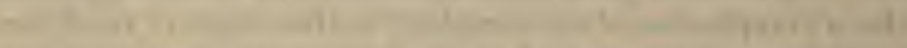

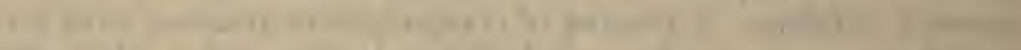

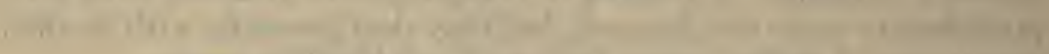

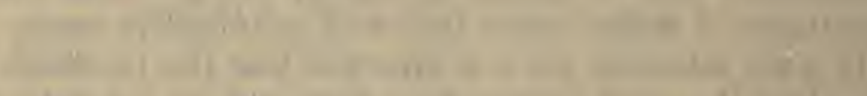

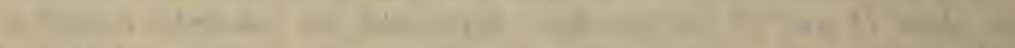

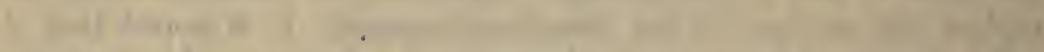

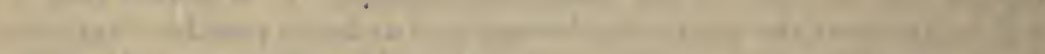

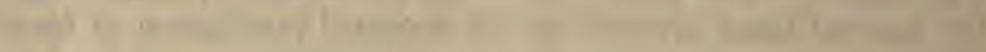

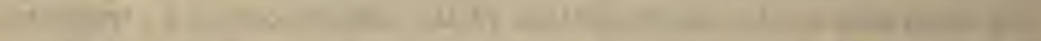

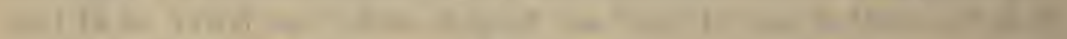

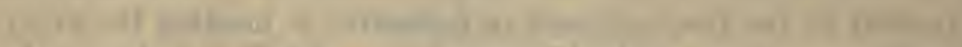

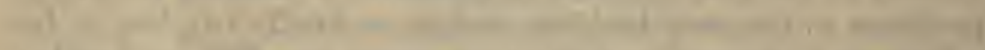

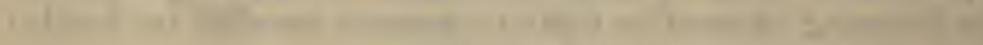
TH

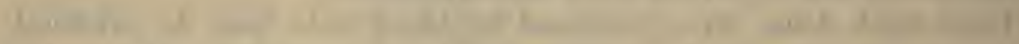

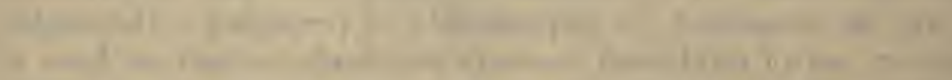

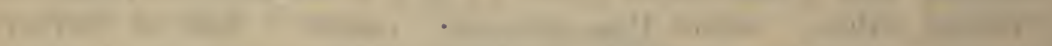

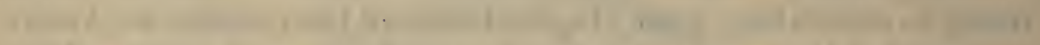

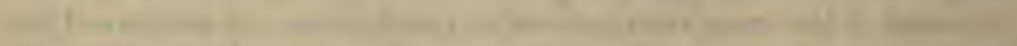

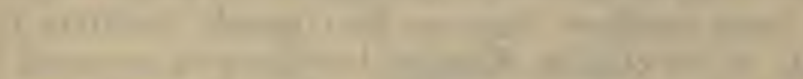

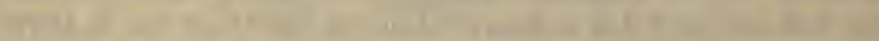

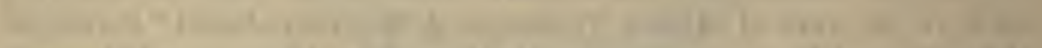

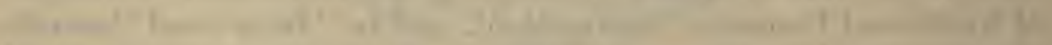
1. nis in

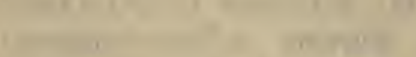

$+1+1$

1

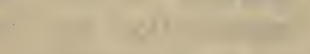

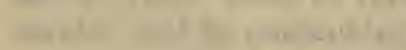

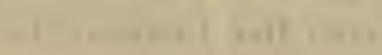




\section{FORMULAS FOR REINFORCED CONCRETE DESIGN}

It is recognized by all authorities on the design of reinforced concrete structures, that the common theory of flexure does not apply for wide ranges of stress. For stresses in excess of those commonly used in design the relation between stress and deformation is not uniform and this divergence becomes more pronounced as the stress increases. Under these conditions the parabola is the curve which most nearly expresses the relation between stress and deformation and is the relation which should be used in the discussion of experimental or test data to obtain accuracy of results.

In the design of structures, however, the stresses used are low, a condition for which it can safely be assumed that the deformation of any compression fibre in a beam is proportional to its distance from the neutral axis. The error in this assumption is small and is on the side of safety.

The formulas which follow are for working loads and assume a straight line variation of stress to deformation of concrete in compression. Tension in the concrete is neglected.

\section{(a) Rectangular Beams.}

\section{*STANDARD NOTATION}

The following notation is recommended:

$f_{\mathrm{B}}=$ tensile unit stress in steel.

$f_{\mathrm{c}}=$ compressive unit stress in concrete.

$E_{\mathrm{s}}=$ modulus of elasticity of steel.

$E_{\mathrm{c}}=$ modulus of elasticity of conerete.

$n=\frac{E_{\mathrm{s}}}{E_{\mathrm{c}}}$

$M=$ moment of resistance, or bending moment in general.

$A_{\mathrm{s}}=$ steel area.

$b=$ breadth of beam.

$d=$ depth of beam to center of steel.

$k=$ ratio of depth of neutral axis to depth $d$.

$z=$ depth below top to resultant of the compressive stresses.

$j=$ ratio of lever arm of resisting couple to depth $d$.

$j d=d-z=$ arm of resisting couple.

$p=$ steel ratio $=\frac{A_{\mathrm{B}}}{b d}$

(b) T-Beams.

$b$ = width of flange.

$b^{\prime}=$ width of stem.

$t=$ thickness of flange.

(c) Beams Reinforced for Compression.

$A^{\prime}=$ area of compressive steel.

$p^{\prime}=$ steel ratio for compressive steel.

$f_{\mathrm{s}}^{\prime}=$ compressive unit stress in steel.

$C=$ total compressive stress in concrete.

* From: Transactions of Am. Soc. of C. E. Vol. IXXXI, December, 1917. 
$C^{\prime}=$ total compressive stress in steel.

$d^{\prime}=$ depth to center of compressive steel.

$z$ = depth to resultant of $C$ and $C^{\prime}$.

(d) Shear, Bond and Web Reinforcement.

$V=$ total shear.

$V^{\prime}=$ total shear producing stress in reinforcement.

0 =shearing unit stress.

$u=$ bond stress per unit area of bar.

$0=$ circumference or perimeter of bar.

$\Sigma_{0}=$ sum of the perimeters of all bars.

$T_{\mathrm{B}}=$ total stress in single reinforcing member.

$s=$ Horizontal spacing of reinforcing members.

\section{(e) Columns.}

$A=$ total net area.

$A_{\mathrm{s}}=$ area of longitudinal steel.

$A_{0}=$ area of concrete.

$P=$ total safe load.

(a) Rectangular Beams.

Position of neutral axis,

Arm of resisting couple,

$$
k=\sqrt{2 p n+(p n)^{2}}-p n .
$$

$$
j=1-\frac{1}{3} k \text {. }
$$

[For $f_{\mathrm{b}}=15,000$ to 16,000 and $f_{0}=600$ to $650, j$ may be taken at $\frac{7}{8} \cdot$ ]

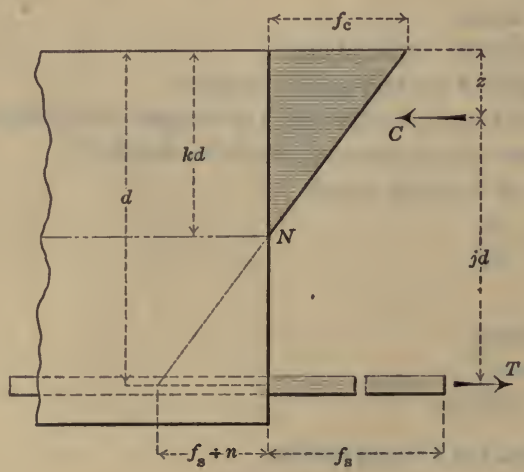

FIG. 1.

Fiber stresses,

$$
\begin{aligned}
& f_{\mathrm{a}}=\frac{M}{A_{\mathrm{o}} j d}=\frac{M}{p j b d^{2}} . \\
& f_{\mathrm{c}}=\frac{2 M}{j k b d^{2}}=\frac{2 p f_{\mathrm{g}}}{k} .
\end{aligned}
$$


Steel ratio, for balanced reinforcement,

(b) T-Beams.

$$
p=\frac{1}{2} \frac{1}{\frac{f_{0}}{f_{0}}\left(\frac{f_{B}}{n f_{0}}+1\right)} .
$$

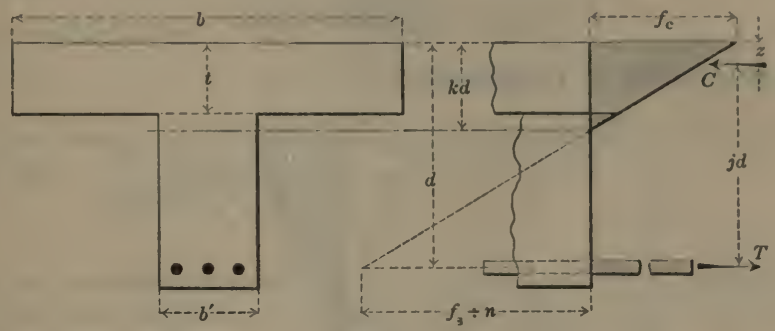

FIG. 2.

Case I. When the neutral axis lies in the flange, use the formulas for rectangular beams.

Case II. When the neutral axis lies in the stem.

The following formulas neglect the compression in the stem.

Position of neutral axis,

$$
k d=\frac{2 n d A_{\mathrm{b}}+b t^{2}}{2 n A_{\mathrm{s}}+2 b t}
$$

Position of resultant compression,

$$
z=\frac{3 k d-2 t}{2 k d-t} \cdot \frac{t}{3}
$$

Arm of resisting couple,

Fiber stresses,

$$
j d=d-z
$$

$$
\begin{aligned}
& f_{\mathrm{B}}=\frac{M}{A_{\mathrm{B}} j d} . \\
& f_{\mathrm{c}}=\frac{M k d}{b t\left(k d-\frac{1}{2} t\right) j d}=\frac{f_{\mathrm{s}}}{n} \cdot \frac{k}{1-k} .
\end{aligned}
$$

(For approximate results the formulas for rectangular beams may be used.)

The following formulas take into account the compression in the stem; they are recommended where the flange is small compared with the stem:

Position of neutral axis,

$$
l k d=\sqrt{\frac{2 n d A_{8}+\left(b-b^{\prime}\right) t^{2}}{b^{\prime}}+\left(\frac{n A_{\mathrm{s}}+\left(b-b^{\prime}\right) t}{b^{\prime}}\right)^{2}}-\frac{n A_{\mathrm{s}}+\left(b-b^{\prime}\right) t .}{b^{\prime}} .
$$

Position of resultant compression,

$$
z=\frac{\left(k d t^{2}-\frac{2}{3} t^{3}\right) b+\left[(k d-t)^{2}\left(t+\frac{1}{3}(k d-t)\right)\right] b^{\prime}}{t(2 k d-t) b+(k d-t)^{2} b^{\prime}}
$$


Arm of resisting couple,

$$
j d=d-z
$$

Fiber stresses,

$$
\begin{aligned}
& f_{\mathrm{s}}=\frac{M}{A_{\mathrm{s}} j d} \\
& f_{\mathrm{c}}=\frac{2 M k d}{\left[(2 k d-t) b t+(k d-t)^{2} b^{\prime}\right] j d}
\end{aligned}
$$

(c) Beams Reinforced for Compression.

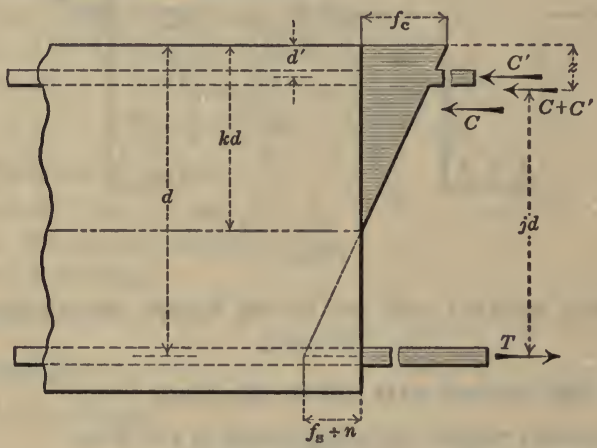

FIg. 3.

Position of neutral axis,

$$
k=\sqrt{2 n\left(p+p^{\prime} \frac{d^{\prime}}{d}\right)+n^{2}\left(p+p^{\prime}\right)^{2}}-n\left(p+p^{\prime}\right)
$$

Position of resultant compression,

$$
z=\frac{\frac{1}{3} k^{3} d+2 p^{\prime} n d^{\prime}\left(k-\frac{d^{\prime}}{d}\right)}{k^{2}+2 p^{\prime} n\left(k-\frac{d^{\prime}}{d}\right)}
$$

Arm of resisting couple,

Fiber stresses,

$$
j d=d-z
$$

$$
\begin{aligned}
& f_{0}=\frac{6 M}{b d^{2}\left[3 k-k^{2}+\frac{6 p^{\prime} n}{k}\left(k-\frac{d^{\prime}}{d}\right)\left(1-\frac{d^{\prime}}{d}\right)\right]} \\
& f_{0}=\frac{M}{p j b d^{2}}=n f_{0} \frac{1-k}{k} \\
& f_{0}^{\prime}=n f_{0} \frac{k-\frac{d^{\prime}}{d}}{k}
\end{aligned}
$$

(d) Shear, Bond, and Web Reinforcement.

For rectangular beams,

$$
v=\frac{V}{b j d}
$$




$$
u=\frac{V}{j d \cdot \Sigma_{0}}
$$

(For approximate results $j$ may be taken at $\frac{7}{8} \cdot$ )

The stresses in web reinforcement may be estimated by means of the following formulas:

Vertical web reinforcement,

$$
T_{\mathrm{B}}=\frac{V^{\prime} s}{j d}
$$

Bars bent up at angles between 20 and 45 deg. with the horizontal and web members inclined at 45 deg.,

$$
T_{\mathrm{s}}=\frac{3}{4} \frac{V^{\prime} s}{j d}
$$

In the text of the report it is recommended that two-thirds of the external vertical shear (total shear) at any section be taken as the amount of total shear producing stress in the web reinforcement. $V^{\prime \prime}$ therefore equals two-thirds of $V$.

The same formulas apply to beams reinforced for compression as regards shear and bond stress for tensile steel.

For T-Beams,

$$
\begin{aligned}
& v=\frac{V}{b^{\prime} j d} \\
& u=\frac{V}{j d \cdot \Sigma, o}
\end{aligned}
$$

[For approximate results $j$ may be taken at $\frac{7}{8} \cdot$ ]

(e) Columns.

Total safe load,

$$
P=f_{\mathrm{c}}\left(A_{\mathrm{o}}+n A_{\mathrm{s}}\right)=f_{\mathrm{c}} A[1+(n-1) p]
$$

Unit stresses,

$$
\begin{aligned}
& f_{0}=\frac{P}{A[1+(n-1) p]} \\
& f_{\mathrm{s}}=n f_{0}
\end{aligned}
$$




\section{EXPLANATION OF THE USE OF DESIGNING DIAGRAMS}

Rectangular beams or slabs, reinforced for tension only, may readily be designed by the aid of Diagram 1, page 16 . This diagram assumes a value for $E_{\mathrm{o}}$ of $2,000,000$ or $n=15$, which is recommended for the design of beams and slabs.

Example 1. - Given a beam having a span of $24 \mathrm{ft}$. simply supported, to carry a load of $4,000 \mathrm{lb}$. per ft. including estimated dead load; $f_{\mathrm{o}}=650, f_{\mathrm{B}}=16,000, E_{\mathrm{o}}=$ $2,000,000, n=15$. Determine size of beam required.

$$
M=\frac{(4,000)(24)(24)}{8}=288,000 \mathrm{ft} . \mathrm{lb} .=3,456,000 \text { in. lb. }
$$

On Diagram 1, page 16, find the intersection of curves for $f_{\mathrm{s}}=16,000$ and $f_{\mathrm{o}}=650$ and $\operatorname{read} K=\frac{M}{b d^{2}}=107.5$ and $p=0.77 \%=0.0077$

$$
b d^{2}=\frac{M}{K}=\frac{3,456,000}{107.5}=32,150
$$

Assuming $b=24$ in., $d=\sqrt{\frac{M}{b K}}=\sqrt{\frac{3,456,000}{(24)(107.5)}}=36.6$

$A_{\mathrm{s}}=p b d=(0.0077)(24)(36.6)=6.76 \mathrm{sq}$. in.

It will be noted that by selecting a value for either $b$ or $d$ the problem may be completely solved. This selection may be governed by the relative cost of steel and concrete or may be limited by clearance.

Care should always be taken to ascertain if the section selected to resist bending moment is satisfactory in shear. In the example:

$$
\begin{gathered}
V=\frac{(4,000)(24)}{2}=48,000 \mathrm{lb} . \\
v=\frac{V}{b j d}=\frac{48,000}{(24)\left(\frac{7}{8}\right)(36.6)}=62.5 \mathrm{lb} . \text { per sq. in. }
\end{gathered}
$$

It will be noted that $j$ has been taken as $\frac{7}{8}$, which is sufficiently close when used in calculations for bond and shear stresses. The result indicates web reinforcement would be required, for a limit of $40 \mathrm{lb}$. per sq. in. shear on the concrete.

Example 2.-Given a beam of $20 \mathrm{ft}$. span having fixed ends and carrying a total load of $1,000 \mathrm{lb}$. per ft.; $b=10$ in., $d=18$ in., $A_{8}=2.20$ sq. in. and $n=15$. Find $f_{8}$, $f_{\mathrm{c}}, j$ and $k$.

$$
\begin{aligned}
& M=\frac{(1,000)(20)(20)(12)}{12}=400,000 \mathrm{in.} \mathrm{lb} . \\
& K=\frac{M}{b d^{2}}=\frac{400,000}{(10)(18)(18)}=123.5 \\
& p=\frac{A_{\mathrm{s}}}{b d}=\frac{2.20}{(10)(18)}=0.0122
\end{aligned}
$$


On Diagram 1 find the intersection of $K=123.5$ and $p=1.22$ and read $f_{0}=11,000$, $f_{\mathrm{c}}=650$. On the upper portion of the diagram for $p=1.22$ find $j=0.846$ and $k=0.452$.

T-Beams. In the design and investigation of T-beams Diagrams 2, 3 and 4, pages 17,18 and 19 will be found useful. These diagrams apply only when the neutral axis falls below the under side of the flange of the T-beam. When the neutral axis falls above, the diagrams for rectangular beams apply.

Diagram 2 can be used only when the area of steel is such that $f_{0}$ is 16,000 and Diagram 3 when $f_{8}$ is 18,000 . From these diagrams, for any assumed value of $d$ and for fixed values of $M, t$ and $b$, we may obtain $j$ for determining the steel area required and also the value of $f_{\mathrm{c}}$. If $f_{\mathrm{c}}$ is fixed, knowing $M$ and $t$, we may find the value of $b$ or $d$, by assuming one of them, and obtain the corresponding steel area. It should be borne in mind that $b$ must not exceed code or specification limits.

Diagram 4 gives values of $k$ and $j$ for T-beams and is useful in checking steel and concrete stresses, the dimensions and reinforcement being known.

Example 1. Given a T-beam having a span of 24 feet, freely supported, to carry a total load of $2,000 \mathrm{lb}$. per foot, $t=5$ in., $b=30$ in., $f_{\mathrm{a}}=16,000, f_{\mathrm{c}}=650, n=15, v=120$.

$$
\begin{aligned}
& M=\frac{(2,000)(24)(24)(12)}{8}=1,728,000 \mathrm{in} . \mathrm{lb} . \\
& V=\frac{(2,000)(24)}{2}=24,000 \mathrm{lb} .
\end{aligned}
$$

Assuming a width of stem of $10 \mathrm{in}$. we find from shear considerations,

$$
\begin{aligned}
& d=\frac{V}{v j b^{\prime}}=\frac{24000}{(120)\left(\frac{7}{8}\right)(10)}=22.85 \mathrm{in} . \\
& \text { then } \frac{t}{d}=\frac{5}{22.85}=0.219
\end{aligned}
$$

From Diagram 2 trace upward from the value of $\frac{t}{d}=0.219$ to intersection with the curve for $f_{\mathrm{c}}=650$ and read $\frac{M}{b d^{2}}=92.0$. Substituting for $M$ and $d$ the values given above we find

$$
\begin{gathered}
\frac{1,728,000}{(b)(22.85)(22.85)}=92.0 \\
b=35.8 \mathrm{in} .
\end{gathered}
$$

This exceeds the fixed dimension of $30 \mathrm{in}$. for $b$ so that it becomes necessary to assume a new value for $d$. Try $d=26$ in.

then $\frac{t}{d}=0.192$

and from Diagram 2

$$
\begin{aligned}
\frac{M}{b d^{2}} & =86.0 \\
b & =29.7 \text { in. }
\end{aligned}
$$

This value is sufficiently close so as to require no further revision.

To find the area of steel required first obtain $j$ from the right-hand side of Diagram 2 by tracing upward from $f_{0}=650$ to intersection of $\frac{M}{b d^{2}}=86.0$ and read $j=0.913$ 


$$
\text { then } A_{\mathrm{s}}=\frac{M}{j d f_{\mathrm{s}}}=\frac{1,728,000}{(0.913)(26)(16,000)}=4.55 \text { sq. in. }
$$

Example 2. Given a T-beam of $20 \mathrm{ft}$. span, ends freely supported, $t=4 \mathrm{in}$., $d=20$ in., $b=30$ in., $A_{\mathrm{a}}=4.0 \mathrm{sq}$. in. Find the total load per foot this beam will carry when $n=15$ and $f_{o}$ and $f_{0}$ are not to exceed 16,000 and 650 pounds per square inch, respectively,

$$
\begin{aligned}
& p=\frac{A_{\mathrm{s}}}{b d}=\frac{4.0}{(20)(30)}=0.00667=0.667 \% \\
& \frac{t}{d}=\frac{4}{20}=0.2
\end{aligned}
$$

On the left of Diagram 4 from the intersection of $\frac{t}{d}=0.2$ and $p=0.667 \%$ read $k=0.40$ and on the right from the intersection of $p=0.667 \%$ and $k=0.40$ read $j=0.912$ $M_{\mathrm{B}}=A_{\mathrm{B}} \mathrm{f}_{\mathrm{B}} j d=(4.0)(16,000)(0.912)(20)=1,167,360$ in. $\mathrm{lb}$.

$$
\begin{aligned}
M_{0}=f_{0} & \left(1-\frac{t}{2 k d}\right) b t j d \\
= & 650\left(1-\frac{1}{(2)(0.40)(20)}\right)(30)(4)(0.912)(20)=1,067,040 \text { in. lb. }
\end{aligned}
$$

The resisting moment of the concrete, being less than that of the steel, will govern the carrying capacity of the beam. Equating the external moment $\left(M=\frac{1}{8} w l^{2}\right)$ in inch pounds to the resisting moment of the concrete and solving for $w$ we find

$$
w=\frac{8 M}{12 l^{2}}=\frac{(8)(1,067,040)}{(12)(20)(20)}=1,778 \mathrm{lb} . \text { per ft. }
$$

Beams Reinforced for Compression. It is sometimes desirable or necessary to place reinforcement in the compression side of a beam in order to maintain the concrete stress within safe limits. Continuous beams of $\mathbf{T}$ section are frequently deficient in concrete area at the supports, where due to the reversal of moment, the stem is in compression. When the stress in the concrete at this point exceeds that specified, the straight bars in the bottom of the beam may be carried through the support and utilized as compressive reinforcement.

A continuous T-beam has the following dimensions:

$$
t=6 \text { in., } b=30 \text { in., } d=34 \text { in., } b^{\prime}=14 \text { in. }
$$

The negative moment is $2,400,000 \mathrm{in}$. $\mathrm{lb}$. It will be assumed that the working stress in the concrete at the center of the beam is 650 pounds per square inch which may be increased $15 \%$ at the support, in accordance with recommendations of the Joint Committee, so that the value at this point is 747 pounds per square inch, steel stress 16,000 pounds per square inch. Determine the amount of compression steel required.

In the top for tension,

$$
\begin{aligned}
& A_{8}=\frac{2,400,000 .}{(16,000)\left(\frac{7}{8}\right)(34)}=5.04 \text { sq. in. } \\
& K=\frac{M}{b d^{2}}=\frac{2,400,000}{(14)(34)(34)}=148
\end{aligned}
$$

Entering Diagram 1 with $K=148$, we find, for $f_{B}=16,000$, that $f_{0}=805$. The reduc- 
tion of $f_{0}$ to 747 will then be $\frac{805-747}{747805}=7.76 \%$. Entering Diagram 5 , on the left margin, with 7.76 and moving to the right to the "concrete curve," thence downward, the amount of compressive steel to effect the reduction in $f_{0}$ is found to be $0.20 \%$ or (14) $(34)(0.002)=0.95$ sq. in.

Combined Bending and Direct Stress. In the design of columns, arch rings, etc., the resultant of the external forces does not always coincide with the center of gravity of the cross section of the member. In such cases consideration must be given to the combined action of bending and direct stress. For reinforced concrete members the general formula for extreme fibre stress where compression exists over the entire section, is

$$
\begin{aligned}
& f_{0}=\frac{W}{A+(n-1) p_{0} A} \pm \frac{M y}{I_{0}+n I_{0}} \\
& W=\text { Total direct load. } \\
& p_{0}=\text { Percentage of reinforcement }=\frac{A_{0}}{A} \\
& y=\text { Distance from center of gravity of section to extreme fibre. } \\
& I_{0}=\text { Moment of inertia of concrete section about the gravity axis } \\
& I_{\mathrm{a}}=\text { Moment of inertia of steel area about the gravity axis. }
\end{aligned}
$$

The other symbols are as given in the standard notation, pages 5 and 6 .

It is in the case of rectangular sections with symmetrical reinforcement, that we most frequently meet with problems involving bending and direct stress and Diagrams $6,7,8 \mathrm{a}$ and $8 \mathrm{~b}$, will be found to greatly facilitate the solution of such problems.

In the case of a homogeneous material no tension exists on the cross section when the resultant falls within the middle third. For a concrete section reinforced with steel bars the conditions are altered somewhat and the resultant may fall slightly outside the middle third without producing tension on the section. In those cases where compression exists over the whole section Diagram 6 may be employed, but where there is tension over part of the section Diagrams 7 and $8 \mathrm{a}$ or $8 \mathrm{~b}$ should be used.

Case I. No Tension on the Cross Section. Consider a column 18 inches square, reinforced with 4-1 in. square bars, carrying a load of 150,000 lb. concentrated 1 in. from the center of the column. Find the maximum unit stress in the concrete.

Percentage of reinforcement, $p_{\mathrm{o}}=\frac{A_{\mathrm{s}}}{b t}=\frac{4}{(18)(18)}=1.23 \%$

The eccentricity $\quad x_{0}=1$ in.

and

$$
\frac{x_{0}}{t}=\frac{1}{18}=0.0555
$$

Entering Diagram 6 with $\frac{x_{0}}{t}=0.0555$, tracing vertically to $p_{0}=1.23 \%$ and then to the left margin find $K^{\prime}=1.09$, a factor by which the average unit stress must be multiplied to find the maximum unit stress.

$$
\text { Max. } f_{c}=\frac{W}{b t} K^{\prime}=\frac{150,000}{(18)(18)}(1.09)=505 \text { lb. per sq. in. }
$$


To obtain the minimum concrete unit stress we know that the minimum is as much below the average as the maximum is above. In this example the average concrete unit stress is $\frac{150,000}{[(18)(18)-4]+(15)(4)}=394 \mathrm{lb}$. per sq in. Thus the minimum unit stress is $394-(394)=2$ pounds per square inch.

Case II. Tension on the Cross-Section. Suppose the column of Case I had an applied moment of $800,000 \mathrm{in}$. $\mathrm{lb}$. in addition to an axial load of $100,000 \mathrm{lb}$. Then

$$
\begin{aligned}
& x_{\mathrm{o}}=\frac{M}{W}=\frac{800,000}{100,000}=8 \mathrm{in} . \\
& \frac{x_{\mathrm{o}}}{t}=\frac{8}{18}=0.444
\end{aligned}
$$

As before $p_{0}=1.23 \%$ entering Diagram 7 with $\frac{x_{0}}{t}=0.444$, tracing vertically to $p_{0}=1.23 \%$, then to the left margin, $k=0.60$. Now entering Diagram 8a with this value of $k$ and tracing to $p_{0}=1.23 \%$, the value of $F$ is found to be 0.139 . Then

Max. $f_{0}=\frac{M}{F b t^{2}}=\frac{800,000}{(0.139)(18)(18)(18)}=987$ pounds per sq. in.

This resulting fibre stress is larger than is usually permitted and would necessitate a redesign of the column section in order to reduce the stress to the limit allowable. 


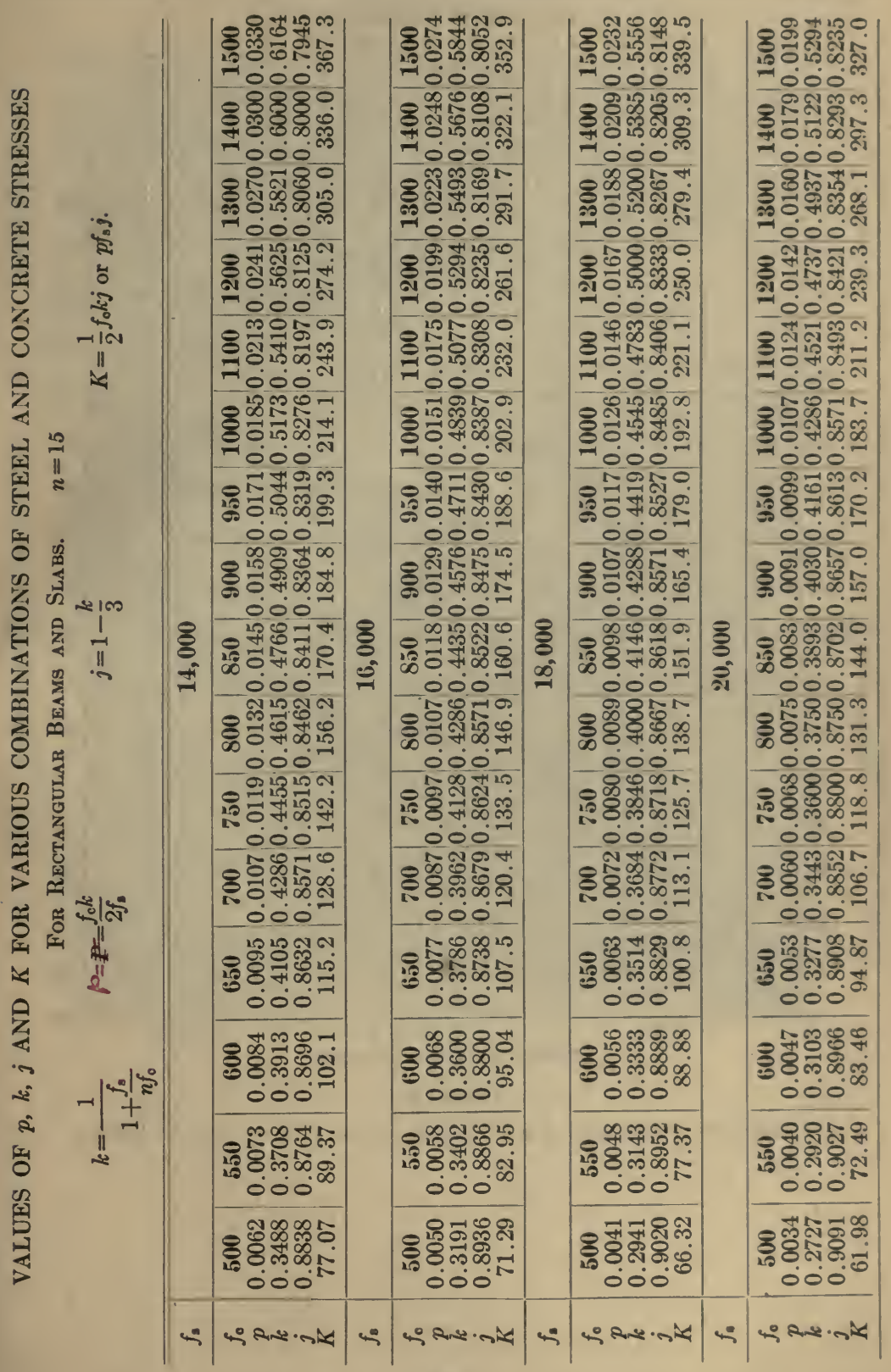


CORRUGATED BAR COMPANY, INC.

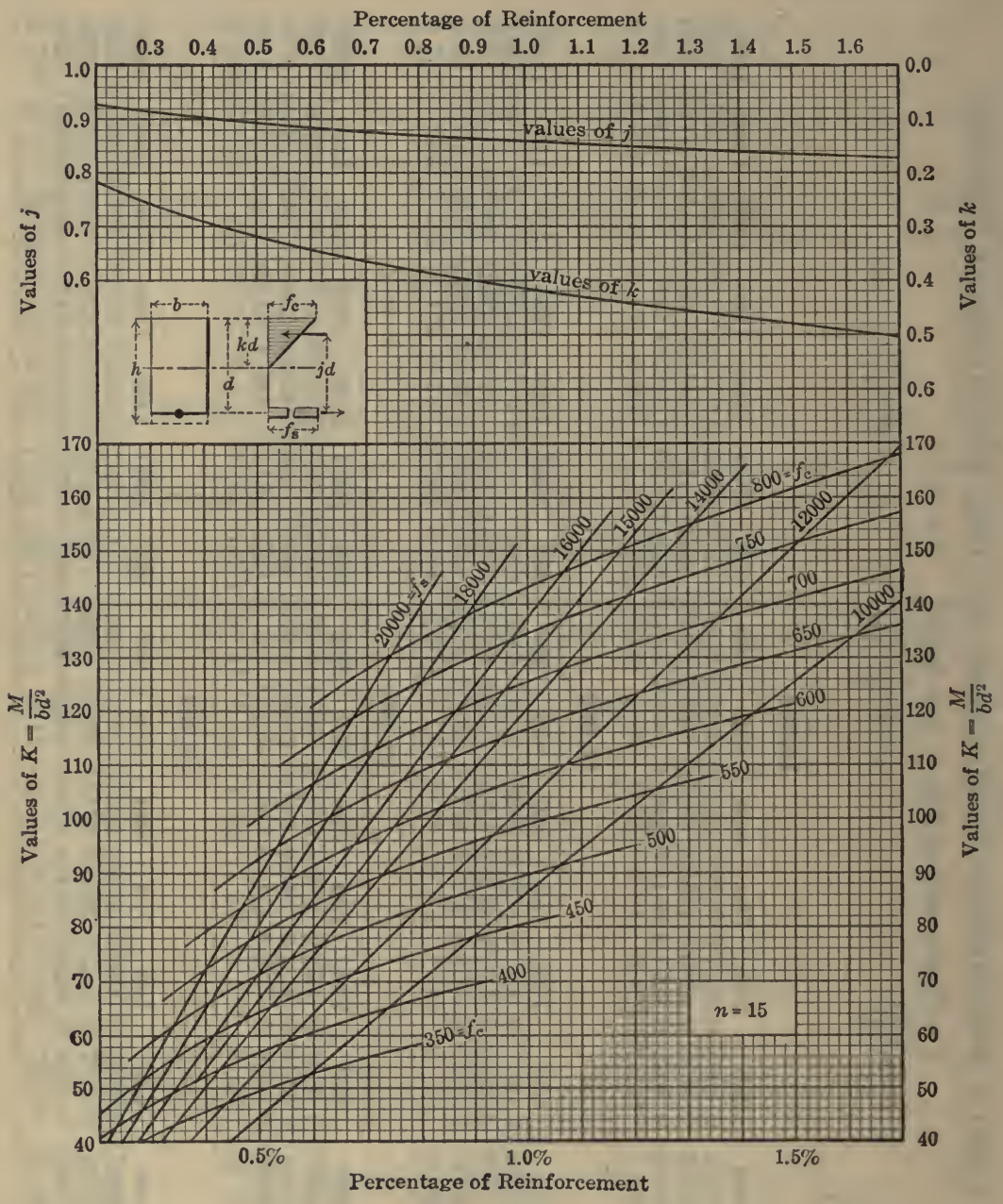

\section{Diagram 1}

Coefficients of resistance $K$ and values of $j$ and $k$ for rectangular beams. 


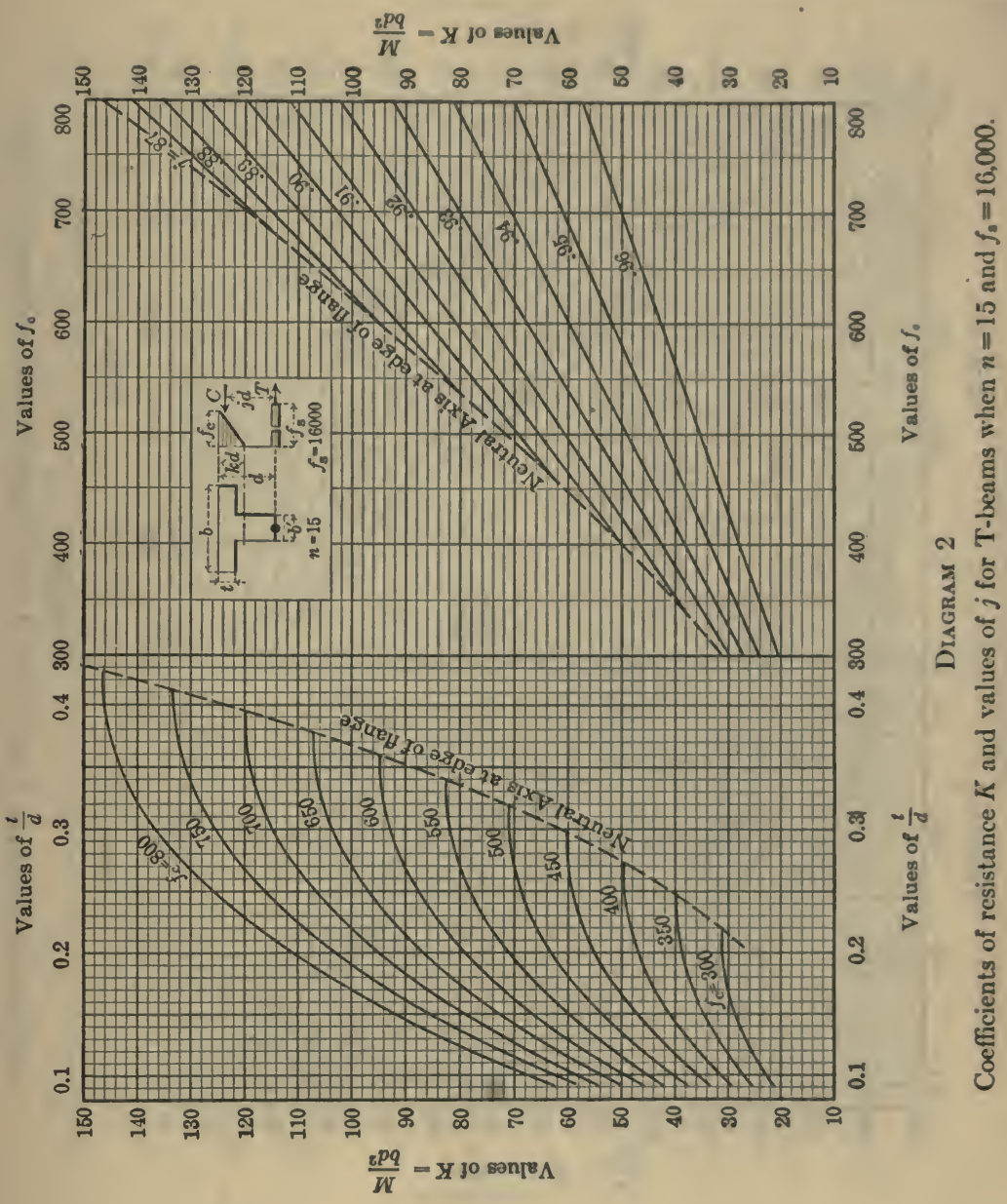




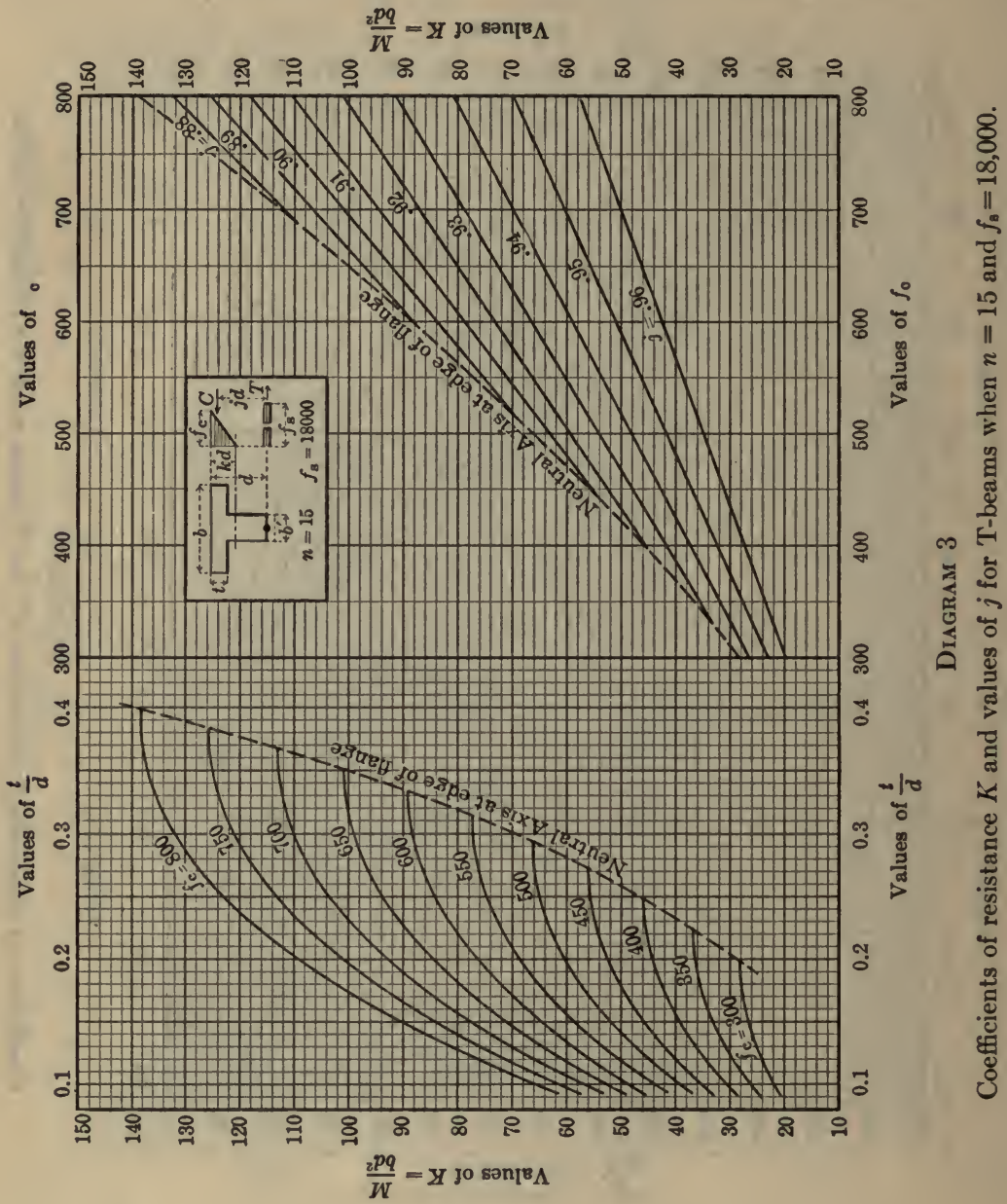


U \& EFUL DATA

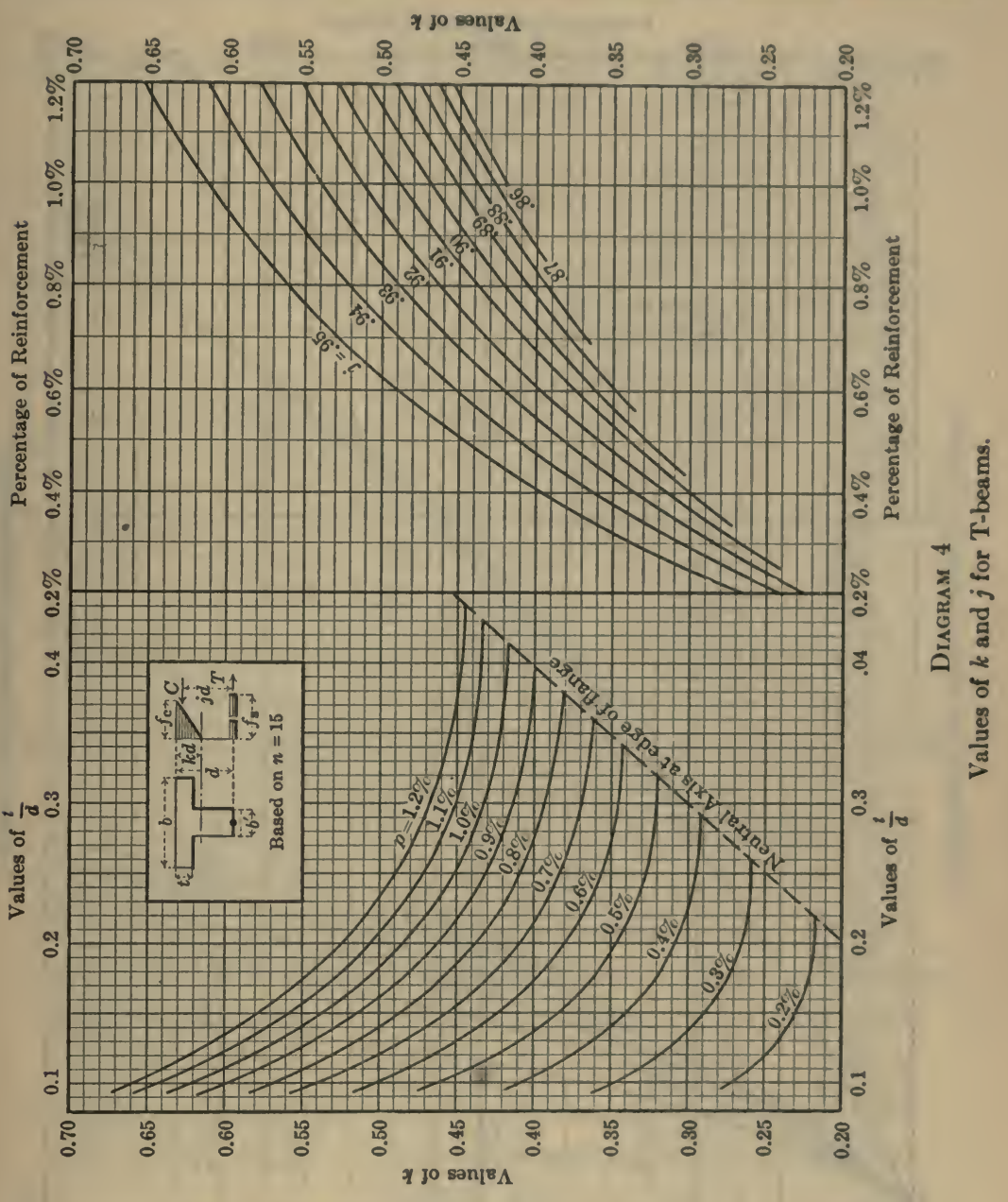


CORRUGATED BAIR COMPANY, INC.

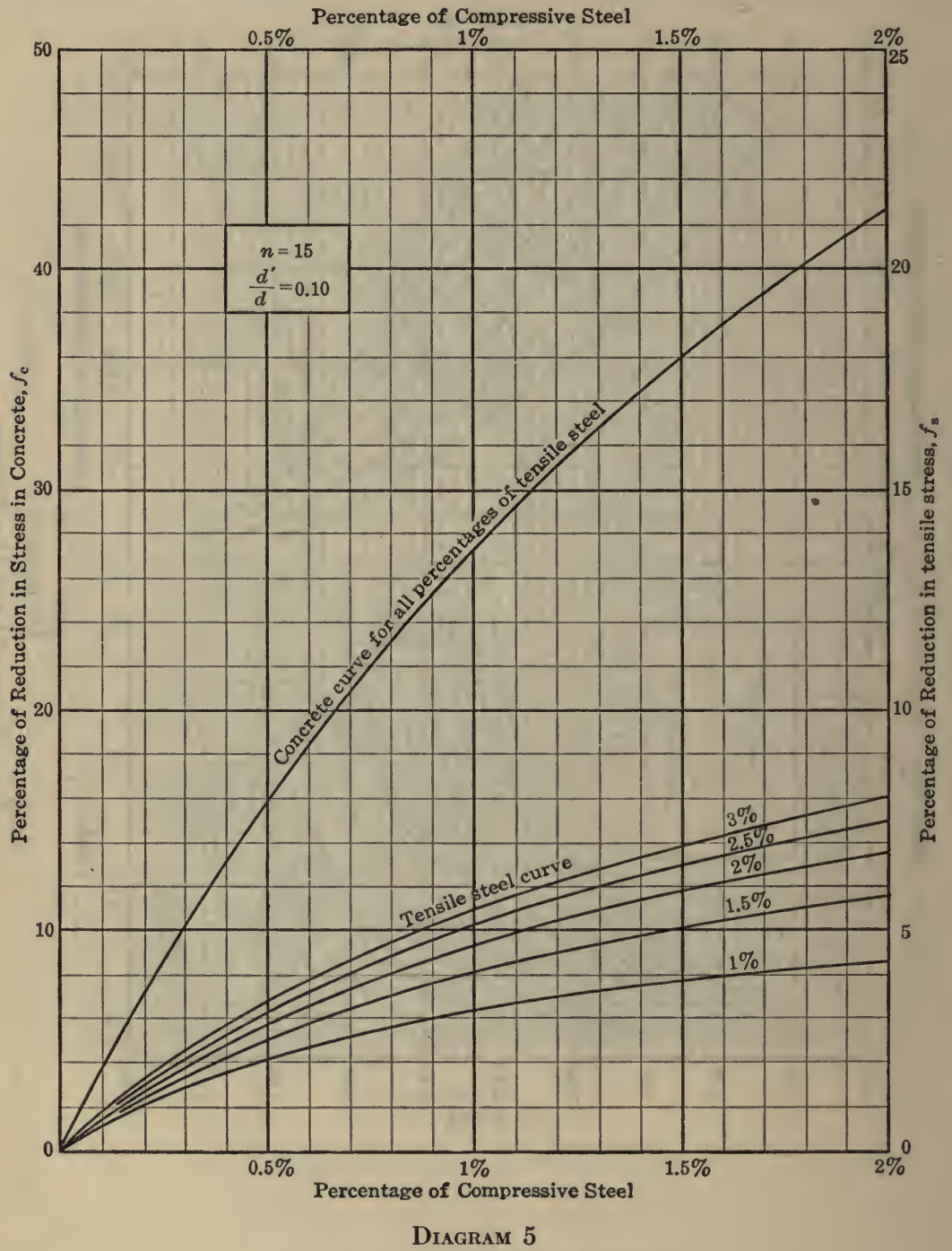

Compressive reinforcement of beams. 
USEFUL DATA

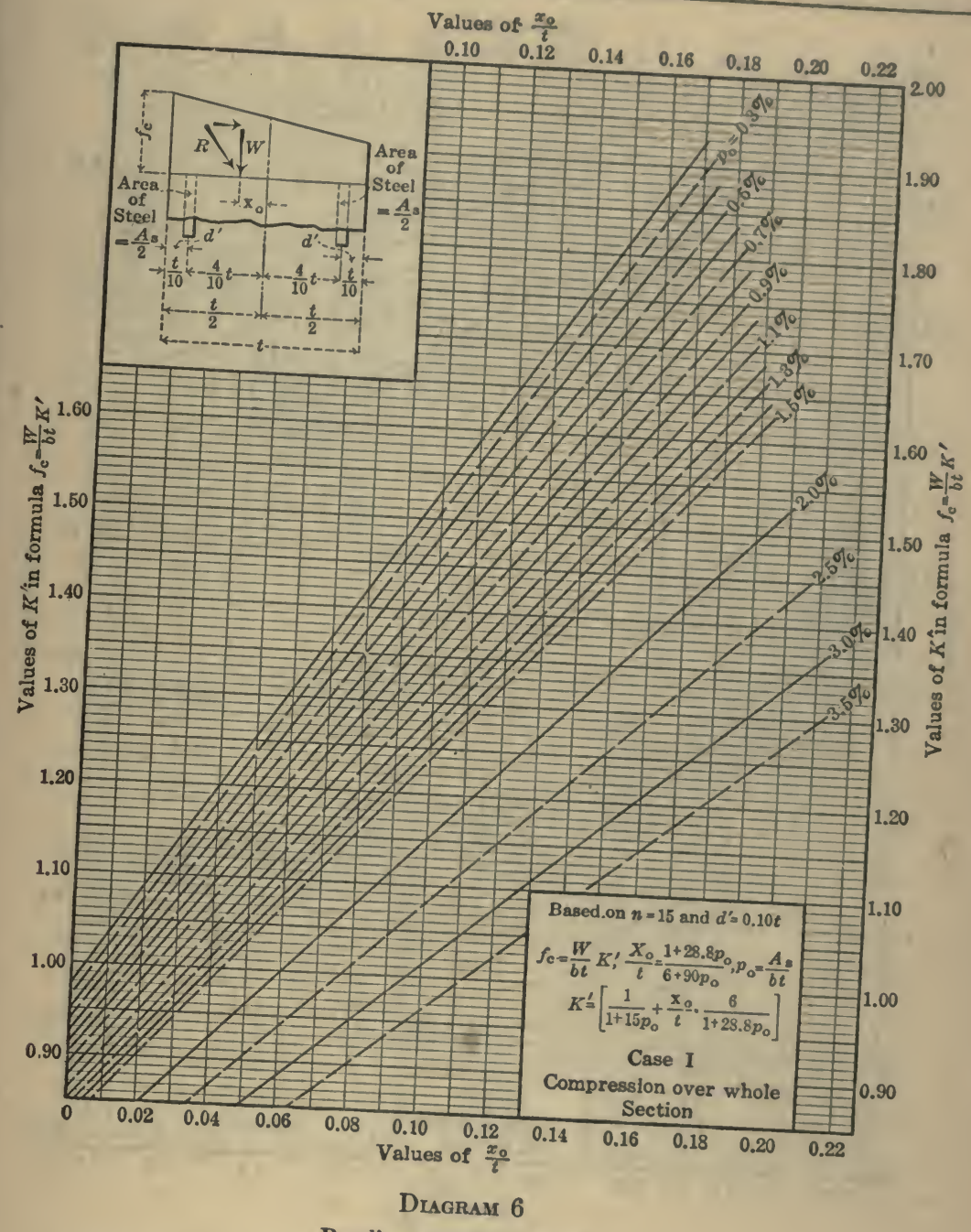

Bending and direct stress.

Values of $K^{\prime}$, a factor by which the average stress is multiplied to obtain the maximum fibre stress. 
CORRUGATED BAR COMPANY, INC.

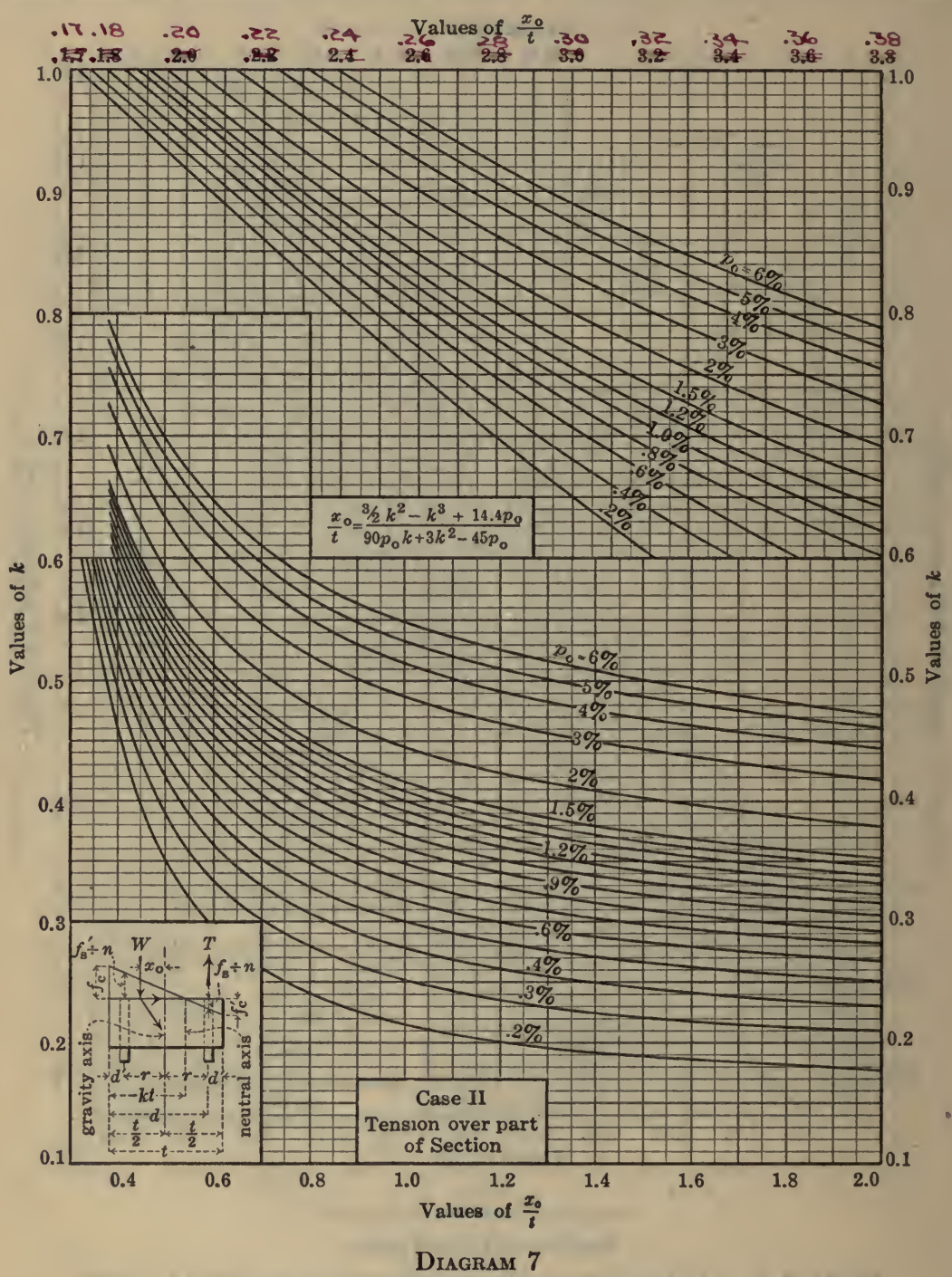

Bending and direct stress.

Values of $k$, the ratio of distance of neutral axis from extreme fibre to total thickness or depth, $t$. 


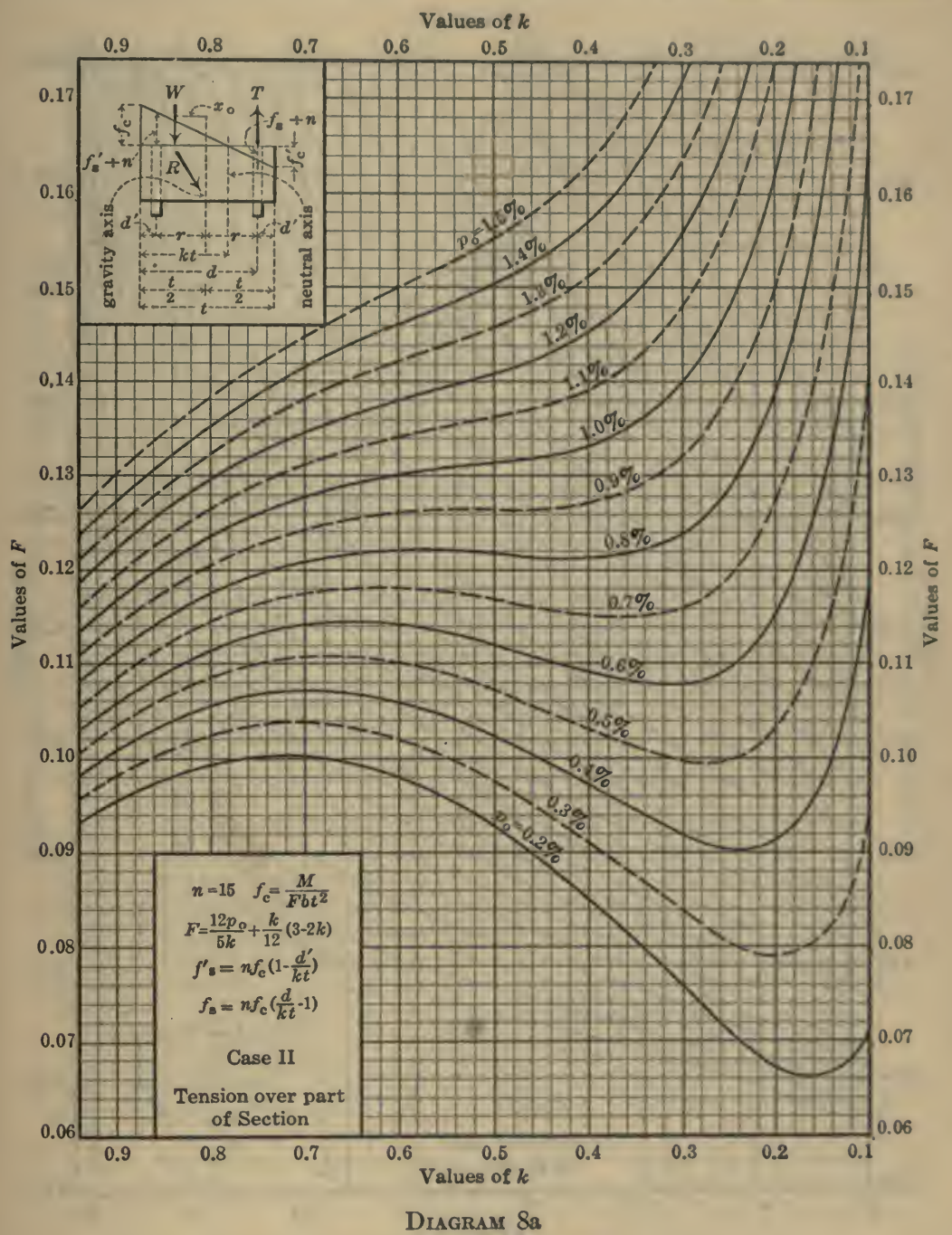

Bending and direct stress.

Values of coefficient $F$ in formula for obtaining extreme fibre stress $f_{0}=\frac{M}{F b t^{2}}$ 
CORRUGATED BAR COMPANY, INC.

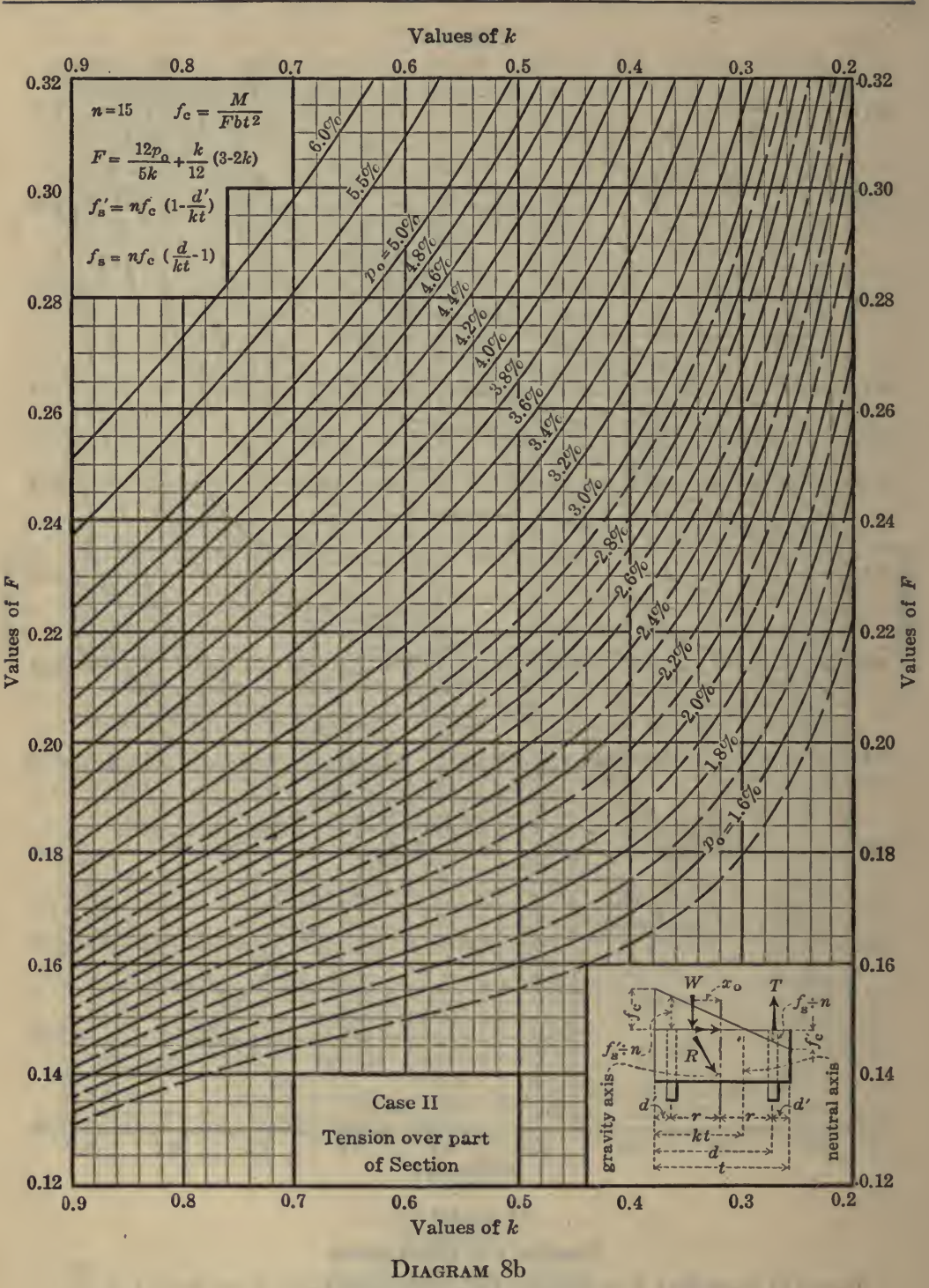

Bending and direct stress.

Values of coefficient $F$ in formula for obtaining extreme fibre stress $f_{0}=\frac{M}{F b t^{2}}$ 
U S EFUL DATA

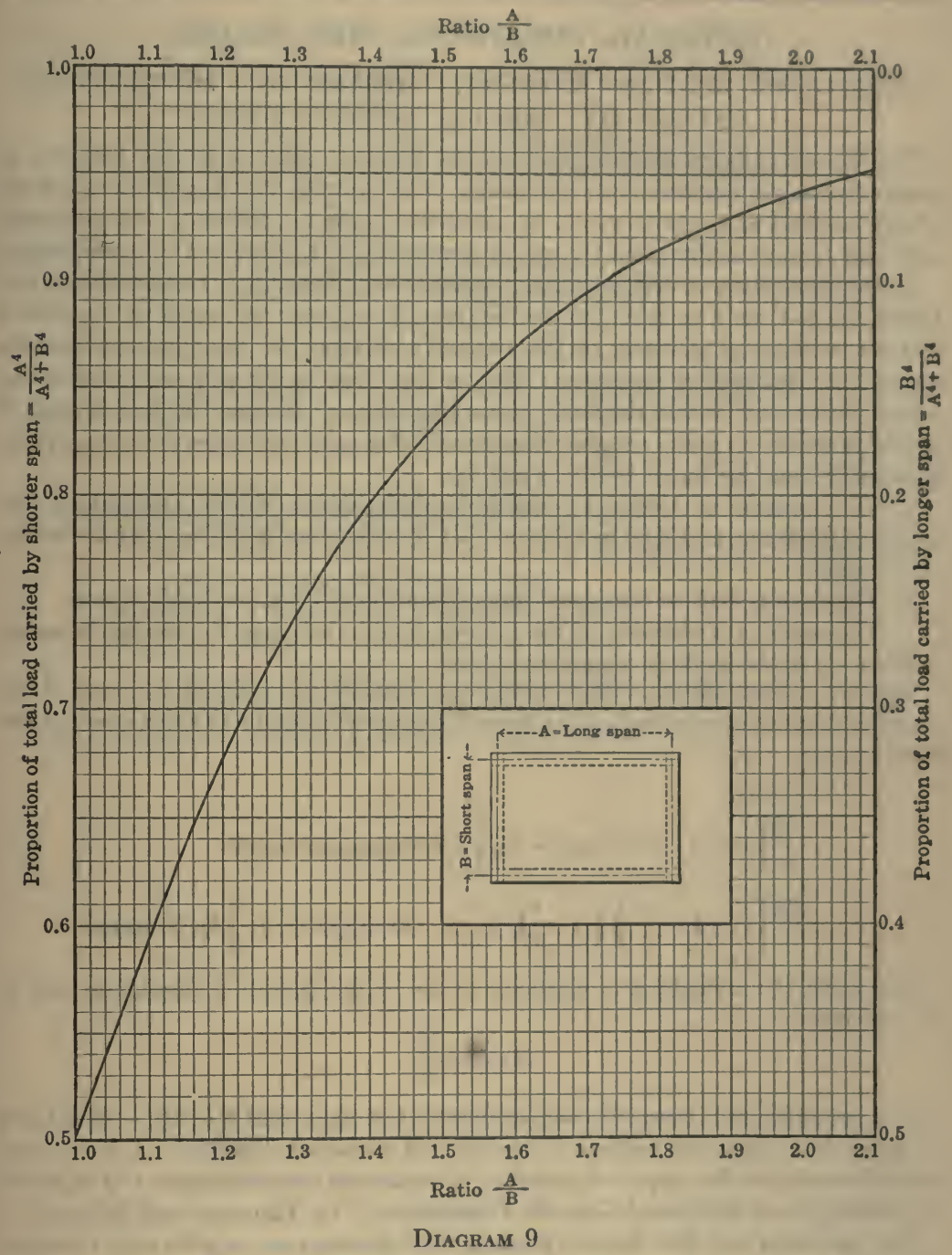

Curve showing distribution of load for rectangular slabs supported at the four edges. 


\section{GENERAL FORMULAS FOR BEAMS}

\section{REACTIONS, BENDING MOMENTS, SHEARS AND DEFLECTIONS CAUSED BY VARIOUS APPLIED LOADS}

The classes of beam loading given on the following pages cover the majority of cases occurring in reinforced concrete design. The formulas may be applied to a beam of any material, although it should be noted that those for deflection and maximum safe load require modification for use in connection with reinforced concrete beams.

In the application of deflection and maximum load formulas to reinforced concrete beams account must be taken of the fact that the moment of inertia of the section and the modulus of elasticity of the material enters into the computations, thereby introducing elements of uncertainty that do not exist in the case of homogeneous beams, at least not within the limits of working stresses. Bearing this fact in mind it will be necessary to make certain assumptions before applying these formulas. These assumptions may be stated briefly as follows:

1. The moment of inertia is considered substantially uniform throughout the length of the beam, and shall be taken as that of the section of the beam at the center of the span.

2. The section shall be considered intact from top of beam to center of steel.

3. The modulus of elasticity of the concrete shall be taken as the average or secant modulus up to the working compressive stress.

For such a beam the moment of inertia of a section is the moment of inertia of the concrete about the neutral axis plus $n$ times the moment of inertia of the steel about the same axis, or

$$
\begin{aligned}
I & =I_{0}+n I_{B} \\
& =\frac{b d^{3}}{3}\left[k^{3}+(1-k)^{3}+3 n p(1-k)_{2}\right] \text { for rectangular beams. } \\
& =\frac{b d^{3}}{3}\left[k^{3}-\left(1-\frac{b^{\prime}}{b}\right)\left(k-\frac{t}{d}\right)^{3}+\frac{b^{\prime}}{b}(1-k)^{3}+3 p n(1-k)^{2}\right] \text { for T-beams }
\end{aligned}
$$

The value of the modulus of elasticity to use in the deflection formula is that of the concrete, or

$$
E=E_{\mathrm{c}}=\frac{E_{\mathrm{8}}}{n}
$$

It is recommenided, from the consideration of test data, that 8 or 10 be used for $n$ to secure fair agreement between computed and measured deflection. A more complete discussion of the subject of deflection of reinforced concrete beams will be found in "Principles of Reinforced Concrete Construction" by Turneaure and Maurer.

The maximum safe load formula given in the following cases applies only to homogeneous beams. To obtain the maximum safe load for a reinforced concrete beam, equate the maximum external moment to the internal resisting moment of the section and solve for WE. For example, in the case of a uniformly loaded rectangular beam supported at the ends, $M_{\mathrm{m}}=\frac{w l^{2}}{8}$ and the resisting moment of the section is $K b d^{2}$, then $W_{m}=\frac{8 K b d^{2}}{12 l^{2}}$ 
Values of $K$ may be obtained from Diagrams 1, 2 and 3 .

Notation. The following notation has been adopted in the formulas:

$P, p=$ Concentrated loads in pounds. unit

$w$ =Superimposed load in pounds per length of beam or slab.

$W=$ Superimposed load supported by beam or slab in pounds.

$l \quad$ =Length of beam in feet.

$L=$ Length of beam in inches.

$R_{1}, R_{2}=$ Reactions at supports of beam, in pounds.

$V_{\mathbf{x}}=$ Total transverse shear in pounds, at distance $x$.

$V_{\mathrm{m}}=$ Total maximum transverse shear in pounds.

$M_{\mathrm{m}}=$ Maximum positive external bending moment in foot-pounds.

$\boldsymbol{M}_{\mathrm{m}}^{\prime}=$ Maximum negative external bending moment in foot-pounds.

$x_{0}=$ Distance in feet to point of zero shear, or to $M_{\mathrm{m}}$.

$w_{m}=$ Maximum safe load in pounds per length of beam or slab for load distribution indicated in each case.

$P_{\mathrm{m}}=$ Maximum safe concentrated load in pounds.

$f=$ Working unit-stress in flexure, in pounds per square inch. (This does not apply to reinforced concrete beams.)

1 = Moment of inertia of cross-section in inches4.

$S=$ Section modulus of cross-section in inches ${ }^{3}$.

(This does not apply to reinforced concrete beams.)

$D=$ Maximum deflection in inches.

$y$ = Distance in fect to point of maximum deflection $D$. 
1. Cantilever Beam. Concentrated load.

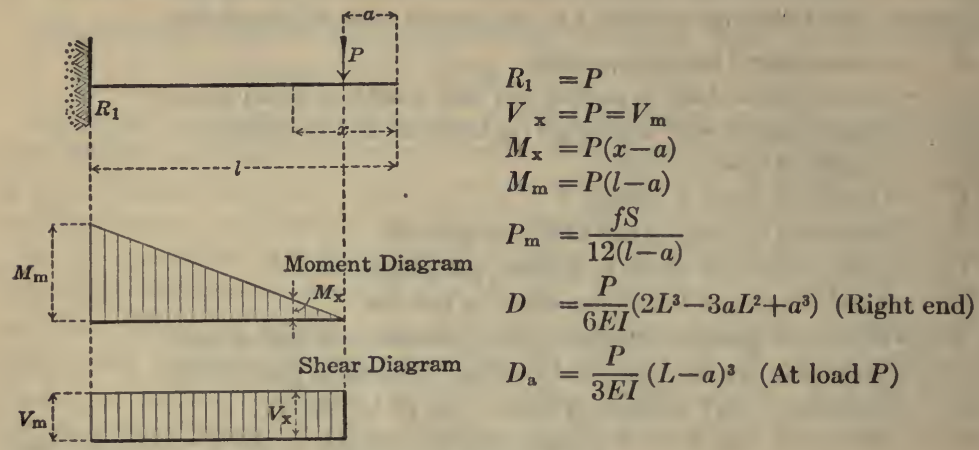

2. Cantilever Beam. Uniformly distributed load.

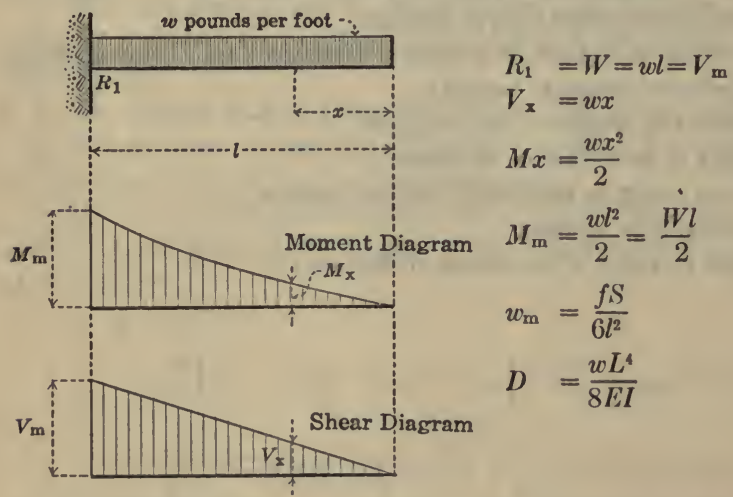

3. Cantilever Beam. Load increasing uniformly to fixed end.

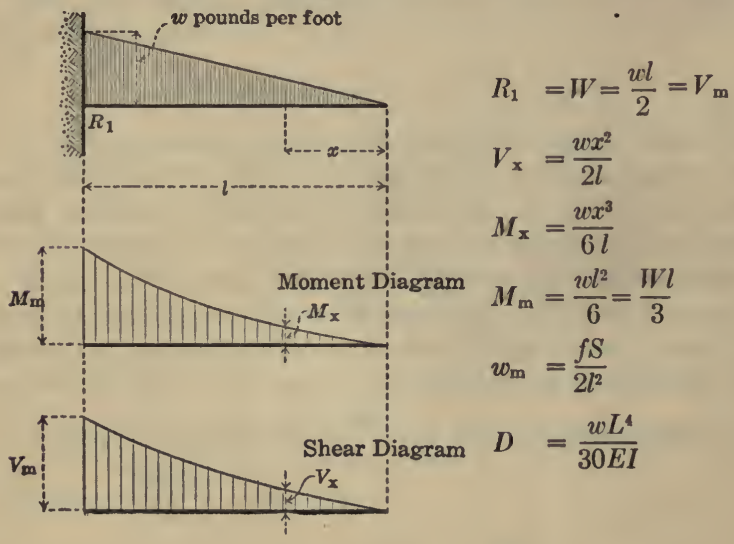


4. Cantilever Beam. Load increasing uniformly to free end.

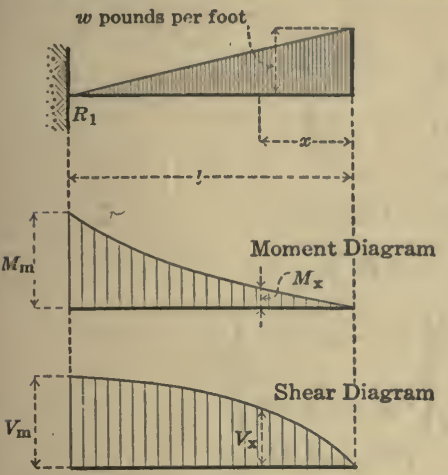

$$
\begin{aligned}
& R_{1}=W=\frac{w l}{2}=V_{\mathrm{m}} \\
& V_{\mathrm{x}}=\frac{w x}{2 l} \frac{w x}{(t-x)} \quad \frac{\omega x}{22}(2 Z-x) \\
& M_{\mathrm{x}}=\frac{w x^{2}}{6 l}(3 l-x) \\
& M_{\mathrm{m}}=\frac{w l^{2}}{3}=\frac{2 W l}{3} \\
& w_{\mathrm{m}}=\frac{f S}{4 l^{2}} \\
& D=\frac{11}{120} \frac{w L^{4}}{E I}
\end{aligned}
$$

5. Beam Supported at Ends. Concentrated load near one end.

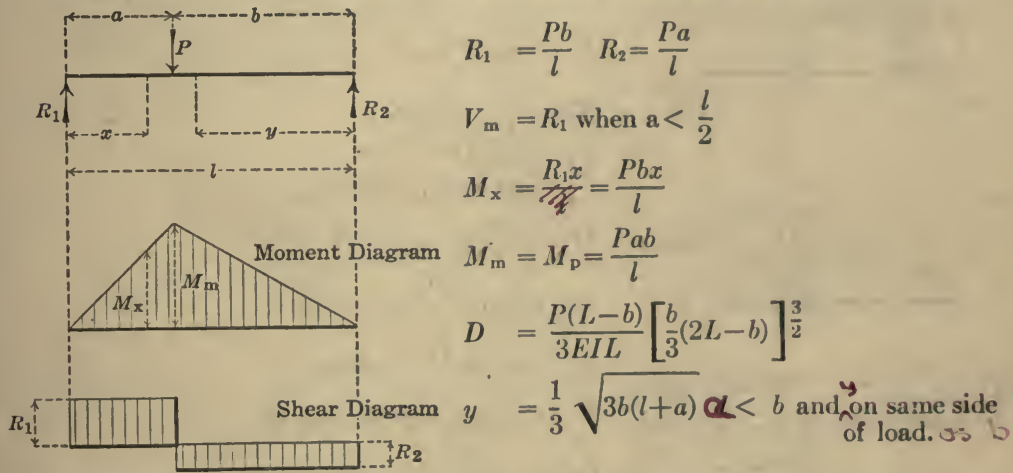

6. Beam Supported at Ends. Concentrated load at center.

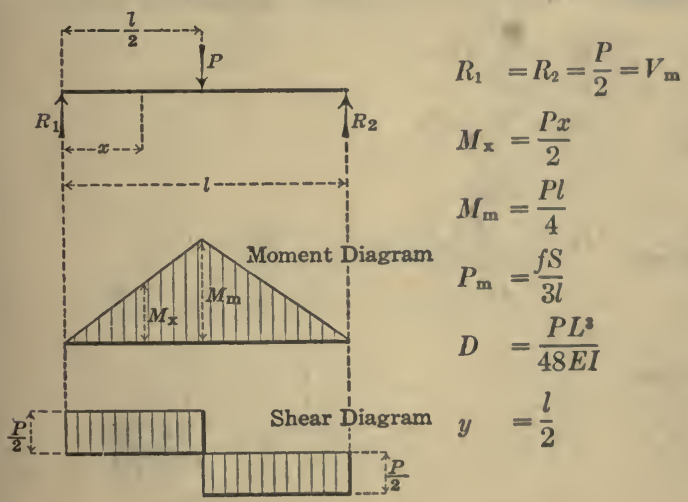


7. Beam Supported at Ends. Two unequal and unsymmetrical concentrated loads

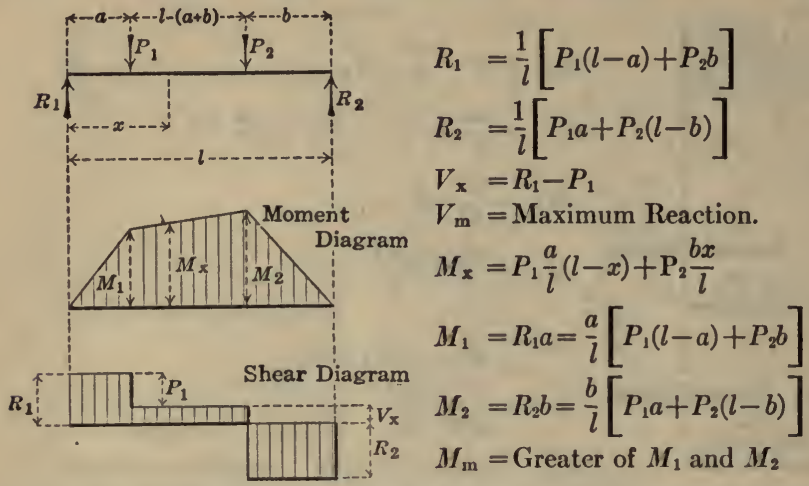

8. Beam Supported at Ends. Two equal unsymmetrical concentrated loads.

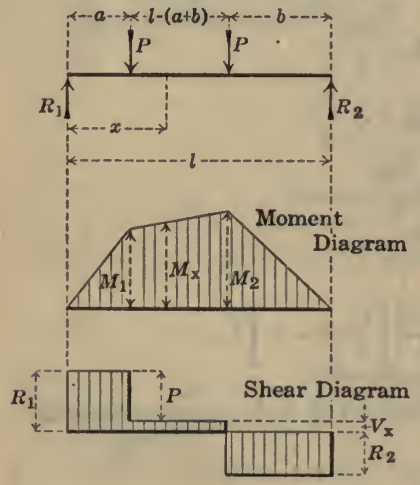

$$
\begin{aligned}
& R_{1}=\frac{P}{l}(l-a+b) \\
& R_{2}=\frac{P}{l}(l-b+a) \\
& V_{\mathrm{x}}=R_{1}-P \\
& V_{\mathrm{m}}=\text { Maximum Reaction } \\
& M_{\mathrm{x}}=\frac{P}{l}(a l-a x+b x) \\
& M_{1}=R_{1} a=\frac{P a}{l}(l-a+b) \\
& M_{2}=R_{2} b=\frac{P b}{l}(l-b+a) \\
& M_{\mathrm{m}}=\mathrm{Greater} \text { of } M_{1} \text { and } M_{2} \\
& P_{\mathrm{m}}=\frac{f l S}{12 b(l-b+a)}(\text { when } b>a)
\end{aligned}
$$

9. Beams Supported at Ends. Two equal symmetrical concentrated loads.

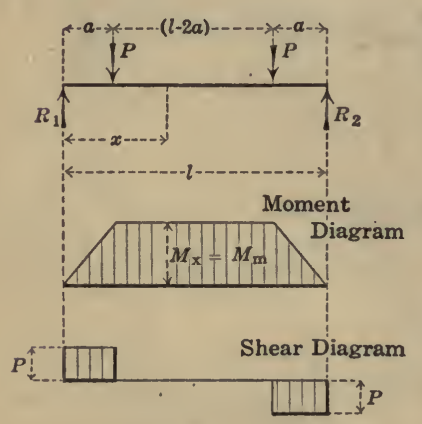

$$
\begin{array}{l|l}
R_{1}= & R_{2}=P=V_{\mathrm{m}} \\
V_{\mathrm{x}}= & \\
M_{\mathrm{x}}= & M_{\mathrm{m}}=P a \\
P_{\mathrm{m}}=\frac{f S}{12 a} & \begin{array}{l}
\text { When } a=\frac{l}{3} \\
D=\frac{P a}{24 E I}\left(3 L^{2}-4 a^{2}\right)
\end{array} \\
& \begin{array}{l}
M_{\mathrm{x}}=M_{\mathrm{m}}=\frac{P l}{3} \\
P_{\mathrm{m}}=\frac{7 f f}{E}=\frac{f \mathrm{~S}}{4 Z} \\
D=\frac{23}{648} \frac{P L}{E I}
\end{array}
\end{array}
$$


10. Beam Supported at Ends. Three unequal unsymmetrical concentrated loads.

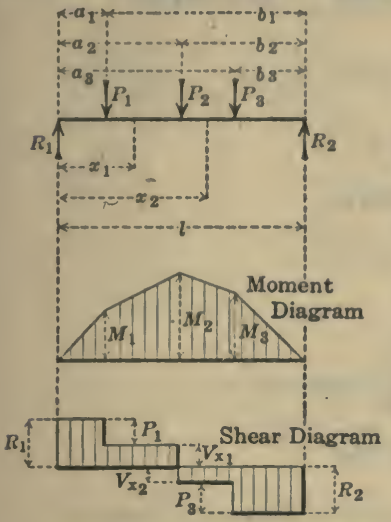

$$
\begin{aligned}
R_{1} & =\frac{\Sigma P b}{l} \quad R_{2}=\frac{\Sigma P a}{l} \\
V_{x_{1}} & =R_{1}-P_{2} \quad V_{x_{2}}=R_{1}-P_{1}-P_{2} \\
V_{m} & =\text { Greater Reaction } \\
M_{1} & =\frac{a_{1}}{l} \Sigma P b \quad M_{2}=\frac{a_{2}}{l} \Sigma P b-P_{1}\left(a_{2}-a_{1}\right) \\
M_{3} & =\frac{b_{2}}{l} \Sigma P a \\
M_{m} & =\text { Greatest of } M_{1} M_{2} \text { and } M_{2} \\
& =M_{2} \text { if } P_{1}>R_{1} \\
& =M_{2} \text { if } P_{1}<R_{1} \text { when }\left(I_{1}+P_{2}\right)>R_{1} \\
& =M_{2} \text { if } P_{2}>R_{2}
\end{aligned}
$$

11. Beam Supported at Ends. Three equal symmetrical concentrated loads.

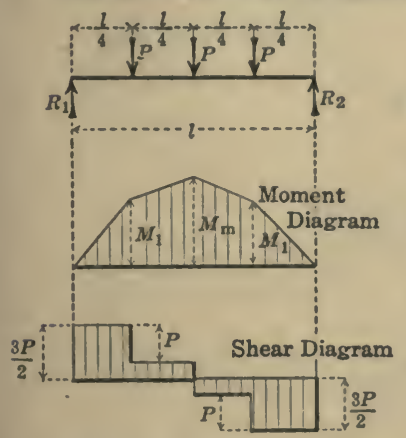

$$
\begin{aligned}
& R_{1}=R_{2}=\frac{3 P}{2}=V_{\mathrm{m}} \\
& M_{1} \stackrel{\doteq}{=} \frac{3}{8} P l \\
& M_{\mathrm{m}}=\frac{P l}{2} \\
& P_{\mathrm{m}}=\frac{f S}{6 l} \\
& D=\frac{19}{384} \frac{P L^{3}}{E I} \text { at center }
\end{aligned}
$$

12. Beam Supported at Ends. Uniform load partially distributed.

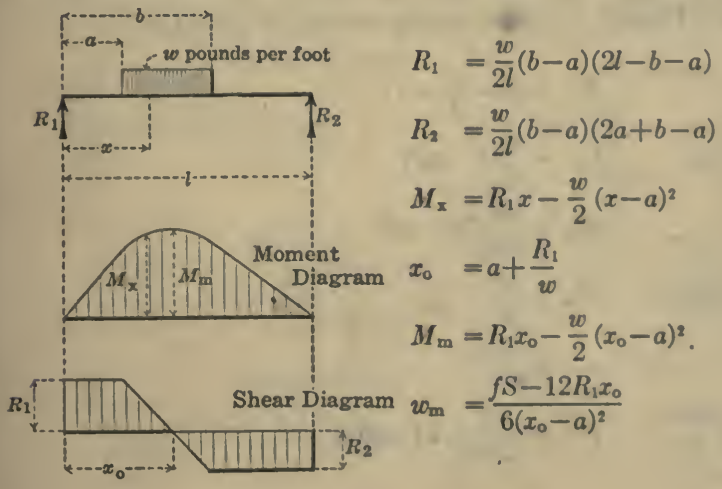


13. Beam Supported at Ends. Uniform load partially discontinuous.

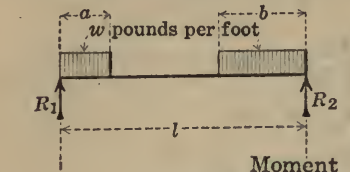

$$
\begin{aligned}
& R_{1}=\frac{w}{2 l}\left(2 a l-a^{2}+b^{2}\right) \\
& R_{2}=\frac{w}{2 l}\left(2 b l-b^{2}+a^{2}\right)
\end{aligned}
$$

$V_{\mathrm{m}}=$ Greater Reaction

$M_{\mathrm{a}}=R_{1} a-\frac{w a^{2}}{2}$

$M_{\mathrm{L}}=R_{2} b-\frac{w b^{2}}{2}$

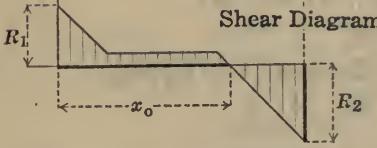

$x_{0}=l-a-b+\frac{R_{1}}{c_{v}}($ when $b>a)$

$M_{\mathrm{m}}=\frac{R_{2}^{2}}{2 w}($ when $b>a)$

14. Beam Supported at Ends. Uniformly distributed load.

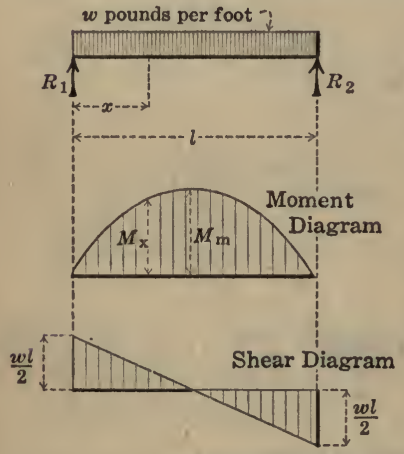

$$
\begin{aligned}
& R_{1}=R_{2}=\frac{w l}{2}=V_{\mathrm{m}} \\
& M_{\mathrm{x}}=\frac{w x}{2}(l-x) \\
& M_{\mathrm{m}}=\frac{w l^{2}}{8}=\frac{W l}{8} \\
& w_{\mathrm{m}}=\frac{2 f S}{3 l^{2}} \\
& D=\frac{5}{384} \frac{w L^{4}}{E I}
\end{aligned}
$$

15. Beam Supported at Ends. Load increasing uniformly to center.

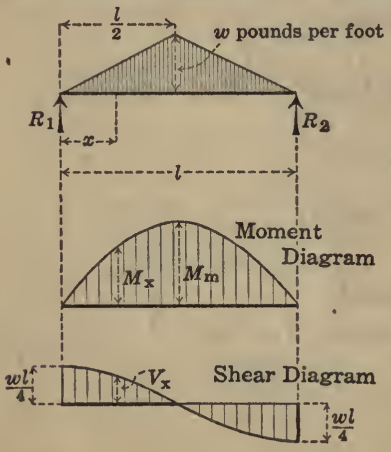

$$
\begin{aligned}
& R_{1}=R_{2}=\frac{w l}{4}=V_{\mathrm{m}} \\
& V_{\mathrm{x}}=\frac{w}{4 l}\left(l^{2}-4 x^{2}\right) \\
& M_{\mathrm{x}}=\frac{w x}{12 l}\left(3 l^{2}-4 x^{2}\right) \\
& M_{\mathrm{m}}=\frac{w l^{2}}{12} \\
& w_{\mathrm{m}}=\frac{f S}{l^{2}} \\
& D=\frac{w L^{4}}{120 E I} \text { at center }
\end{aligned}
$$


16. Beam Supported at Ends. Load decreasing uniformly to center.

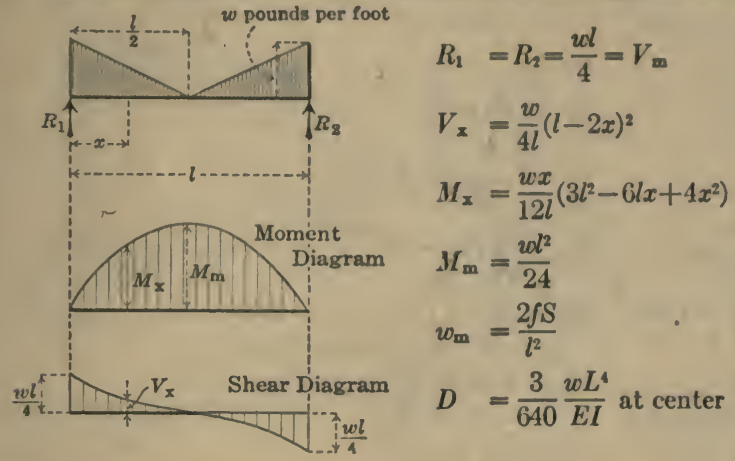

17. Beam Supported at Ends. Load increasing uniformly to one end.

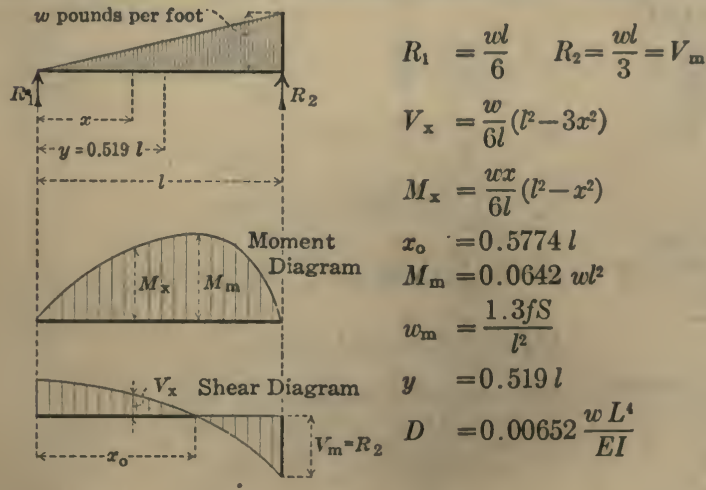

18. Beam Fixed at Ends. Load increasing uniformly to center.

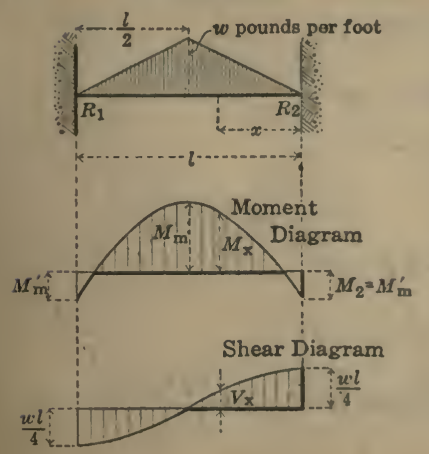

$$
\begin{aligned}
& R_{1}=R_{2}=\frac{w l}{4} \\
& V_{\mathrm{x}}=\frac{w l}{4}-\frac{w x^{2}}{l} \\
& M_{\mathrm{x}}=M I_{2}+\frac{w l x}{4}-\frac{w x^{2}}{3 l} \\
& M_{2}=M_{\mathrm{m}}^{\prime}=-\frac{5 w l^{2}}{96} \\
& M_{\mathrm{m}}=\frac{g w l^{2}}{32}
\end{aligned}
$$


19. Beam Fixed at Ends. Load decreasing uniformly to center.

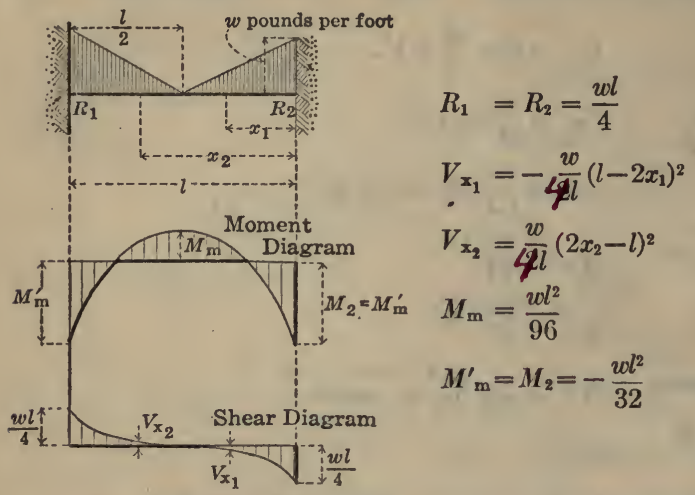

20. Beam Supported at Ends. Uniformly distributed load plus load increasing uniformly to one end.

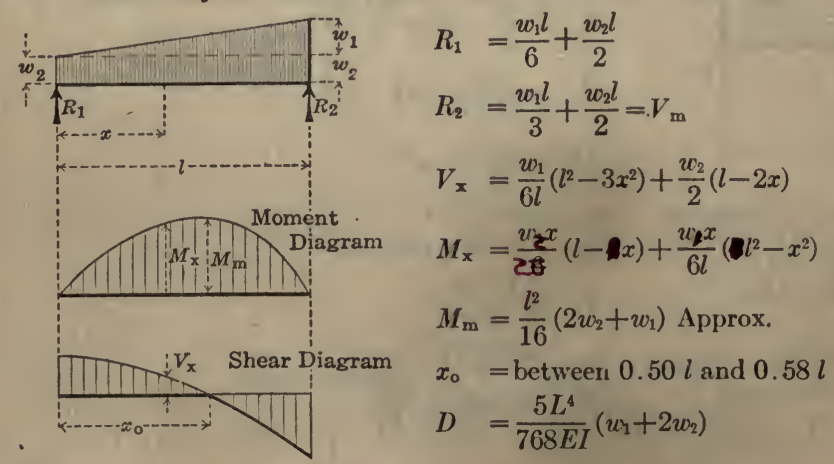

21. Beam Fixed at One End, Supported at Other. Concentrated load.

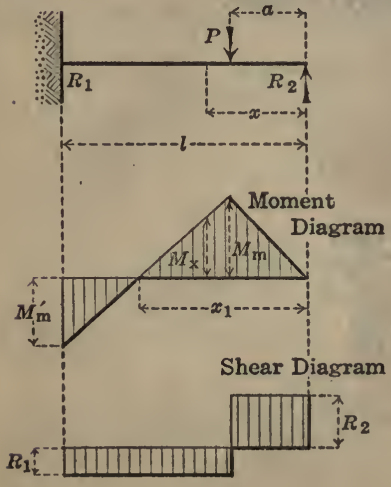

$$
\begin{aligned}
& R_{1}=\frac{P}{2 l^{3}}\left(3 a l^{2}-a^{3}\right) \\
& R_{2}=\frac{P}{2 l^{3}}\left(2 l^{3}-3 a l^{2}+a^{3}\right) \\
& M_{\mathrm{x}}=R_{2} x-P(x-a) \\
& x_{1}=\frac{P a}{P-R_{2}} \\
& M_{\mathrm{m}}=\frac{P a}{2 l^{3}}\left(2 l^{3}-3 a l^{2}+a^{3}\right) \\
& M_{\mathrm{m}}^{\prime}=\frac{P}{2 l^{2}}\left(a l^{2}-a^{3}\right) \\
& \text { when } y=a=0.414 l, D=0.0098 \frac{P L^{3}}{E I}
\end{aligned}
$$


22. Beam Fixed at One End, Supported at Other. Load increasing uniformly

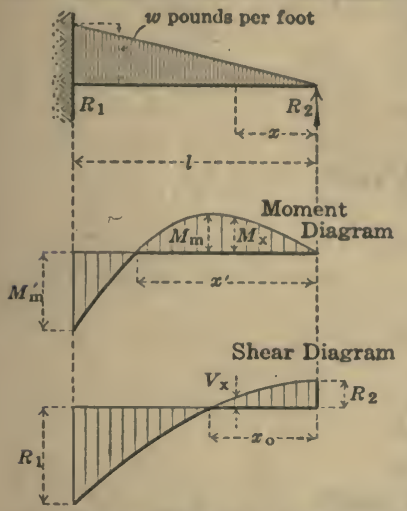
to fixed end.

$$
\begin{aligned}
& R_{1}=\frac{4 w l}{10} \\
& R_{2}=\frac{w l}{10} \\
& V_{\mathrm{x}}=\frac{w l}{10}-\frac{w x^{2}}{2 l} \\
& M_{\mathrm{x}}=\frac{w l x}{10}-\frac{w x^{3}}{6 l} \\
& x_{\mathrm{o}}=0.4474 l \\
& M_{\mathrm{m}}=0.031 w l^{2} \\
& M_{\mathrm{m}}^{\prime}=-\frac{w l^{2}}{15} \\
& x^{\prime}=0.775 l
\end{aligned}
$$

23. Beam Fixed at One End, Supported at Other. Uniformly distributed load.

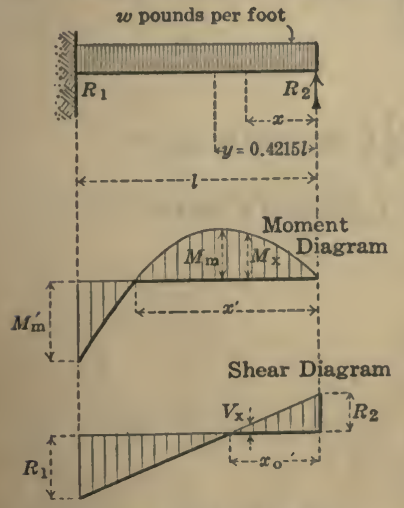

$$
\begin{array}{ll}
R_{1}=\frac{5}{8} w l \\
R_{2}=\frac{3}{8} w l \\
V_{\mathrm{x}}=\frac{3}{8} w l-w x \\
M_{\mathrm{x}}=\frac{w x}{8}(3 l-4 x) \\
M_{\mathrm{m}}=\frac{9}{128} w l^{2} & x^{\prime}=\frac{3}{4} l \\
M_{\mathrm{m}}^{\prime}=-\frac{1}{8} w l^{2} & y=0.4215 l \\
x_{0}=\frac{3}{8} l & D=\frac{0.0054 w L^{6}}{E I}
\end{array}
$$

24. Beam Fixed at Ends. Load increasing uniformly to one end.

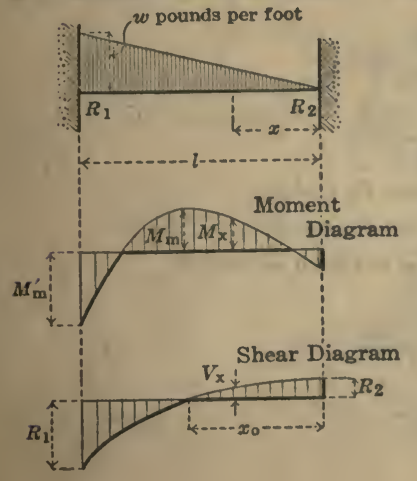

$$
\begin{aligned}
& R_{1}=\frac{7 w l}{20} \\
& R_{2}=\frac{3 w l}{20} \\
& V_{\mathrm{x}}=\frac{3 w l}{20}-\frac{w x^{2}}{2 l} \\
& M_{\mathrm{x}}=\frac{3 l^{2}}{30}+\frac{3 x d x}{20}=-\frac{m x^{3}}{6 t}=\frac{3 \omega 2 x}{20}-\frac{\omega l^{2}}{30}-\frac{\omega x}{62} \\
& M_{1}=M_{\mathrm{m}}^{\prime}=-\frac{u l^{2}}{20} \\
& x_{\mathrm{o}}=0.548 l \\
& M_{\mathrm{m}}=0.0215 w l^{2}
\end{aligned}
$$


25. Beam Fixed at Ends. Uniformly distributed load.

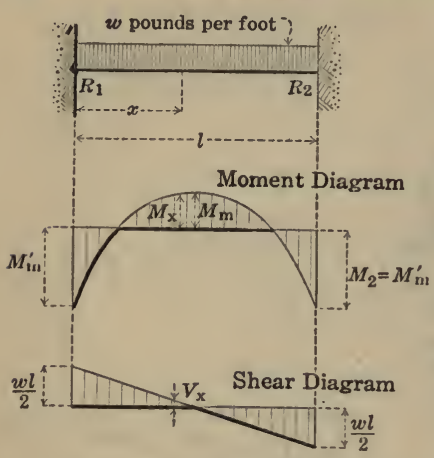

$$
\begin{aligned}
& R_{1}=R_{2}=\frac{1}{2} w l \\
& V_{\mathrm{x}}=\frac{1}{2} w l-w x \\
& M_{\mathrm{x}}=\frac{w}{2}\left(-\frac{l^{2}}{6}+l x-x^{2}\right) \\
& M_{\mathrm{m}}=\frac{1}{24} w l^{2} \\
& x_{\mathrm{o}}=\frac{1}{2} l \\
& M_{\mathrm{m}}^{\prime}=\frac{1}{12} w l^{2} \\
& D=\frac{1}{384} \frac{w L^{4}}{E I}
\end{aligned}
$$

26. Beam Fixed at Ends. Concentrated load at center.

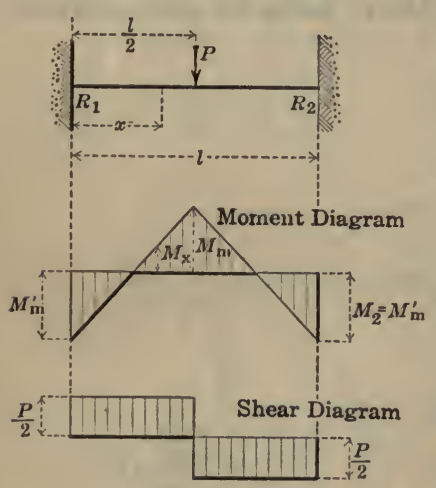

$$
\begin{aligned}
R_{1} & =R_{2}=\frac{1}{2} P \\
V_{\mathrm{x}} & =\frac{1}{2} P \\
M_{\mathrm{x}} & =\frac{1}{2} P\left(x-\frac{1}{4} l\right) \text { between } R_{1} \text { and } P \\
& =\frac{1}{2} P\left(\frac{3}{4} l-x\right) \text { between } P \text { and } R_{2} \\
M_{\mathrm{m}} & =\frac{1}{8} P l \\
x_{0} & =\frac{1}{2} l \\
M_{\mathrm{m}}^{\prime} & =\frac{1}{8} P l \\
D & =\frac{1}{192} \frac{P l}{E I}
\end{aligned}
$$

27. Beam Fixed at Ends. Concentrated load.

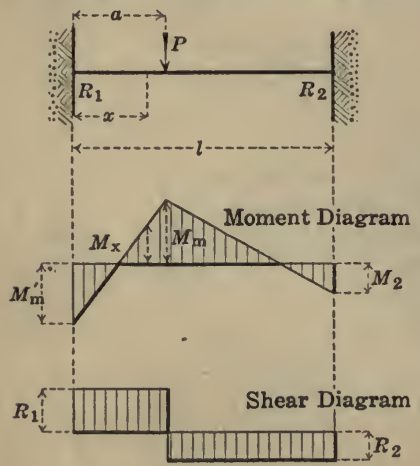

$$
\begin{aligned}
& R_{1}=P \frac{(l-a)^{2}(2 a+l)}{l^{3}} \\
& R_{2}=P \frac{a^{2}(3 l-2 a)}{l^{3}} \\
& V_{\mathrm{x}}=R_{1} \text { between } R_{1} \text { and } P \\
& V_{\mathrm{x}_{1}}=R_{2} \text { between } P \text { and } R_{2} \\
& M_{\mathrm{x}}=R_{1} x+M^{\prime}{ }_{\mathrm{m}} \text { between } R_{1} \text { and } P \\
& M_{\mathrm{m}}=R_{1} a+M_{\mathrm{m}}^{\prime} \\
& M_{\mathrm{m}}^{\prime}=-P \frac{a(l-a)^{2}}{l^{2}} \\
& M_{2}=-P \frac{a^{2}(l-a)}{l^{2}}
\end{aligned}
$$


Concentrated Moving Loads. Position for maximum moment and shear.

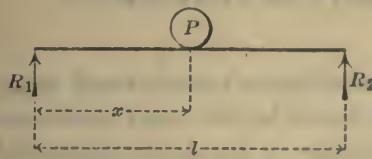

Max. $V$ when $x=0$

Max. $M$ when $x=\frac{l}{2}$

$V_{\mathrm{m}}=R_{1}=P$

$$
\begin{aligned}
R_{\mathrm{l}} & =\frac{P}{2} \\
M_{\mathrm{m}} & =\frac{P l}{4}(\text { at } P \text { ) }
\end{aligned}
$$

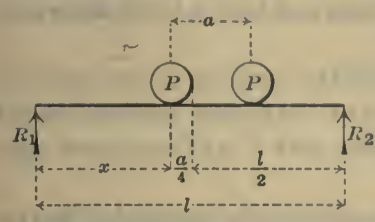

Max. $V$ when $x=0$

Max. M when $x=\frac{1}{4}(2 l-a) \quad R_{1}=\frac{P}{2 l}(2 l-a)$

$V_{\mathrm{m}}=R_{1}=\frac{P}{l}(2 l-a)$ $\left.M_{\mathrm{m}}=\frac{B}{2 l}(2)+2\right) \frac{P}{8 L}(2 ?-i)$

When $a$ exceeds $0.586 l$, use case of one load only, for $\boldsymbol{M}_{\mathrm{m}}$.

Max. $V$ when $x=a$

\begin{tabular}{l|l} 
Max. $M$ when $x=\frac{l}{2}$ & $R_{1}=\frac{3}{2} P$
\end{tabular}

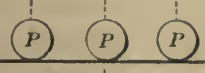

$V_{\mathrm{m}}=R_{1}=\frac{3 P}{l}(l-a) \quad M_{\mathrm{m}}=\frac{P}{4}(3 l-4 a)$

When $a$ exceeds $0.45 l$, use case of two loads only, for $M_{\mathrm{m}}$.

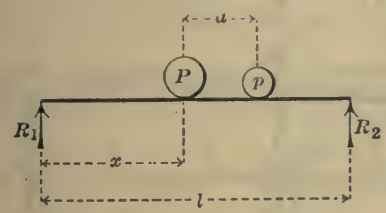

Max. $V$ when $x=0$

$\operatorname{Max} . M$ when $x=\frac{1}{2}\left[l-\frac{p a}{\Sigma P}\right] \mid M_{\mathrm{m}}=\frac{x^{2}}{l} \Sigma P$

Maximum moment may occur under one load only.

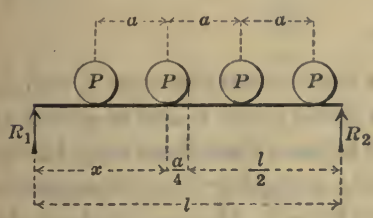

Max. $V$ when $x=a$

Max. $M$ when $x=\frac{1}{4}(2 l-a) \mid R_{1}=\frac{P}{l}(2 l-a)$

$V_{\mathrm{m}}=R_{1}=\frac{4 \mathrm{P}}{l}\left(2-\frac{3 a}{2}\right) M_{\mathrm{m}}=\frac{P}{4 l}\left(4 l^{2}-8 a l+a^{2}\right)$

When $a$ exceeds $0.268 l$, use case of three loads only, for $\boldsymbol{M}_{\mathrm{m}}$.

Max. $V$ when $x=a$
Max. $M$ when $x=\frac{1}{2 \sum\langle(p h+R a)}=\frac{7}{2}+\frac{1}{2 \sum P}(p b-P a$

$V_{\mathrm{m}}=R_{\mathrm{l}}=P+[P(l-a)+p(l-a-b)] \frac{1}{l}$

$M_{\mathrm{m}}=\frac{x^{2} \Sigma P}{l}-P a \quad$ Maximum moment may occur for two loads only

Max. $V$ when $x=b$

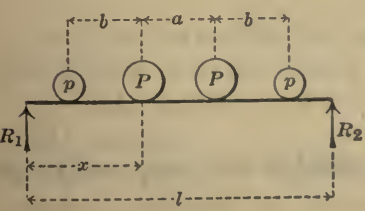

Max. $M$ when $x=\frac{1}{4}(2 l-a)=\begin{aligned} & V_{\mathrm{m}}=R_{1}=\frac{\Sigma P}{2 l}(2 l-2 b-a) \\ & M_{\mathrm{m}}=\frac{\Sigma P}{16 l}(2 l-a)^{2}-p b\end{aligned}$

Maximum moment may occur for preceding case. 


\section{MOMENTS AND SHEARS FOR CONTINUOUS BEAMS}

The moment factors commonly specified for continuous beams assume equal spans and uniform loads. While these factors are within safe limits for the usual conditions met with in building design, cases arise where it is advisable to investigate the actual moments and shears produced, through inequality of span and load, by the theorem of three moments.

This theorem may be employed in problems involving either uniform or concentrated loads or combinations of the two, but a full discussion of the theory involved would be out of place in a book of this character and, therefore, only a brief statement covering its application will be given.

In the formulas and diagrams which follow it is assumed that the moment of inertia is constant throughout the length of the beam and that the supports retain their same relative position after the beam is loaded as before.

\section{UNIFORM LOAD}

For uniform load the theorem is expressed by the formula,

$$
M_{1} l_{1}+2 M_{2}\left(l_{1}+l_{2}\right)+M_{3} l_{2}=-\frac{w_{1} l_{1}^{3}}{4}-\frac{w_{2} l_{2}^{3}}{4}
$$

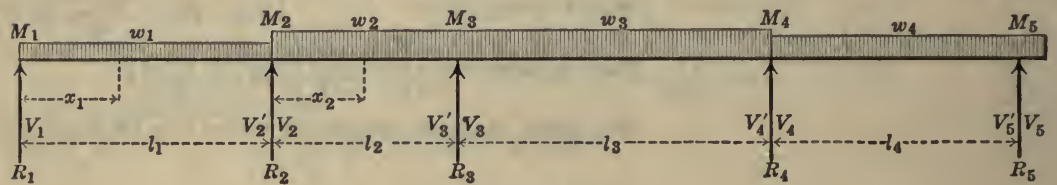

Fig. 4

The equation as written applies to the moments at the supports in span $l_{1}$ and $l_{2}$; by increasing all the subscripts by one it will apply to spans $l_{2}$ and $l_{3}$ and so on for as many spans as there are in the structure. It will thus be seen that there may be obtained as many equations as there are unknown moments, assuming that the moments at the first and last support are zero or their values are known. Having obtained the moments at the supports, the shears and moments at any other section of the beam may be found by the following equations.

Consider for example, spans $l_{1}$ and $l_{2}$ in Fig. 4 .

$$
\begin{aligned}
& V_{1}=\frac{M_{2}-M_{1}}{l_{1}}+\frac{w_{1} l_{1}}{2} \\
& V_{2}^{\prime}=V_{1}-w_{1} l_{1} \\
& V_{2}=\frac{M_{3}-M_{2}}{l_{2}}+\frac{w_{2} l_{2}}{2} \\
& V_{3}^{\prime}=V_{2}-w_{2} l_{3}
\end{aligned}
$$

The reaction at any support $\left(R_{1}, R_{2}\right.$, etc.) will be equal to the shear on its right plus that on its left with the sign reversed. 
The distance to the point of zero shear, provided the shear changes sign in the span, is

$$
\begin{aligned}
& x_{1}=\frac{V_{1}}{w_{1}} \text { for } \operatorname{span} l_{1} \\
& x_{2}=\frac{V_{2}}{w_{3}} \text { for } \operatorname{span} l_{2}
\end{aligned}
$$

The bending moment at this point,

$$
\begin{aligned}
& M=M_{1}+V_{1} x_{1}-\frac{w_{1} x_{1}^{2}}{2} \text { for span } l_{1} \\
& M=M_{2}+V_{2} x_{2}-\frac{w_{2} x_{2}^{2}}{2} \text { for span } l_{2}
\end{aligned}
$$

If the sign of this moment is plus, it is the maximum positive moment. If the sign is minus, it is the minimum negative moment and indicates that no positive moment exists at any point of the span.

By changing the subscripts as previously mentioned the formulas may be applied to any span.

\section{CONCENTRATED LOADS}

For concentrated loads the theorem is expressed by the formula,

$$
M_{1} l_{1}+2 M_{2}\left(l_{1}+l_{2}\right)+M_{3} l_{2}=-\Sigma P_{1} l_{1}^{2}\left(k_{1}-k_{1}^{3}\right)-\Sigma P_{3} l_{2}^{2}\left(2 k_{2}-3 k_{2}^{2}+k_{2}^{3}\right)
$$

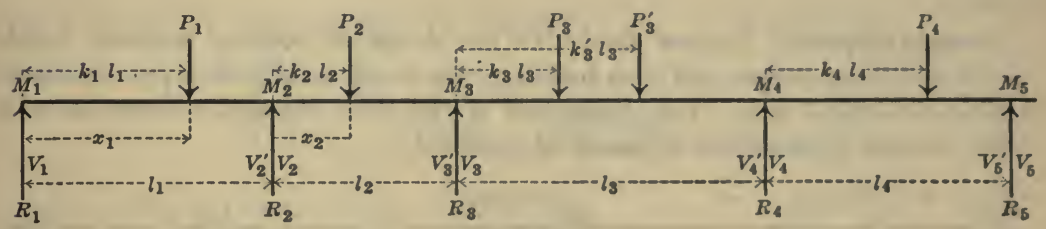

Fig. 5

Applied to spans $l_{3}$ and $l_{4}$, Fig. 5 , the formula would be written as follows:

$M_{3} l_{3}+2 M_{4}\left(l_{3}+l_{4}\right)+M_{5} l_{4}=-\left[P_{3} l_{3}^{2}\left(k_{3}-k_{3}^{3}\right)+P^{\prime} l_{3}^{2}\left(k_{3}^{\prime}-k_{3}^{\prime} 3^{3}\right)\right]-\left[P_{4} l_{4}^{2}\left(2 k_{4}-3 k_{4}^{2}+k_{4}^{3}\right)\right]$

The shears will then be:

$$
\begin{aligned}
& V_{3}=\frac{M_{4}-M_{3}}{l_{3}}+P_{3}\left(1-k_{3}\right)+P_{3}^{\prime}\left(1-k_{3}^{\prime}\right) \\
& V_{4}^{\prime}=V_{3}-\left(P_{3}+P_{3}^{\prime}\right) \\
& V_{4}=\frac{M_{5}-M_{4}}{l_{4}}+P_{4}\left(1-k_{4}\right) \\
& V_{5}^{\prime}=V_{4}-P_{4}
\end{aligned}
$$

Knowing the moments at the supports, the shears and moments at any section may be obtained as in the case of continuous beams with uniformly distributed loads.

Careful attention must be paid to the use of the proper algebraic signs in the foregoing equations.

Equal Spans. Uniform load over all spans: Diagram 10, page 45, gives moment coefficients of $w l^{2}$ at eritical sections of continuous beams of from two to 
seven spans and Diagram 11 gives shear coefficients of $w l$ at supports for the same case.

Example. For a beam of three spans the negative moment at the first interior support is $0.10 w l^{2}$. The shear at the end of the middle span is $\frac{5}{10} w l$ and at the inner support of the end span it is $\frac{6}{10} w l$.

Equal Concentrated Loads on All Spans: Diagram 12, page 46, shows three cases of loading. (1) Loads at middle points. (2) Loads at third points. (3) Loads at middle and quarter points. The full irregular line is the moment curve and the broken line represents the shear line for each case of loading. The ordinates to the moment line are coefficients of $P l$ and the ordinates to the shear line are coefficients of $P$. The numerical coefficients are given at critical sections.

Example: At the central span of a five-span girder loaded at the third points, the negative moment at the adjacent support is $0.211 \mathrm{Pl}$; the positive moment at either of the loads is $0.122 \mathrm{Pl}$ and the reaction at the adjacent support is $1.93 \mathrm{P}$.

The moments and shears of any uniform load should be combined with those of the concentrated loads on the girder.

Partial Uniform Load: Diagrams 13, 14, 15 and 16, pages 47 and 48, for two and three span beams, give moment and shear coefficients that are maximum for the indicated positions of the uniform load; the ends of the beams being either free or fixed.

Unequal Spans. Uniform Load: In the design of schools, hospitals, hotels public buildings, garages and shop buildings, the layout usually involves continuous beams of unequal span. The application of the three moment theorem in such cases can best be illustrated by means of problems.

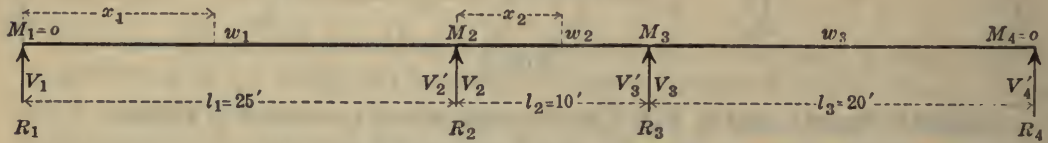

Live load per foot of $\operatorname{span}=800 \mathrm{lbs}$.

Dead " " " " " $=1000 "$

Frg. 6

Problem I. Assume a beam of three unequal spans as shown in Fig. 6, carrying a live load of 800 pounds per linear foot and a dead load of 1,000 pounds per linear foot. Find the critical moments, shears and reactions, the ends being assumed simply supported.

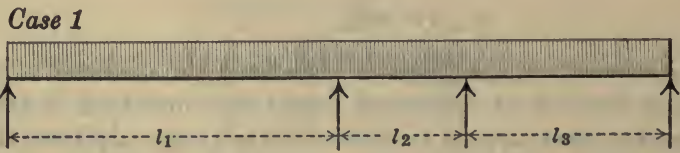

Live Load on All Spans. The ends being simply supported $M_{1}=M_{4}=0$.

The moments $M_{2}$ and $M_{3}$ at the intermediate supports can now be found. 
From page $38, M_{1} l_{1}+2 M_{2}\left(l_{1}+l_{2}\right)+M_{3} l_{2}=-\frac{w_{1} l_{1}^{3}}{4}-\frac{w_{2} l_{2}^{2}}{4}$

$$
M_{2} l_{2}+2 M_{3}\left(l_{2}+l_{3}\right)+M_{4} l_{3}=-\frac{w_{2} l_{2}{ }^{3}}{4}-\frac{w_{3} l_{2}{ }^{3}}{4}
$$

Substituting numerical values in these two equations:

$$
\begin{aligned}
& -70 M_{2}+10 M_{3}=-\frac{(1,800)(25)^{3}}{4}-\frac{(1,800)(10)^{3}}{4}=-7,481,250 \ldots \\
& 10 M_{2}+60 M_{3}=-\frac{(1,800)(10)^{3}}{4}-\frac{(1,800)(20)^{3}}{4}=-4,050,000 \ldots
\end{aligned}
$$

Multiplying equation (1) by 6 and subtracting equation (2)

$$
410 M_{2}=-40,837,500
$$

$M_{2}=-99,604 \mathrm{ft}$. lb. and we find $M_{3}=-50,899 \mathrm{ft}$. lb.

Substituting in Eq. (2) $M_{3}=-50,899 \mathrm{ft}$. lb.

From page $38, V_{1}=\frac{M_{2}-M_{1}}{l_{1}}+\frac{w_{1} l_{1}}{2}$

$$
=\frac{(-99,604)}{25}+\frac{(1,800)(25)}{2}=18,516 \mathrm{lb} \text {. }
$$

$$
\begin{aligned}
& V_{2}^{\prime}=V_{1}-w_{1} l_{1}=18,516-(1,800)(25) \\
& =-26,484 \mathrm{lb} \text {. } \\
& V_{2}=\frac{M_{3}-M_{2}}{l_{2}}+\frac{w_{2} l_{2}}{2} \\
& =\frac{(-50,899)-(-99,604)}{10}+\frac{(1,800)(10)}{2}=13,870 \mathrm{lb} . \\
& V_{8}^{\prime}=V_{2}-w_{2} l_{2}=13,870-(1,800)(10) \quad=-4,130 \mathrm{lb} \\
& V_{3}=\frac{M_{4}-M_{8}}{l_{3}}+\frac{w_{3} l_{3}}{2} \\
& =\frac{-(-50,899)}{20}+\frac{(1,800)(20)}{2} \quad=20,545 \mathrm{lb} . \\
& V_{4}^{\prime}=V_{3}-w_{3} l_{3}=20,545-(1,800)(20) \quad=-15,455 \mathrm{lb} \text {. } \\
& R_{1}=V_{1}=18,516 \text { lbs. } \\
& R_{2}=V_{2}+V_{2}^{\prime}=13,870+26,484=40,354 \mathrm{lb} \text {. } \\
& R_{3}=V_{s}+V^{\prime}=20,545+4,130=24,675 \mathrm{lb} \text {. } \\
& R_{4}=V^{\prime}{ }_{4}=15,455 \mathrm{lbs} \text {. }
\end{aligned}
$$

Distance from left support to point of zero shear,

$$
\begin{aligned}
& x_{1}=\frac{V_{1}}{w_{1}}=\frac{18,516}{1,800}=10.29 \mathrm{ft} . \text { for span } l_{1} \\
& x_{2}=\frac{V_{2}}{w_{2}}=\frac{13,870}{1,800}=7.71 \mathrm{ft} . \text { for span } l_{2} \\
& x_{3}=\frac{V_{3}}{w_{3}}=\frac{20,545}{1,800}=11.41 \mathrm{ft} . \text { for span } l_{3}
\end{aligned}
$$


Moment at point $x_{1}, M=M_{1}+V_{1} x_{1}-\frac{w_{1} x_{1}^{2}}{2}$

$$
=(18,516)(10.29)-\frac{(1,800)(10.29)^{2}}{2}=+95,243 \mathrm{ft} . \mathrm{lb} \text {. }
$$

This is the maximum positive moment in span $l_{1}$ for this condition of loading. Moment at point $x_{2}$,

$$
\begin{aligned}
M & =M_{2}+V_{2} x_{2}-\frac{w_{2} x_{2}{ }^{2}}{2} \\
& =(-99,604)+(13,870)(7.71)-\frac{(1,800)(7.71)^{2}}{2}=-46,166 \mathrm{ft} . \mathrm{lb} .
\end{aligned}
$$

This is the minimum negative moment in span $l_{2}$ and indicates that no positive moment exists in the span for this condition of loading, a point that is worthy of notice, as quite commonly this span is designed for positive moment only. Moment at point $x_{3}$,

$$
\begin{aligned}
M & =M_{3}+V_{3} x_{3}-\frac{w_{3} x_{3}^{2}}{2} \\
& =(-50,899)+(20,545)(11.41)-\frac{(1,800)(11.41)^{2}}{2}=+66,350 \mathrm{ft} . \mathrm{lb} .
\end{aligned}
$$

If the beam is subjected to partial loading larger moments, shears and reactions may be obtained than in Case 1. The maximum values for this problem are given in the following cases when the live load is placed as shown.

Case 2

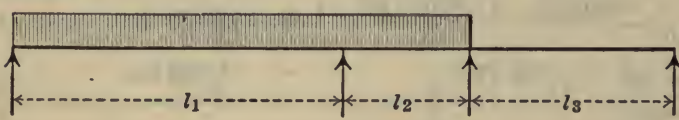

Case 3

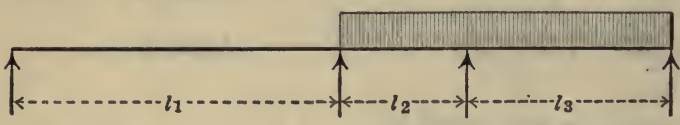

Max. $M_{3}=-58,521 \mathrm{ft}$. lb. Max. $V_{3}^{\prime}=-9,465 \mathrm{lb}$. Max. $V_{3}=+20,926 \mathrm{lb}$. Max. $R_{3}=+30,391 \mathrm{lb}$.
Max. $M_{2}=-103,506 \mathrm{ft}$. $\mathrm{lb}$. Max. $V_{2}^{\prime}=-26,640 \mathrm{lb}$. Max. $V_{2}=+16,992 \mathrm{lb}$. Max. $R_{2}=+43,632 \mathrm{lb}$.
Case \&

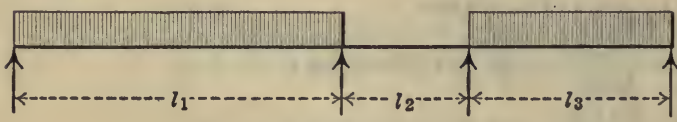

Max. positive moment in $\operatorname{span} l_{1}=+97,300 \mathrm{ft}$. $\mathrm{lb}$. Max. positive moment in $\operatorname{span} l_{3}=+67,300 \mathrm{ft}$. lb.

Case 4 also gives the position of live load for maximum negative moment at the center of $\operatorname{span} l_{2}$.

Max. $V_{1}=+18,614 \mathrm{lb}$.

Max. $V_{4}^{\prime}=-15,606 \mathrm{lb}$.

Max. $R_{1}=+18,614 \mathrm{lb}$.

Max. $R_{\mathrm{f}}=+15,606 \mathrm{lb}$. 
Problem II. Assume a beam having a long central span and two shorter end spans of equal length, as shown in Fig. 7, carrying a live load of 2,500 pounds per linear foot and a dead load of 2,200 pounds per linear foot. To find the critical moments, shears and reactions, assuming the ends simply supported.

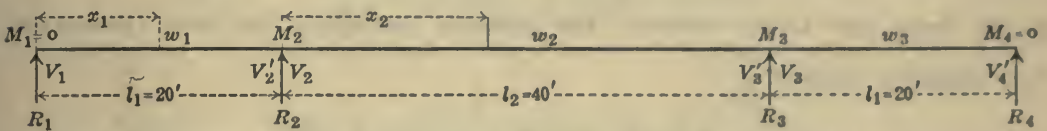

Live load per $\mathrm{ft}$. of span $=2500 \mathrm{lb}$.

Dead " " " " " $=2200 "$

Fig. 7

The solution of this problem involves the same procedure as that carried out in detail for Problem I, Case 1, and, therefore, the results only are tabulated below for the various cases of critical loading.

Case 1

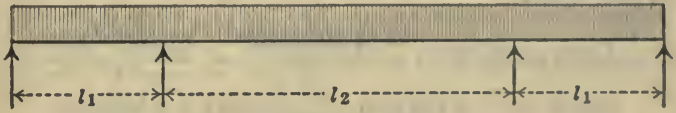

$$
M_{2}=M_{3}=-528,750 \mathrm{ft} \text {. lb. }
$$

Positive moment at point $x_{1}=+44,978 \mathrm{ft}$. lb.

Positive moment at point $x_{2}=+411,250 \mathrm{ft}$. lb.

$V_{1}=+20,562$ lbs. $\quad V_{2}^{\prime}=-73,438$ lbs. $V_{2}=+94,000 \mathrm{lb}$.

$R_{1}=20,562 \mathrm{lbs} . \quad R_{2}=167,438 \mathrm{lb}$.

Case 2

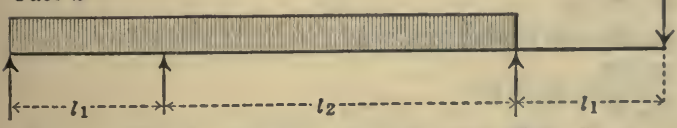

NoтE.-This loading gives negative reaction, $R_{\mathfrak{l}}=-2,094 \mathrm{lb}$.

\section{Case 3}

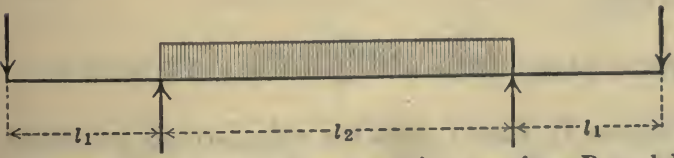

Max. $M_{2}=-544,375 \mathrm{ft}$. lb. Max. $V_{2}^{\prime}=-71,219 \mathrm{lb}$. Max. $V_{2}=+95,562 \mathrm{lb}$. Max. $R_{2}=+169,781 \mathrm{lb}$.

Note.-This loading gives negative reactions $R_{1}$ and $R_{4}=-2,875 \mathrm{lb}$.

Case 4

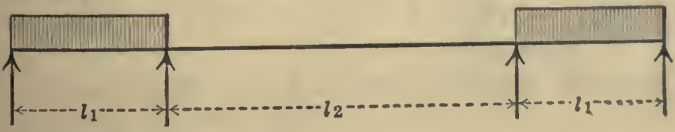

Max. positive moment in span $l_{1}=+116,294 \mathrm{ft}$. lb.

Max. $V_{1}=+33,063 \mathrm{lb}$.

Max. $R_{1}=+33,063 \mathrm{lb}$. 
The condition of simply supported ends assumed in Problems I and II is that which occurs when the beam frames into a brick wall. If, however, the ends frame into a column or girder, the moment at the end support will not equal zero, but will have a value depending upon the degree of fixity. This may be as large as $\frac{w l^{2}}{12}$ where small beams frame into heavy columns. For ordinary conditions the Joint Committee recommends a value of $\frac{w l^{2}}{16}$

We will now take Problem II, Case 1 , and substitute for $M_{1}$, a value $\frac{w_{1} l_{1}^{2}}{16}$ and for $M_{4}, \frac{w_{3} l_{3}^{2}}{16}$ and note the effect on the various moments and shears.

$$
M_{1}=M_{4}=-\frac{w_{1} l_{1}^{2}}{16}=-\frac{(4,700)(20)^{2}}{16}=-117,500 \mathrm{ft} . \mathrm{lb} .
$$

From symmetry $M_{2}=M_{3}$

$$
M_{1} l_{1}+2 M_{2}\left(l_{1}+l_{2}\right)+M_{3} l_{2}=-\frac{w_{1} l_{1}^{3}}{4}-\frac{w_{2} l_{2}^{8}}{4}
$$

Inserting numerical values,

$$
\begin{aligned}
(-117,500)(20)+120 & M_{2}+40 M_{2}=-\frac{(4,700)(20)^{3}}{4}-\frac{(4,700)(40)^{3}}{4} \\
160 & M_{2}=(-9,400,000-75,200,000)+2,350,000 \\
M_{2} & =M_{3}=\frac{(-84,600,000+2,350,000)}{160}=-514,063 \mathrm{ft} . \mathrm{lb} . \\
V_{1} & =\frac{(-514,063)-(-117,500)}{20}+\frac{(4,700)(20)}{2}=+27,172 \mathrm{lb} . \\
V_{2}^{\prime} & =27,172-(4,700)(20)=-66,828 \mathrm{lb} . \\
V_{2} & =\frac{(-514,063)-(-514,063)}{40}+\frac{(4,700)(40)}{2}=+94,000 \mathrm{lb} . \\
R_{1} & =27,172 \mathrm{lb} . \\
R_{2} & =94,000+66,828=160,828 \mathrm{lb} . \\
x_{1} & =\frac{27,172}{4,700}=5.78 \mathrm{ft} .
\end{aligned}
$$

Moment at $x_{1}, M=(-117,500)+(27,172)(5.78)-\frac{(4,700)(5.78)^{2}}{2}=-38,955 \mathrm{ft} . \mathrm{lb}$.

$$
x_{2}=\frac{94,000}{4,700}=20 \mathrm{ft} \text {. }
$$

Moment at $x_{2}, M=(-514,063)+(94,000)(20)-\frac{(4,700)(20)^{2}}{2}=+425,937 \mathrm{ft} . \mathrm{lb}$.

It will be noted that for Problem II, Case 1, with the ends partially restrained, negative moment exists throughout $\operatorname{span} l_{1}$. 


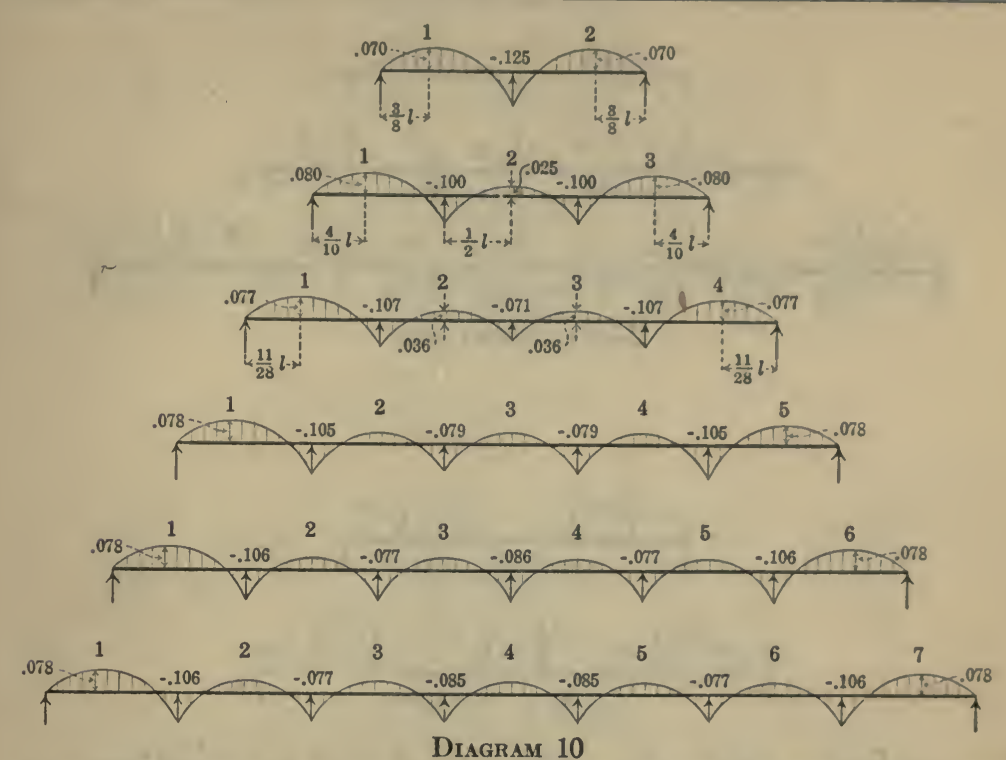

Moment coefficients of $w l^{2}$ for continuous beams of equal spans supported at the ends and uniformly loaded.

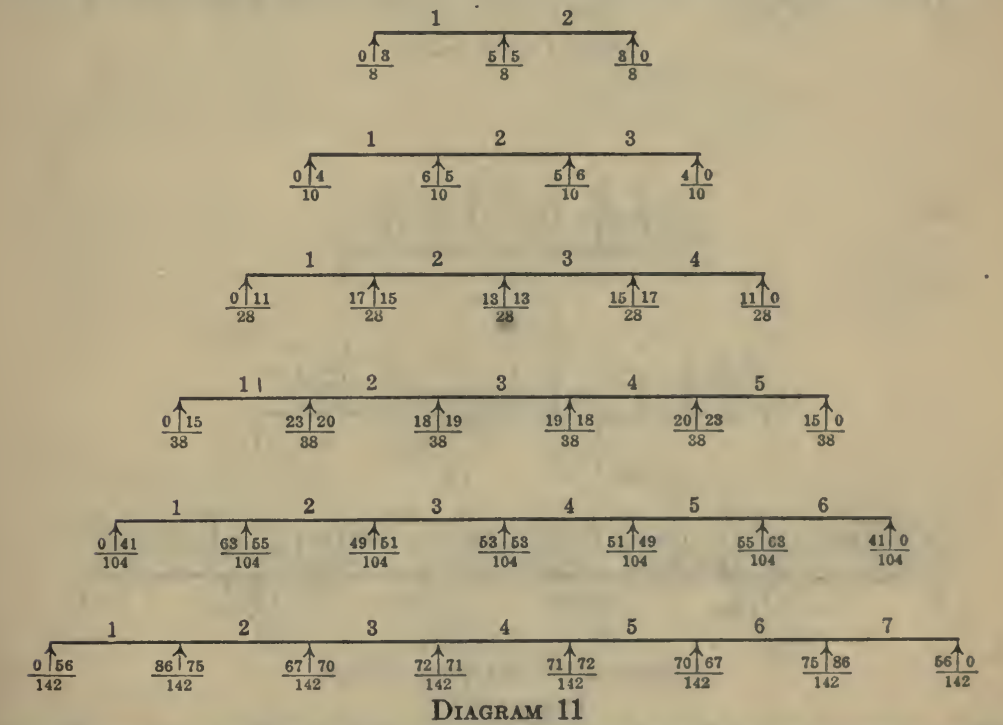

Shear coefficients of $v l$ for continuous beams of equal spans supported at the ends and uniformly loaded. 


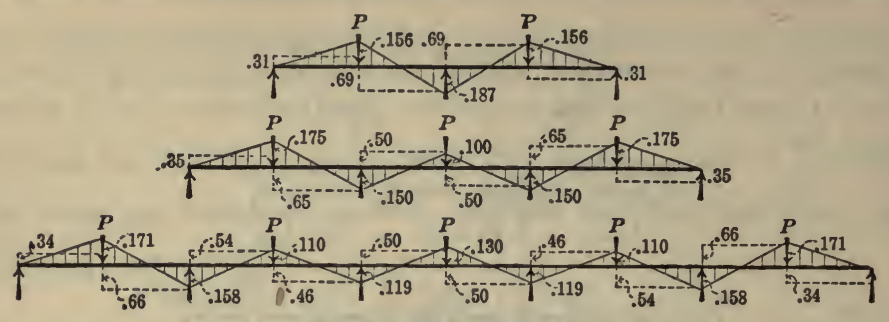

Loads at Middle Points

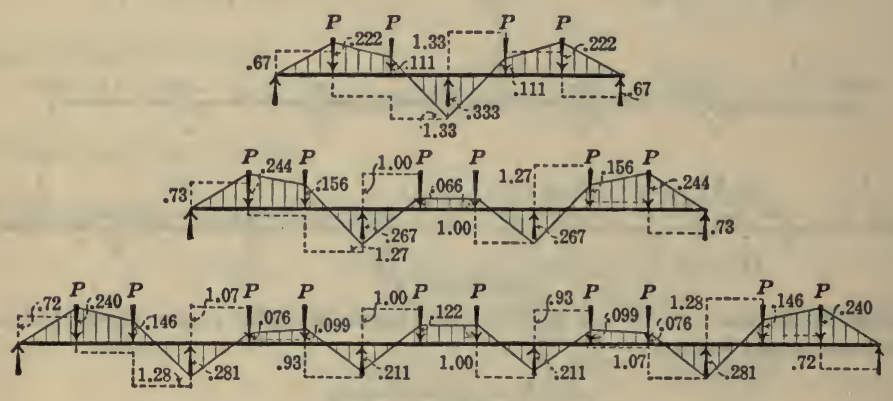

Loads at Third Points

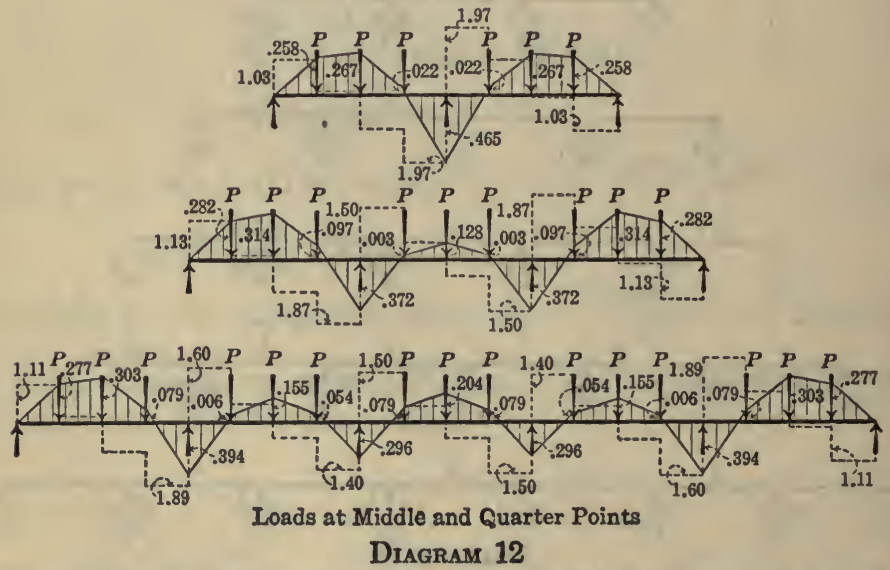

Moment coefficient of $P l$ and shear coefficient of $P$ for continuous beams of equal spans supported at the ends and loaded as indicated. 


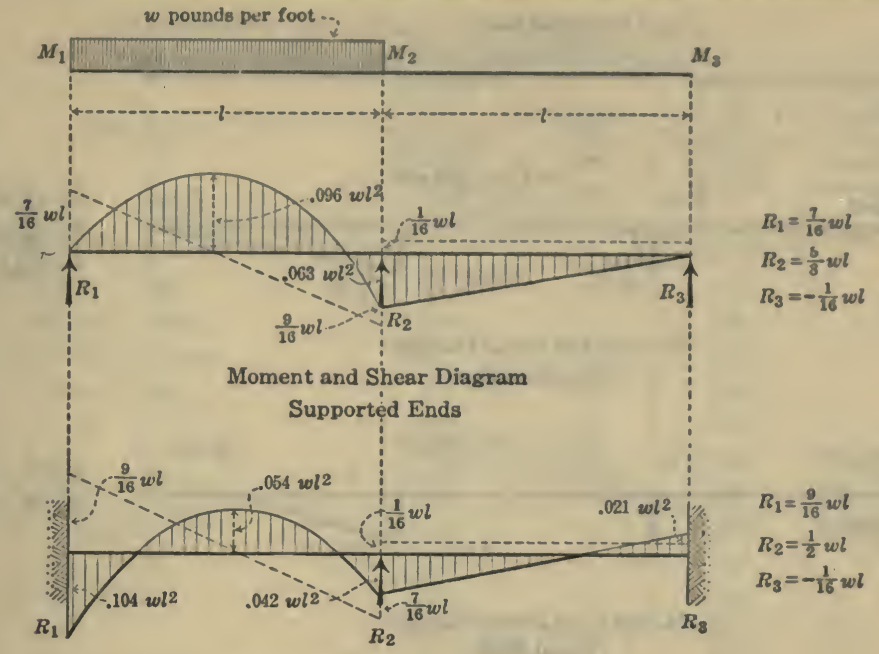

\section{Moment and Shear Diagram}

Fixed Ends

Diagram 13

Moment and shear coeffieients for continuous beams of two equal spans with uniformly distributed load on one span only.

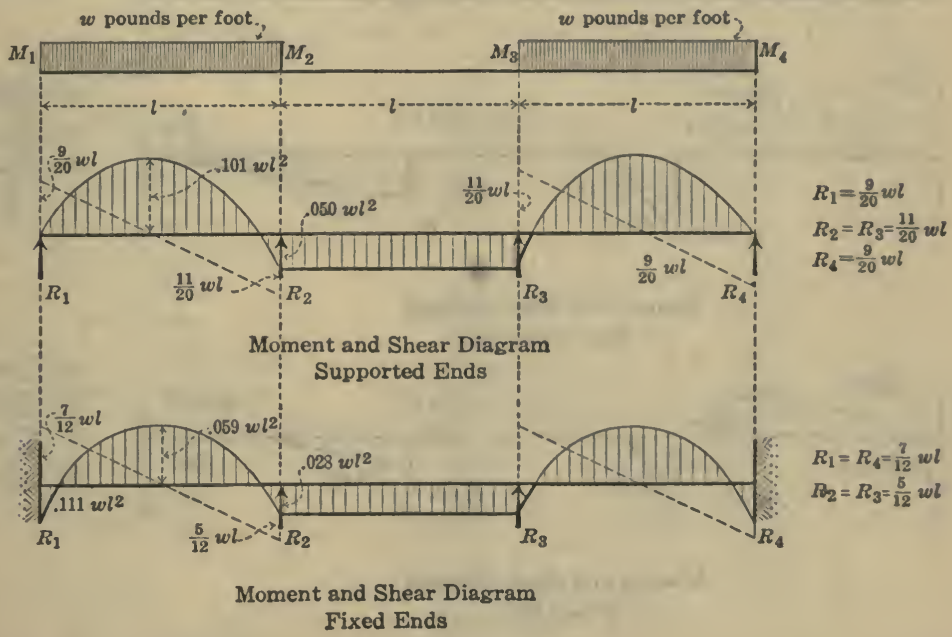

\section{Diagram 14}

Moment and shear coefficients for continuous beams of three equal spans with uniformly distributed load on two end spans only. 


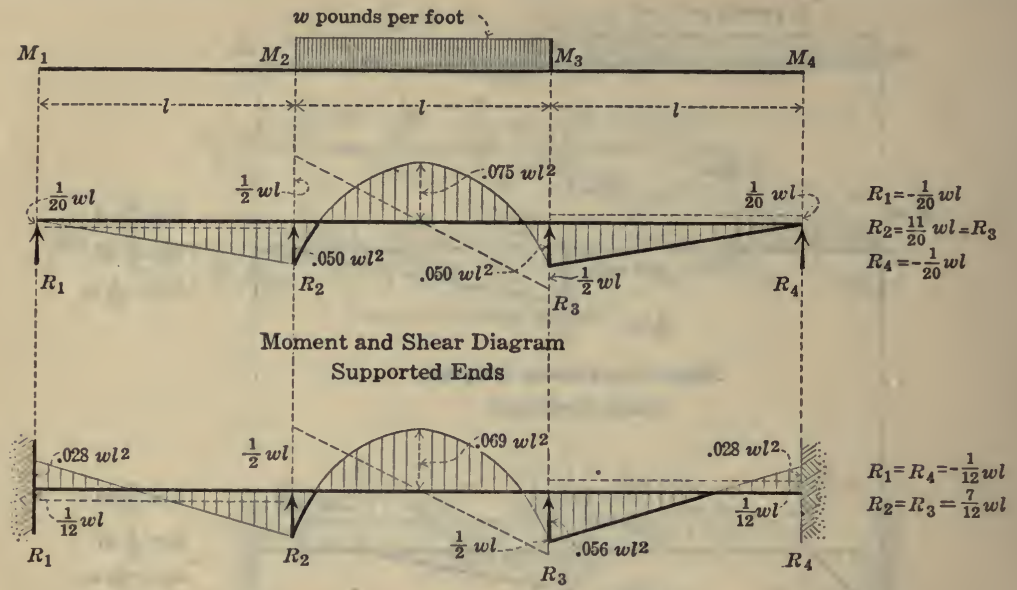

Moment and Shear Diagram

Fixed Ends

Diagram 15

Moment and shear coefficients for continuous beams of three equal spans with uniformly distributed load on center span only.

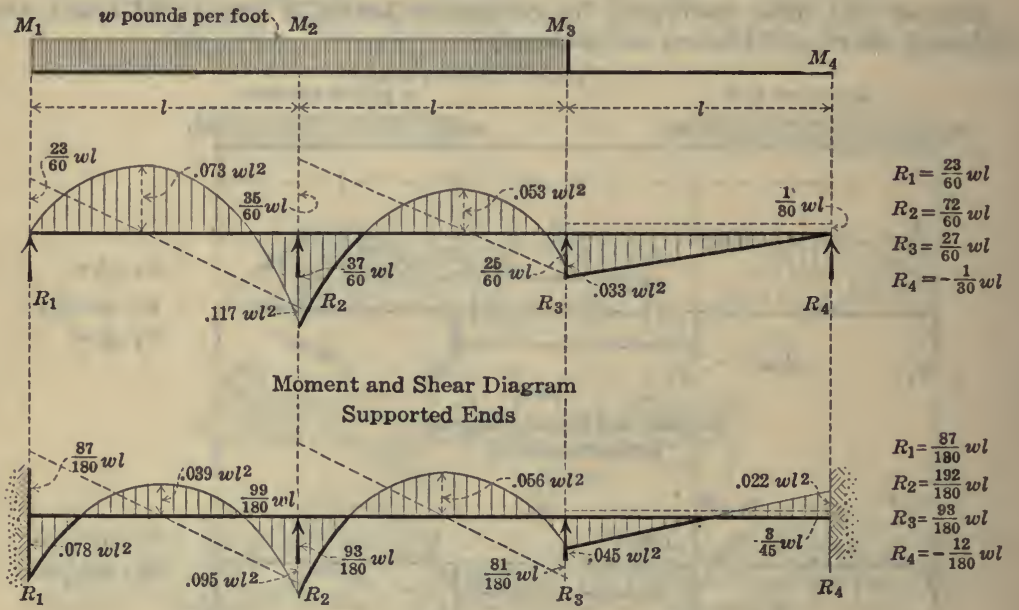

Moment and Shear Diagram

Fixed Ends

\section{Diagram 16}

Moment and shear coefficients for continuous beams of three equal spans with uniformly distributed load on center and one end span only 


\section{CONTENTS OF STORAGE WAREHOUSES}

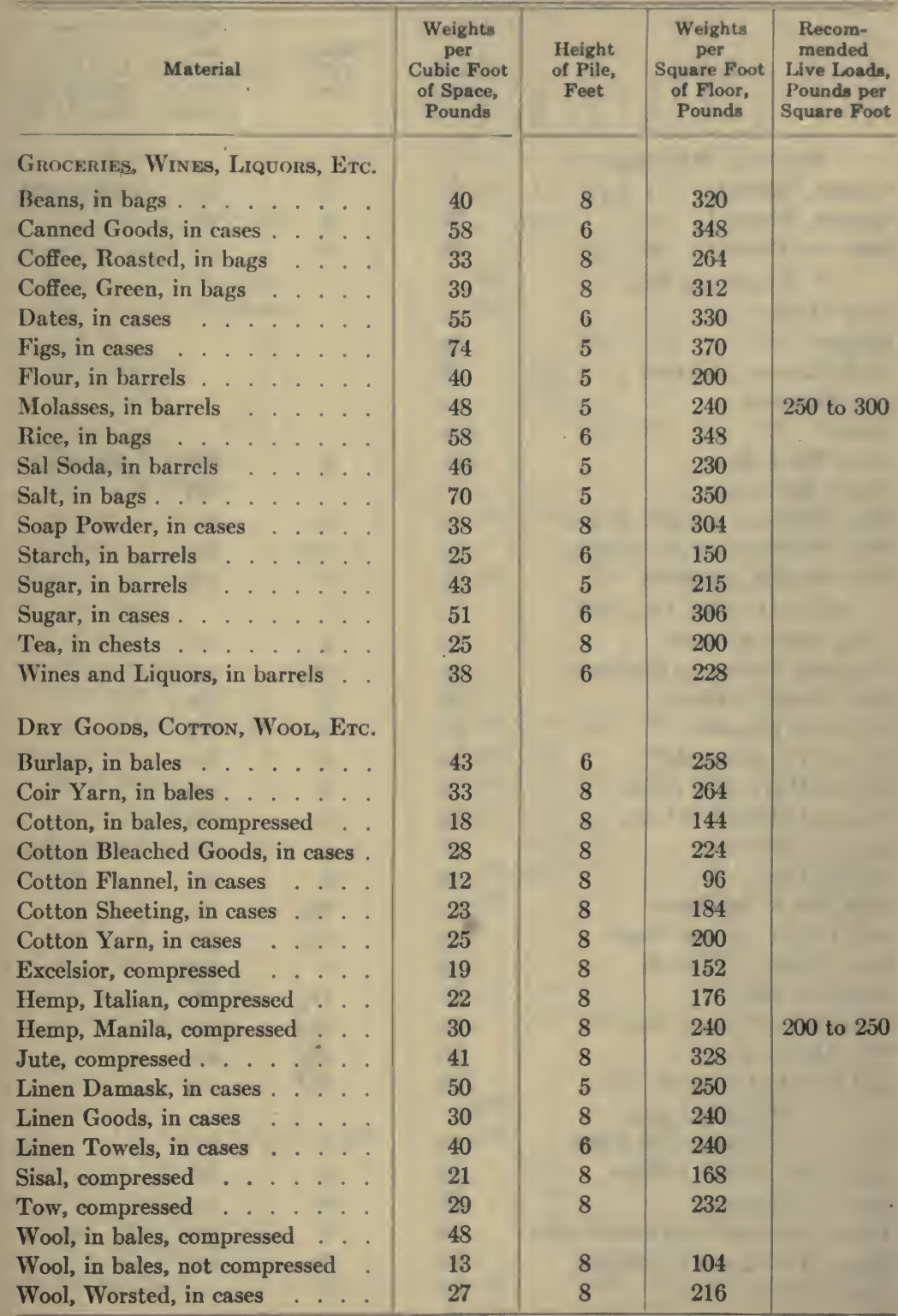




\section{CONTENTS OF STORAGE WAREHOUSES}

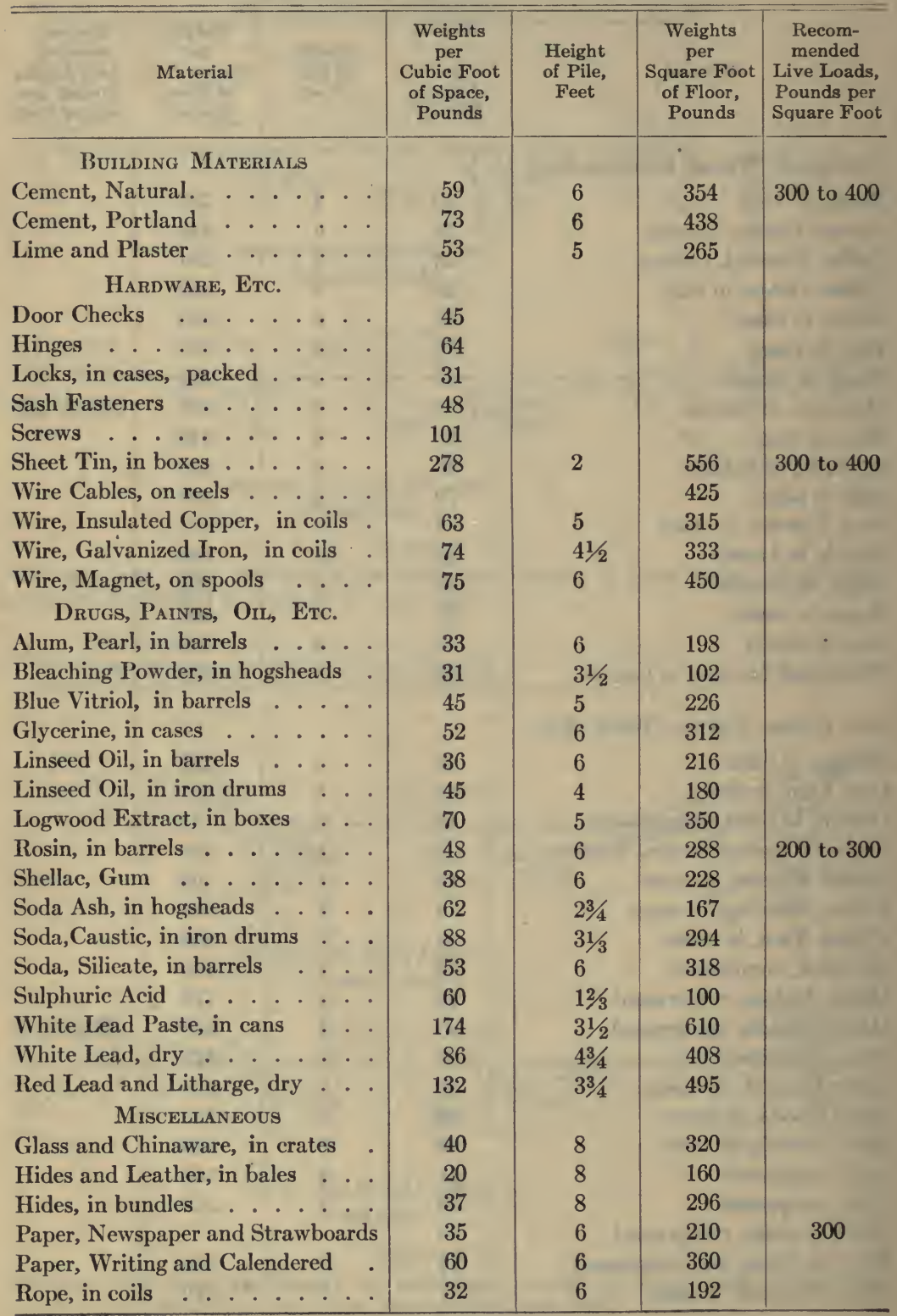


BUILDING CODE REQUIREMENTS FOR LIVE LOAD

Structure

\begin{tabular}{|c|c|c|c|c|c|c|c|}
\hline 总 & 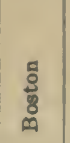 & 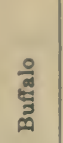 & $\begin{array}{l}\text { \& } \\
\text { हुँ } \\
\text { తึ }\end{array}$ & 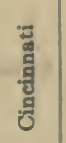 & 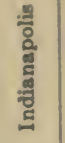 & 荧 & $\begin{array}{l}\frac{2}{\circ} \\
\frac{2}{2} \\
\frac{0}{2}\end{array}$ \\
\hline \multirow[t]{3}{*}{60} & 50 & 70 & 40 & 40 & 50 & 30 & 50 \\
\hline & 100 & & 100 & & & & \\
\hline & & 100 & 100 & 100 & 125 & & 125 \\
\hline \multirow{7}{*}{$\begin{array}{r}75 \\
125\end{array}$} & & & 100 & & & 50 & \\
\hline & & & & & & 80 & \\
\hline & & 100 & 100 & & 125 & 50 & 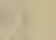 \\
\hline & 200 & & 100 & 150 & & 100 & 17 \\
\hline & 200 & & & & & 100 & \\
\hline & & 100 & 100 & 100 & 125 & 50 & \\
\hline & & & & & & 80 & \\
\hline \multirow[t]{2}{*}{60} & 50 & 40 & 40 & 40 & 50 & 30 & 50 \\
\hline & & 70 & 50 & & 50 & 30 & 50 \\
\hline \multirow[t]{3}{*}{60} & & 70 & 50 & 40 & 75 & 30 & 50 \\
\hline & & & & 100 & & & \\
\hline & & & 50 & & & & \\
\hline 175 & & & & 150 & 200 & & \\
\hline 125 & 125 & 120 & 100 & 100 & 100 & 100 & 100 \\
\hline 125 & 250 & & 100 & & & & \\
\hline 125 & $12 \bar{j}$ & 120 & 100 & 100 & 100 & 100 & 100 \\
\hline \multirow[t]{2}{*}{250} & 250 & & & 150 & 200 & & 1 \\
\hline & 250 & 150 & & 150 & 200 & & Hr \\
\hline 75 & 100 & 70 & 50 & 50 & 75 & 40 & 75 \\
\hline \multirow{2}{*}{150} & & & & 100 & 150 & 80 & 100 \\
\hline & & & & 100 & & & \\
\hline \multirow[t]{2}{*}{75} & 60 & & & 60 & 100 & 40 & 100 \\
\hline & 125 & 100 & 75 & & & $\begin{array}{l}60 \\
60 \\
60\end{array}$ & \\
\hline 200 & & & & 300 & 300 & 150 & 300 \\
\hline \multirow[t]{4}{*}{100} & & 120 & 100 & 75 & S5 & SO & 85 \\
\hline & 70 & & 100 & 80 & -1 & 60 & \\
\hline & 70 & & & & & & \\
\hline & 40 & 40 & 25 & 25 & 30 & 30 & $\begin{array}{l}50 \\
50\end{array}$ \\
\hline 30 & & 30 & 20 & 20 & & 30 & 30 \\
\hline
\end{tabular}

Apartments

Public Rooms and Halls

Assembly Halls

Fxd. Seat Auditoriums

Mov. Seat Auditoriums

Churches

Dance Halls

Drill Rooms

Theaters

Theater Balconies

Theater Stairways

Dwellings

Hospitals

Hotels.

\section{(2)}

First Floors

Corridors

Office Rooms

Manufacturing

Light Manufacturing

Mercantile

Retail Stores

Heavy Storehouses

Warehouses

Offices .

First Floor

Corridors

Public Buildings

Schools-Class Rooms

Assembly Rooms

Corridors

\section{Stairways}

Sidewalks

Stables, Carriage Ilouses, Garages

Stairways, General

Fire Escapes

Roofs-Slope Under $20^{\circ}$

Over $20^{\circ}$ (IIor. Proj.)

Wind Pressures 


\section{BUILDING CODE REQUIREMENTS FOR LIVE LOAD}

\begin{tabular}{|c|c|c|c|c|c|c|c|c|c|c|}
\hline Structure & & & 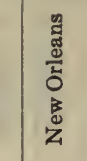 & 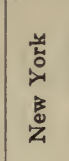 & 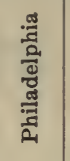 & 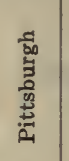 & $\begin{array}{l}\stackrel{n}{3} \\
\stackrel{\leftrightarrow}{\circ} \\
\dot{\omega}\end{array}$ & 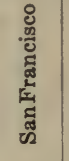 & 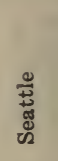 & 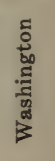 \\
\hline & $\cdots \cdots$ & & 40 & & 70 & & 50 & 60 & 40 & 50 \\
\hline Public Rooms and Halls & • • • & & 70 & & & & & & & \\
\hline $\begin{array}{l}\text { Assembly Halls } \\
\text { Fxd. Seat Auditoriums }\end{array}$ & $\begin{array}{l}\cdots \\
\therefore\end{array}$ & $\therefore$ & & 100 & 120 & 150 & 100 & 75 & 75 & \\
\hline Mov. Seat Auditoriums & $\ldots$ & . & & & & & & 125 & 100 & \\
\hline Churches ..... & . . . & . . & & & & & 75 & & 75 & \\
\hline Dance Halls . . . . & . . . . & . . & 150 & & & & & & 100 & \\
\hline Drill Rooms . . . . & ... & . . & - 150 & & & & & & 250 & \\
\hline Theaters..... & . . . & . . & & & & & 100 & 75 & 75 & \\
\hline Theater Balconies . . . & . . . & . . & & & & & & & & \\
\hline Theater Stairways . & . . . & . . & & & & & & & 100 & \\
\hline Dwellings ..... & ... . & . . & 40 & 40 & 70 & 70 & 50 & 60 & 40 & 50 \\
\hline Hospitals . . . . . & . . . . & . . & & & 70 & & 50 & 60 & 50 & \\
\hline Hotels . . . . . . . & . . . & . . & 40 & & 70 & & 50 & 60 & 40 & 50 \\
\hline First Floors .... . & . . . & . . & & & & & & & 100 & 75 \\
\hline Corridors ..... & . . . . & . . & & & & & & 125 & 100 & 75 \\
\hline Office Rooms . . . . . & . . . . & . . & & & & & & & & 75 \\
\hline Manufacturing ... & . . . . & . . & & & 150 & 200 & 150 & 250 & & \\
\hline Light Manufacturing . & . . . & . $\cdot$ & 125 & & 120 & & 100 & 125 & 125 & \\
\hline Mercantile ..... & . . & . . & 200 & & & & 150 & & & \\
\hline Retail Stores . . . . & ... & . . & 125 & 120 & 120 & & 150 & 125 & 125 & 110 \\
\hline Heavy Storehouses . . & . . . . & . . & & & 150 & & & 250 & & 150 \\
\hline Warehouses .... & . . . & . . & 200 & & 150 & 200 & 150 & 250 & & 150 \\
\hline Offices . . . . . . & . . . & . . & 70 & 60 & 100 & & 60 & 60 & 50 & 75 \\
\hline First Floor . . . . & . . . & . . & & & & & 100 & & 125 & \\
\hline Corridors & . . . & . . & & & & & 100 & & & 110 \\
\hline Public Buildings . . . & . . . & . . & 125 & & & & 100 & & & 110 \\
\hline Schools-Class Rooms . & ... & . & 60 & 75 & & & 75 & 75 & 50 & 75 \\
\hline Assembly Rooms . . . & . . . & . . & 125 & & & & & & 75 & \\
\hline Corridors ..... & . . . & . . & & & & & & 125 & 100 & \\
\hline Stairways . . . . & . . . . & . & & & & & & & & \\
\hline Sidewalks ... & $\ldots$ & 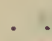 & 300 & 300 & & & & 150 & & \\
\hline Stables, Carriage Houses, & Garages & $0^{\circ}$ & & & & & 100 & 75 & 75 & \\
\hline Stairways, General . . . & . . . & . . & 70 & & & & & & 100 & \\
\hline Fire Escapes . . . . & . . . & . . & 70 & & & & & & 100 & \\
\hline Roofs-Slope under $20^{\circ}$ & . . . & . & 30 & 40 & 30 & 50 & 30 & 30 & 40 & 25 \\
\hline Over $20^{\circ}$ (Hor. Proj.) & . . . & . . & & & 30 & & & 20 & 40 & 25 \\
\hline Wind Pressures . . . . & ... & 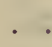 & & 30 & 30 & & 30 & 20 & & 30 \\
\hline
\end{tabular}




\section{WEIGH'TS OF MATERIAL}

\begin{tabular}{|c|c|c|c|}
\hline Substance & \begin{tabular}{|c|} 
Weight, \\
Pounds \\
per \\
Cubic Foot
\end{tabular} & Substance & $\begin{array}{c}\begin{array}{c}\text { Weight, } \\
\text { Pounds } \\
\text { per } \\
\text { Cubic Foot }\end{array} \\
\end{array}$ \\
\hline Ashlar Masontr & 165 & $\begin{array}{c}\text { Earth, Etc., Excavated. } \\
\text { (CONtinded) }\end{array}$ & \\
\hline $\begin{array}{l}\text { Granite, syenite, gneiss . } \\
\text { Limestone, marble . }\end{array}$ & $\begin{array}{l}165 \\
160\end{array}$ & Earth, dry, loose & 76 \\
\hline Sandstone, bluestone . . . . & 140 & Earth, dry, packed . & 95 \\
\hline Mortar Rubble Masonry & & $\begin{array}{l}\text { Earth, moist, loose } \\
\text { Earth, moist, packed }\end{array}$ & $\begin{array}{l}78 \\
96\end{array}$ \\
\hline Granite, syenite, gneiss . . . & 155 & Earth, mud, flowing . . . . & 108 \\
\hline Limestone, marble & 150 & Earth, mud, packed . & 115 \\
\hline Sandstone, bluestone & 130 & Riprap, limestone . . . . & $80-115$ \\
\hline Dry Rubble Masonry & & Riprap, sandstone & 90 \\
\hline Granite, syenite, gneiss . . . & 130 & Riprap, shale . . . & 105 \\
\hline Limestone, marble . . . . & 125 & Sand, gravel, dry, loose . & $90-105$ \\
\hline Sandstone, bluestone . . . & 110 & $\begin{array}{l}\text { Sand, gravel, dry, packed } \\
\text { Sand, gravel, dry, wet }\end{array}$ & $\begin{array}{l}100-120 \\
118-120\end{array}$ \\
\hline Brick MasonRY & & 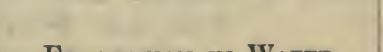 & \\
\hline ed bri & 140 & Excavation in Water & \\
\hline Common brick & 120 & Sand or gravel & 60 \\
\hline Soft brick. . & 100 & Sand or gravel and clay & 65 \\
\hline Concrete Masonry & & $\begin{array}{l}\text { Clay . . . . . } \\
\text { River mud . . . }\end{array}$ & $\begin{array}{l}80 \\
90\end{array}$ \\
\hline Cement, stone, sand . . . . & 144 & Soil . . . . & 70 \\
\hline Cement, slag, etc. . . . . & 130 & Stone riprap . & 65 \\
\hline Cement, cinder, etc. . . & 100 & & \\
\hline Various Building Materials & & Mrnerats & \\
\hline Ashes, cin & $40-45$ & Asbestos . . & 153 \\
\hline and, loose & 90 & Barytes . . . . & 281 \\
\hline Cement, Portland, set . . & 18 & Basalt . . & 184 \\
\hline Lime, gypsum, loose . . . . & $53-6$ & Bauxite. . . . & 159 \\
\hline Mortar, set . . . . . . & 10 & Borax & 109 \\
\hline Slags, bank slag . . . . & $67-72$ & Chalk & 137 \\
\hline Slags, bank screenings . . . & $98-117$ & Clay, marl . . . & 137 \\
\hline Slags, machine slag. . . . . & 9 & Dolomite . . . . . & 181 \\
\hline Slags, slag sand . . . . . & $49-55$ & $\begin{array}{l}\text { Feldspar, orthoclase . . } \\
\text { Gneiss, serpentine . . }\end{array}$ & $\begin{array}{l}159 \\
159\end{array}$ \\
\hline Excavated & & Granite, syenite . . & 175 \\
\hline Clay, dry . . . . & 63 & Greenstone, trap. & 187 \\
\hline Clay, damp, plastic & 11 & Gypsum, alabaster & 159 \\
\hline Clay and gravel, dry . . . & 100 & Hornblende . . . . . & 187 \\
\hline
\end{tabular}




\section{WEIGHTS OF MATERIAL}

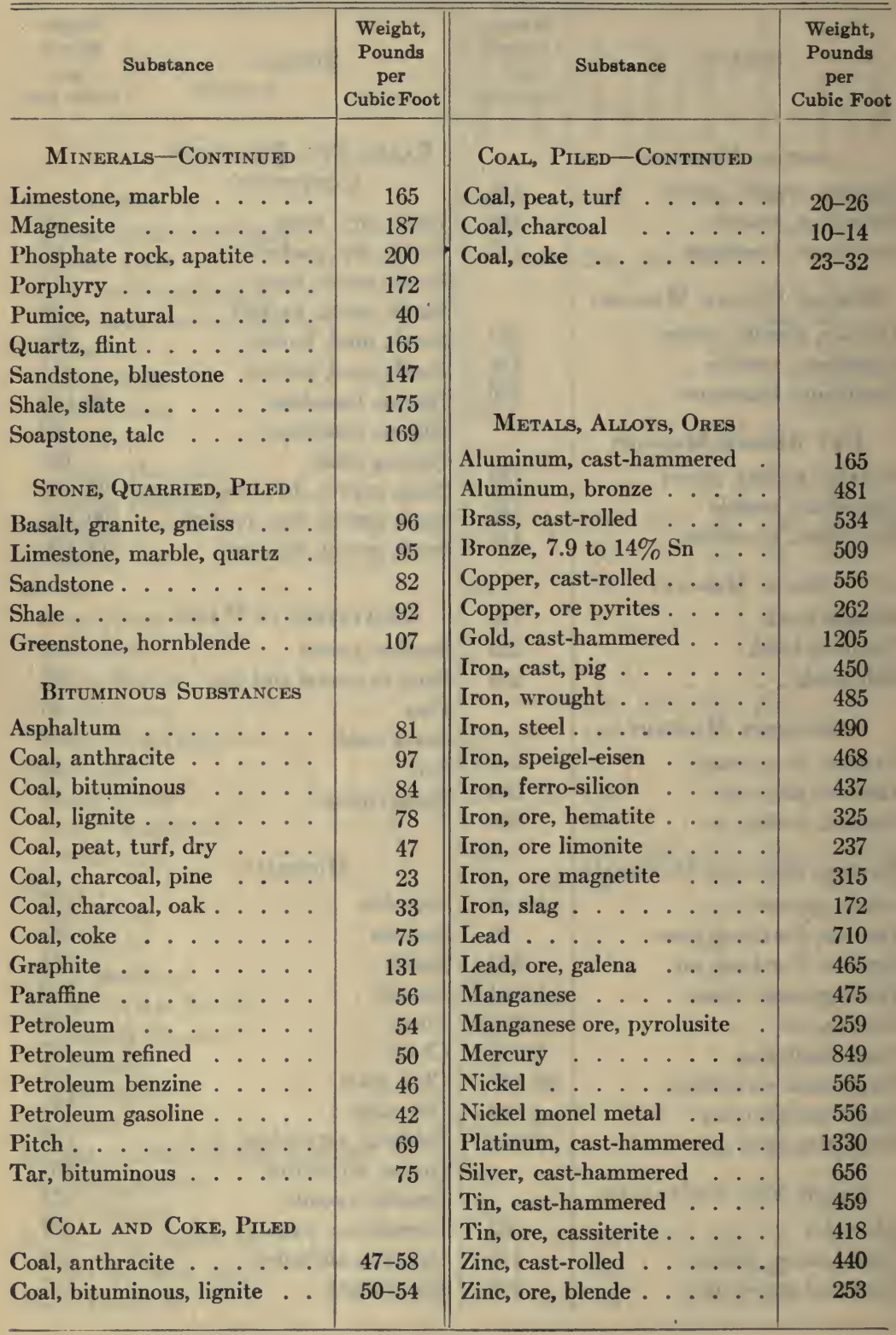


WEIGHTS OF MATERIAL

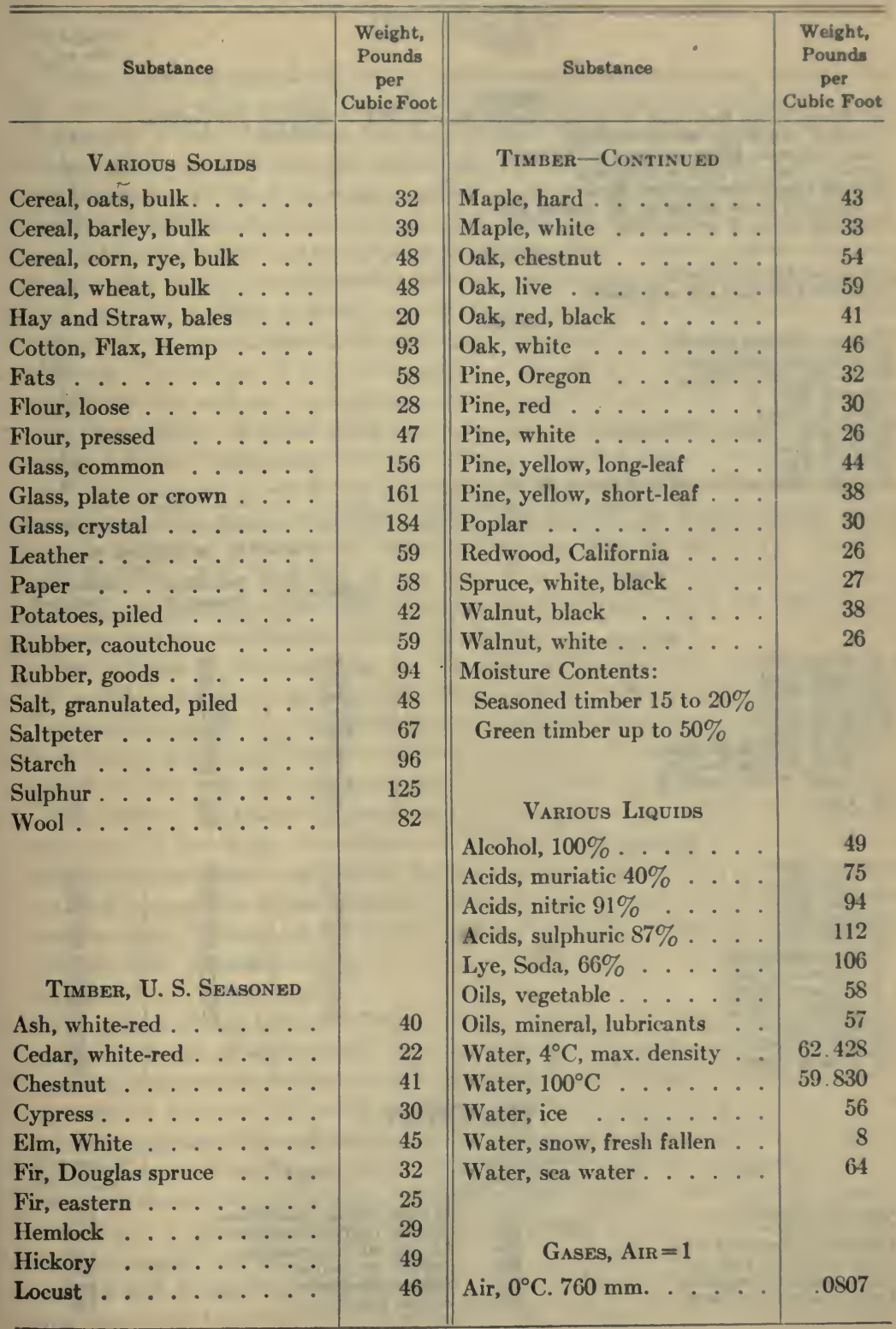




\section{WEIGHTS OF BUILDING MATERIALS}

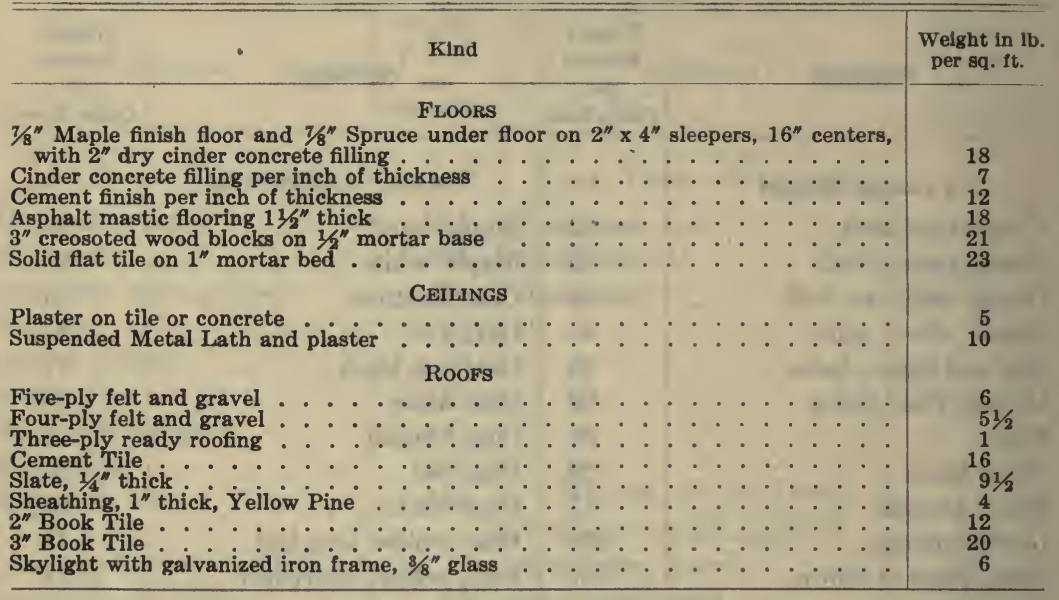

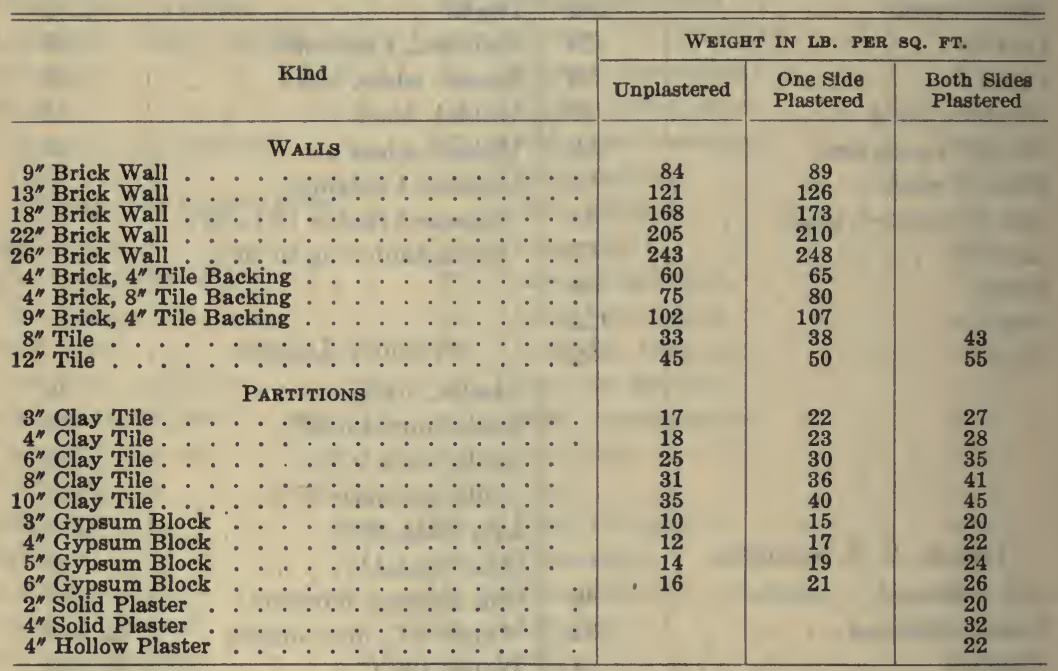

\section{MASONRY}

\begin{tabular}{|c|c|c|c|}
\hline Kind & $\begin{array}{l}\text { Welght in lb. } \\
\text { per cu. ft. }\end{array}$ & Kind & $\begin{array}{c}\text { Welght in lb. } \\
\text { per cu. } \mathrm{ft} \text {. }\end{array}$ \\
\hline $\begin{array}{l}\text { Concrete, cinder . . : } \\
\text { Concrete, stone : . } \\
\text { Concrete, reinforced stone } \\
\text { Brick masonry, soft . } \\
\text { Brick masonry, common : } \\
\text { Brick masonry, pressed. }\end{array}$ & $\begin{array}{r}140 \text { to } 150 \\
150 \\
100 \\
125 \\
140\end{array}$ & 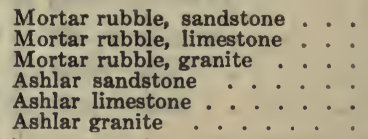 & $\begin{array}{l}130 \\
150 \\
155 \\
140 \\
160 \\
165\end{array}$ \\
\hline
\end{tabular}




\section{FLOORS AND ROOFS-WITH EXPLANATION}

\section{OF TABLES}

Types. The selection of the best type of floor and roof construction depends upon the spans, loads to be carried, character of the building and local conditions. Buildings readily divide themselves into two general groups,-those primarily for the housing of people, and those for warehousing and industrial purposes.

In structures of the first group, comprising office and public buildings, schools, hospitals, hotels, apartments, dwellings and garages the majority of the spans are long and the loads light (40 to 125 pounds per square foot). Such conditions require a greater depth of slab, to avoid undue deflection, than would be demanded for strength alone, and the problem is usually solved through the use of a concrete ribbed floor employing clay or composition tile, metal or wood forms of whatever depth may be necessary. In the case of clay or composition tile they always remain a part of the permanent floor and may be said to represent the best type of form or filler for concrete ribbed slabs; they add stiffness and strength to the construction and in no way detract from its fireproofness. To reduce the cost, however, metal forms are frequently used and may be of either the removable or permanent type. Permanent metal forms are of light gauge steel sheets, stiffened transversely by corrugations, to enable them to withstand the loads and impacts of service; they add nothing to the structural efficiency of the floor and, in fact, may damage the concrete through expansion of the exposed metal in case of fire. For this reason, if metal forms are used, those of a removable type would seem the proper selection.

For buildings in the warehouse and industrial group the loads usually vary from 125 to 500 pounds per square foot. Where the panels are square, or approximately so, the flat slab type of floor presents the utmost advantages structurally and economically. If, however, the ratio of length of short to long side of panel exceeds 1:11/3, a beam and girder floor with solid concrete slabs should generally be used.

Treatment. All beams, including the ribs in concrete ribbed slabs, may be classed under one of two types-rectangular or T-section. In rectangular beams, the concrete above the neutral axis within the limits of the width of the beam must resist the total compressive stress (assisted in special cases by additional steel in the compression area); whereas, in the T-section beam the flange, when built monolithically with the web, materially increases the compressive resistance of the beam and consequently its carrying capacity.

The tables of safe loads for ribbed slabs, continuous or partially continuous, are based on a length of span equal to the distance center to center of supports, with the condition that the tile or form, as the case may be, shall extend to within not less than twelve inches of the center of support as indicated by the illustrations at the head of the tables. The critical section for bending is usually at this point and as the ratio between the bending moment here and at the center of span varies with each change of span, it would be uneconomical to maintain a constant steel area for each fixed depth of slab on all spans. In these tables the proper steel area for each depth and span length is given.

Load and Moment Conditions. The carrying capacity of beams and slabs is dependent upon the condition of fixity at the supports and the stresses allowed in the 
steel and concrete. The safe load tables, pages 61 to 106 inclusive, have been prepared on the basis of two different stress combinations and for moments of $\frac{w l^{2}}{8}, \frac{w l^{2}}{10}$ and $\frac{w l^{2}}{12}$. The governing conditions are stated in the heading of each table.

It is important to note that in the case of ribbed slabs or of T-beams continuous over supports, that the critical compressive stress usually occurs at the supports where the flange is in tension and the stem of the beam is in compression, thereby resolving the problem into that of a beam of rectangular section. However, as only a short section of the beam is under maximum compression at this point it is considered entirely permissible to employ a higher unit stress in the concrete here than at the center of span. This feature has been carefully considered in preparing the tables for continuous or semi-continuous ribbed slabs and T-beams and the maximum fibre stress in the concrete at supports has been held to a value not exceeding $15 \%$ greater than the fibre stress noted in the table. This is in accordance with the recommendations of the Joint Committee on Concrete and Reinforced Concrete.

In the case of T-beams continuous over supports the straight bars in the bottom of the beam are considered to act as compressive reinforcement and should be carried past the face of the support a sufficient distance to develop their stress in bond.

Shear. After selecting from the tables the proper slab or beam to be used for any particular load, special attention should be given to the shear reinforcemcnt required. For the solid concrete slabs the loads given produce shears of less than forty pounds per square inch on the area $b j d$. For the concrete ribbed slabs loads to the left of the heavy stepped line produce shears in excess of ninety pounds, pcr square inch on $b j d$ and vertical stirrups should be used in addition to the bent up bars in the ribs.

In the case of the beam tables, to satisfy the majority of code requirements, stirrups will have to be provided. Particular attention is called to the fact tha $i$ all loads to the left of the heavy stepped line produce shears in excess of 120 pounds per square inch on bjd.

Fireproofing. In preparing the tables the depth of fireproofing under the reinforcement was taken at $3 / 4$ in. for solid and ribbed slabs and $11 / 2$ in. for beams. As the fireproofing determines the effective depth for any given slab or beam it will be necessary to increase or reduce the table load should a change be made in the amount of fireproofing. This may be effected by multiplying the sum of the superimposed and dead loads of the table by the square of the ratio of the new effective depth to the given effective depth. This gives the new total load from which should be deducted the dead load in order to obtain the new superimposed load.

The following examples illustrate the use of the tables:

Solid Concrete Slabs. Given a floor layout consisting of solid concrete slabs, continuous over beams spaced $12^{\prime}-0^{\prime \prime}$ in the clear. The floor to carry a live load of 150 pounds per square foot, a wood finish on sleepers embedded in 2 inches of cinder concrete and a plastered ceiling; the finish and plaster weighing together 25 pounds per square foot, giving a total superimposed load of 175 pounds per square foot. The stress in the steel not to exceed 16,000 pounds per square inch and in the concrete 650 pounds per square inch. 
The table on page 63 for slabs continuous over supports and based on the specified unit stresses shows that a $61 / 2$-inch slab reinforced with $5 / 8$ " round bars, $71 / 2$ inches on centers, will carry 178 pounds per square foot.

Concrete Ribbed Slabs-Clay Tile Fillers: Given a floor of $22^{\prime}-0$ " span, noncontinuous. This floor is to carry a live load of 70 pounds per square foot, a wood floor on cinder concrete fill and a plastered ceiling; the finish and plaster weighing together 27 pounds per square foot, thus giving a total superimposed load of 97 pounds per square foot. Steel stress 16,000 pounds per square inch and concrete 650 pounds per square inch.

The table on page 67 for non-continuous slabs shows that a $10^{\prime \prime}+2 \frac{1}{2}$ " slab with 1.15 square inches of steel in each rib will carry 106 pounds per square foot and a $12^{\prime \prime}+2^{\prime \prime}$ slab with 1.04 square inches of steel will carry 112 pounds per square foot. If the $10^{\prime \prime}+2 \frac{1}{2}$ " slab is selected the area of steel called for in the table may be reduced in the ratio of actual total load per square foot to the total carrying capacity per square foot or,

New steel area $=(1.15)\left(\frac{97+87}{106+87}\right)=1.10$ sq. in. per rib.

This area may be secured by using $11 / 4^{\prime \prime}$ rounds and $11 / 8^{\prime \prime}$ rounds in alternate ribs or $2-3 / 4^{\prime \prime}$ squares in each rib. If the $12^{\prime \prime}+2^{\prime \prime}$ slab is used the table area of 1.04 square inches may be similarly reduced to 0.96 square inch and a $11 / 8$ " round bar placed in each rib.

Concrete Ribbed Slabs-Steel or Wood Forms. Consider a floor slab of $21^{\prime}-0^{\prime \prime}$ span continuous on one end only. The floor is to carry a live load of 80 pounds per square foot, a wood floor on cinder concrete fill weighing 18 pounds per square foot and a ceiling of plaster on metal lath weighing 10 pounds per square foot, giving a total superimposed load of 108 pounds per square foot.

The table on page 74 gives for a $12^{\prime \prime}+3^{\prime \prime}$ slab reinforced with 1.30 square inches of steel in each rib a carrying capacity of 122 pounds per square foot. As this is in excess of the requirements the table area of steel may be reduced as in the previous example and an area of 1.20 square inches used. This area is secured by using $2-7 / 8$ " round bars in each rib.

Tee-Beams, - Continuous over supports. Determine size and reinforcement of a beam in a floor construction assuming the span to be 22 feet, the superimposed load 200 pounds per square foot, the floor slab $4 \frac{1}{2} 2^{\prime \prime}$ thick and the distance center to center of beams as $7^{\prime}-0^{\prime \prime}$. The beam is continuous over supports and the unit stresses employed are to be as follows: $f_{\mathrm{B}}, 16,000 ; f_{\mathrm{c}}, 650$; and $v, 120$. Haunches are not to be used at the ends of the beams. This represents the type of beam usually encountered in building construction commonly referred to as a T-beam, but due to the continuity at supports its capacity must necessarily be rated on the section of the web of the beam rather than upon the T-section at center of span.

From the data given the total load per square foot of floor, including the weight of the slab, is found to be 256 pounds per square foot, or 1,792 pounds per linear foot of beam. By referring to the table on page $9 S$ it will be found that a $10^{\prime \prime} \times 26^{\prime \prime}$ beam reinforced with $4-1^{\prime \prime}$ round bars will carry, exclusive of the weight of beam, 1,927 pounds, which from a practical standpoint fulfills the conditions of the problem. 
If it is required to maintain a minimum depth of beam it will be found that a $14^{\prime \prime} \times 22^{\prime \prime}$ beam, reinforced with $6-7 / 8^{\prime \prime}$ round bars will carry the load. It will be noted, however, that the shallower beam requires more steel and concrete.

To determine the number of stirrups required for the $10^{\prime \prime} \times 26^{\prime \prime}$ beam, find the end shear.

$$
v=\frac{(2,062)\left(\frac{22}{2}\right)}{\left(\frac{7}{8}\right)(10)(23)}=113 \mathrm{lb} . \text { per sq. in. }
$$

From the table on page 107 for an end shear of 120 pounds per square inch a 10 -inch beam of 22 foot span requires $20-3 / 8^{\prime \prime}$ round stirrups, and for an end shear of 100 pounds per square inch, $14-3 / 8$ " round stirrups. As the actual end shear is 113 pounds per square inch, by interpolation, $18-3 / 8$ " round stirrups will be sufficient.

In the tables which follow the endeavor has been made to give a fairly wide range of values from which to make the desired selection of size of member and reinforcement required, so that knowing the load and span the designer may enter the tables and choose the beam or slab which best meets the needs of his particular case, much as he would select a beam from a safe load table in a structural steel handbook. 


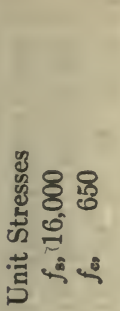

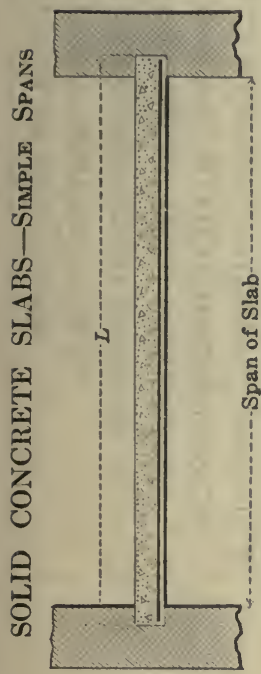

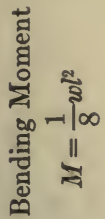

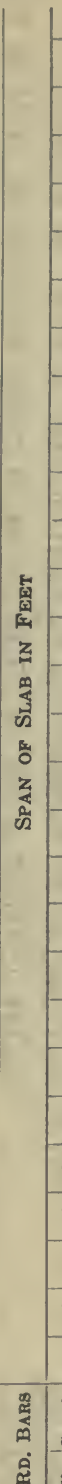

เค 요

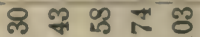
की तี 8 ॠ क न न ๓ ศั

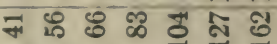
○ 90 c 00 a $\pi$

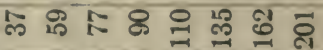

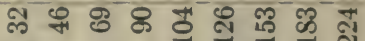

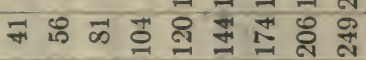

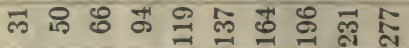
\% 8 \% 8 \% 요

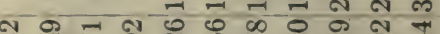
잉하

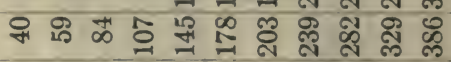

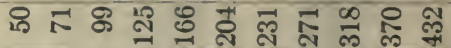

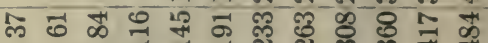
10 0000000 下饮 8

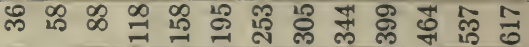

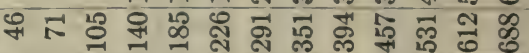

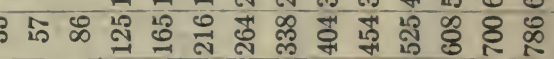

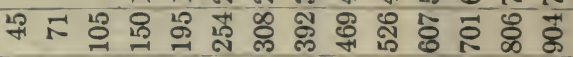

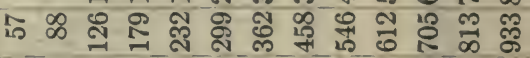

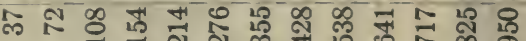
म 1 त त

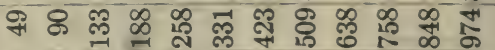
तु ज्ञ ఐ

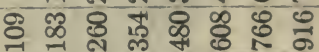

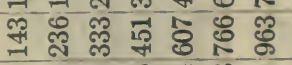

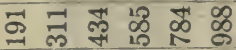

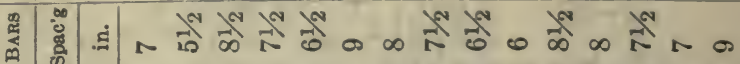

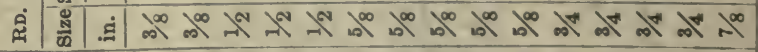

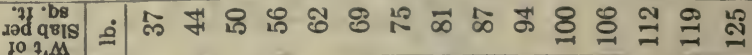
政

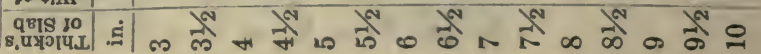




\section{Concrete} Slabs

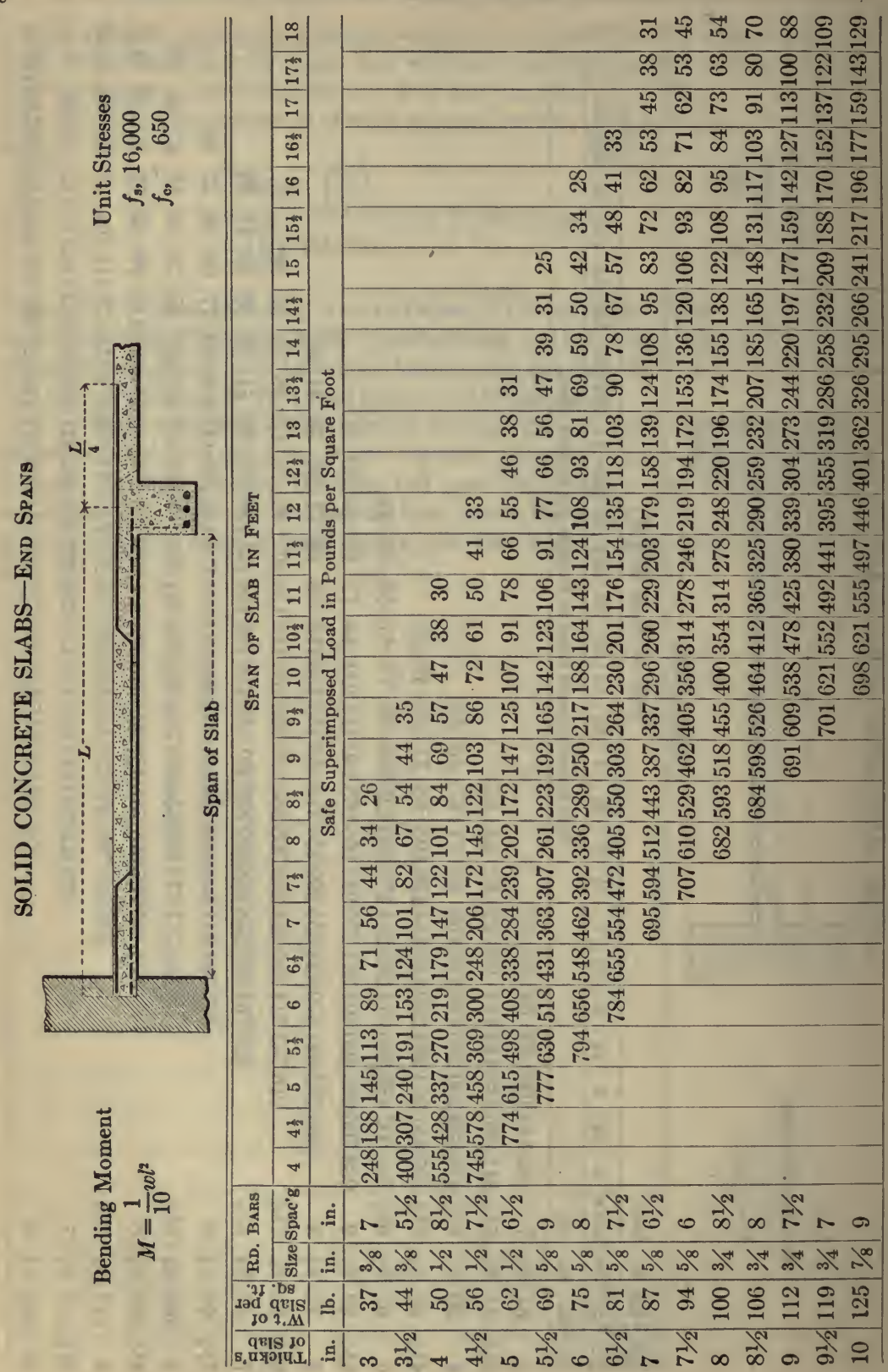




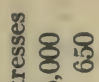

芩

言
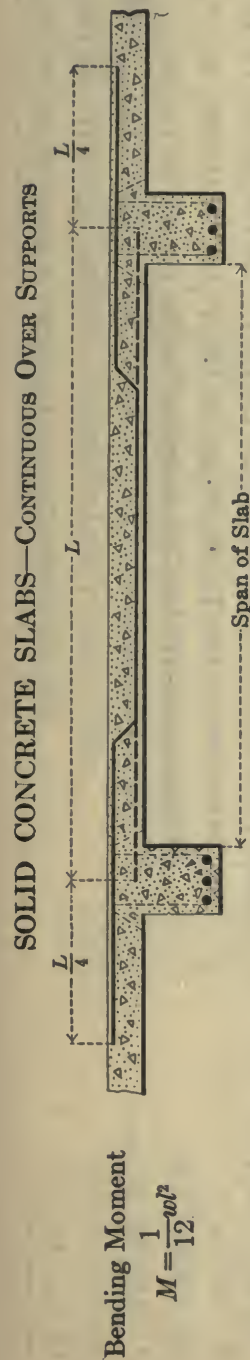

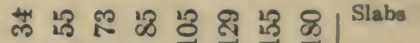
₹ एँ क्ष न

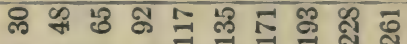
ధొ

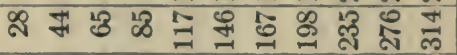
ल

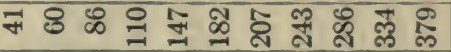

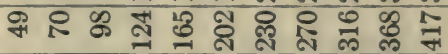
艁

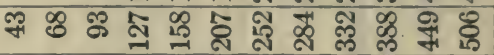

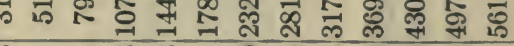

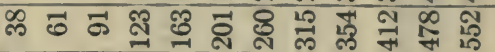

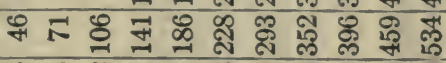
ג施

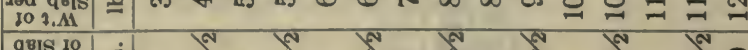

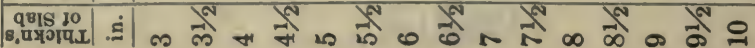




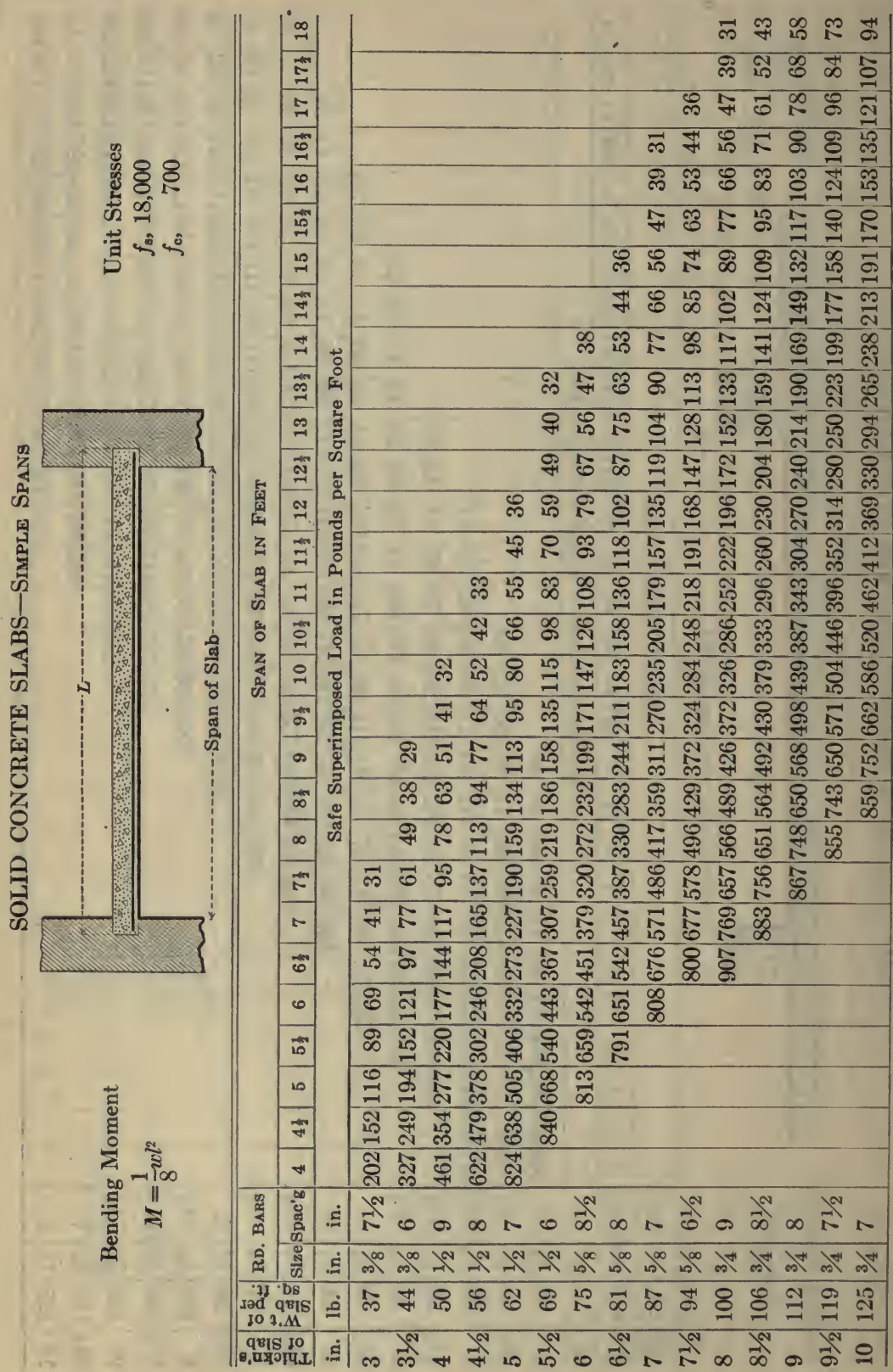



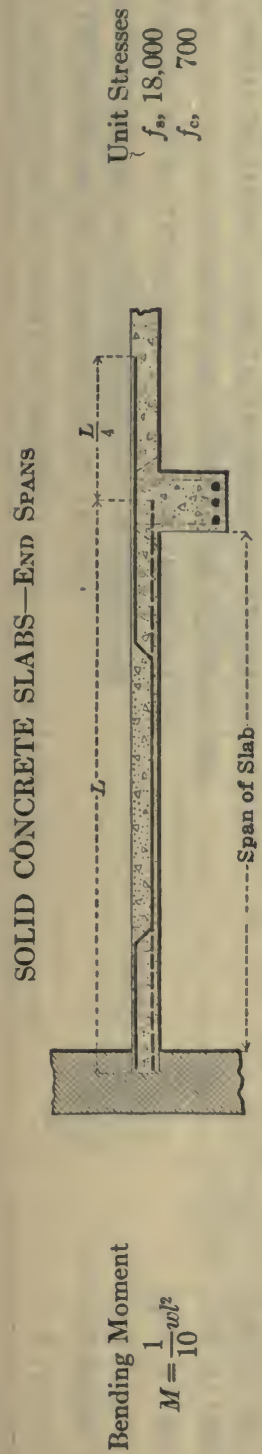

ผิ भु 8 ณ ส

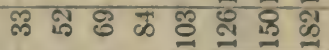
유

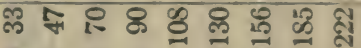
유 ๓ क 18 है 0 की

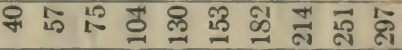

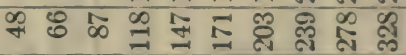

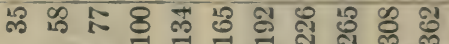
क 50 क

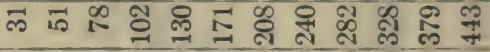
क

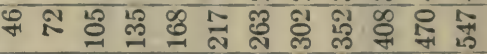

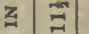
क क 露

응

$\circ$

$\infty$

$\infty$ 放

$\infty$ \%

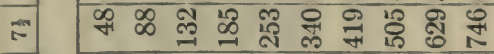

- उ 음

- 0 त్

ஜ ญ

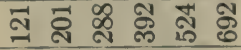

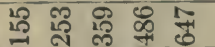

है तै 10 मे

न ने

त्ष

离 ณ

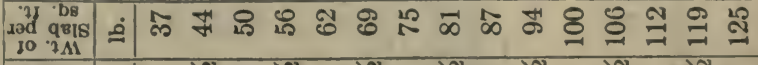

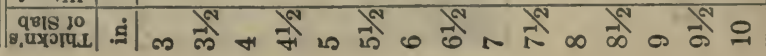



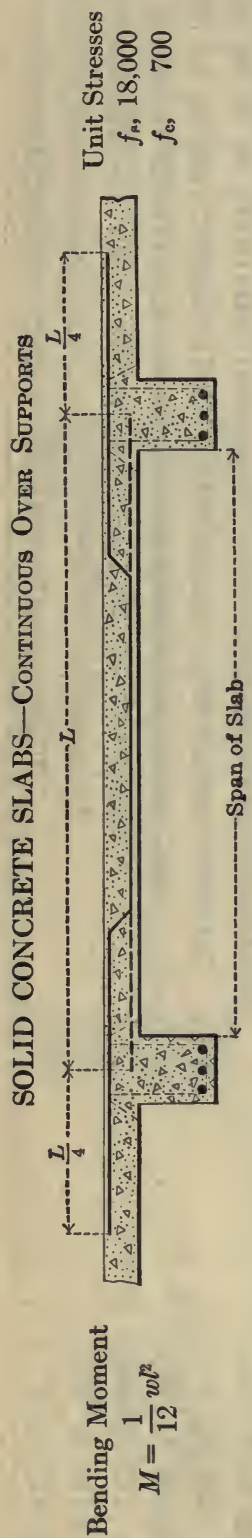

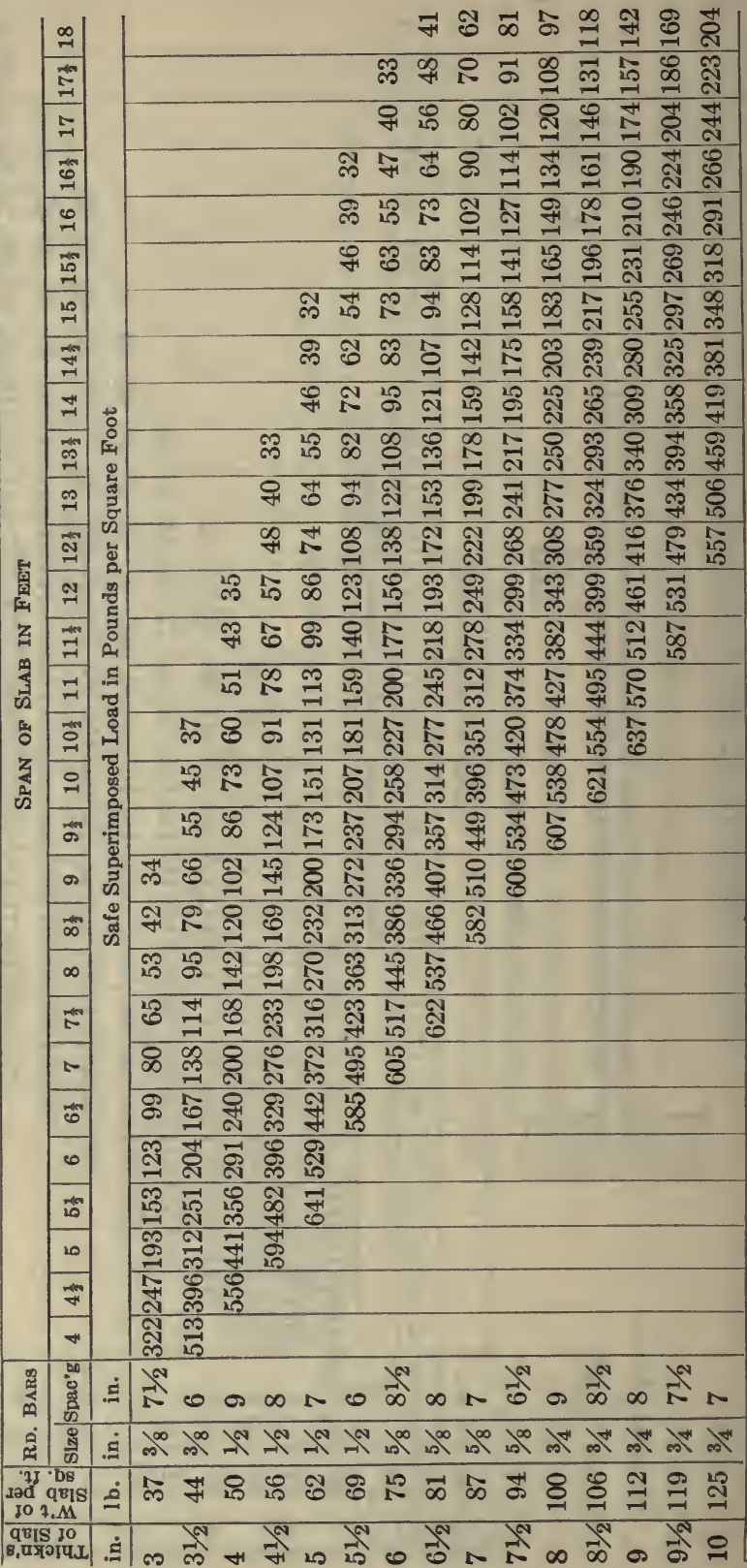




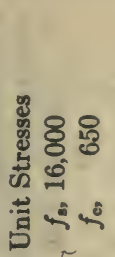

लै के मे है थ

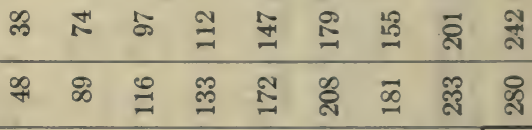

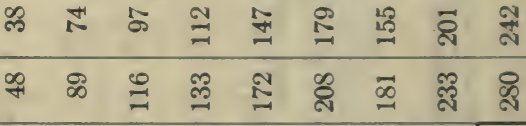

म

7

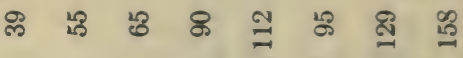

ஒ

8 ळ

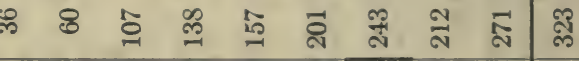

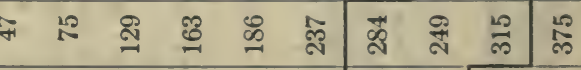
ஜ 8 \%

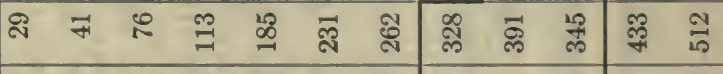
ㄴ 18 \&

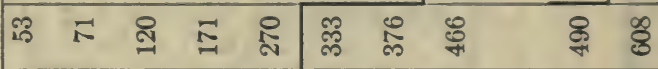
R \& ๙ิ ๙

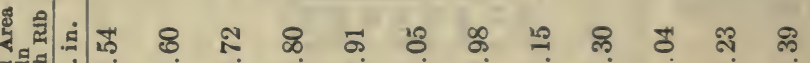

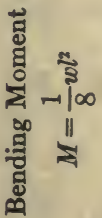

母ี

这离

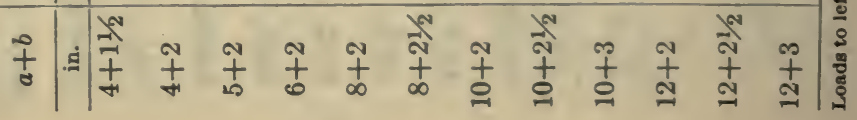




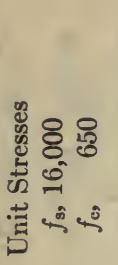

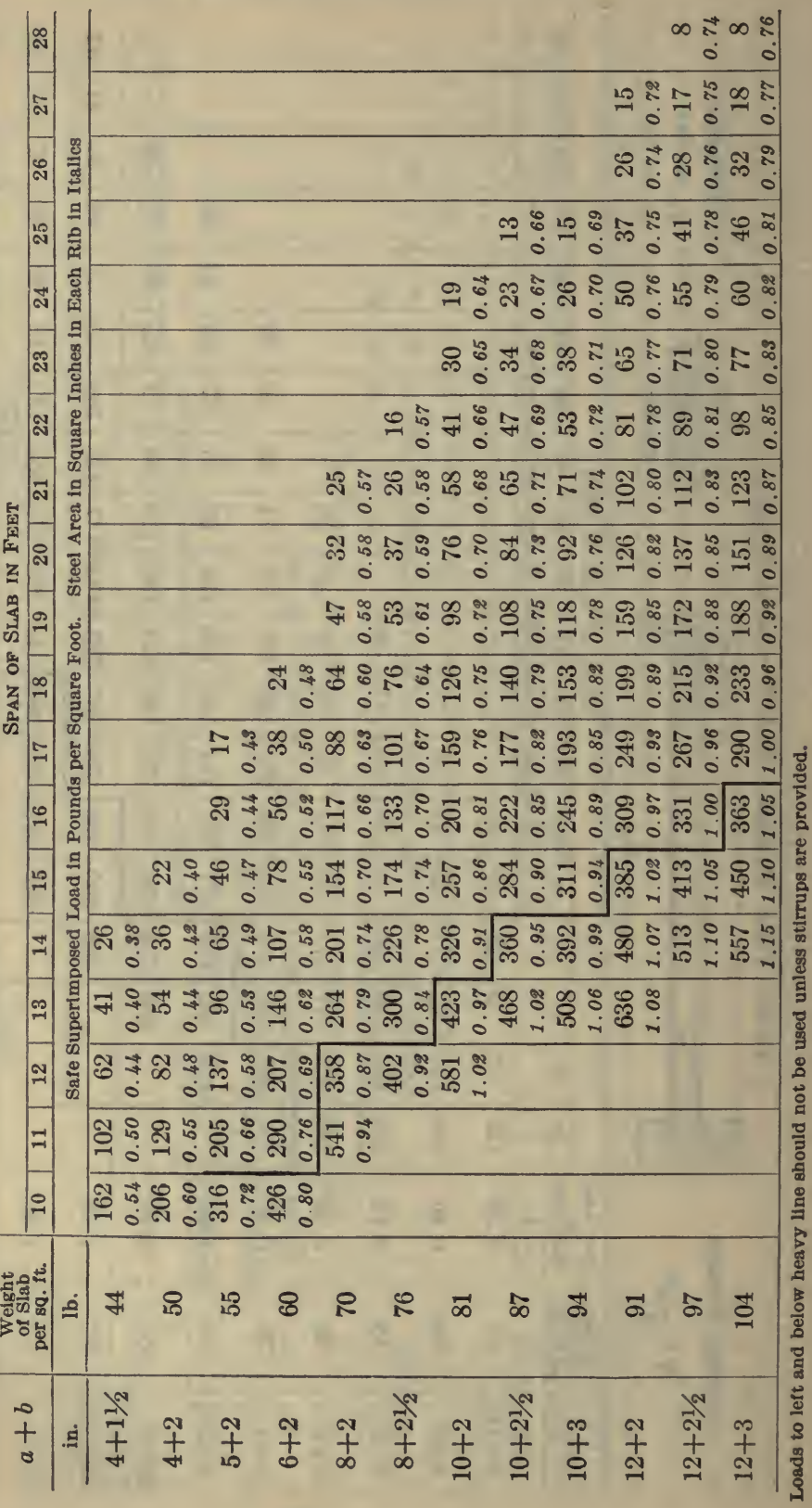



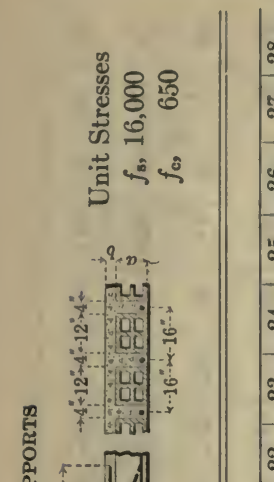

ลี

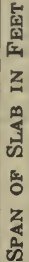

พิ

ก

范

N

ง

参

I

8



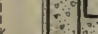

$+010$

(1)... 4

ह

(

离

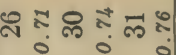
ஸे ₹ 000

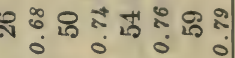

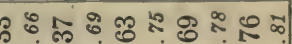

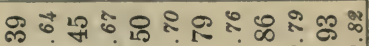
$\begin{array}{llllll}0 & 0 & 0 & 0 & 0\end{array}$ กิ่ ณ - 0 0 0 元

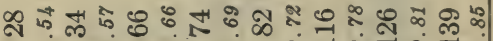
$\begin{array}{lllllll}0 & 0 & 0 & 0 & 0 & 0 & 0\end{array}$ 윤

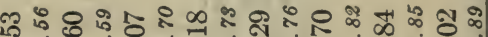
0 : 0 :

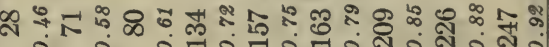
० 0 ० 0 月

10 ㄴำำㅇำ

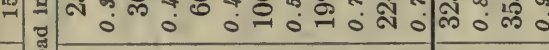

\# 역

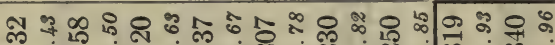

m

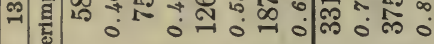

귝

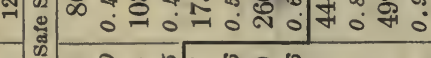

is

$\exists$

กี นกำ

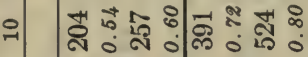

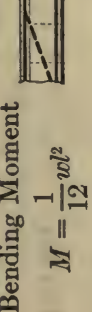

\section{$\because$}

둥워 땜ำ

H

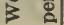

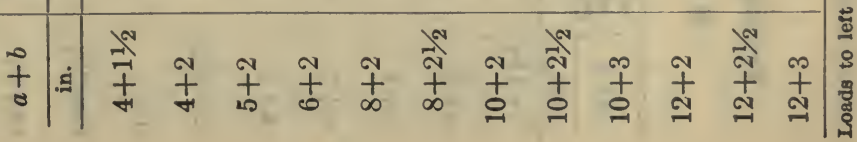


CORRUGATED BAR COMPANY, INC.

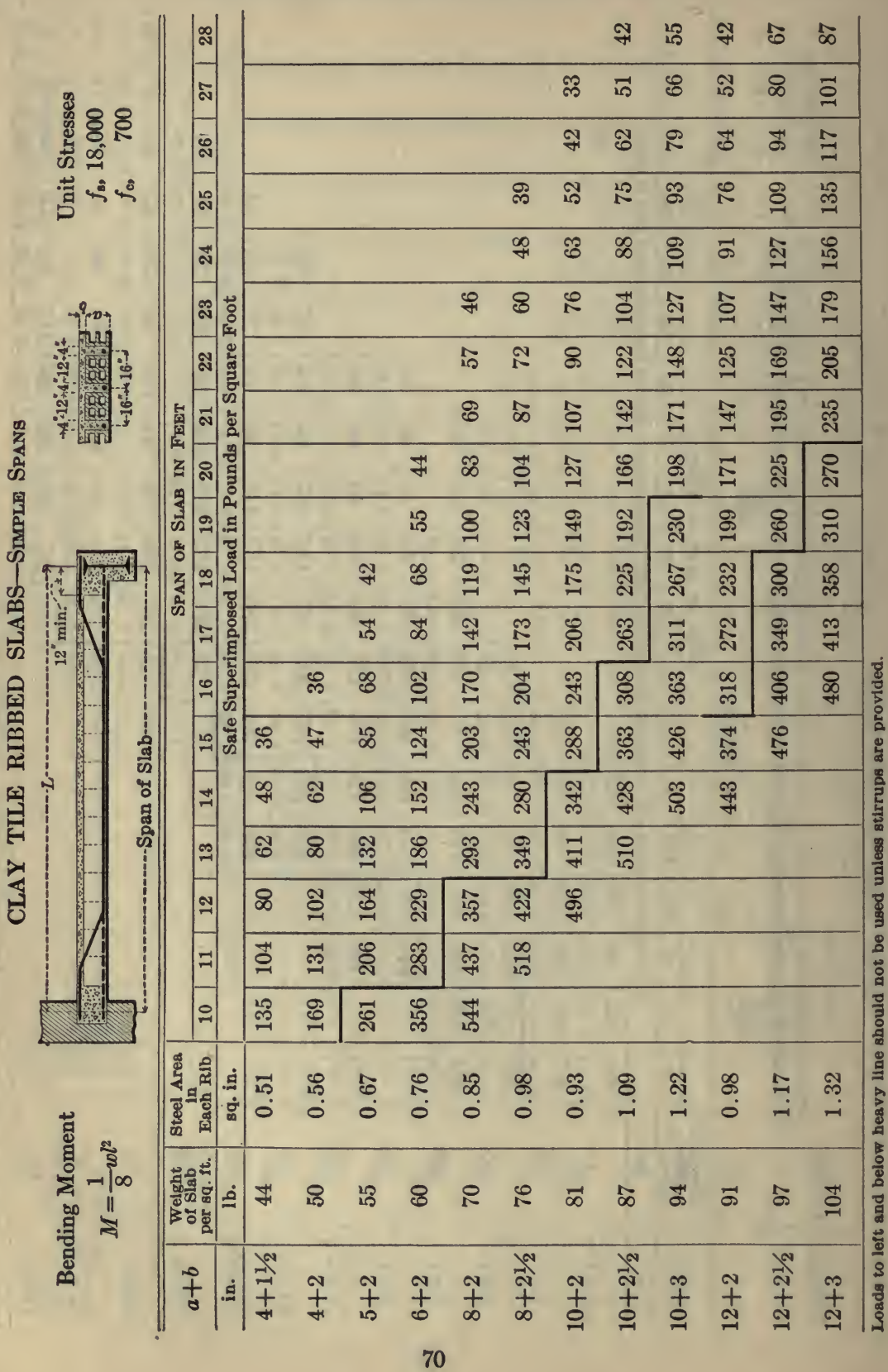



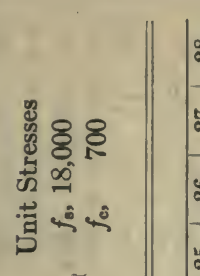

$=$ 숭

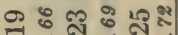
- $0^{\circ} 0$

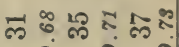
- 0 0

is

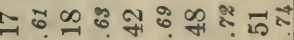

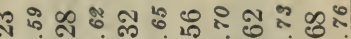
- 0 o 0 0.

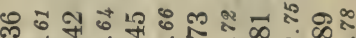
24 * - 엉

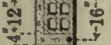
- 0 ० 0 ० กิ - 000000 ๙

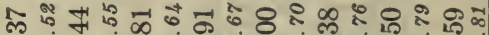

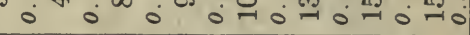
ก็ แี่ os 0 0 0 0 :

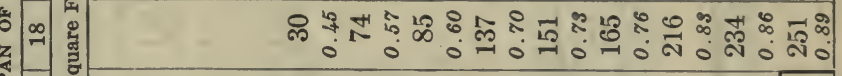

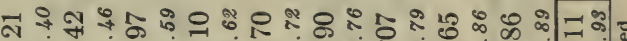
$=$

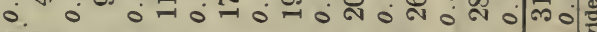

䄈

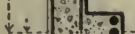
, $x$ o 1: $4-1$ . if .
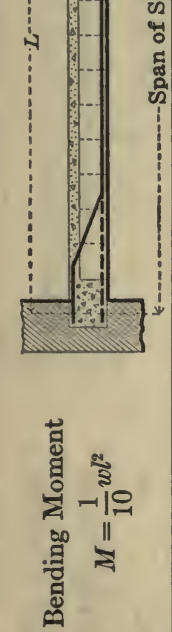

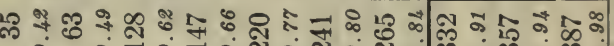

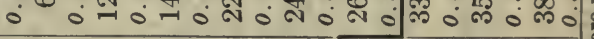

드 พ

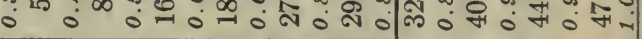

శ్ 然

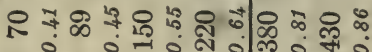

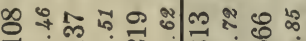

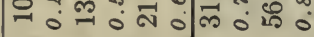

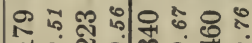
- 0 ล 
USEF UL DATA
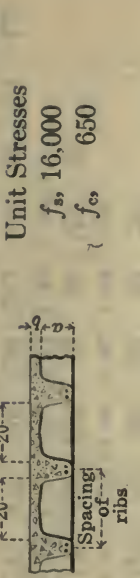


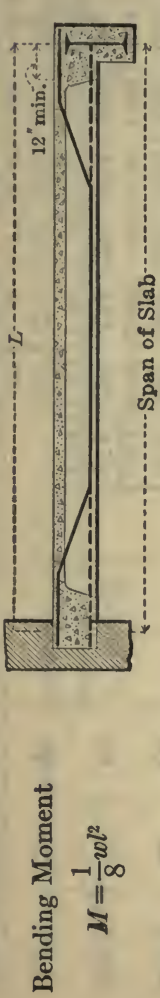

उ

\begin{tabular}{|c|c|c|c|c|c|c|c|c|c|c|c|}
\hline 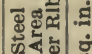 & సे. & ণै & ஸे & $\stackrel{\mathscr{m}}{\stackrel{2}{*}}$ & से & $\infty$ & ช & ๒్ & ? & $\stackrel{\sim}{\sim}$ & ণै \\
\hline . & & & & & -1 & $\pi$ & $N$ & & -1 & N & $N$ \\
\hline 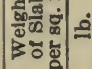 & $\mathscr{f}$ & $\stackrel{\infty}{\sim}$ & กิ & เกิ & ญ & $\infty$ & 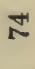 & 오 & $\stackrel{0}{N}$ & ஜ & $\infty$ \\
\hline
\end{tabular}

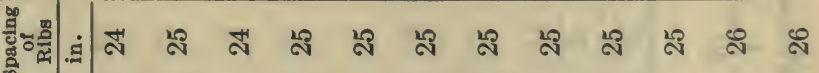

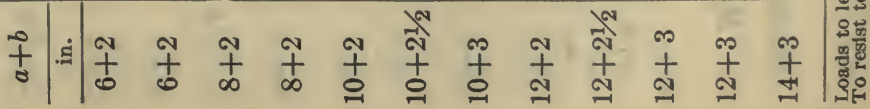



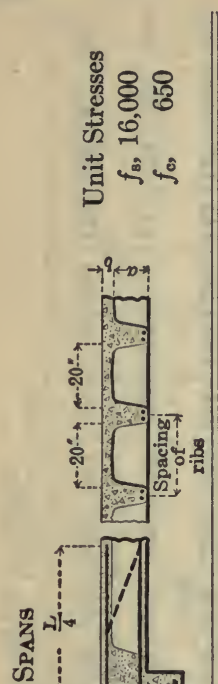

至

空

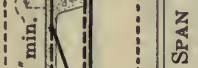

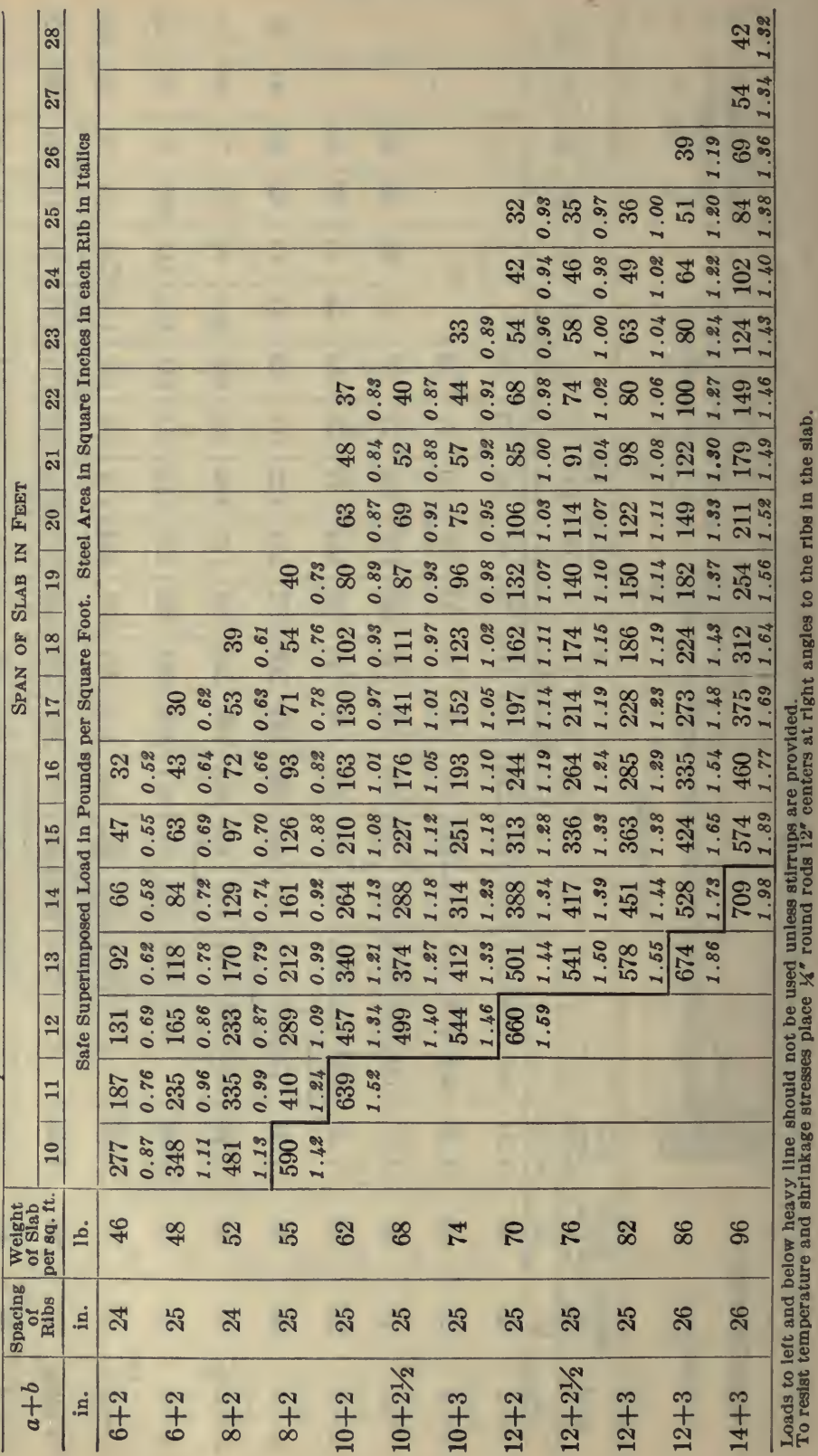




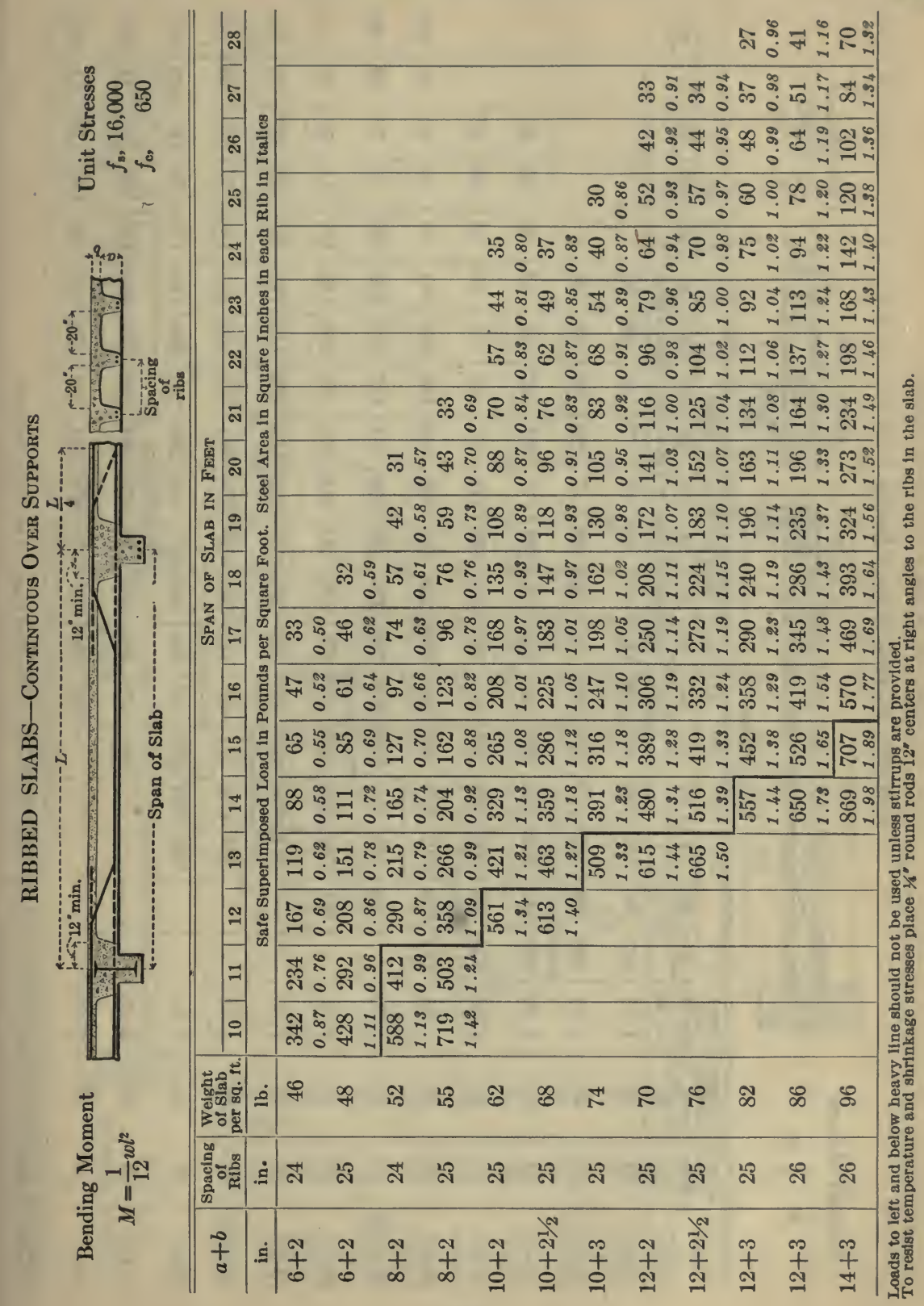




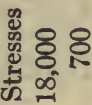

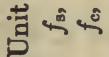
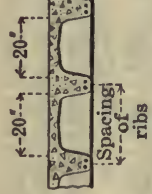

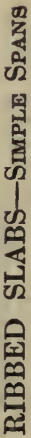
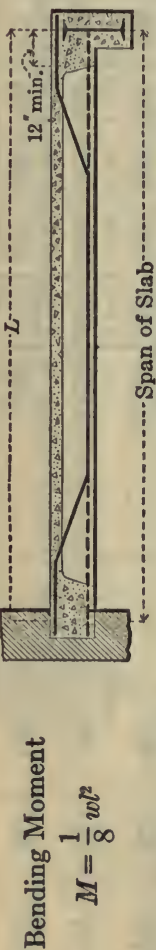

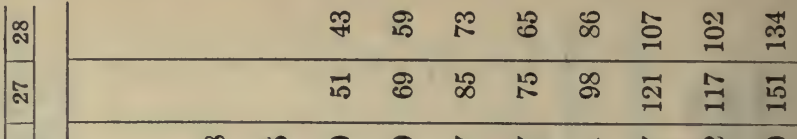

$\stackrel{\leftrightarrow}{*}$ $\infty$ ద \& \& भ भ

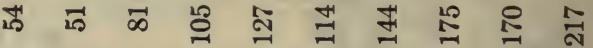

เึ ๓

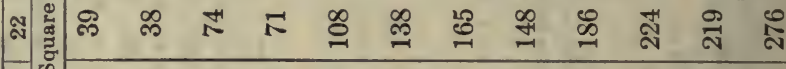
สี z

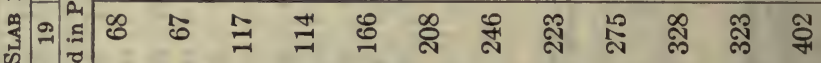
部敬

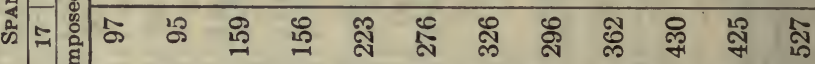
-

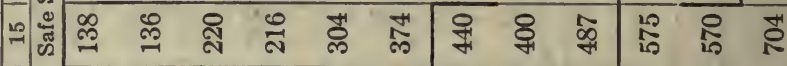
ㄱ|

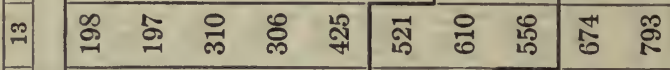

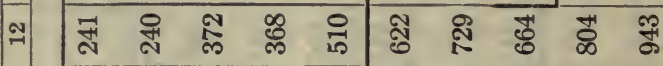

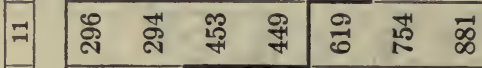
$\therefore$ 일

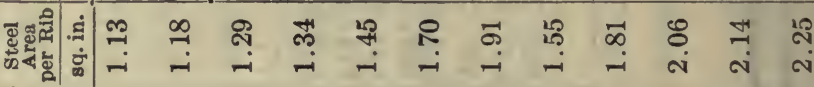
वृำ

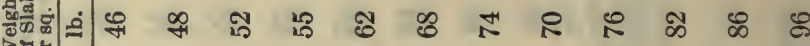
ऐ능 क्षे

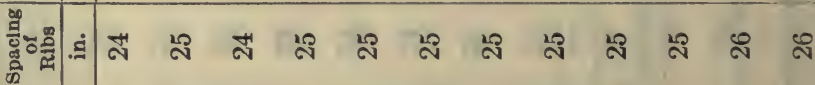

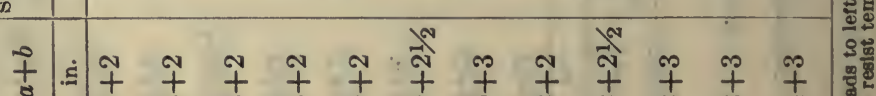

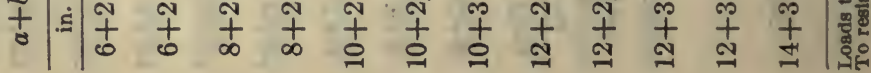




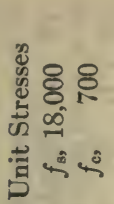

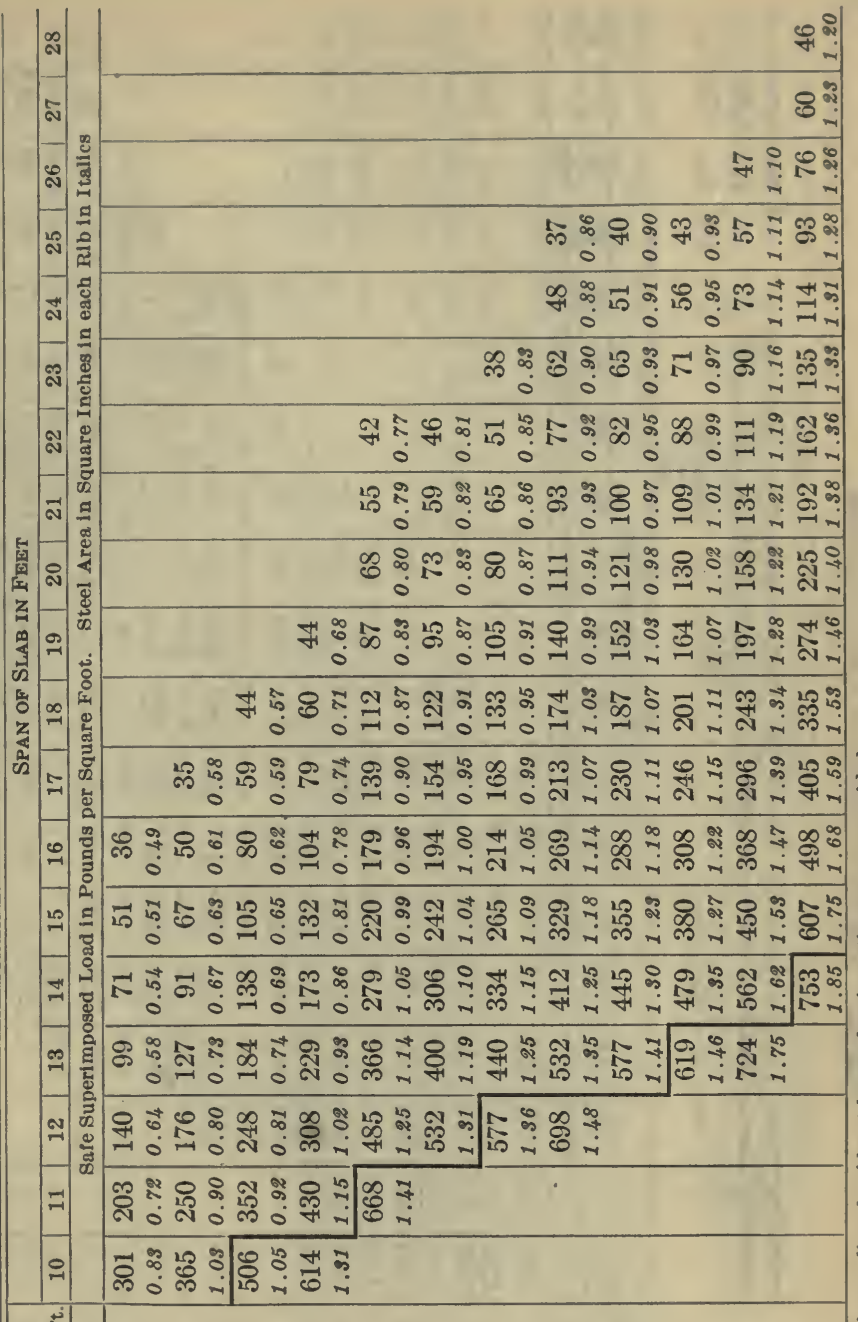

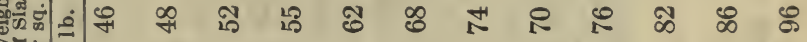

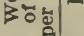

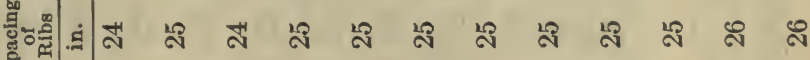

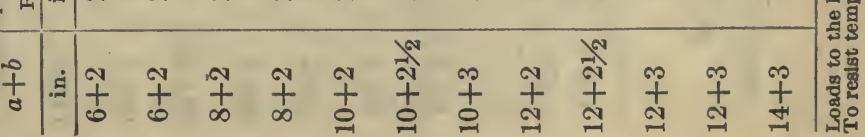




\section{造8 8}
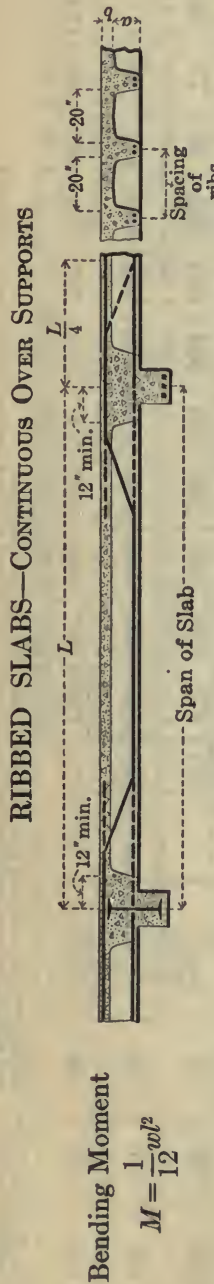

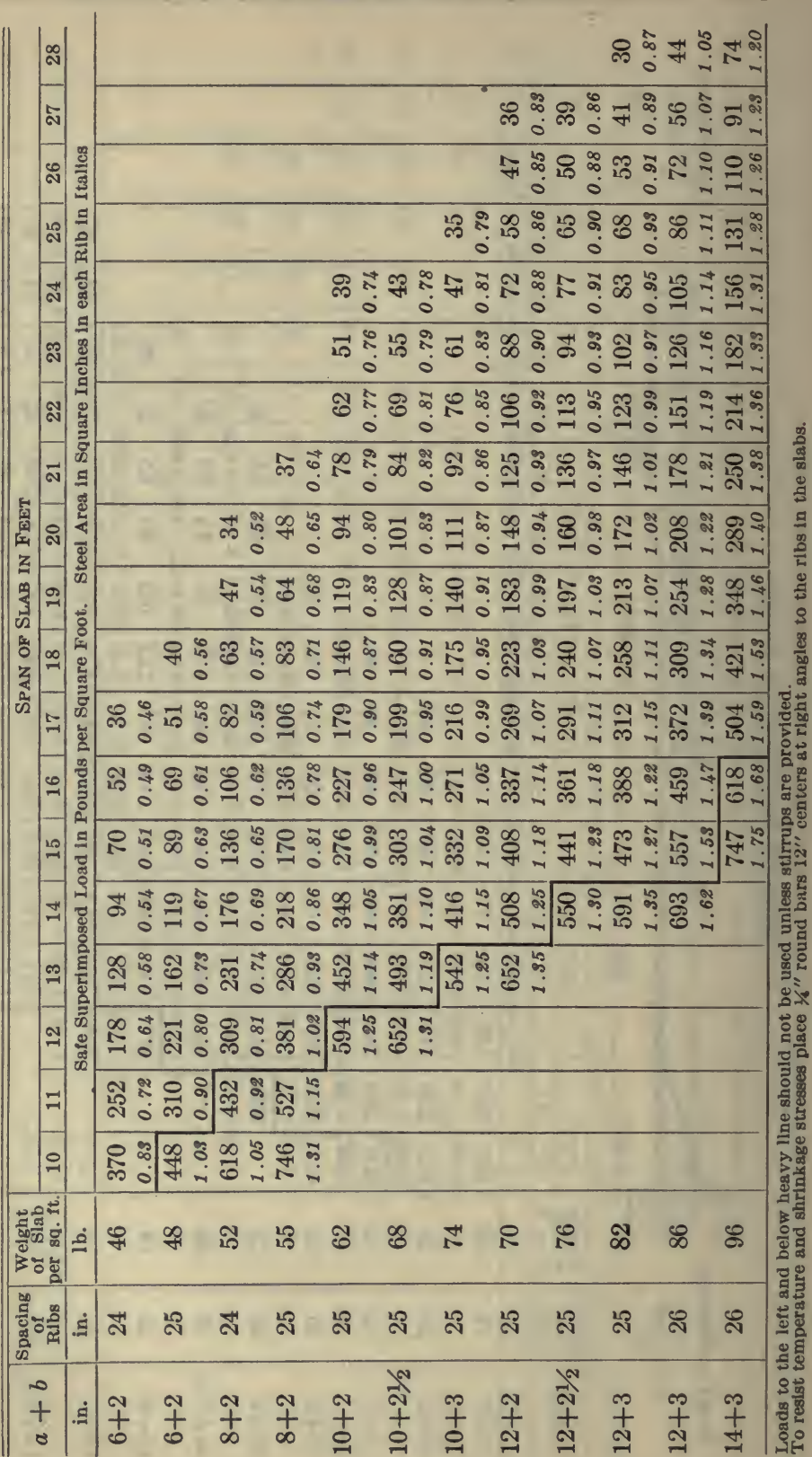




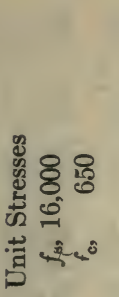

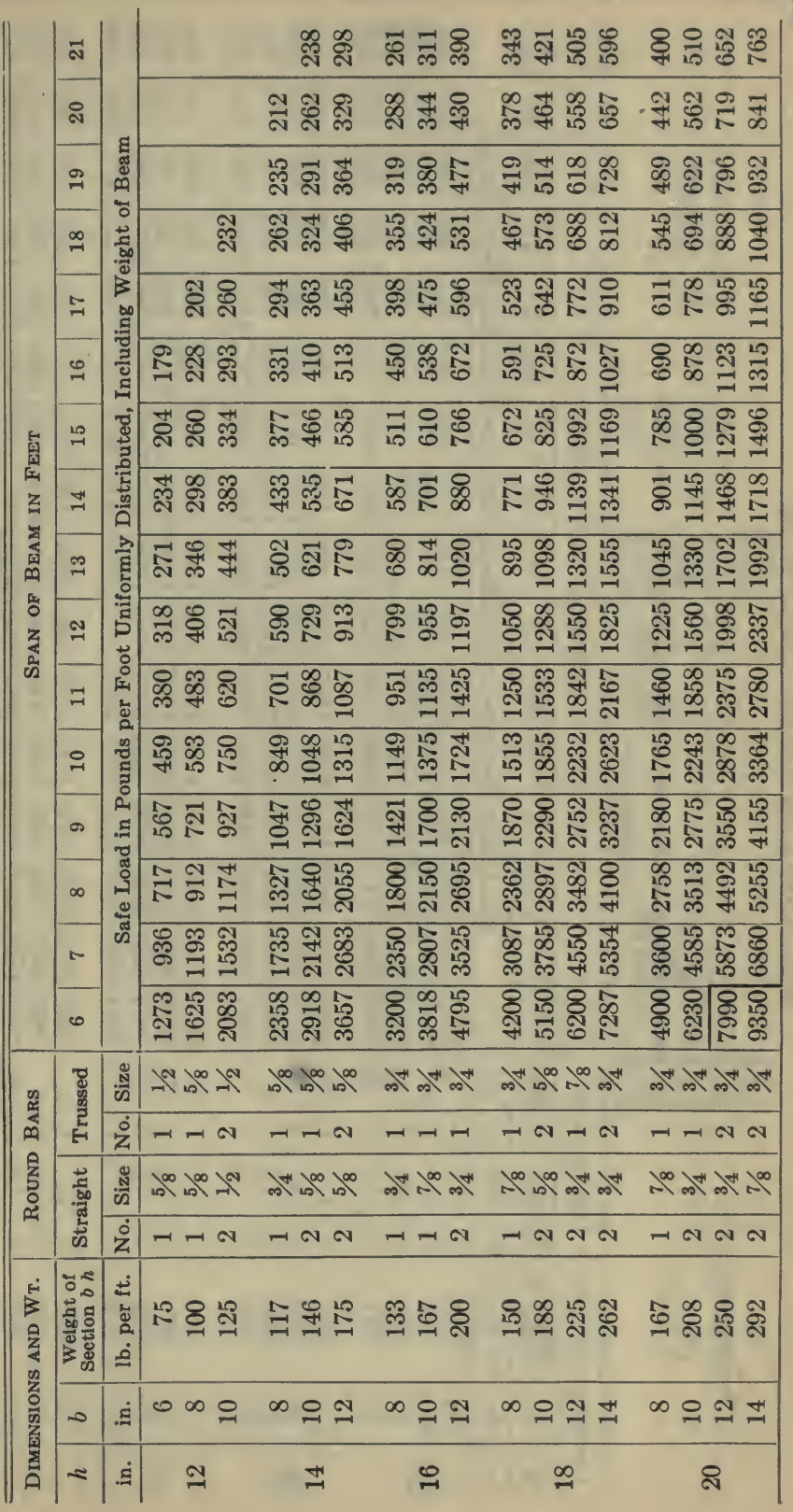


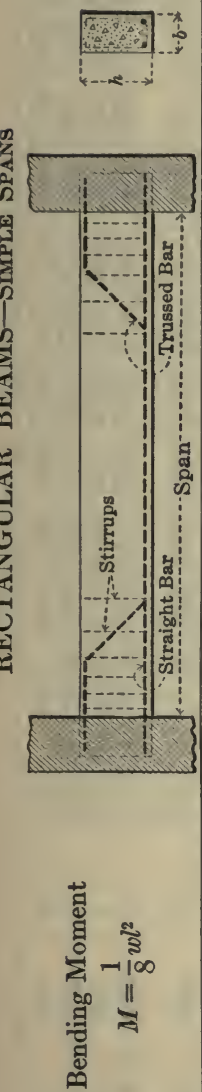


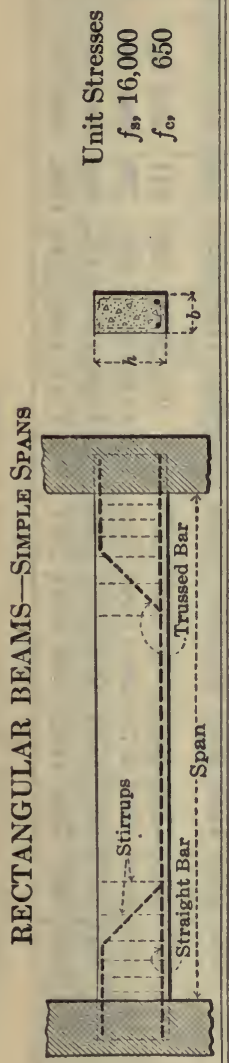

\begin{tabular}{|c|c|c|c|c|c|c|c|}
\hline & เ2 & & 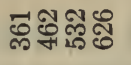 & 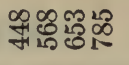 & 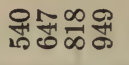 & 응్ㅗำ & 으ㅇㅝㅡ \\
\hline & సే & & 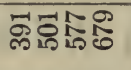 & 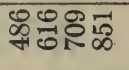 & 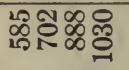 & ๑ొన్ & की \\
\hline & ลึ & ตू๊ & 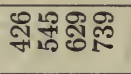 & คํㅠํำ & 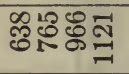 & 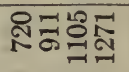 & 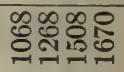 \\
\hline & สิ & $\overrightarrow{\overparen{L}}$ & 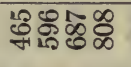 & 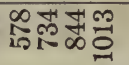 & స్రిల్నిని & 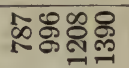 & 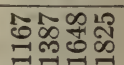 \\
\hline & $\vec{N}$ & हี & 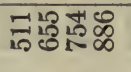 & 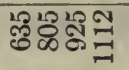 & 이요 & ర్రొนิ ผิ & 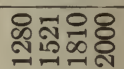 \\
\hline & สิ & כूँّ & 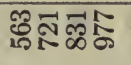 & క్రం శ్రిస్త & 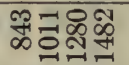 & 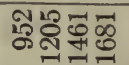 & 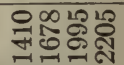 \\
\hline & 9 & 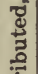 & 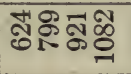 & 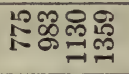 & 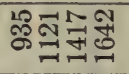 & 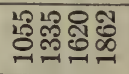 & 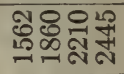 \\
\hline & $\stackrel{\infty}{-1}$ & $\begin{array}{l}5 \\
\text { 总 } \\
\text { ดी }\end{array}$ & 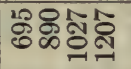 & 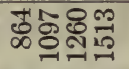 & $\begin{array}{l}\text { 유융유 } \\
\text { 엄서메 }\end{array}$ & $\begin{array}{l}\text { 요유 } \\
\text { 멈윰유 }\end{array}$ & $\begin{array}{l}\text { 융ㅇㅇ } \\
\text { 듀유 }\end{array}$ \\
\hline 8 & $\Xi$ & हू & 요요 & 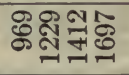 & 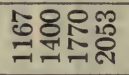 & 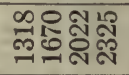 & 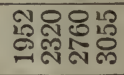 \\
\hline & $\stackrel{\oplus}{-1}$ & है & 유요 & 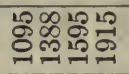 & 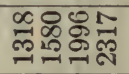 & 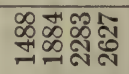 & 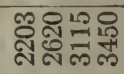 \\
\hline & 10 & 后 & 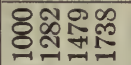 & 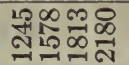 & 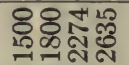 & 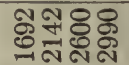 & 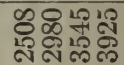 \\
\hline & 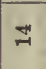 & 몰 & 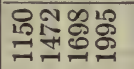 & 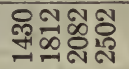 & 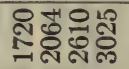 & 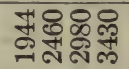 & 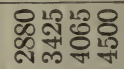 \\
\hline & $\stackrel{2}{\sim}$ & D & 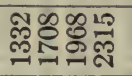 & 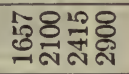 & 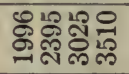 & 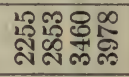 & 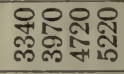 \\
\hline & N & 3 & 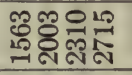 & 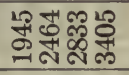 & 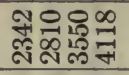 & 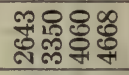 & 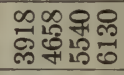 \\
\hline & 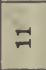 & & 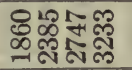 & 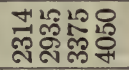 & 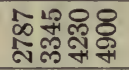 & 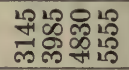 & 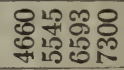 \\
\hline & 우 & & 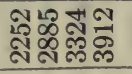 & 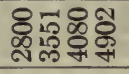 & 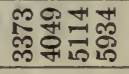 & 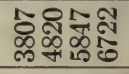 & 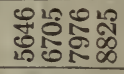 \\
\hline & 总 & 䒩 & $\mathrm{N}^{\infty}$ のர் & $\mathrm{N}^{\infty}{ }^{+1} \mathrm{~N}^{\infty}$ & $-{ }^{2}$ & $-\pi^{2}$ & $\stackrel{\infty}{=}=$ \\
\hline & $\underset{E}{E}$ & $\dot{\mathrm{z}}$ & - INN & -NーN & ПーNN & ーーNN & - \\
\hline$z$ & 苛 & 苋 & $N^{\infty} \infty \mathbb{N}^{\infty}$ & $-{ }^{2}$ & $n^{\infty} N_{-1}^{\infty}$ & & \\
\hline & 总 & $\dot{0}$ & $\mathrm{NON}$ & ーNNN & TONON & -NNN & NNNM \\
\hline 宓 & 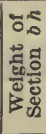 & 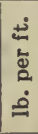 & 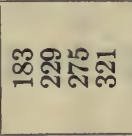 & 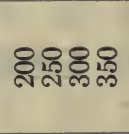 & 그슈ำ & 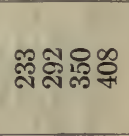 & 뚱요 \\
\hline tis & $\infty$ & . & $\infty \subseteq$ & $\infty$ & 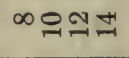 & $\infty$ & \pm 0 \\
\hline & s & $\cong$ & ลี & $\sim$ & N & 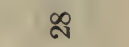 & లి \\
\hline
\end{tabular}

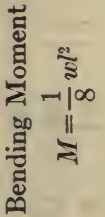




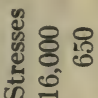

㤩

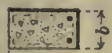

(k-......
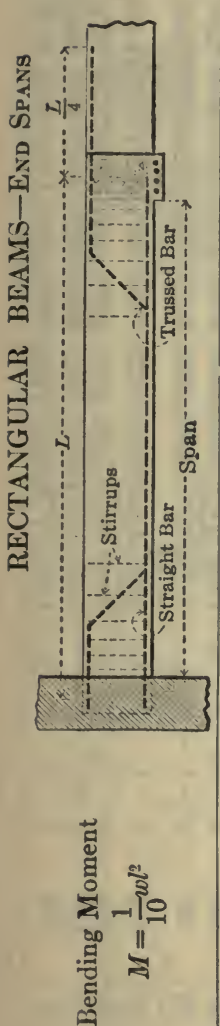

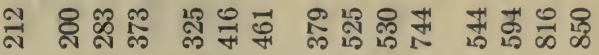

๓ 유 ิㅗ

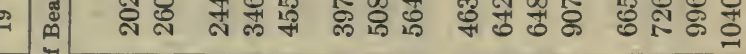

N

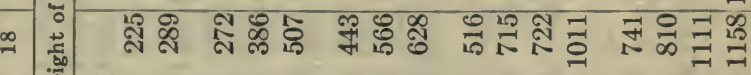
$\Rightarrow$ त

$=$ ๙

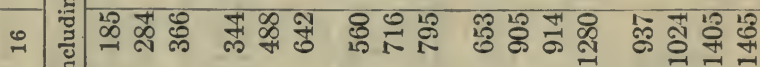

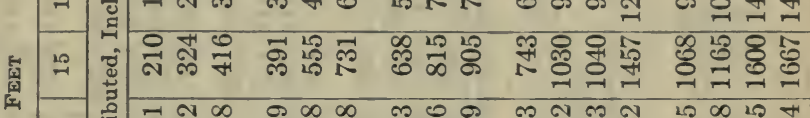
z

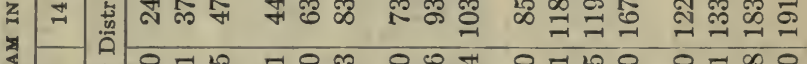

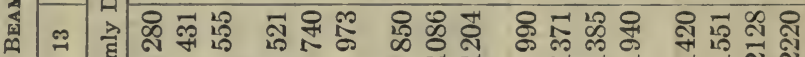
约 竞

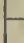

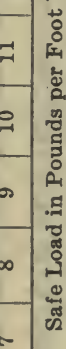
๙ิ

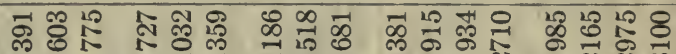

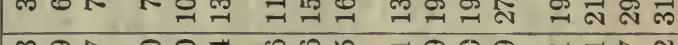
๓ -1-1 - -

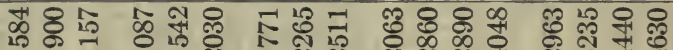
म

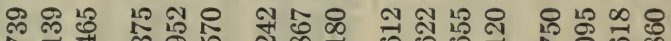
퍼

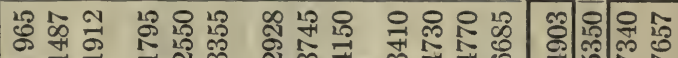

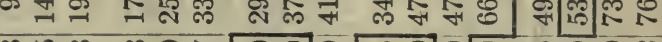

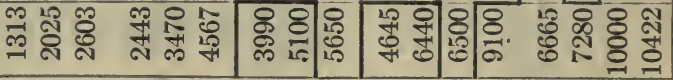

\begin{tabular}{|c|c|c|c|c|c|c|}
\hline T & $\stackrel{\mathbb{N}}{\sqrt{2}}$ & $\left.\lambda^{N}\right)^{\infty} \lambda^{N}$ & $\omega^{\infty} \infty \pi^{\infty}$ & $\infty \mathbb{N}^{\infty} \mathrm{N}^{\infty}$ & का मी $^{\infty}$ की लो & 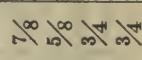 \\
\hline Е & ¿ & 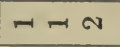 & 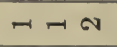 & TrN & $\neg N N C$ & $\neg N N N$ \\
\hline 蕗 & घี & $\lambda^{N} \infty^{\infty} \overbrace{}^{N}$ & $\infty^{\infty} \omega^{+1} \boldsymbol{D}^{\infty}$ & $\infty \mathbb{N}^{\infty} \mathrm{N}^{\infty}$ & 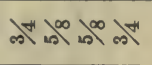 & 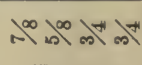 \\
\hline 峁 & z & $r-N$ & 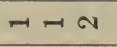 & $\neg-\infty$ & - N N N & $\neg N \propto N$ \\
\hline
\end{tabular}
सं

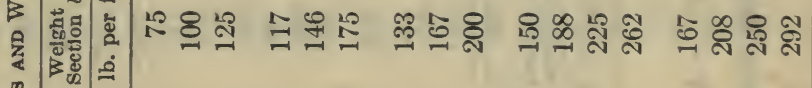
量

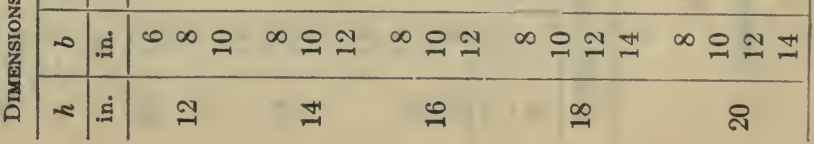



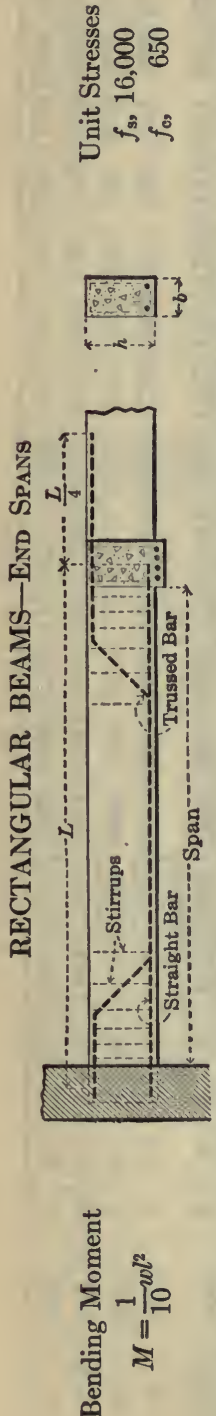

\begin{tabular}{|c|c|c|c|c|c|c|}
\hline & \multirow{6}{*}{ 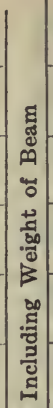 } & 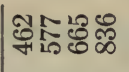 & Ұำ & ธำ 응 & $1 \infty$ 든 & ొ స్లిన్సిన్స \\
\hline & & ํํำ సิ & న్⿵⺆⿻上丨ీర్లి & 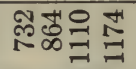 & 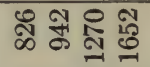 & $\begin{array}{l}\infty \\
0 \\
0\end{array}$ \\
\hline & & 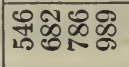 & 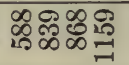 & 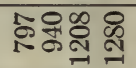 & 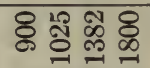 & 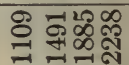 \\
\hline & & 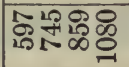 & 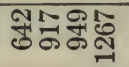 & 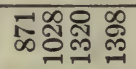 & 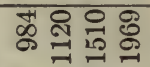 & 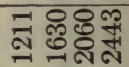 \\
\hline & & 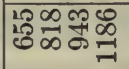 & 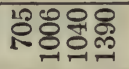 & 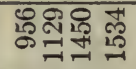 & $\begin{array}{l}0 \\
\text { की } \\
0 \\
0\end{array}$ & 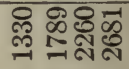 \\
\hline & & 충융్ㅇ & 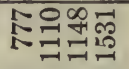 & 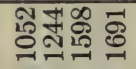 & 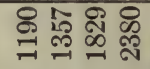 & 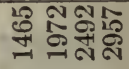 \\
\hline & 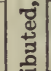 & 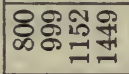 & 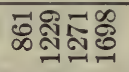 & 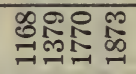 & 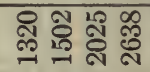 & 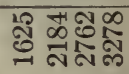 \\
\hline & 常 & 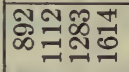 & 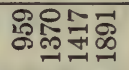 & 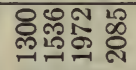 & 욤ำ & 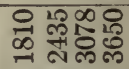 \\
\hline & हू & 용휴역염어 & 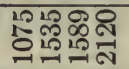 & 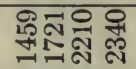 & 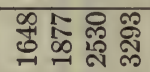 & 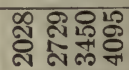 \\
\hline & हี & 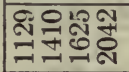 & 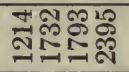 & 屯ี & 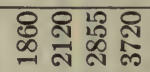 & 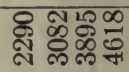 \\
\hline & $\begin{array}{c}: \\
0 \\
4 \\
4\end{array}$ & 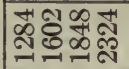 & "-유윰ำ & 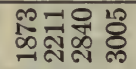 & 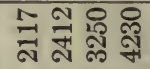 & 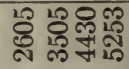 \\
\hline & $\begin{array}{l}n \\
z \\
z\end{array}$ & 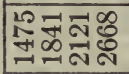 & 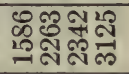 & 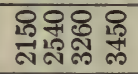 & 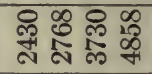 & 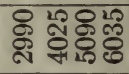 \\
\hline & $\begin{array}{l}0 \\
\text { म } \\
. \approx\end{array}$ & 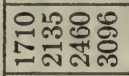 & 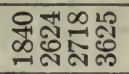 & 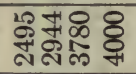 & 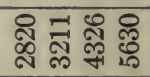 & 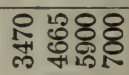 \\
\hline & 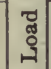 & 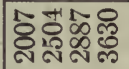 & 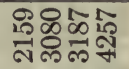 & 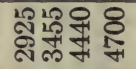 & 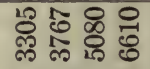 & 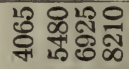 \\
\hline & 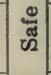 & 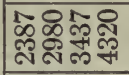 & 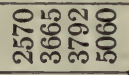 & 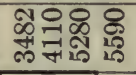 & 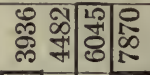 & 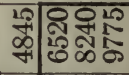 \\
\hline & & 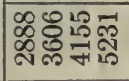 & 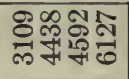 & 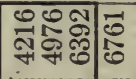 & 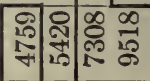 & 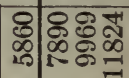 \\
\hline & 茎 & $\infty^{\infty}$ का & 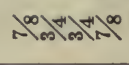 & & & \\
\hline & $\dot{0}$ & N & NON & N $\mathrm{N}$ & - & 6 \\
\hline & 站 & $\infty^{\infty} \infty^{+\pi}$ & నొळ & - लाँ! & & $\stackrel{\infty}{\infty}$ \\
\hline & ż & $\mathrm{N}-1$ & ПNONN & ПNO N & - N N & 016 \\
\hline & 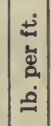 & 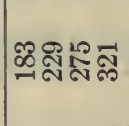 & 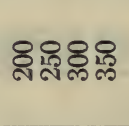 & 듯ำ & ๓ึ क् & 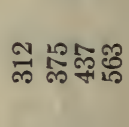 \\
\hline &. & $\infty$ 의 & $\infty$ 으표 & $\infty$ 의 & $\infty \stackrel{ }{N} \subseteq$ & 의 거표 \\
\hline & ஹ્ & ถิ & ฟ่ & สั & & సృ \\
\hline
\end{tabular}


密 80 芯

苞

का (2)

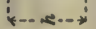
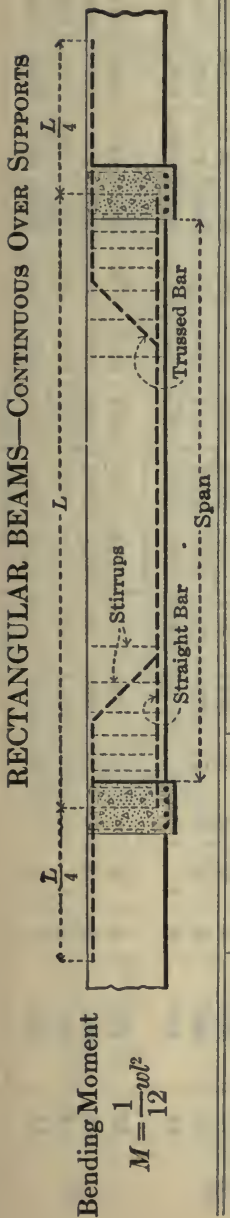

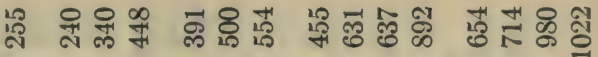

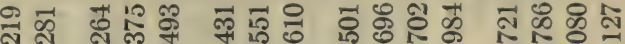
ลำ

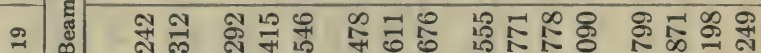

1 다의

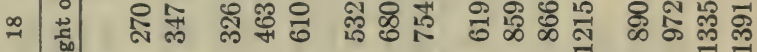
1 舟 $\therefore$ 势

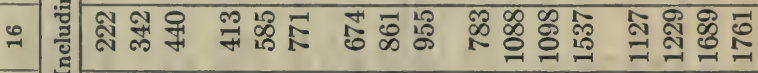

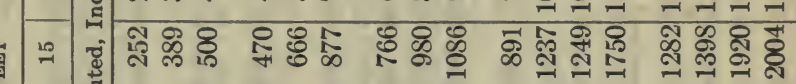
舟 z $\because-\ddot{Q}$

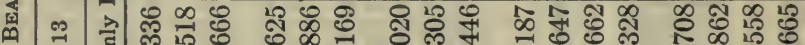
\% 点

ง 空 욤 $\circ$ a. . च 西 $=0,0 \%$

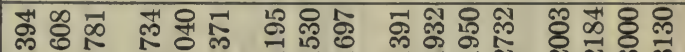

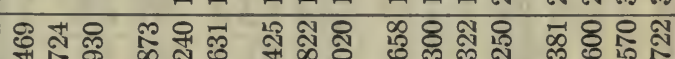
W 舟

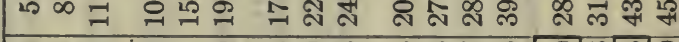
\&8.

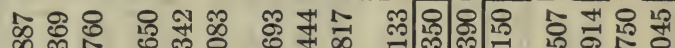

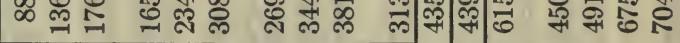
응

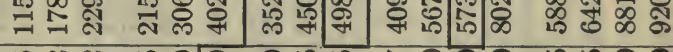

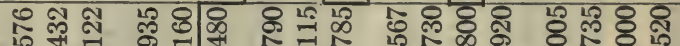
被

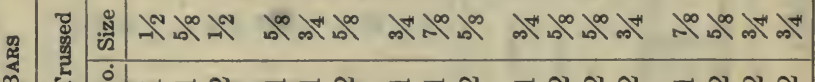

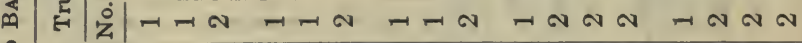

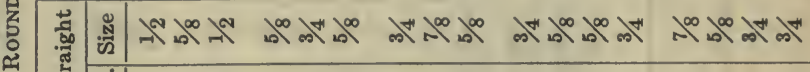

蔗

空 हैं :

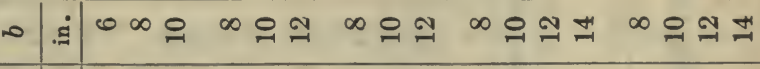
$\sim . \leq$

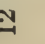

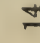
이 $\infty$ \& 

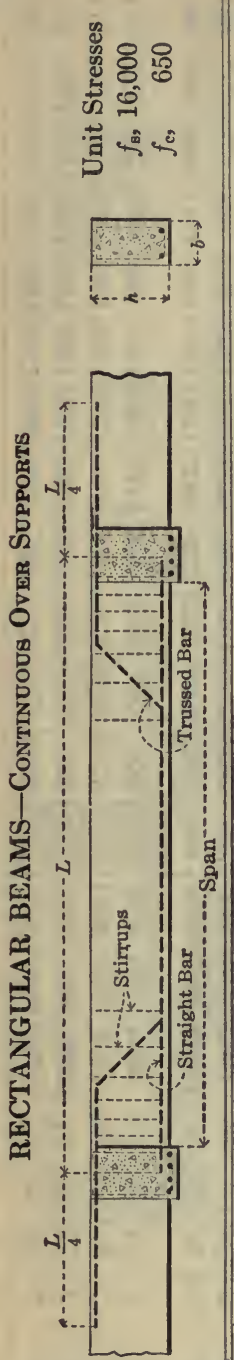

हैं

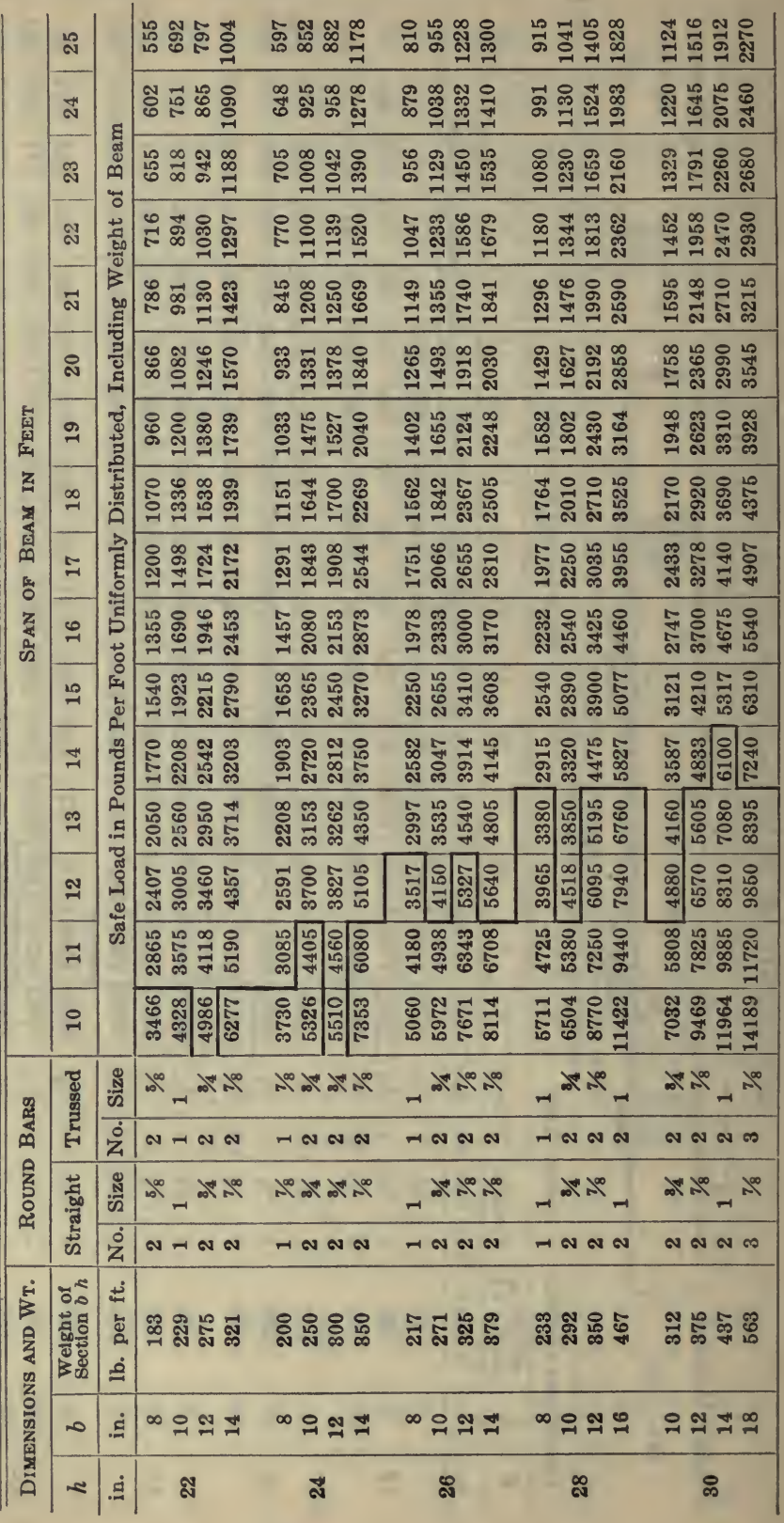




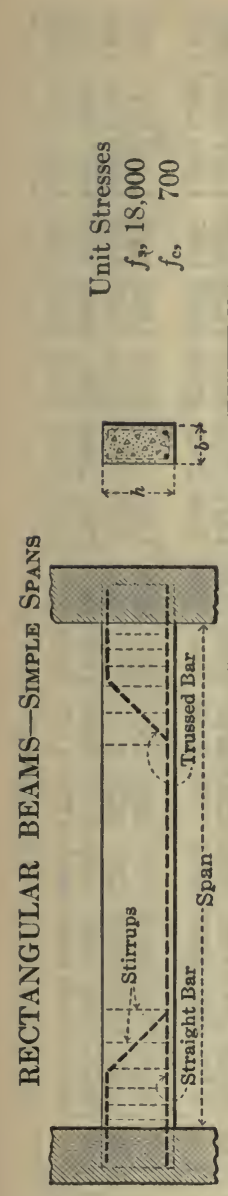

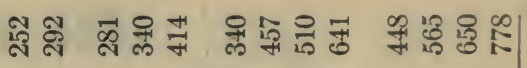

స̃ สิ

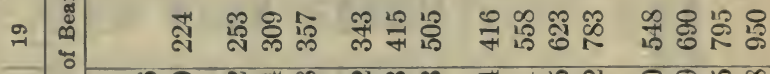
\&

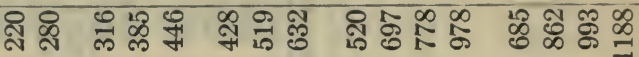
๙ तี ณू 용 8 \% 然

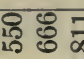
ลู่ สุ

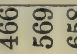
要 范范

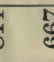
20

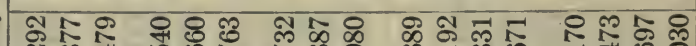

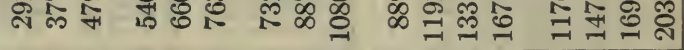

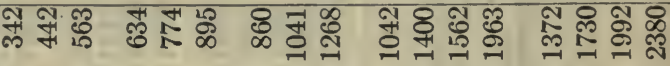

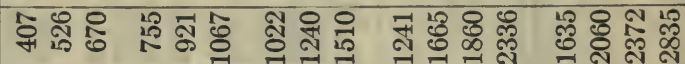
¿ 우 os

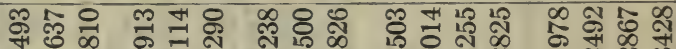
क 88 क 잉유 억

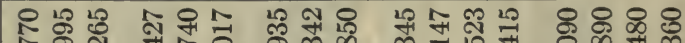

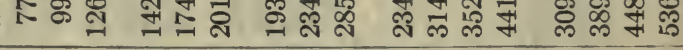

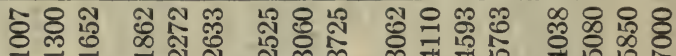

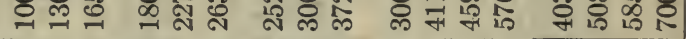

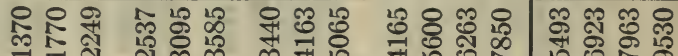

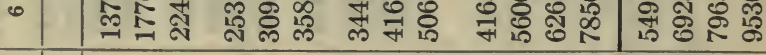

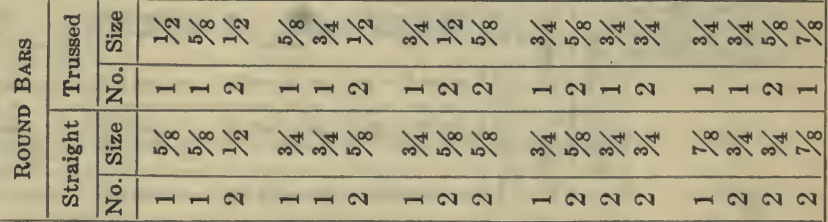
荧

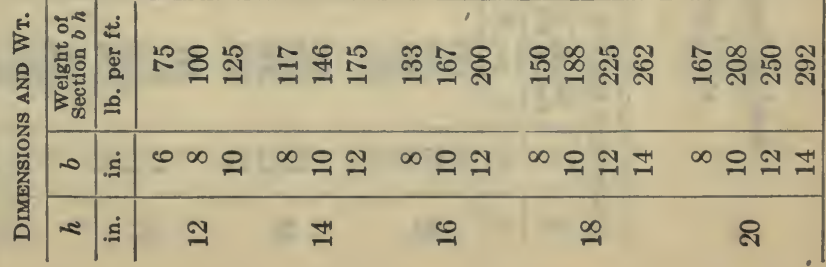


gular

Beams

\section{总 88 \\ ऊू}

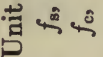

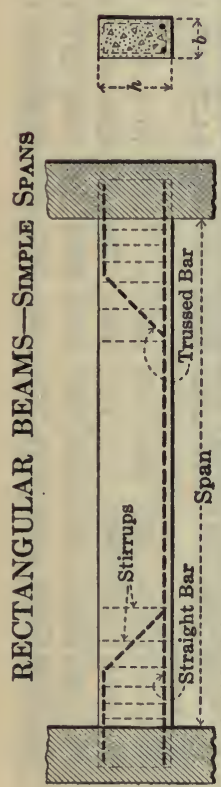

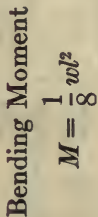

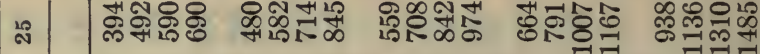

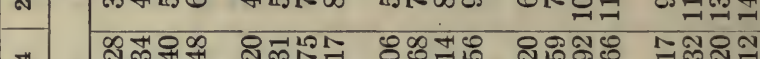

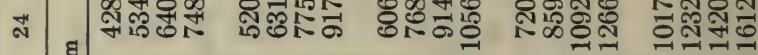

ส

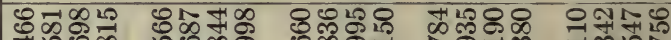

ล

สื

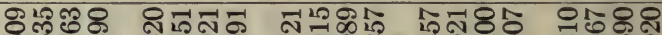

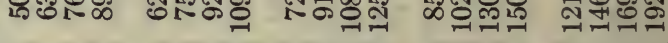

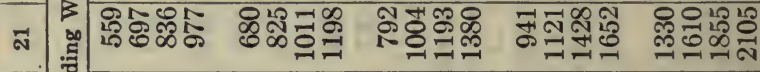

우

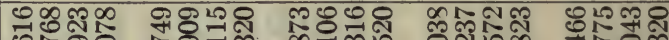

○ा

4. छ্

夏 2 逮 6000.

$\stackrel{\infty}{-1}$

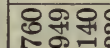

ต

โอำ

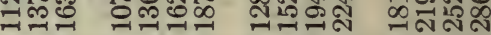

$\%$

究

20

$\pm$

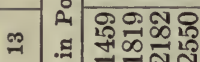

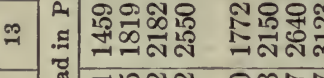

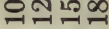

프는

สำลㄴำ

నิస్ ผ

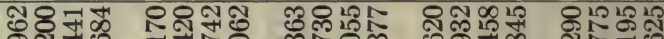

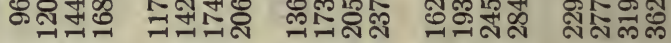

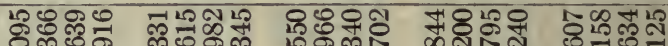

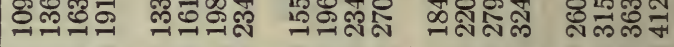

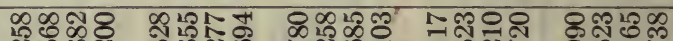

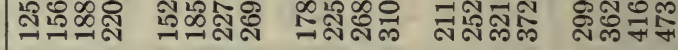

ง

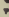

Nㅡำ

ลิ่ง ติ

సै్ల్ भ

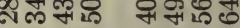

ต

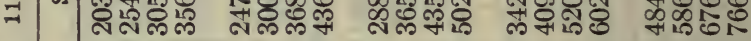

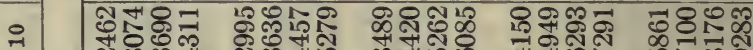

न तै

\begin{tabular}{|c|c|c|c|c|c|c|c|}
\hline \multirow{4}{*}{ 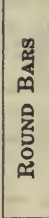 } & \multirow{2}{*}{ 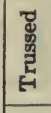 } & 约 & 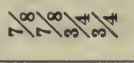 & $\infty_{-1}^{+} \stackrel{N}{\infty}^{\infty}$ & $\mathbf{N}_{\infty}^{\infty}$ & $\mathrm{N}^{\infty} \mathrm{N}^{\infty}$ & $\mathrm{N}^{\infty}$ \\
\hline & & ì & - & 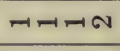 & -NNN & - & -NNN \\
\hline & \multirow{2}{*}{ 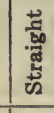 } & $\frac{\pi}{\sqrt{2}}$ & నొఱాలులున & 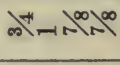 & $-\infty{ }^{-2}$ & $n^{\infty} N^{\infty}-$ & $N^{\infty}-1$ \\
\hline & & z & HNONA & $\mathrm{N} \neg \mathrm{NN}$ & INNN & MNON & NNAN \\
\hline \multirow{4}{*}{ 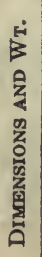 } & .4: & : & , & & & & \\
\hline & 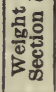 & $\begin{array}{l}\stackrel{8}{\not} \\
\varrho\end{array}$ & ఇ & 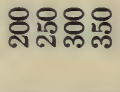 & స్ససี & 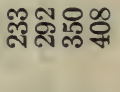 & 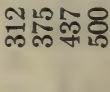 \\
\hline & $\infty$ &. & $\infty$ 엎ㄱ & $\infty$ 의 & 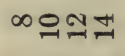 & $\infty \varrho \underset{\sim}{\infty}$ & $\varrho \stackrel{ }{N} \underset{-}{ }$ \\
\hline & 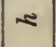 &. & ลั & जี & $\stackrel{\oplus}{\circ}$ & ผ & คి \\
\hline
\end{tabular}


递 8 웅

की

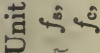
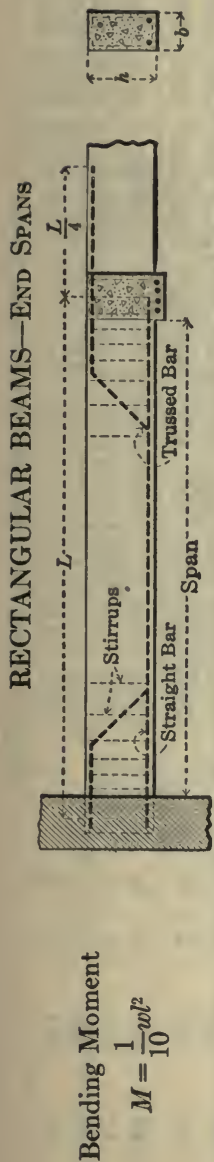

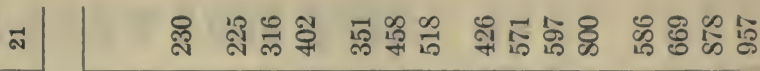

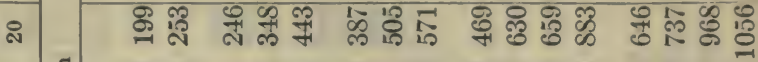

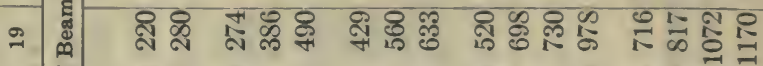

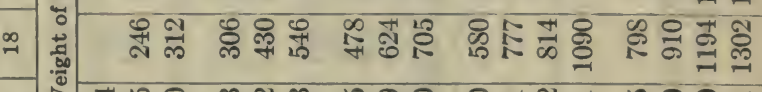

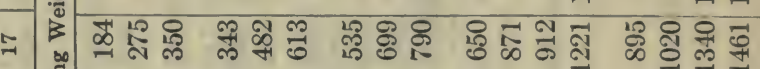
策 $\because$ 焉

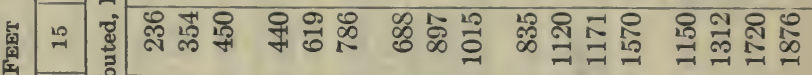
士 ₹窝 $\rightarrow$ (1) 发 근 $\Rightarrow$ $\Rightarrow$

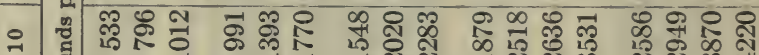
-

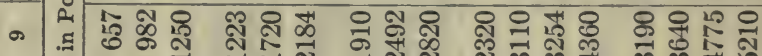
- 5 o ×

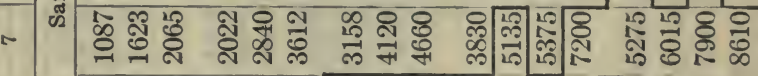

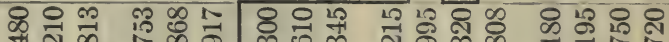

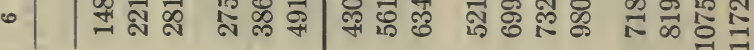

\begin{tabular}{|c|c|c|c|c|c|}
\hline \multirow{2}{*}{$\mid \begin{array}{ll}0 \\
0\end{array}$} & 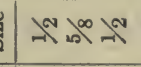 & $\infty^{\infty} \infty^{+\infty}$ & $\mathrm{m}^{4} \infty \mathrm{\infty}^{\infty}$ & 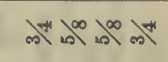 & $\mathrm{x}^{\infty} \infty^{\infty} \mathrm{m}^{-1} \mathrm{~m}^{+1}$ \\
\hline & 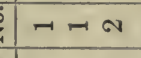 & 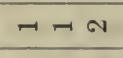 & $\rightarrow \infty 0$ & $N \rightarrow N$ N N & $-\infty \omega$ \\
\hline \multirow{2}{*}{ 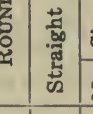 } & 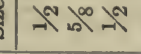 & $n^{\infty} m^{+\infty} \mathrm{l}^{\infty}$ & $x^{\prime \prime} \operatorname{los}^{\infty}$ & का & ஸி \\
\hline & $-\pi-\infty$ & $-\pi \infty$ & ta d & ー ๙ N N & - \\
\hline
\end{tabular}

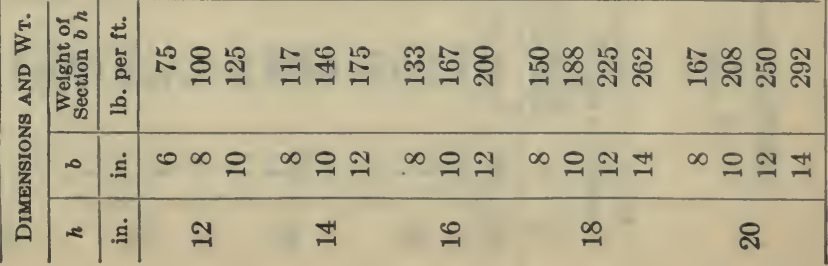


遇 88

की

范离官
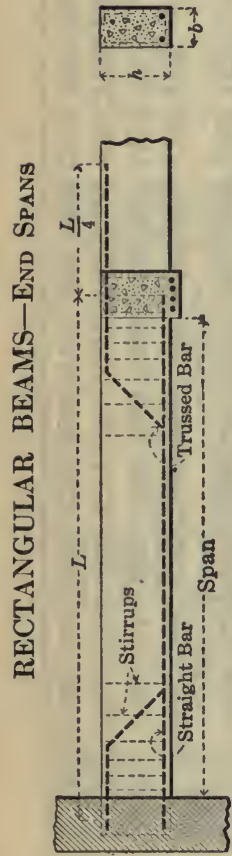

है
เ

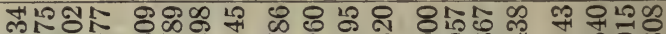
⿰习习

\%

A

-

ส

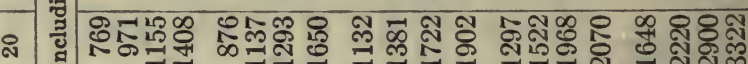

๙

궁웅 유.

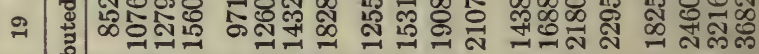

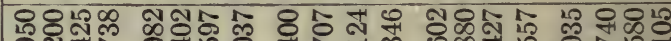

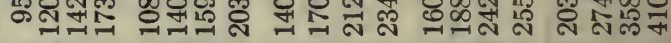

น马Н

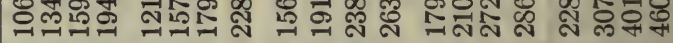

ศు⿻

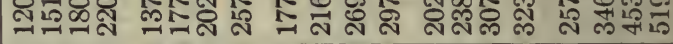

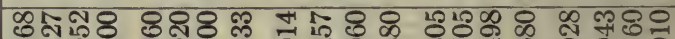

แ

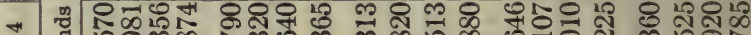

-

4

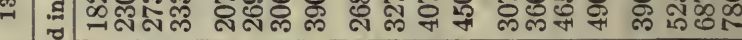

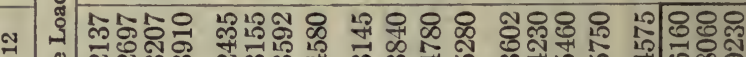

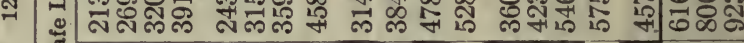

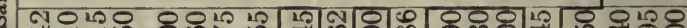

1

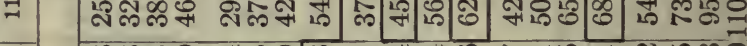

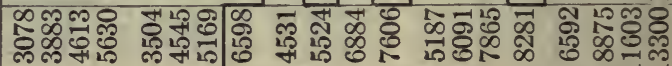

离

卷

๓ E

营

萢

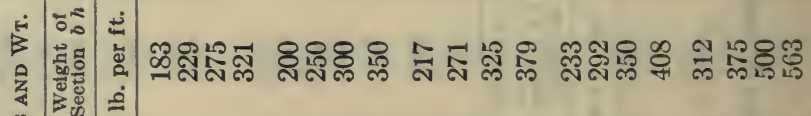

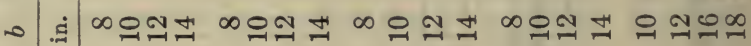

$-\dot{a}$

สี

ぶ

คิ

๙

ㅇำ 
衷 8 \% 8

品

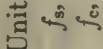

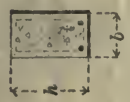

(⿸丆口
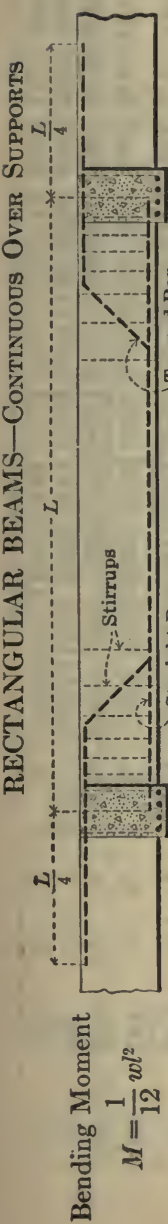

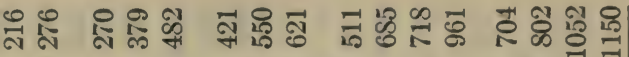

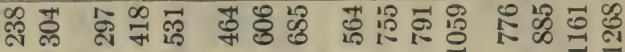

ลิ

$\rightarrow$

$\div$

$\stackrel{\infty}{\sim}$

$\pm$

鼻

的

z

간

ค

등

$z$
$\frac{\alpha}{2}$

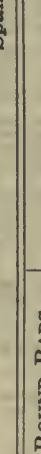

$\stackrel{2}{-1}$

ำ

$=$

$\circ$

a)

a

$\infty$

5

$\bullet$

N

4⿻

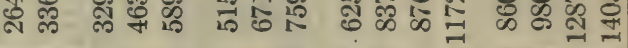

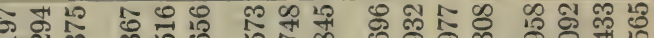

-

궁ำ 규

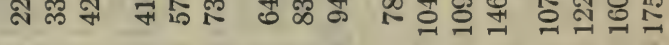

ผำ

구유

ผ भै ผ

ผิ

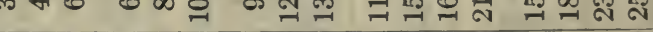

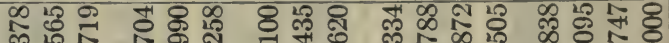

ஸे

सु

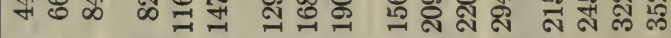

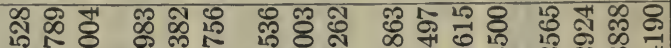

เก

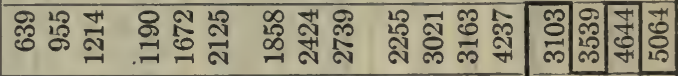

\%

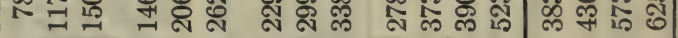
\& $\$$ क ॠ

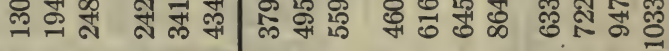

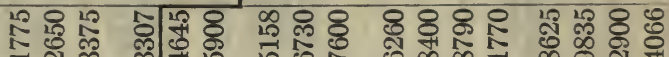
드 \% ल

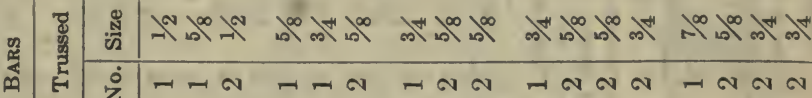

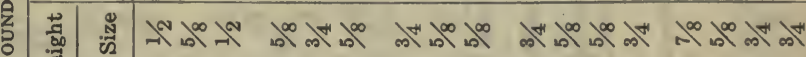

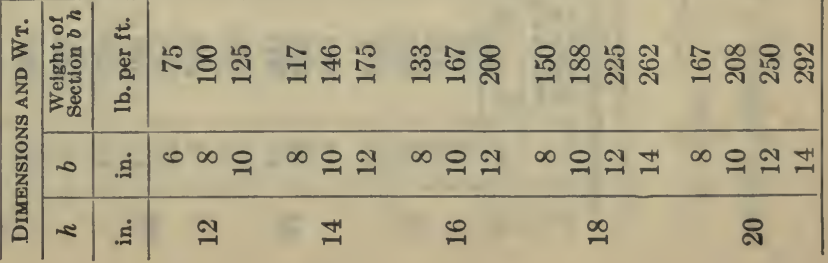




\section{gular}

Beams
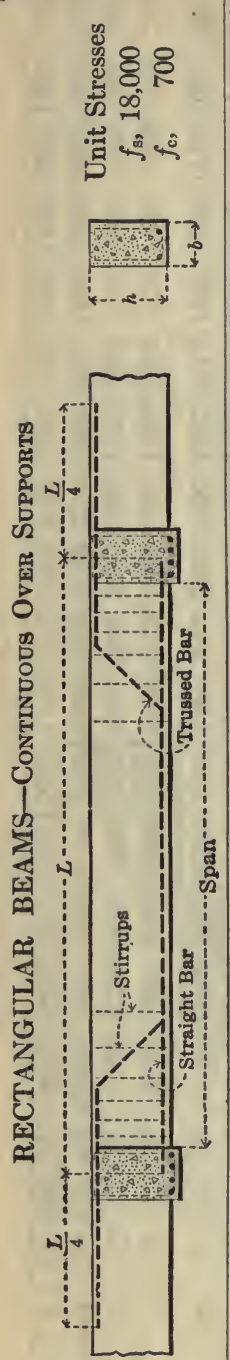

范

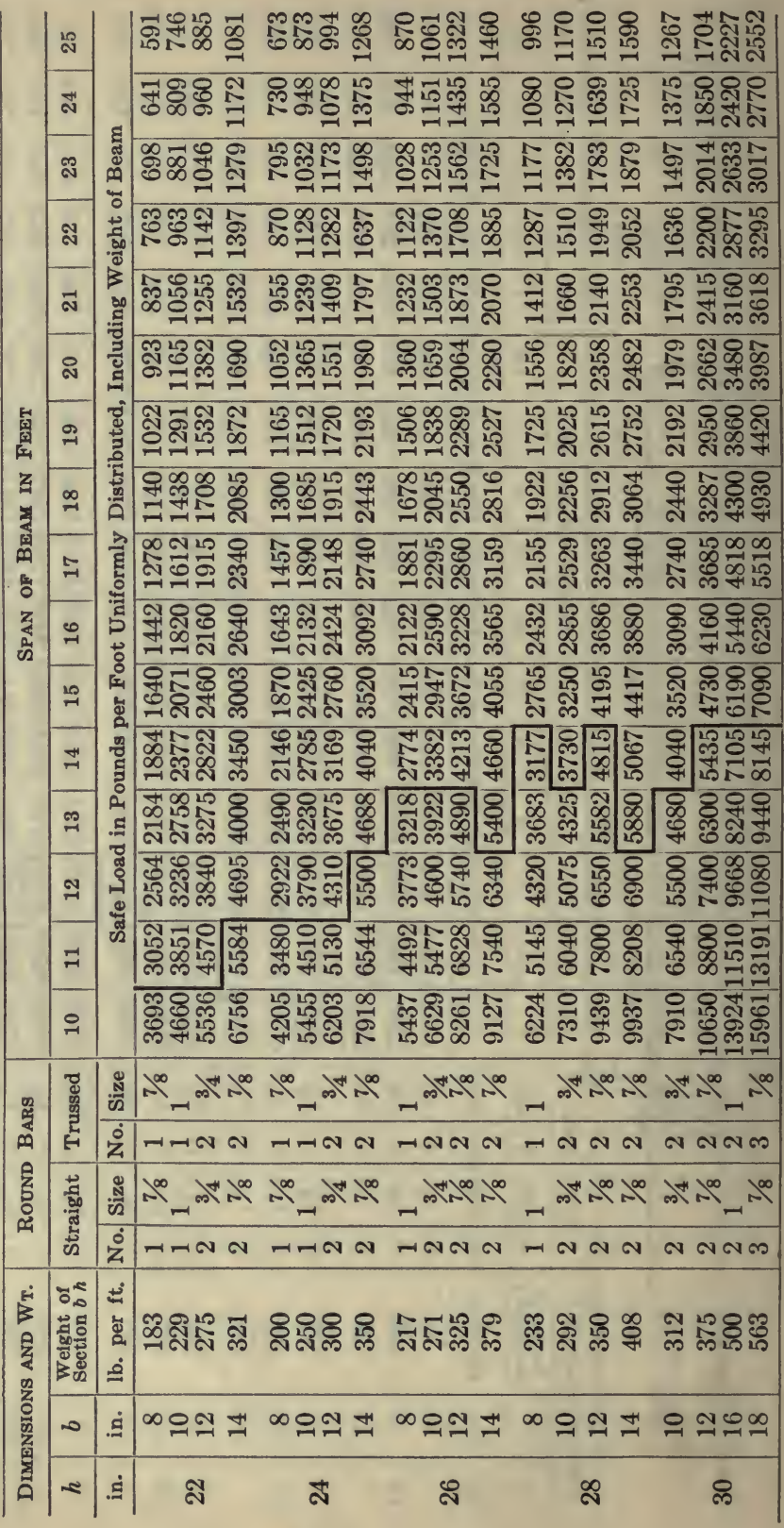




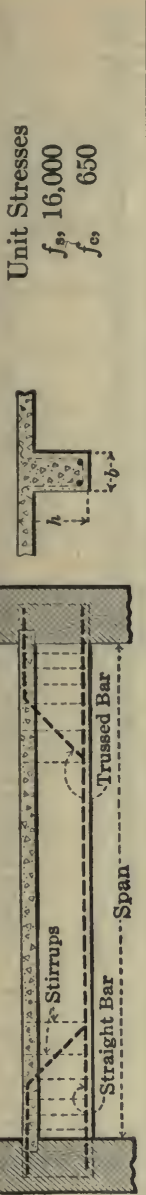

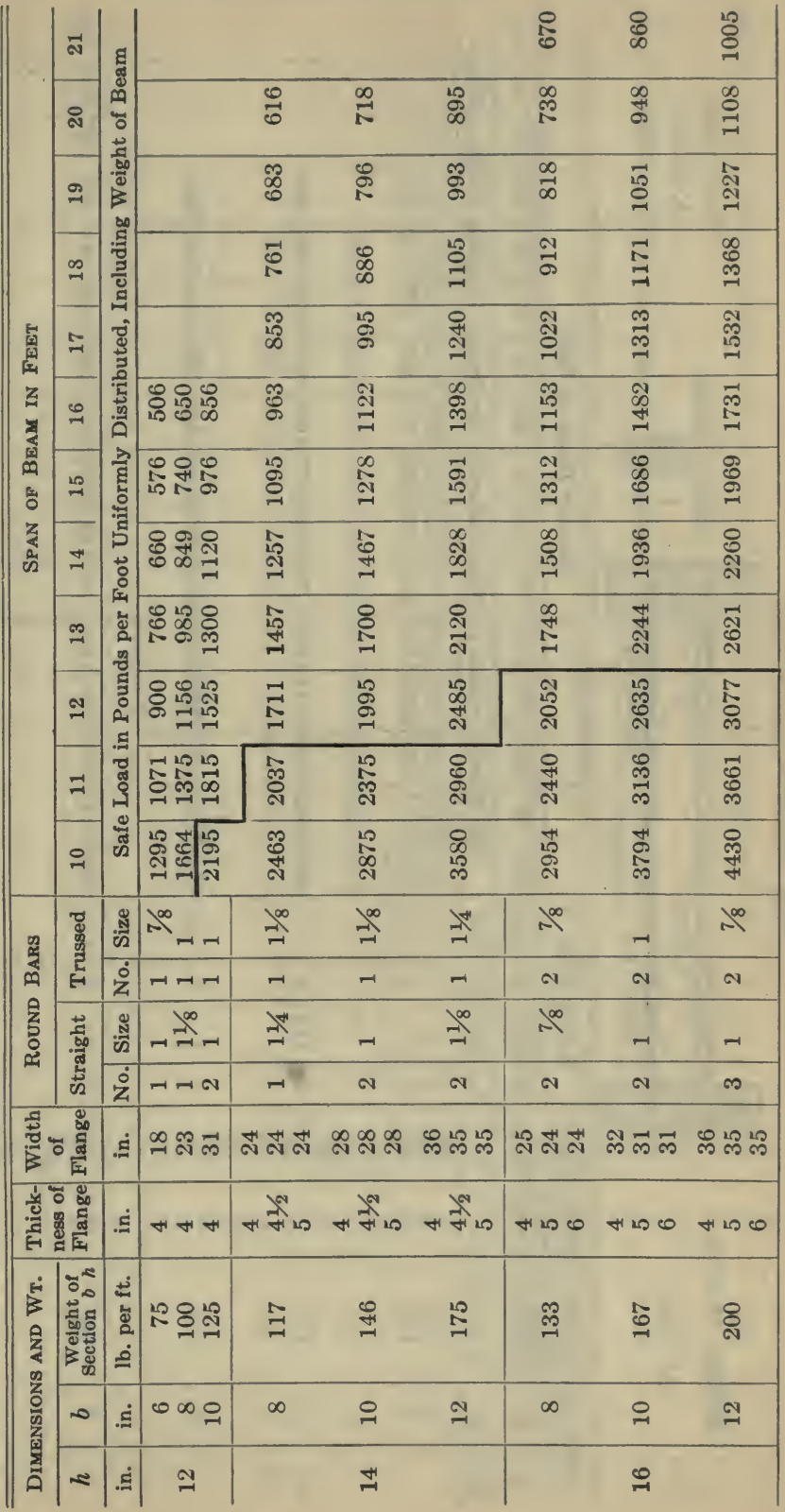




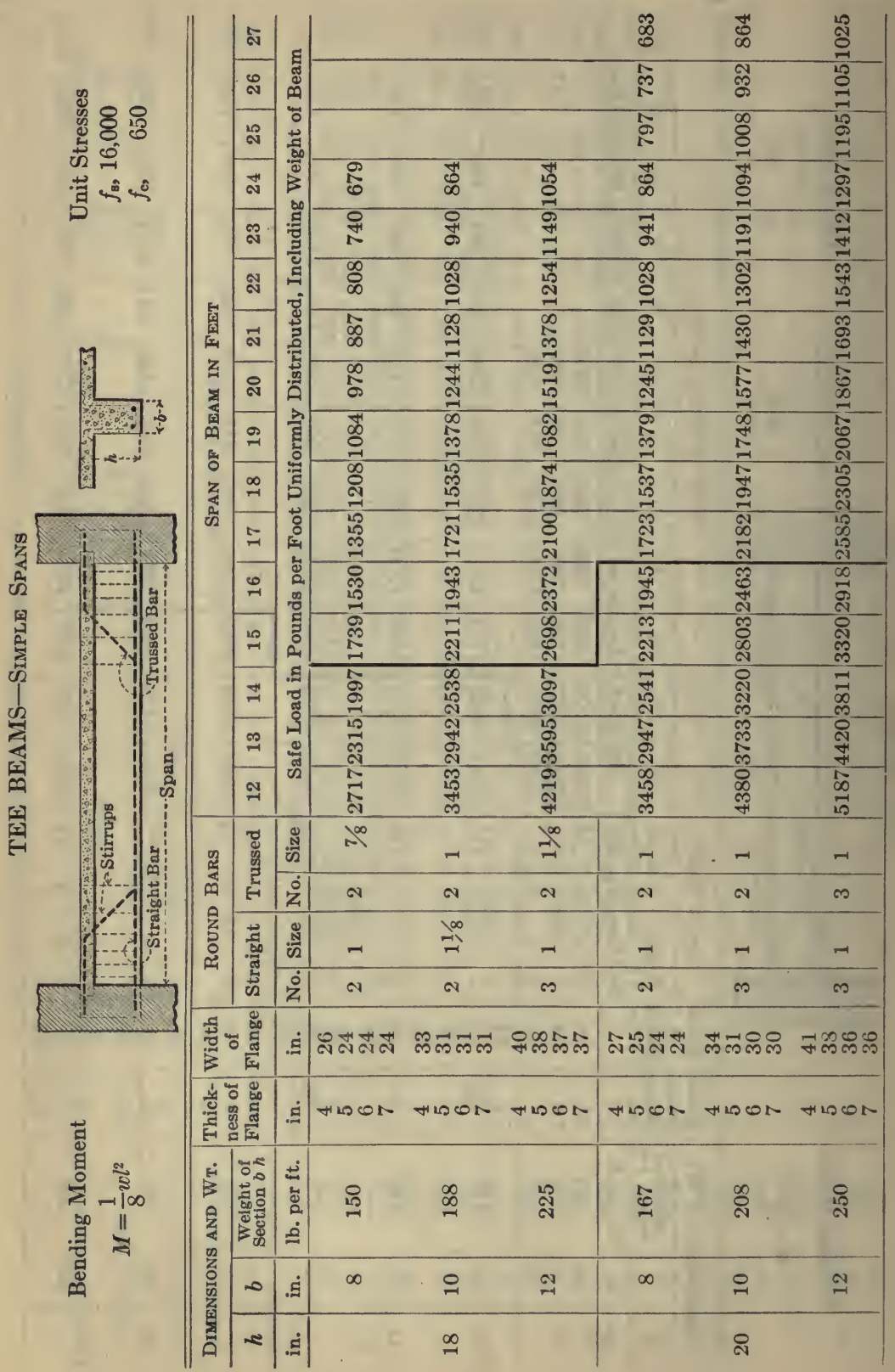




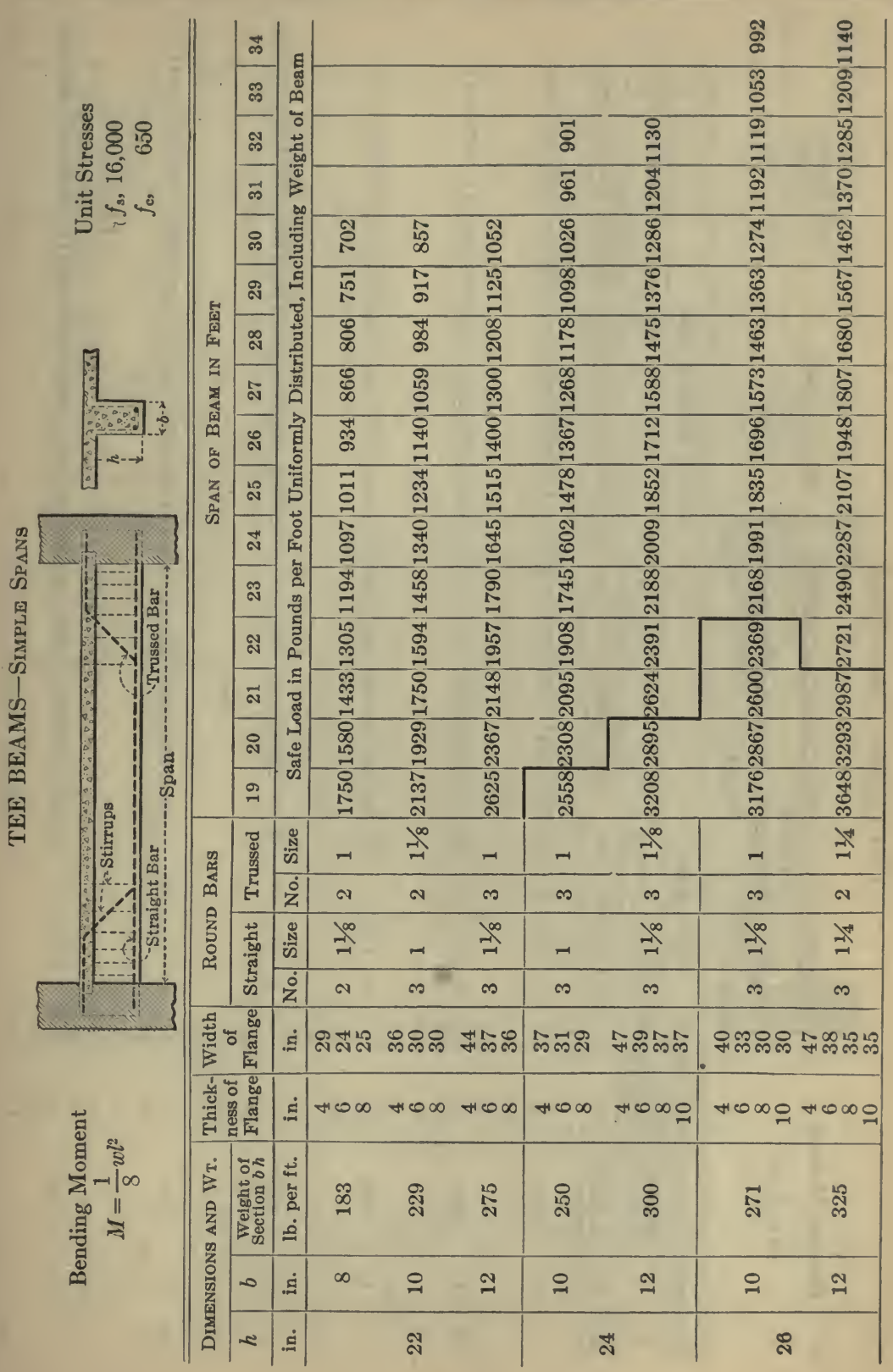




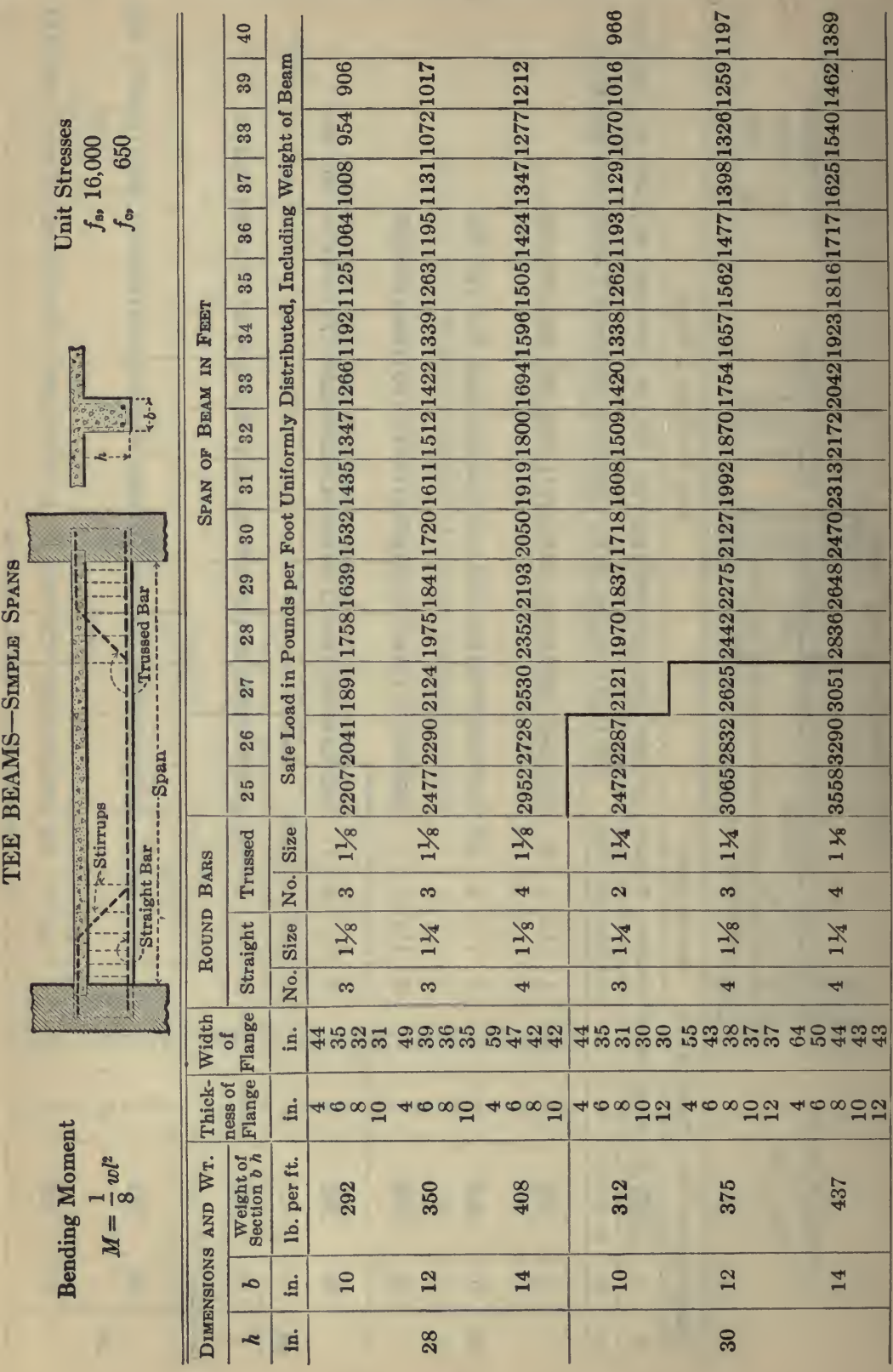



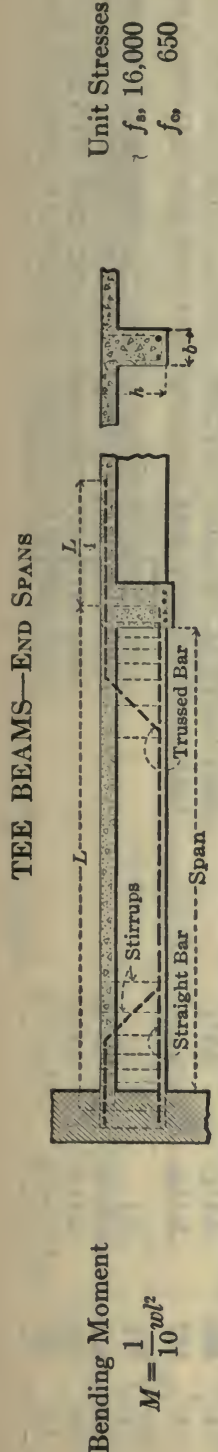

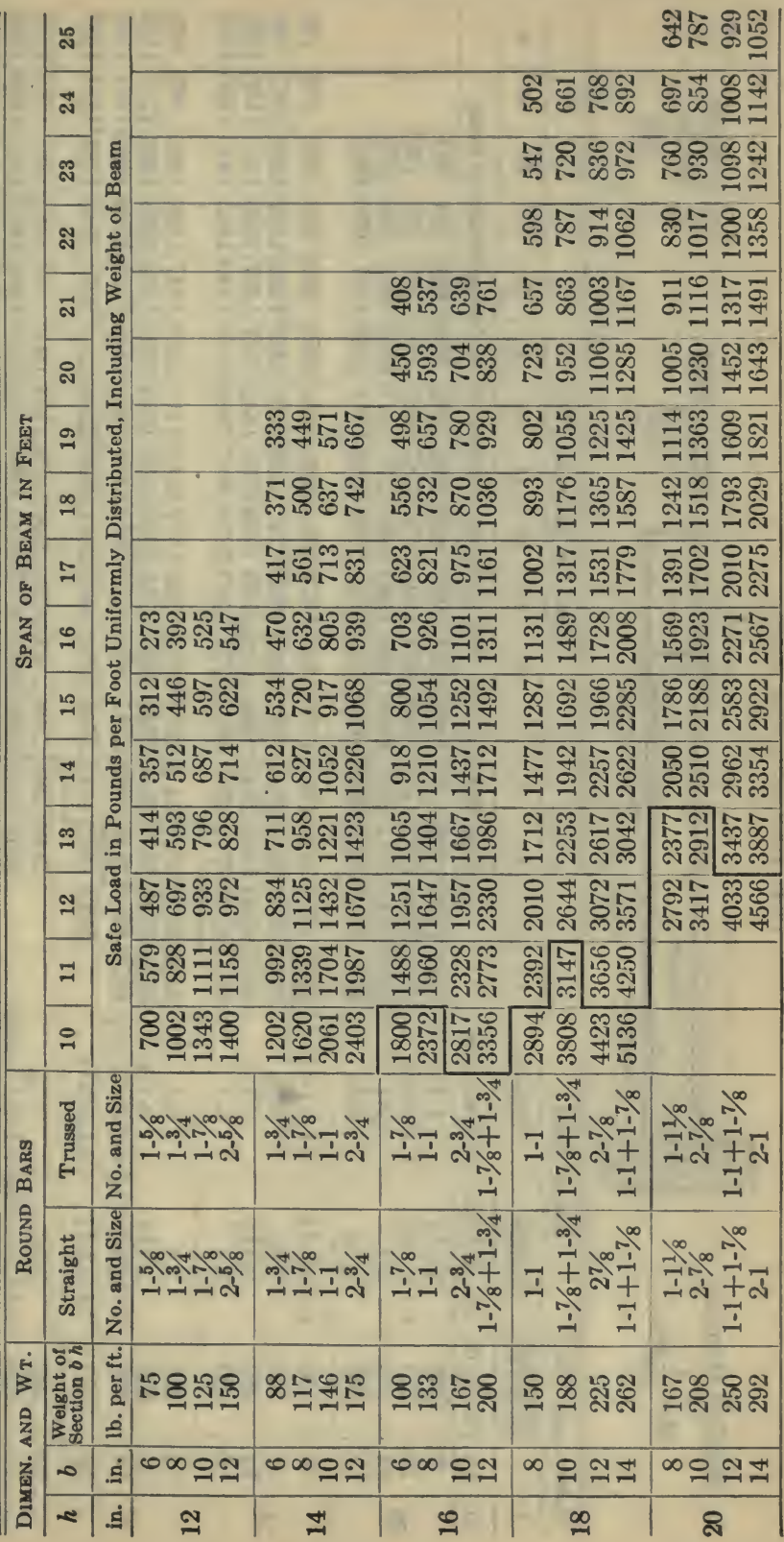



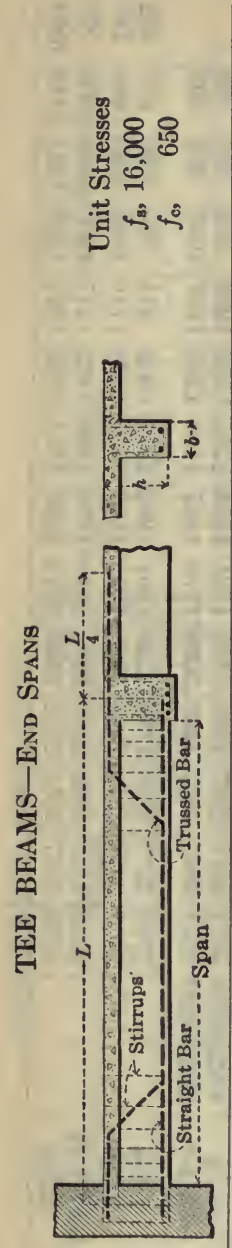

\begin{tabular}{|c|c|c|c|c|c|c|c|}
\hline & $\vec{\infty}$ & & & 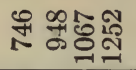 & 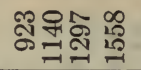 & 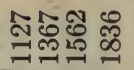 & ฌ2వి \\
\hline & ణ & ह్ర & & คํํำ & 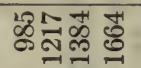 & 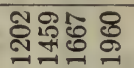 & 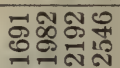 \\
\hline & స్ & $\frac{m}{\circ}$ & 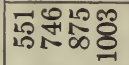 & 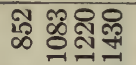 & 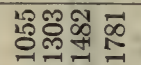 & 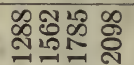 & 음그ำฟ \\
\hline & 脑 & 营 & 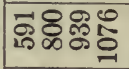 & m లో & 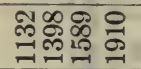 & 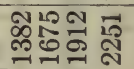 & 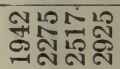 \\
\hline & สิ & . & 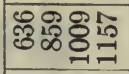 & 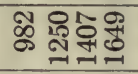 & 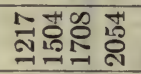 & 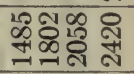 & 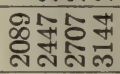 \\
\hline & N & 总 & ஜ요유 & 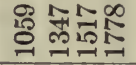 & 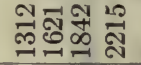 & 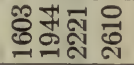 & 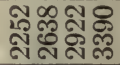 \\
\hline 嘼 & 㓠 & 蛋 & พैชิ도ำ & 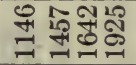 & 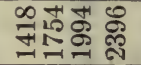 & 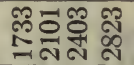 & 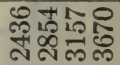 \\
\hline z & సే & $\begin{array}{l}5 \\
\text { s } \\
0 \\
0\end{array}$ & 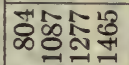 & 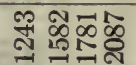 & 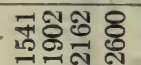 & Dర్లి & 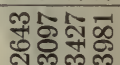 \\
\hline ๓્ต & :ึ & हू & 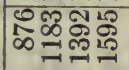 & 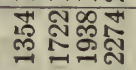 & 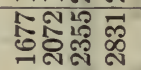 & 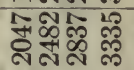 & 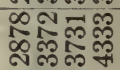 \\
\hline & สี & : & 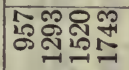 & 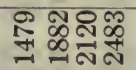 & 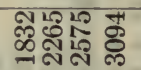 & 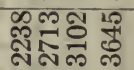 & 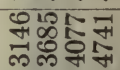 \\
\hline & $\bar{N}$ & $\begin{array}{c}8 \\
8 \\
\vdots \\
0\end{array}$ & 주유 & 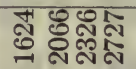 & 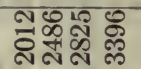 & 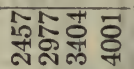 & 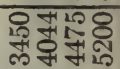 \\
\hline & ผิ & ă & 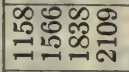 & 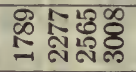 & 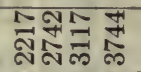 & 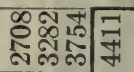 & 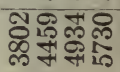 \\
\hline & $\stackrel{7}{-1}$ & . & 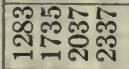 & 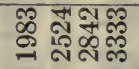 & 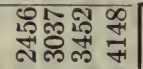 & 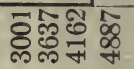 & \\
\hline & $\stackrel{\infty}{\sim}$ & ర్đ & 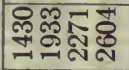 & 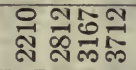 & 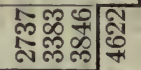 & 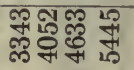 & \\
\hline & 5 & ัँّ & 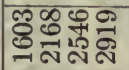 & 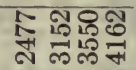 & 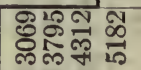 & & \\
\hline & $\stackrel{\bullet}{\sim}$ & & 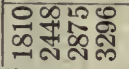 & 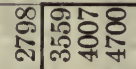 & 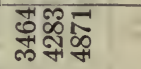 & & \\
\hline 告 & 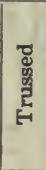 & 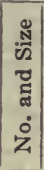 & 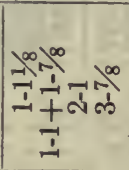 & 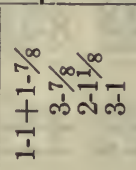 & 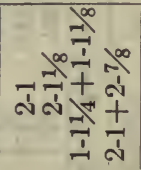 & 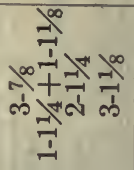 & 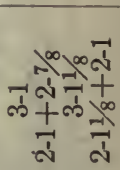 \\
\hline 成 & 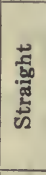 & $\begin{array}{l}\tilde{N} \\
\dot{n} \\
\dot{n} \\
\dot{\varpi} \\
\dot{0} \\
z\end{array}$ & 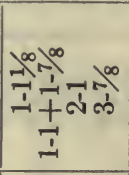 & $\begin{array}{l}I^{\infty} \\
\frac{1}{ \pm} \\
\frac{1}{-}\end{array}$ & 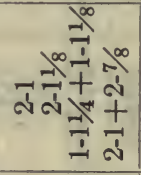 & के & 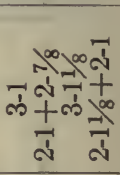 \\
\hline 莫 & 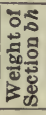 & 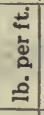 & ஐ్ׁస: స్స & 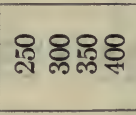 & 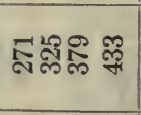 & 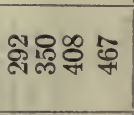 & 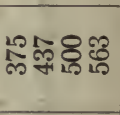 \\
\hline 畜 & 0 & $\dot{\Xi}$ & $\infty \subseteq$ & 으워 & 으멥 & 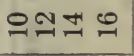 & $\cong \pm \varrho \infty$ \\
\hline & & . & ฟี & 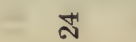 & ๙ & 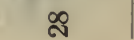 & \\
\hline
\end{tabular}

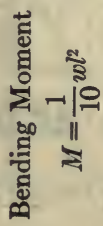



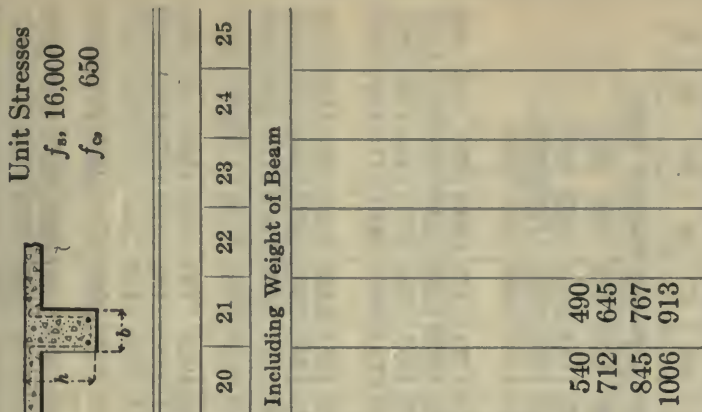

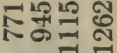

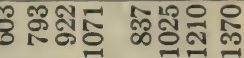

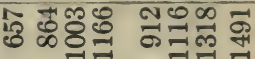

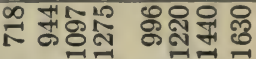

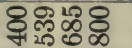

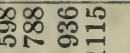

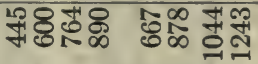

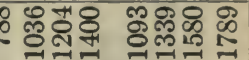

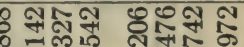

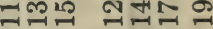

8m心

开

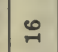

(1)

औ

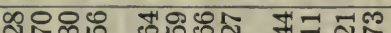

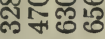

以ก⿻

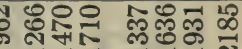

건

เ

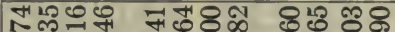

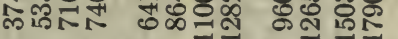

종

- $\rightarrow-\mathbb{N}_{-\pi} \mathrm{N}$

๕

$\exists$

:

มูสำก

10

17

주요

걱

\&

$\rightarrow$

-

|

रूis

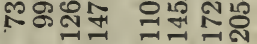

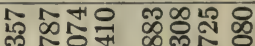

๓్ન స్సఁ

ง

+ 00 (

땡

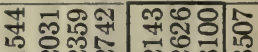

นี่ ลิล สิล

$\rightarrow$ कँ

-1 -

$=$

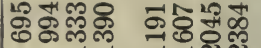

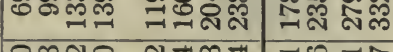

ก

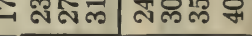

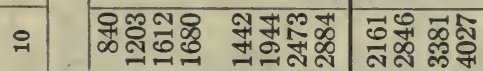

(1)

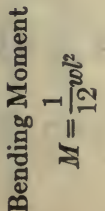

(1)

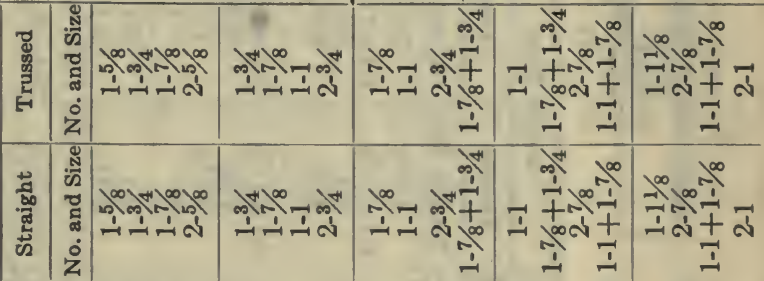

\begin{tabular}{|c|c|c|c|c|c|c|}
\hline 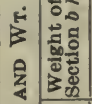 & 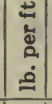 & 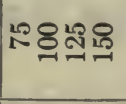 & 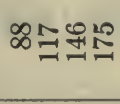 & 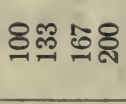 & เి జ & స్రీ \\
\hline$\infty$ & . & 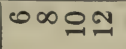 & 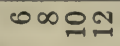 & $0 \infty$ 으 & $\infty$ 으쿼 & $\infty \varrho$ \\
\hline$\alpha$ &. & $\cong$ & \pm & $\stackrel{\overbrace{}}{\mathscr{}}$ & $\stackrel{\infty}{\sim}$ & $N$ \\
\hline
\end{tabular}



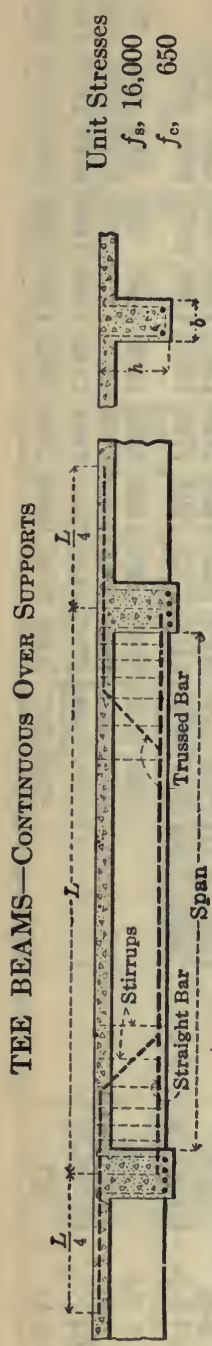

范

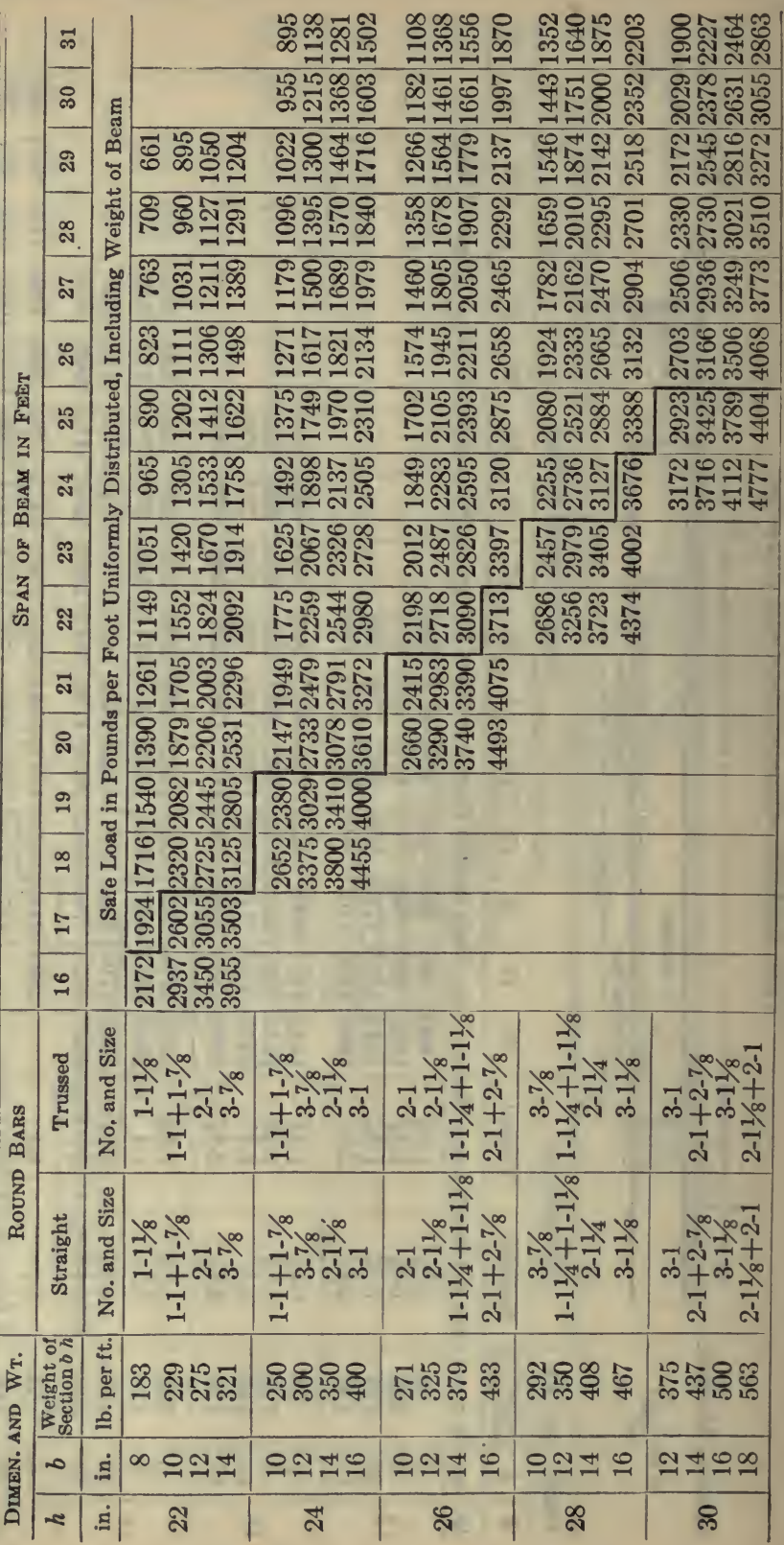




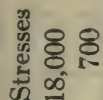

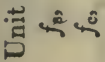
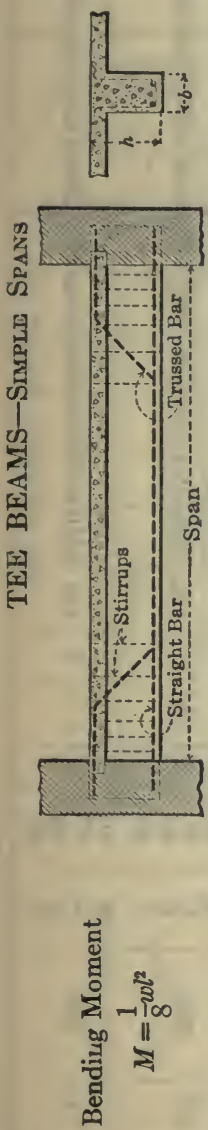

\begin{tabular}{|c|c|c|c|c|c|c|c|c|}
\hline & 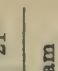 & & & & & $\bar{\infty}$ & ळ & 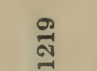 \\
\hline & ๑ّ & & స్ㅇㅇ & & $\overline{8}$ & 茫 & 哭 & 萝 \\
\hline & 要 & & 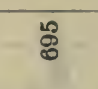 & $\stackrel{\infty}{\infty}$ & 兽 & ஓ & $\underset{\Xi}{\mathbb{\Xi}}$ & 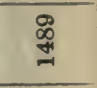 \\
\hline & $\infty$ & & 莣 & $\stackrel{\infty}{\infty}$ & జ్ర్ & $\stackrel{\Xi}{g}$ & 总 & $\stackrel{8}{\stackrel{0}{2}}$ \\
\hline & 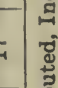 & 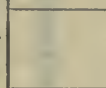 & 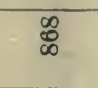 & $\stackrel{\infty}{\Xi}$ & 跑 & 怘 & 兽 & 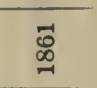 \\
\hline 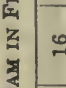 & 4 & 颌距要 & 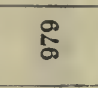 & : & 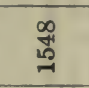 & 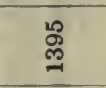 & 呑 & \\
\hline 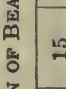 & 4 & 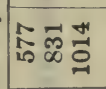 & $\stackrel{2}{\Xi}$ & 垔 & 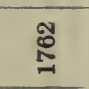 & 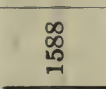 & 芦 & \\
\hline$=$ & 4 & 잉ㅁㅇㅇㅁㅛ & 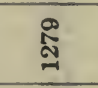 & 要 & कू్ & 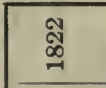 & 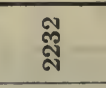 & 䒺 \\
\hline & ఫ & 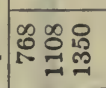 & 蔡 & $\stackrel{m}{\Phi}$ & 氮 & $\ddot{z}$ & 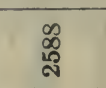 & ס \\
\hline & & 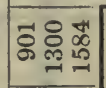 & 胥 & 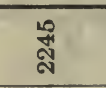 & 哭 & $\begin{array}{l}\overrightarrow{0} \\
\stackrel{d}{d} \\
\end{array}$ & $\begin{array}{l}\infty \\
\text { o. } \\
\end{array}$ & 䇹 \\
\hline & & 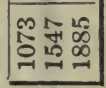 & 答 & 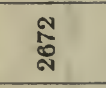 & 勇 & 䟵 & 足 & 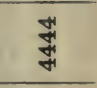 \\
\hline & 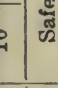 & 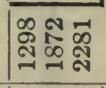 & 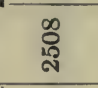 & 跑 & 颉 & 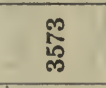 & 芯 & \\
\hline & 递 & $\mid x^{2}-x^{\infty}$ & $\stackrel{\infty}{\Rightarrow}$ & $\stackrel{\infty}{\Rightarrow}$ & - & $\nexists$ & $\Rightarrow$ & 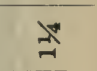 \\
\hline 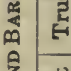 & $\dot{\dot{z}}$ & $|-m-|$ & $\rightarrow$ & - & N & - & - & \\
\hline & 部 & $\mid \rightarrow{ }^{\infty}-1$ & $\nexists^{\infty}$ & - & - & 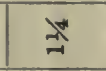 & $\Rightarrow$ & 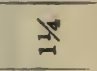 \\
\hline & $\dot{z}$ & $|-\infty|$ & - & $\omega$ & N & - & $\omega$ & $w$ \\
\hline & & $\approx \stackrel{2}{1}$ & ఇহ্ণ & ஜ్లిణ & 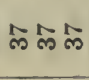 & ชัส ส & "ెదల్ & बิ \\
\hline 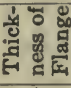 & & ササー & + & सें & में & $+\infty$ & $+\infty 0$ & \\
\hline- & & 28 & $\exists$ & I & 뫀 & ๓ & $\Phi$ & 8 \\
\hline$\infty$ & 0.9 & 1000 & $\infty$ & 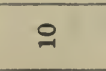 & 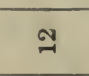 & $\infty$ & 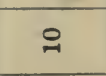 & $\cong$ \\
\hline 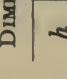 & & $\cong$ & 2 & $\Xi$ & & & 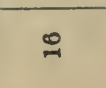 & \\
\hline
\end{tabular}




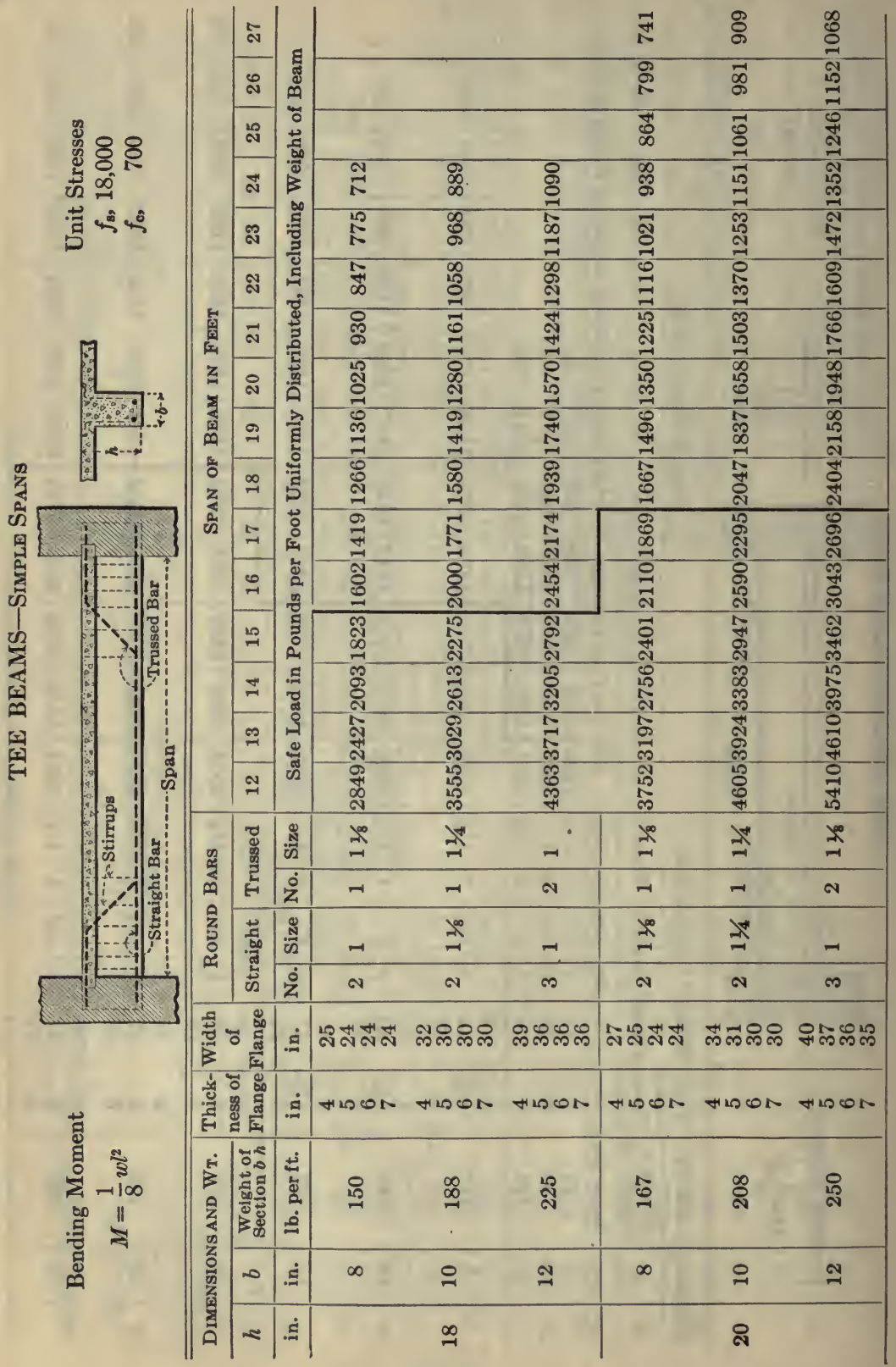



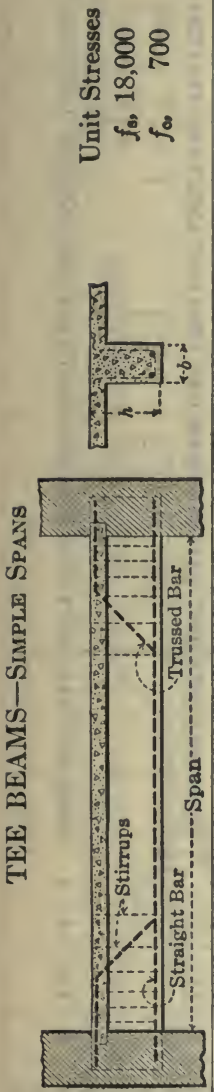

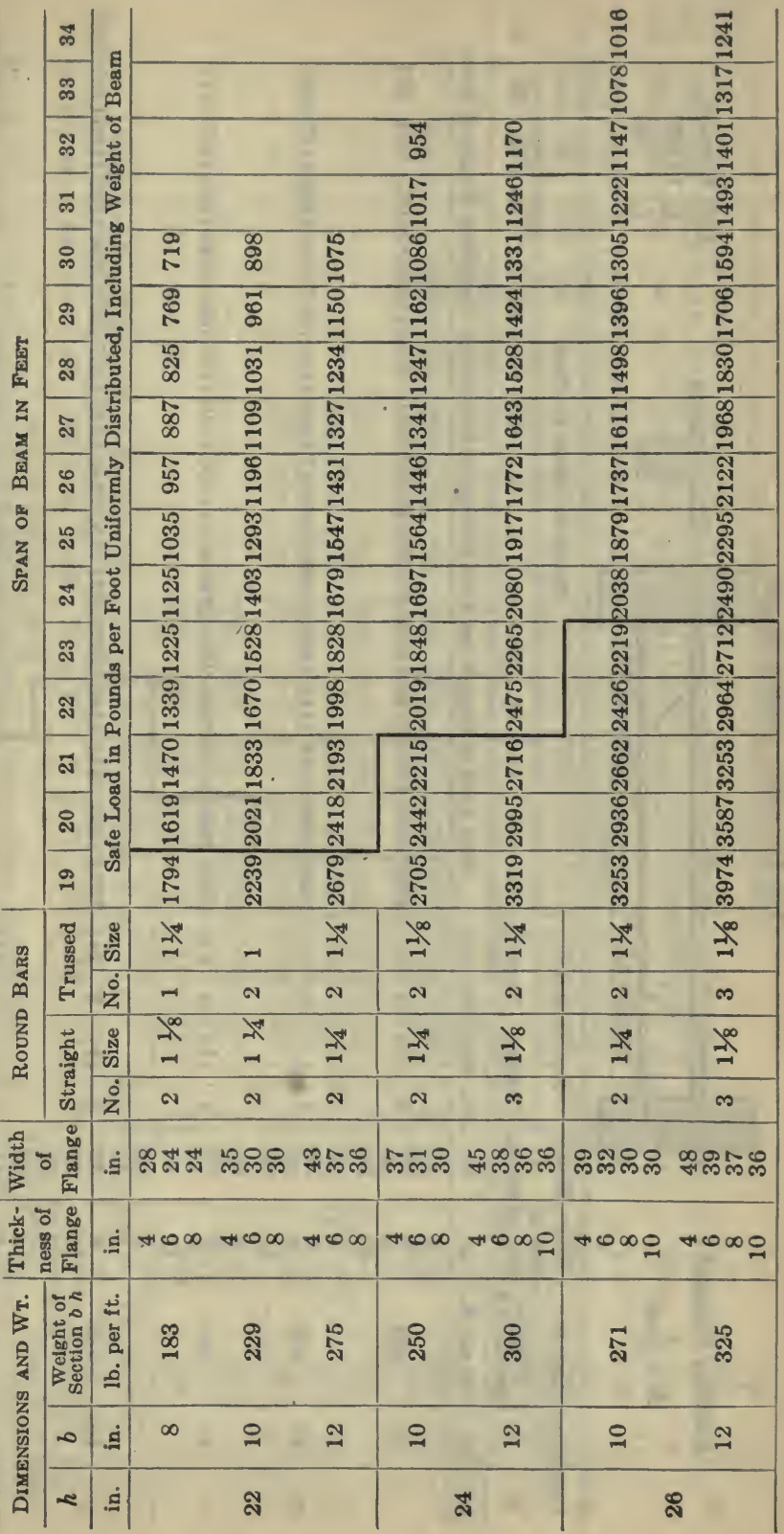




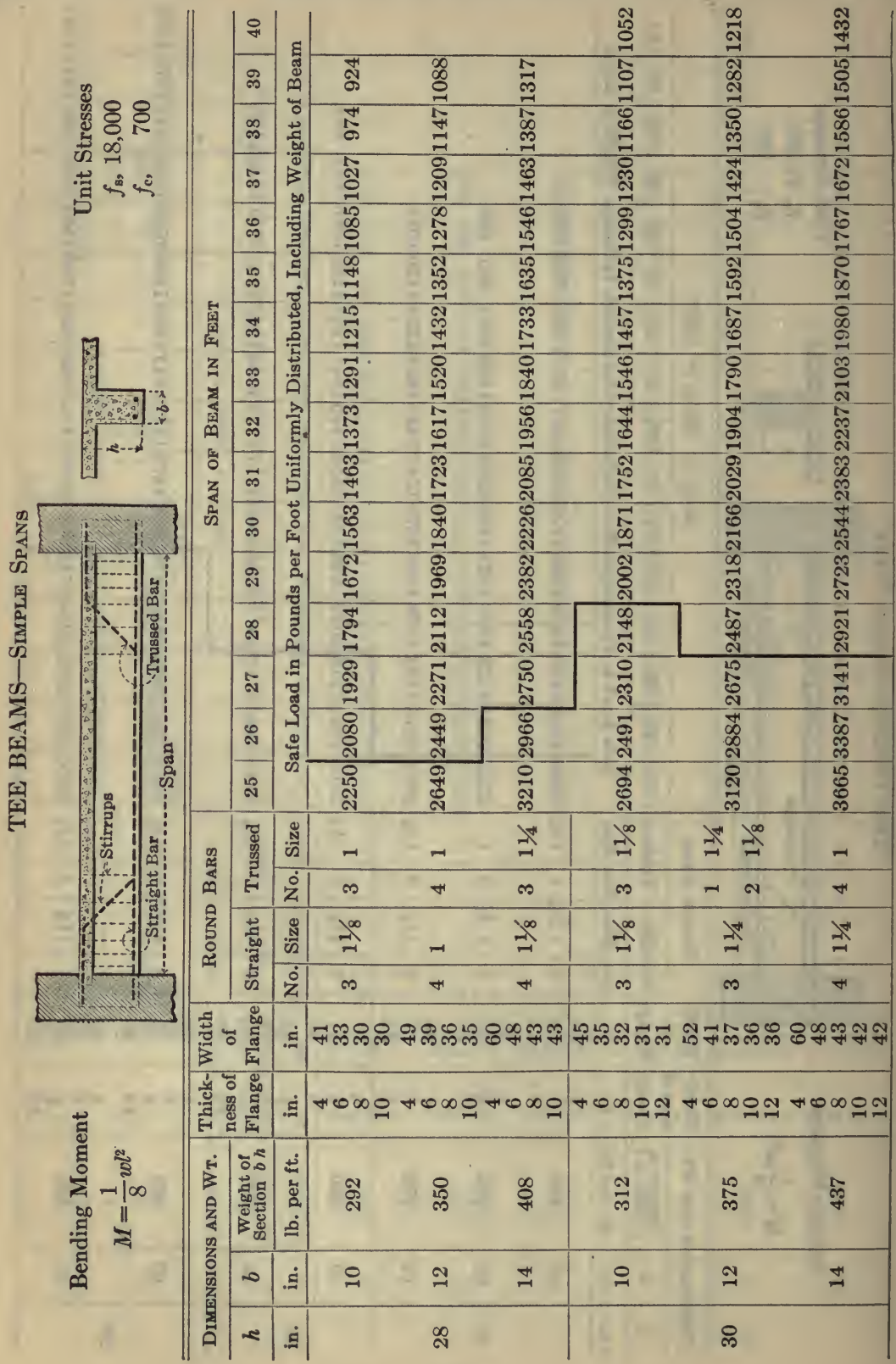



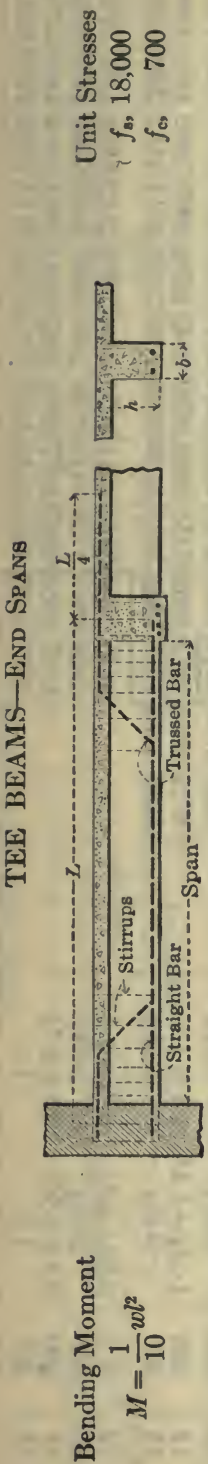

융 윤영웍

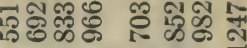

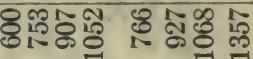

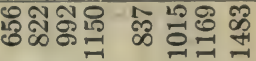

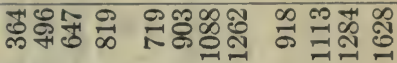

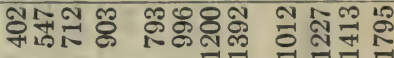

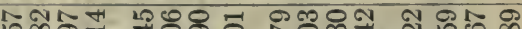

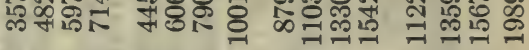

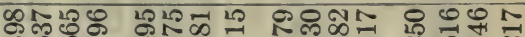

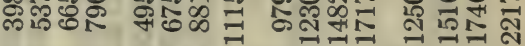

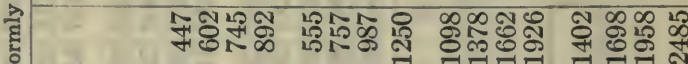

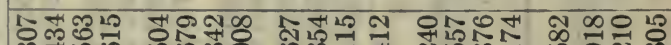

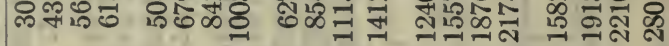

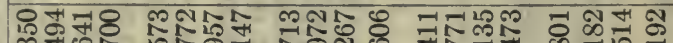

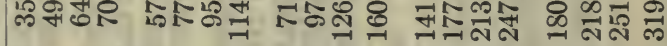

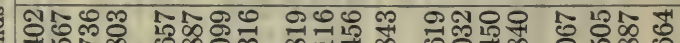
\# \%下贸

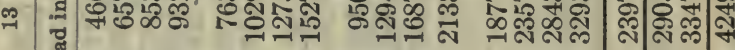

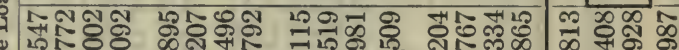
-

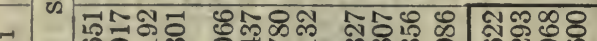

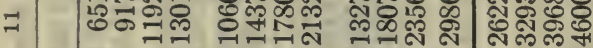

- त 인

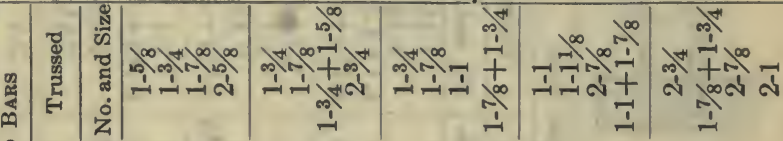

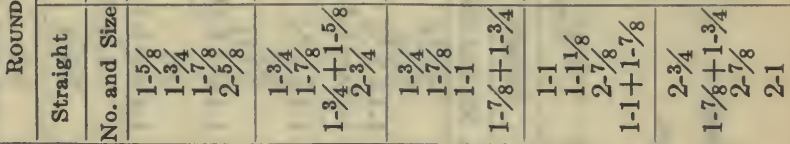
+5:

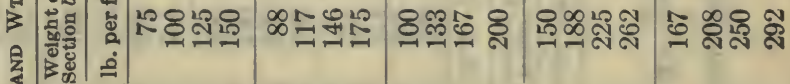

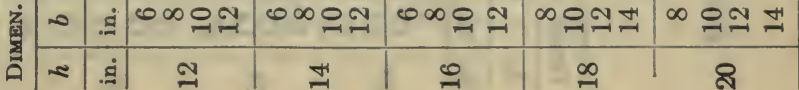



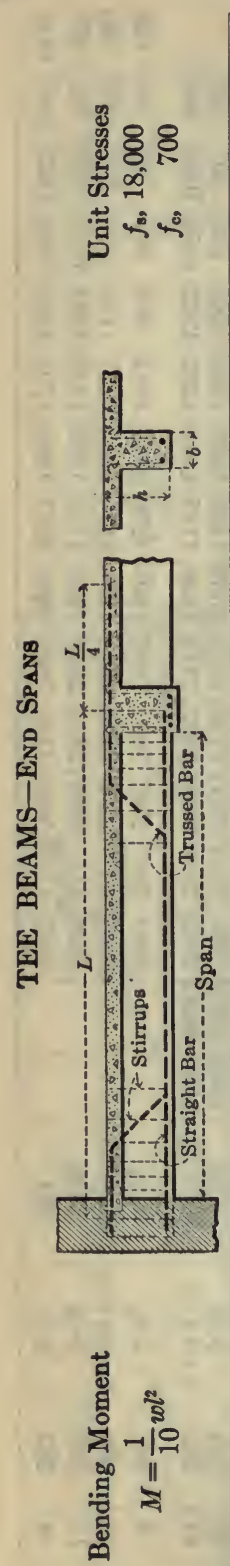

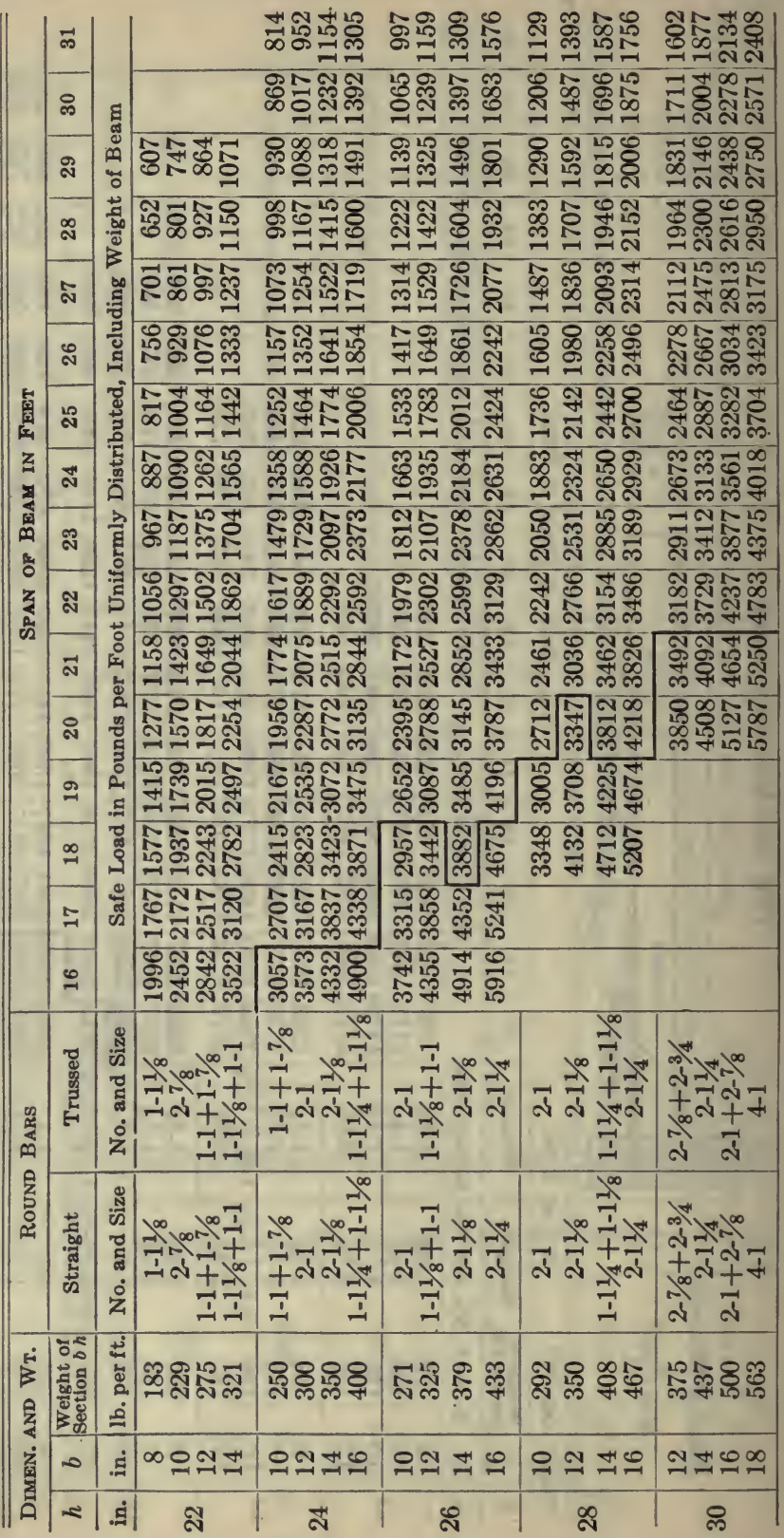



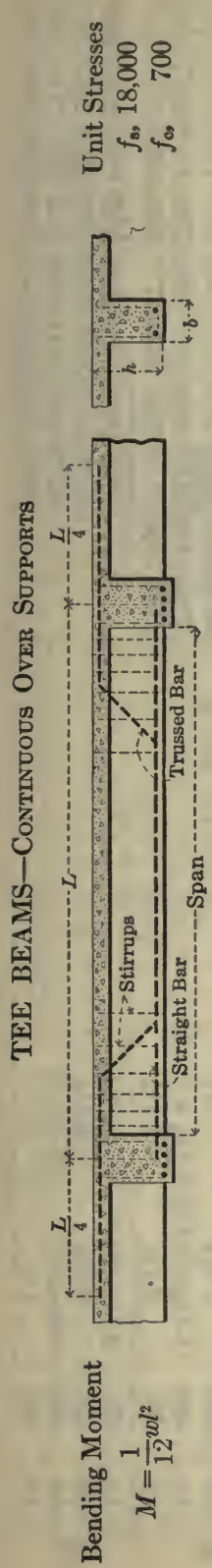

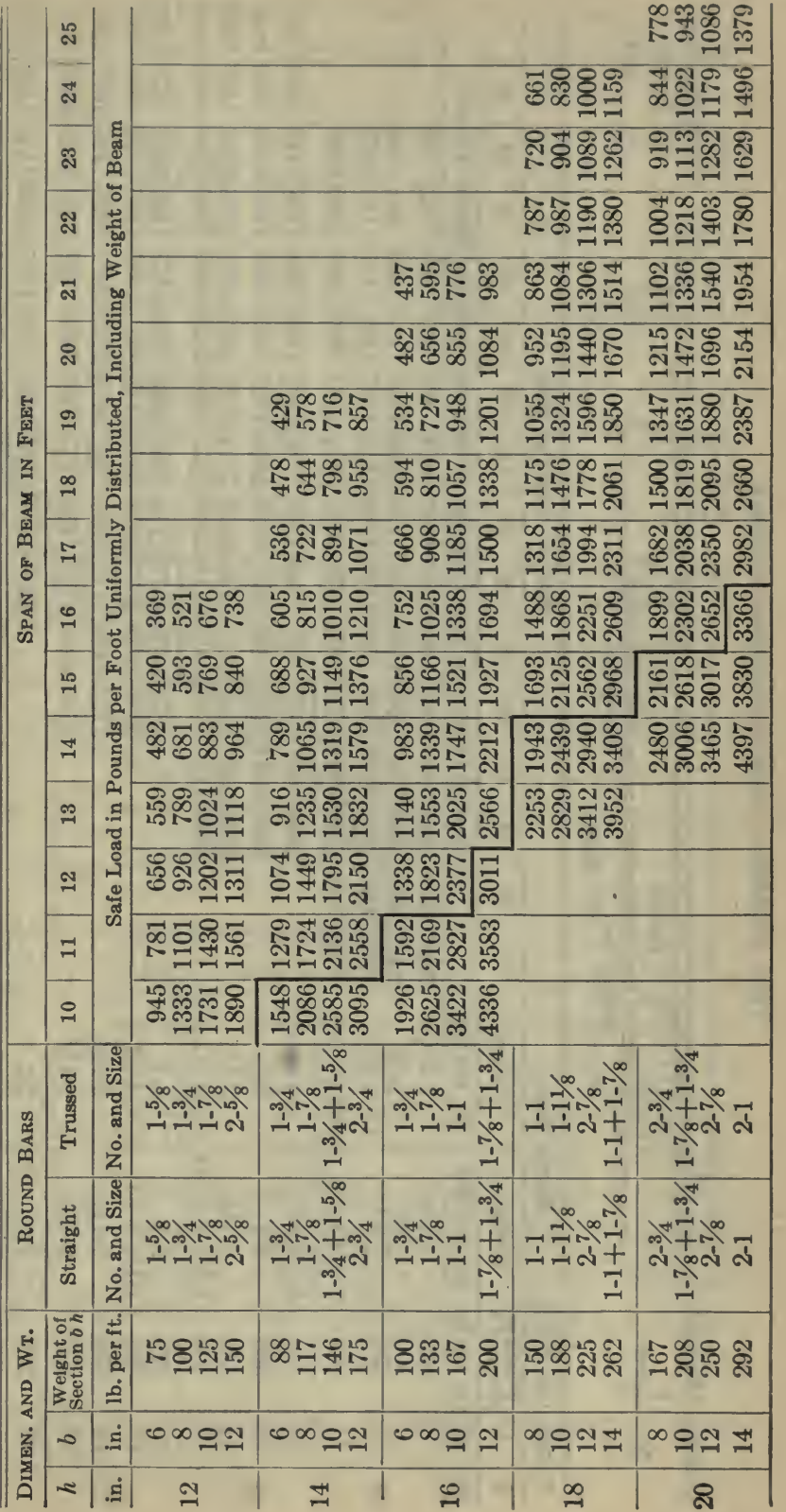



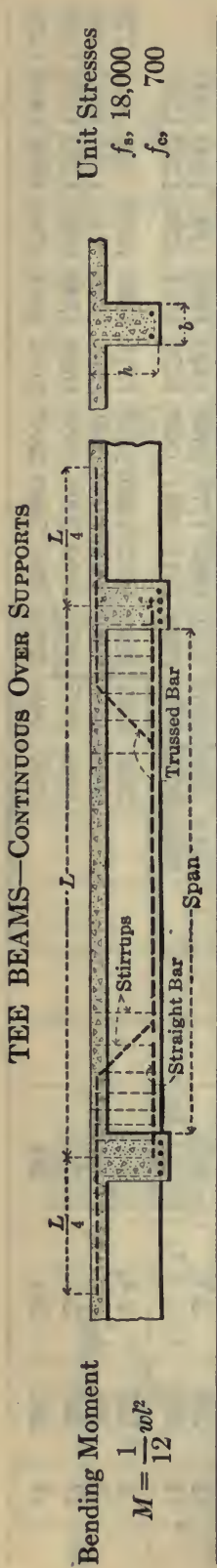

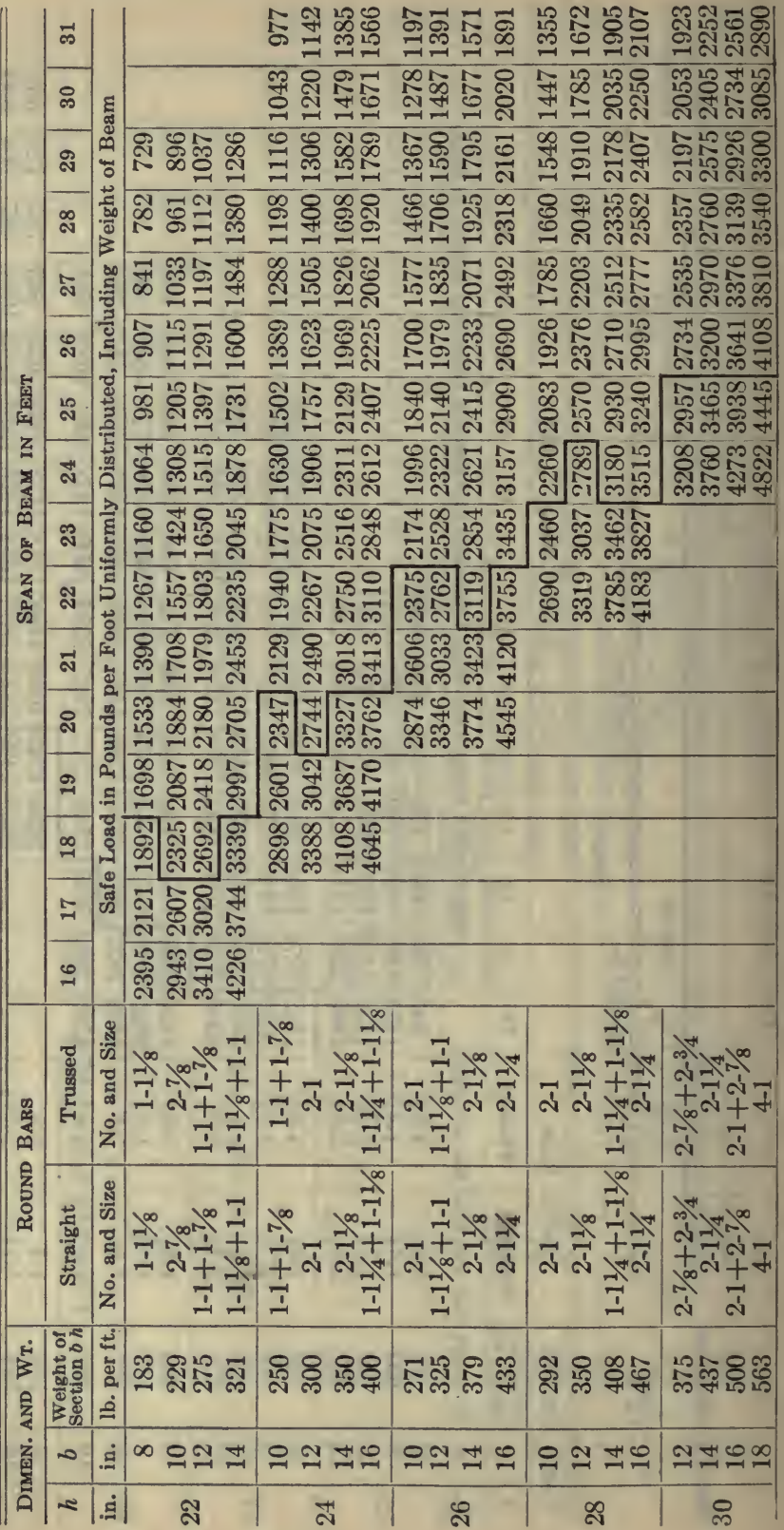




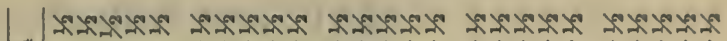

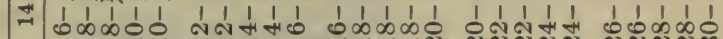

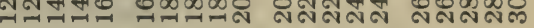

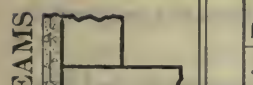

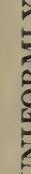

?



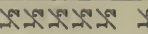

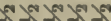
$-x^{-x-1}-x-1$ 에메규

อัดง

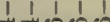

1 1 11

1 1 1

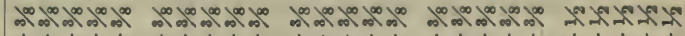

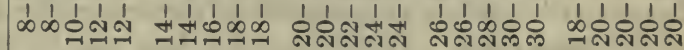

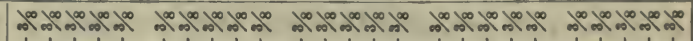

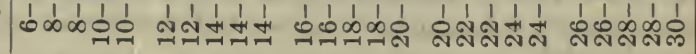

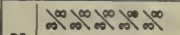
(1)

Non

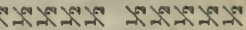

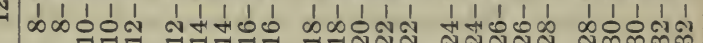

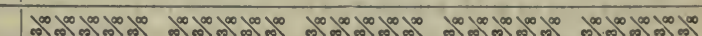

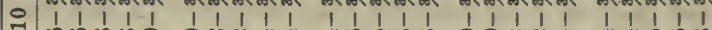

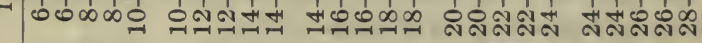

-

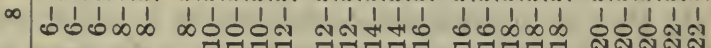

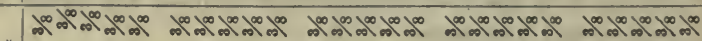

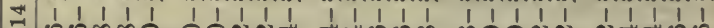

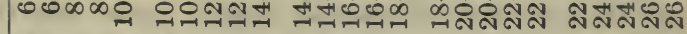

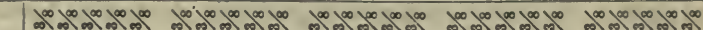

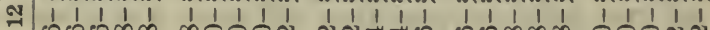

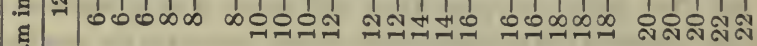

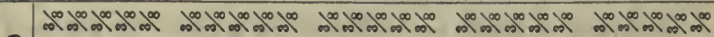

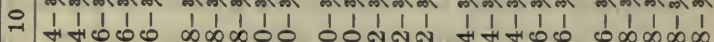

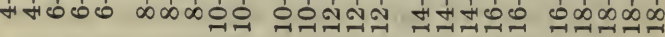

-

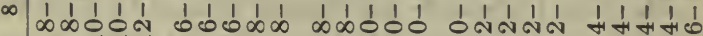

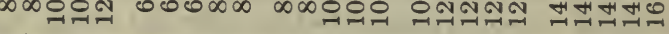

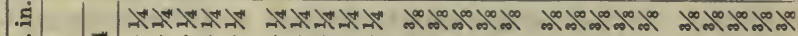

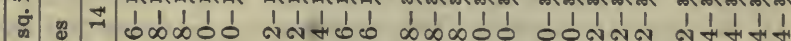
के

: ઘ

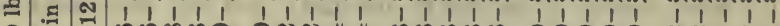

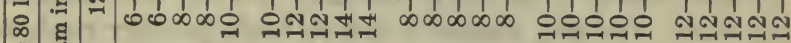

II

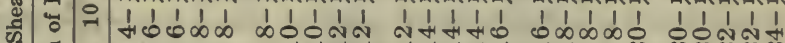
뮴

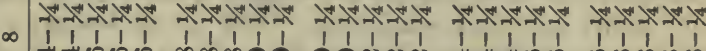

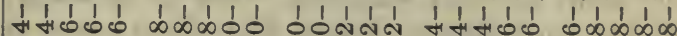
⿷匚⿳丨コ丨卜 


\section{FLAT SLAB TABLES}

\section{CORR-PLATE FLOORS}

(PATENTED)

Flat slab floors, or floors in which beam elements are omitted and the slab supported directly on the columns, are the result of the ever-present demand for efficiency and economy of construction, particularly for buildings in the warehouse and industrial group. This demand has been met by numerous "systems" of flat slab floors, each reflecting the ideas of the designer as to the distribution of the reinforcement; some approaching the ideal distribution and still others varying widely from the mark.

The Corrugated Bar Company, Inc., has developed through its research and laboratory work, accompanied by field tests, a method of design and a system of steel distribution which it is believed meets the conditions of the problem in the most logical manner. This construction is known as Corr-Plate-a flat slab reinforced in two directions in such manner as to conform to the variation in moment that exists in a flat plate on point supports when subjected to load, and for which is claimed the following engineering advantages:

(a) That it can be accurately designed.

(b). That the arrangement of reinforcement in two directions, parallel to the sides of the panel, is the best arrangement to meet the stress distribution as observed in laboratory and field tests.

(c) That the steel distribution adopted varies with the bending moment, being heaviest at the panel margin and gradually decreasing toward mid-panel.

(d) That having no more than two layers of reinforcement at any one point the length of arm of the moment couple at any section is the greatest possible, thus affording maximum strength and stiffness for a given thickness of concrete.

(e) The factor of safety being substantially uniform throughout, there results a saving in quantity of concrete and reinforcement.

Aside from the specific advantages claimed for Corr-Plate Floors, flat slab construction in general appeals to the owner and builder from the economic and service standpoint. The advantages may be summed up in the following brief paragraphs:

(a) No beams or girders. This means saving in forms and by virtue of the flat ceiling, greater economy in the installation of the sprinkler system.

(b) Saves space. From one to one and a half feet of actual free space is gained in every story. This may amount to a story height in every eight, depending upon the standard of ceiling heights used in the building. The reduction in total height of building means a saving in walls, columns, piping, stairways, elevator structure and in every item in a building that is affected by a change in story height.

(c) Better light and ventilation. There are no beams to cast shadows or interfere with a thorough diffusion of light. Flat ceilings remove all obstacles to the free movement of air currents and to that extent assist in maintaining uniform conditions as to temperature, humidity and the removal of vitiated air.

(d) Better fire protection. The damage a concrete building sustains by fire bears a direct relation to the number of corners exposed to the action of heat and water. In this respect it is clear that the beamless floor has a decided advantage over the beam 
and girder type of construction. Again, beams serve to deflect hose streams and to form pockets in the ceiling that collect and intensify heat.

(e) Speed and economy. A flat slab building may be erected in less time and with greater economy than one of the beam and girder type.

The moment factors used in the design of Corr-Plate Floors, as previously stated, are based on the result of experiment in the laboratory supplemented by tests on actual structures where steel and concrete deformations were measured and stress and moment distribution determined therefrom. The distribution of moment, and similarly of reinforcement, is given in Fig. 8, where, to avoid confusion, the distribution is given for only one set of reinforcements. The same methods are applied to the reinforcements at right angles to the ones shown. The figures in the circles are the denominators of the moment coefficient per foot width of slab for the band in which they appear; thus the positive moment per foot of width at the center of the band extending between columns is, $M=\frac{W S^{2}}{20}$ and the negative moment over the column for the same band is $M=\frac{W S^{2}}{10}$

Similarly for the remaining bands into which the panel is divided. The clear span

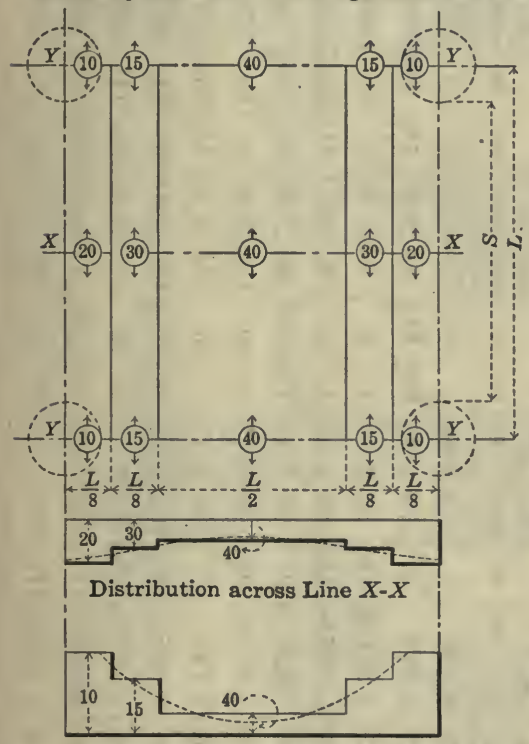

Distribution across Line $Y \cdot Y$ between the column heads in feet, is $S$, and $w$ is the total dead and live load per square foot of floor. In Fig. 8, the heavy stepped line shows the practical distribution of moment while the dashed curved line shows the actual distribution as determined by experiment.

The tables on pages 110 and 111 are based on standard Corr-Plate design for stresses of $f_{\mathrm{B}}=16,000, f_{\mathrm{o}}=650$; and $f_{\mathrm{B}}=$ $18,000, f_{\mathrm{c}}=700$, respectively, and are for square interior panels. Similar tables are given on pages 112 and 113 to meet the requirements of the Chicago Flat Slab ruling and the Final Report of the Joint Committee. The latter report is given in detail on pages 194 to 211 .

The approximate weight of reinforcement per square foot of floor area is given for each span and load and includes steel required for the support of the negative moment reinforcement.

Fig. 8 


\begin{tabular}{|c|c|c|c|c|c|c|c|c|c|c|c|c|c|}
\hline : & 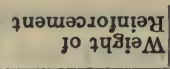 & $\mid$ & ஸ் & ก่ & $\ddot{n}$ & ले & की & in & Fi & + & & 2 & 6 \\
\hline : & J0 748 & $\doteq \underset{\square}{0}$ & $\Xi$ & İ & సิ & ణ్ & \& & $\stackrel{\infty}{=}$ & सै & ญี & O & $\stackrel{10}{1}$ & \\
\hline छ & $\mathbb{E}$ &.$\Xi$ & $\stackrel{\sim}{\sim}$ & m & लू & $\Xi$ & ती & 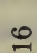 & छा & $\stackrel{\infty}{\simeq}$ & $\underset{-1}{\infty}$ & $=$ & \\
\hline 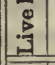 & 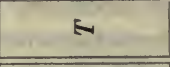 &.$\Xi$ & $-\infty$ & a & के & 의 & $0^{2}$ & $=$ & $\Rightarrow$ & $\stackrel{\text { I }}{ }$ & $\cong$ & $=$ & \\
\hline 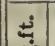 & 7นวนวәอ & 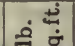 & ชิ & $\stackrel{0}{0}$ & जั & gr & $E$ & $\infty$ & $\stackrel{0}{=}$ & $D_{-1}^{\infty}$ & 2 & 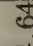 & \\
\hline 。্ळ & & -0 & $N$ & $\infty$ & $\infty$ & $\infty$ & $\infty$ & $n$ & $\forall$ & + & t & $\nabla$ & \\
\hline 5 & 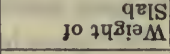 & 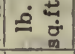 & ชิ & $\stackrel{2}{0}$ & $\Xi$ & స్- & ลิ & ๓్ & ణ్లి & F & 起 & @ & \\
\hline : & $\approx$ & $\Xi$ & 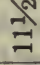 & 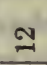 & ले & $\stackrel{\infty}{\sim}$ & $\stackrel{-2}{2}$ & \pm & $\stackrel{ \pm}{ \pm}$ & $\stackrel{0}{-1}$ & $=$ & $\propto$ & \\
\hline 总 & Fi & $\leqq$ & $\mathrm{n}^{2}$ & 0 & $\hat{\infty}^{N}$ & o & $\omega^{2}$ & 음 & $0_{0}^{-1}$ & $\nexists$ & $\Rightarrow$ & - & \\
\hline 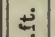 & y & & $\infty$ & $\Re$ & $\bar{\sigma}$ & 28 & ஸ & 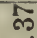 & $\overline{6}$ & $\infty$ & $\infty$ & $\vec{J}$ & \\
\hline$\dot{\varnothing}$ & M & $=\sigma_{0}^{\circ}$ & N & ง & N & ก & r & $?$ & 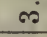 & ๓ & 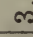 & $\nabla$ & \\
\hline$\frac{0}{8}$ & $\begin{array}{r}q \varepsilon i S \\
\text { jo } 743 ! \rho_{\Lambda} \\
\end{array}$ & 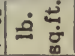 & \& & $\stackrel{\infty}{\infty}$ & $\stackrel{8}{0}$ & $\stackrel{N}{\Xi}$ & $\stackrel{N}{=}$ & ิำ & N & ๓్- & f & $\stackrel{2}{\leftarrow}$ & \\
\hline : & $\approx$ &. & $\infty$ & $\vec{\sigma}^{\alpha}$ & $=$ & 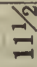 & $=$ & 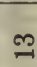 & $\stackrel{\text { m- }}{-1}$ & \pm & 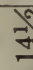 & 5 & \\
\hline$\stackrel{0}{3}$ & E & ఏ & $N$ & $N^{2}$ & $\infty$ & $\vec{\infty}^{N}$ & $\vec{\infty}^{N}$ & 0 & $0^{-1}$ & 으 & 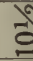 & $\subseteq$ & \\
\hline 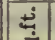 & 7) पәш & \pm & $\stackrel{\infty}{-}$ & $\dot{m}$ & in & ? & $\infty$ & $\mathscr{8}$ & ติ & $\bar{m}$ & ? & \& & \\
\hline ర్ & 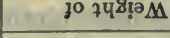 & $=\dot{\infty}_{\infty}^{\circ}$ & N & ง & ஸ & ก & N & N & ம & ம & 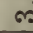 & $\infty$ & \\
\hline 용 & jo $74^{\circ}$ & 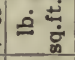 & R & $ஜ$ & ๙ิ & ชิ & $\infty$ & $\ddot{0}$ & $\stackrel{0}{0}$ & $\stackrel{N}{\Xi}$ & & 5 & \\
\hline : & $\approx$ &. & $\infty$ & $\overbrace{}^{N}$ & a & $\infty$ & $\sigma^{N}$ & 음 & 을 & 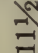 & I & 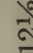 & \\
\hline 站 & E & .7 & $\infty$ & $\hat{0}^{-1}$ & $N$ & $N$ & $\stackrel{N}{N}^{N}$ & $\infty$ & $\infty$ & $\infty$ & $\sigma$ & 0 & \\
\hline 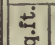 & quə & $\stackrel{5}{\varrho}$ & চ & $\stackrel{20}{?}$ & O & भ. & F & 8 & R & 5 & I & & \\
\hline & & ס & -1 & N & i & N & N & ஸ & N & ต & $\infty$ & $\infty$ & 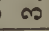 \\
\hline 옥 & $\begin{array}{r}q^{8} \mathrm{IS} \\
\text { jo } 74^{9 ! \partial} M\end{array}$ & $\triangleq \stackrel{0}{\dot{0}}$ & R & R & $\notin$ & $\infty$ & ชิ & $\infty$ & 으 & +1 & $\equiv$ & $=$ & \\
\hline 융 & $\mathbb{Z}$ & . & $\infty$ & $\infty$ & $-\infty$ & $\vec{\infty}^{-}$ & C & के & 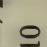 & 을 & 인 & - & \\
\hline$\frac{5}{3}$ & E &. & & & $\frac{1}{6}$ & $\omega^{N}$ & & $N^{2}$ & & & $\vec{\infty}$ & & \\
\hline 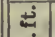 & y & $=$ & లి & ๓ & ஸे & F & กิ & 18 & N & $\infty$ & $\sigma$ & e & \\
\hline 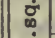 & I & ष्ठ & $-i$ & $\sim$ & - & - & $-\dot{-}$ & - & - & $-i$ & - & ค & \\
\hline 0 & $\begin{array}{c}\text { qeis } \\
\text { jo } 74^{8} \operatorname{li}^{\circ} \Lambda\end{array}$ & 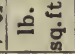 & $\Re$ & $\Re$ & $n$ & R & R & R & $\infty$ & $\infty$ & 8 & $\mathscr{c}$ & \\
\hline 0 & $\nabla$ & $\Xi$ & $\mathrm{N}^{2}$ & $\lambda^{N}$ & $\lambda^{2}$ & $\infty$ & $\infty$ & $\infty$ & $\vec{\infty}^{N}$ & $\vec{\infty}$ & $\sigma$. & $\sigma$ & \\
\hline 3 & E &. & -12 & in & $\mathrm{in}^{2}$ & 0 & & & $\overrightarrow{0}^{2}$ & 0 & $\theta$ & & \\
\hline & 6 &. & $\Rightarrow$ & $\mathscr{H}$ & $\mathscr{H}$ & $\frac{9}{4}$ & กิ & Iก & 웅 & 8 & है & ${ }^{26}$ & $\begin{array}{l}\alpha \\
e \\
e\end{array}$ \\
\hline & 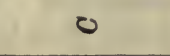 & ـ & @ & $\begin{array}{l}0 \\
0\end{array}$ & 0 & $\stackrel{p}{n}$ & $\underset{1}{1}$ & \& & $\stackrel{0}{\infty}$ & $\infty$ & $\sigma_{j}$ & 0 & \\
\hline & & 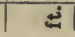 & 20 & $\oplus$ & 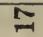 & $\infty$ & 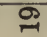 & 요 & $\bar{N}$ & c & 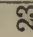 & $\bar{J}$ & \\
\hline
\end{tabular}




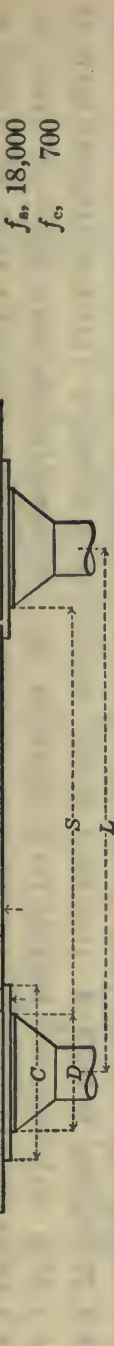

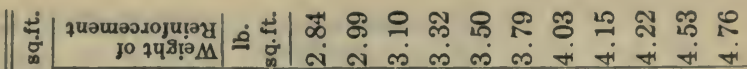

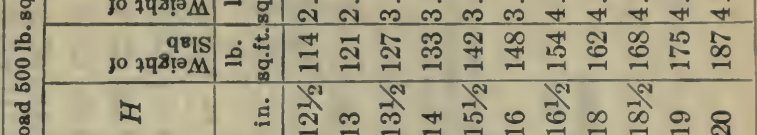

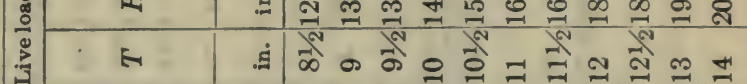

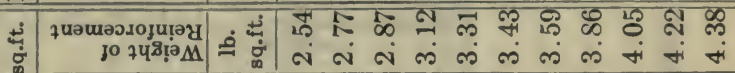
ఏ رо

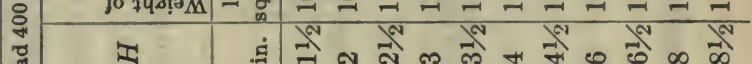
च $\quad$ I :

\section{西}

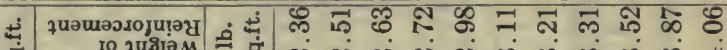

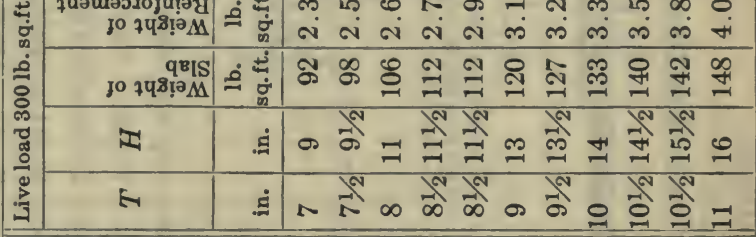

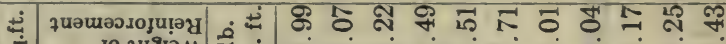
Ð0

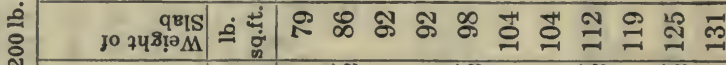

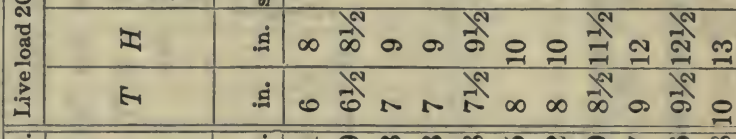

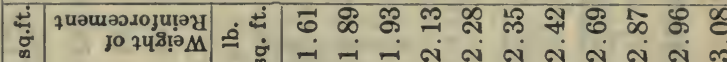

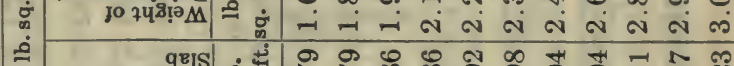

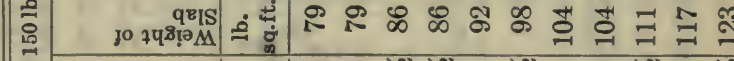

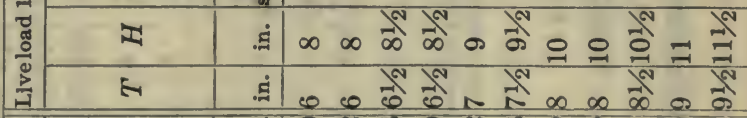

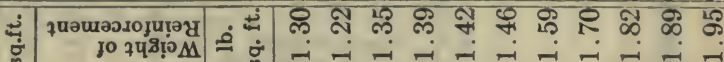
㶾

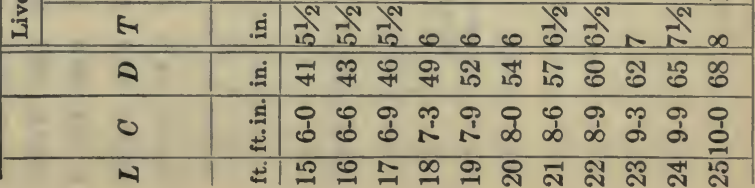




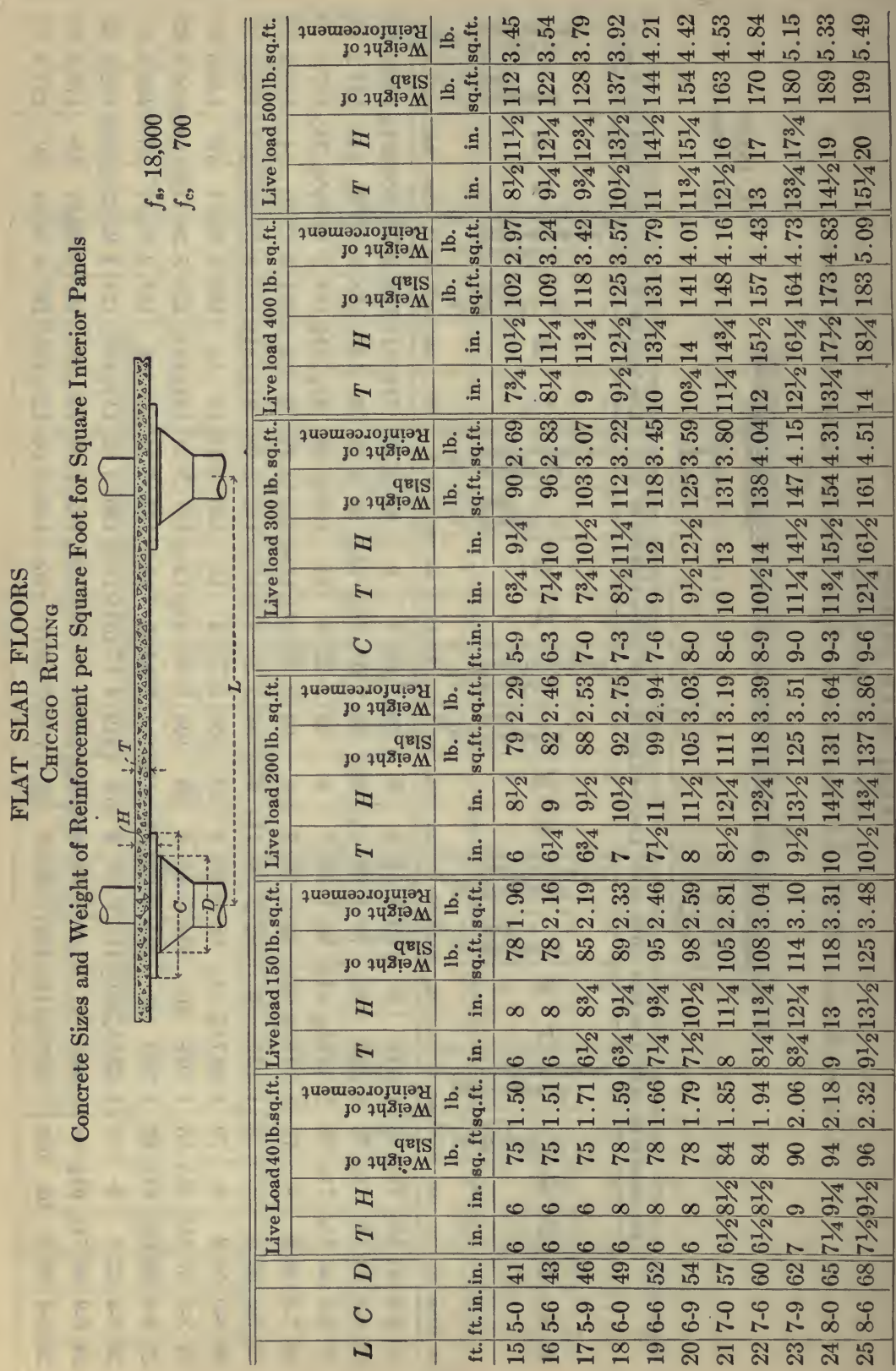




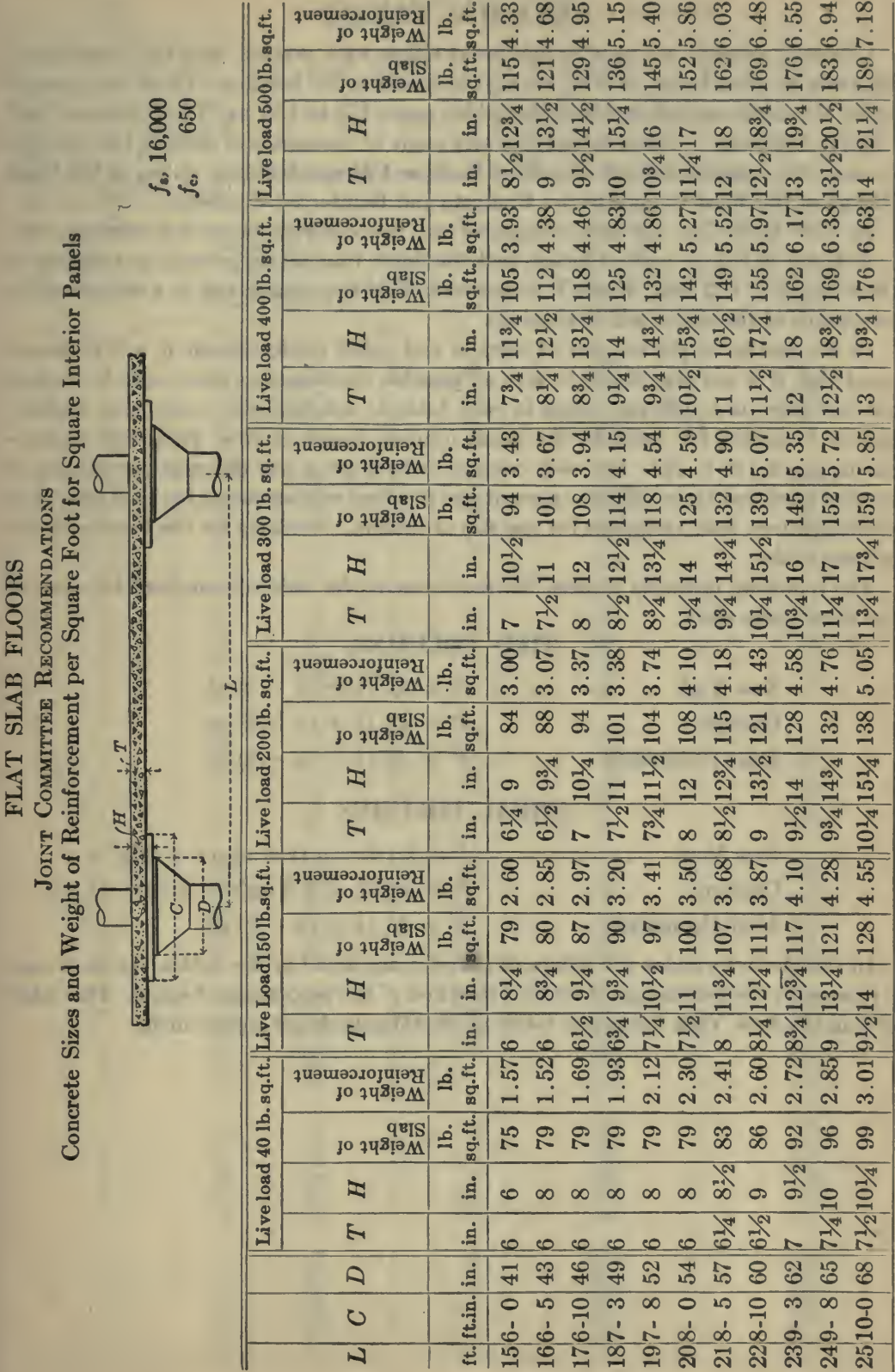




\section{COLUMN TABLES}

Concrete columns are usually reinforced either with vertical bars tied together at intervals by steel hoops or with vertical bars and spiral hooping. These two general types of column are referred to in the tables, pages 115 to 132, as "Tied Columns" and "Spiral Columns." These tables give safe loads in thousands of pounds for columns to meet the requirements of the New York or Chicago building codes, or the Final Report of the Joint Committee on Concrete and Reinforced Concrete.

In New York City the code recognizes but two concrete mixes for columns, viz., 1: $2: 4$ and $1: 1 \frac{1}{2}: 3$, while Chicago and the Joint Committee permit, in addition to these two, a 1:1:2 mix. Where it is desired to hold the column size to a minimum the advantage of the richer mix is apparent.

In the matter of percentages of vertical and spiral reinforcement it will be recognized that it is not practicable to give all possible combinations that could be worked out but there is a sufficient range in each table to satisfactorily cover most requirements. Consider, for example, the spiral columns based on New York code requirements, pages 122 to 124 . For each column there is given five different percentages of vertical steel and three different percentages of spiral reinforcement for each mix, thus yielding fifteen load variations for one size of column. Similarly for the balance of the column tables.

The following formulas express the requirements for safe column load for each of the three codes used:

\section{TIED COLUMNS}

New York . . . P P $=A f_{\mathrm{c}}[1+(n-1) p]$
Chicago . . . P P $=A f_{\mathrm{c}}[1+(n-1) p]$
Joint Committee . P $P=A f_{\mathrm{c}}[1+(n-1) p]$

\section{SPIRAL COLUMNS}

New York .....P $P=f_{\mathrm{c}}(A-p A)+n f_{\mathrm{c}} p A+2 f_{\mathrm{s}} p^{\prime} A$

Chicago . . . . . P $P=A f_{\mathrm{c}}\left(1+2.5 n p^{\prime}\right)[1+(n-1) p]$

Joint Committee ....P $P=A f_{\mathrm{c}}[1+(n-1) p]$

In the above formulas the values of $f_{\mathrm{c}}$ and $n$ are noted in the tables. In each case $p$ represents the percentage of vertical steel and $p^{\prime}$ the percentage of spiral. The value of $f_{\mathrm{a}}$ in the New York formula is taken at 20,000 pounds per square inch. 
Column

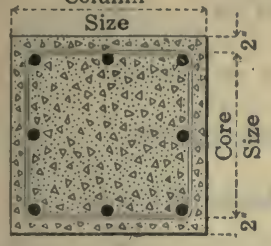

SQUARE TIED COLUMNS

Safe Axial Loads in Thousands of Pounds

New York City Building Code Requirements

Ratio of Length of Column to its Side, limited to 15

\begin{tabular}{|c|c|c|c|c|c|c|c|}
\hline \multirow{3}{*}{$\begin{array}{c}\begin{array}{c}\text { Column } \\
\text { Size }\end{array} \\
\text { in. } \\
\end{array}$} & \multirow{3}{*}{$\begin{array}{l}\text { Core } \\
\text { Size } \\
\text { in. }\end{array}$} & \multicolumn{2}{|c|}{$\begin{array}{c}\text { ROUND BAR } \\
\text { TIES } \\
\end{array}$} & \multirow{2}{*}{\multicolumn{2}{|c|}{$\begin{array}{c}\text { ROUND BAR } \\
\text { VERTICALS }\end{array}$}} & \multirow{3}{*}{$\begin{array}{c}\text { 1: 2: } 4 \text { Concrete } \\
f_{\mathrm{c}}=500 \mathrm{lb} . \text { per sq. in. } \\
n=15\end{array}$} & \multirow{3}{*}{$\begin{array}{c}1: 11 / 2: 3 \text { Concrete } \\
f_{0}=600 \mathrm{lb} . \text { per sq. in. } \\
n=12\end{array}$} \\
\hline & & Size & Spacing & & & & \\
\hline & & in. & in. & No. & Size & & \\
\hline 12 & 8 & $\begin{array}{l}1 / 4 \\
1 / 4 \\
1 / 4 \\
1 / 4 \\
1 / 4\end{array}$ & $\begin{array}{r}5 \\
7 \\
9 \\
11 \\
12\end{array}$ & $\begin{array}{l}4 \\
4 \\
4 \\
4 \\
4\end{array}$ & $\begin{array}{l}3 / 8 \\
1 / 2 \\
5 / 8 \\
3 / 4 \\
7 / 8\end{array}$ & $\begin{array}{l}35 \\
38 \\
41 \\
44 \\
49\end{array}$ & $\begin{array}{l}41 \\
44 \\
46 \\
50 \\
54\end{array}$ \\
\hline 13 & 9 & $\begin{array}{l}1 / 4 \\
11 / 4 \\
11 / 4 \\
1 / 4 \\
1 / 4 \\
1 / 4\end{array}$ & $\begin{array}{r}5 \\
7 \\
9 \\
11 \\
12 \\
12\end{array}$ & $\begin{array}{l}4 \\
4 \\
4 \\
4 \\
4 \\
4\end{array}$ & $\begin{array}{l}8 / 8 \\
1 / 2 \\
5 / 8 \\
8 / 4 \\
1^{7 / 8}\end{array}$ & $\begin{array}{l}44 \\
46 \\
49 \\
53 \\
57 \\
62\end{array}$ & $\begin{array}{l}52 \\
54 \\
57 \\
60 \\
64 \\
69\end{array}$ \\
\hline 14 & 10 & $\begin{array}{l}1 / 4 \\
1 / 4 \\
1 / 4 \\
1 / 4 \\
1 / 4 \\
1 / 4\end{array}$ & $\begin{array}{r}7 \\
9 \\
11 \\
12 \\
12 \\
12\end{array}$ & $\begin{array}{l}4 \\
4 \\
4 \\
4 \\
4 \\
4\end{array}$ & $\begin{array}{r}1 / 2 \\
3 / 8 \\
8 / 4 \\
7 / 8 \\
11 \\
11 / 8\end{array}$ & $\begin{array}{l}55 \\
59 \\
62 \\
67 \\
72 \\
78\end{array}$ & $\begin{array}{l}65 \\
68 \\
72 \\
76 \\
81 \\
86\end{array}$ \\
\hline 15 & 11 & $\begin{array}{l}1 / 4 \\
1 / 4 \\
1 / 4 \\
1 / 4 \\
1 / 4 \\
1 / 4\end{array}$ & $\begin{array}{r}7 \\
9 \\
11 \\
12 \\
12 \\
12\end{array}$ & $\begin{array}{l}4 \\
4 \\
4 \\
4 \\
4 \\
6\end{array}$ & $\begin{array}{l}1 / 2 \\
\frac{1}{3 / 8} \\
3 / 4 \\
1^{7 / 8} \\
1\end{array}$ & $\begin{array}{l}66 \\
69 \\
73 \\
77 \\
82 \\
93\end{array}$ & $\begin{array}{r}78 \\
81 \\
84 \\
88 \\
93 \\
104\end{array}$ \\
\hline 16 & 12 & $\begin{array}{l}1 / 4 \\
1 / 4 \\
1 / 4 \\
1 / 4 \\
1 / 4 \\
1 / 4\end{array}$ & $\begin{array}{r}7 \\
11 \\
12 \\
12 \\
12 \\
12\end{array}$ & $\begin{array}{l}4 \\
4 \\
4 \\
4 \\
4 \\
6 \\
6\end{array}$ & $\begin{array}{l}1 / 2 \\
8 / 4 \\
7 / 8 \\
1 \\
11 / 8 \\
11 / 8\end{array}$ & $\begin{array}{r}77 \\
84 \\
89 \\
94 \\
100 \\
114\end{array}$ & $\begin{array}{r}92 \\
98 \\
102 \\
107 \\
113 \\
126\end{array}$ \\
\hline 17 & 13 & $\begin{array}{l}1 / 4 \\
1 / 4 \\
1 / 4 \\
1 / 4 \\
1 / 4 \\
1 / 4\end{array}$ & $\begin{array}{r}9 \\
11 \\
12 \\
12 \\
12 \\
12\end{array}$ & $\begin{array}{l}4 \\
4 \\
4 \\
6 \\
6 \\
8 \\
8\end{array}$ & $\begin{array}{l}\frac{8}{8 / 8} \\
3 / 4 \\
7 / 8 \\
1^{7 / 8} \\
1\end{array}$ & $\begin{array}{r}93 \\
97 \\
101 \\
110 \\
117 \\
128\end{array}$ & $\begin{array}{l}110 \\
113 \\
117 \\
125 \\
133 \\
143\end{array}$ \\
\hline 18 & 14 & $\begin{array}{l}1 / 4 \\
1 / 4 \\
1 / 4 \\
1 / 4 \\
1 / 4 \\
1 / 4\end{array}$ & $\begin{array}{r}9 \\
12 \\
12 \\
12 \\
12 \\
12\end{array}$ & $\begin{array}{l}4 \\
4 \\
4 \\
6 \\
6 \\
8\end{array}$ & $\begin{array}{l}\frac{5}{3 / 8} \\
13 / 8 \\
17 / 8 \\
11 / 8 \\
11 / 8\end{array}$ & $\begin{array}{l}107 \\
115 \\
120 \\
123 \\
140 \\
154\end{array}$ & $\begin{array}{l}126 \\
134 \\
139 \\
142 \\
158 \\
171\end{array}$ \\
\hline 19 & 15 & $\begin{array}{l}1 / 4 \\
1 / 4 \\
1 / 4 \\
1 / 4 \\
11 / 4 \\
1 / 4 \\
\end{array}$ & $\begin{array}{r}9 \\
12 \\
12 \\
12 \\
12 \\
12 \\
\end{array}$ & $\begin{array}{l}4 \\
4 \\
6 \\
6 \\
8 \\
8\end{array}$ & $\begin{array}{l}5 / 8 \\
7 / 8 \\
7 / 8 \\
1 \\
111 / 8 \\
\end{array}$ & $\begin{array}{l}121 \\
129 \\
138 \\
145 \\
156 \\
168 \\
\end{array}$ & $\begin{array}{l}143 \\
151 \\
159 \\
166 \\
176 \\
188\end{array}$ \\
\hline
\end{tabular}


CORRUGATED BAR COMPANY, INC.

Tied

Columns

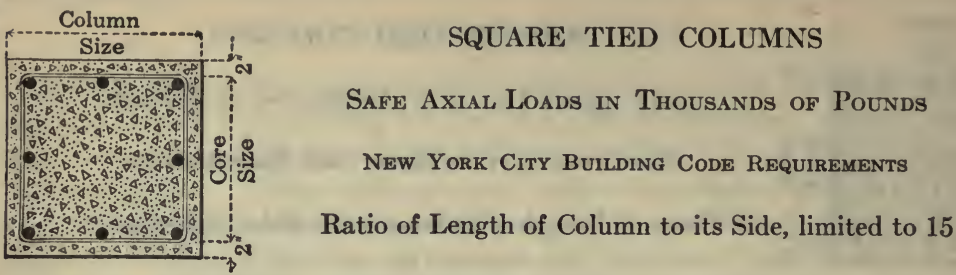

\begin{tabular}{|c|c|c|c|c|c|c|c|}
\hline \multirow{3}{*}{$\begin{array}{c}\begin{array}{c}\text { Column } \\
\text { Size }\end{array} \\
\text { in. }\end{array}$} & \multirow{3}{*}{$\begin{array}{r}\text { Core } \\
\text { Size }\end{array}$} & \multicolumn{2}{|c|}{$\begin{array}{c}\text { ROUND BAR } \\
\text { TIES }\end{array}$} & \multirow{2}{*}{\multicolumn{2}{|c|}{$\begin{array}{c}\text { ROUND BAR } \\
\text { VeRTICALS }\end{array}$}} & \multirow{3}{*}{$\begin{array}{c}\text { 1: } 2: 4 \text { Concrete } \\
f_{\mathrm{c}}=500 \mathrm{lb} . \text { per sq. in. } \\
n=15\end{array}$} & \multirow{3}{*}{$\begin{array}{c}1: 11 / 2: 3 \text { Concrete } \\
f_{\mathrm{o}}=600 \mathrm{lb} . \text { per sq. in. } \\
n=12\end{array}$} \\
\hline & & Size & Spacing & & & & \\
\hline & & in. & in. & No. & Size & & \\
\hline 20 & 16 & $\begin{array}{l}1 / 4 \\
1 / 4 \\
1 / 4 \\
1 / 4 \\
1 / 4 \\
1 / 4\end{array}$ & $\begin{array}{l}11 \\
12 \\
12 \\
12 \\
12 \\
12\end{array}$ & $\begin{array}{r}4 \\
4 \\
6 \\
8 \\
8 \\
10\end{array}$ & $\begin{array}{l}13 / 4 \\
7 / 8 \\
7 / 8 \\
11 / 8 \\
11 / 8\end{array}$ & $\begin{array}{l}140 \\
150 \\
153 \\
162 \\
184 \\
197\end{array}$ & $\begin{array}{l}165 \\
174 \\
177 \\
185 \\
206 \\
219\end{array}$ \\
\hline 21 & 17 & $\begin{array}{l}1 / 4 \\
1 / 4 \\
1 / 4 \\
1 / 4 \\
1 / 4 \\
1 / 4\end{array}$ & $\begin{array}{l}11 \\
12 \\
12 \\
12 \\
12 \\
12\end{array}$ & $\begin{array}{r}4 \\
4 \\
6 \\
8 \\
8 \\
8 \\
12\end{array}$ & $\begin{array}{l}1^{8 / 4} \\
1 \\
1 \\
11 / 8 \\
11 / 8\end{array}$ & $\begin{array}{r}157 \\
166 \\
177 \\
188 \\
200 \\
228\end{array}$ & $\begin{array}{l}185 \\
194 \\
204 \\
215 \\
226 \\
252\end{array}$ \\
\hline 22 & 18 & $\begin{array}{l}1 / 4 \\
1 / 4 \\
1 / 4 \\
1 / 4 \\
1 / 4 \\
1 / 4\end{array}$ & $\begin{array}{l}11 \\
12 \\
12 \\
12 \\
12 \\
12\end{array}$ & $\begin{array}{r}4 \\
6 \\
6 \\
8 \\
8 \\
12\end{array}$ & $\begin{array}{l}\frac{8 / 4}{1 / 8} \\
1 \\
1 \\
11 / 4 \\
11 / 8\end{array}$ & $\begin{array}{l}174 \\
187 \\
195 \\
206 \\
231 \\
245\end{array}$ & $\begin{array}{l}206 \\
218 \\
226 \\
236 \\
259 \\
273\end{array}$ \\
\hline 23 & 19 & $\begin{array}{l}1 / 4 \\
1 / 4 \\
1 / 4 \\
1 / 4 \\
1 / 4 \\
1 / 4\end{array}$ & $\begin{array}{r}9 \\
12 \\
12 \\
12 \\
12 \\
12\end{array}$ & $\begin{array}{r}6 \\
6 \\
6 \\
10 \\
10 \\
12\end{array}$ & $\begin{array}{l}5 / 8 \\
7 / 8 \\
11 / 8 \\
1 \\
11 / 8 \\
11 / 4\end{array}$ & $\begin{array}{l}193 \\
206 \\
222 \\
235 \\
250 \\
284\end{array}$ & $\begin{array}{l}229 \\
240 \\
256 \\
268 \\
282 \\
314\end{array}$ \\
\hline 24 & 20 & $\begin{array}{l}1 / 4 \\
1 / 4 \\
1 / 4 \\
1 / 4 \\
1 / 4 \\
1 / 4\end{array}$ & $\begin{array}{l}11 \\
12 \\
12 \\
12 \\
12 \\
12\end{array}$ & $\begin{array}{r}6 \\
6 \\
6 \\
10 \\
12 \\
16\end{array}$ & $\begin{array}{l}1^{8 / 4} \\
11 / 8 \\
11 \\
11 / 8 \\
11 / 8\end{array}$ & $\begin{array}{l}219 \\
233 \\
242 \\
255 \\
284 \\
311\end{array}$ & $\begin{array}{l}258 \\
271 \\
279 \\
292 \\
319 \\
345\end{array}$ \\
\hline 25 & 21 & $\begin{array}{l}1 / 4 \\
1 / 4 \\
1 / 4 \\
1 / 4 \\
1 / 4 \\
1 / 4\end{array}$ & $\begin{array}{l}11 \\
12 \\
12 \\
12 \\
12 \\
12\end{array}$ & $\begin{array}{r}6 \\
6 \\
8 \\
12 \\
14 \\
18\end{array}$ & $\begin{array}{l}1^{8 / 4} \\
1 \\
1 \\
11 / 8 \\
11 / 8\end{array}$ & $\begin{array}{l}239 \\
254 \\
265 \\
286 \\
318 \\
346\end{array}$ & $\begin{array}{l}282 \\
296 \\
306 \\
327 \\
356 \\
383\end{array}$ \\
\hline 26 & 22 & $\begin{array}{l}1 / 4 \\
1 / 4 \\
1 / 4 \\
1 / 4 \\
1 / 4 \\
1 / 4\end{array}$ & $\begin{array}{r}9 \\
12 \\
12 \\
12 \\
12 \\
12\end{array}$ & $\begin{array}{r}8 \\
8 \\
10 \\
12 \\
14 \\
18\end{array}$ & $\begin{array}{l}5 / 8 \\
1^{7 / 8} \\
1 \\
11 / 8 \\
11 / 8\end{array}$ & $\begin{array}{l}259 \\
276 \\
297 \\
308 \\
340 \\
367\end{array}$ & $\begin{array}{l}307 \\
322 \\
342 \\
353 \\
382 \\
409\end{array}$ \\
\hline 27 & 23 & $\begin{array}{l}1 / 4 \\
1 / 4 \\
1 / 4 \\
1 / 4 \\
11 / 4 \\
1 / 4\end{array}$ & $\begin{array}{r}9 \\
12 \\
12 \\
12 \\
12 \\
12\end{array}$ & $\begin{array}{l}10 \\
10 \\
10 \\
14 \\
16 \\
20\end{array}$ & $\begin{array}{l}5 / 8 \\
7 / 8 \\
1 \\
11 / 8 \\
11 / 8\end{array}$ & $\begin{array}{l}286 \\
307 \\
320 \\
\mathbf{3 4 1} \\
\mathbf{3 7 6} \\
\mathbf{4 0 4}\end{array}$ & $\begin{array}{l}338 \\
357 \\
369 \\
390 \\
422 \\
449\end{array}$ \\
\hline
\end{tabular}


Column

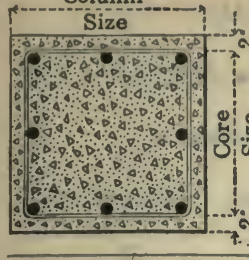

SQUARE TIED COLUMNS

Ratio of Length of Column to its Side, limited to 15

New York City Building Code Requirements

\section{Safe Axial Loads in Thousands of Pounds}

\section{$\frac{8}{2}$}

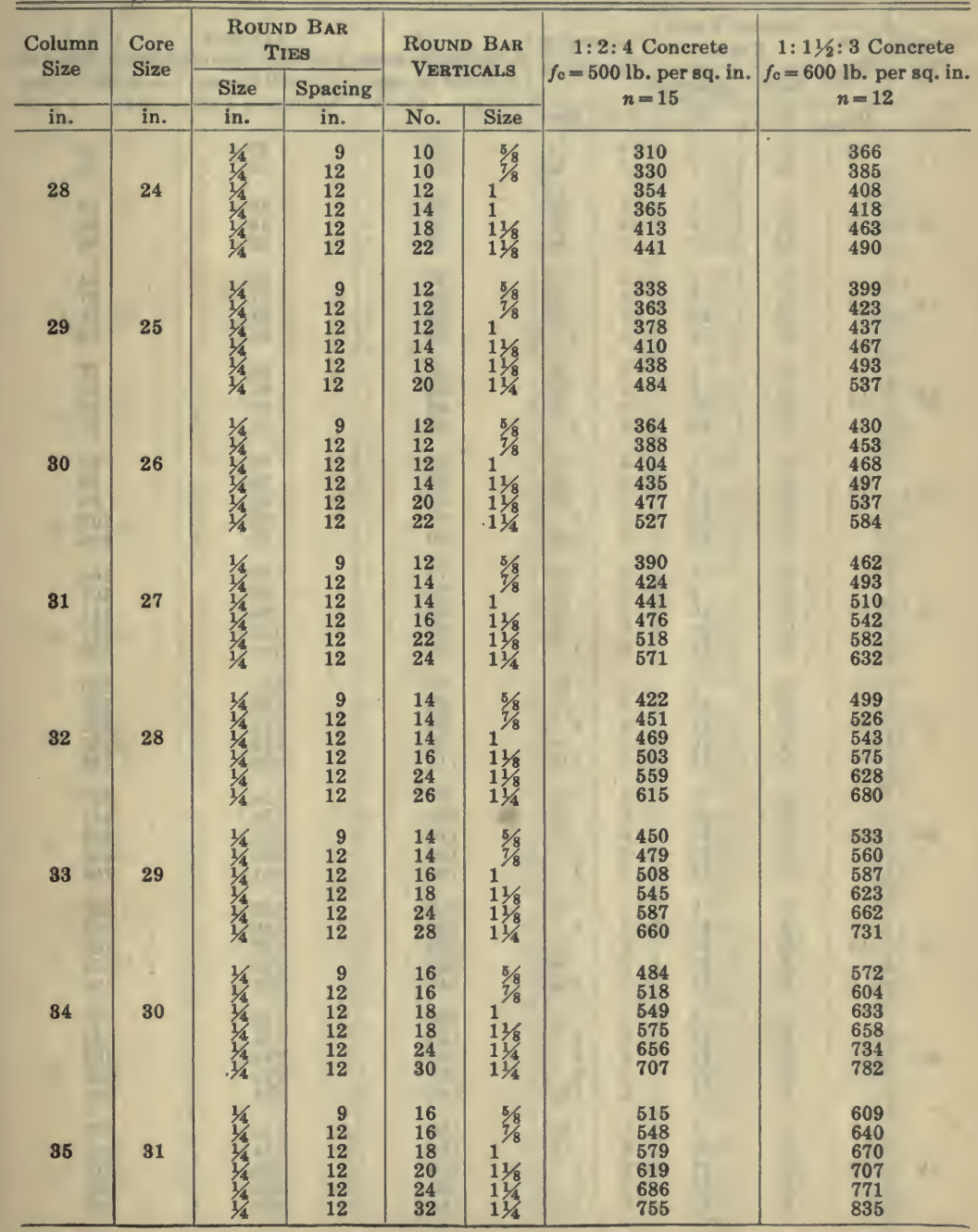


CORRUGATED BAR COMPANY, INC.

Tied

Columns

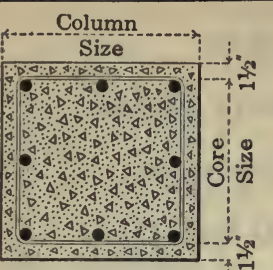

SQUARE TIED COLUMNS

Safe Axial Loads in Thousands of Pounds

Chicago Building Code Requirements

Ratio of Length of Column to its Side, limited to 12

\begin{tabular}{|c|c|c|c|c|c|c|c|c|}
\hline \multirow{3}{*}{$\begin{array}{c}\text { Column } \\
\text { Size } \\
\text { in. }\end{array}$} & \multirow{3}{*}{$\begin{array}{l}\text { Core } \\
\text { Size } \\
\text { in. }\end{array}$} & \multicolumn{2}{|c|}{$\begin{array}{c}\text { ROUND BAR } \\
\text { TIES }\end{array}$} & \multirow{2}{*}{\multicolumn{2}{|c|}{$\begin{array}{l}\text { ROUND BAR } \\
\text { VERTICALS }\end{array}$}} & \multirow{3}{*}{$\begin{array}{c}1: 2: 4 \\
2,000 \text { lb.Concrete } \\
f_{\mathrm{c}}=400 \mathrm{lb} . \text { per } \\
\text { sq. in. } \quad n=15\end{array}$} & \multirow{3}{*}{$\begin{array}{c}1: 11 / 2: 3 \\
2,400 \text { lb.Concrete } \\
f_{\mathrm{c}}=480 \text { lb. per } \\
\text { sq. in. } n=12\end{array}$} & \multirow{3}{*}{$\begin{array}{c}1: 1: 2 \\
\text { 2,900 lb. Concrete } \\
f_{c}=580 \text { lb. per } \\
\text { sq. in. } \quad n=10\end{array}$} \\
\hline & & \multirow{2}{*}{$\begin{array}{c}\text { Size } \\
\text { in. }\end{array}$} & \multirow{2}{*}{\begin{tabular}{|c|} 
Spacing \\
in. \\
\end{tabular}} & & & & & \\
\hline & & & & No. & Size & & & \\
\hline 11 & 8 & $\begin{array}{l}1 / 4 \\
1 / 4\end{array}$ & $\begin{array}{l}7 \\
9\end{array}$ & $\begin{array}{l}4 \\
4\end{array}$ & $\begin{array}{l}5 / 8 \\
3 / 4\end{array}$ & $\begin{array}{l}32 \\
35\end{array}$ & $\begin{array}{l}37 \\
40\end{array}$ & $\begin{array}{l}44 \\
46\end{array}$ \\
\hline 12 & 9 & $\begin{array}{l}1 / 4 \\
1 / 4 \\
1 / 4\end{array}$ & $\begin{array}{r}7 \\
9 \\
10\end{array}$ & $\begin{array}{l}4 \\
4 \\
4\end{array}$ & $\begin{array}{l}5 / 8 \\
3 / 4 \\
7 / 8\end{array}$ & $\begin{array}{l}39 \\
42 \\
46\end{array}$ & $\begin{array}{l}45 \\
48 \\
52\end{array}$ & $\begin{array}{l}53 \\
56 \\
60\end{array}$ \\
\hline 13 & 10 & $\begin{array}{l}1 / 4 \\
1 / 4 \\
1 / 4 \\
1 / 4\end{array}$ & $\begin{array}{r}7 \\
9 \\
10 \\
12\end{array}$ & $\begin{array}{l}4 \\
4 \\
4 \\
4\end{array}$ & $1^{\frac{5}{8 / 8}}{ }^{7 / 8}$ & $\begin{array}{l}47 \\
50 \\
53 \\
58\end{array}$ & $\begin{array}{l}54 \\
57 \\
61 \\
65\end{array}$ & $\begin{array}{l}64 \\
67 \\
71 \\
74\end{array}$ \\
\hline 14 & 11 & $\begin{array}{l}1 / 4 \\
1 / 4 \\
1 / 4 \\
1 / 4\end{array}$ & $\begin{array}{r}7 \\
9 \\
10 \\
12\end{array}$ & $\begin{array}{l}4 \\
4 \\
4 \\
4\end{array}$ & $1^{5 / 8} \frac{8 / 4}{7 / 8}$ & $\begin{array}{l}55 \\
58 \\
62 \\
66\end{array}$ & $\begin{array}{l}65 \\
67 \\
71 \\
75\end{array}$ & $\begin{array}{l}77 \\
79 \\
83 \\
87\end{array}$ \\
\hline 15 & 12 & $\begin{array}{l}1 / 4 \\
1 / 4 \\
1 / 4 \\
1 / 4 \\
1 / 4\end{array}$ & $\begin{array}{r}7 \\
9 \\
10 \\
12 \\
13\end{array}$ & $\begin{array}{l}4 \\
4 \\
4 \\
4 \\
4\end{array}$ & $\begin{array}{l}5 / 8 \\
8 / 4 \\
7 / 8 \\
11 \\
11 / 8\end{array}$ & $\begin{array}{l}64 \\
68 \\
71 \\
75 \\
80\end{array}$ & $\begin{array}{l}76 \\
78 \\
82 \\
86 \\
90\end{array}$ & $\begin{array}{r}90 \\
93 \\
96 \\
100 \\
104\end{array}$ \\
\hline 16 & 13 & $\begin{array}{l}1 / 4 \\
1 / 4 \\
1 / 4 \\
1 / 4 \\
1 / 4\end{array}$ & $\begin{array}{r}7 \\
9 \\
10 \\
10 \\
12\end{array}$ & $\begin{array}{l}4 \\
4 \\
4 \\
6 \\
6\end{array}$ & $\begin{array}{l}5 / 8 \\
8 / 4 \\
7 / 8 \\
7 / 8 \\
1^{7 / 8}\end{array}$ & $\begin{array}{l}74 \\
77 \\
81 \\
88 \\
94\end{array}$ & $\begin{array}{r}88 \\
90 \\
94 \\
100 \\
106\end{array}$ & $\begin{array}{l}104 \\
107 \\
111 \\
117 \\
123\end{array}$ \\
\hline 17 & 14 & $\begin{array}{l}1 / 4 \\
1 / 4 \\
1 / 4 \\
1 / 4 \\
1 / 4\end{array}$ & $\begin{array}{r}7 \\
10 \\
12 \\
10 \\
13\end{array}$ & $\begin{array}{l}4 \\
4 \\
4 \\
6 \\
6\end{array}$ & $\begin{array}{l}\frac{8}{8} \\
1 / 8 \\
11 / 8 \\
11 / 8\end{array}$ & $\begin{array}{r}85 \\
92 \\
96 \\
99 \\
112\end{array}$ & $\begin{array}{l}101 \\
107 \\
111 \\
113 \\
125\end{array}$ & $\begin{array}{l}120 \\
126 \\
130 \\
133 \\
145\end{array}$ \\
\hline 18 & 15 & $\begin{array}{l}1 / 4 \\
1 / 4 \\
1 / 4 \\
1 / 4 \\
1 / 4\end{array}$ & $\begin{array}{r}7 \\
10 \\
10 \\
12 \\
12\end{array}$ & $\begin{array}{l}4 \\
4 \\
6 \\
6 \\
8\end{array}$ & $\begin{array}{l}5 / 8 \\
7 / 8 \\
1^{7 / 8} \\
1\end{array}$ & $\begin{array}{r}97 \\
104 \\
110 \\
116 \\
125\end{array}$ & $\begin{array}{l}114 \\
121 \\
127 \\
133 \\
141\end{array}$ & $\begin{array}{l}137 \\
143 \\
149 \\
155 \\
163\end{array}$ \\
\hline 19 & 16 & $\begin{array}{l}\frac{6}{16} \\
\frac{8}{18} \\
\frac{8}{18} \\
\frac{1}{18} \\
\frac{1}{16}\end{array}$ & $\begin{array}{r}9 \\
12 \\
10 \\
10 \\
13\end{array}$ & $\begin{array}{l}4 \\
4 \\
6 \\
8 \\
8\end{array}$ & $\begin{array}{l}13 / 4 \\
7 / 8 \\
7 / 8 \\
11 / 8\end{array}$ & $\begin{array}{l}112 \\
120 \\
123 \\
129 \\
147\end{array}$ & $\begin{array}{l}132 \\
139 \\
142 \\
148 \\
165\end{array}$ & $\begin{array}{r}158 \\
165 \\
167 \\
174 \\
190\end{array}$ \\
\hline 20 & 17 & $\begin{array}{l}\frac{5}{16} \\
\frac{8}{18} \\
\frac{8}{16} \\
\frac{8}{18} \\
\frac{8}{16}\end{array}$ & $\begin{array}{r}9 \\
12 \\
12 \\
12 \\
13\end{array}$ & $\begin{array}{l}4 \\
4 \\
6 \\
8 \\
8\end{array}$ & $\begin{array}{l}1^{3 / 4} \\
1 \\
1 \\
11 / 8\end{array}$ & $\begin{array}{l}126 \\
133 \\
142 \\
151 \\
160\end{array}$ & $\begin{array}{l}148 \\
155 \\
164 \\
172 \\
181\end{array}$ & $\begin{array}{l}177 \\
184 \\
192 \\
200 \\
209\end{array}$ \\
\hline 21 & 18 & $\begin{array}{l}\frac{8}{16} \\
\frac{8}{18} \\
\frac{8}{16} \\
\frac{8}{18} \\
\frac{1}{18}\end{array}$ & $\begin{array}{r}9 \\
10 \\
12 \\
12 \\
15\end{array}$ & $\begin{array}{l}4 \\
6 \\
6 \\
8 \\
8\end{array}$ & $\begin{array}{l}\frac{8 / 4}{7 / 8} \\
1^{71 / 4}\end{array}$ & $\begin{array}{l}139 \\
150 \\
156 \\
165 \\
185\end{array}$ & $\begin{array}{l}165 \\
174 \\
179 \\
189 \\
207\end{array}$ & $\begin{array}{l}197 \\
207 \\
213 \\
221 \\
239\end{array}$ \\
\hline
\end{tabular}


U BEFUL DATA

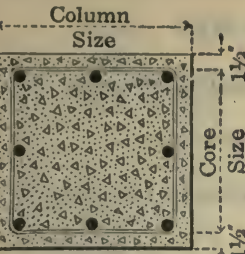

SQUARE TIED COLUMNS

\begin{tabular}{|c|c|c|c|c|c|c|c|c|c|}
\hline \multirow{3}{*}{$\begin{array}{c}\begin{array}{c}\text { Column } \\
\text { Size }\end{array} \\
\text { in. }\end{array}$} & \multirow{3}{*}{$\begin{array}{l}\text { Core } \\
\text { Size } \\
\text { in. }\end{array}$} & \multicolumn{2}{|c|}{$\begin{array}{c}\text { ROUND BAR } \\
\text { TIES }\end{array}$} & \multirow{2}{*}{\multicolumn{2}{|c|}{$\begin{array}{l}\text { Round BAR } \\
\text { VERTICALS }\end{array}$}} & \multirow{3}{*}{$\begin{array}{l}1: 2: 4 \\
2,000 \text { lb.Concrete } \\
f_{c}=400 \text { lb. per } \\
\text { sq. in. } \quad n=15\end{array}$} & \multirow{3}{*}{\multicolumn{2}{|c|}{$\begin{array}{c}1: 11 / 2: 3 \\
2,400 \mathrm{lb} \text {.Concrete } \\
\text { fo }=480 \mathrm{lb} \text {. per } \\
\text { sq. in. } \quad n=12\end{array}$}} & \multirow{3}{*}{$\begin{array}{l}1: 1: 2 \\
2,900 \text { lb.Conerete } \\
f_{\mathrm{c}}=580 \mathrm{lb} . \text { per } \\
\text { sq. in. } \quad n=10\end{array}$} \\
\hline & & \multirow{2}{*}{$\begin{array}{c}\text { Size } \\
\text { in. }\end{array}$} & \multirow{2}{*}{$\frac{\text { Spacing }}{\text { in. }}$} & & & & & & \\
\hline & & & & No. & Size & & & & \\
\hline 22 & 19 & $\begin{array}{l}\frac{5}{16} \\
\frac{8}{16} \\
\frac{6}{16} \\
\frac{5}{16} \\
\frac{8}{16}\end{array}$ & $\begin{array}{r}7 \\
10 \\
13 \\
12 \\
13\end{array}$ & $\begin{array}{r}6 \\
6 \\
6 \\
10 \\
10\end{array}$ & $\begin{array}{l}5 / 8 \\
7 / 8 \\
11 / 8 \\
11 / 8 \\
11 / 8\end{array}$ & $\begin{array}{l}155 \\
165 \\
178 \\
188 \\
200\end{array}$ & $\begin{array}{l}183 \\
192 \\
205 \\
215 \\
226\end{array}$ & & $\begin{array}{l}219 \\
228 \\
241 \\
250 \\
261\end{array}$ \\
\hline 23 & 20 & $\begin{array}{l}\frac{8}{18} \\
\frac{8}{18} \\
\frac{8}{18} \\
\frac{8}{18} \\
\frac{8}{18}\end{array}$ & $\begin{array}{r}9 \\
12 \\
13 \\
12 \\
13\end{array}$ & $\begin{array}{r}6 \\
6 \\
6 \\
10 \\
12\end{array}$ & $\begin{array}{l}1^{2 / 4} \\
11 / 8 \\
11 / 8 \\
11 / 8\end{array}$ & $\begin{array}{r}175 \\
186 \\
193 \\
204 \\
227\end{array}$ & $\begin{array}{l}206 \\
217 \\
223 \\
233 \\
255\end{array}$ & & $\begin{array}{l}246 \\
257 \\
263 \\
273 \\
294\end{array}$ \\
\hline 24 & 21 & $\begin{array}{l}\frac{5}{16} \\
\frac{8}{18} \\
\frac{8}{18} \\
\frac{8}{18}\end{array}$ & $\begin{array}{r}9 \\
12 \\
12 \\
12 \\
13\end{array}$ & $\begin{array}{r}6 \\
6 \\
8 \\
12 \\
14\end{array}$ & $\begin{array}{l}1^{8 / 4} \\
1 \\
1 \\
11 / 8\end{array}$ & $\begin{array}{l}191 \\
203 \\
212 \\
229 \\
254\end{array}$ & $\begin{array}{l}226 \\
237 \\
245 \\
261 \\
285\end{array}$ & & $\begin{array}{l}270 \\
280 \\
289 \\
305 \\
328\end{array}$ \\
\hline 25 & 22 & $\begin{array}{l}\frac{1}{18} \\
\frac{8}{16} \\
\frac{8}{18} \\
\frac{8}{16} \\
\frac{3}{16}\end{array}$ & $\begin{array}{r}7 \\
10 \\
12 \\
12 \\
13\end{array}$ & $\begin{array}{r}8 \\
8 \\
10 \\
12 \\
14\end{array}$ & $\begin{array}{l}\frac{5}{7 / 8} \\
1^{7 / 8} \\
11 / 8\end{array}$ & $\begin{array}{l}207 \\
221 \\
238 \\
246 \\
272\end{array}$ & $\begin{array}{l}245 \\
258 \\
274 \\
282 \\
306\end{array}$ & & $\begin{array}{l}294 \\
306 \\
322 \\
330 \\
353\end{array}$ \\
\hline 26 & 23 & $\begin{array}{l}3 / 8 \\
3 / 8 \\
3 / 8 \\
3 / 8 \\
3 / 8\end{array}$ & $\begin{array}{r}7 \\
10 \\
12 \\
12 \\
13\end{array}$ & $\begin{array}{l}10 \\
10 \\
10 \\
14 \\
16\end{array}$ & $\begin{array}{l}\frac{5}{8 / 8} \\
1^{7 / 8} \\
11 / 8\end{array}$ & $\begin{array}{l}229 \\
245 \\
256 \\
273 \\
301\end{array}$ & $\begin{array}{l}270 \\
286 \\
296 \\
312 \\
338\end{array}$ & & $\begin{array}{l}323 \\
338 \\
348 \\
364 \\
390\end{array}$ \\
\hline 27 & 24 & $\begin{array}{l}\frac{3}{3 / 8} \\
3 / 8 \\
3 / 8 \\
3 / 8 \\
3 / 8\end{array}$ & $\begin{array}{r}7 \\
10 \\
12 \\
12 \\
13\end{array}$ & $\begin{array}{l}10 \\
10 \\
12 \\
14 \\
18\end{array}$ & $\begin{array}{l}\frac{8 / 8}{7 / 8} \\
1^{7 / 8} \\
11 / 8\end{array}$ & $\begin{array}{l}248 \\
264 \\
283 \\
292 \\
330\end{array}$ & $\begin{array}{l}293 \\
308 \\
326 \\
335 \\
371\end{array}$ & & $\begin{array}{l}350 \\
365 \\
383 \\
392 \\
427\end{array}$ \\
\hline 28 & 25 & $\begin{array}{l}\frac{2}{8} 8 \\
8 / 8 \\
28 / 8 \\
3 / 8 \\
3 / 8\end{array}$ & $\begin{array}{r}7 \\
10 \\
12 \\
13 \\
13\end{array}$ & $\begin{array}{l}12 \\
12 \\
12 \\
14 \\
18\end{array}$ & $\begin{array}{l}5 / 8 \\
7 / 8 \\
111 / 8 \\
11 / 8\end{array}$ & $\begin{array}{l}271 \\
291 \\
303 \\
328 \\
350\end{array}$ & $\begin{array}{l}320 \\
338 \\
350 \\
374 \\
395\end{array}$ & & $\begin{array}{l}382 \\
400 \\
412 \\
435 \\
456\end{array}$ \\
\hline 29 & 26 & $\begin{array}{l}3 / 8 \\
\frac{3}{8} \\
\frac{3}{8} \\
3 / 8 \\
3 / 8\end{array}$ & $\begin{array}{r}7 \\
10 \\
12 \\
13 \\
13\end{array}$ & $\begin{array}{l}12 \\
12 \\
12 \\
14 \\
20\end{array}$ & $\begin{array}{l}5 / 8 \\
5 / 8 \\
11 / 8 \\
11 / 8\end{array}$ & $\begin{array}{l}291 \\
311 \\
323 \\
348 \\
382\end{array}$ & $\begin{array}{l}344 \\
362 \\
374 \\
398 \\
429\end{array}$ & & $\begin{array}{l}411 \\
430 \\
441 \\
465 \\
495\end{array}$ \\
\hline . & 27 & $\begin{array}{l}8 / 8 \\
8 / 8 \\
3 / 8 \\
3 / 8 \\
3 / 8\end{array}$ & $\begin{array}{r}7 \\
10 \\
12 \\
13 \\
13\end{array}$ & $\begin{array}{l}12 \\
14 \\
14 \\
16 \\
22\end{array}$ & $\begin{array}{l}8 / 8 \\
7 / 8 \\
11 / 8 \\
11 / 8 \\
11 / 8\end{array}$ & $\begin{array}{l}312 \\
339 \\
353 \\
381 \\
414\end{array}$ & $\begin{array}{l}370 \\
394 \\
408 \\
434 \\
465\end{array}$ & & $\begin{array}{l}442 \\
467 \\
480 \\
506 \\
537\end{array}$ \\
\hline 31 & 28 & $\begin{array}{l}3 / 8 \\
3 / 8 \\
3 / 8 \\
3 / 8 \\
3 / 8\end{array}$ & $\begin{array}{r}7 \\
10 \\
12 \\
13 \\
13\end{array}$ & $\begin{array}{l}14 \\
14 \\
14 \\
16 \\
24\end{array}$ & $\begin{array}{l}5 / 8 \\
7 / 8 \\
11 \\
11 / 8 \\
11 / 8\end{array}$ & $\begin{array}{l}338 \\
361 \\
375 \\
403 \\
447\end{array}$ & $\begin{array}{l}399 \\
421 \\
434 \\
460 \\
502\end{array}$ & & $\begin{array}{l}477 \\
499 \\
512 \\
538 \\
579\end{array}$ \\
\hline
\end{tabular}

Ratio of Length of Column to its Side, limited to 12

Safe Axial loads in Thougands of Pounds

Chicago Building Code Requirements 


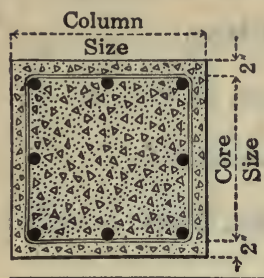

\section{SQUARE TIED COLUMNS}

\section{Safe Axial Loads in Thousands of Pounds \\ Joint Committee Recommendations}

Ratio of Unsupported Length of Column to its Side, limited to 15

\begin{tabular}{|c|c|c|c|c|c|c|c|c|}
\hline \multirow{3}{*}{$\begin{array}{c}\begin{array}{c}\text { Column } \\
\text { Size }\end{array} \\
\text { in. } \\
\end{array}$} & \multirow{3}{*}{$\begin{array}{l}\text { Core } \\
\text { Size } \\
\text { in. } \\
\end{array}$} & \multicolumn{2}{|c|}{$\begin{array}{c}\text { ROUND BAR } \\
\text { TIES } \\
\end{array}$} & \multirow{2}{*}{\multicolumn{2}{|c|}{$\begin{array}{l}\text { ROUND BAR } \\
\text { VERTICALS }\end{array}$}} & \multirow{3}{*}{$\begin{array}{c}1: 2: 4 \\
2,000 \text { lb. Concrete } \\
f_{c}=450 \text { lb. per } \\
\text { sq. in. } n=15\end{array}$} & \multirow{3}{*}{$\begin{array}{c}1: 11 / 2: 3 \\
2,500 \text { lb.Concret } \\
f_{\mathrm{c}}=562.5 \mathrm{lb} \text {. per } \\
\text { sq. in. } \quad n=12\end{array}$} & \multirow{3}{*}{$\begin{array}{l}1: 1: 2 \\
3,000 \text { lb. Concrete } \\
f \mathrm{c}=675 \mathrm{lb} . \text { per } \\
\text { sq. in. } n=10\end{array}$} \\
\hline & & \multirow{2}{*}{$\begin{array}{c}\text { Size } \\
\text { in. }\end{array}$} & \multirow{2}{*}{$\frac{\text { Spacing }}{\text { in. }}$} & & & & & \\
\hline & & & & No. & Size & & & \\
\hline 12 & 8 & $\begin{array}{l}1 / 4 \\
1 / 4 \\
1 / 4 \\
1 / 4\end{array}$ & $\begin{array}{r}8 \\
10 \\
12 \\
12\end{array}$ & $\begin{array}{l}4 \\
4 \\
4 \\
4\end{array}$ & $\begin{array}{l}1 / 2 \\
5 / 8 \\
3 / 4 \\
7 / 8\end{array}$ & $\begin{array}{l}34 \\
37 \\
40 \\
44\end{array}$ & $\begin{array}{l}41 \\
44 \\
47 \\
51\end{array}$ & $\begin{array}{l}48 \\
51 \\
54 \\
58\end{array}$ \\
\hline 13 & 9 & $\begin{array}{l}1 / 4 \\
1 / 4 \\
1 / 4 \\
1 / 4 \\
1 / 4\end{array}$ & $\begin{array}{r}8 \\
10 \\
12 \\
12 \\
12\end{array}$ & $\begin{array}{l}4 \\
4 \\
4 \\
4 \\
4\end{array}$ & $\begin{array}{r}1 / 2 \\
5 / 8 \\
8 / 4 \\
1^{7 / 8}\end{array}$ & $\begin{array}{r}41 \\
44 \\
-\quad 48 \\
52 \\
56\end{array}$ & $\begin{array}{l}50 \\
53 \\
56 \\
60 \\
65\end{array}$ & $\begin{array}{l}59 \\
62 \\
65 \\
69 \\
74\end{array}$ \\
\hline 14 & 10 & $\begin{array}{l}1 / 4 \\
1 / 4 \\
1 / 4 \\
1 / 4 \\
1 / 4\end{array}$ & $\begin{array}{l}10 \\
12 \\
12 \\
12 \\
12\end{array}$ & $\begin{array}{l}4 \\
4 \\
4 \\
4 \\
4\end{array}$ & $\begin{array}{l}5 / 8 \\
8 / 4 \\
1 / 8 \\
11 / 8 \\
11 / 8\end{array}$ & $\begin{array}{l}53 \\
56 \\
60 \\
65 \\
70\end{array}$ & $\begin{array}{l}64 \\
67 \\
71 \\
76 \\
81\end{array}$ & $\begin{array}{l}75 \\
78 \\
82 \\
87 \\
92\end{array}$ \\
\hline 15 & 11 & $\begin{array}{l}1 / 4 \\
1 / 4 \\
1 / 4 \\
1 / 4 \\
1 / 4\end{array}$ & $\begin{array}{l}10 \\
12 \\
12 \\
12 \\
12\end{array}$ & $\begin{array}{l}4 \\
4 \\
4 \\
4 \\
6\end{array}$ & $1^{\frac{5}{8 / 8}} 1^{1 / 8}$ & $\begin{array}{l}62 \\
66 \\
70 \\
74 \\
84\end{array}$ & $\begin{array}{l}76 \\
79 \\
83 \\
87 \\
97\end{array}$ & $\begin{array}{r}89 \\
92 \\
96 \\
101 \\
110\end{array}$ \\
\hline 16 & 12 & $1 / 4$ & 12 & $\begin{array}{l}4 \\
4 \\
4 \\
4 \\
6\end{array}$ & $\begin{array}{l}8 / 4 \\
7 / 8 \\
11 \\
11 / 8 \\
11 / 8\end{array}$ & $\begin{array}{r}76 \\
80 \\
85 \\
90 \\
102\end{array}$ & $\begin{array}{r}92 \\
96 \\
100 \\
106 \\
118\end{array}$ & $\begin{array}{l}108 \\
112 \\
116 \\
121 \\
133\end{array}$ \\
\hline 17 & 13 & $1 / 4$ & 12 & $\begin{array}{l}4 \\
4 \\
6 \\
6 \\
8\end{array}$ & $1^{\frac{8}{7 / 8}}$ & $\begin{array}{r}87 \\
91 \\
99 \\
106 \\
116\end{array}$ & $\begin{array}{l}106 \\
110 \\
117 \\
124 \\
134\end{array}$ & $\begin{array}{l}125 \\
129 \\
136 \\
143 \\
152\end{array}$ \\
\hline 18 & 14 & $1 / 4$ & 12 & $\begin{array}{l}4 \\
4 \\
6 \\
6 \\
8\end{array}$ & $\begin{array}{l}1 / 8 \\
1 / 8 \\
11 / 8 \\
11 / 8\end{array}$ & $\begin{array}{l}103 \\
108 \\
111 \\
126 \\
138\end{array}$ & $\begin{array}{l}125 \\
130 \\
133 \\
147 \\
159\end{array}$ & $\begin{array}{l}147 \\
151 \\
154 \\
169 \\
181\end{array}$ \\
\hline 19 & 15 & to & 12 & $\begin{array}{l}4 \\
6 \\
6 \\
8 \\
8\end{array}$ & $1^{\frac{7 / 8}{7 / 8}}$. & $\begin{array}{l}116 \\
124 \\
131 \\
141 \\
151\end{array}$ & $\begin{array}{l}141 \\
149 \\
156 \\
165 \\
176\end{array}$ & $\begin{array}{l}166 \\
174 \\
181 \\
190 \\
200\end{array}$ \\
\hline 20 & 16 & It & 12 & $\begin{array}{r}4 \\
6 \\
8 \\
8 \\
10\end{array}$ & $\begin{array}{l}17 / 8 \\
7 / 8 \\
11 / 8 \\
11 / 8\end{array}$ & $\begin{array}{l}135 \\
138 \\
145 \\
165 \\
178\end{array}$ & $\begin{array}{l}163 \\
166 \\
174 \\
193 \\
205\end{array}$ & $\begin{array}{l}192 \\
195 \\
202 \\
221 \\
233\end{array}$ \\
\hline 21 & 17 & if & 12 & $\begin{array}{r}4 \\
6 \\
8 \\
8 \\
12\end{array}$ & $\begin{array}{l}1 \\
1 \\
1 \\
11 / 8 \\
11 / 8\end{array}$ & $\begin{array}{l}150 \\
160 \\
170 \\
180 \\
205\end{array}$ & $\begin{array}{l}182 \\
192 \\
201 \\
212 \\
236\end{array}$ & $\begin{array}{l}214 \\
224 \\
233 \\
243 \\
268\end{array}$ \\
\hline
\end{tabular}




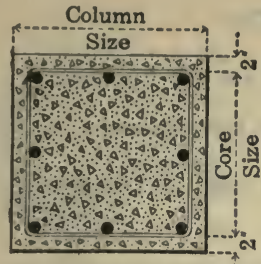

SQUARE TIED COLUMNS

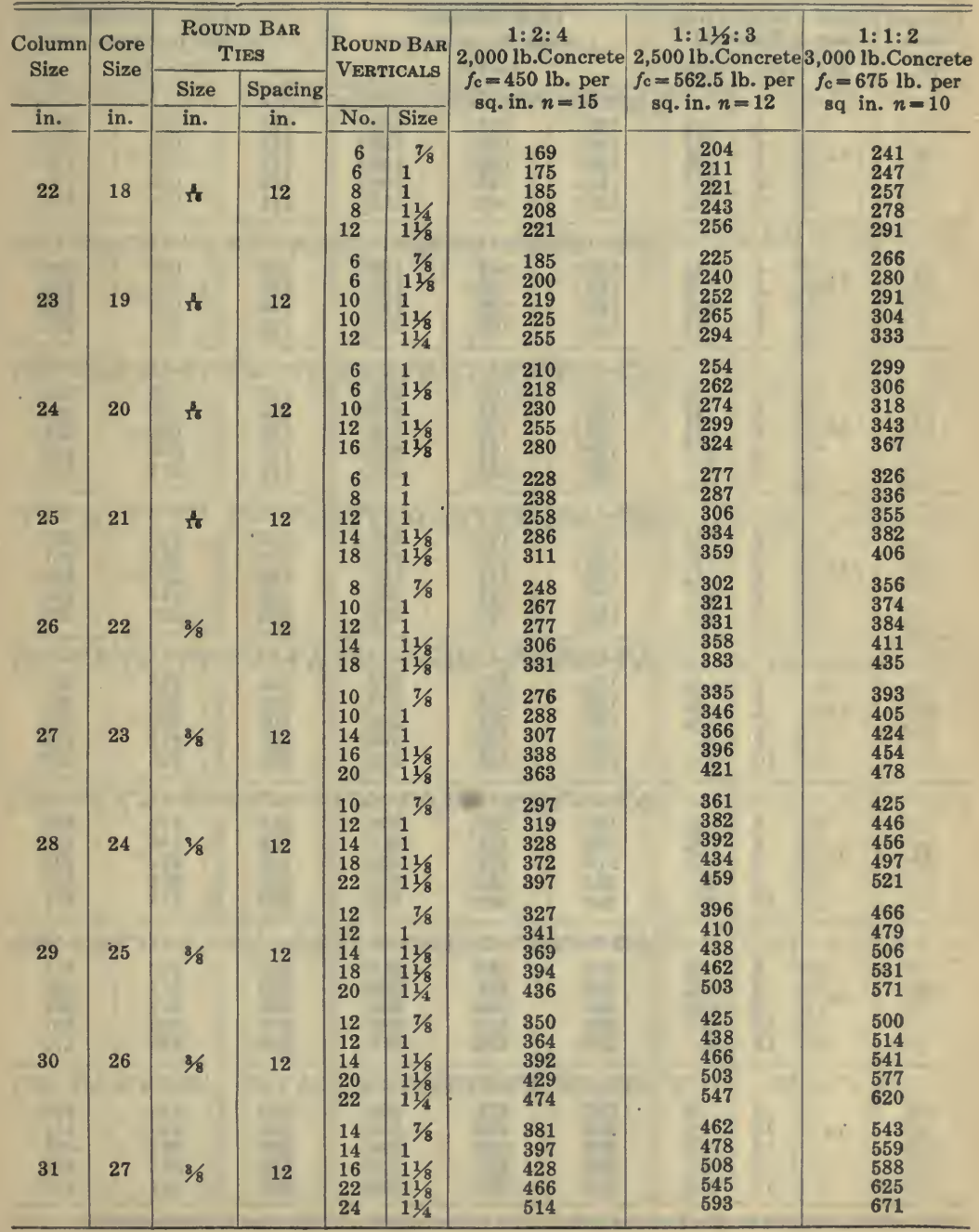

Ratio of Unsupported Length of Column to its Side,

\section{Safe Axial Loads in Thousands of Pounds \\ JoInt Committee Recommendations}

\section{limited to 15}


Column

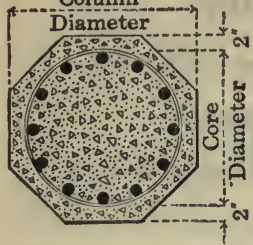

\section{SPIRAL COLUMNS}

\section{Safe Axial Loads in Thousands of Pounds}

New York City ButLding Code Requirements

Ratio of Length of Column to its Side or Diameter, limited to 15 .

\begin{tabular}{|c|c|c|c|c|c|c|c|c|c|}
\hline \multirow{3}{*}{$\begin{array}{c}\text { Column } \\
\text { Diam. } \\
\text { in. } \\
\end{array}$} & \multirow{2}{*}{$\begin{array}{c}\text { Core } \\
\text { Diam. } \\
\text { in. }\end{array}$} & \multicolumn{2}{|c|}{$\begin{array}{c}\text { ROUND BAR } \\
\text { VERTICALS } \\
\end{array}$} & \multirow{2}{*}{\multicolumn{3}{|c|}{$\begin{array}{c}1: 2: 4 \text { Concrete } \\
f_{c}=500 \text { lb. per sq. in. } \\
n=15\end{array}$}} & \multirow{2}{*}{\multicolumn{3}{|c|}{$\begin{array}{c}1: 11 / 2: 3 \text { Concrete } \\
f_{\mathrm{c}}=600 \mathrm{lb} . \text { per } \mathrm{sq} . \text { in. } \\
n=12\end{array}$}} \\
\hline & & No. & Size & & & & & & \\
\hline & & & & $1 / 4^{\prime \prime} \phi-2^{\prime \prime} p$ & $\frac{5}{16} " \phi-17 / 8 n p$ & $3 / 8^{\prime \prime} \phi-13 / 4 " p$ & $1 / 4 " \phi-2^{\prime \prime} p$ & $\frac{5}{16} " \phi-17 / 8 " p$ & $3 / 8^{\prime \prime} \phi-13 / 4^{\prime \prime}$ \\
\hline 16 & 12 & $\begin{array}{l}4 \\
4 \\
4 \\
4 \\
7\end{array}$ & $\begin{array}{r}5 / 8 \\
8 / 4 \\
7 / 8 \\
17 / 8\end{array}$ & $\begin{array}{l}100 \\
104 \\
109 \\
114 \\
122\end{array}$ & $\begin{array}{l}124 \\
128 \\
133 \\
138 \\
146\end{array}$ & $\begin{array}{l}154 \\
158 \\
162 \\
168 \\
175\end{array}$ & $\begin{array}{l}111 \\
115 \\
119 \\
124 \\
131\end{array}$ & $\begin{array}{l}135 \\
139 \\
143 \\
148 \\
155\end{array}$ & $\begin{array}{l}165 \\
169 \\
173 \\
178 \\
185\end{array}$ \\
\hline 17 & 13 & $\begin{array}{l}4 \\
4 \\
5 \\
8 \\
\end{array}$ & $\begin{array}{r}8 / 4 \\
1 / 8 \\
7 / 8 \\
\end{array}$ & $\begin{array}{l}" \phi-17 / 8^{\prime \prime} p \\
120 \\
124 \\
135 \\
141\end{array}$ & $\begin{array}{c}\frac{8}{16} " \phi-13 / 4 " p \\
148 \\
152 \\
163 \\
169\end{array}$ & $\begin{array}{c}3^{\prime \prime} \phi-15 / 8 n \\
182 \\
187 \\
197 \\
204\end{array}$ & $\begin{array}{c}1 / 4^{\prime \prime} \phi-17 / 8^{\prime \prime} p \\
132 \\
136 \\
146 \\
152\end{array}$ & $\begin{array}{c}\frac{3}{16} \phi-18 / 4^{\prime \prime} p \\
160 \\
165 \\
175 \\
180\end{array}$ & $\begin{array}{l}195 \\
199 \\
209 \\
215\end{array}$ \\
\hline 18 & 14 & $\begin{array}{l}5 \\
5 \\
5 \\
6 \\
8 \\
\end{array}$ & $1^{5 / 8} 87 / 8$ & $\begin{array}{c}1 / 4^{\prime \prime} \phi-18 / 4^{\prime \prime} p \\
134 \\
139 \\
145 \\
157 \\
168\end{array}$ & $\begin{array}{c}3 / 8-21 / 4^{\prime \prime} p \\
168 \\
173 \\
179 \\
191 \\
202\end{array}$ & $\begin{array}{c}\frac{7}{16} " \phi-21 / 8^{n} p \\
208 \\
213 \\
219 \\
231 \\
242\end{array}$ & $\begin{array}{c}1 / 4^{\prime \prime} \phi-13 / 4^{n} p \\
149 \\
154 \\
159 \\
170 \\
181\end{array}$ & $\begin{array}{r}8 / 8 " \phi-2 \\
18 \\
18 \\
19 \\
2 \\
21\end{array}$ & 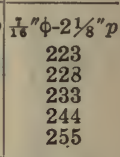 \\
\hline 19 & 15 & $\begin{array}{l}6 \\
6 \\
6 \\
7 \\
0\end{array}$ & $1^{\frac{5}{8} 8}$ & $\begin{array}{c}1 / 4^{\prime \prime} \phi-184^{\prime \prime} p \\
151 \\
157 \\
164 \\
177 \\
188\end{array}$ & $\begin{array}{r}8 / 8 " \phi \\
19 \\
20 \\
21 \\
22 \\
28\end{array}$ & $\begin{array}{r}\frac{7}{16} " \phi \\
2 \\
2 \\
2 \\
2 \\
2\end{array}$ & $\begin{array}{r}1 / 4 " \phi-1 \\
16 \\
17 \\
18 \\
19 \\
20\end{array}$ & $\begin{array}{r}3 / 8^{\prime \prime} \phi \\
21 \\
22 \\
22 \\
24 \\
25\end{array}$ & $\begin{array}{c}\frac{7}{16} \phi-2^{\prime \prime} p \\
255 \\
261 \\
267 \\
279 \\
290\end{array}$ \\
\hline 20 & 16 & $\begin{array}{r}7 \\
7 \\
7 \\
8 \\
10 \\
\end{array}$ & $1^{5 / 8} \frac{8}{3 / 4}$ & $\begin{array}{c}\frac{5}{10} " \phi-21 / 2 " p \\
175 \\
182 \\
189 \\
204 \\
215\end{array}$ & $\begin{array}{c}3 / 8^{n} \phi-17 / 8^{n} p \\
227 \\
233 \\
241 \\
255 \\
266\end{array}$ & $\begin{array}{r}\frac{7}{16} " \phi- \\
2 \\
2 \\
2 \\
3 \\
3\end{array}$ & $\begin{array}{c}\frac{5}{16} " \phi-21 / 2^{n} p \\
194 \\
200 \\
208 \\
222 \\
232\end{array}$ & $\begin{array}{r}3 / 8 " \phi- \\
2 \\
2 \\
2 \\
2 \\
2 \\
\end{array}$ & 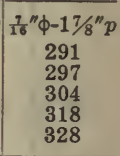 \\
\hline 21 & 17 & $\begin{array}{r}8 \\
8 \\
8 \\
8 \\
11\end{array}$ & $\begin{array}{l}5 / 8 \\
\frac{5}{4} \\
1^{7 / 8} \\
1\end{array}$ & $\begin{array}{c}\frac{5}{16} " \phi-23 / 8 n \\
197 \\
204 \\
213 \\
224 \\
240 \\
\end{array}$ & $\begin{array}{c}3 / 8 " \phi-18 / 4 " p \\
257 \\
264 \\
273 \\
284 \\
300 \\
\end{array}$ & \begin{tabular}{|r}
$\frac{7}{16} " \phi-$ \\
3 \\
3 \\
3 \\
3 \\
3 \\
\end{tabular} & $\begin{array}{c}{ }^{8} " \phi-23 / 8 " p \\
219 \\
226 \\
234 \\
244 \\
259\end{array}$ & $\begin{array}{r}3 / 8 " \phi- \\
2 \\
2 \\
2 \\
3 \\
3 \\
\end{array}$ & \begin{tabular}{|r|}
$\frac{7}{16} " \phi-$ \\
3 \\
3 \\
3 \\
3 \\
3 \\
\end{tabular} \\
\hline 22 & 18 & $\begin{array}{r}8 \\
9 \\
9 \\
10 \\
12 \\
\end{array}$ & $\begin{array}{r}5 / 8 \\
8 / 4 \\
1^{7 / 8}\end{array}$ & \begin{tabular}{|c|}
$\frac{5}{16} " \phi-21 / 4 " p$ \\
219 \\
229 \\
239 \\
256 \\
267
\end{tabular} & \begin{tabular}{|c|}
$\frac{7}{16} " \phi-23 / 8 " p$ \\
283 \\
294 \\
304 \\
321 \\
332
\end{tabular} & $\begin{array}{r}\frac{7}{16} " \phi-1 \\
3 \\
35 \\
36 \\
38 \\
39\end{array}$ & \begin{tabular}{|c|}
$\frac{5}{16} " \phi-21 / 4^{n} p$ \\
243 \\
253 \\
263 \\
279 \\
289
\end{tabular} & \begin{tabular}{|c|}
$\frac{7}{16} " \phi-23 / 8 " p$ \\
307 \\
317 \\
327 \\
343 \\
353
\end{tabular} & $\begin{array}{c}\frac{7}{16} " \phi-15 / 8 " p \\
371 \\
382 \\
391 \\
407 \\
417\end{array}$ \\
\hline 23 & 19 & $\begin{array}{r}8 \\
10 \\
10 \\
11 \\
11 \\
\end{array}$ & $\begin{array}{r}8 / 4 \\
8 / 4 \\
1^{7 / 8} \\
11 / 8 \\
\end{array}$ & $\begin{array}{c}\frac{5}{16} " \phi-2 " p \\
255 \\
261 \\
272 \\
291 \\
307 \\
\end{array}$ & \begin{tabular}{|c|}
$\frac{7}{16} " \phi-21 / 4 " p$ \\
321 \\
327 \\
338 \\
357 \\
373 \\
\end{tabular} & \begin{tabular}{|c|}
$\frac{7}{16} " \phi-15 / 8^{n} p$ \\
381 \\
387 \\
398 \\
417 \\
433 \\
\end{tabular} & $\begin{array}{c}\frac{5}{16} " \phi-2^{\prime \prime} p \\
282 \\
288 \\
298 \\
316 \\
331\end{array}$ & \begin{tabular}{|c|}
$\frac{7}{16} " \phi-21 / 4^{\prime \prime} p$ \\
348 \\
353 \\
364 \\
381 \\
397 \\
\end{tabular} & $\begin{array}{c}\frac{7}{16} \phi-15 / 8^{\prime \prime} p \\
408 \\
414 \\
424 \\
441 \\
457\end{array}$ \\
\hline
\end{tabular}

NoTw-Size and pitch of spiral wire is given at head of each group of loads for each size column. 
USEFUL DATA

Column

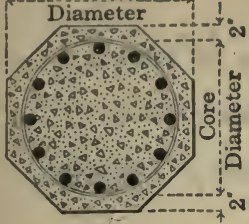

SPIRAL COLUMNS

\section{Safe Axial Loads in Thousands of Pounds}

\section{New York City Building Code Requirements}

Ratio of Length of Column to its Side or Diameter, limited to 15.

\begin{tabular}{|c|c|c|c|c|c|c|c|c|c|}
\hline \multirow{2}{*}{$\begin{array}{c}\text { Column } \\
\text { Diam. } \\
\text { in. }\end{array}$} & \multirow{2}{*}{$\begin{array}{c}\text { Core } \\
\text { Diam. } \\
\text { in. }\end{array}$} & \multicolumn{2}{|c|}{$\begin{array}{c}\text { ROUND BAR } \\
\text { VERTICALS }\end{array}$} & \multirow{2}{*}{\multicolumn{3}{|c|}{$\begin{array}{c}1: 2: 4 \text { Concrete } \\
f_{\mathrm{c}}=500 \mathrm{lb} . \text { per } \mathrm{sq} . \text { in. } \\
n=15\end{array}$}} & \multirow{2}{*}{\multicolumn{3}{|c|}{$\begin{array}{c}\begin{array}{c}1: 11 / 2: 3 \text { Concrete } \\
f_{0}=600 \mathrm{lb} . \text { per sq. in. } \\
n=12\end{array}\end{array}$}} \\
\hline & & No. & Size & & & & & & \\
\hline 24 & 20 & $\begin{array}{r}8 \\
10 \\
10 \\
12 \\
12\end{array}$ & $\begin{array}{l}8 / 4 \\
8 / 4 \\
7 / 8 \\
1 \\
11 / 8\end{array}$ & $\begin{array}{c}\frac{8}{18} " \phi-2^{\prime \prime} p \\
275 \\
281 \\
292 \\
316 \\
333\end{array}$ & $\begin{array}{c}\frac{7}{16} " \phi-21 / 4^{\prime \prime} p \\
344 \\
350 \\
361 \\
385 \\
403\end{array}$ & $\begin{array}{c}1 / 2^{\prime \prime} \phi-2^{\prime \prime} p \\
419 \\
426 \\
437 \\
461 \\
478\end{array}$ & $\begin{array}{c}18 " \phi-2 " p \\
305 \\
311 \\
321 \\
344 \\
360\end{array}$ & \begin{tabular}{|c|}
$\frac{7}{10} \phi-21 / 4 "$ \\
374 \\
380 \\
390 \\
413 \\
429 \\
\end{tabular} & $\begin{array}{c}1 / 2^{\prime \prime} \phi-2^{\prime \prime} p \\
449 \\
455 \\
466 \\
488 \\
505\end{array}$ \\
\hline 25 & 21 & $\begin{array}{r}8 \\
10 \\
10 \\
13 \\
14\end{array}$ & $\begin{array}{l}\frac{8 / 4}{9 / 8} \\
1 \\
1 \\
11 / 8\end{array}$ & $\begin{array}{c}8 / 8^{\prime \prime} \phi-25 / 8^{\prime \prime} p \\
302 \\
319 \\
332 \\
349 \\
375\end{array}$ & $\begin{array}{c}\frac{7}{18} " \phi-21 / 8 " p \\
378 \\
395 \\
408 \\
425 \\
451\end{array}$ & $\begin{array}{c}1 / 2^{\prime \prime} \phi-17 / 8^{\prime \prime} p \\
464 \\
481 \\
494 \\
511 \\
537\end{array}$ & $\begin{array}{c}3 / 8 " \phi-25 / 8^{\prime \prime} p \\
335 \\
351 \\
363 \\
379 \\
404\end{array}$ & $\begin{array}{c}\frac{7}{16} " \phi-21 / 8 " p \\
411 \\
428 \\
440 \\
456 \\
480\end{array}$ & $\begin{array}{c}1 / 2 " \phi-17 / 8 " p \\
497 \\
514 \\
526 \\
541 \\
566\end{array}$ \\
\hline 26 & 22 & $\begin{array}{r}9 \\
10 \\
10 \\
13 \\
15 \\
\end{array}$ & $\begin{array}{r}8 / 4 \\
1 / 8 \\
11 / 8 \\
11 / 8 \\
\end{array}$ & $\begin{array}{c}3 / 8^{\prime \prime} \phi-21 / 2^{\prime \prime} p \\
332 \\
346 \\
359 \\
395 \\
408 \\
\end{array}$ & $\begin{array}{c}\frac{7}{16} \phi-2^{\prime \prime} p \\
419 \\
433 \\
446 \\
481 \\
495 \\
\end{array}$ & \begin{tabular}{|c|}
$1 / 2^{\prime \prime} \phi-18 / 4^{\prime \prime} p$ \\
516 \\
530 \\
543 \\
579 \\
592
\end{tabular} & $\begin{array}{c}8 / 8 " \phi-21 / 2 p^{\prime \prime} \\
368 \\
382 \\
394 \\
427 \\
441\end{array}$ & $\begin{array}{c}7 \overline{7}^{\prime \prime} \phi-2^{\prime \prime} p \\
455 \\
468 \\
481 \\
514 \\
527\end{array}$ & $\begin{array}{c}1 / 2^{\prime \prime} \phi-184^{n} p \\
552 \\
566 \\
578 \\
611 \\
625\end{array}$ \\
\hline 27 & 23 & $\begin{array}{l}10 \\
10 \\
11 \\
13 \\
16\end{array}$ & $\begin{array}{l}3 / 4 \\
7 / 8 \\
11 / 8 \\
11 / 8\end{array}$ & $\begin{array}{c}3 / 8^{\prime \prime} \phi-23 / 8^{\prime \prime} p \\
365 \\
376 \\
394 \\
424 \\
445\end{array}$ & $\begin{array}{r}1 / 2 " \phi-2 \\
45 \\
46 \\
48 \\
51 \\
53 \\
\end{array}$ & $\begin{array}{c}1 / 2 " \phi-13 / 4 \\
551 \\
562 \\
581 \\
611 \\
631\end{array}$ & $\begin{array}{c}8 / 8=-23 / 8 " p \\
405 \\
415 \\
433 \\
461 \\
481\end{array}$ & $\begin{array}{c}1 / 2 " \phi-21 / 2 \\
496 \\
507 \\
524 \\
552 \\
572\end{array}$ & $\begin{array}{l}\phi-18 / 4 " p \\
591 \\
601 \\
619 \\
647 \\
667\end{array}$ \\
\hline 28 & 24 & $\begin{array}{l}10 \\
10 \\
12 \\
14 \\
15 \\
\end{array}$ & $\begin{array}{l}1^{2 / 4} \\
1 \\
11 / 8 \\
11 / 4\end{array}$ & $\begin{array}{c}8 / 8^{\prime \prime} \phi-21 / 4^{\prime \prime} p \\
395 \\
419 \\
430 \\
461 \\
493 \\
\end{array}$ & $\begin{array}{l}45 \\
5 \\
5 \\
56 \\
59 \\
\end{array}$ & $\begin{array}{r}1 / 2 " \phi-1 \\
60 \\
63 \\
64 \\
67 \\
70 \\
\end{array}$ & $\begin{array}{l}\phi-21 / 4 " p \\
438 \\
461 \\
471 \\
501 \\
531 \\
\end{array}$ & $\begin{array}{l}5 \\
5 \\
5 \\
6 \\
6 \\
\end{array}$ & 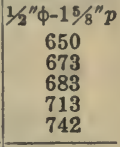 \\
\hline 29 & 25 & $\begin{array}{l}10 \\
10 \\
12 \\
15 \\
16\end{array}$ & $\begin{array}{l}1^{7 / 8} \\
1 \\
11 / 8 \\
11 / 4\end{array}$ & $\begin{array}{c}3 / 8 " \phi-21 / 8^{\prime \prime} p \\
439 \\
452 \\
463 \\
501 \\
534 \\
\end{array}$ & $\begin{array}{r}1 / 2 " \phi-2 \\
5 \\
5 \\
5 \\
6 \\
6 \\
\end{array}$ & $\begin{array}{c}1 / 2^{\prime \prime} \phi-15 / 8^{\prime \prime} p \\
653 \\
666 \\
677 \\
715 \\
748\end{array}$ & $\begin{array}{c}3 / 8^{\prime \prime} \phi-21 / 8^{\prime \prime} p \\
485 \\
497 \\
508 \\
544 \\
575\end{array}$ & $4 / 4^{\prime \prime} p$ & $3 / p$ \\
\hline 30 & 26 & $\begin{array}{l}10 \\
10 \\
12 \\
16 \\
17 \\
\end{array}$ & $\begin{array}{l}7 / 8 \\
11 / 8 \\
11 / 8 \\
11 / 4\end{array}$ & $\begin{array}{c}3 / 8 " \phi-21 / 8 " p \\
467 \\
480 \\
508 \\
536 \\
571 \\
\end{array}$ & $\begin{array}{c}1 / 2^{\prime \prime} \phi-21 / 4^{\prime \prime} p \\
581 \\
594 \\
623 \\
651 \\
685 \\
\end{array}$ & $\begin{array}{c}1 / 2 " \phi-11 / 2^{\prime \prime} p \\
717 \\
730 \\
759 \\
787 \\
821 \\
\end{array}$ & $\begin{array}{c}a / 8 " \phi-21 / 8 " p \\
518 \\
530 \\
556 \\
583 \\
615 \\
\end{array}$ & $\begin{array}{c}1 / 2^{\prime \prime} \phi-21 / 4^{\prime \prime} p \\
632 \\
644 \\
671 \\
697 \\
730\end{array}$ & $\begin{array}{c}\phi-11 / 2 " p \\
768 \\
780 \\
807 \\
833 \\
866 \\
\end{array}$ \\
\hline 31 & 27 & $\begin{array}{l}10 \\
11 \\
12 \\
18 \\
19 \\
\end{array}$ & $\begin{array}{l}7 / 8 \\
11 / 8 \\
118 \\
11 / 4 \\
\end{array}$ & $\begin{array}{c}3 / 8^{\prime \prime} \phi-2^{\prime \prime} p \\
502 \\
521 \\
544 \\
585 \\
623 \\
\end{array}$ & $\begin{array}{c}1 / 2^{\prime \prime} \phi-21 / 8^{\prime \prime} p \\
631 \\
649 \\
672 \\
714 \\
752 \\
\end{array}$ & $\begin{array}{c}1 / 2^{\prime \prime} \phi-11 / 2 " p \\
754 \\
773 \\
796 \\
837 \\
875 \\
\end{array}$ & $\begin{array}{c}8 / 8 " \phi-2^{\prime \prime} p \\
557 \\
575 \\
596 \\
636 \\
672 \\
\end{array}$ & $\begin{array}{c}1 / 2^{\prime \prime} \phi-21 / 8^{\prime \prime} p \\
685 \\
703 \\
725 \\
764 \\
800 \\
\end{array}$ & $\begin{array}{c}1 / 2^{\prime \prime} \phi-11 / 2^{\prime \prime} p \\
809 \\
827 \\
848 \\
887 \\
923 \\
\end{array}$ \\
\hline
\end{tabular}

Nots-Size and pltch of Bptral wire is glven at head of each group of loads for each size column. 
Column

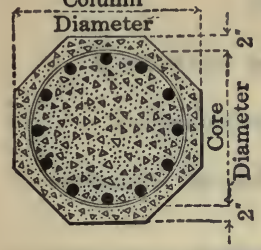

\section{SPIRAL COLUMNS}

\section{Safe Axial Loads in Thousands of Pounds}

\section{New York City Building Code Requirements}

Ratio of Length of Column to its Side or Diameter, limited to 15 .

\begin{tabular}{|c|c|c|c|c|c|c|c|c|c|}
\hline $\begin{array}{l}\text { Column } \\
\text { Diam. }\end{array}$ & $\begin{array}{c}\text { Core } \\
\text { Diam. }\end{array}$ & VE & & $\begin{array}{r}1 \\
f_{c}=5\end{array}$ & $4 \mathrm{C}$ & e. & $f_{\mathrm{c}}=\epsilon^{1}$ & 0 & e \\
\hline in. & in. & No. & Size & & & & & & \\
\hline & & 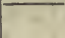 & & $3 / 8^{\prime \prime} \phi-2^{\prime \prime} p$ & $1 / 2^{\prime \prime} \phi-21 / 8^{\prime \prime} p$ & 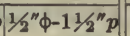 & $3 / 8^{\prime \prime} \phi-2^{\prime \prime} p$ & $1 / 2^{m} \phi$ & $6 \mathrm{mo}$ \\
\hline 32 & 28 & $\begin{array}{l}10 \\
12 \\
13 \\
18\end{array}$ & $\begin{array}{l}1^{7 / 8} \\
11 / 8 \\
11 / 8 \\
11 / 4\end{array}$ & $\begin{array}{l}556 \\
581 \\
615 \\
662\end{array}$ & $\begin{array}{l}68 \\
71 \\
74 \\
79\end{array}$ & $\begin{array}{l}8 \\
8 \\
8 \\
9\end{array}$ & 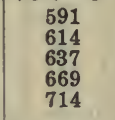 & $\begin{array}{l}7 \\
8 \\
8\end{array}$ & $\begin{array}{l}5 \\
8 \\
1 \\
5\end{array}$ \\
\hline 33 & 29 & $\begin{array}{l}11 \\
13 \\
14 \\
18\end{array}$ & $\begin{array}{l}1^{2 / 8} \\
11 / 8 \\
11 / 4 \\
11 / 4\end{array}$ & $\begin{array}{r}8 / 8 " \phi \\
56 \\
58 \\
61 \\
67 \\
70\end{array}$ & $\begin{array}{r}1 / 2 " \phi- \\
6 \\
7 \\
7 \\
8 \\
8\end{array}$ & $\begin{array}{l}8 \\
8 \\
8 \\
9 \\
9\end{array}$ & $\begin{array}{c}3 / 8^{\prime \prime} \phi-2^{\prime \prime} p \\
627 \\
651 \\
676 \\
729 \\
762\end{array}$ & $\begin{array}{r}1 / 2 " \phi- \\
7 \\
7 \\
8 \\
8 \\
8\end{array}$ & $\begin{array}{c}\delta^{\prime \prime} \phi-11 / 2 \\
897 \\
921 \\
945 \\
999 \\
1031\end{array}$ \\
\hline 34 & 30 & $\begin{array}{l}12 \\
14 \\
15 \\
18 \\
23\end{array}$ & $\begin{array}{l}1^{7 / 8} \\
11 / 8 \\
11 / 4 \\
11 / 4\end{array}$ & $\begin{array}{r}3 / 8 " \phi-2 \\
58 \\
61 \\
64 \\
69 \\
73\end{array}$ & $\begin{array}{l}73 \\
76 \\
79 \\
84 \\
88\end{array}$ & $\begin{array}{r}1 / 2 m \phi- \\
8 \\
9 \\
9 \\
9 \\
10\end{array}$ & 8 & $\begin{array}{l}8 \\
9 \\
9\end{array}$ & $\begin{array}{r}9 \\
9 \\
10 \\
10\end{array}$ \\
\hline 35 & 31 & $\begin{array}{l}13 \\
15 \\
15 \\
18 \\
25 \\
\end{array}$ & $\begin{array}{l}1^{7 / 8} \\
11 / 8 \\
11 / 4 \\
11 / 4 \\
\end{array}$ & $\begin{array}{c}8 / 8-21 / 8 " p \\
622 \\
650 \\
672 \\
722 \\
782\end{array}$ & $\begin{array}{r}1 / 2 " \phi-2 \\
77 \\
80 \\
82 \\
87 \\
93\end{array}$ & $\begin{array}{r}1 / 2 n \phi \\
9 \\
9 \\
9\end{array}$ & $\begin{array}{c}8^{\prime \prime} \phi-21 / 8^{n} p \\
695 \\
721 \\
741 \\
789 \\
845\end{array}$ & $\begin{array}{r}1 / 2 n \phi- \\
8 \\
8 \\
8 \\
9 \\
10\end{array}$ & $\begin{array}{r}1 / 2 " \phi \\
9 \\
10 \\
10 \\
10 \\
11\end{array}$ \\
\hline 36 & 32 & $\begin{array}{l}14 \\
15 \\
16 \\
20 \\
26\end{array}$ & $\begin{array}{l}1^{7 / 8} \\
11 / 8 \\
11 / 4 \\
11 / 4\end{array}$ & $\begin{array}{c}8 / 8^{\prime \prime} \phi-21 / 4 " p \\
644 \\
668 \\
697 \\
757 \\
809\end{array}$ & $\begin{array}{r}1 / 2 m \phi- \\
7 \\
8 \\
8 \\
9 \\
9\end{array}$ & $\begin{array}{r}1 / 2^{\prime \prime} \phi \\
9 \\
9\end{array}$ & $\begin{array}{c}8^{\prime \prime} \phi-21 / 4 " p \\
721 \\
744 \\
771 \\
828 \\
877\end{array}$ & \begin{tabular}{|r|}
$1 / 2 " \phi-$ \\
8 \\
8 \\
9 \\
9 \\
10
\end{tabular} & $\begin{array}{l}10 \\
10 \\
11 \\
11\end{array}$ \\
\hline 37 & 33 & $\begin{array}{l}14 \\
15 \\
16 \\
21 \\
28 \\
\end{array}$ & $\begin{array}{l}7 / 8 \\
11 / 8 \\
11 / 4 \\
11 / 4 \\
11 / 4 \\
\end{array}$ & $\begin{array}{l}678 \\
724 \\
756 \\
802 \\
860 \\
\end{array}$ & $\begin{array}{r}1 / 2 n \phi-2 \\
83 \\
88 \\
91 \\
95 \\
101\end{array}$ & $g^{\prime \prime} p$ & $\begin{array}{l}76 \\
80 \\
83 \\
87 \\
93\end{array}$ & $\begin{array}{r}9 \\
9 \\
9 \\
10\end{array}$ & $\begin{array}{l}1088 \\
1132 \\
1162 \\
1205 \\
1260 \\
\end{array}$ \\
\hline 38 & 34 & $\begin{array}{l}14 \\
15 \\
16 \\
22 \\
29\end{array}$ & $\begin{array}{l}1 \\
11 / 8 \\
11 / 4 \\
11 / 4 \\
11 / 4\end{array}$ & $\begin{array}{c}8 / 8^{\prime \prime} \phi-21 / 4 \\
727 \\
754 \\
788 \\
839 \\
899\end{array}$ & $\begin{array}{r}1 / 2 " \phi- \\
8 \\
9 \\
9 \\
10\end{array}$ & $\begin{array}{l}1068 \\
1096 \\
1129 \\
1180 \\
1241\end{array}$ & $\begin{array}{r}\text { " } \phi-2 \\
81 \\
83 \\
87 \\
91 \\
97\end{array}$ & $\begin{array}{r}1 / 2 " \phi- \\
9 \\
10 \\
10 \\
10 \\
11\end{array}$ & $\begin{array}{l}2^{\prime \prime} \phi-11 / 2^{\prime \prime} p \\
1155 \\
1181 \\
1212 \\
1261 \\
1317\end{array}$ \\
\hline 39 & $\bar{*}$ & $\begin{array}{l}14 \\
15 \\
16 \\
24 \\
31\end{array}$ & $\begin{array}{l}1 \\
11 / 8 \\
11 / 4 \\
11 / 4 \\
11 / 4\end{array}$ & $\begin{array}{r}8 / 8 " \phi-21 / 4 \\
758 \\
786 \\
819 \\
887 \\
948\end{array}$ & $\begin{array}{c}1 / 2 " \phi-21 / 4 \\
927 \\
955 \\
988 \\
1056 \\
1117\end{array}$ & $\begin{array}{l}n-11 \\
1112 \\
1140 \\
1173 \\
1241 \\
1302\end{array}$ & $\begin{array}{r}\phi-21 / 4 \\
850 \\
876 \\
907 \\
972 \\
1029\end{array}$ & $\begin{array}{l}1019 \\
1045 \\
1077 \\
1141 \\
1198\end{array}$ & $\begin{array}{l}2^{\prime \prime} \phi-11 / 2 \\
1204 \\
1230 \\
1261 \\
1326 \\
1383 \\
\end{array}$ \\
\hline
\end{tabular}

Notz-Size and pitch of splral wire is given at head of each group of loads for each size column. 


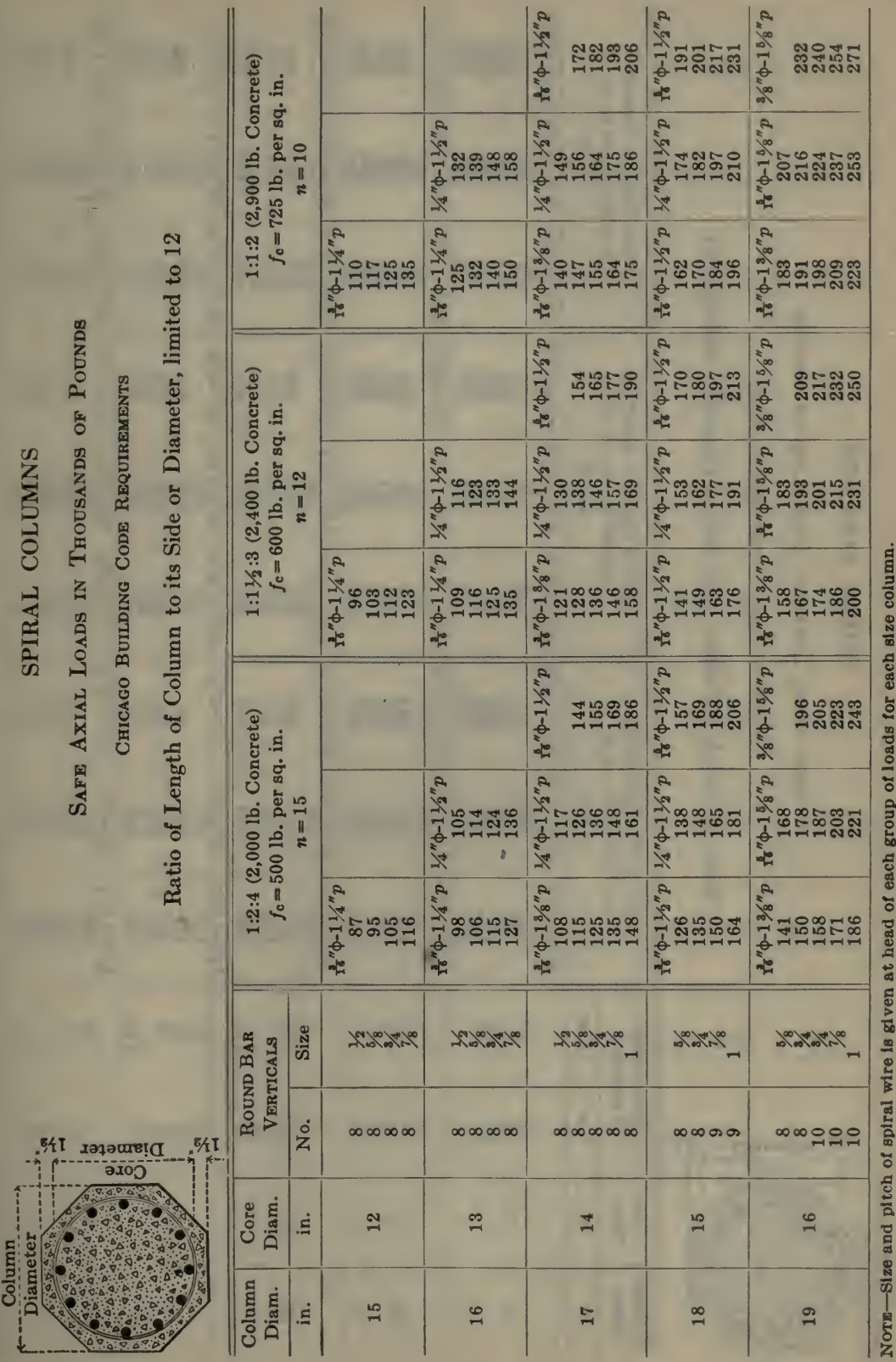




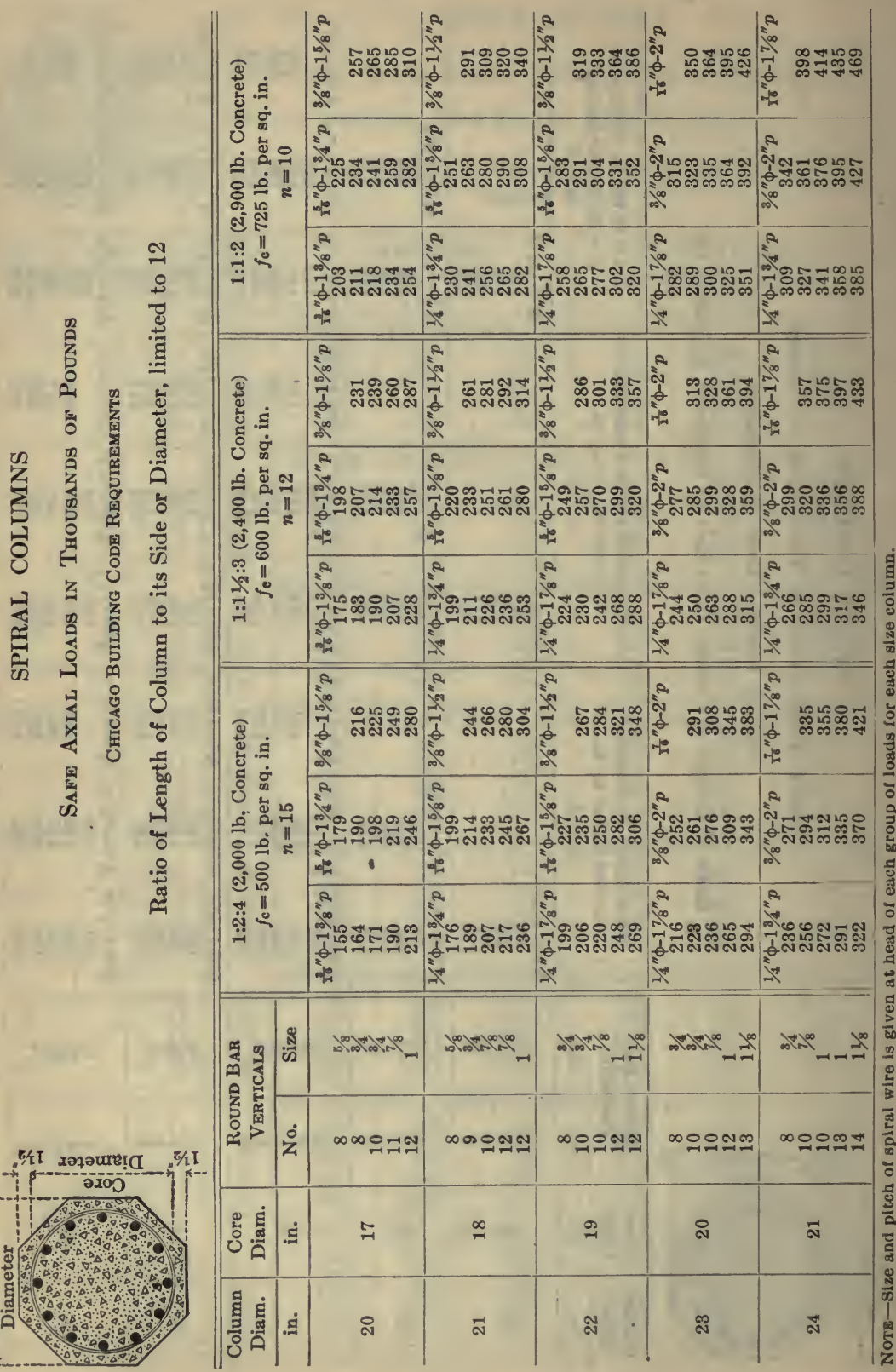




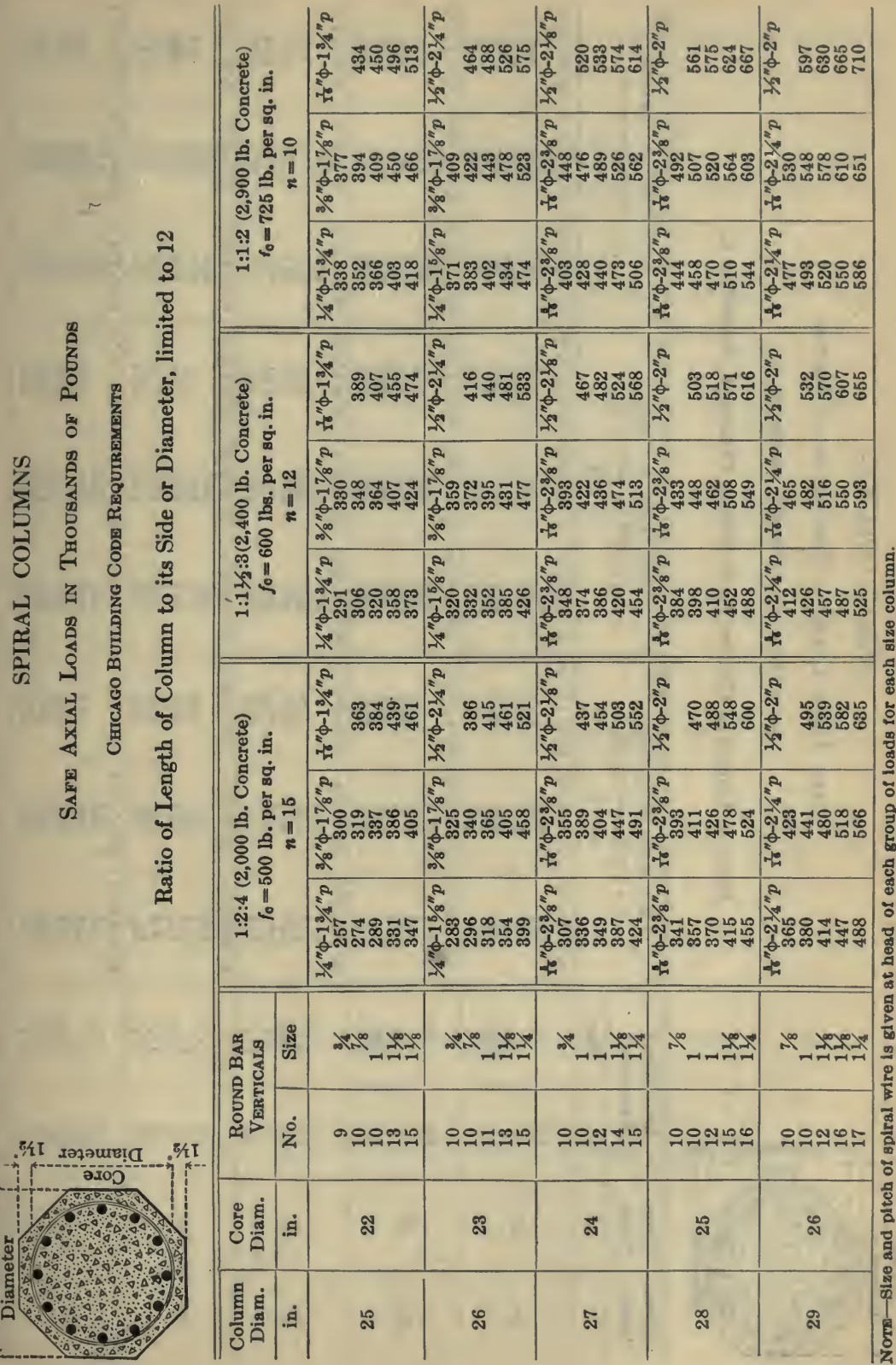




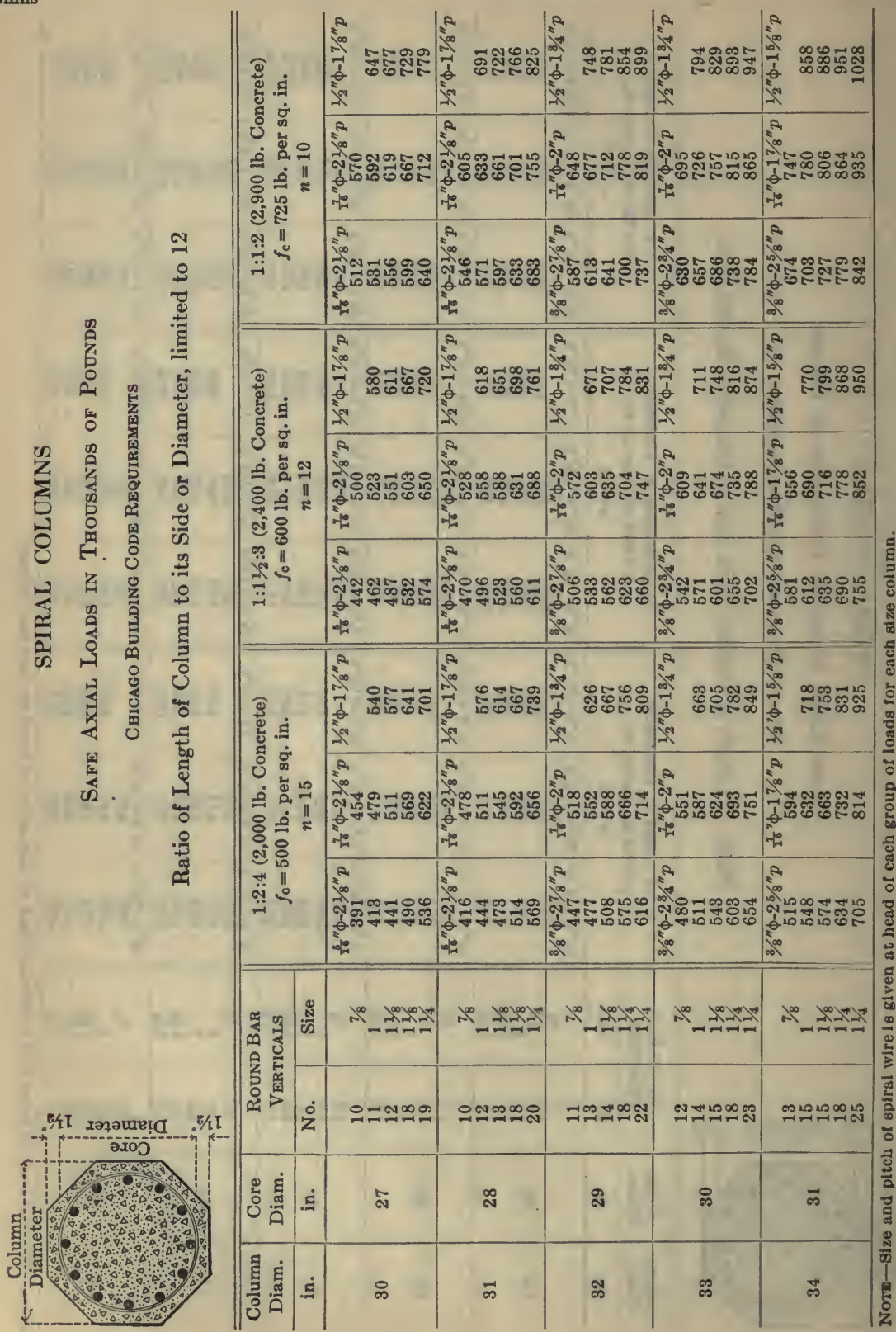




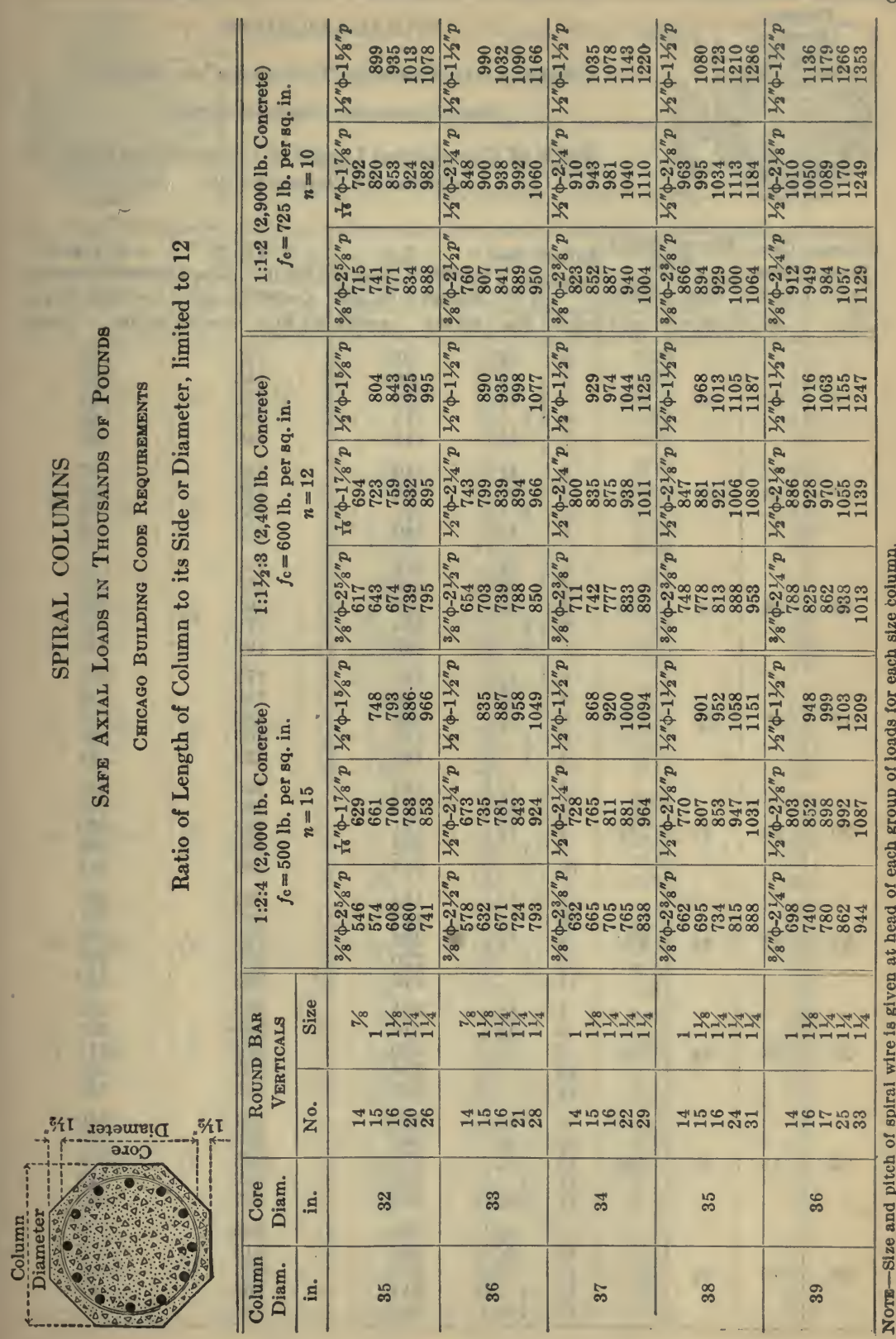


CORRUGATED BAR COMPANY, INC.

Column

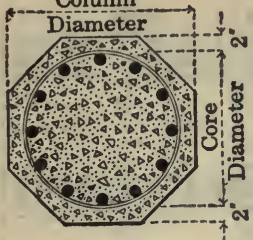

\section{SPIRAL COLUMNS}

Safe Axial Loads in Thougands of Pounds

\section{Joint CoMmittee Recommendations}

Ratio of Unsupported Length of Column to its Core

Diameter, limited to 10

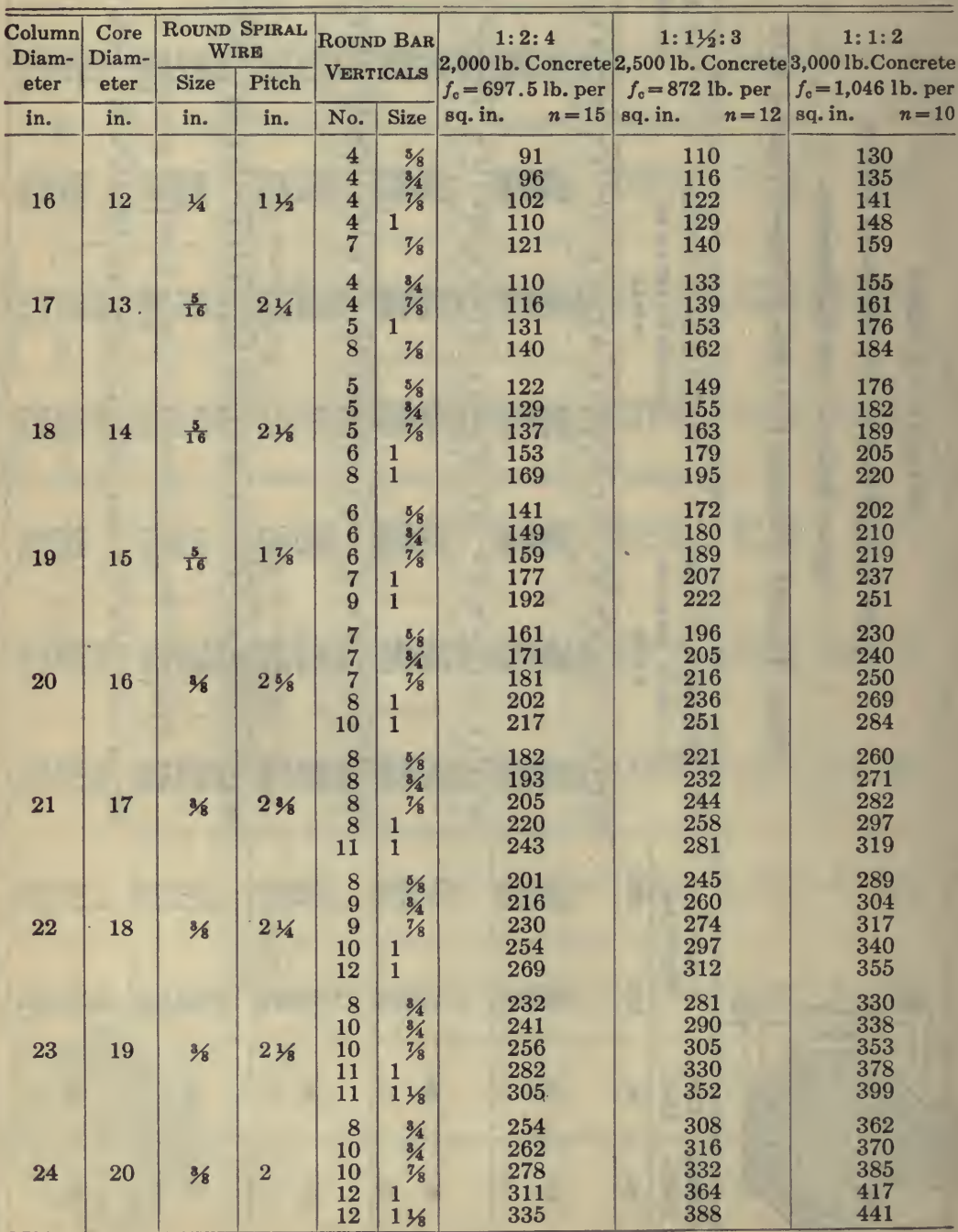


Column

Diameter

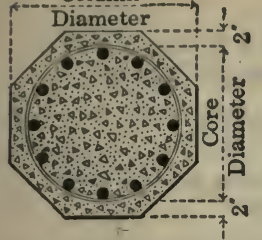

\section{SPIRAL COLUMNS}

\section{Safe Axial Loads in Thousands of Pounds} JOINT COMMITTEE RECOMMENDATIONS

\section{Ratio of Unsupported Length of Column to its Core} Diameter, limited to 10

\begin{tabular}{|c|c|c|c|c|c|c|c|c|}
\hline \multirow{3}{*}{$\begin{array}{c}\text { Column } \\
\text { Diam- } \\
\text { eter }\end{array}$} & \multirow{3}{*}{\begin{tabular}{|c|}
$\begin{array}{c}\text { Core } \\
\text { Diam- } \\
\text { eter }\end{array}$ \\
in.
\end{tabular}} & \multicolumn{2}{|c|}{$\begin{array}{c}\text { ROUND SPIRAL } \\
\text { WIRE }\end{array}$} & \multirow{2}{*}{\multicolumn{2}{|c|}{$\begin{array}{l}\text { ROUND BAR } \\
\text { VERTICALS }\end{array}$}} & \multirow{3}{*}{\begin{tabular}{|c|}
$1: 2: 4$ \\
2,000 lb. Concrete \\
$f_{0}=697.5$ lb. per \\
sq. in. $\quad n=15$
\end{tabular}} & \multirow{3}{*}{$\begin{array}{c}1: 11 / 2: 3 \\
2,500 \mathrm{lb} \text {. Concrete } \\
f_{0}=872 \text { lb. per } \\
\text { sq. in. } \quad n=12\end{array}$} & \multirow{3}{*}{$\begin{array}{c}1: 1: 2 \\
3,000 \text { lb. Concrete } \\
f_{0}=1,046 \text { lb. per } \\
\text { sq. in. } \quad n=10\end{array}$} \\
\hline & & \multirow{2}{*}{$\begin{array}{l}\text { Size } \\
\text { in. }\end{array}$} & \multirow{2}{*}{$\frac{\text { Pitch }}{\text { in. }}$} & & & & & \\
\hline & & & & No. & Size & & & \\
\hline 25 & 21 & $\frac{7}{16}$ & $23 / 4$ & $\begin{array}{l}8 \\
10 \\
10 \\
13 \\
14\end{array}$ & $\begin{array}{l}\frac{8 / 4}{7 / 8} \\
1^{71 / 8}\end{array}$ & $\begin{array}{l}276 \\
300 \\
318 \\
341 \\
378\end{array}$ & $\begin{array}{l}336 \\
360 \\
377 \\
400 \\
436\end{array}$ & $\begin{array}{l}396 \\
419 \\
436 \\
459 \\
493\end{array}$ \\
\hline 26 & 22 & $\frac{7}{16}$ & $25 / 8$ & $\begin{array}{r}9 \\
10 \\
10 \\
13 \\
15\end{array}$ & $\begin{array}{l}\frac{8}{4} \\
1 / 8 \\
11 / 8 \\
11 / 8\end{array}$ & $\begin{array}{l}304 \\
324 \\
342 \\
391 \\
411\end{array}$ & $\begin{array}{l}370 \\
389 \\
407 \\
455 \\
475\end{array}$ & $\begin{array}{l}435 \\
454 \\
472 \\
519 \\
538\end{array}$ \\
\hline 27 & 23 & $\frac{7}{16}$ & $21 / 2$ & $\begin{array}{l}10 \\
10 \\
11 \\
13 \\
16\end{array}$ & $\begin{array}{l}3 / 4 \\
7 / 8 \\
1 \\
11 / 8 \\
11 / 8\end{array}$ & $\begin{array}{l}333 \\
348 \\
374 \\
416 \\
445\end{array}$ & $\begin{array}{l}405 \\
420 \\
445 \\
486 \\
515\end{array}$ & $\begin{array}{l}476 \\
491 \\
516 \\
556 \\
585\end{array}$ \\
\hline 28 & 24 & $\frac{7}{16}$ & $23 / 8$ & $\begin{array}{l}10 \\
10 \\
12 \\
14 \\
15\end{array}$ & $\begin{array}{l}1^{3 / 4} \\
1 \\
11 / 8 \\
11 / 4\end{array}$ & $\begin{array}{l}359 \\
392 \\
408 \\
452 \\
495\end{array}$ & $\begin{array}{l}437 \\
470 \\
485 \\
528 \\
571\end{array}$ & $\begin{array}{l}515 \\
547 \\
562 \\
604 \\
646\end{array}$ \\
\hline 29 & 25 & $\frac{7}{16}$ & $23 / 8$ & $\begin{array}{l}10 \\
10 \\
12 \\
15 \\
16\end{array}$ & $\begin{array}{l}7 / 8 \\
1 \\
11 / 8 \\
11 / 4\end{array}$ & $\begin{array}{l}401 \\
419 \\
434 \\
488 \\
534\end{array}$ & $\begin{array}{l}486 \\
503 \\
518 \\
571 \\
616\end{array}$ & $\begin{array}{l}570 \\
587 \\
602 \\
654 \\
698\end{array}$ \\
\hline 30 & 26 & $\frac{7}{16}$ & $21 / 4$ & $\begin{array}{l}10 \\
10 \\
12 \\
16 \\
17\end{array}$ & $\begin{array}{l}7 / 8 \\
11 / 8 \\
11 / 8 \\
11 / 4\end{array}$ & $\begin{array}{l}429 \\
447 \\
487 \\
525 \\
574\end{array}$ & $\begin{array}{l}521 \\
538 \\
577 \\
615 \\
663\end{array}$ & $\begin{array}{l}612 \\
629 \\
668 \\
705 \\
752\end{array}$ \\
\hline 31 & 27 & $\frac{7}{16}$ & $21 / 8$ & $\begin{array}{l}10 \\
11 \\
12 \\
18 \\
19\end{array}$ & $\begin{array}{l}7 / 8 \\
11 / 8 \\
11 / 8 \\
11 / 4\end{array}$ & $\begin{array}{l}458 \\
484 \\
516 \\
574 \\
627\end{array}$ & $\begin{array}{l}557 \\
582 \\
614 \\
671 \\
723\end{array}$ & $\begin{array}{l}656 \\
680 \\
711 \\
767 \\
819\end{array}$ \\
\hline 32 & 28 & $\frac{7}{16}$ & 2 & $\begin{array}{l}10 \\
12 \\
13 \\
18 \\
20\end{array}$ & $\begin{array}{l}7 / 8 \\
11 / 8 \\
11 / 8 \\
11 / 4\end{array}$ & $\begin{array}{l}488 \\
521 \\
556 \\
604 \\
669\end{array}$ & $\begin{array}{l}594 \\
627 \\
661 \\
708 \\
773\end{array}$ & $\begin{array}{l}701 \\
733 \\
766 \\
812 \\
875\end{array}$ \\
\hline 33 & 29 & $1 / 2$ & $25 / 8$ & $\begin{array}{l}11 \\
13 \\
14 \\
18 \\
22\end{array}$ & \begin{tabular}{l|}
1 \\
1 \\
$11 / 8$ \\
$11 / 4$ \\
$11 / 4$
\end{tabular} & $\begin{array}{l}525 \\
560 \\
597 \\
676 \\
724\end{array}$ & $\begin{array}{l}639 \\
674 \\
710 \\
787 \\
835\end{array}$ & $\begin{array}{l}753 \\
787 \\
821 \\
899 \\
945\end{array}$ \\
\hline
\end{tabular}


Column

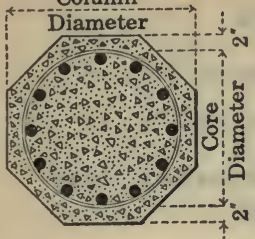

\section{SPIRAL COLUMNS}

Safe Axial Loads in Thousands of Pounds

JoINT COMMItTEE ReCommendations

Ratio of Unsupported Length of Column to its Core

Diameter, limited to 10

\begin{tabular}{|c|c|c|c|c|c|c|c|c|}
\hline \multirow{3}{*}{$\begin{array}{c}\begin{array}{c}\text { Column } \\
\text { Diam- } \\
\text { eter }\end{array} \\
\text { in. }\end{array}$} & \multirow{3}{*}{\begin{tabular}{|c|}
$\begin{array}{c}\text { Core } \\
\text { Diam- } \\
\text { eter }\end{array}$ \\
in. \\
\end{tabular}} & \multicolumn{2}{|c|}{$\begin{array}{c}\text { ROUND SPIRAL } \\
\text { WIRE } \\
\end{array}$} & \multirow{2}{*}{\multicolumn{2}{|c|}{$\begin{array}{l}\text { ROUND BAR } \\
\text { VERTICALS }\end{array}$}} & \multirow{3}{*}{$\begin{array}{c}1: 2: 4 \\
2,000 \text { lb. Concrete } \\
f_{0}=697.5 \text { lb. per } \\
\text { sq. in. } \quad n=15\end{array}$} & \multirow{2}{*}{$\begin{array}{c}1: 11 / 2: 3 \\
2,500 \text { lb. Concrete } \\
f_{\mathrm{c}}=872 \text { lb. per }\end{array}$} & \multirow{3}{*}{$\begin{array}{c}1: 1: 2 \\
3,000 \text { lb. Concrete } \\
f_{\mathrm{c}}=1,046 \text { lb. per } \\
\text { sq. in. } \quad n=10\end{array}$} \\
\hline & & \multirow{2}{*}{$\begin{array}{l}\text { Size } \\
\text { in. }\end{array}$} & \multirow{2}{*}{$\begin{array}{c}\text { Pitch } \\
\text { in. }\end{array}$} & & & & & \\
\hline & & & & No. & Size & & sq. in. $n=12$ & \\
\hline 34 & 30 & $1 / 2$ & $21 / 2$ & $\begin{array}{l}12 \\
14 \\
15 \\
18 \\
23\end{array}$ & $\begin{array}{l}1^{7 / 8} \\
11 / 8 \\
11 / 4 \\
11 / 4\end{array}$ & $\begin{array}{l}564 \\
601 \\
638 \\
708 \\
769\end{array}$ & $\begin{array}{l}685 \\
722 \\
759 \\
828 \\
887\end{array}$ & $\begin{array}{r}807 \\
843 \\
880 \\
947 \\
1005\end{array}$ \\
\hline 35 & 31 & $1 / 2$ & $23 / 8$ & $\begin{array}{l}13 \\
15 \\
15 \\
18 \\
25\end{array}$ & $\begin{array}{l}1^{7 / 8} \\
11 / 8 \\
11 / 4 \\
11 / 4\end{array}$ & $\begin{array}{l}603 \\
641 \\
672 \\
742 \\
826\end{array}$ & $\begin{array}{l}733 \\
771 \\
801 \\
870 \\
952\end{array}$ & $\begin{array}{r}863 \\
900 \\
930 \\
997 \\
1078\end{array}$ \\
\hline 36 & 32 & $1 / 2$ & $23 / 8$ & $\begin{array}{l}14 \\
15 \\
16 \\
20 \\
26\end{array}$ & $\begin{array}{l}1^{7 / 8} \\
11 / 8 \\
11 / 4 \\
11 / 4\end{array}$ & $\begin{array}{l}643 \\
676 \\
716 \\
800 \\
873\end{array}$ & $\begin{array}{r}782 \\
814 \\
854 \\
937 \\
1008\end{array}$ & $\begin{array}{r}920 \\
952 \\
991 \\
1073 \\
1142\end{array}$ \\
\hline 37 & 33 & $1 / 2$ & $21 / 4$ & $\begin{array}{l}14 \\
15 \\
16 \\
21 \\
28\end{array}$ & $\begin{array}{r}7 / 8 \\
11 / 8 \\
11 / 4 \\
11 / 4 \\
11 / 4\end{array}$ & $\begin{array}{l}679 \\
742 \\
788 \\
851 \\
932\end{array}$ & $\begin{array}{r}826 \\
889 \\
934 \\
996 \\
1075\end{array}$ & $\begin{array}{r}974 \\
1035 \\
1080 \\
1140 \\
1219\end{array}$ \\
\hline 38 & 34 & $1 / 2$ & $21 / 4$ & $\begin{array}{l}14 \\
15 \\
16 \\
22 \\
29\end{array}$ & $\begin{array}{l}1 \\
11 / 8 \\
11 / 4 \\
11 / 4 \\
11 / 4\end{array}$ & $\begin{array}{l}740 \\
779 \\
825 \\
897 \\
981\end{array}$ & $\begin{array}{r}897 \\
935 \\
980 \\
1051 \\
1133\end{array}$ & $\begin{array}{l}1053 \\
1090 \\
1135 \\
1203 \\
1285\end{array}$ \\
\hline 39 & 35 & $1 / 2$ & $21 / 8$ & $\begin{array}{l}14 \\
15 \\
16 \\
24 \\
31\end{array}$ & $\begin{array}{l}1 \\
11 / 8 \\
11 / 4 \\
11 / 4 \\
11 / 4\end{array}$ & $\begin{array}{r}778 \\
817 \\
863 \\
958 \\
1043\end{array}$ & $\begin{array}{r}945 \\
982 \\
1028 \\
1122 \\
1205\end{array}$ & $\begin{array}{l}1110 \\
1146 \\
1192 \\
1283 \\
1365\end{array}$ \\
\hline 40 & 36 & $1 / 2$ & $21 / 8$ & $\begin{array}{l}14 \\
16 \\
17 \\
25 \\
33\end{array}$ & $\begin{array}{l}1 \\
11 / 8 \\
11 / 4 \\
11 / 4 \\
11 / 4\end{array}$ & $\begin{array}{r}817 \\
865 \\
914 \\
1010 \\
1106\end{array}$ & $\begin{array}{r}993 \\
1040 \\
1087 \\
1182 \\
1276\end{array}$ & $\begin{array}{l}1168 \\
1215 \\
1262 \\
1353 \\
1446\end{array}$ \\
\hline 41 & 37 & $1 / 2$ & 2 & $\begin{array}{l}14 \\
16 \\
18 \\
26 \\
35\end{array}$ & $\begin{array}{ll}1 & 1 \\
1 & 1 / 8 \\
1 & 1 / 4 \\
1 & 1 / 4 \\
1 & 1 / 4\end{array}$ & $\begin{array}{r}857 \\
905 \\
965 \\
1061 \\
1169\end{array}$ & $\begin{array}{l}1043 \\
1090 \\
1149 \\
1243 \\
1349\end{array}$ & $\begin{array}{l}1228 \\
1274 \\
1333 \\
1425 \\
1528\end{array}$ \\
\hline 42 & 38 & $1 / 2$ & 2 & $\begin{array}{l}15 \\
17 \\
19 \\
28 \\
37\end{array}$ & $\begin{array}{l}1 \\
11 / 8 \\
11 / 4 \\
11 / 4 \\
11 / 4\end{array}$ & $\begin{array}{r}906 \\
956 \\
1019 \\
1126 \\
1234\end{array}$ & $\begin{array}{l}1102 \\
1151 \\
1212 \\
1318 \\
1424\end{array}$ & $\begin{array}{l}1298 \\
1345 \\
1406 \\
1509 \\
1613\end{array}$ \\
\hline
\end{tabular}




\section{FOOTING TABLES}

The purpose of a footing is to distribute the column load uniformly over the soil so that unequal settlements may be avoided. To accomplish this one of three general types of foundation may be used: (a) square spread footings; (b) combined spread footings; (c) spread footings supported by piles. Tables covering these three types
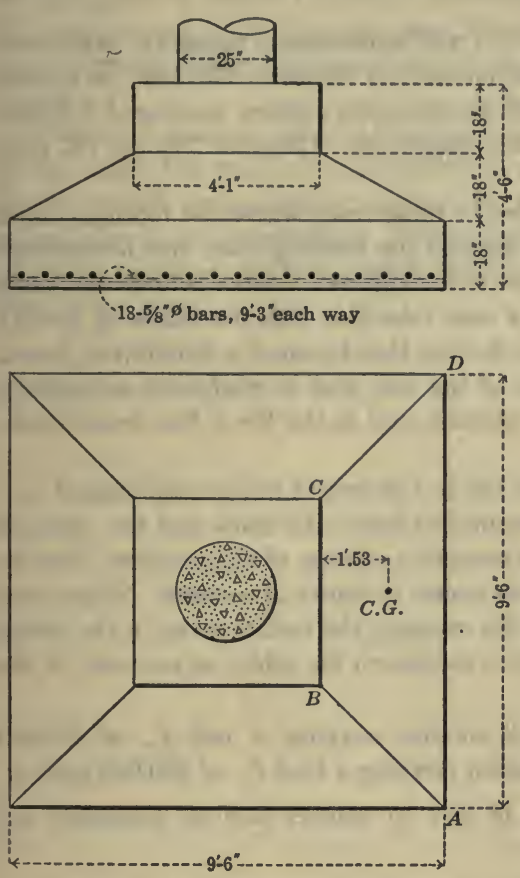

FIG. 9 are given on pages 136 to 142 . In special cases there may be employed cantilever footings or a raft foundation extending over the entire lot area. To cover these in tabular form, however, would be difficult.

In the table for square column footings the method of design outlined in Bulletin 67 , University of Illinois, has been followed and its application is illustrated in the following problem.

Problem-Given a column load of 500,000 pounds. Design a square spread footing, reinforced in two directions, for an allowable soil pressure of 6,000 pounds per square foot; diameter of column 25 inches; punching shear not to exceed 120 pounds per square inch; bond stress limited to 100 pounds per square inch; $f_{\mathrm{s}}=16,000$ and $f_{\mathrm{c}}=650$.

Approximating the weight of the footing at 400 pounds per square foot, the net soil reaction will be $6,000-$ $400=5,600$ pounds per square foot. The area of the footing will be, 500 ,$000 \div 5,600=90.4$ sq. ft. and we will use a footing $9^{\prime} 6^{\prime \prime} \times 9^{\prime} 6^{\prime \prime}$.

The effective depth of footing is found by dividing the column load, less the net soil reaction directly under the column area, by the perimeter of the column multiplied by the unit punching shear, or Eff. depth $=\frac{500,000-19,000}{(25)(3.14)(120)}=51 \mathrm{in}$.

To the effective depth should be added 3 inches of concrete for the protection of the metal, giving a total depth of footing of $4^{\prime} 6^{\prime \prime}$.

The area of steel, in one direction, to resist the moment of the net soil reaction about the edge $B C$ of the cap, is

$$
A_{\mathrm{s}}=\frac{\left(\frac{4.08+9.50}{2}\right)(2.71)(5,600)(1.53)(12)}{\left(\frac{7}{8}\right)(33)(16,000)}=4.10 \text { sq. in. }
$$

This area is equivalent to $14-5 / 8$ " round bars.

As it is required to maintain the bond stress at 100 pounds per square inch 
(assuming the use of deformed bars), the number of bars selected for moment considerations may not be sufficient to accomplish this. In this case the unit bond stress,

$$
u=\frac{\left(\frac{4.08+9.50}{2}\right)(2.71)(5,600)}{(14)(1.965)\left(\frac{7}{8}\right)(33)}=130 \mathrm{lb} \text {. per sq. in. }
$$

The allowable bond stress is exceeded and it will be necessary, therefore, to increase the number of $5 / 8$ " round bars required for moment in the ratio $130 / 100$; or a total of $18-5 / 8$ " round bars will be needed in each direction at a uniform spacing of 6 inches. The quantities of material required by this design are: Concrete $233 \mathrm{cu}$. $\mathrm{ft}$., steel $36-5 / 8$ " round bars $9^{\prime} 3^{\prime \prime}$ long.

The combined spread footing is employed in those cases where the footings under wall columns are not permitted to extend beyond the building line; this necessitates extending the footing under the wall column to the adjacent interior column and making it of such dimensions that its center of area coincides with the center of gravity of the column loads that bear upon it. The footing thus becomes a distributing beam, uniformly loaded by the upward reaction of the soil, and is reinforced accordingly in the upper face longitudinally between columns, and in the lower face transversely under each column.

The designs given in the tables on pages 136 to 139 cover a fairly wide range of conditions and as will be noted it is only required to know the loads and the distance center to center of the columns to obtain a complete solution of the problem. Results are all given in terms of the distance $l$ in feet center to center of columns. Where conditions depart from what might be called the average, the results given in the tables will be slightly in error but not sufficiently so to disturb the safety or economy of the design.

Example-Given a 24-inch square wall column carrying a load $P_{1}$, of 350,000 pounds and a 26-inch diameter interior column carrying a load $P_{2}$, of 450,000 pounds, or $P_{2} / P_{1}=1.3$. The columns are spaced 18 feet on centers and the allowable soil pressure is 6,000 pounds per square foot.

The sum of the loads is 800,000 pounds and the distance $c$ is one foot. Entering the table on page 138 with $P_{1}+P_{2}=800,000$ we find opposite the ratio $P_{2} / P_{1}=1.3$; the following dimensions and steel areas:

$$
\begin{aligned}
& L=1.13 l+2 c=(1.13)(18)+(2)(1)=22.34 \mathrm{ft} . \\
& B=\frac{121}{l}=\frac{121}{18}=6.72 \mathrm{ft} . \\
& H=0.2 l+0.25=(0.2)(18)+(0.25)=3.85 \mathrm{ft} . \\
& h=3.6-\frac{l}{6}=3.6-\frac{18}{6}=0.60 \mathrm{ft} . \\
& A_{\mathrm{s}}=27.3 \text { sq. in. } \\
& A_{\mathrm{s}}^{\prime}=\frac{2,465}{l^{2}}=\frac{2,465}{(18)^{2}}=7.60 \mathrm{sq} . \text { in. } \\
& A_{\mathrm{s}}^{\prime \prime}=\frac{1,895}{l^{2}}=\frac{1,895}{\left(18^{2}\right)}=5.85 \mathrm{sq} . \text { in. }
\end{aligned}
$$


The disposition of the reinforcing steel is indicated in the cuts at the head of the table.

Where soil conditions are of such a nature that spread footings cannot be used, resort must be had to piles of either wood or concrete. If the piles are cut off below the low water line, wooden piles may be used, but if exposed to alternate wet and dry conditions wood is subject to rot and concrete piles should be employed. The tables of pile caps given on pages 141 and 142 are designed for concrete piles having an assumed carrying capacity of 30 tons per pile. The style and reinforcement of cap and number of concrete piles required are determined from the tables, when the column load is known.

Designs for concrete piles of the pre-cast type are given in the table on page 143. Except under the most adverse conditions as regards surface and sub-soil, a load of 30 tons per pile may be safely used and if the piles are driven to rock a $50 \%$ increase in load should be permissible. The amount of reinforcement used is considered a minimum consistent with successful handling of the pile from the casting yard to its place in the work. 


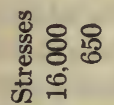

苛

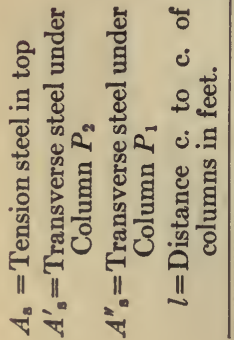

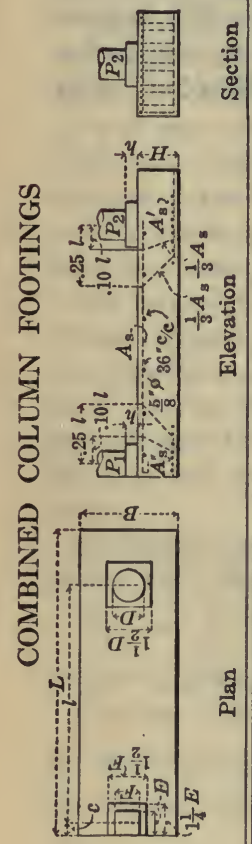

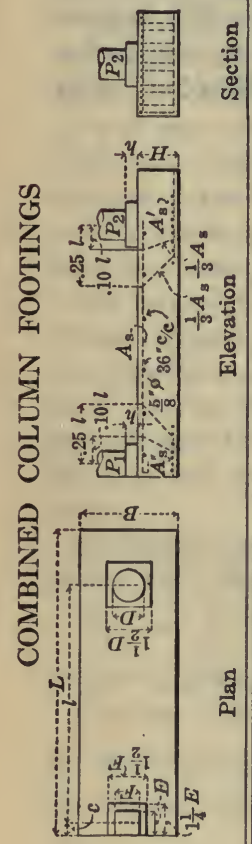

(2)

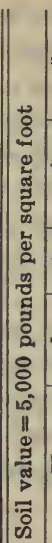

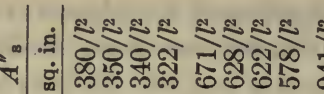

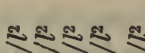

(1)
:

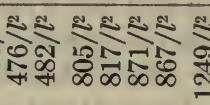

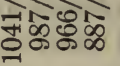

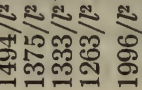
$\leqslant \leqslant \leqslant \leqslant \leqslant \leqslant$

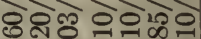

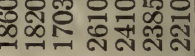

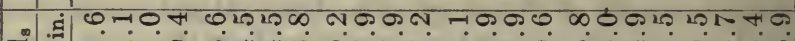
|

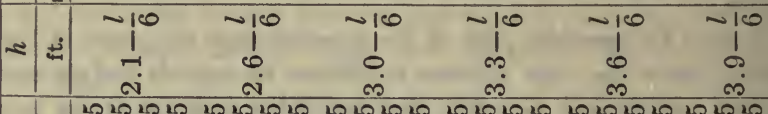

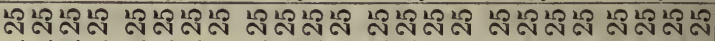

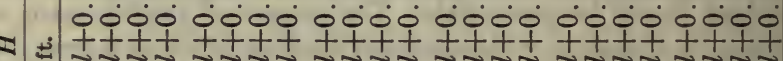
$\vec{\omega} \vec{\infty} \vec{\omega} \vec{\omega} \vec{\omega} \vec{\omega} \vec{\infty} \vec{\omega} \vec{\omega} \vec{\infty} \vec{\omega} \vec{\infty} \vec{\omega} \vec{\omega} \vec{\infty}$ 0000 $000 \dot{0} 000$ 00்

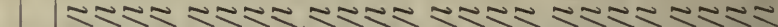
๑ : ×.

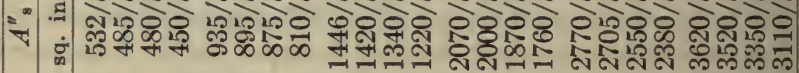

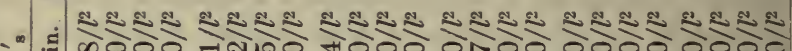
7 = क.

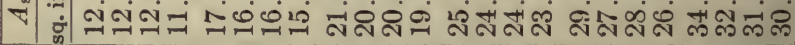
$\sim-10 \sim 10 \sim 10 \sim 10 \sim 10 \sim 10$

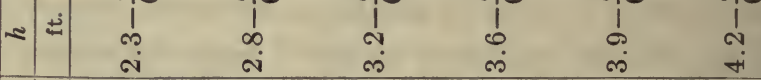

ง. 000 0000 000 000

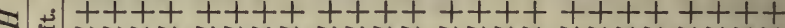

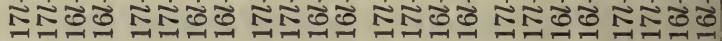
0000 0000 0000 0000 0000000

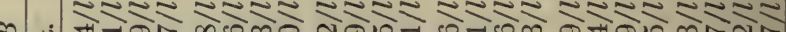

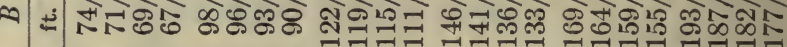

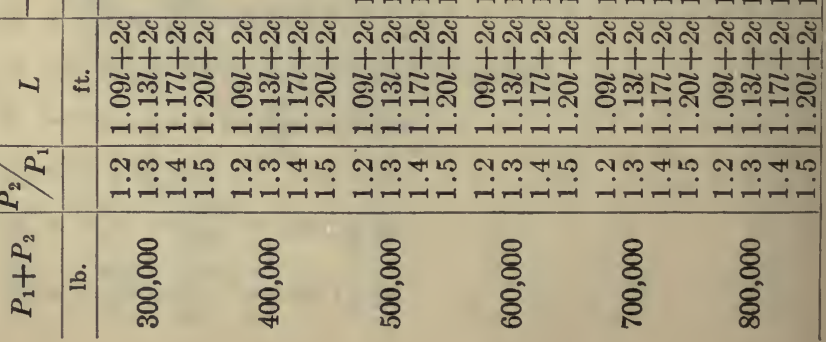




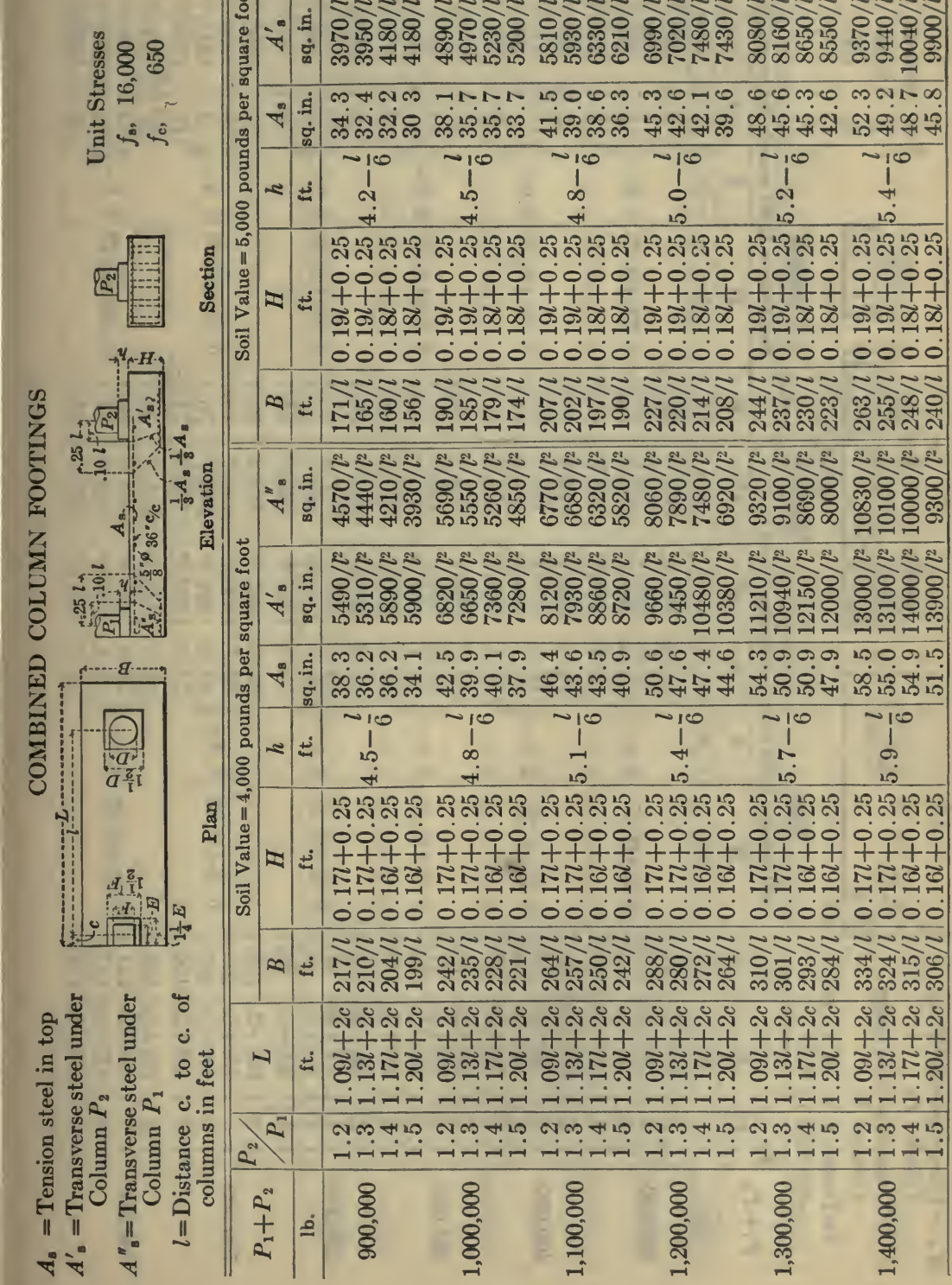

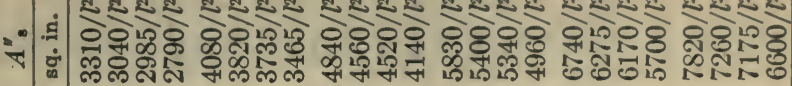

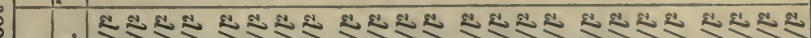

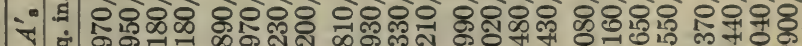
manm nNN

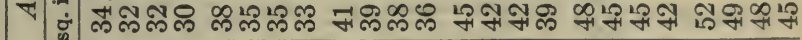

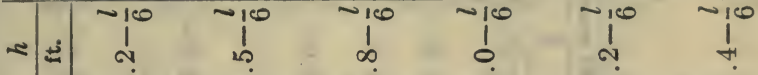

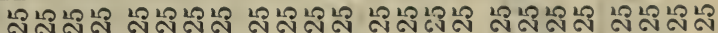
000 000 000 000 0000 0000

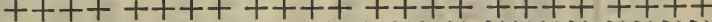

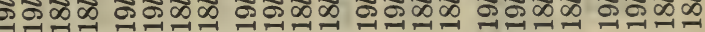
000000000000000000000000 10:0

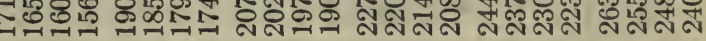
$\checkmark$ 品

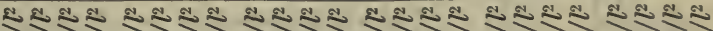

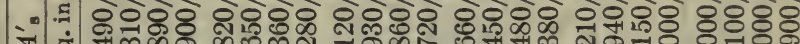
4 - mกN-10070 H

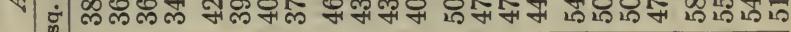
$\approx \pm$ । 


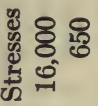

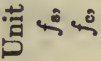
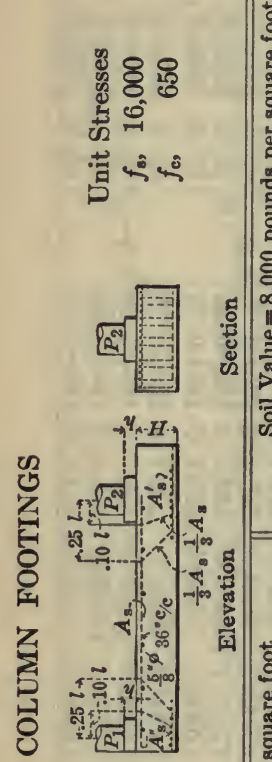

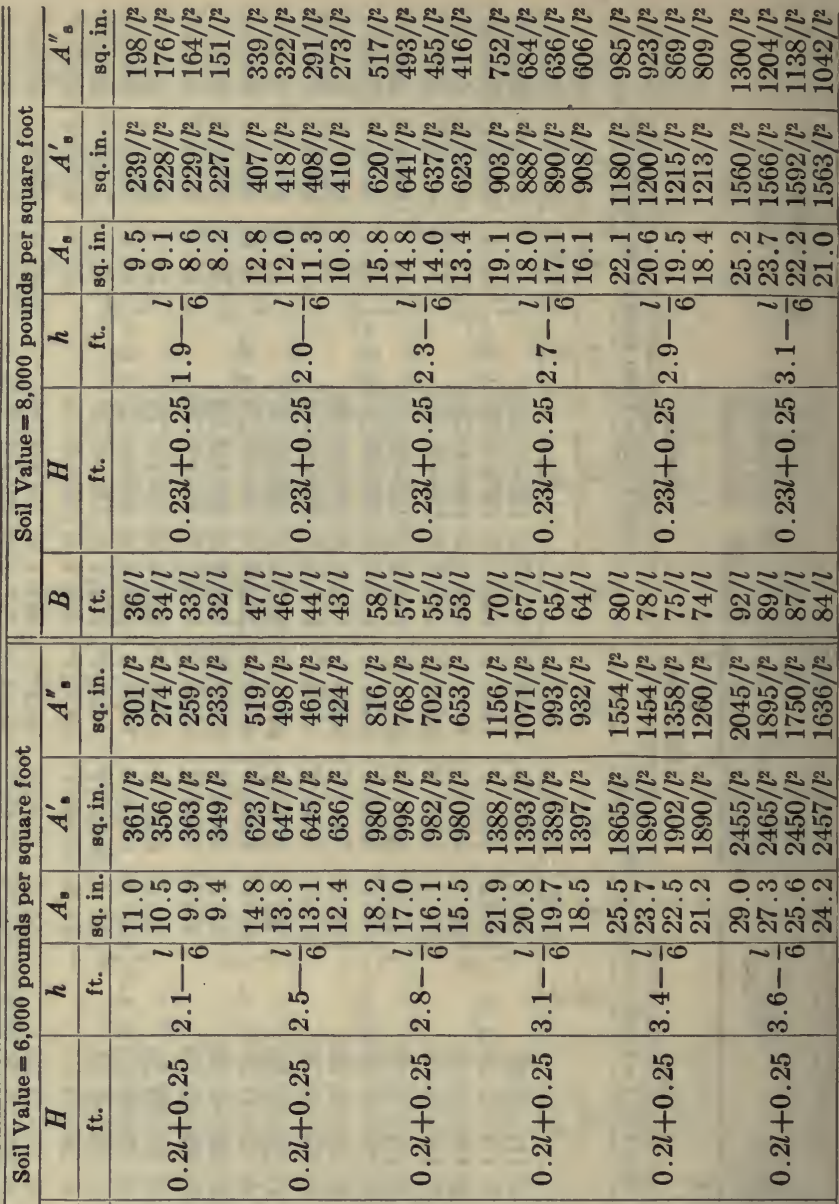

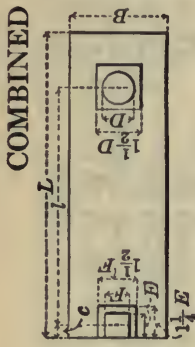

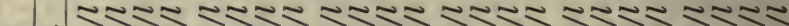

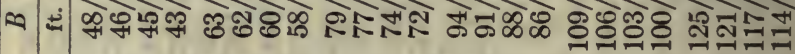

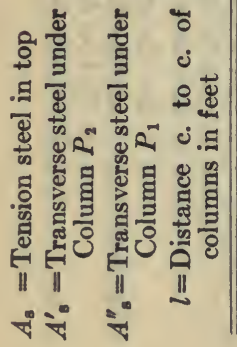

\begin{tabular}{|c|c|c|c|c|c|c|c|}
\hline$r$ & 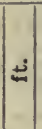 & 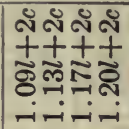 & 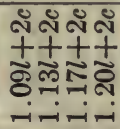 & 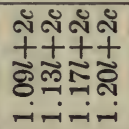 & 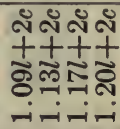 & 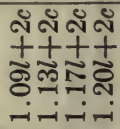 & 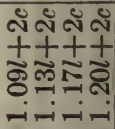 \\
\hline & & 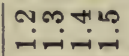 & 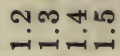 & & 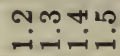 & 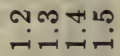 & \\
\hline$\frac{R^{*}}{+}$ & $\cong$ & §ి & 용 & $\begin{array}{l}8 \\
8 \\
8\end{array}$ & $\begin{array}{l}8 \\
8 \\
8 \\
8\end{array}$ & $\begin{array}{l}8 \\
8 \\
8\end{array}$ & $\begin{array}{l}8 \\
8 \\
8\end{array}$ \\
\hline
\end{tabular}




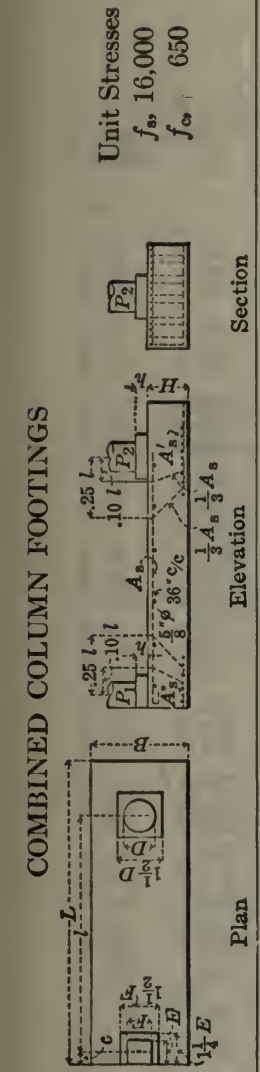

\begin{tabular}{|c|c|c|c|c|c|c|c|c|}
\hline \multirow{11}{*}{ 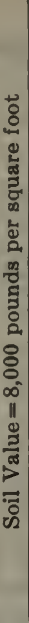 } & $=$ & 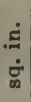 & 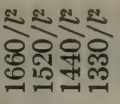 & 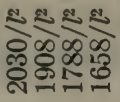 & 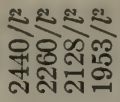 & 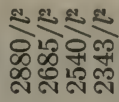 & 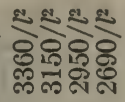 & 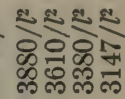 \\
\hline & $\ddot{*}$ & $\begin{array}{l}\Xi \\
\dot{\delta}\end{array}$ & 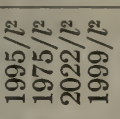 & 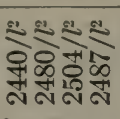 & 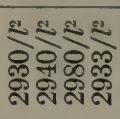 & 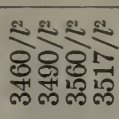 & 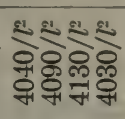 & 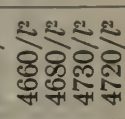 \\
\hline & & $\dot{\Xi}$ & ๓ $\infty$ ! & मோ サ & ำกษ & m-100 & नफ्म & N $0-\infty$ \\
\hline & $\nabla$ & $\dot{\square}$ & 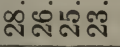 & 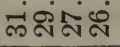 & ஸ் ஸें & 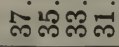 & 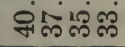 & జ్ํำ \\
\hline & & & -10 & -10 & $=10$ & -100 & -10 & -10 \\
\hline & $\approx$ & $\rightleftarrows$ & 1 & $\begin{array}{l}1 \\
25\end{array}$ & 1 & 1 & $\stackrel{1}{\infty}$ & $\begin{array}{l}1 \\
20\end{array}$ \\
\hline & & & 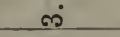 & $\infty$ & $\dot{\infty}$ & $\dot{\nabla}$ & $\dot{H}$ & $\dot{\theta}$ \\
\hline & & & งิ & ติ & งิ & กิ & ติ & ลุ \\
\hline & 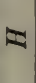 & \pm & 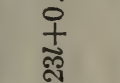 & $\frac{0}{ \pm}$ & $\stackrel{0}{+}$ & $\stackrel{0}{ \pm}$ & $\begin{array}{l}\dot{0} \\
\dot{\mathfrak{n}}\end{array}$ & $\begin{array}{l}\dot{0} \\
\stackrel{+}{\pi}\end{array}$ \\
\hline & & & $0^{\circ}$ & 0 & 0 & $\dot{0}$ & $\dot{0}$ & $\dot{0}$ \\
\hline & $\infty$ & $\stackrel{\Delta}{ }$ & 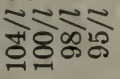 & 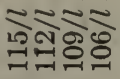 & 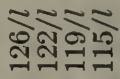 & 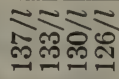 & 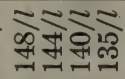 & 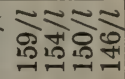 \\
\hline \multirow[b]{7}{*}{ 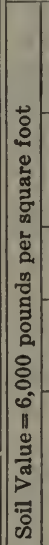 } & $=\frac{\infty}{4}$ & $\frac{\tilde{\Omega}}{\dot{\square}}$ & 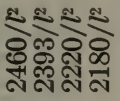 & 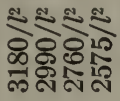 & 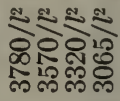 & 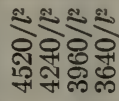 & 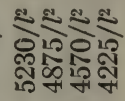 & 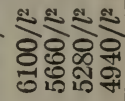 \\
\hline & $=$ & \begin{tabular}{l}
$\stackrel{\text { \} }{\dot{\sigma}}} \\
{ }$ & 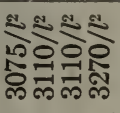 & 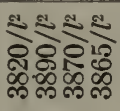 & 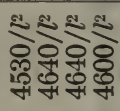 & 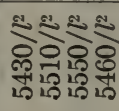 & 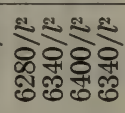 & 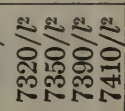 \\
\hline & &.$\beth$ & $0,00 \%$ & NONH & $\forall-\infty N$ & $0 \operatorname{CoN}$ & $-m \infty \forall$ & NNos \\
\hline & $\nabla$ & $\dot{\square}$ & ผ่ల్ణ & ణొఱ్ణ ஸిల్ & लెल लే దี & भ워 & & 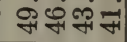 \\
\hline & $\sim$ & $\doteq$ & $\begin{array}{l}10 \\
1 \\
\infty \\
\infty\end{array}$ & $\begin{array}{c}100 \\
1 \\
0 \\
4\end{array}$ & $\begin{array}{c}-10 \\
1 \\
\text { भ } \\
+1\end{array}$ & $\begin{array}{c}10 \\
1 \\
0 \\
4 \\
4\end{array}$ & $\begin{array}{l}10 \\
1 \\
0 \\
4\end{array}$ & $\begin{array}{l}100 \\
1 \\
10\end{array}$ \\
\hline & & & ลิ & ลิ & ลิ & ลิ & ลิ & คి \\
\hline & $\mathbb{Z}$ & 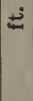 & $\begin{array}{l}0 \\
\frac{1}{\omega} \\
0\end{array}$ & $\begin{array}{l}\dot{1} \\
\dot{0} \\
\dot{0}\end{array}$ & $\begin{array}{l}\dot{0} \\
\dot{\pi} \\
\dot{0}\end{array}$ & 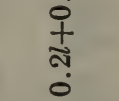 & $\begin{array}{l}\dot{0} \\
\dot{+} \\
\dot{0}\end{array}$ & 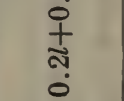 \\
\hline & & & & & & & & \\
\hline
\end{tabular}
\end{tabular}

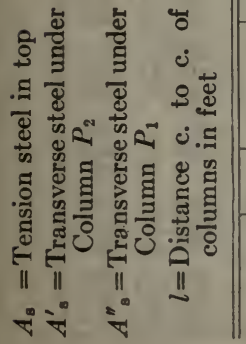

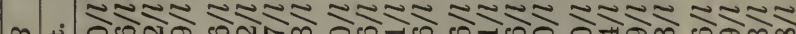

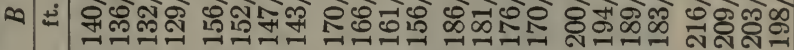

\begin{tabular}{|c|c|c|c|c|c|c|c|}
\hline$\checkmark$ & $\rightleftarrows$ & 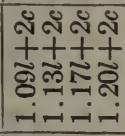 & 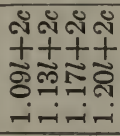 & 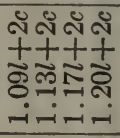 & 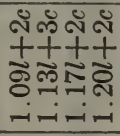 & 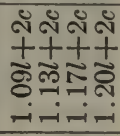 & 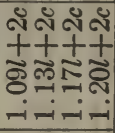 \\
\hline & & 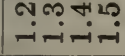 & 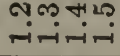 & חי- & 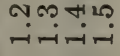 & 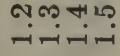 & 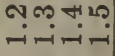 \\
\hline & $\cong$ & $\begin{array}{l}8 \\
8 \\
8\end{array}$ & $\begin{array}{l}8 \\
8 \\
8 \\
8 \\
-1\end{array}$ & $\begin{array}{l}8 \\
8 \\
8 \\
8 \\
-1\end{array}$ & $\begin{array}{l}8 \\
8 \\
8 \\
\text { ণิ } \\
-1\end{array}$ & $\begin{array}{l}8 \\
8 \\
8 \\
8 \\
\text { - }\end{array}$ & $\begin{array}{l}8 \\
8 \\
\text { ही } \\
-1\end{array}$ \\
\hline
\end{tabular}




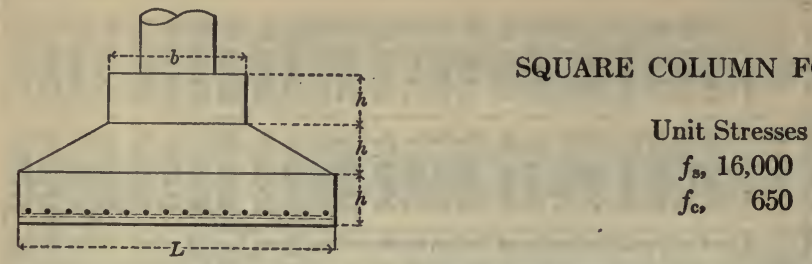

\begin{tabular}{|c|c|c|c|c|c|c|c|c|c|c|c|c|}
\hline \multicolumn{2}{|c|}{$L$} & \multirow{2}{*}{ 1b. per sq. ft. } & \multirow{2}{*}{$\frac{\begin{array}{c}\text { Column } \\
\text { Load }\end{array}}{\text { in } 10001 \mathrm{~b} .}$} & \multirow{2}{*}{$\begin{array}{c}\begin{array}{c}\text { Minimum } \\
\text { Column } \\
\text { Diameter }\end{array} \\
\text { in. }\end{array}$} & \multicolumn{2}{|c|}{$h$} & \multicolumn{2}{|c|}{$b$} & \multicolumn{2}{|c|}{$\begin{array}{l}\text { Reinforcement } \\
\text { Round Bars } \\
\text { Each Way }\end{array}$} & \multirow{2}{*}{$\begin{array}{c}\begin{array}{c}\text { Weight } \\
\text { of } \\
\text { steel }\end{array} \\
\text { lb. }\end{array}$} & \multirow{2}{*}{$\begin{array}{c}\begin{array}{c}\text { Volume of } \\
\text { Concrete }\end{array} \\
\text { cu. ft. }\end{array}$} \\
\hline ft. & in. & & & & it. & In. & ft. & In. & No. & Size & & \\
\hline $\begin{array}{l}5 \\
5 \\
5\end{array}$ & $\begin{array}{l}0 \\
0 \\
0\end{array}$ & $\begin{array}{l}4000 \\
6000 \\
8000\end{array}$ & $\begin{array}{r}96 \\
145 \\
194\end{array}$ & $\begin{array}{l}13 \\
16 \\
18\end{array}$ & $\begin{array}{l}0 \\
0 \\
0\end{array}$ & $\begin{array}{r}7 \\
8 \\
10\end{array}$ & $\begin{array}{l}1 \\
2 \\
2\end{array}$ & $\begin{array}{r}10 \\
3 \\
7\end{array}$ & $\begin{array}{l}14 \\
16 \\
15\end{array}$ & $\begin{array}{l}1 / 2 \\
1 / 2 \\
1 / 2\end{array}$ & $\begin{array}{r}88.8 \\
100.3 \\
94.1\end{array}$ & $\begin{array}{l}23.7 \\
29.3 \\
38.8\end{array}$ \\
\hline $\begin{array}{l}5 \\
5 \\
5\end{array}$ & $\begin{array}{l}6 \\
6 \\
6\end{array}$ & $\begin{array}{l}4000 \\
6000 \\
8000\end{array}$ & $\begin{array}{l}116 \\
175 \\
234\end{array}$ & $\begin{array}{l}14 \\
17 \\
19\end{array}$ & $\begin{array}{l}0 \\
0 \\
0\end{array}$ & $\begin{array}{r}8 \\
10 \\
11\end{array}$ & $\begin{array}{l}2 \\
2 \\
2\end{array}$ & $\begin{array}{r}1 \\
6 \\
10\end{array}$ & $\begin{array}{l}14 \\
15 \\
16\end{array}$ & $\begin{array}{l}1 / 3 \\
1 / 2 \\
1 / 2\end{array}$ & $\begin{array}{r}97.0 \\
104.0 \\
110.9\end{array}$ & $\begin{array}{l}33.3 \\
44.4 \\
51.6\end{array}$ \\
\hline $\begin{array}{l}6 \\
6 \\
6\end{array}$ & $\begin{array}{l}0 \\
0 \\
0\end{array}$ & $\begin{array}{l}4000 \\
6000 \\
8000\end{array}$ & $\begin{array}{l}137 \\
207 \\
278\end{array}$ & $\begin{array}{l}15 \\
18 \\
20\end{array}$ & $\begin{array}{l}0 \\
0 \\
1\end{array}$ & $\begin{array}{r}9 \\
11 \\
0\end{array}$ & $\begin{array}{l}2 \\
2 \\
2\end{array}$ & $\begin{array}{r}3 \\
9 \\
11\end{array}$ & $\begin{array}{l}14 \\
16 \\
18\end{array}$ & $\begin{array}{l}1 / 2 \\
1 / 2 \\
1 / 2\end{array}$ & $\begin{array}{l}106.3 \\
121.4 \\
136.6\end{array}$ & $\begin{array}{l}44.5 \\
58.2 \\
65.2\end{array}$ \\
\hline $\begin{array}{l}6 \\
6 \\
6\end{array}$ & $\begin{array}{l}6 \\
6 \\
6\end{array}$ & $\begin{array}{l}4000 \\
6000 \\
8000\end{array}$ & $\begin{array}{l}161 \\
242 \\
324\end{array}$ & $\begin{array}{l}16 \\
19 \\
21\end{array}$ & $\begin{array}{l}0 \\
1 \\
1\end{array}$ & $\begin{array}{l}9 \\
0 \\
2\end{array}$ & $\begin{array}{l}2 \\
2 \\
3\end{array}$ & $\begin{array}{r}4 \\
10 \\
4\end{array}$ & $\begin{array}{l}17 \\
17 \\
17\end{array}$ & $\begin{array}{l}1 / 2 \\
1 / 2 \\
1 / 2\end{array}$ & $\begin{array}{l}140.3 \\
140.3 \\
140.3\end{array}$ & $\begin{array}{l}51.6 \\
73.2 \\
91.5\end{array}$ \\
\hline $\begin{array}{l}7 \\
7 \\
7\end{array}$ & $\begin{array}{l}0 \\
0 \\
0\end{array}$ & $\begin{array}{l}4000 \\
6000 \\
8000\end{array}$ & $\begin{array}{l}186 \\
280 \\
375\end{array}$ & $\begin{array}{l}17 \\
20 \\
23\end{array}$ & $\begin{array}{l}0 \\
1 \\
1\end{array}$ & $\begin{array}{r}10 \\
1 \\
3\end{array}$ & $\begin{array}{l}2 \\
3 \\
3\end{array}$ & $\begin{array}{l}6 \\
1 \\
7\end{array}$ & $\begin{array}{l}17 \\
18 \\
19\end{array}$ & $\begin{array}{l}1 / 2 \\
1 / 2 \\
1 / 2\end{array}$ & $\begin{array}{l}151.5 \\
160.4 \\
169.3\end{array}$ & $\begin{array}{r}66.2 \\
92.3 \\
113.6\end{array}$ \\
\hline $\begin{array}{l}7 \\
7 \\
7\end{array}$ & $\begin{array}{l}6 \\
6 \\
6\end{array}$ & $\begin{array}{l}4000 \\
6000 \\
8000\end{array}$ & & $\begin{array}{l}18 \\
22 \\
24\end{array}$ & $\begin{array}{l}0 \\
1 \\
1\end{array}$ & $\begin{array}{r}11 \\
1 \\
4\end{array}$ & $\begin{array}{l}2 \\
3 \\
3\end{array}$ & $\begin{array}{l}9 \\
3 \\
9\end{array}$ & $\begin{array}{l}18 \\
20 \\
20\end{array}$ & $\begin{array}{l}1 / 2 \\
1 / 2 \\
1 / 2\end{array}$ & $\begin{array}{l}172.3 \\
191.4 \\
191.4\end{array}$ & $\begin{array}{r}84.3 \\
105.2 \\
137.4\end{array}$ \\
\hline $\begin{array}{l}8 \\
8 \\
8\end{array}$ & $\begin{array}{l}0 \\
0 \\
0\end{array}$ & & & & $\begin{array}{l}1 \\
1 \\
1\end{array}$ & $\begin{array}{l}0 \\
3 \\
5\end{array}$ & $\begin{array}{l}2 \\
3 \\
4\end{array}$ & $\begin{array}{r}10 \\
6 \\
0\end{array}$ & $\begin{array}{l}18 \\
20 \\
21\end{array}$ & $\begin{array}{l}1 / 3 \\
1 / 2 \\
1 / 2\end{array}$ & $\begin{array}{l}184.1 \\
204.6 \\
214.8\end{array}$ & $\begin{array}{l}103.6 \\
138.7 \\
166.3\end{array}$ \\
\hline $\begin{array}{l}8 \\
8 \\
8\end{array}$ & $\begin{array}{l}6 \\
6 \\
6\end{array}$ & $\begin{array}{l}4000 \\
6000 \\
8000\end{array}$ & $\begin{array}{l}272 \\
410 \\
546\end{array}$ & $\begin{array}{l}20 \\
24 \\
26\end{array}$ & $\begin{array}{l}1 \\
1 \\
1\end{array}$ & $\begin{array}{l}0 \\
3 \\
7\end{array}$ & $\begin{array}{l}2 \\
3 \\
4\end{array}$ & $\begin{array}{r}11 \\
8 \\
3\end{array}$ & $\begin{array}{l}21 \\
23 \\
21\end{array}$ & $\begin{array}{l}1 / 2 \\
1 / 2 \\
1 / 2\end{array}$ & $\begin{array}{l}228.7 \\
250.5 \\
228.7\end{array}$ & $\begin{array}{l}116.0 \\
155.8 \\
209.7\end{array}$ \\
\hline $\begin{array}{l}9 \\
9 \\
9\end{array}$ & $\begin{array}{l}0 \\
0 \\
0\end{array}$ & & & $\begin{array}{l}21 \\
24 \\
27\end{array}$ & $\begin{array}{l}1 \\
1 \\
1\end{array}$ & $\begin{array}{l}1 \\
5 \\
8\end{array}$ & $\begin{array}{l}3 \\
3 \\
4\end{array}$ & $\begin{array}{r}2 \\
11 \\
6\end{array}$ & $\begin{array}{l}21 \\
22 \\
22\end{array}$ & $\begin{array}{l}1 / 2 \\
1 / 2 \\
1 / 2\end{array}$ & $\begin{array}{l}242.6 \\
254.1 \\
254.1\end{array}$ & $\begin{array}{l}141.8 \\
198.5 \\
247.4\end{array}$ \\
\hline $\begin{array}{l}9 \\
9 \\
9\end{array}$ & $\begin{array}{l}6 \\
6 \\
6\end{array}$ & & & $\begin{array}{l}21 \\
25 \\
29\end{array}$ & $\begin{array}{l}1 \\
1 \\
1\end{array}$ & $\begin{array}{l}3 \\
6 \\
9\end{array}$ & $\begin{array}{l}3 \\
4 \\
4\end{array}$ & $\begin{array}{l}5 \\
1 \\
9\end{array}$ & $\begin{array}{l}16 \\
18 \\
19\end{array}$ & $\begin{array}{l}5 / 8 \\
5 / 8 \\
5 / 8\end{array}$ & $\begin{array}{l}310.8 \\
349.7 \\
369.1\end{array}$ & $\begin{array}{r}183.4 \\
233.2 \\
289.5\end{array}$ \\
\hline 0 & $\begin{array}{l}0 \\
0 \\
0\end{array}$ & & & & $\begin{array}{l}1 \\
1 \\
1\end{array}$ & $\begin{array}{r}3 \\
7 \\
10\end{array}$ & $\begin{array}{l}3 \\
4 \\
4\end{array}$ & $\begin{array}{r}6 \\
3 \\
11\end{array}$ & $\begin{array}{l}17 \\
19 \\
20\end{array}$ & $\begin{array}{l}5 / 8 \\
5 / 8 \\
5 / 8\end{array}$ & & $\begin{array}{l}201.7 \\
271.6 \\
333.5\end{array}$ \\
\hline 0 & $\begin{array}{l}6 \\
6 \\
6\end{array}$ & & $\begin{array}{l}405 \\
614 \\
822\end{array}$ & $\begin{array}{l}23 \\
28 \\
30\end{array}$ & $\begin{array}{l}1 \\
1 \\
2\end{array}$ & $\begin{array}{l}4 \\
8 \\
0\end{array}$ & $\begin{array}{l}3 \\
4 \\
5\end{array}$ & $\begin{array}{l}8 \\
7 \\
2\end{array}$ & $\begin{array}{l}18 \\
19 \\
20\end{array}$ & $\begin{array}{l}5 / 8 \\
5 / 8 \\
5 / 8\end{array}$ & $\begin{array}{l}387.5 \\
409.0 \\
430.5\end{array}$ & $\begin{array}{l}237.0 \\
318.5 \\
401.3\end{array}$ \\
\hline 11 & $\begin{array}{l}0 \\
0 \\
0\end{array}$ & & & $\begin{array}{l}24 \\
28 \\
33\end{array}$ & $\begin{array}{l}1 \\
1 \\
2\end{array}$ & $\begin{array}{l}5 \\
9 \\
0\end{array}$ & $\begin{array}{l}3 \\
4 \\
5\end{array}$ & $\begin{array}{r}11 \\
8 \\
5\end{array}$ & $\begin{array}{l}18 \\
20 \\
22\end{array}$ & $\begin{array}{l}5 / 8 \\
5 / 8 \\
5 / 8\end{array}$ & & $\begin{array}{l}277.8 \\
363.0 \\
440.7\end{array}$ \\
\hline 1 & $\begin{array}{l}6 \\
6 \\
6\end{array}$ & & $\begin{array}{l}484 \\
731 \\
983\end{array}$ & $\begin{array}{l}25 \\
30 \\
34\end{array}$ & $\begin{array}{l}1 \\
1 \\
2\end{array}$ & $\begin{array}{r}5 \\
10 \\
1\end{array}$ & $\begin{array}{l}4 \\
4 \\
5\end{array}$ & $\begin{array}{r}0 \\
11 \\
7\end{array}$ & $\begin{array}{l}20 \\
21 \\
22\end{array}$ & $\begin{array}{l}5 / 8 \\
5 / 8 \\
5 / 8\end{array}$ & $\begin{array}{l}472.5 \\
496.1 \\
519.8\end{array}$ & $\begin{array}{l}301.8 \\
416.8 \\
498.5\end{array}$ \\
\hline 2 & $\begin{array}{l}0 \\
0 \\
0\end{array}$ & $\begin{array}{l}4000 \\
6000 \\
8000\end{array}$ & $\begin{array}{r}524 \\
792 \\
1067\end{array}$ & $\begin{array}{l}26 \\
31 \\
35\end{array}$ & $\begin{array}{l}1 \\
1 \\
2\end{array}$ & $\begin{array}{r}6 \\
11 \\
2\end{array}$ & $\begin{array}{l}4 \\
5 \\
5\end{array}$ & $\begin{array}{r}2 \\
2 \\
10\end{array}$ & $\begin{array}{l}22 \\
22 \\
24\end{array}$ & $\begin{array}{l}5 / 8 \\
5 / 8 \\
5 / 8\end{array}$ & $\begin{array}{l}542.9 \\
542.9 \\
592.2\end{array}$ & $\begin{array}{l}347.8 \\
475.9 \\
564.8\end{array}$ \\
\hline $\begin{array}{l}12 \\
12 \\
12\end{array}$ & $\begin{array}{l}6 \\
6 \\
6\end{array}$ & $\begin{array}{l}4000 \\
6000 \\
8000\end{array}$ & $\begin{array}{r}566 \\
857 \\
1158\end{array}$ & $\begin{array}{l}26 \\
32 \\
38\end{array}$ & $\begin{array}{l}1 \\
2 \\
2\end{array}$ & $\begin{array}{l}7 \\
0 \\
2\end{array}$ & $\begin{array}{l}4 \\
5 \\
6\end{array}$ & $\begin{array}{l}3 \\
4 \\
1\end{array}$ & $\begin{array}{l}23 \\
22 \\
26\end{array}$ & $\begin{array}{l}5 / 8 \\
5 / 8 \\
5 / 8\end{array}$ & $\begin{array}{l}591.7 \\
566.0 \\
668.9\end{array}$ & $\begin{array}{l}396.0 \\
537.0 \\
611.4\end{array}$ \\
\hline
\end{tabular}




\section{REINFORCED CONCRETE PILE CAPS}

Carrting Capactity of Each Pile $=30$ Tons

$f_{\mathrm{s}}=16,000 \mathrm{lb}$. per sq. in.

$f_{0}=$ less than $650 \mathrm{lb}$. per sq. in.

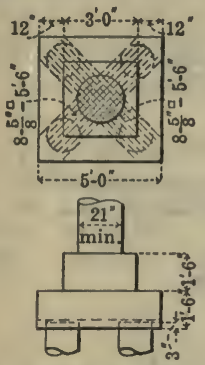

\section{Prles}

Column load $=232,000 \mathrm{lb}$.

Concrete $=1.89 \mathrm{cu}$. yd. Reinforcement $=\mathbf{1 1 8} \mathbf{l b}$.
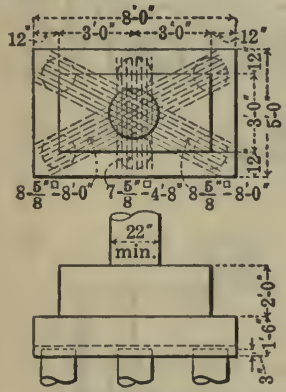

6 Piles

Column load $=346,000 \mathrm{lb}$.

Concrete $=3.55 \mathrm{cu}$. yd.

Reinforcement $=217 \mathbf{l b}$.
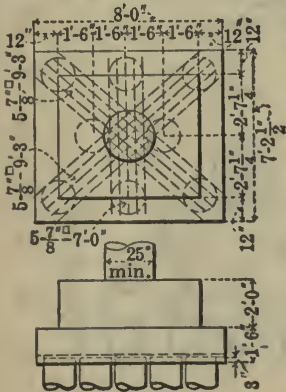

8 Pilus

Column load $=457,000 \mathrm{lb}$.

Concrete $=5.56 \mathrm{cu} . \mathrm{yd}$.

Reinforcement $=337 \mathrm{lb}$.
Punching shear $=120 \mathrm{lb}$. per sq. in.

Bond stress $=100 \mathrm{lb}$. per sq. in.
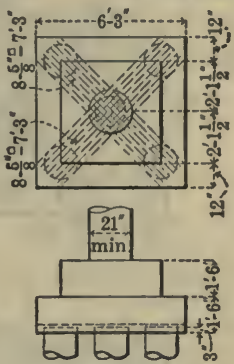

5 Piles

Column load $=287,000 \mathrm{lb}$.

Concrete $=3.17 \mathrm{cu}$. yd.

Reinforcement $=156 \mathrm{lb}$.
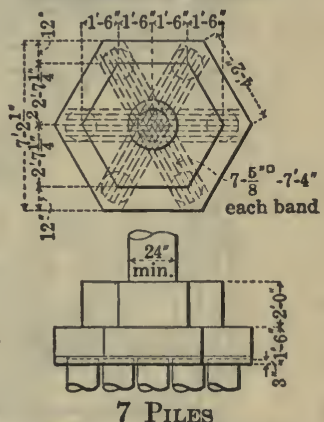

Column load $=403,000 \mathrm{lb}$.

Concrete $=42.4 \mathrm{~cm}$ d. A . $A$ Cu. Y d Reinforcement $=208 \mathrm{lb}$.

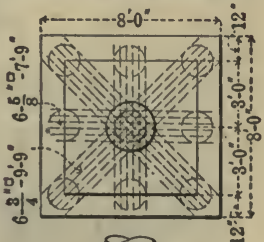

$26^{\prime \prime}$

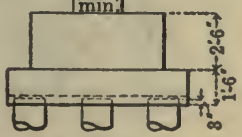

9 Pilles

Column load $=512,000 \mathrm{lb}$.

Concrete $=6.89 \mathrm{cu}$. yd.

Reinforcement $=352 \mathrm{lb}$. 


\section{REINFORCED CONCRETE PILE CAPS}

Carrying Capacity of Each Pile $=30$ Tons

$f_{\mathrm{s}}=16,000 \mathrm{lb}$. per sq. in.

Punching shear $=120 \mathrm{lb}$. per sq. in.

$f_{\mathrm{c}}=$ less than $650 \mathrm{lb}$. per sq. in.

Bond stress $=100 \mathrm{lb}$. per sq. in.
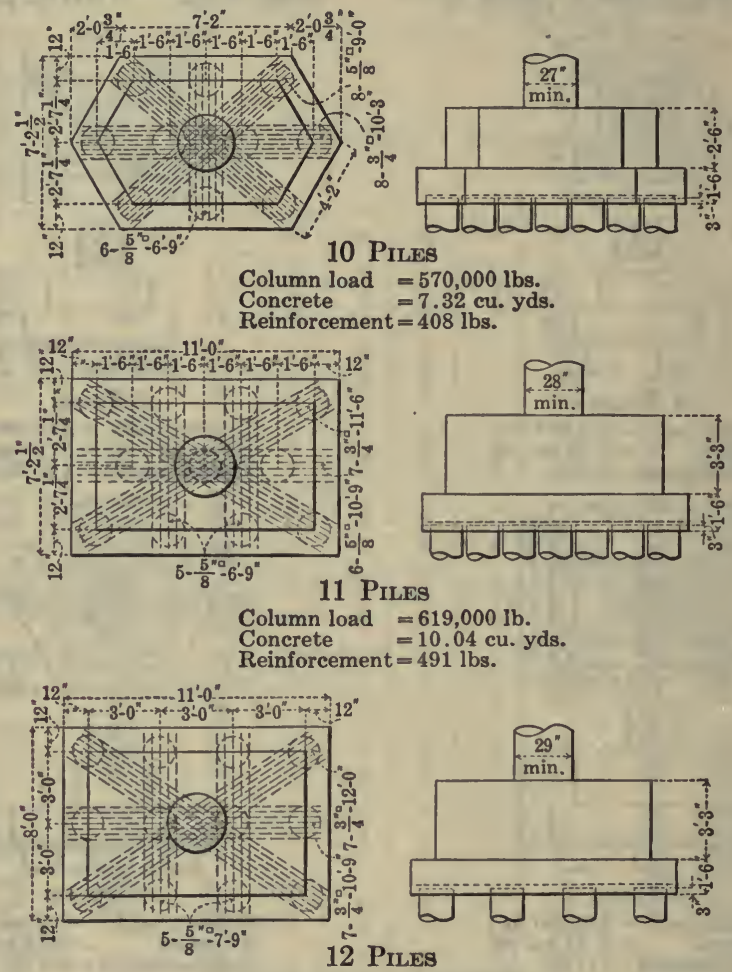

Column load $=674,000 \mathrm{lbs}$.

Concrete $=11.39 \mathrm{cu}$. yds.

Reinforcement $=577$ lbs.

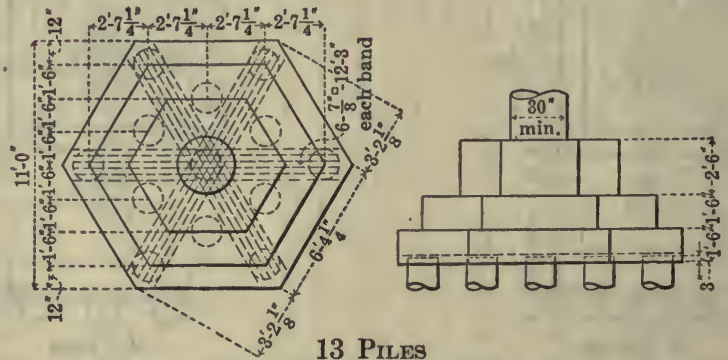

Column load $=729,000 \mathrm{lbs}$.

Concrete $=12.63 \mathrm{cu}$. yds.

Reinforcement $=583 \mathrm{lbs}$. 


\section{REINFORCED CONCRETE PILES}

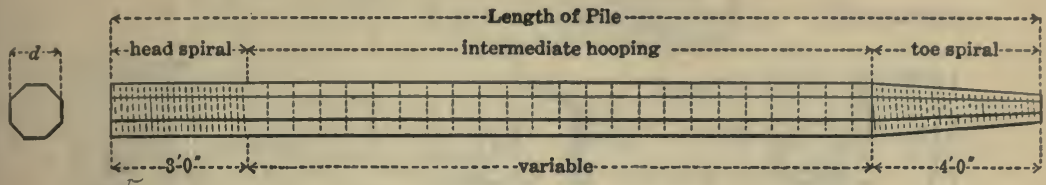

Concrete Mixture: 1: 2: 4

\begin{tabular}{|c|c|c|c|c|c|c|c|c|c|c|}
\hline \multirow{3}{*}{$\begin{array}{c}\begin{array}{c}\text { Length } \\
\text { of Pile }\end{array} \\
\text { ft. } \\
\end{array}$} & \multirow{3}{*}{\begin{tabular}{|c|}
$\begin{array}{c}\text { Diameter } \\
\text { of Pile }\end{array}$ \\
in. \\
\end{tabular}} & \multirow{2}{*}{\multicolumn{2}{|c|}{$\begin{array}{l}\text { Round Bar } \\
\text { Verticals }\end{array}$}} & \multicolumn{2}{|c|}{ HEAd SPIRAL } & \multicolumn{2}{|c|}{ Hooping } & \multicolumn{2}{|c|}{ Toe Spiral } & \multirow{3}{*}{$\begin{array}{c}\begin{array}{c}\text { Volume of } \\
\text { Concrete } \\
\text { per Pile }\end{array} \\
\text { cu. ft. }\end{array}$} \\
\hline & & & & \multirow{2}{*}{\begin{tabular}{|c|} 
Rounds \\
Size
\end{tabular}} & \multirow{2}{*}{$\begin{array}{c}\text { Pitch } \\
\text { in. }\end{array}$} & \multirow{2}{*}{$\frac{\text { Rounds }}{\text { Size }}$} & \multirow{2}{*}{\begin{tabular}{|c|} 
Spacing \\
in. \\
\end{tabular}} & \multirow{2}{*}{\begin{tabular}{|c|} 
Rounds \\
Size \\
\end{tabular}} & \multirow{2}{*}{$\begin{array}{c}\text { Pitch } \\
\text { in. }\end{array}$} & \\
\hline & & No. & Size & & & & & & & \\
\hline 14 & 14 & 6 & $5 / 8$ & $1 / 4$ & 2 & $1 / 4$ & 12 & $1 / 4$ & $11 / 2$ & 14.2 \\
\hline 16 & 14 & 6 & $5 / 8$ & $1 / 4$ & 2 & $1 / 4$ & 12 & $1 / 4$ & $11 / 2$ & 16.4 \\
\hline 18 & 14 & 6 & $5 / 8$ & $1 / 4$ & 2 & $1 / 4$ & 12 & $1 / 4$ & $11 / 2$ & 18.7 \\
\hline 20 & 14 & 6 & $5 / 8$ & $1 / 4$ & 2 & $1 / 4$ & 12 & $1 / 4$ & $11 / 2$ & 209 \\
\hline 22 & 16 & 6 & $5 / 8$ & $\frac{5}{16}$ & 2 & $1 / 4$ & 12 & $1 / 4$ & $11 / 2$ & 29.9 \\
\hline 24 & 16 & 6 & $3 / 4$ & $\frac{5}{16}$ & 2 & $1 / 4$ & 12 & $1 / 4$ & $11 / 2$ & 32.8 \\
\hline 26 & 16 & 6 & $3 / 4$ & $\frac{5}{16}$ & 2 & $1 / 4$ & 12 & $1 / 4$ & $11 / 2$ & 35.8 \\
\hline 28 & 16 & 6 & $7 / 8$ & $\frac{5}{16}$ & 2 & $1 / 4$ & 12 & $1 / 4$ & $11 / 2$ & 38.7 \\
\hline 30 & 16 & 6 & $7 / 8$ & $\frac{5}{16}$ & 2 & $1 / 4$ & 12 & $1 / 4$ & $11 / 2$ & 41.6 \\
\hline 32 & 18 & 8 & $3 / 4$ & $3 / 8$ & 2 & $3 / 8$ & 12 & $3 / 8$ & $11 / 2$ & 56.2 \\
\hline 34 & 18 & 8 & $7 / 8$ & $3 / 8$ & 2 & $3 / 8$ & 12 & $3 / 8$ & $11 / 2$ & 59.9 \\
\hline 36 & 18 & 8 & $7 / 8$ & $3 / 8$ & 2 & $3 / 8$ & 12 & $3 / 8$ & $11 / 2$ & 63.6 \\
\hline 38 & 18 & 8 & 1 & $3 / 8$ & 2 & $3 / 8$ & 12 & $3 / 8$ & $11 / 2$ & 67.4 \\
\hline 40 & 18 & 8 & 1 & $3 / 8$ & 2 & $3 / 8$ & 12 & $3 / 8$ & $11 / 2$ & 71.1 \\
\hline 42 & 20 & 8 & $11 / 8$ & $3 / 8$ & $11 / 2$ & $3 / 8$ & 12 & $3 / 8$ & $11 / 2$ & 92.3 \\
\hline 44 & 20 & 8 & $11 / 8$ & $3 / 8$ & $11 / 2$ & $3 / 8$ & 12 & $3 / 8$ & $11 / 2$ & 96.9 \\
\hline 46 & 20 & 8 & $11 / 4$ & $3 / 8$ & $11 / 2$ & $3 / 8$ & 12 & $3 / 8$ & $11 / 2$ & 101.5 \\
\hline 48 & 20 & 8 & $11 / 4$ & $3 / 8$ & $11 / 2$ & $3 / 8$ & 12 & $3 / 8$ & $11 / 2$ & 106.1 \\
\hline 50 & 20 & 8 & $11 / 4$ & $3 / 8$ & $11 / 2$ & $3 / 8$ & 12 & $3 / 8$ & $11 / 2$ & 110.7 \\
\hline 52 & 22 & 8 & $11 / 4$ & $3 / 8$ & $11 / 2$ & $3 / 8$ & 12 & $3 / 8$ & $11 / 2$ & 138.8 \\
\hline
\end{tabular}


and

Water

Pressures
EARTH AND WATER PRESSURES

$p=$ intensity of pressure at any depth $h$. $H=$ total pressure above $A-B$.

$M=$ moment at section $A-B$.

\begin{tabular}{|c|c|c|c|c|c|c|c|c|c|}
\hline \multirow{2}{*}{$h$} & \multicolumn{3}{|c|}{$\begin{array}{c}\text { EARTH HORIZONTAL } \\
w=100 \mathrm{lb} .\end{array}$} & \multicolumn{3}{|c|}{$\begin{array}{l}\text { EARTH SURCHARGED } \\
w=100 \mathrm{lb} .\end{array}$} & \multicolumn{3}{|c|}{$\begin{array}{c}\text { WATER } \\
w=62.5 \mathrm{lb} .\end{array}$} \\
\hline & $\begin{array}{c}p= \\
0.2948 w h\end{array}$ & $\begin{array}{c}H= \\
0.1474 w h^{2}\end{array}$ & $\begin{array}{c}M= \\
0.5896 w h^{3}\end{array}$ & $\begin{array}{c}p= \\
0.7034 w h\end{array}$ & $\begin{array}{c}H \\
0.3517 w h^{2}\end{array}$ & $\begin{array}{c}M= \\
1.4068 w h^{3}\end{array}$ & $\underset{w h}{p=}$ & $\begin{array}{l}H= \\
0.5 p h\end{array}$ & $\begin{array}{l}M= \\
4 H h\end{array}$ \\
\hline $\mathrm{ft}$. & lb. & lb. & in. lb. & lb. & lb. & in. lb. & lb. & Ib. & in. $1 \mathrm{~b}$. \\
\hline $\begin{array}{l}1 \\
2 \\
3 \\
4 \\
5\end{array}$ & $\begin{array}{r}29 \\
59 \\
88 \\
118 \\
147\end{array}$ & $\begin{array}{r}15 \\
59 \\
132 \\
236 \\
368\end{array}$ & $\begin{array}{r}59 \\
472 \\
1,592 \\
3,773 \\
7,370\end{array}$ & $\begin{array}{r}70 \\
141 \\
211 \\
281 \\
352\end{array}$ & $\begin{array}{r}35 \\
141 \\
317 \\
563 \\
879\end{array}$ & $\begin{array}{r}141 \\
1,125 \\
3,798 \\
9,003 \\
17,584\end{array}$ & $\begin{array}{r}63 \\
125 \\
188 \\
250 \\
313\end{array}$ & $\begin{array}{r}31 \\
125 \\
281 \\
500 \\
781\end{array}$ & $\begin{array}{r}125 \\
1,000 \\
3,375 \\
8,000 \\
15,625\end{array}$ \\
\hline $\begin{array}{r}6 \\
7 \\
8 \\
9 \\
10\end{array}$ & $\begin{array}{l}177 \\
206 \\
236 \\
265 \\
295\end{array}$ & $\begin{array}{r}531 \\
721 \\
944 \\
1,193 \\
1,475\end{array}$ & $\begin{array}{l}12,735 \\
20,223 \\
30,187 \\
42,982 \\
58,960\end{array}$ & $\begin{array}{l}422 \\
492 \\
563 \\
633 \\
703\end{array}$ & $\begin{array}{l}1,266 \\
1,723 \\
2,251 \\
2,849 \\
3,517\end{array}$ & $\begin{array}{r}30,385 \\
48,251 \\
72,025 \\
102,551 \\
140,673\end{array}$ & $\begin{array}{l}375 \\
438 \\
500 \\
563 \\
625\end{array}$ & $\begin{array}{l}1,125 \\
1,531 \\
2,000 \\
2,531 \\
3,125\end{array}$ & $\begin{array}{r}27,000 \\
42,87 j \\
64,000 \\
91,125 \\
125,000\end{array}$ \\
\hline $\begin{array}{l}11 \\
12 \\
13 \\
14 \\
15\end{array}$ & $\begin{array}{l}324 \\
354 \\
383 \\
413 \\
442\end{array}$ & $\begin{array}{l}1,782 \\
2,124 \\
2,490 \\
2,891 \\
3,315\end{array}$ & $\begin{array}{r}78,476 \\
101,883 \\
129,535 \\
161,786 \\
198,990\end{array}$ & $\begin{array}{r}774 \\
844 \\
914 \\
985 \\
1,055\end{array}$ & $\begin{array}{l}4,255 \\
5,064 \\
5,943 \\
6,893 \\
7,913\end{array}$ & $\begin{array}{l}187,236 \\
243,084 \\
309,060 \\
386,008 \\
474,773\end{array}$ & $\begin{array}{l}688 \\
750 \\
813 \\
875 \\
938\end{array}$ & $\begin{array}{l}3,781 \\
4,500 \\
5,281 \\
6,125 \\
7,031\end{array}$ & $\begin{array}{l}166,375 \\
216,000 \\
274,625 \\
343,000 \\
421,875\end{array}$ \\
\hline $\begin{array}{l}16 \\
17 \\
18 \\
19 \\
20\end{array}$ & $\begin{array}{l}472 \\
501 \\
531 \\
560 \\
590\end{array}$ & $\begin{array}{l}3,77.6 \\
4,259 \\
4,779 \\
5,320 \\
5,900\end{array}$ & $\begin{array}{l}241,500 \\
289,670 \\
343,855 \\
404,407 \\
471,680\end{array}$ & $\begin{array}{l}1,125 \\
1,196 \\
1,266 \\
1,336 \\
1,407\end{array}$ & $\begin{array}{r}9,003 \\
10,164 \\
11,395 \\
12,696 \\
14,067\end{array}$ & $\begin{array}{r}576,199 \\
691,129 \\
820,408 \\
964,879 \\
1,125,388\end{array}$ & $\begin{array}{l}1,000 \\
1,063 \\
1,125 \\
1,188 \\
1,250\end{array}$ & $\begin{array}{r}8,000 \\
9,031 \\
10,125 \\
11,281 \\
12,500\end{array}$ & $\begin{array}{r}512,000 \\
614,125 \\
729,000 \\
857,375 \\
1,000,000\end{array}$ \\
\hline $\begin{array}{l}21 \\
22 \\
23 \\
24 \\
25\end{array}$ & $\begin{array}{l}619 \\
649 \\
678 \\
708 \\
737\end{array}$ & $\begin{array}{l}6,500 \\
7,139 \\
7,797 \\
8,496 \\
9,213\end{array}$ & $\begin{array}{l}546,029 \\
627,806 \\
717,366 \\
815,063 \\
921,250\end{array}$ & $\begin{array}{l}1,477 \\
1,547 \\
1,618 \\
1,688 \\
1,758\end{array}$ & $\begin{array}{l}15,509 \\
17,021 \\
18,604 \\
20,257 \\
21,980\end{array}$ & $\begin{array}{l}1,302,777 \\
1,497,891 \\
1,711,574 \\
1,944,670 \\
2,198,023\end{array}$ & $\begin{array}{l}1,313 \\
1,375 \\
1,438 \\
1,500 \\
1,563\end{array}$ & $\begin{array}{l}13,781 \\
15,125 \\
16,531 \\
18,000 \\
19,531\end{array}$ & $\begin{array}{l}1,157,625 \\
1,331,000 \\
1,520,875 \\
1,728,000 \\
1,953,125\end{array}$ \\
\hline $\begin{array}{l}26 \\
27 \\
28 \\
29 \\
30\end{array}$ & $\begin{array}{l}766 \\
796 \\
825 \\
855 \\
884\end{array}$ & $\begin{array}{r}9,958 \\
10,746 \\
11,550 \\
12,398 \\
13,260\end{array}$ & $\begin{array}{l}1,036,280 \\
1,160,509 \\
1,294,290 \\
1,437,975 \\
1,591,920\end{array}$ & $\begin{array}{l}1,829 \\
1,899 \\
1,969 \\
2,040 \\
2,110\end{array}$ & $\begin{array}{l}23,774 \\
25,638 \\
27,572 \\
29,577 \\
31,652\end{array}$ & $\begin{array}{l}2,472,479 \\
2,768,876 \\
3,088,064 \\
3,430,885 \\
3,798,184\end{array}$ & $\begin{array}{l}1,625 \\
1,688 \\
1,750 \\
1,813 \\
1,875\end{array}$ & $\begin{array}{l}21,125 \\
22,781 \\
24,500 \\
26,281 \\
28,125\end{array}$ & $\begin{array}{l}2,197,000 \\
2,460,375 \\
2,744,000 \\
3,048,625 \\
3,375,000\end{array}$ \\
\hline $\begin{array}{l}31 \\
32 \\
33 \\
34 \\
35\end{array}$ & $\begin{array}{r}914 \\
943 \\
973 \\
1,002 \\
1,032 \\
\end{array}$ & $\begin{array}{l}14,167 \\
15,088 \\
16,045 \\
17,034 \\
18,060 \\
\end{array}$ & $\begin{array}{l}1,756,477 \\
1,932,001 \\
2,118,846 \\
2,317,364 \\
2,527,910 \\
\end{array}$ & $\begin{array}{l}2,180 \\
2,251 \\
2,321 \\
2,391 \\
2,462 \\
\end{array}$ & $\begin{array}{l}33,797 \\
36,012 \\
38,298 \\
40,655 \\
43,081\end{array}$ & $\begin{array}{l}4,190,803 \\
4,609,588 \\
5,055,382 \\
5,529,030 \\
6,031,375\end{array}$ & $\begin{array}{l}1,938 \\
2,000 \\
2,063 \\
2,125 \\
2,188 \\
\end{array}$ & $\begin{array}{l}30,031 \\
32,000 \\
34,031 \\
36,125 \\
38,281 \\
\end{array}$ & $\begin{array}{l}3,723,875 \\
4,096,000 \\
4,492,125 \\
4,913,000 \\
5,359,375 \\
\end{array}$ \\
\hline
\end{tabular}

Angle of repose $=33^{\circ}$ 


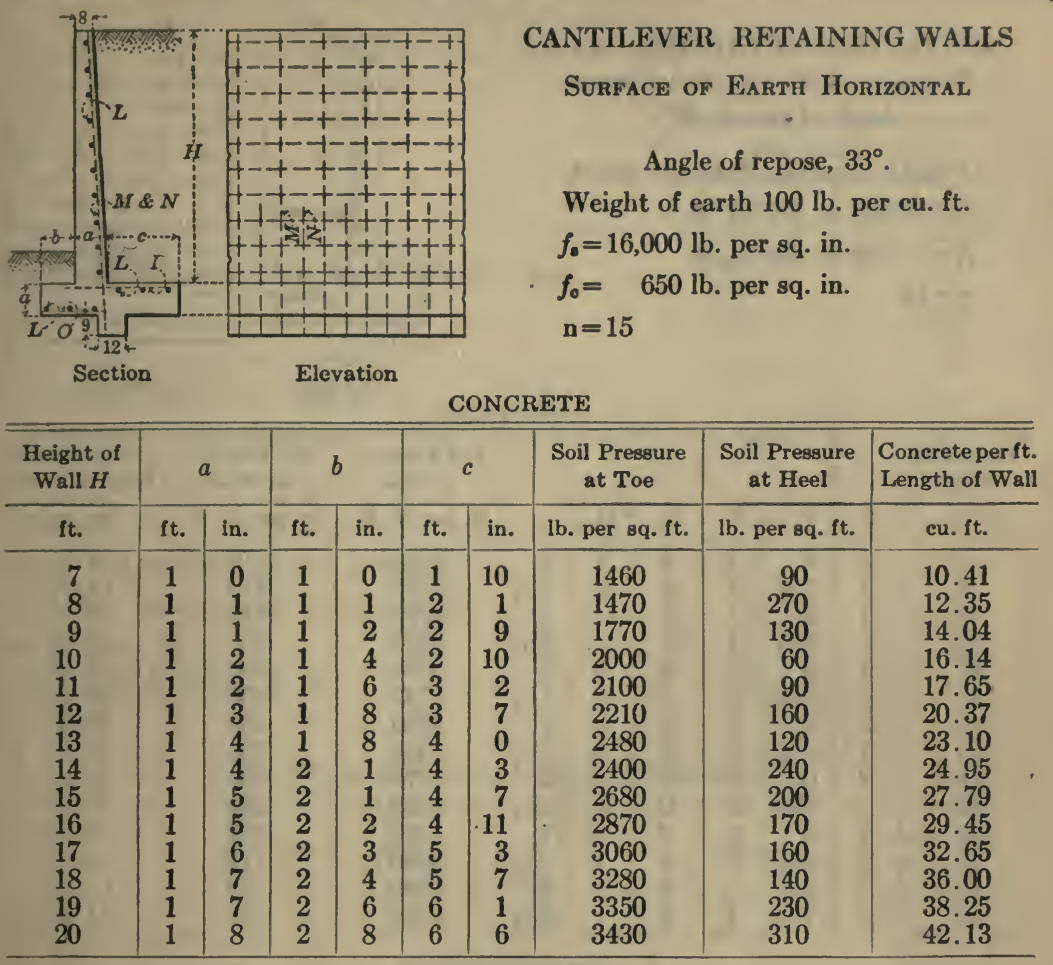

\section{REINFORCEMENT}

Bars in all Cases of Round Section

\begin{tabular}{|c|c|c|c|c|c|c|c|c|c|c|c|c|c|c|c|c|c|c|c|}
\hline \multirow[b]{2}{*}{ 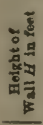 } & \multicolumn{3}{|c|}{$M$ BARS } & \multicolumn{4}{|c|}{$N$ BARs } & \multicolumn{4}{|c|}{$O$ BARB } & \multicolumn{4}{|c|}{$P$ BARS } & \multicolumn{3}{|c|}{$L$ BARS } & \multirow{2}{*}{ 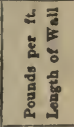 } \\
\hline & 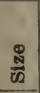 & 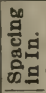 & ఏ్ & $\stackrel{ \pm}{*}$ & 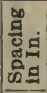 & 명 & 궁 & 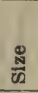 & 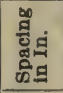 & 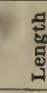 & & $\frac{\Perp}{\pi}$ & 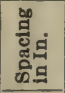 & $\begin{array}{l}\text { 我 } \\
\text { Фี } \\
\Phi\end{array}$ & 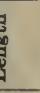 & $\begin{array}{l}0 \\
2 \\
\end{array}$ & 츨 & 咅 & \\
\hline 7 & $1 / 2$ & 24 & $8^{\prime}$ & $1 / 2$ & 24 & 8 & & 6 & 12 & ? & & 6 & 12 & 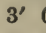 & & 12 & , & & 14.4 \\
\hline 8 & $1 / 2$ & 24 & $9^{\prime} 6^{\prime \prime}$ & $1 / 2$ & 24 & & & & & . & & & 12 & : & & & & & \\
\hline 9 & $1 / 2$ & 17 & $10^{\prime} 6^{\prime \prime}$ & 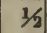 & 17 & $4^{\prime}$ & 9 & & 1 & & & & $111 / 2$ & & & & & & 1. \\
\hline 10 & $8 / 8$ & 21 & 9 & 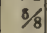 & 21 & $5^{\prime}$ & & & & & & & & & & & & & 6.8 \\
\hline 11 & $5 / 8$ & 16 & 8 & $5 / 8$ & 16 & $5^{\prime}$ & & & $71 / 2$ & $2^{\prime}$ & 9 & & 8 & $4^{\prime}$ & & & & & 2.8 \\
\hline 18 & $8 / 4$ & 20 & a & $3 / 4$ & 20 & $6^{\prime}$ & & & 11 & $3^{\prime}$ & & & 1 & ' & & & & & 1.8 \\
\hline 18 & $3 / 4$ & 16 & $15^{\prime} 3$ & & 16 & 0 & & & $101 / 2$ & $3^{\prime}$ & & & 1 & ' & & & & & 3.4 \\
\hline 14 & $3 / 4$ & 13 & $16^{\prime} 3$ & 8 & 13 & $6^{\prime}$ & & & 11 & $4^{\prime}$ & 0 & $3 /$ & 1 & $6^{\prime}$ & & & & & 5.4 \\
\hline 16 & $7 / 8$ & 1 & 6 & 7 & 16 & $7^{\prime}$ & & & 10 & $4^{\prime}$ & 0 & 7 & 11 & $7^{\prime}$ & 6 & & & & 8.9 \\
\hline 16 & $2 / 8$ & 1 & $18^{\prime} 6^{\prime \prime}$ & $7 / 8$ & 18 & $7^{\prime}$ & & & 9 & $4^{\prime}$ & 3 & 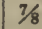 & 91 & $7^{\prime}$ & & & & & 5.2 \\
\hline 1 & & 1 & $19^{\prime} 6^{\prime \prime}$ & 1 & 1 & $8^{\prime}$ & & & 81 & $4^{\prime}$ & 3 & 7 & & $8^{\prime}$ & 0 & & & & 2.2 \\
\hline 1 & & 1 & $20^{\prime} 6^{\prime \prime}$ & & 1 & $8^{\prime}$ & 3 & & 73 & $4^{\prime}$ & 3 & & & $8^{\prime}$ & & & & & 9.8 \\
\hline 15 & 13 & 14 & $21^{\prime} 6^{\prime \prime}$ & & 14 & $8^{\prime}$ & & & & $5^{\prime}$ & $0^{n}$ & 1 & 172 & $9^{\prime}$ & & 33 & & & 4.7 \\
\hline 20 & $11 / 8$ & 13 & $22^{\prime} 6^{\prime \prime}$ & & 13 & $9^{\prime}$ & $0^{n}$ & & $81 / 2$ & $5^{\prime}$ & $0^{\prime \prime}$ & , & 7 & $9^{\prime}$ & $9^{n}$ & 34 & & 12 & 192.9 \\
\hline
\end{tabular}

Hooks are required at lower end of $M$ and $N$ bars for walls over 11' $0^{\prime \prime}$ in height. 
CANTILEVER RETAINING WALLS

Surface of Earth Surcharged

Angle of repose, $33^{\circ}$.

Weight of earth $100 \mathrm{lb}$. per cu. $\mathrm{ft}$.

$f_{\mathrm{s}}=16,000 \mathrm{lb}$. per sq. in.

$f_{\mathrm{c}}=650 \mathrm{lb}$. per sq. in.

$n=15$

CONCRETE

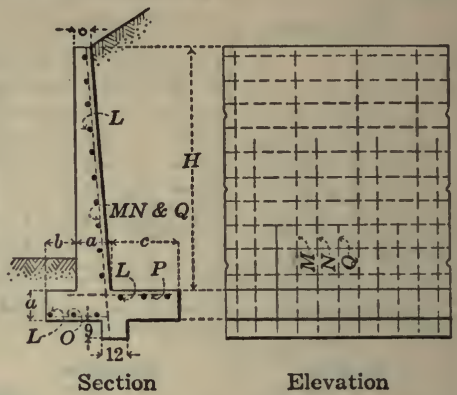

\begin{tabular}{c|c|c|c|c|c|c|c|c|c|c}
\hline $\begin{array}{c}\text { Height of } \\
\text { Wall } H\end{array}$ & \multicolumn{2}{|c|}{$a$} & \multicolumn{2}{|c|}{$b$} & \multicolumn{2}{|c|}{$c$} & $\begin{array}{c}\text { Soil Pressure } \\
\text { at Toe }\end{array}$ & $\begin{array}{c}\text { Soil Pressure } \\
\text { at Heel }\end{array}$ & $\begin{array}{c}\text { Concrete perft. } \\
\text { Length of Wall }\end{array}$ \\
\cline { 1 - 5 } ft. & ft. & in. & ft. & in. & ft. & in. & lb. per sq. ft. & lb. per sq. ft. & cu. ft. \\
\hline 7 & 1 & 0 & 1 & 4 & 2 & 8 & 2230 & 270 & 11.55 \\
8 & 1 & $11 / 2$ & 1 & 6 & 3 & $71 / 2$ & 2370 & 510 & 14.79 \\
9 & 1 & 3 & 1 & 8 & 4 & 1 & 2700 & 540 & 18.12 \\
10 & 1 & $41 / 2$ & 1 & 10 & 4 & $61 / 2$ & 3020 & 580 & 21.61 \\
11 & 1 & 6 & 2 & 0 & 4 & 8 & 3300 & 640 & 24.90 \\
12 & 1 & $71 / 2$ & 2 & 3 & 5 & $11 / 2$ & 3730 & 550 & 29.12 \\
13 & 1 & 9 & 2 & 5 & 5 & 7 & 4060 & 580 & 33.50 \\
14 & 1 & $101 / 2$ & 2 & 8 & 5 & $91 / 2$ & 4340 & 620 & 37.90 \\
15 & 2 & 0 & 2 & 10 & 6 & 2 & 4770 & 550 & 42.75 \\
16 & 2 & $11 / 2$ & 3 & 0 & 6 & $51 / 2$ & 5170 & 510 & 47.70 \\
17 & 2 & 3 & 3 & 2 & 6 & 11 & 5490 & 550 & 53.28 \\
18 & 2 & $41 / 2$ & 3 & 4 & 7 & $21 / 2$ & 5930 & 470 & 58.85 \\
19 & 2 & 6 & 3 & 8 & 7 & 6 & 6090 & 550 & 65.05 \\
20 & 2 & $71 / 2$ & 4 & 0 & 7 & $81 / 2$ & 6330 & 630 & 71.30 \\
\hline
\end{tabular}

REINFORCEMENT

Bars in all Cases of Round Section

\begin{tabular}{|c|c|c|c|c|c|c|c|c|c|c|c|c|c|c|c|c|c|c|c|}
\hline \multirow[b]{2}{*}{ 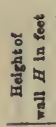 } & \multicolumn{3}{|c|}{$M$ BARS } & \multicolumn{3}{|c|}{$N$ BARS } & \multicolumn{3}{|c|}{$Q$ BARS } & \multicolumn{3}{|c|}{$O$ BARS } & \multicolumn{3}{|c|}{$P$ BARS } & \multicolumn{3}{|c|}{$L \mathrm{BARS}$} & \multirow{2}{*}{ 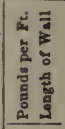 } \\
\hline & 쁌 & 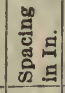 & 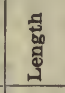 & $\frac{\mathbb{N}}{\pi}$ & 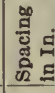 & 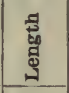 & 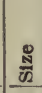 & 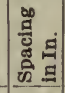 & 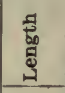 & ॠँ & 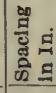 & 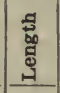 & 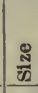 & 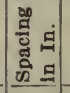 & 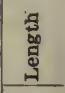 & 字 & $\frac{\mathbb{N}}{\pi}$ & : & \\
\hline 7 & $1 / 2$ & 14 & $8^{\prime} 6^{\prime \prime}$ & $1 / 2$ & 14 & $4^{\prime} 0^{\prime \prime}$ & & & & $8 / 8$ & $91 / 2$ & $2^{\prime} 6^{\prime \prime}$ & $8 / 8$ & 11 & $4^{\prime} 0^{\prime \prime}$ & 13 & $1 / 2$ & 12 & 18.9 \\
\hline 8 & & 17 & & & 17 & $4^{\prime}$ & & & & & 8 & $2^{\prime} 9^{\prime \prime}$ & $8 / 8$ & 8 & $4^{\prime} 9^{\prime \prime}$ & 14 & $1 / 2$ & 12 & 4 \\
\hline 9 & $5 / 8$ & 13 & $10^{\prime} 9^{\prime \prime}$ & 118 & 13 & $5^{\prime} 0^{\prime \prime}$ & & & & $1 / 2$ & 12 & $3^{\prime} 3^{\prime \prime}$ & $1 / 2$ & 10 & $5^{\prime} 9^{\prime \prime}$ & 17 & $1 / 2$ & 12 & 33.8 \\
\hline 10 & $8 / 4$ & 16 & $12^{\prime} 6^{\prime \prime}$ & $8 / 4$ & 16 & $6^{\prime} 0^{\prime \prime}$ & & & & $1 / 2$ & $81 / 2$ & $3^{\prime} 6^{\prime \prime}$ & $1 / 2$ & $81 / 2$ & $6^{\prime} 3^{\prime \prime}$ & 19 & $1 / 2$ & 2 & 43.8 \\
\hline 11 & $8 / 4$ & $191 / 2$ & $13^{\prime} 6^{\prime \prime}$ & $1 / 4$ & $191 / 2$ & $7^{\prime} 6^{\prime \prime}$ & $8 / 4$ & $191 / 2$ & $5^{\prime} 6^{\prime \prime}$ & $5 / 8$ & 12 & $4^{\prime} 0^{\prime \prime}$ & $1 / 2$ & $81 / 2$ & $6^{\prime} 3^{\prime \prime}$ & 21 & $1 / 2$ & 12 & 49.5 \\
\hline 12 & $8 / 4$ & $161 / 2$ & $14^{\prime} 9^{\prime \prime}$ & $1 / 4$ & $161 / 2$ & $7^{\prime} 9^{\prime \prime}$ & $8 / 4$ & $161 / 2$ & $5^{\prime} 9^{\prime \prime}$ & $5 / 8$ & 9 & $4^{\prime} 3^{\prime \prime}$ & $5 / 8$ & 11 & $7^{\prime} 3^{\prime \prime}$ & 23 & $1 / 2$ & 12 & 61. \\
\hline 13 & $7 / 8$ & 19 & $15^{\prime} 9^{\prime \prime}$ & $7 / 8$ & $191 / 2$ & $8^{\prime} 6^{\prime \prime}$ & $7 / 8$ & $191 / 2$ & $6^{\prime} 3^{\prime \prime}$ & $5 / 8$ & 8 & $4^{\prime} 6^{\prime \prime}$ & $5 / 8$ & 8 & $7^{\prime}$ & 24 & $5 / 8$ & 12 & 85.0 \\
\hline 14 & $7 / 8$ & $161 / 2$ & $17^{\prime} 0^{\prime \prime}$ & $17 / 8$ & $161 / 2$ & $\begin{array}{ll}9^{\prime} & 0^{\prime \prime}\end{array}$ & $7 / 8$ & $161 / 2$ & $6^{\prime} 6^{\prime \prime}$ & $5 / 8$ & $51 / 2$ & $4^{\prime} 9^{\prime \prime}$ & $5 / 8$ & $71 / 2$ & $7^{\prime} 9^{\prime \prime}$ & 25 & $5 / 8$ & 12 & 100.8 \\
\hline 15 & 1 & 18 & $18^{\prime} 0^{\prime \prime}$ & & 18 & $9^{\prime} 6^{\prime \prime}$ & & 18 & $6^{\prime} 9^{\prime \prime}$ & $3 / 4$ & 8 & $\mid \begin{array}{ll}5^{\prime} & 3^{\prime \prime}\end{array}$ & $8 / 4$ & 9 & $8^{\prime} 9^{\prime \prime}$ & 28 & $5 / 8$ & 12 & 122.4 \\
\hline 16 & 1 & $161 / 2$ & $19^{\prime} 3^{\prime \prime}$ & & $161 / 2$ & $10^{\prime} 0^{\prime \prime}$ & & $161 / 2$ & $7^{\prime} 0^{\prime \prime}$ & $8 / 4$ & 7 & $\begin{array}{ll}5^{\prime} & 6^{\prime \prime}\end{array}$ & $8 / 4$ & 8 & $\begin{array}{llll}9^{\prime} & 0^{\prime \prime}\end{array}$ & 30 & $5 / 8$ & 12 & 139.2 \\
\hline 17 & $11 / 8$ & 18 & $20^{\prime} 3^{\prime \prime}$ & $11 / 8$ & 18 & $10^{\prime} 6^{\prime \prime}$ & $11 / 8$ & 818 & $7^{\prime} 6^{\prime \prime}$ & $8 / 4$ & 6 & $5^{\prime} 9$ & $8 / 4$ & $\gamma$ & $9^{\prime} 6^{\prime \prime}$ & 31 & $5 / 8$ & 12 & 164.0 \\
\hline 18 & $11 / 8$ & $161 / 2$ & $21^{\prime} 6^{\prime \prime}$ & $11 / 8$ & $161 / 2$ & $11^{\prime}$ & $11 / 8$ & $8161 / 2$ & $7^{\prime} 9^{\prime \prime}$ & $7 / 8$ & 8 & $\left|\begin{array}{ll}6^{\prime} & 3^{\prime \prime}\end{array}\right|$ & $7 / 8$ & $81 / 21$ & $10^{\prime} 0$ & 34 & $5 / 8$ & 12 & 185.8 \\
\hline 15 & $11 / 4$ & 18 & $22^{\prime} 6^{\prime \prime}$ & $11 / 4$ & 18 & $11^{\prime} 9^{\prime \prime}$ & $11 / 4$ & 18 & $8^{\prime} 3^{\prime \prime}$ & $7 / 8$ & $61 / 2$ & $6^{\prime} 6$ & $7 / 8$ & 8 & $10^{\prime} 6^{\prime \prime}$ & 35 & $5 / 8$ & 12 & 215.7 \\
\hline 20 & $11 / 4$ & $161 / 2$ & $23^{\prime} 9^{\prime \prime}$ & 1 & $161 / 2$ & $12^{\prime} 3^{\prime \prime}$ & $11 / 4$ & $161 / 2$ & $8^{\prime} 6^{\prime \prime}$ & 7 & 6 & $7^{\prime} 0^{\prime \prime}$ & $7 / 8$ & $71 / 21$ & $10^{\prime} 6^{\prime \prime}$ & 37 & $5 / 8$ & 12 & 241.0 \\
\hline
\end{tabular}

Hooks are required at lower end of $M, N$ and $Q$ bars for walls over $9^{\prime} 0^{\prime \prime}$ in height. 


\section{FOUNDATIONS}

Bearing Capacity of Solls

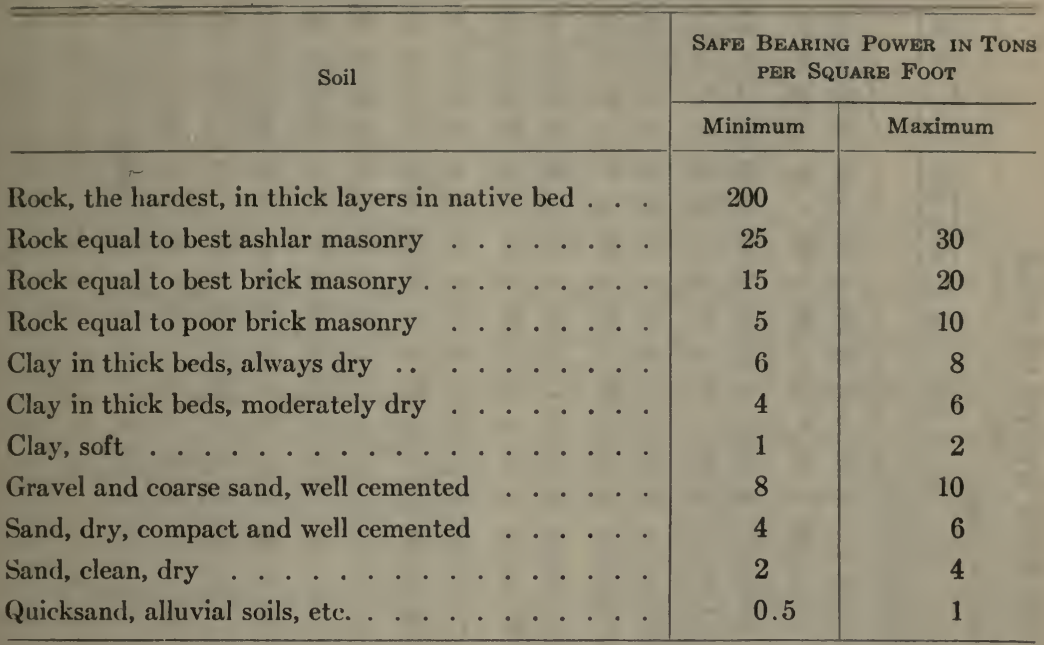

\section{Coefficien's and Angles of Friction}

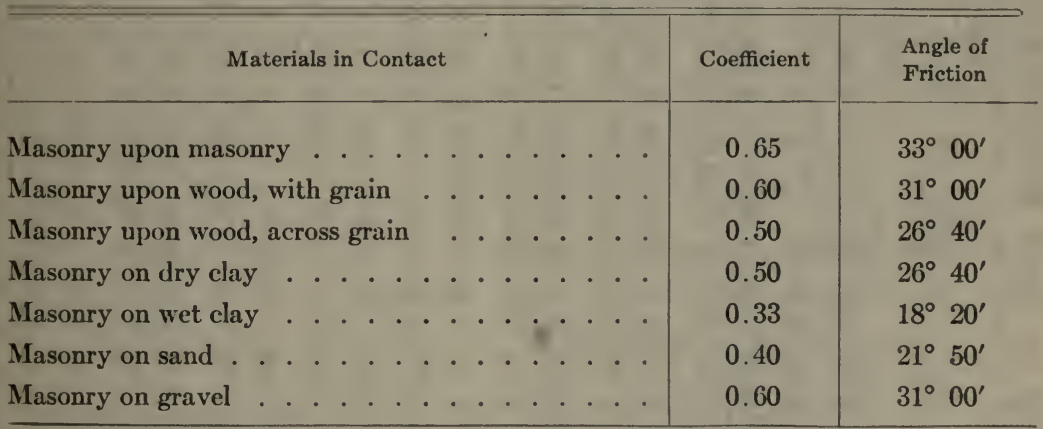

From "A Treatise on Masonry Construction," by Prof. Ira O. Baker. 


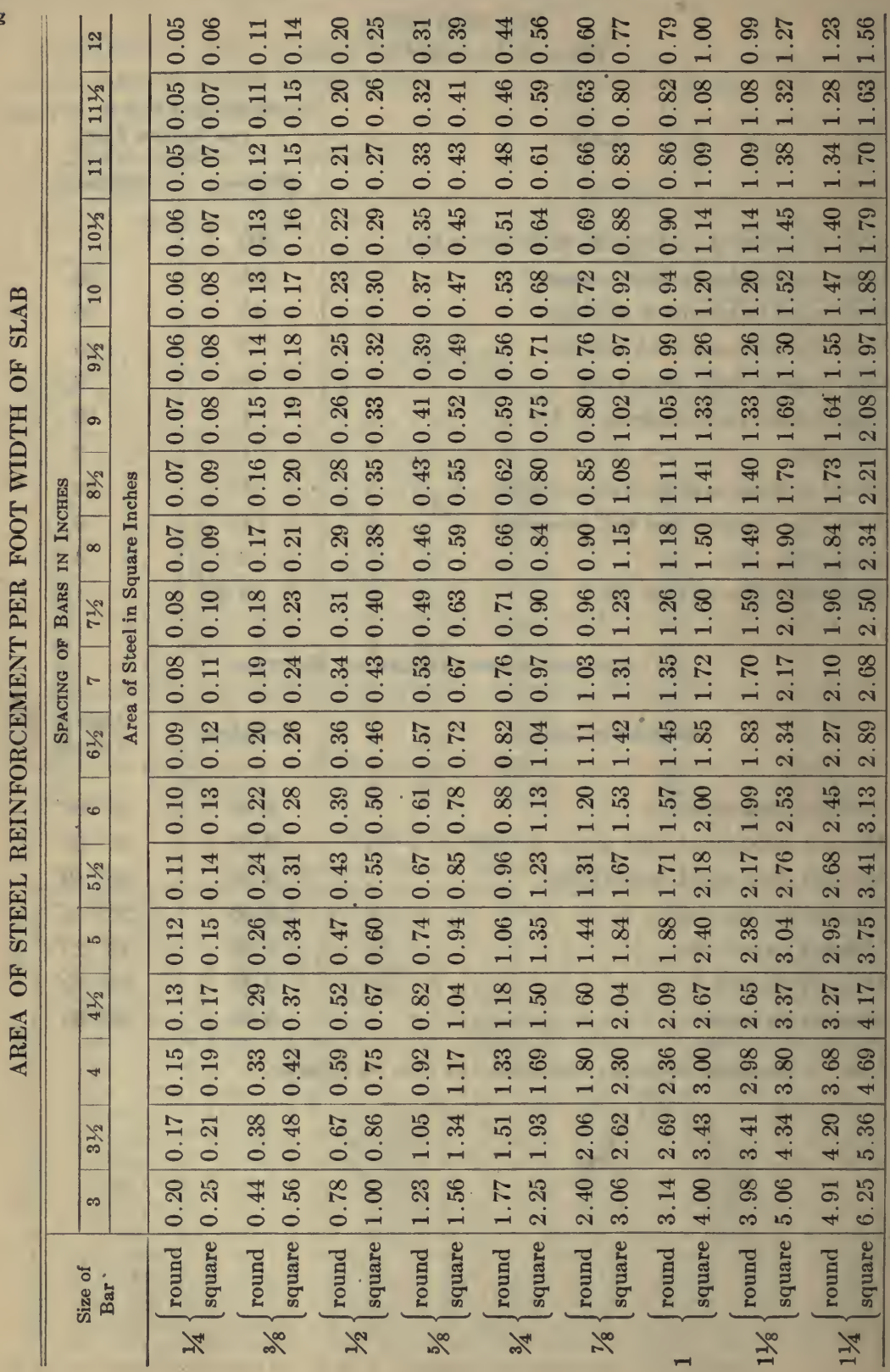


VERTICAL STEEL REQUIRED FOR GIVEN PERCENTAGES OF ROUND COLUMN CORE AREAS

\begin{tabular}{|c|c|c|c|c|c|c|c|c|c|c|c|c|c|c|}
\hline \multirow{2}{*}{$\begin{array}{l}\text { Core } \\
\text { Dia. }\end{array}$} & \multirow{2}{*}{$\begin{array}{l}\text { Core } \\
\text { Area }\end{array}$} & \multicolumn{13}{|c|}{ PERCEnt of Core AREa } \\
\hline & & 0.5 & 0.75 & 1.0 & 1.5 & 2.0 & 2.5 & 3.0 & 3.5 & 4.0 & 5.0 & 60 & 7.0 & 8.0 \\
\hline in. & sq. in. & \multicolumn{13}{|c|}{ Area of Vertical Steel in Square Inches } \\
\hline 12 & 113 & 0.57 & 0.85 & 1.13 & 1.70 & 2.26 & 2.83 & 3.39 & 3.96 & $6 \quad 4.52$ & 5.65 & 6.79 & 7.92 & 9.05 \\
\hline 13 & 133 & 0.66 & 1.00 & 1.33 & 1.99 & 2.65 & 3.32 & 3.98 & 4.65 & 5.31 & 6.64 & 7.96 & 9.29 & 10.62 \\
\hline 14 & 154 & 0.77 & 1.15 & 1.54 & 2.31 & 3.08 & 3.85 & 4.62 & 5.39 & 6.16 & 7.70 & 9.24 & 10.78 & 12.32 \\
\hline 15 & 177 & 0.88 & 1.33 & 1.77 & 2.65 & 3.53 & 4.42 & 5.30 & 6.19 & $\begin{array}{l}9.07 \\
\end{array}$ & 8.84 & 10.60 & 12.37 & 14.14 \\
\hline 16 & 201 & 1.01 & 1.51 & 2.01 & 3.02 & 4.02 & 5.03 & 6.03 & 7.04 & 8.04 & 10.05 & 12.06 & 14.07 & 16.08 \\
\hline 17 & 227 & 1.13 & 1.70 & 2.27 & 3.40 & 4.54 & 5.67 & 6.81 & 7.94 & 9.08 & 11.35 & 13.62 & 15.89 & 18.16 \\
\hline 18 & 254 & 1.27 & 1.91 & 2.54 & 3.82 & 5.09 & 6.36 & 7.63 & 8.91 & 10.18 & 12.72 & 15.27 & 17.81 & 20 \\
\hline 19 & 284 & 1.42 & 2.13 & 2.84 & 4.25 & 5.67 & 7.09 & 8.51 & 9.92 & 11.34 & 14.18 & 17.01 & 19.85 & 22.68 \\
\hline 20 & 314 & 1.57 & 2.36 & 3.14 & 4.71 & 6.28 & 7.85 & 9.42 & 11.00 & 12.57 & 15.71 & 18.85 & 21.99 & 25.13 \\
\hline 21 & 346 & 1.73 & 2.60 & 3.46 & 5.20 & 6.93 & 8.66 & 10.39 & 12.12 & 13.85 & 17.32 & 20.78 & 24.25 & 27 \\
\hline 22 & 380 & 1.90 & 2.85 & 3.80 & 5.70 & 7.60 & 9.50 & 11.40 & 13.30 & 15.21 & 19.01 & 22.81 & 26.61 & 30.41 \\
\hline 23 & 415 & 2.08 & 3.12 & 4.15 & 6.23 & 8.31 & 10.39 & 12.46 & 14.54 & 16.62 & 20.77 & 24.93 & 29.08 & 33.24 \\
\hline 24 & 452 & 2.26 & 3.39 & 4.52 & 6.79 & 9.05 & 11.31 & 13.57 & 15.83 & 18.10 & 22.62 & 27.14 & 31.67 & 36. \\
\hline 25 & 491 & 2.45 & 3.68 & 4.91 & 7.36 & 9.82 & 12.27 & 14.73 & 17.18 & \begin{tabular}{|l|l|}
3 & 19.63
\end{tabular} & 24.54 & 29.45 & 34.36 & 39 \\
\hline 26 & 531 & 2.65 & 3.98 & 5.31 & 7.96 & 10.62 & 13.27 & 15.93 & 18.58 & 21.24 & 26.55 & 31.86 & 37.17 & 42.47 \\
\hline 27 & 573 & 2.86 & 4.29 & 5.73 & 8.59 & 11.45 & 14.31 & 17.18 & 20.04 & 22.90 & 28.63 & 34.35 & 40.08 & 45 \\
\hline 28 & 616 & 3.08 & 4.62 & 6.16 & 9.24 & 12.32 & 15.39 & 18.47 & 21.55 & 24.63 & 30.79 & 36.95 & 43.10 & \\
\hline 29 & 661 & 3.30 & 4.95 & 6.61 & 9.91 & 13.21 & 16.51 & 19.82 & 23.12 & 26.42 & 33.03 & 39.63 & 46.24 & 52.84 \\
\hline 30 & 707 & 3.53 & 5.30 & 7.07 & 10.60 & 14.14 & 17.67 & 21.21 & 24.74 & 28.27 & 35.34 & 42.41 & 49.48 & 56.55 \\
\hline 31 & 755 & 3.77 & 5.66 & 7.55 & 11.32 & 15.10 & 18.87 & 22.64 & 26.42 & 30.19 & 37.74 & 45.29 & 52.83 & 60 \\
\hline 32 & 804 & 4.02 & 6.08 & 8.04 & 12.06 & 16.08 & 20.11 & 24.13 & 28.15 & 32.17 & 40.21 & 48.25 & 56.30 & 64.34 \\
\hline 33 & 855 & 4.28 & 6.41 & 8.55 & 12.83 & 17.11 & 21.38 & 25.66 & 29.94 & 34.21 & 42.76 & 51.32 & 59.87 & 68.42 \\
\hline 34 & 908 & 4.54 & 6.81 & 9.08 & 13.62 & 18.16 & 22.70 & 27.24 & 31.78 & 36.32 & 45.40 & 54.48 & 63.55 & 72.63 \\
\hline 35 & 962 & 4.81 & 7.22 & 9.62 & 14.43 & 19.24 & 24.05 & 28.86 & 33.67 & 38.48 & 48.11 & 57.73 & 67.35 & 76.97 \\
\hline 36 & 1018 & 5.09 & 7.63 & 10.18 & 15.27 & 20.36 & 25.45 & 30.54 & 35.63 & 40.72 & 50.89 & 61.07 & 71.25 & 81.43 \\
\hline
\end{tabular}




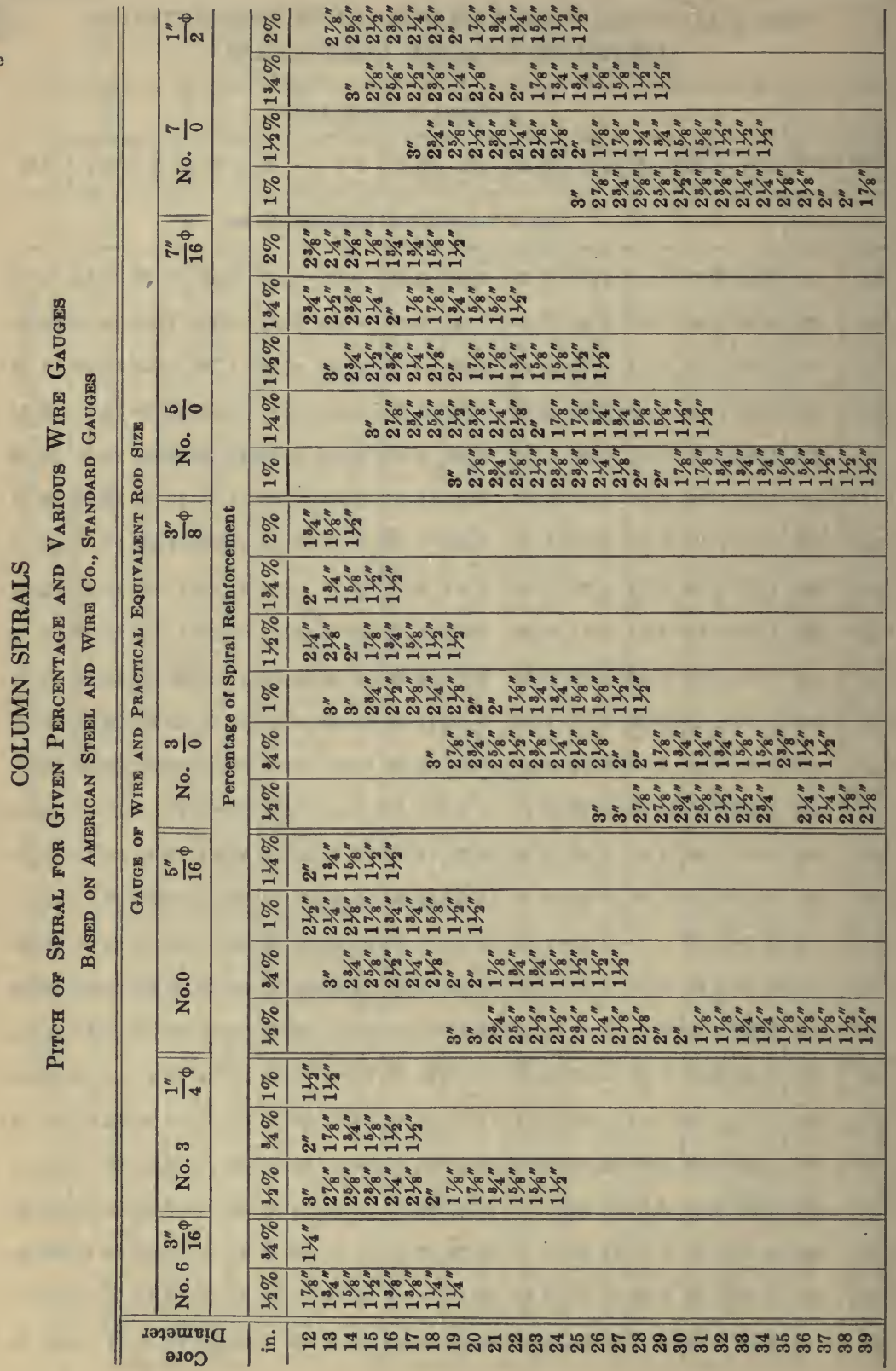


Weight in Pounds per Foot of Height

(Weights do not Include Spacers)

No. 3 Wire-Equivalent $\frac{1}{4}$ "Round Bar

Based on American Steel and Wire Co.'s Standard Gauge

\begin{tabular}{|c|c|c|c|c|c|c|c|c|c|c|}
\hline \multirow{2}{*}{$\begin{array}{c}\begin{array}{c}\text { Diameter } \\
\text { of } \\
\text { Spiral }\end{array} \\
\text { in. }\end{array}$} & \multicolumn{9}{|c|}{ Pitch of SPIRAL in Inches } & \multirow{2}{*}{ 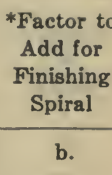 } \\
\hline & $11 / 2$ & $13 / 4$ & 2 & $21 / 4$ & $21 / 2$ & $23 / 4$ & 3 & $31 / 2$ & 4 & \\
\hline 12 & 4.10 & 3.51 & 3.08 & 2.74 & 2.47 & 2.24 & 2.06 & 1.77 & 1.55 & 1.30 \\
\hline 13 & 4.43 & 3.80 & 3.32 & 2.96 & 2.66 & 2.42 & 2.22 & 1.91 & 1.68 & 1.40 \\
\hline 14 & 4.76 & 4.08 & 3.57 & 3.18 & 2.86 & 2.60 & 2.39 & 2.05 & 1.80 & 1.50 \\
\hline 15 & 5.09 & 4.36 & 3.82 & 3.39 & 3.06 & 2.78 & 2.55 & 2.19 & 1.92 & 1.55 \\
\hline 16 & 5.42 & 4.65 & 4.07 & 3.61 & 3.26 & 2.96 & 2.72 & 2.33 & 2.05 & 1.60 \\
\hline 17 & 5.75 & 4.93 & 4.31 & 3.83 & 3.46 & 3.14 & 2.88 & 2.47 & 2.17 & 1.70 \\
\hline 18 & 6.08 & 5.21 & 4.56 & 4.05 & 3.65 & 3.32 & 3.05 & 2.61 & 2.29 & 1.80 \\
\hline 19 & 6.41 & 5.49 & 4.81 & 4.27 & 3.85 & 3.50 & 3.21 & 2.75 & 2.41 & 1.90 \\
\hline 20 & 6.74 & 5.78 & 5.06 & 4.49 & 4.05 & 3.68 & 3.38 & 2.90 & 2.54 & 2.00 \\
\hline 21 & 7.07 & 6.06 & 5.31 & 4.71 & 4.25 & 3.86 & 3.54 & 3.04 & 2.66 & 2.05 \\
\hline 22 & 7.40 & 6.34 & 5.55 & 4.92 & 4.45 & 4.04 & 3.71 & 3.18 & 2.78 & 2.10 \\
\hline 23 & 7.73 & 6.63 & 5.80 & 5.16 & 4.64 & 4.22 & 3.87 & 3.32 & 2.91 & 2.20 \\
\hline 24 & 8.06 & 6.91 & 6.05 & 5.38 & 4.84 & 4.40 & 4.04 & 3.46 & 3.03 & 2.30 \\
\hline 25 & 8.39 & 7.19 & 6.30 & 5.60 & 5.04 & 4.58 & 4.20 & 3.60 & 3.15 & 2.40 \\
\hline 26 & 8.72 & 7.47 & 6.54 & 5.82 & 5.24 & 4.76 & 4.37 & 3.74 & 3.28 & 2.50 \\
\hline 27 & 9.05 & 7.76 & 6.79 & 6.04 & 5.43 & 4.94 & 4.53 & 3.88 & 3.40 & 2.55 \\
\hline 28 & 9.38 & 8.04 & 7.04 & 6.26 & 5.63 & 5.12 & 4.70 & 4.02 & 3.52 & 2.60 \\
\hline 29 & 9.71 & 8.33 & 7.29 & 6.48 & 5.83 & 5.30 & 4.86 & 4.17 & 3.65 & 2.70 \\
\hline 30 & 10.04 & 8.61 & 7.53 & 6.70 & 6.03 & 5.48 & 5.03 & 4.31 & 3.77 & 2.80 \\
\hline 31 & 10.37 & 8.89 & 7.78 & 6.92 & 6.23 & 5.66 & 5.19 & 4.45 & 3.89 & 2.90 \\
\hline 32 & 10.70 & 9.17 & 8.03 & 7.14 & 6.42 & 5.84 & 5.36 & 4.59 & 4.02 & 3.00 \\
\hline 33 & 11.03 & 9.46 & 8.28 & 7.36 & 6.62 & 6.02 & 5.52 & 4.73 & 4.14 & 3.05 \\
\hline 34 & 11.36 & 9.74 & 8.52 & 7.58 & 6.82 & 6.20 & 5.68 & 4.87 & 4.26 & 3.10 \\
\hline 35 & 11.69 & 10.02 & 8.77 & 7.80 & 7.02 & 6.38 & 5.85 & 5.01 & 4.39 & 3.20 \\
\hline 36 & 12.02 & 10.31 & 9.02 & 8.02 & 7.22 & 6.56 & 6.01 & 5.15 & 4.51 & 3.30 \\
\hline 37 & 12.35 & 10.59 & 9.27 & 8.24 & 7.42 & 6.74 & 6.18 & 5.30 & 4.64 & 3.40 \\
\hline 38 & 12.68 & 10.87 & 9.52 & 8.46 & 7.61 & 6.92 & 6.35 & 5.44 & 4.76 & 3.50 \\
\hline
\end{tabular}

* Weight of one extra turn at top and bottom of spiral. 


\section{COLUMN SPIRALS}

Weight in Pounds per Foot of Height (Weights do not Include Spacers)

No. 0 Wire-Equivalent $\frac{5^{\prime \prime}}{16}$ Round Bar

Based on American Steel and Wire Co.'s Standard Gauge

\begin{tabular}{|c|c|c|c|c|c|c|c|c|c|c|}
\hline \multirow{2}{*}{$\begin{array}{c}\begin{array}{c}\text { Diameter } \\
\text { of } \\
\text { Spiral }\end{array} \\
\text { in. }\end{array}$} & \multicolumn{9}{|c|}{ Pitch of SpIRal in INCHES } & \multirow{2}{*}{ 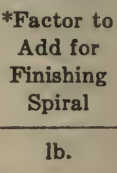 } \\
\hline & $11 / 2$ & $18 / 4$ & 2 & $21 / 4$ & $21 / 2$ & $23 / 4$ & 3 & $31 / 2$ & 4 & \\
\hline 12 & 6.48 & 5.56 & 4.87 & 4.33 & 3.90 & 3.55 & 3.26 & 2.80 & 2.46 & 2.05 \\
\hline 13 & 7.00 & 6.01 & 5.26 & 4.68 & 4.22 & 3.83 & 3.52 & 3.02 & 2.65 & 2.20 \\
\hline 14 & 7.53 & 6.46 & 5.65 & 5.03 & 4.53 & 4.12 & 3.78 & 3.25 & 2.85 & 2.40 \\
\hline 15 & 8.05 & 6.90 & 6.04 & 5.36 & 4.84 & 4.40 & 4.04 & 3.47 & 3.04 & 2.45 \\
\hline 16 & 8.57 & 7.35 & 6.44 & 5.72 & 5.15 & 4.69 & 4.30 & 3.69 & 3.24 & 2.50 \\
\hline 17 & 9.10 & 7.80 & 6.83 & 6.07 & 5.47 & 4.97 & 4.56 & 3.92 & 3.43 & 2.70 \\
\hline 18 & 9.62 & 8.25 & 7.22 & 6.44 & 5.78 & 5.26 & 4.82 & 4.14 & 3.63 & 2.85 \\
\hline 19 & 10.14 & 8.70 & 7.61 & 6.77 & 6.09 & 5.54 & 5.08 & 4.36 & 3.82 & 3.00 \\
\hline 20 & 10.66 & 9.14 & 8.00 & 7.10 & 6.41 & 5.83 & 5.34 & 4.59 & 4.02 & 3.15 \\
\hline 21 & 11.19 & 9.59 & 8.39 & 7.46 & 6.72 & 6.11 & 5.60 & 4.81 & 4.21 & 3.25 \\
\hline 22 & 11.71 & 10.04 & 8.78 & 7.78 & 7.03 & 6.40 & 5.86 & 5.03 & 4.41 & 3.30 \\
\hline 23 & 12.23 & 10.49 & 9.18 & 8.16 & 7.35 & 6.68 & 6.13 & 5.26 & 4.60 & 3.50 \\
\hline 24 & 12.75 & 10.93 & 9.57 & 8.51 & 7.66 & 6.97 & 6.39 & 5.48 & 4.80 & 3.65 \\
\hline 25 & 13.28 & 11.38 & 9.96 & 8.88 & 7.97 & 7.25 & 6.65 & 5.70 & 4.99 & 3.80 \\
\hline 26 & 13.80 & 11.83 & 10.35 & 9.20 & 8.29 & 7.53 & 6.91 & 5.93 & 5.19 & 3.95 \\
\hline 27 & 14.32 & 12.28 & 10.74 & 9.55 & 8.60 & 7.82 & 7.17 & 6.15 & 5.39 & 4.05 \\
\hline 28 & 14.84 & 12.73 & 11.13 & 9.90 & 8.91 & 8.10 & 7.43 & 6.37 & 5.58 & 4.10 \\
\hline 29 & 15.37 & 13.17 & 11.53 & 10.25 & 9.22 & 8.39 & 7.69 & 6.60 & 5.78 & 4.30 \\
\hline 30 & 15.89 & 13.62 & 11.92 & 10.60 & 9.54 & 8.67 & 7.95 & 6.82 & 5.97 & 4.40 \\
\hline 31 & 16.41 & 14.07 & 12.31 & 10.95 & 9.85 & 8.96 & 8.21 & 7.04 & 6.15 & 4.60 \\
\hline 32 & 16.94 & 14.52 & 12.70 & 11.29 & 10.16 & 9.24 & 8.47 & 7.27 & 6.36 & 4.75 \\
\hline 33 & 17.46 & 14.97 & 13.09 & 11.64 & 10.48 & 9.53 & 8.73 & 7.49 & 6.56 & 4.80 \\
\hline 34 & 17.98 & 15.41 & 13.48 & 11.99 & 10.79 & 9.81 & 8.99 & 7.71 & 6.75 & 4.90 \\
\hline 35 & 18.50 & 15.86 & 13.88 & 12.34 & 11.10 & 10.10 & 9.26 & 7.94 & 6.95 & 5.05 \\
\hline 36 & 19.02 & 16.31 & 14.27 & 12.69 & 11.42 & 10.38 & 9.52 & 8.16 & 7.14 & 5.20 \\
\hline 37 & 19.54 & 16.75 & 14.67 & 13.04 & 11.74 & 10.66 & 9.78 & 8.38 & 7.34 & 5.40 \\
\hline 38 & 20.06 & 17.20 & 15.06 & 13.38 & 12.04 & 10.95 & 10.04 & 8.61 & 7.53 & 5.55 \\
\hline
\end{tabular}

* Weight of one extra turn at top and bottom of spiral. 


\section{COLUMN SPIRALS}

Weight in Pounds per Foot of Height

(Weights do not Include Spacers)

No. $\frac{3}{0}$ Wire-Equivalent $\frac{3 "}{8}$ Round Bar

Based on American Steel and Wire Co.'s Standard Gauge

\begin{tabular}{|c|c|c|c|c|c|c|c|c|c|c|}
\hline \multirow{2}{*}{$\begin{array}{c}\begin{array}{c}\text { Diameter } \\
\text { of } \\
\text { Spiral }\end{array} \\
\text { in. }\end{array}$} & \multicolumn{9}{|c|}{ Pitch of SpIRal in Inches } & \multirow{2}{*}{$\begin{array}{c}\text { *Factor to } \\
\text { Add for } \\
\begin{array}{c}\text { Finishing } \\
\text { Spiral }\end{array} \\
\text { lb. }\end{array}$} \\
\hline & $11 / 2$ & $18 / 4$ & 2 & $21 / 4$ & $21 / 2$ & $23 / 4$ & 3 & $31 / 2$ & 4 & \\
\hline 12 & 9.02 & 7.73 & 6.77 & 6.02 & 5.43 & 4.94 & 4.53 & 3.89 & 3.42 & 2.85 \\
\hline 13 & 9.74 & 8.36 & 7.32 & 6.51 & 5.85 & 5.33 & 4.89 & 4.21 & 3.69 & 3.05 \\
\hline 14 & 10.47 & 8.98 & 7.86 & 6.97 & 6.30 & 5.73 & 5.26 & 4.52 & 3,96 & 3.30 \\
\hline 15 & 11.20 & 9.60 & 8.41 & 7.46 & 6.73 & 6.13 & 5.62 & 4.83 & 4.23 & 3.40 \\
\hline 16 & 11.92 & 10.22 & 8.95 & 7.94 & 7.17 & 6.52 & 5.98 & 5.14 & 4.50 & 3.50 \\
\hline 17 & 12.65 & 10.85 & 9.49 & 8.43 & 7.60 & 6.92 & 6.35 & 5.45 & 4.77 & 3.75 \\
\hline 18 & 13.38 & 11.47 & 10.04 & 8.91 & 8.04 & 7.31 & 6.71 & 5.74 & 5.05 & 3.95 \\
\hline 19 & 14.11 & 12.09 & 10.59 & 9.40 & 8.48 & 7.71 & 7.07 & 6.05 & 5.32 & 4.20 \\
\hline 20 & 14.83 & 12.72 & 11.13 & 9.88 & 8.91 & 8.10 & 7.43 & 6.38 & 5.59 & 4.40 \\
\hline 21 & 15.56 & 13.34 & 11.68 & 10.37 & 9.35 & 8.50 & 7.80 & 6.69 & 5.86 & 4.50 \\
\hline 22 & 16.29 & 13.96 & 12.22 & 10.83 & 9.78 & 8.90 & 8.16 & 6.99 & 6.13 & 4.60 \\
\hline 23 & 17.01 & 14.59 & 12.77 & 11.36 & 10.22 & 9.29 & 8.52 & 7.31 & 6.40 & 4.85 \\
\hline 24 & 17.74 & 15.21 & 13.31 & 11.83 & 10.65 & 9.69 & 8.88 & 7.62 & 6.67 & 5.10 \\
\hline 25 & 18.47 & 15.83 & 13.86 & 12.32 & 11.09 & 10.09 & 9.25 & 7.93 & 6.93 & 5.30 \\
\hline 26 & 19.19 & 16.45 & 14.40 & 12.80 & 11.53 & 10.48 & 9.61 & 8.24 & 7.22 & 5.50 \\
\hline 27 & 19.92 & 17.08 & 14.95 & 13.29 & 11.96 & 10.88 & 9.95 & 8.55 & 7.49 & 5.60 \\
\hline 28 & 20.65 & 17.70 & 15.49 & 13.77 & 12.40 & 11.27 & 10.34 & 8.86 & 7.76 & 5.70 \\
\hline 29 & 21.37 & 18.32 & 16.04 & 14.26 & 12.83 & 11.67 & 10.70 & 9.17 & 8.03 & 6.00 \\
\hline 30 & 22.10 & 18.95 & 16.58 & 14.74 & 13.27 & 12.06 & 11.08 & 9.48 & 8.30 & 6.10 \\
\hline 31 & 22.83 & 19.57 & 17.12 & 15.22 & 13.70 & 12.46 & 11.42 & 9.79 & 8.57 & 6.40 \\
\hline 32 & 23.55 & 20.19 & 17.67 & 15.71 & 14.14 & 12.86 & 11.79 & 10.11 & 8.85 & 6.60 \\
\hline 33 & 24.28 & 20.82 & 18.21 & 16.19 & 14.57 & 13.25 & 12.15 & 10.42 & 9.12 & 6.70 \\
\hline 34 & 25.01 & 21.44 & 18.76 & 16.68 & 15.01 & 13.65 & 12.51 & 10.73 & 9.37 & 6.80 \\
\hline 35 & 25.74 & 22.06 & 19.30 & 17.17 & 15.45 & 14.04 & 12.89 & 11.04 & 9.66 & 7.00 \\
\hline 36 & 26.45 & 22.68 & 19.85 & 17.65 & 15.88 & 14.44 & 13.24 & 11.35 & 9.93 & 7.20 \\
\hline 37 & 27.18 & 23.31 & 20.40 & 18.13 & 16.33 & 14.83 & 13.59 & 11.66 & 10.21 & 7.50 \\
\hline 38 & 27.91 & 23.92 & 20.95 & 18.62 & 16.75 & 15.23 & 13.99 & 11.96 & 10.47 & 7.70 \\
\hline
\end{tabular}

* Weight of one extra turn at top and bottom of spiral. 


\section{COLUMN SPIRALS}

Weight in Pounds Per Foot of Height

(Weights do not Include Spacers)

No. $\frac{5}{0}$ Wire-Equivalent $\frac{7 "}{16}$ Round Bar

Based on American Steel and Wire Co.'s Standard Gauge

\begin{tabular}{|c|c|c|c|c|c|c|c|c|c|c|}
\hline \multirow{2}{*}{$\begin{array}{c}\begin{array}{c}\text { Diameter } \\
\text { of } \\
\text { Spiral }\end{array} \\
\text { in. }\end{array}$} & \multicolumn{9}{|c|}{ PITCH of SPIRAL in INCHES } & \multirow{2}{*}{$\begin{array}{c}\text { *Factor to } \\
\begin{array}{c}\text { Add for } \\
\text { Finishing } \\
\text { Spiral }\end{array} \\
\text { lb. }\end{array}$} \\
\hline & $11 / 2$ & $1 \% / 4$ & 2 & $21 / 4$ & $21 / 2$ & $23 / 4$ & 3 & $31 / 2$ & 4 & \\
\hline 12 & 12.72 & 10.91 & 9.56 & 8.50 & 7.66 & 6.97 & 6.40 & 5.49 & 4.82 & 4.00 \\
\hline 13 & 13.75 & 11.79 & 10.33 & 9.18 & 8.27 & 7.53 & 6.91 & 5.93 & 5.21 & 4.30 \\
\hline 14 & 14.78 & 12.67 & 11.09 & 9.87 & 8.89 & 8.09 & 7.42 & 6.37 & 5.59 & 4.70 \\
\hline 15 & 15.80 & 13.55 & 11.86 & 10.55 & 9.50 & 8.65 & 7.93 & 6.81 & 5.97 & 4.80 \\
\hline 16 & 16.83 & 14.43 & 12.63 & 11.23 & 10.12 & 9.20 & 8.44 & 7.24 & 6.36 & 4.90 \\
\hline 17 & 17.85 & 15.31 & 13.40 & 11.92 & 10.73 & 9.76 & 8.95 & 7.67 & 6.74 & 5.30 \\
\hline 18 & 18.88 & 16.19 & 14.17 & 12.60 & 11.35 & 10.32 & 9.46 & 8.11 & 7.12 & 5.60 \\
\hline 19 & 19.91 & 17.07 & 14.94 & 13.28 & 11.96 & 10.88 & 9.98 & 8.54 & 7.50 & 5.90 \\
\hline 20 & 20.93 & 17.95 & 15.71 & 13.97 & 12.58 & 11.44 & 10.49 & 9.00 & 7.89 & 6.20 \\
\hline 21 & 21.96 & 18.82 & 16.48 & 14.65 & 13.19 & 12.00 & 11.00 & 9.44 & 8.27 & 6.40 \\
\hline 22 & 22.98 & 19.70 & 17.25 & 15.33 & 13.81 & 12.56 & 11.51 & 9.88 & 8.63 & 6.50 \\
\hline 23 & 24.01 & 20.58 & 18.01 & 16.02 & 14.42 & 13.11 & 12.02 & 10.32 & 9.04 & 6.90 \\
\hline 24 & 25.03 & 21.46 & 18.78 & 16.70 & 15.04 & 13.67 & 12.54 & 10.75 & 9.42 & 7.20 \\
\hline 25 & 26.06 & 22.34 & 19.55 & 17.38 & 15.65 & 14.23 & 13.05 & 11.18 & 9.78 & 7.50 \\
\hline 26 & 27.09 & 23.22 & 20.32 & 18.07 & 16.26 & 14.79 & 13.56 & 11.62 & 10.19 & 7.75 \\
\hline 27 & 28.11 & 24.10 & 21.09 & 18.75 & 16.88 & 15.35 & 14.07 & 12.05 & 10.57 & 7.95 \\
\hline 28 & 29.14 & 24.98 & 21.86 & 19.43 & 17.49 & 15.91 & 14.60 & 12.49 & 10.93 & 8.05 \\
\hline 29 & 30.16 & 25.86 & 22.63 & 20.12 & 18.11 & 16.47 & 15.09 & 12.95 & 11.34 & 8.45 \\
\hline 30 & 31.19 & 26.74 & 23.40 & 20.80 & 18.72 & 17.02 & 15.61 & 13.39 & 11.70 & 8.65 \\
\hline 31 & 32.21 & 27.61 & 24.17 & 21.48 & 19.34 & 17.58 & 16.12 & 13.82 & 12.08 & 9.05 \\
\hline 32 & 33.24 & 28.49 & 24.93 & 22.17 & 19.95 & 18.13 & 16.65 & 14.26 & 12.48 & 9.30 \\
\hline 33 & 34.22 & 29.37 & 25.70 & 22.85 & 20.57 & 18.70 & 17.14 & 14.70 & 12.87 & 9.40 \\
\hline 34 & 35.26 & 30.25 & 26.47 & 23.53 & 21.18 & 19.26 & 17.65 & 15.14 & 13.23 & 9.60 \\
\hline 35 & 36.30 & 31.13 & 27.24 & 24.22 & 21.80 & 19.82 & 18.17 & 15.56 & 13.63 & 9.90 \\
\hline 36 & 37.30 & 32.01 & 28.01 & 24.90 & 22.41 & 20.38 & 18.68 & 16.00 & 14.00 & 10.20 \\
\hline 37 & 38.32 & 32.90 & 28.80 & 25.60 & 23.05 & 20.93 & 19.20 & 16.46 & 14.41 & 10.60 \\
\hline 38 & 39.35 & 33.77 & 29.58 & 26.29 & 23.62 & 21.49 & 19.72 & 16.90 & 14.78 & 10.90 \\
\hline
\end{tabular}

* Weight of one extra turn at top and bottom of spiral. 


\section{COLUMN SPIRALS}

Weight in Pounds per Foot of Height

(Weights do not Include Spacers)

No. $\frac{7}{0}$ Wire-Equivalent $\frac{1}{2}$ "Round Bar

Based on American Steel and Wire Co.'s Standard Gauge

\begin{tabular}{|c|c|c|c|c|c|c|c|c|c|c|}
\hline \multirow{2}{*}{$\begin{array}{c}\begin{array}{c}\text { Diameter } \\
\text { of } \\
\text { Spiral }\end{array} \\
\text { in. }\end{array}$} & \multicolumn{9}{|c|}{ Pitch of Spiral in Inches } & \multirow{2}{*}{$\begin{array}{c}\text { *Factor to } \\
\begin{array}{c}\text { Add for } \\
\text { Finishing } \\
\text { Spiral }\end{array} \\
\text { lb. }\end{array}$} \\
\hline & $11 / 2$ & $18 / 4$ & 2 & $21 / 4$ & $21 / 2$ & $23 / 4$ & 3 & $31 / 2$ & 4 & \\
\hline 12 & 16.52 & 14.15 & 12.41 & 11.04 & 9.96 & 9.03 & 8.30 & 7.14 & 6.26 & 5.20 \\
\hline 13 & 17.85 & 15.31 & 13.39 & 11.92 & 10.74 & 9.77 & 8.96 & 7.70 & 6.76 & 5.60 \\
\hline 14 & 19.18 & 16.45 & 14.40 & 12.81 & 11.54 & 10.48 & 9.63 & 8.27 & 7.26 & 6.10 \\
\hline 15 & 20.52 & 17.59 & 15.40 & 13.67 & 12.34 & 11.22 & 10.28 & 8.84 & 7.75 & 6.25 \\
\hline 16 & 21.85 & 18.75 & 16.40 & 14.56 & 13.14 & 11.93 & 10.96 & 9.39 & 8.27 & 6.40 \\
\hline 17 & 23.18 & 19.88 & 17.38 & 15.44 & 13.95 & 12.67 & 11.61 & 9.96 & 8.75 & 6.90 \\
\hline 18 & 24.52 & 21.01 & 18.40 & 16.33 & 14.73 & 13.40 & 12.29 & 10.52 & 9.23 & 7.25 \\
\hline 19 & 25.84 & 22.14 & 19.40 & 17.22 & 15.53 & 14.11 & 12.94 & 11.09 & 9.72 & 7.65 \\
\hline 20 & 27.17 & 23.30 & 20.40 & 18.10 & 16.33 & 14.85 & 13.62 & 11.69 & 10.24 & 8.00 \\
\hline 21 & 28.51 & 24.44 & 21.41 & 18.99 & 17.13 & 15.56 & 14.28 & 12.26 & 10.74 & 8.30 \\
\hline 22 & 29.84 & 25.56 & 22.38 & 19.84 & 17.94 & 16.30 & 14.95 & 12.83 & 11.21 & 8.40 \\
\hline 23 & 31.17 & 26.73 & 23.39 & 20.79 & 18.72 & 17.03 & 15.61 & 13.39 & 11.73 & 8.90 \\
\hline 24 & 32.50 & 27.86 & 24.39 & 21.68 & 19.52 & 17.75 & 16.28 & 13.96 & 12.23 & 9.30 \\
\hline 25 & 33.83 & 29.00 & 25.40 & 22.57 & 20.32 & 18.48 & 16.94 & 14.53 & 12.70 & 9.70 \\
\hline 26 & 35.16 & 30.12 & 26.37 & 23.46 & 21.12 & 19.20 & 17.61 & 15.08 & 13.22 & 10.00 \\
\hline 27 & 36.50 & 31.29 & 27.38 & 24.34 & 21.89 & 19.93 & 18.27 & 15.64 & 13.72 & 10.30 \\
\hline 28 & 37.83 & 32.43 & 28.38 & 25.23 & 22.71 & 20.65 & 18.94 & 16.21 & 14.19 & 10.40 \\
\hline 29 & 39.16 & 33.59 & 29.38 & 26.12 & 23.51 & 21.38 & 19.60 & 16.81 & 14.72 & 11.00 \\
\hline 30 & 40.49 & 34.71 & 30.36 & 27.00 & 24.31 & 22.10 & 20.28 & 17.38 & 15.21 & 11.20 \\
\hline 31 & 41.82 & 35.85 & 31.37 & 27.89 & 25.11 & 22.83 & 20.93 & 17.95 & 15.68 & 11.70 \\
\hline 32 & 43.14 & 36.97 & 32.38 & 28.78 & 25.90 & 23.55 & 21.61 & 18.52 & 16.21 & 12.10 \\
\hline 33 & 44.47 & 38.14 & 33.38 & 29.67 & 26.70 & 24.28 & 22.26 & 19.08 & 16.70 & 12.20 \\
\hline 34 & 45.80 & 39.28 & 34.35 & 30.55 & 27.50 & 25.00 & 22.90 & 19.65 & 17.18 & 12.50 \\
\hline 35 & 47.13 & 40.40 & 35.36 & 31.44 & 28.30 & 25.73 & 23.59 & 20.20 & 17.70 & 12.90 \\
\hline 36 & 48.46 & 41.57 & 36.37 & 32.33 & 29.10 & 26.46 & 24.23 & 20.76 & 18.18 & 13.30 \\
\hline 37 & 49.80 & 42.70 & 37.38 & 33.22 & 29.92 & 27.18 & 24.92 & 21.37 & 18.71 & 13.80 \\
\hline 38 & 51.13 & 43.83 & 38.38 & 34.11 & 30.68 & 27.90 & 25.60 & 21.93 & 19.19 & 14.20 \\
\hline
\end{tabular}

* Weight of one extra turn at top and bottom of spiral. 
CORRUGATED BAR COMPANY, INC.

\section{Spirals}

Wire and Spacers

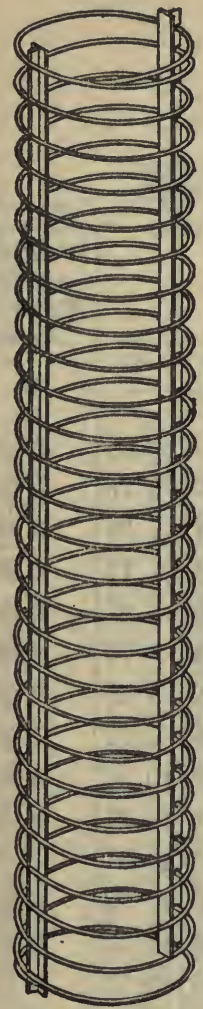

\section{COLUMN SPIRALS}

Standard Wire and Spacers

STANDARD WIRE

\begin{tabular}{c|c|c|c|c}
\hline \hline Gauge & $\begin{array}{c}\text { Practical } \\
\text { Equiv. }\end{array}$ & Diameter & Area & Wt. per lin.ft. \\
\cline { 1 - 2 } \cline { 5 - 5 } No. & Rod & in. & sq. in. & lb. \\
\hline 3 & $1 / 4^{\prime \prime} \phi$ & 0.2437 & 0.0467 & 0.1578 \\
0 & $\frac{5}{16}{ }^{\prime \prime} \phi$ & 0.3065 & 0.0740 & 0.2497 \\
$\frac{8}{0}$ & $3 / 8^{\prime \prime} \phi$ & 0.3625 & 0.1029 & 0.3473 \\
$\frac{5}{0}$ & $\frac{7}{16}{ }^{\prime \prime} \phi$ & 0.4305 & 0.1453 & 0.4901 \\
$\frac{7}{0}$ & $1 / 2^{\prime \prime} \phi$ & 0.4900 & 0.1886 & 0.6363 \\
\hline
\end{tabular}

American Steel \& Wire Co. Gauges.

STANDARD SPACERS

\begin{tabular}{|c|c|c|c|c|c|}
\hline \multicolumn{2}{|c|}{ SPIRAL } & \multicolumn{2}{|c|}{ WIRE } & \multicolumn{2}{|c|}{ T-SEction SPacers } \\
\hline $\begin{array}{l}\text { Diam. } \\
\text { in. }\end{array}$ & $\begin{array}{l}\text { Height } \\
\text { ft. }\end{array}$ & Gauge & $\begin{array}{c}\text { Practical } \\
\text { Equiv. }\end{array}$ & Size & $\begin{array}{l}\text { Wt. per ft. } \\
\text { for two } \\
\text { Spacers }\end{array}$ \\
\hline 9 to 15 & 1 to 15 & 3 & $1 / 4^{\prime \prime} \phi$ & $1 \times 1 \times 1 / 8$ & 1.60 \\
\hline 16 to 30 & 1 to 20 & 3 & $1 / 4^{\prime \prime} \phi$ & $11 / 4 \times 11 / 4 \times 1 / 8$ & 2.00 \\
\hline 9 to 15 & 1 to 15 & 0 & $\frac{5}{16} " \phi$ & $11 / 4 \times 11 / 4 \times 1 / 8$ & 2.00 \\
\hline 16 to 30 & 1 to 20 & 0 & $\frac{5}{16} " \phi$ & $11 / 4 \times 11 / 4 \times \frac{3}{16}$ & 2.96 \\
\hline 9 to 15 & 1 to 15 & $\frac{8}{0}$ & $3 / 8^{\prime \prime} \phi$ & $11 / 4 \times 11 / 4 \times 1 / 8$ & 2.00 \\
\hline 16 to 30 & 1 to 20 & $\frac{3}{0}$ & $3 / 8 " \phi$ & $11 / 4 \times 11 / 4 \times \frac{3}{16}$ & 2.96 \\
\hline 9 to 30 & 1 to 20 & $\frac{5}{0}$ & $\frac{7}{16} " \phi$ & $11 / 2 \times 11 / 2 \times \frac{3}{16}$ & 3.60 \\
\hline 9 to 30 & 1 to 20 & $\frac{7}{0}$ & $1 / 2^{\prime \prime} \phi$ & $11 / 2 \times 1 \frac{1}{2} \times \frac{3}{16}$ & 3.60 \\
\hline
\end{tabular}

Nore.-For spirals over 30 inches in diameter use twice the number of spacers specified for spirals under 30 inches in diameter. 


\section{COLUMNS}

Areas, Perimeters, Weights, Volumes and Moments of Inkrtia

Note-Moments of Inertia calculated about Axis $\boldsymbol{A}-\boldsymbol{A}$

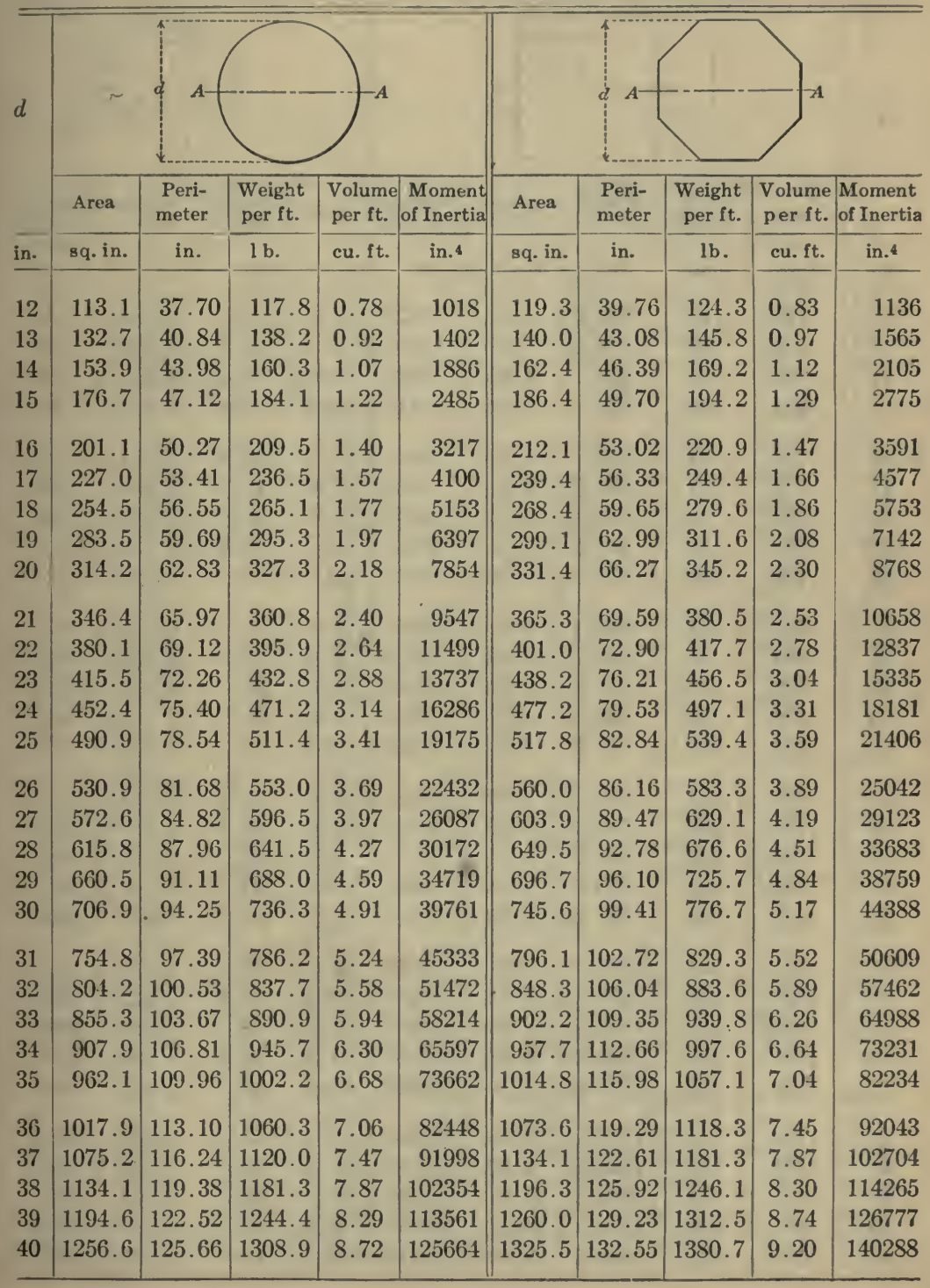


COLUMNS

Areas, Perimeters, Weights, Volumes and Moments of Inertia.

Note.-Moments of Inertia calculated about Axis $A-A$

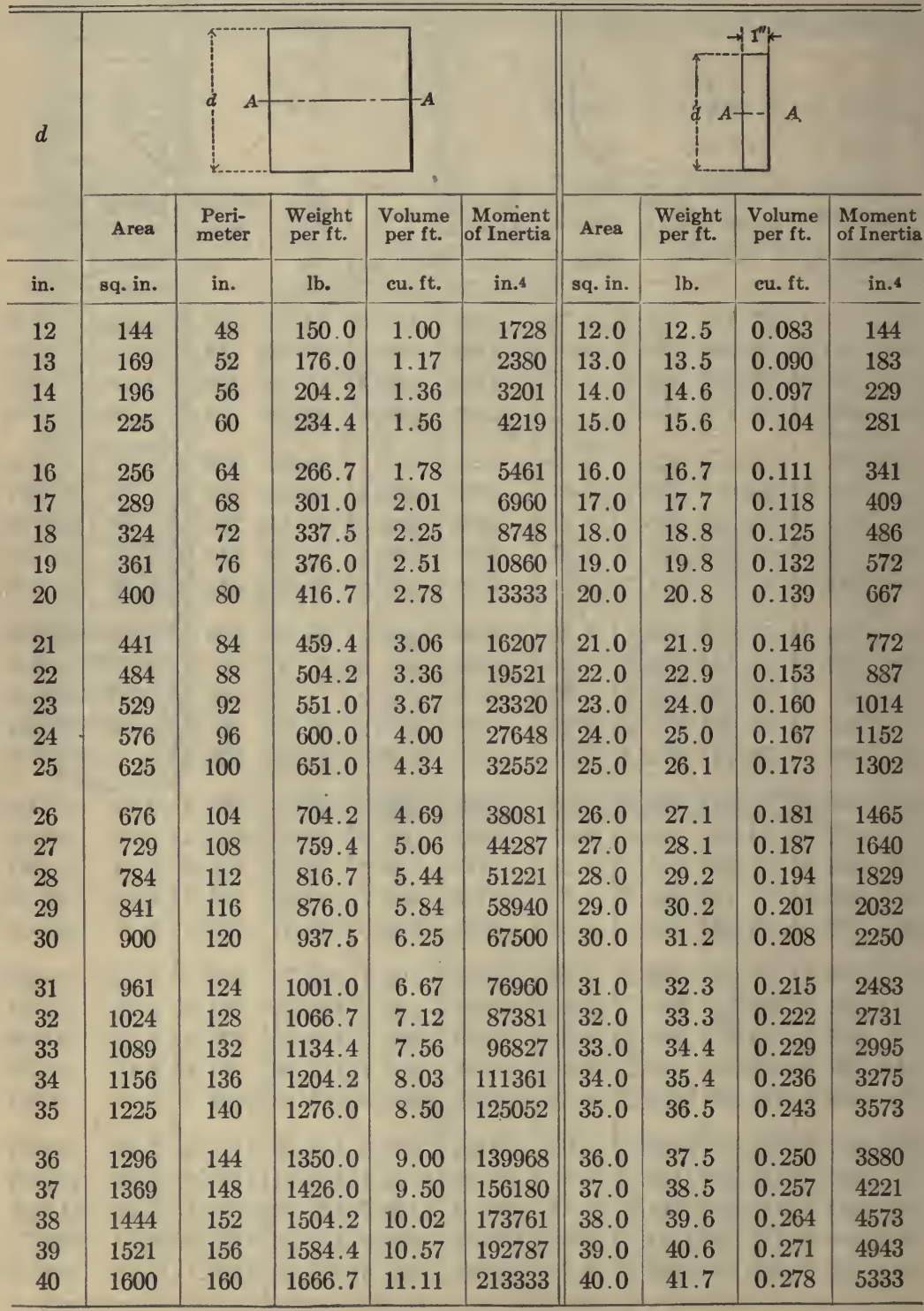




\begin{tabular}{|c|c|c|c|c|c|c|c|c|}
\hline \multicolumn{2}{|c|}{ Circle } & & OF $\mathrm{Co}$ & PRESSED & $\begin{array}{l}\text { Terms } \\
\text { Inches }^{4} \\
I=n I_{\mathrm{a}}\end{array}$ & $\begin{array}{l}\text { NERTIA } \\
\text { NGED IN } \\
\text { F CONC }\end{array}$ & RETE & \\
\hline Diameter & \multicolumn{4}{|c|}{$n=12$} & \multicolumn{4}{|c|}{$n=15$} \\
\hline Circle & \multicolumn{4}{|c|}{ Percentage of Column Verțicals } & \multicolumn{4}{|c|}{ Percentage of Column Verticals } \\
\hline in. & $1 \%$ & $2 \%$ & $3 \%$ & $4 \%$ & $1 \%$ & $2 \%$ & $3 \%$ & $4 \%$ \\
\hline 12 & 244 & 488 & 732 & 976 & 305 & 610 & 915 & 1220 \\
\hline 13 & 336 & 672 & 1008 & 1344 & 420 & 840 & 1261 & 1681 \\
\hline 14 & 452 & 904 & 1356 & 1808 & 565 & 1130 & 1696 & 2261 \\
\hline 15 & 596 & 1192 & 1787 & 2383 & 745 & 1491 & 2234 & 2979 \\
\hline 16 & 771 & 1543 & 2314 & 3085 & 964 & 1928 & 2893 & 3857 \\
\hline 17 & 983 & 1966 & 2949 & 3932 & 1229 & 2458 & 3686 & 4915 \\
\hline 18 & 1235 & 2471 & 3706 & 4942 & 1544 & 3089 & 4633 & 6178 \\
\hline 19 & 1534 & 3067 & 4601 & 6135 & 1917 & 3835 & 5752 & 7669 \\
\hline 20 & 1883 & 3766 & 5649 & 7532 & 2354 & 4708 & 7062 & 9416 \\
\hline 21 & 2289 & 4578 & 6866 & 9155 & 2861 & 5723 & 8584 & 11445 \\
\hline 22 & 2757 & 5514 & 8271 & 11028 & 3446 & 6893 & 10339 & 13786 \\
\hline 23 & 3293 & 6587 & 9880 & 13173 & 4117 & 8234 & 12351 & 16469 \\
\hline 24 & 3905 & 7809 & 11714 & 15618 & 4881 & 9763 & 14644 & 19525 \\
\hline 25 & 4597 & 9194 & 13791 & 18389 & 5747 & 11494 & 17241 & 22988 \\
\hline 26 & 5378 & 10756 & 16134 & 21512 & 6723 & 13447 & 20170 & 26893 \\
\hline 27 & 6254 & 12509 & 18763 & 25018 & 7819 & 15638 & 23456 & 31275 \\
\hline 28 & 7234 & 14467 & 21701 & 28935 & 9043 & 18086 & 27129 & 36173 \\
\hline 29 & 8324 & 16648 & 24971 & 33295 & 10406 & 20812 & 31218 & 41623 \\
\hline 30 & 9533 & 19065 & 28598 & 38131 & 11917 & 23834 & 35751 & 47669 \\
\hline 31 & 10869 & 21737 & 32606 & 43475 & 13587 & 27175 & 40762 & 54349 \\
\hline 32 & 12340 & 24681 & 37021 & 49362 & 15427 & 30854 & 48282 & 61709 \\
\hline 33 & 13957 & 27914 & 41870 & 55827 & 17448 & 34896 & 52344 & 69747 \\
\hline 34 & 15727 & 31454 & 47181 & 62908 & 19661 & 39322 & 58983 & 78643 \\
\hline 35 & 17660 & 35321 & 52981 & 70642 & 22078 & 44156 & 66234 & 88312 \\
\hline 36 & 19767 & 39534 & 59301 & 79068 & 24711 & 49423 & 74134 & 98845 \\
\hline 37 & 22057 & 44113 & 66170 & 88226 & 27574 & 55147 & 82721 & 110294 \\
\hline 38 & 24539 & 49079 & 73618 & 98158 & 30678 & 61355 & 92033 & 122710 \\
\hline 39 & 27226 & 54453 & 81679 & 108905 & 34036 & 68073 & 102109 & 136146 \\
\hline 40 & 30128 & 60256 & 90384 & 120512 & 37664 & 75328 & 112992 & 150656 \\
\hline
\end{tabular}

Note. - For calculation of the moment of inertia the bars are assumed transformed into a continuous cylinder having a sectional area equivalent to the sum of the area of the bars. 
CORRUGATED BAR COMPANY, INC.

of Inertia

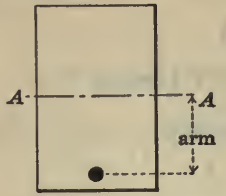

\section{MOMENTS OF INERTIA OF BARS}

INCHES 4

For Various Distances From An Axis $A-A$

Values Expressed in Nearest Whole Numbers

\begin{tabular}{|c|c|c|c|c|c|c|c|c|c|c|c|c|c|c|}
\hline \multirow{2}{*}{$\begin{array}{c}\text { Arm } \\
\text { in. }\end{array}$} & \multicolumn{7}{|c|}{ SQUARE BARS } & \multicolumn{7}{|c|}{ ROUND BARS } \\
\hline & $1 / 2^{\prime \prime}$ & $5 / 8^{\prime \prime}$ & $3 / 4^{\prime \prime}$ & $7 / 8^{\prime \prime}$ & $1^{\prime \prime}$ & $11 / 8^{\prime \prime}$ & $11 / 4^{n}$ & $1 / 2^{\prime \prime}$ & $5 / 8^{\prime \prime}$ & $8 / 4^{\prime \prime}$ & $7 / 8^{\prime \prime}$ & $1^{\prime \prime}$ & $11 / 8^{\prime \prime}$ & $11 / 4^{\prime \prime}$ \\
\hline 2 & 1 & 2 & 2 & 3 & 4 & 5 & 6 & 1 & 1 & 2 & 2 & 3 & 4 & 0 \\
\hline 21 & 2 & 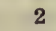 & 4 & 5 & 6 & 8 & 10 & & 2 & $=$ & 4 & 5 & 6 & 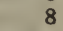 \\
\hline 3 & 2 & 4 & 5 & 7 & 9 & 12 & 14 & ? & 3 & 4 & 5 & 7 & 9 & 11 \\
\hline $31 / 2$ & 3 & 0 & 7 & 9 & 12 & 16 & 19 & 2 & 4 & 5 & 7 & 10 & 12 & 15 \\
\hline 4 & 4 & 6 & 9 & 12 & 16 & 20 & 25 & 3 & 5 & 7 & 10 & 13 & 16 & 20 \\
\hline $41 / 2$ & 5 & 8 & 11 & 16 & 20 & 26 & 32 & 4 & 6 & 9 & 12 & 16 & 20 & 25 \\
\hline & 6 & 10 & 14 & 19 & 25 & & & 5 & 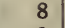 & 11 & 15 & 20 & 25 & 1 \\
\hline $51 / 2$ & 8 & 12 & 17 & 23 & 30 & & 81 & & 9 & 13 & 18 & 24 & 30 & 37 \\
\hline 6 & 9 & 14 & 20 & 28 & 36 & 4 & 56 & 7 & 11 & 16 & 22 & 28 & 36 & 44 \\
\hline $61 / 2$ & 11 & 17 & 24 & 32 & 42 & 5 & 6 & 8 & 13 & 19 & 25 & 33 & 42 & 59 \\
\hline 7 & 12 & 19 & 28 & 38 & 49 & 62 & 77 & 10 & 15 & 22 & 29 & 39 & 49 & 60 \\
\hline $71 / 2$ & 1 & 22 & 3 & 43 & 56 & 71 & 88 & 1 & 17 & 25 & 34 & 44 & 56 & 69 \\
\hline 8 & 1 & 25 & 3 & 10 & 64 & 81 & 100 & 1 & 20 & 28 & 39 & 50 & 64 & 79 \\
\hline $81 / 2$ & 1 & 28 & 1 & 55 & 72 & 92 & 113 & 1 & & 32 & 43 & 57 & 72 & 89 \\
\hline 9 & 20 & 32 & 4 & 62 & 81 & 103 & 127 & 16 & 25 & 36 & 49 & 64 & 81 & 100 \\
\hline $91 / 2$ & 23 & 35 & 51 & 69 & 90 & 114 & 141 & 18. & 28 & 40 & 54 & 71 & 90 & 111 \\
\hline & 2 & 3 & F & 77 & 100 & & & & 3 & & 60 & 79 & 99 & 123 \\
\hline & 2 & 4 & 6 & 84 & 110 & 1 & 172 & & & 49 & 66 & 87 & 110 & 135 \\
\hline 11 & 3 & 47 & 6 & 93 & 121 & & 189 & 24 & 3 & 53 & 73 & 95 & 120 & 149 \\
\hline $111 / 2$ & 38 & 52 & 7 & 101 & 132 & 16 & 207 & 26 & 4 & 58 & 80 & 104 & 132 & 162 \\
\hline 12 & 36 & 56 & 81 & 110 & 144 & 182 & 225 & 28 & 44 & 64 & 87 & 113 & 143 & 177 \\
\hline 15 & 4 & 6 & & & & & 264 & & 5 & 75 & 102 & 33 & & 208 \\
\hline 14 & 4 & 77 & 11 & 150 & 196 & & 306 & 38 & 6 & 87 & 118 & 154 & & 241 \\
\hline 15 & 5 & 88 & 12 & 172 & 225 & & 352 & 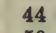 & 0 & 99 & 135 & 177 & 224 & 276 \\
\hline 16 & 6 & 100 & 14 & 196 & 256 & & 400 & 50 & 79 & 113 & 154 & 201 & 255 & 314 \\
\hline 17 & 72 & 113 & 16 & 221 & 289 & 3 & 4 & 57 & 8 & 128 & 174 & 227 & 287 & 355 \\
\hline 18 & 8 & & & 248 & 324 & & 5 & 64 & 0 & & 1 & 255 & & 398 \\
\hline 19 & 9 & 141 & & 276 & 361 & 4 & 5 & 71 & 11 & 160 & 217 & 28 & 9 & 443 \\
\hline 20 & 10 & 1 & & 306 & 400 & 50 & 625 & 79 & & 177 & 241 & 314 & 8 & 491 \\
\hline 21 & 11 & 172 & 2 & 3 & 441 & 55 & 689 & 87 & 135 & 195 & 265 & 346 & 438 & 541 \\
\hline 22 & 12 & 1 & & & 4 & 6 & 756 & 95 & 14 & 214 & 291 & 380 & 481 & 594 \\
\hline 23 & & 206 & & & 5 & & 827 & 104 & & & 31 & & & 649 \\
\hline 24 & & 2 & & & 5 & 72 & 900 & 1 & & 254 & 346 & 45 & & 707 \\
\hline 25 & 15 & 244 & 35 & 47 & 625 & 791 & 977 & 123 & & 276 & 376 & 491 & & 767 \\
\hline 26 & 16 & 264 & 38 & 518 & 676 & 856 & 1056 & 133 & 207 & 299 & 407 & 531 & 672 & 830 \\
\hline 27 & 18 & 28 & 4 & & 729 & 923 & 1 & 143 & 22 & 322 & 438 & 573 & 725 & 895 \\
\hline 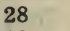 & & & & & 784 & & & & & 346 & 471 & 16 & 779 & 962 \\
\hline 29 & & 32 & 4 & 6 & 841 & 10 & 1314 & 1 & & & 506 & 661 & 836 & 1032 \\
\hline 30 & 22 & 352 & 50 & 689 & 900 & & & 17 & 27 & 3 & 541 & 707 & 895 & 1105 \\
\hline 32 & 25 & 400 & $5 ?$ & 784 & 1024 & 1296 & 1600 & 201 & 314 & 452 & 616 & 804 & 1018 & 1257 \\
\hline 34 & 28 & 45 & & 885 & & 1463 & 1 & - & 3 & 5 & 695 & 908 & 149 & 1419 \\
\hline 36 & & 5 & & & & & & & & & 779 & 1018 & & 1591 \\
\hline 38 & 36 & 5 & 8. & 1106 & 1444 & 1828 & 22 & 2 & 44 & 8 & 868 & 1134 & & 1772 \\
\hline 40 & 400 & 625 & 900 & 1225 & 1600 & 2025 & & 314 & 491 & 707 & 962 & 1257 & 1590 & 1964 \\
\hline 42 & 441 & 689 & 992 & 1351 & 1764 & 2233 & 2756 & 346 & 541 & 779 & 1061 & 1385 & 1753 & 2165 \\
\hline 44 & 48 & 75 & 108 & 1482 & 1936 & 2450 & & 38 & 59 & 855 & 34 & 1521 & 1924 & 2376 \\
\hline 46 & 52 & 82 & 119 & 1620 & 2116 & 2678 & 330 & 4 & 649 & 935 & 1272 & 1662 & 2103 & 2597 \\
\hline 48 & 576 & 900 & 1296 & 1764 & 2304 & 2916 & 3600 & 452 & 707 & 1018 & 1385 & 1810 & 2290 & 2828 \\
\hline
\end{tabular}




\section{BEAM QUANTITIES}

Cubic Feft of Concrete per Linear Foot of Beam

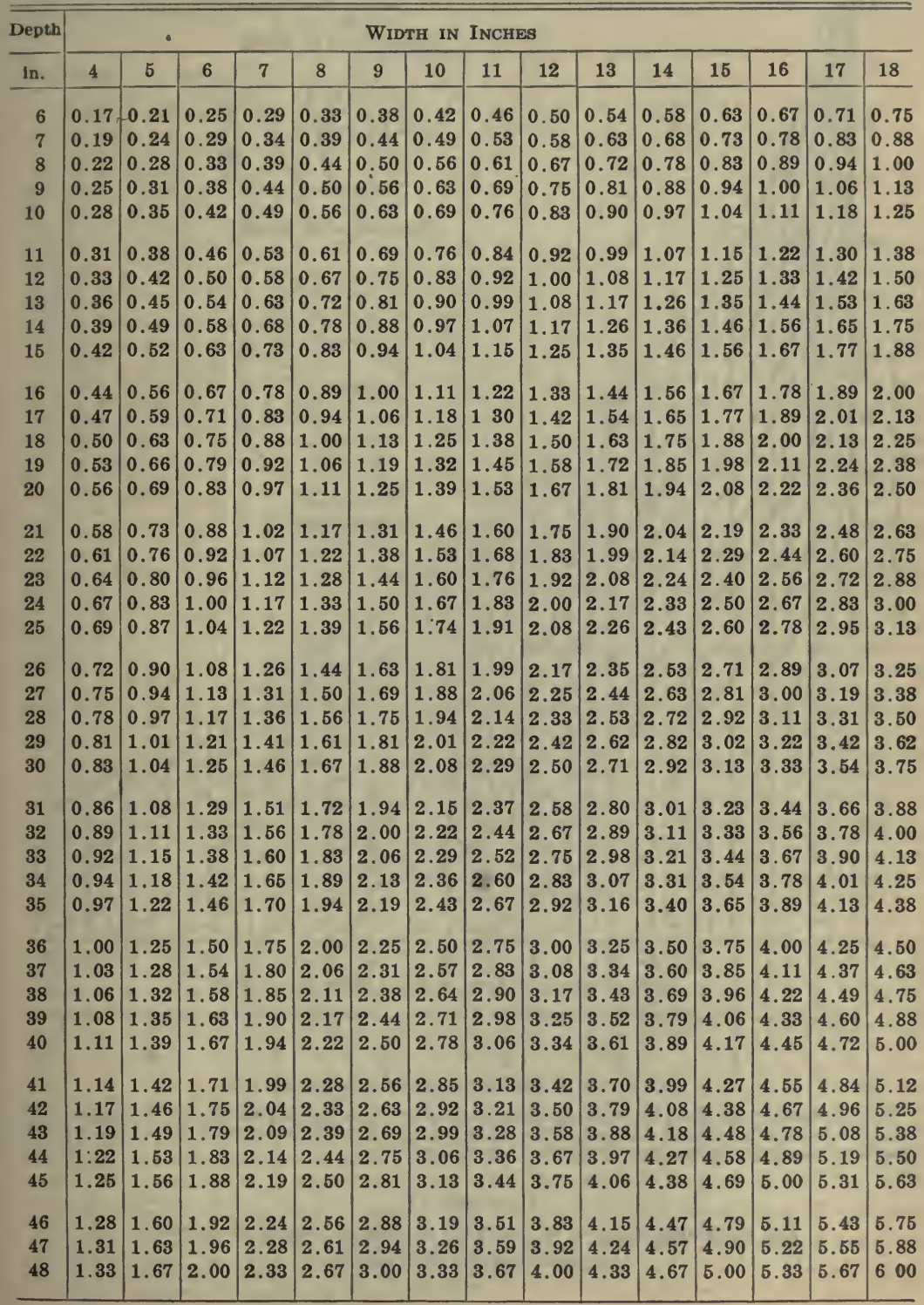




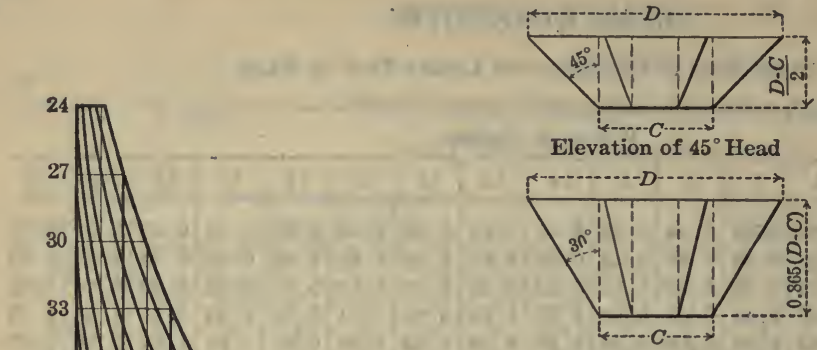

Elevation of $20^{\circ} \mathrm{Head}$

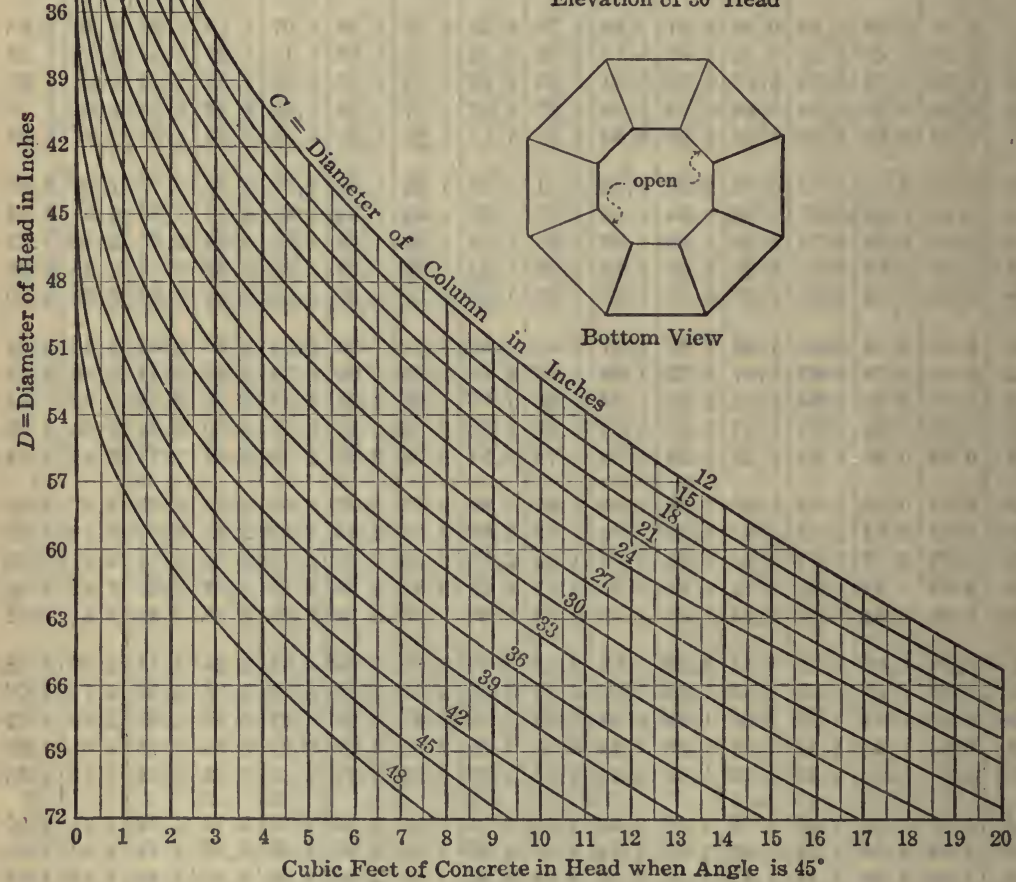

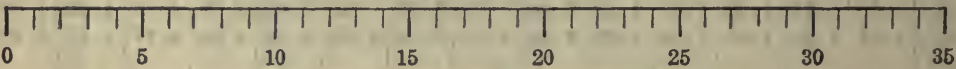

Cubic Feet of Concrete in Head when Angle is $30^{\circ}$

The above diagram gives the volume of the ring of concrete forming the head. Volumes given are for octagonal heads and columns. For square heads and columns multiply the volume by 1.21 For round heads and columns multiply the volume by 0.95 .

\section{Diagram 17}

For obtaining volume of concrete in column heads of columns supporting flat slab floors. 
QUANTITIES OF MATERIALS FOR ONE CUBIC YARD OF RAMMED CONCRETE BASED ON A BARREL OF 3.8 CUBIC FEET

The following table glves the quantitles of materials required for one yard of concrete. The results given have been taken from a simliar table in Concrete Plain and Relnforced by Taylor and Thompson, with tho author's permission, to use this copyrlghted matter.

\begin{tabular}{|c|c|c|c|c|c|c|c|c|c|c|c|c|c|c|c|c|c|}
\hline \multirow{2}{*}{\multicolumn{3}{|c|}{$\begin{array}{c}\text { Proportions } \\
\text { by } \\
\text { Parts }\end{array}$}} & \multirow{2}{*}{\multicolumn{3}{|c|}{$\begin{array}{c}\text { Proportlons } \\
\text { by } \\
\text { Volumes }\end{array}$}} & \multirow{2}{*}{\multicolumn{6}{|c|}{ Percentages }} & \multirow{2}{*}{\multicolumn{3}{|c|}{\begin{tabular}{|c|} 
BROKEN STONE OR \\
$\begin{array}{c}40 \% \\
\text { Gravel or } \\
\text { Mlxed }\end{array}$
\end{tabular}}} & \multicolumn{3}{|c|}{ GRA VEL } \\
\hline & & & & & & & & & & & & & & & & ed & \\
\hline & \multirow{2}{*}{ 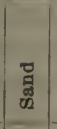 } & \multirow{2}{*}{ 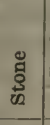 } & 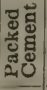 & 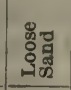 & 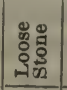 & Еี & 总 & 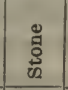 & 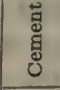 & 맳 & 苛 & 嵌 & 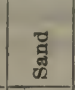 & : & 嵌 & 司 & $\begin{array}{l}0 \\
\text { ¿ } \\
\text { ט. }\end{array}$ \\
\hline & & & Bbl. & t. & Cu. & Bы. & Cu Yd. & C & Bbl. & u. Yd. & Cu.Yd. & bl. & . & c & bl. & 1. & $\mathrm{y}$ \\
\hline 1 & 1 & 1. & 1 & .8 & 5.7 & 3. & 0.45 & 7 & 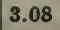 & & 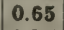 & 97 & & .63 & . & 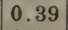 & .59 \\
\hline 1 & 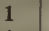 & 2 & 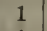 & & & & & & & & & & & & & & \\
\hline 1 & 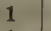 & 2.5 & 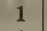 & 98 & 9.5 & & & & & & & & & & & & 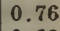 \\
\hline 1 & 1 & 3 & 1 & 3. & 11.4 & 2. & 0 & & & & & & & & & & .8 \\
\hline 1 & 1.5 & 2 & 1 & & & 2.49 & 0 . & & & & & & & & & & .6 \\
\hline 1 & .5 & .5 & 1 & & 9.5 & & & & & & & & & & & & 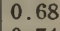 \\
\hline 1 & 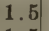 & 3 & 1 & & 1.4 & & & & & & & & & & & & .7 \\
\hline 1 & 1.5 & 3.5 & 1 & & 13.3 & & & & & & & & & & & & .75 \\
\hline & 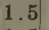 & 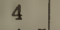 & 1 & & 15.2 & & & & & & & & & & & & 0 \\
\hline & 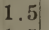 & 4 & & & 7.1 & & & & & & & & & & & & 0 \\
\hline & .5 & 5 & 1 & & & & & & & & & & & & & & 0.9 \\
\hline & & 3 & 1 & & 11.4 & 1. & & & & & & & & 0.74 & & & 0.68 \\
\hline & & ? & 1 & & & & & & & & & & & & & & 0.7 \\
\hline & & A & 1 & & & 1. & & & & & & & & & & & .7 \\
\hline & & 4. & 1 & 7. & 17.1 & 1. & 0. & & & & & & & 0.89 & .28 & & 0.81 \\
\hline & & E & 1 & & & & & & & & & & & 0.93 & 20 & & 0.8 \\
\hline & & & 1 & & & & & & & & & & & & & & 0.8 \\
\hline & & 6 & 1 & 7 & 8 & 1. & & & & & & & & & & & .8 \\
\hline & .5 & 3 & 1 & 9 & .4 & 1.72 & 0. & & & & & & & 8 & & & 0.6 \\
\hline & & & 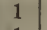 & & & 1. & & & & & & & & & & & 0.6 \\
\hline & & 4 & 1 & & & 1. & & & & & & & & & .29 & & 7 \\
\hline & 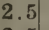 & 4. & 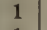 & 9 & 1 & 1. & & & & & & & & 33 & .20 & & 0.76 \\
\hline 1 & 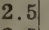 & & 1 & & & 1. & 0. & & & & & & & 0. & 13 & & 0.80 \\
\hline & & .5 & 1 & & & & & & & & & & & & & & \\
\hline & & 0 & 1 & & & 1. & & & & & & & & & & & 0.8 \\
\hline 1 & 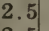 & 6 & 1 & & & 1.18 & & & & & & & & 0.97 & .96 & & 0.8 \\
\hline & & & 1 & & & 1.1 & & & & & & & & & & & 0.9 \\
\hline & & 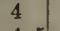 & 1 & 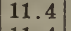 & & 1.42 & & & & & & & & & & & \\
\hline & & 4. & 1 & & & 1. & & & & & & & & & 13 & & 0.72 \\
\hline 1 & & 5 & 1 & & & 1.28 & & & & & & 7 & & 0.82 & & & 0.7 \\
\hline 1 & & 5. & 1 & & & 1. & & & & & & 1 & & & & & \\
\hline 1 & & 6 & 1 & & & & & & & & & & & & & & .81 \\
\hline 1 & & 6. & 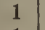 & & & & & & & & & & & & 92 & & 0.84 \\
\hline 1 & & . & 1 & & & & & & & & & & & & & & . \\
\hline 1 & & 7.5 & 1 & & & & & & & & & & & & 3 & & \\
\hline 1 & & 8 & 1 & & & & & & & & & & & & 30 & & 0.90 \\
\hline 1 & & 5 & 1 & & & & & & & & & & & & & & \\
\hline 1 & & 6 & 1 & & & & & & & & & & & & & & \\
\hline 3 & & . & 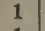 & & & & & & & & & & & & & & 0.79 \\
\hline 1 & & 8 & 1 & & & & & & & & & & & & 0.74 & & 0.83 \\
\hline 1 & & 0 & 1 & & & & & & & & & & & & & & \\
\hline 1 & 4 & & 1 & & & & & & & & & & & & & & \\
\hline 1 & 5 & 10 & 1 & & .0 & & & & & & & & & & & & 0.84 \\
\hline 1 & & & 1 & 2.8 & 5.5 & & 0.52 & 1.04 & 58 & 0.20 & 0.98 & 0.56 & $0: 47$ & 0.94 & 0.50 & 0.42 & 0.84 \\
\hline
\end{tabular}

Notk.-Variations in the fineness of the sand and the compacting of the concrete may affect the quantitles by $10 \%$ in either direction.

Use $45 \%$ column for average conditions and for broken stone with dust sereened out.

Use $50 \%$ column for broken stone screened to uniform size

Use $40 \%$ column for gravel or mixed stone and gravel.

Use $30 \%$ column for scientifically graded mixtures. 


\section{BARS}

One of the assumptions always made in connection with the design of reinforced concrete structures is that the steel and concrete are so intimately united by means of the bond that the two materials act together as a single new material. For many years it was insisted upon that the adhesion between concrete and plain bars was sufficient, but as the art of reinforced concrete construction developed the sufficiency of this adhesion began to be questioned and various methods were devised, such as hooking or splitting the ends of the bars, to prevent their slipping in the concrete. Such methods are but makeshifts at best, as bond to be effective must be continuous, and in practically all reinforced concrete designs of to-day the demand is for a deformed bar of proper design - a bar that grips the concrete in a positive manner by means of projecting ribs normal to the direction of stress.

The design tables appearing in this book are based on the employment of a properly designed deformed bar, and their use in connection with other types of bars is not recommended.

\section{CORRUGATED ROUNDS}

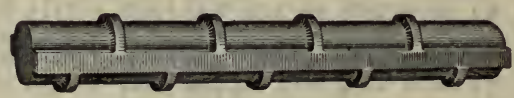

Standard Sizes

\begin{tabular}{l|c|c|c|c|c|c|c|c}
\hline \hline Size IN INCHES . . . . & $3 / 8$ & $1 / 2$ & $5 / 8$ & $3 / 4$ & $7 / 8$ & 1 & $11 / 8$ & $11 / 4$ \\
\hline Net Area in Square Inches . & 0.11 & 0.19 & 0.30 & 0.44 & 0.60 & 0.78 & 0.99 & 1.22 \\
Weight per Foot in Pounds. & 0.38 & 0.66 & 1.05 & 1.52 & 2.06 & 2.69 & 3.41 & 4.21 \\
Perimeter in Inches . . . & 1.23 & 1.66 & 2.10 & 2.53 & 2.95 & 3.36 & 3.80 & 4.23 \\
\hline
\end{tabular}

\section{CORRUGATED SQUARES}

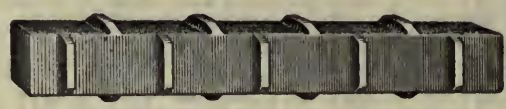

Standard Sizes

\begin{tabular}{l|c|c|c|c|c|c|c|c|c}
\hline \hline Stze IN INCHES . . . . . & $1 / 4$ & $3 / 8$ & $1 / 2$ & $5 / 8$ & $3 / 4$ & $7 / 8$ & 1 & $11 / 8$ & $11 / 4$ \\
\hline Net Area in Square Inches . & 0.06 & 0.14 & 0.25 & 0.39 & 0.56 & 0.76 & 1.00 & 1.26 & 1.55 \\
Weight per Foot in Pounds. & 0.22 & 0.49 & 0.86 & 1.35 & 1.94 & 2.64 & 3.43 & 4.34 & 5.35 \\
Perimeter in Inches . . . & 1.00 & 1.50 & 2.00 & 2.50 & 3.00 & 3.50 & 4.00 & 4.50 & 5.00 \\
\hline
\end{tabular}


Specifications. Purchasers can greatly influence the prompt shipment of orders for ordinary "mill shipment" by considering, in the preparation of their specifications and material bills, the factors connected with the methods and internal organization of a steel mill.

Adherence to the Manufacturers' Standard Specifications for Deformed Concrete Reinforcing Bars (see page 188), will always facilitate prompt shipment. It is, of course, possible to furnish any class of material that is within the power of the mill to roll, but where specifications are in any way special the entire order must be made from special heats. The process is one out of the ordinary routine of the mill, billets already prepared cannot be used, and delay in the filling of the order is certain.

Sizes. It is necessary in the rolling of steel bars, for a mill to finish rolling all of the bars on its schedule of one particular size before changing the rolls for other sizes of bars. In mill parlance, what is known as a "rolling" extends over a period of several days and an order containing a large number of sizes might be compelled to remain in the mill until the completion of the entire rolling, so that where quick shipment is desired, the number of sizes on an order should be kept as low as possible.

In addition to confining the order to as few sizes as may be consistent with the requirements, every endeavor should be made to avoid specifying bars in $\frac{1}{16}$ th sizes. This is an error frequently made by inexperienced designers in an effort to meet a theoretical steel area required by their calculations, and can only result in delay at the mill and increased labor and confusion in the field through the necessity of handling a multiplicity of bars of slightly varying size.

Lengths. In ordinary mill practice the bars are rolled to lengths varying from 100 to 300 feet and sheared into lengths called for by the material bills as they come from the rolls. When, however, the number of lengths are very large and where there are only a small number of bars of one length, the shearing cannot be done as fast as the bars are rolled, consequently the bars must be laid to one side and sheared after the conclusion of the rolling in order that the operation of the mill may not be delayed. It is, therefore, always desirable to keep the number of lengths as low as possible where quick shipment is a necessary requirement.

The lengths should always be given to the nearest inch as bars are not ordinarily sheared to a greater degree of accuracy. Where it is important that the length called for be exact, a note to this effect should be placed opposite the item on the order.

Fabrication. For all reinforced concrete structures there is usually a considerable amount of fabricated reinforcement to be furnished. Sometimes this fabrication is done in the field but through the use of special machinery and methods of operation all classes of bending and other fabrication of bar reinforcement can be accomplished with greater accuracy and advantage in the shop than in the field, and in the majority of instances it is advisable for the purchaser to specify "shop fabrication."

Accompanying all orders for fabricated material there should be furnished in addition to the list of number of pieces, size and length of bar, a sketch of each differently fabricated piece with the dimensions plainly marked thereon. A few of the details 
most frequently encountered in practice are given below, showing in each case the detail dimensions required by the shop.
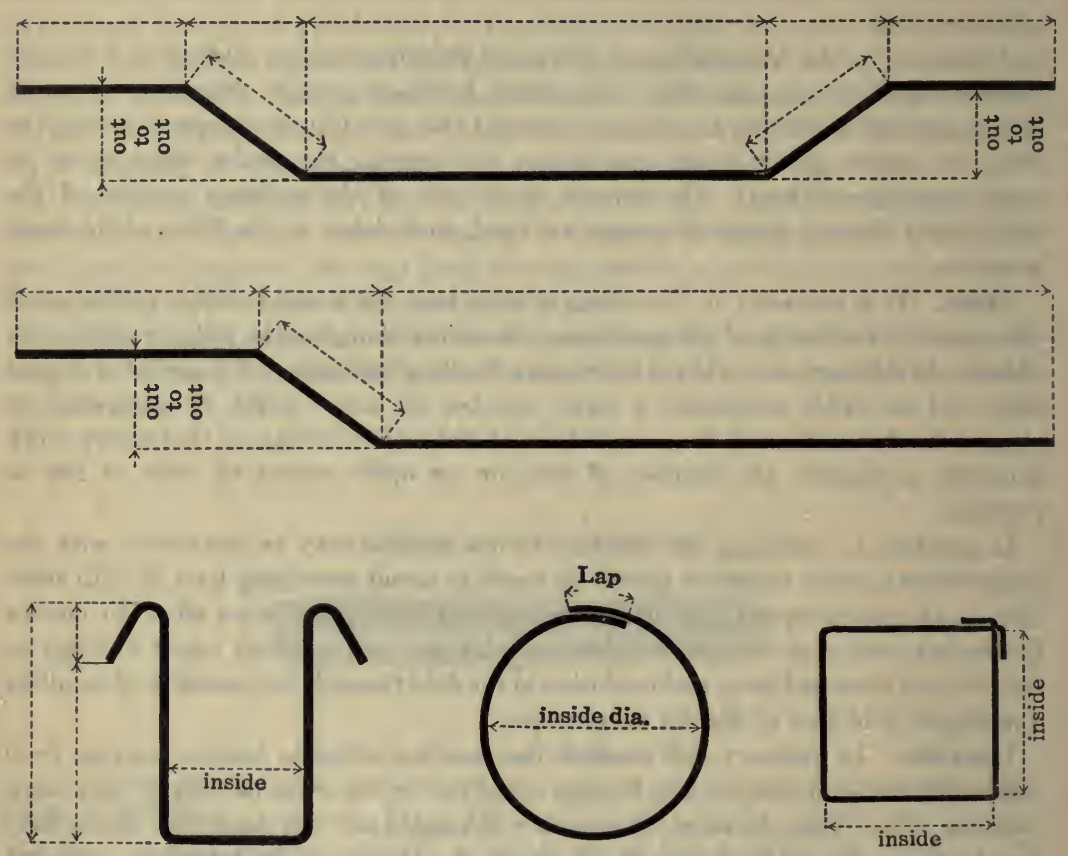
AMERICAN STEEL AND WIRE CO.'S STEEL AND IRON WIRE GAUGE AND DIFFERENT SIZES OF WIRE

\begin{tabular}{|c|c|c|c|c|c|c|}
\hline $\begin{array}{l}\text { Diameter } \\
\text { Inches }\end{array}$ & $\begin{array}{l}\text { Steel Wire } \\
\text { Gauge }\end{array}$ & $\begin{array}{l}\text { Diameter } \\
\text { Inches }\end{array}$ & $\begin{array}{c}\text { Area, Square } \\
\text { Inches }\end{array}$ & $\begin{array}{l}\text { Pounds } \\
\text { per Foot }\end{array}$ & $\begin{array}{c}\text { Feet } \\
\text { per Pound }\end{array}$ & $\begin{array}{c}\text { Feet per } \\
2,000 \mathrm{Lbs} .\end{array}$ \\
\hline $\begin{array}{l}1 / 2 \\
\frac{15}{32} \sim\end{array}$ & $\frac{7}{0}$ & $\begin{array}{l}0.500 \\
0.490 \\
0.468\end{array}$ & $\begin{array}{l}0.19635 \\
0.18857 \\
0.17202\end{array}$ & $\begin{array}{l}0.6625 \\
0.6363 \\
0.5804\end{array}$ & $\begin{array}{l}1.50 \\
1.51 \\
1.72\end{array}$ & $\begin{array}{l}3018 \\
3023 \\
3445\end{array}$ \\
\hline$\frac{7}{16}$ & $\begin{array}{l}\frac{6}{0} \\
\frac{5}{8}\end{array}$ & $\begin{array}{l}0.460 \\
0.437 \\
0.430\end{array}$ & $\begin{array}{l}0.16619 \\
0.14998 \\
0.14532\end{array}$ & $\begin{array}{l}0.5608 \\
0.5061 \\
0.4901\end{array}$ & $\begin{array}{l}1.78 \\
1.97 \\
2.04\end{array}$ & $\begin{array}{l}3566 \\
3952 \\
4081\end{array}$ \\
\hline $3 / 8$ & $\begin{array}{l}4 \\
0\end{array}$ & $\begin{array}{l}0.406 \\
0.393 \\
0.375\end{array}$ & $\begin{array}{l}0.12946 \\
0.12130 \\
0.11044\end{array}$ & $\begin{array}{l}0.4368 \\
0.4094 \\
0.3726\end{array}$ & $\begin{array}{l}2.28 \\
2.44 \\
2.68\end{array}$ & $\begin{array}{l}4578 \\
4885 \\
5367\end{array}$ \\
\hline$\frac{11}{32}$ & $\begin{array}{l}\frac{8}{0} \\
\frac{2}{0}\end{array}$ & $\begin{array}{l}0.362 \\
0.343 \\
0.331\end{array}$ & $\begin{array}{l}0.10292 \\
0.09240 \\
0.08604\end{array}$ & $\begin{array}{l}0.3473 \\
0.3117 \\
0.2904\end{array}$ & $\begin{array}{l}2.87 \\
3.20 \\
3.44\end{array}$ & $\begin{array}{l}5758 \\
6412 \\
6887\end{array}$ \\
\hline$\frac{5}{16}$ & $\begin{array}{l}0 \\
1\end{array}$ & $\begin{array}{l}0.312 \\
0.307 \\
0.283\end{array}$ & $\begin{array}{l}0.07645 \\
0.07402 \\
0.06290\end{array}$ & $\begin{array}{l}0.2579 \\
0.2497 \\
0.2123\end{array}$ & $\begin{array}{l}3.87 \\
4.00 \\
4.71\end{array}$ & $\begin{array}{l}7755 \\
8011 \\
9420\end{array}$ \\
\hline $\begin{array}{l}\frac{2}{32} \\
1 / 4\end{array}$ & 2 & $\begin{array}{l}0.281 \\
0.263 \\
0.250\end{array}$ & $\begin{array}{l}0.06210 \\
0.05432 \\
0.04908\end{array}$ & $\begin{array}{l}0.2092 \\
0.1834 \\
0.1656\end{array}$ & $\begin{array}{l}4.78 \\
5.45 \\
6.03\end{array}$ & $\begin{array}{r}9560 \\
10905 \\
12077\end{array}$ \\
\hline$\frac{7}{32}$ & $\begin{array}{l}3 \\
4\end{array}$ & $\begin{array}{l}0.244 \\
0.225 \\
0.218\end{array}$ & $\begin{array}{l}0.04675 \\
0.03976 \\
0.03732\end{array}$ & $\begin{array}{l}0.1578 \\
0.1342 \\
0.1259\end{array}$ & $\begin{array}{l}6.33 \\
7.45 \\
7.94\end{array}$ & $\begin{array}{l}12674 \\
14903 \\
15885\end{array}$ \\
\hline$\frac{3}{16}$ & $\begin{array}{l}5 \\
6\end{array}$ & $\begin{array}{l}0.207 \\
0.192 \\
0.187\end{array}$ & $\begin{array}{l}0.03365 \\
0.02895 \\
0.02746\end{array}$ & $\begin{array}{l}0.1135 \\
0.0977 \\
0.0926\end{array}$ & $\begin{array}{r}8.81 \\
10.23 \\
10.79\end{array}$ & $\begin{array}{l}17621 \\
20471 \\
21598\end{array}$ \\
\hline$\frac{5}{32}$ & $\begin{array}{l}7 \\
8\end{array}$ & $\begin{array}{l}0.177 \\
0.162 \\
0.156\end{array}$ & $\begin{array}{l}0.02460 \\
0.02061 \\
0.01911\end{array}$ & $\begin{array}{l}0.0830 \\
0.0696 \\
0.0644\end{array}$ & $\begin{array}{l}12.04 \\
14.36 \\
15.52\end{array}$ & $\begin{array}{l}24096 \\
28735 \\
31056\end{array}$ \\
\hline $1 / 8$ & $\begin{array}{r}9 \\
10\end{array}$ & $\begin{array}{l}0.148 \\
0.135 \\
0.125\end{array}$ & $\begin{array}{l}0.01720 \\
0.01431 \\
0.01227\end{array}$ & $\begin{array}{l}0.0580 \\
0.0483 \\
0.0414\end{array}$ & $\begin{array}{l}17.24 \\
20.70 \\
24.15\end{array}$ & $\begin{array}{l}34482 \\
41408 \\
48309\end{array}$ \\
\hline$\frac{3}{32}$ & $\begin{array}{l}11 \\
12\end{array}$ & $\begin{array}{l}0.120 \\
0.105 \\
0.093\end{array}$ & $\begin{array}{l}0.01130 \\
0.00865 \\
0.00679\end{array}$ & $\begin{array}{l}0.0382 \\
0.0292 \\
0.0229\end{array}$ & $\begin{array}{l}26.17 \\
34.24 \\
43.66\end{array}$ & $\begin{array}{l}52356 \\
68493 \\
87336\end{array}$ \\
\hline & $\begin{array}{l}13 \\
14 \\
15 \\
16\end{array}$ & $\begin{array}{l}0.092 \\
0.080 \\
0.072 \\
0.063\end{array}$ & $\begin{array}{l}0.00664 \\
0.00502 \\
0.00407 \\
0.00311\end{array}$ & $\begin{array}{l}0.0224 \\
0.0169 \\
0.0137 \\
0.0105\end{array}$ & $\begin{array}{l}44.64 \\
59.17 \\
72.99 \\
95.23\end{array}$ & $\begin{array}{r}89286 \\
118343 \\
145985 \\
190476\end{array}$ \\
\hline
\end{tabular}




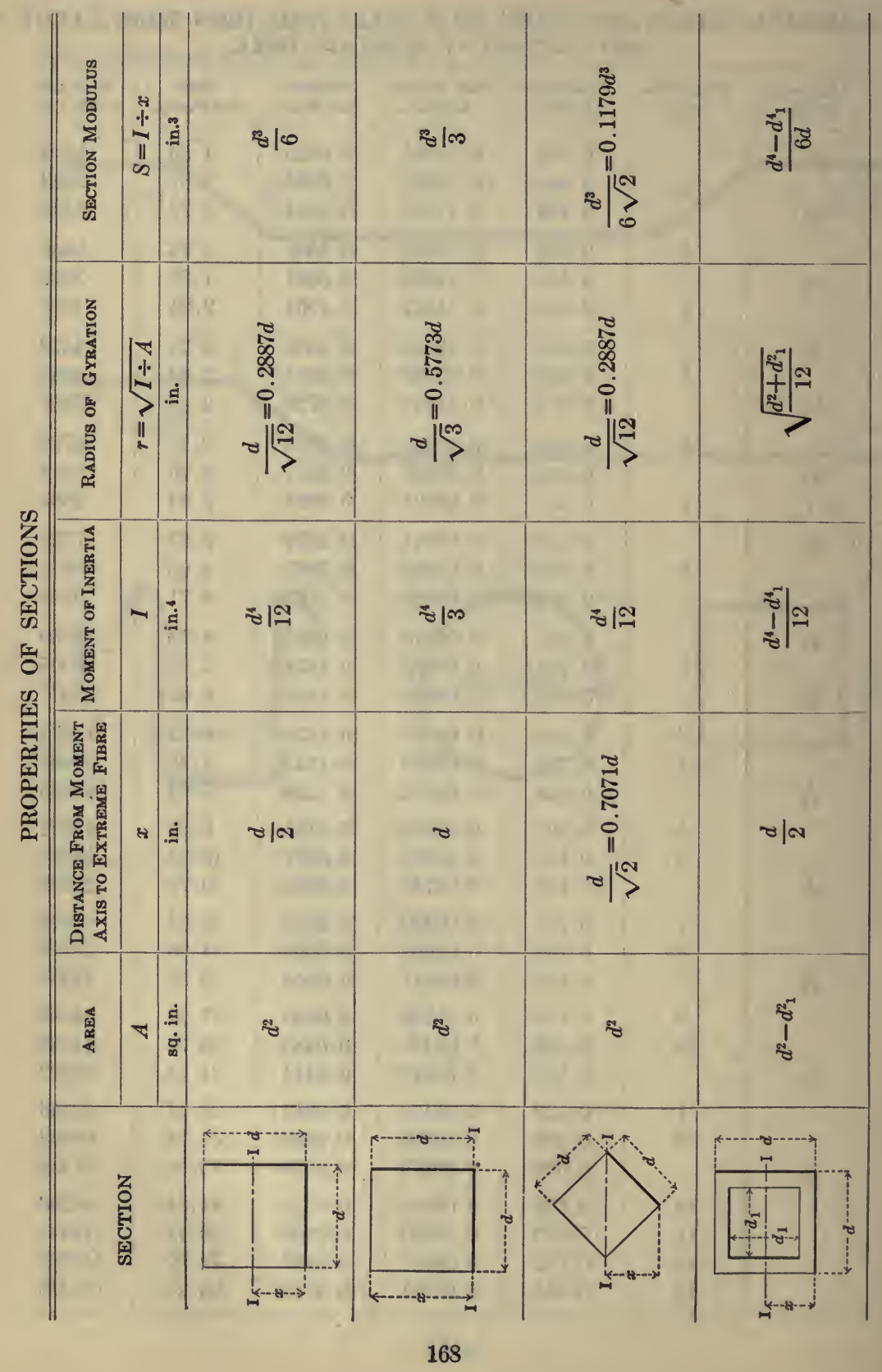




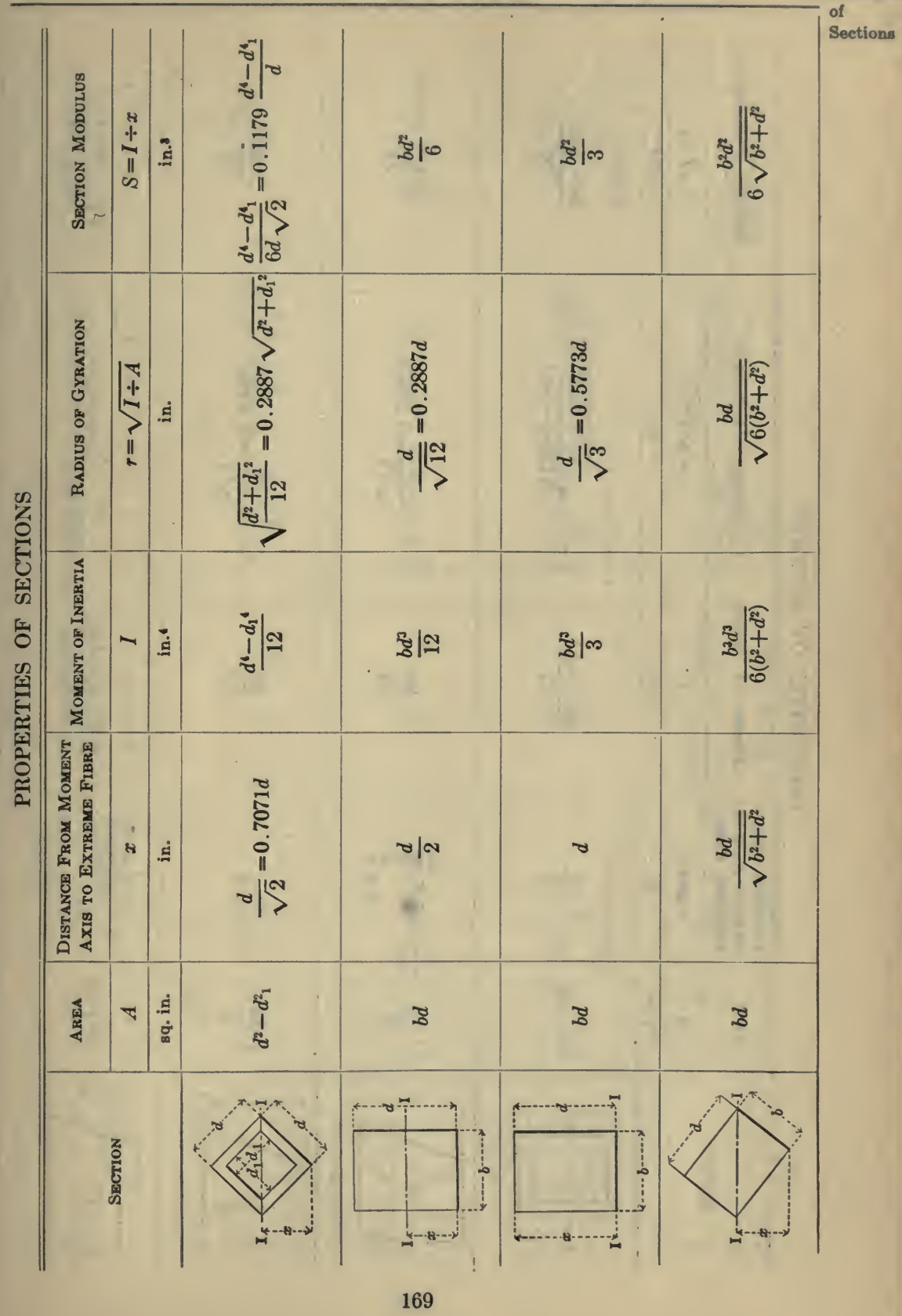




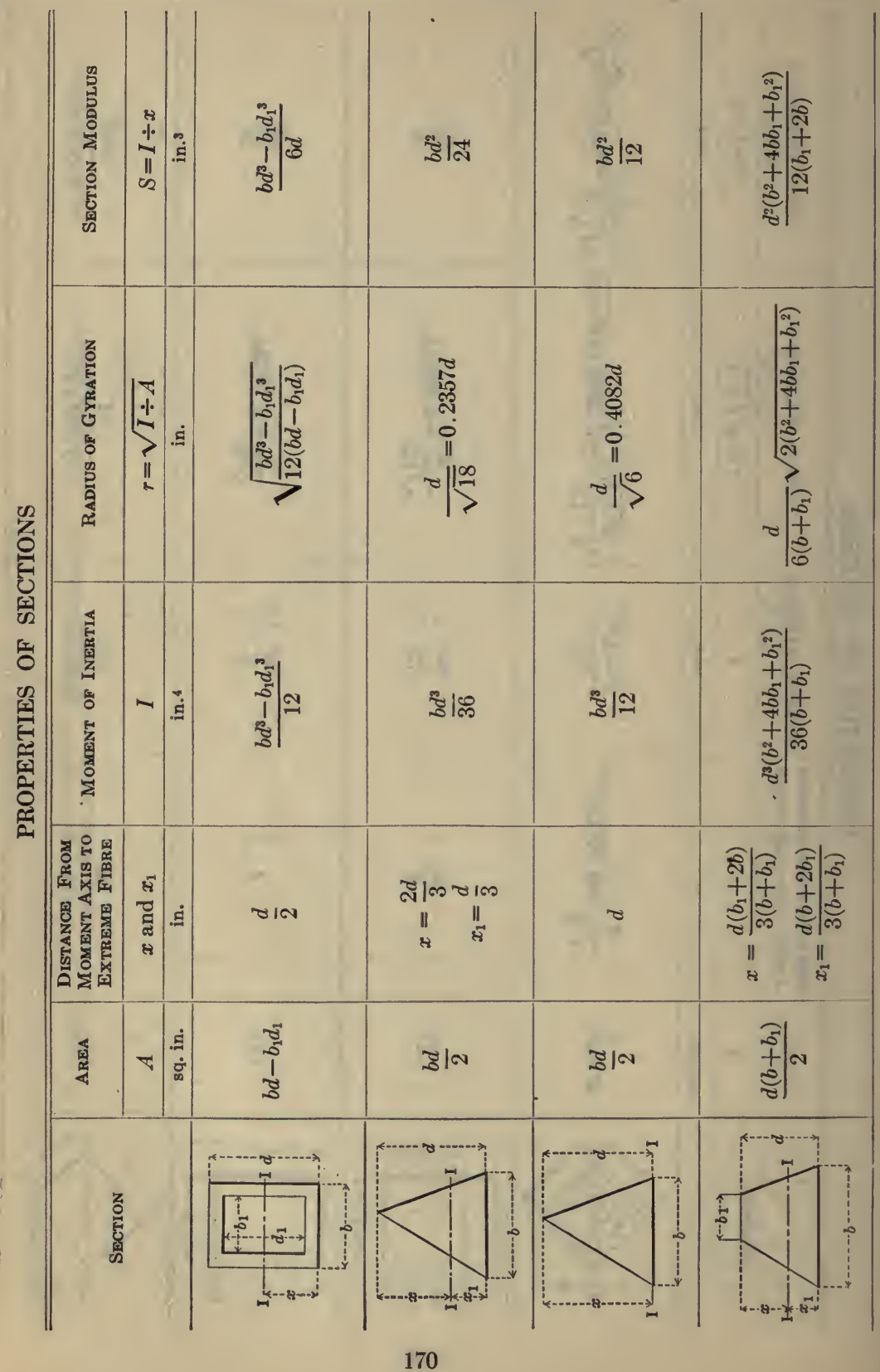




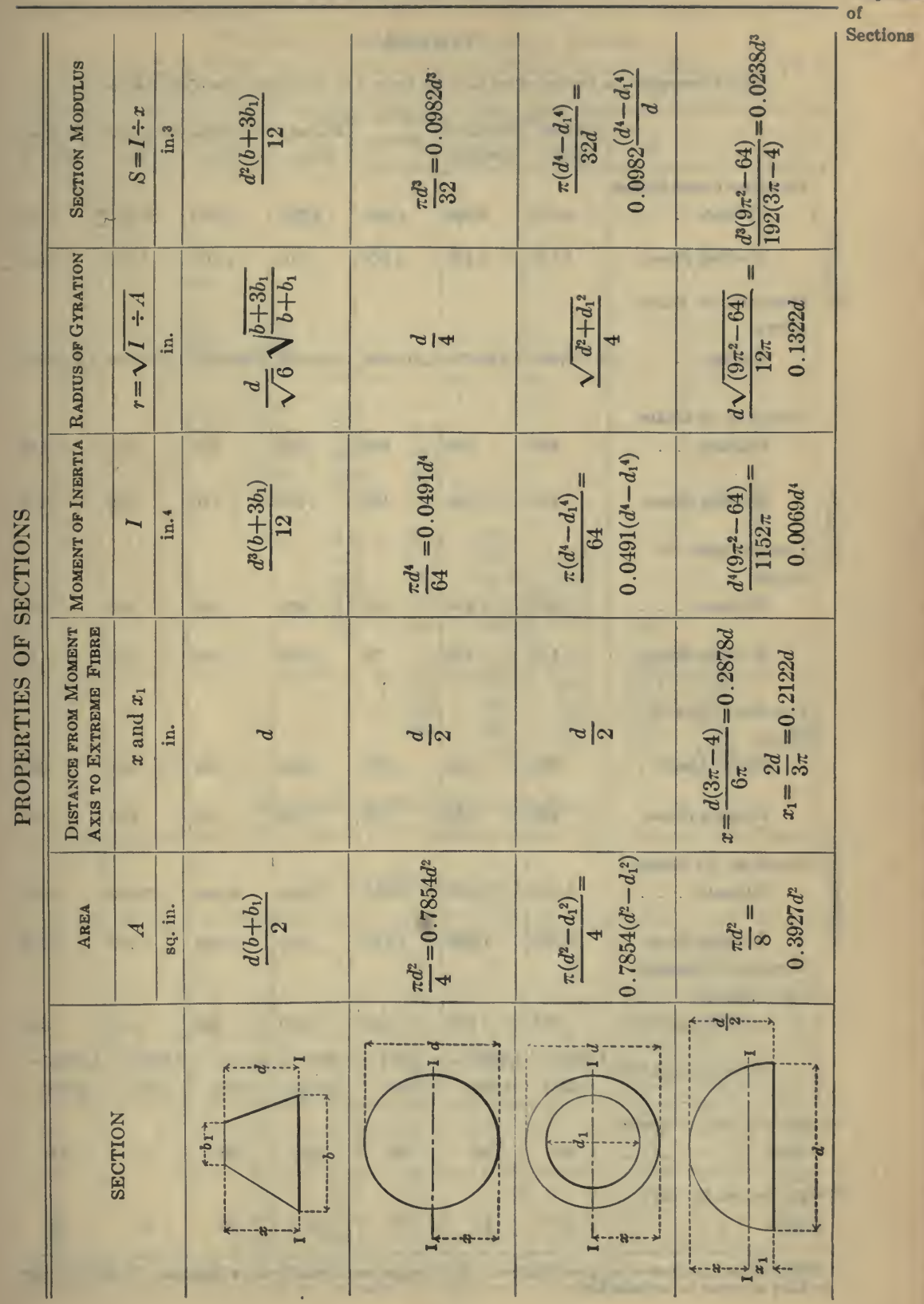




\section{TIMBER}

Unit Stresses In Pounds Per Square Inch And Weights Per Cubic Foot

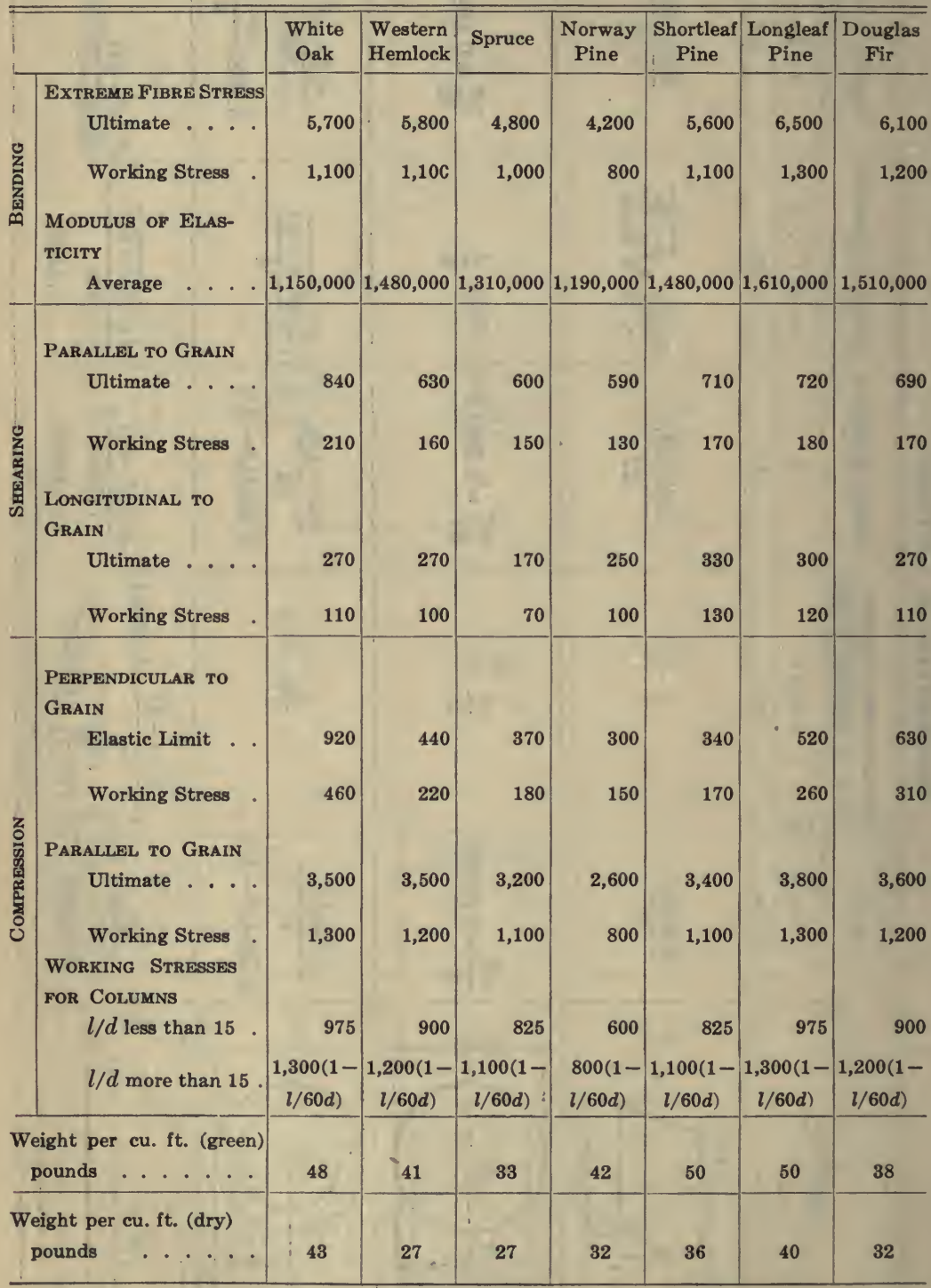

The stresses given are for green timber. For temporary structures an increase of $50 \%$ in the working stresses is permissible. 


\section{WOODEN BEAMS-UNIFORMLY LOADED}

Loads given are total loads for a beam one inch thick and for a maximum bending stress of 1,000 pounds per square inch.

\begin{tabular}{|c|c|c|c|c|c|c|c|c|c|c|}
\hline \multirow{2}{*}{$\begin{array}{c}\text { Span } \\
\text { in } \\
\text { Feet }\end{array}$} & \multicolumn{10}{|c|}{ DEPTH OF BEAM IN INCHES } \\
\hline & 2 & 4 & 6 & 8 & 10 & 12 & 14 & 16 & 18 & 20 \\
\hline $\begin{array}{l}2 \\
3 \\
4 \\
5\end{array}$ & $\begin{array}{r}187 \\
148 \\
-111 \\
89\end{array}$ & 356 & & & & & & & & \\
\hline $\begin{array}{r}6 \\
7 \\
8 \\
9 \\
10\end{array}$ & $\begin{array}{l}74 \\
63 \\
56\end{array}$ & $\begin{array}{l}296 \\
254 \\
222 \\
198 \\
178\end{array}$ & $\begin{array}{l}500 \\
444 \\
400\end{array}$ & 711 & & & & & & . \\
\hline $\begin{array}{l}11 \\
12 \\
13 \\
14 \\
15\end{array}$ & & $\begin{array}{l}162 \\
148\end{array}$ & $\begin{array}{l}364 \\
333 \\
308 \\
286 \\
267\end{array}$ & $\begin{array}{l}646 \\
593 \\
547 \\
508 \\
474\end{array}$ & $\begin{array}{l}926 \\
855 \\
794 \\
741\end{array}$ & 1067 & I & n & $\frac{1}{4}$ & \\
\hline $\begin{array}{l}16 \\
17 \\
18 \\
19 \\
20\end{array}$ & & & 250 & $\begin{array}{l}444 \\
418 \\
395 \\
374 \\
356\end{array}$ & $\begin{array}{l}694 \\
654 \\
617 \\
585 \\
556\end{array}$ & $\begin{array}{r}1000 \\
941 \\
889 \\
842 \\
800\end{array}$ & $\begin{array}{l}1281 \\
1210 \\
1146 \\
1089\end{array}$ & 1422 & $\frac{11}{45}$ & 1 \\
\hline $\begin{array}{l}21 \\
22 \\
23 \\
24 \\
25\end{array}$ & & & 18 & $=$ & $\begin{array}{l}529 \\
505 \\
483 \\
463\end{array}$ & $\begin{array}{l}762 \\
727 \\
696 \\
667 \\
640\end{array}$ & $\begin{array}{r}1037 \\
990 \\
947 \\
907 \\
871\end{array}$ & $\begin{array}{l}1354 \\
1293 \\
1237 \\
1185 \\
1138\end{array}$ & $\begin{array}{l}1636 \\
1565 \\
1500 \\
1440\end{array}$ & $\begin{array}{l}1852 \\
1778\end{array}$ \\
\hline
\end{tabular}

SQUARE WOODEN COLUMNS

Loads given are in thousands of pounds for a working stress parallel to the grain of 1,000 pounds per square inch.

$$
P=1,000\left(1-\frac{l}{60 d}\right)
$$

\begin{tabular}{c|c|c|c|c|c|c|c|c|c|c}
\hline \hline $\begin{array}{c}\text { Height } \\
\text { of } \\
\text { Column }\end{array}$ & \multicolumn{10}{c}{ Side OF SquARE IN INCHES } \\
\cline { 2 - 11 } & 4 & 6 & 8 & 10 & 12 & 14 & 16 & 18 & 20 & $\mathbf{2 4}$ \\
\hline 5 & 12.0 & 27.0 & 48.0 & 75.0 & 108.0 & 147.0 & 192.0 & 243.0 & 300.0 & 432.0 \\
6 & 11.2 & 27.0 & 48.0 & 75.0 & 108.0 & 147.0 & 192.0 & 243.0 & 300.0 & 432.0 \\
7 & 10.4 & 27.0 & 48.0 & 75.0 & 108.0 & 147.0 & 192.0 & 243.0 & 300.0 & 432.0 \\
8 & 9.6 & 26.4 & 48.0 & 75.0 & 108.0 & 147.0 & 192.0 & 243.0 & 300.0 & 432.0 \\
9 & 8.8 & 25.2 & 48.0 & 75.0 & 108.0 & 147.0 & 192.0 & 243.0 & 300.0 & 432.0 \\
10 & 8.0 & 24.0 & 48.0 & 75.0 & 108.0 & 147.0 & 192.0 & 243.0 & 300.0 & 432.0 \\
11 & & 22.8 & 46.4 & 75.0 & 108.0 & 147.0 & 192.0 & 243.0 & 300.0 & 432.0 \\
12 & & 21.6 & 44.8 & 75.0 & 108.0 & 147.0 & 192.0 & 243.0 & 300.0 & 432.0 \\
14 & & 19.2 & 41.6 & 72.0 & 108.0 & 147.0 & 192.0 & 243.0 & 300.0 & 432.0 \\
16 & & & 38.4 & 68.0 & 105.6 & 147.0 & 192.0 & 243.0 & 300.0 & 432.0 \\
18 & & & 35.2 & 64.0 & 100.8 & 145.6 & 192.0 & 243.0 & 300.0 & 432.0 \\
20 & & & 32.0 & 60.0 & 96.0 & 140.0 & 192.0 & 243.0 & 300.0 & 432.0 \\
\hline
\end{tabular}

To obtain the carrying capacity of beams or columns where the unit stress is other than 1,000 pounds per square inch, increase or decrease the table loads proportionately. 


\section{AREAS OF CIRCULAR SEGMENTS}

\section{For Ratios of Rise and Chord}

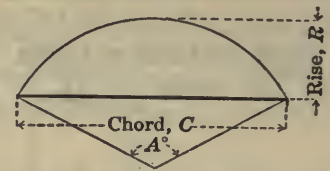

Area $=C \times R \times$ Coefficient

\begin{tabular}{|c|c|c|c|c|c|c|c|c|c|c|c|}
\hline$A^{\circ}$ & $\begin{array}{l}\text { Coeffi- } \\
\text { cient }\end{array}$ & $\frac{R}{C}$ & $A^{\circ}$ & $\begin{array}{c}\text { Coeffi- } \\
\text { cient }\end{array}$ & $\frac{R}{C}$ & $A^{\circ}$ & $\begin{array}{l}\text { Coeffi- } \\
\text { cient }\end{array}$ & $\frac{R}{C}$ & $A^{\circ}$ & $\begin{array}{c}\text { Coeffi- } \\
\text { cient }\end{array}$ & $\frac{R}{C}$ \\
\hline 1 & 6667 & 0.0022 & 46 & 6722 & 0.1017 & 91 & 6895 & 0.2097 & 136 & 7239 & 0.3373 \\
\hline 2 & 667 & 0.0044 & 47 & 6724 & 0.1040 & . & 6901 & 0.2122 & 137 & 0.7249 & 0.3404 \\
\hline 3 & 0.6667 & 0.0066 & 48 & 6727 & 0.1063 & 93 & 0.6906 & 0.2148 & 138 & 0.7260 & 0.3436 \\
\hline 4 & 0.6667 & 0.0087 & 49 & 6729 & 0.1086 & 94 & 0.6912 & 0.2174 & 139 & 0.7270 & 0.3469 \\
\hline 5 & 0.6667 & 0.0109 & 50 & 0.6732 & 0.1109 & 95 & 0.6918 & 0.2200 & 140 & 0.7281 & 0.3501 \\
\hline 6 & 0.6667 & 0.0131 & 51 & 6734 & 0.1131 & 96 & 6924 & 0.2226 & 141 & 7292 & 0.3534 \\
\hline 7 & 0.6668 & 0.0153 & 52 & 3737 & 0.1154 & 97 & 6930 & 0.2252 & 142 & 303 & 0.3567 \\
\hline 8 & 0.6668 & 0.0175 & 53 & 6740 & 0.1177 & 98 & 0.6936 & 0.2279 & & 314 & 0.3600 \\
\hline 9 & 0.6669 & 0.0197 & 54 & 6743 & 0.1200 & 99 & 6942 & 0.2305 & 144 & 325 & 0.3633 \\
\hline 10 & 0.6670 & 0.0218 & 55 & 3746 & 0.1224 & 100 & 0.6948 & 0.2332 & 145 & 0.7336 & 0.3666 \\
\hline 11 & 0.6670 & 0.0240 & 56 & 6749 & 0.1247 & 101 & 3954 & 0.2358 & 146 & & 0.3700 \\
\hline 12 & 671 & & 57 & 6752 & 0.1270 & 102 & 961 & 0.2385 & & & 0.3734 \\
\hline 13 & 0.6672 & 0.0284 & 58 & 0.6755 & 0.1293 & 103 & 6967 & 0.2412 & 148 & 372 & 0.3768 \\
\hline 14 & $0.6672 \mid$ & 0.0306 & 59 & & 0.1316 & & & 0.2439 & & & 0.3802 \\
\hline 15 & 0.6673 & 0.0328 & 60 & 761 & 0.1340 & 105 & 980 & 0.2466 & 150 & 396 & 0.3837 \\
\hline 16 & 674 & 350 & 61 & 764 & 1363 & 106 & 987 & .2493 & 151 & & 126 \\
\hline 17 & 674 & & 62 & 768 & & 107 & 994 & 0.2520 & & & 3901 \\
\hline 18 & 0.6675 & 0.0394 & 63 & 5771 & 0.1410 & 108 & 001 & 0.2548 & 153 & 434 & 0.3946 \\
\hline 19 & 0.6676 & 0.0416 & 64 & 775 & & 109 & & 0.2575 & & 47 & 0.3977 \\
\hline 20 & 0.6677 & 0.0 & 65 & 79 & 0. & 110 & & 0.2603 & 155 & 60 & 0.4013 \\
\hline 21 & 0.6678 & 459 & 66 & 3782 & 0.1481 & 111 & 022 & 0.2631 & 15 & & 049 \\
\hline 22 & 679 & & 67 & & & 1. & & 0.2659 & & & 085 \\
\hline 23 & 0.6680 & 0.0 & 68 & 790 & 29 & 11 & 37 & 0.2687 & & 00 & 0.4122 \\
\hline 24 & 0.6681 & 0.0 & 6 & & & 1 & & 0.2715 & & & 0.4159 \\
\hline 25 & 0.6682 & & 70 & 797 & 0.1577 & 115 & 052 & 0.2743 & 160 & 528 & 0.4196 \\
\hline 26 & 0.6684 & 0.0570 & 71 & 1 & 1 & 1 & 60 & .2772 & 1 & 42 & 0 \\
\hline 27 & & & 7 & & & & & 800 & & & 270 \\
\hline 28 & 0.6687 & 0.0614 & 73 & 809 & 0. & 11 & 076 & 0.2829 & 16 & 571 & 0.4308 \\
\hline 29 & 0.6688 & 0.0 & 74 & & 0. & 11 & 084 & 0.2858 & 16 & & 0.4346 \\
\hline 30 & 0.6690 & & 75 & & 0.1697 & 120 & 0.7092 & 0.2887 & 165 & 01 & 0.4385 \\
\hline 31 & & & 76 & & & 121 & & 0.291 & & & 0.4 \\
\hline 32 & & 0.0 & 7 & & 46 & & & & & & \\
\hline 3 & 594 & 0. & 7 & & 11 & 1 & 7 & 0.2975 & 1 & 48 & 02 \\
\hline 34 & 0.6696 & 0.0 & 79 & & & 12 & & 0.3004 & 169 & 364 & 0.4542 \\
\hline 35 & 0.6698 & & 80 & & 0.1820 & 125 & 0.7134 & 0.3034 & 170 & 680 & 0.4582 \\
\hline 36 & & & 8 & & & & & & & & \\
\hline 37 & & & 82 & & & & & 94 & & & 663 \\
\hline 38 & 0.6704 & 0.0 & 83 & & & & & & & & \\
\hline 39 & 0.6706 & 0.0859 & 84 & & & 12 & & 0.3155 & 17 & 746 & 0.4745 \\
\hline 40 & 0.6708 & 0.08 & 85 & 64 & 0.1 & 130 & 80 & 0.3185 & 175 & 0.7763 & 0.4787 \\
\hline 41 & 0.6710 & 0. & 86 & & 0.1 & 18 & & & 176 & & \\
\hline 42 & & & 87 & & & 1 & & & & & 77 \\
\hline 45 & 0.6714 & 0.0 & 88 & & & 1 & & & 17 & 17 & 0. \\
\hline 44 & 0.6717 & 0.0972 & 89 & 0.6884 & 0.2046 & 134 & 0.7 & 0.3309 & 179 & & 0.4957 \\
\hline 45 & 0.6719 & 0.0995 & 90 & 06890 & 0.2071 & 135 & 0.7229 & 0.3341 & 180 & 0.7854 & 0.5000 \\
\hline
\end{tabular}




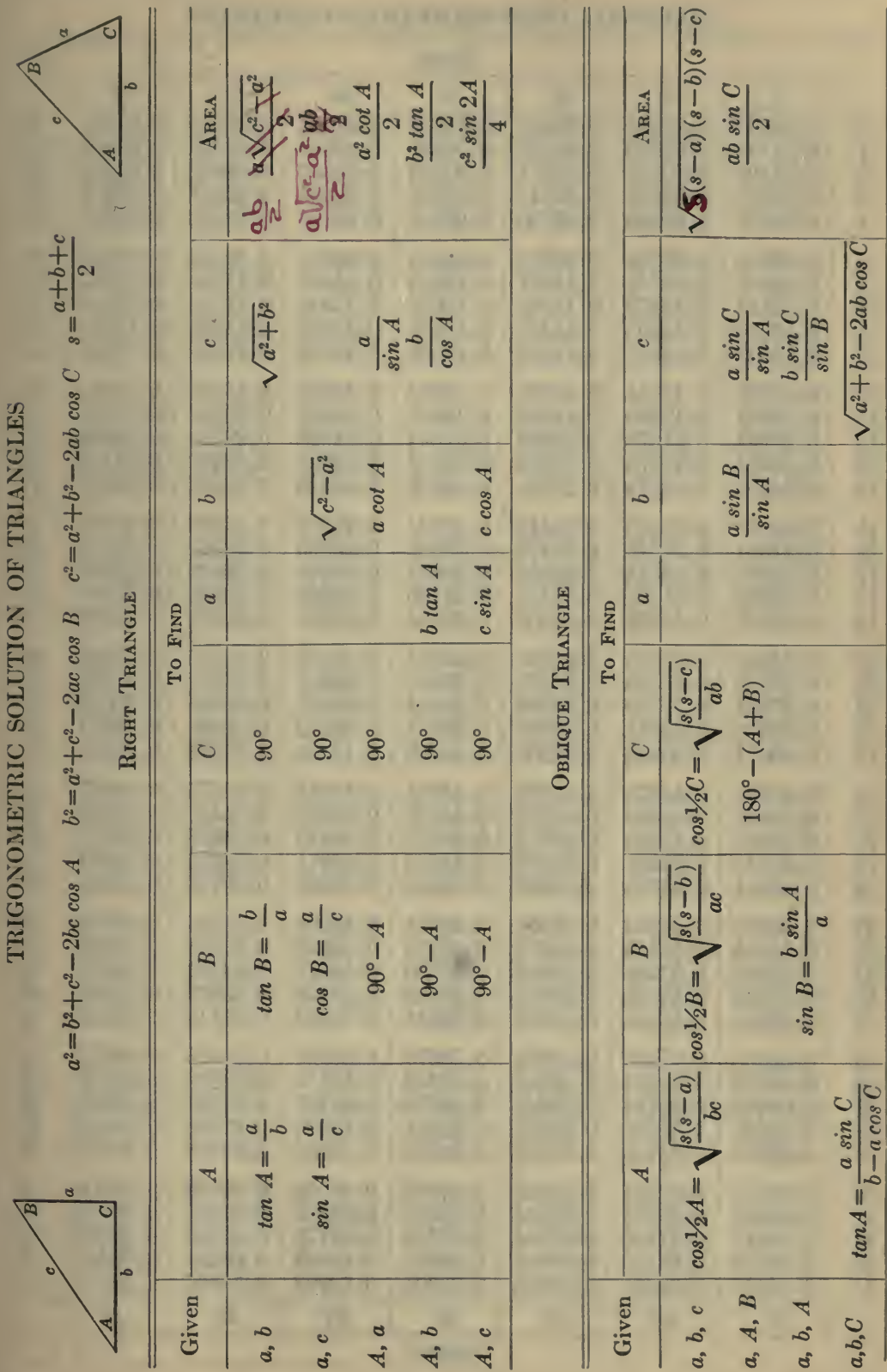


NATURAL TRIGONOMETRIC FUNCTIONS

\begin{tabular}{|c|c|c|c|c|c|c|c|c|}
\hline & \multicolumn{8}{|c|}{ SINES } \\
\hline & $0^{\prime}$ & $10^{\prime}$ & $20^{\prime}$ & $30^{\prime}$ & $40^{\prime}$ & $50^{\prime}$ & $60^{\prime}$ & \\
\hline 0 & 0.00000 & 0.00291 & 0.00582 & 0.00873 & 0.01164 & 0.01454 & 0.01745 & 89 \\
\hline 1 & & 0.02036 & 0.02327 & 0.02618 & & & & 88 \\
\hline 2 & 3490 & 0.03781 & 0.04071 & 0.04362 & 0.04653 & 4943 & 0.05234 & 87 \\
\hline 3 & 0.05234 & 0.05524 & 0.05814 & 0.06105 & 0.06395 & 0.06685 & 0.06976 & \\
\hline 4 & 0.06976 & 0.07266 & 0.07556 & 0.07846 & 0.08136 & 0.08426 & 0.08716 & 85 \\
\hline 5 & 16 & 005 & 295 & 35 & 74 & 0. & 53 & 84 \\
\hline 6 & & 0.10742 & 1031 & 0. & & & & - \\
\hline 7 & 187 & 0.12476 & 0.12764 & 0.13053 & 0.13341 & 3629 & 0.13917 & 82 \\
\hline 8 & 917 & 0.14205 & 0.14493 & 0.14781 & 0.15069 & 0.15356 & 0.15643 & 81 \\
\hline 9 & 0.15643 & 0.15931 & 0.16218 & 0.16505 & 0.16792 & 0.17078 & 0.17365 & 80 \\
\hline 10 & UE & 1 & 37 & & & & & 79 \\
\hline 11 & 081 & 0.19366 & 0. & & & & & 78 \\
\hline 12 & 0.20791 & 0.21076 & 0.21360 & 644 & 0. & & & 77 \\
\hline 13 & 2495 & 0.22778 & 0.23062 & 0.23345 & 0.23627 & & & 76 \\
\hline 14 & 0.24192 & 0.24474 & 0.24756 & 0.25038 & 5320 & & & 75 \\
\hline 15 & & & & & & & & 74 \\
\hline 16 & 564 & 0.27843 & 0.2 & & & & & 73 \\
\hline 17 & 0.29237 & 0.29515 & 0.29793 & 0. & 0 . & & & 72 \\
\hline 18 & 0902 & 0.31178 & 0.31454 & 0 . & & & & 71 \\
\hline 19 & 0.32557 & 0.32832 & 6 & & & & & 70 \\
\hline 20 & & & & & & & & 69 \\
\hline 21 & & 0.3 & & 0. & & & & 68 \\
\hline 22 & 0.37461 & 0.37730 & 0.3 & & & & & 67 \\
\hline 23 & 0.39073 & 0.39341 & 0.39608 & & & & & 66 \\
\hline 24 & 0.40674 & 0.40939 & 0. & 0. & 0. & 8 & 2 & 65 \\
\hline 25 & 0.4226 & 0 & -0 & 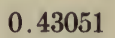 & & & & 64 \\
\hline 26 & 0.43837 & 0.440 & & & & & & 63 \\
\hline 27 & 0.45399 & 0.45658 & & & & & & 62 \\
\hline 28 & & & & & & & & 61 \\
\hline 29 & 0.48481 & 0.48735 & 0.48989 & 0.49242 & 0.49495 & 0.49748 & 0.50000 & 60 \\
\hline 30 & & & & & & & & 59 \\
\hline 31 & 504 & & & & & & & 58 \\
\hline 32 & & & & & & & & 57 \\
\hline 33 & & & & & & & & 56 \\
\hline 34 & 6919 & 160 & 401 & 0 . & 0.56880 & 0.57119 & & 55 \\
\hline 35 & & & & & & & & 54 \\
\hline 36 & 79 & & & & & & & \\
\hline 37 & & & & & & & & 52 \\
\hline 38 & & & & & & & & 51 \\
\hline 39 & 932 & 0. & 0. & 3608 & 0.6 & 4056 & 0.64279 & 50 \\
\hline 40 & & & & & & & & \\
\hline 41 & & & & & & & & 48 \\
\hline 42 & & & & & & & & 47 \\
\hline $4:$ & & & & & & & & 46 \\
\hline 44 & 0.69466 & 0.69675 & 0.69883 & 0.70091 & 0.70298 & 0.70505 & 0.70711 & 45 \\
\hline & $60^{\prime}$ & $50^{\prime}$ & 40 & 30 & $20^{\prime}$ & $10^{\prime}$ & 0 & \\
\hline
\end{tabular}

Cosines 
NATURAL TRIGONOMETRIC FUNCTIONS

\begin{tabular}{|c|c|c|c|c|c|c|c|c|}
\hline & & & & Cosines & & & & \\
\hline & $0^{\prime}$ & $10^{\prime}$ & $20^{\prime}$ & $30^{\prime}$ & $40^{\prime}$ & $50^{\prime}$ & $60^{\prime}$ & \\
\hline 0 & 1.00000 & 1.00000 & 0.99998 & 0.99996 & 0.99993 & 0.99989 & 0.99985 & 89 \\
\hline 1 & 0.99985 & 0.99979 & 0.99973 & 0.99966 & 0.99958 & 0.99949 & 0.99939 & 88 \\
\hline 2 & 0.99939 & 0.99929 & 0.99917 & 0.99905 & 0.99892 & 0.99878 & 0.99863 & 87 \\
\hline 3 & 0.99863 & 0.99847 & 0.99831 & 0.99813 & 0.99795 & 0.99776 & 0.99756 & 86 \\
\hline 4 & 0.99756 & 0.99736 & 0.99714 & 0.99692 & 0.99668 & 0.99644 & 0.99619 & 85 \\
\hline 5 & 0.99619 & 0.99594 & 0.99567 & 0.99540 & 0.99511 & 0.99482 & 452 & 84 \\
\hline 6 & 0.99452 & 0.99421 & 0.99390 & 0.99357 & 0.99324 & 0.99290 & 0. & 83 \\
\hline 7 & 0.99255 & 0.99219 & 0.99182 & 0.99144 & 0.99106 & 0.99067 & 0.99027 & 82 \\
\hline 8 & 0.99027 & 0.98986 & 0.98944 & 0.98902 & 0.98858 & 0.98814 & 0.98769 & 81 \\
\hline 9 & 0.98769 & 0.98723 & 0.98676 & 0.98629 & 0.98580 & 0.98531 & 0.98481 & 80 \\
\hline 10 & 3481 & 30 & 78 & 325 & 3272 & 18 & 63 & 79 \\
\hline 11 & 0.98163 & 0.98107 & 8050 & 7992 & 0.97934 & & 15 & 78 \\
\hline 12 & 0.97815 & 0.97754 & 0.97692 & 0.97630 & 0.97566 & 0.97502 & 0. & 77 \\
\hline 13 & 0.97437 & 0.97371 & 0.97304 & 0.97237 & 0.97169 & 0.97100 & 0.9 & 76 \\
\hline 14 & 0.97030 & 0.96959 & 0.96887 & 0.96815 & 0.96742 & 0.96667 & 93 & 75 \\
\hline 15 & 593 & & & & & & & 74 \\
\hline 16 & 0.96126 & 0.96046 & 0.95964 & 5882 & 0.95799 & 715 & 330 & 73 \\
\hline 17 & 0.95630 & 0.95545 & 0.95459 & 0.95372 & 0.95284 & 0.95195 & 0.9 & 72 \\
\hline 18 & 0.95106 & 0.95015 & 0.94924 & 0.94832 & 0.94740 & 0.94646 & 0.9 & 71 \\
\hline 19 & 0.94552 & 0.94 & 0.94361 & 264 & 0.94 & 0.940 & 0.93 & 70 \\
\hline 20 & 969 & & & & & & & 69 \\
\hline 21 & 0.93358 & 0.93253 & 148 & 042 & 0.9 & 27 & 18 & 68 \\
\hline 22 & 0.92718 & 0.92609 & 0.92499 & 388 & 0.92276 & 64 & 0.9 & 67 \\
\hline 23 & 0.92050 & 0.91936 & 822 & 706 & 0. & & 0. & 66 \\
\hline 24 & 0.91355 & & 16 & & & & & 65 \\
\hline 25 & 0.90631 & 0.90507 & 383 & & & & & 64 \\
\hline 26 & 0.89879 & 0.89752 & 623 & 493 & 63 & 32 & 01 & 63 \\
\hline 27 & 0.89101 & 0.88968 & 0.88835 & 701 & 566 & 431 & 0. & 62 \\
\hline 28 & 0.88295 & 0.88158 & 3020 & 0. & 0. & & 0. & 61 \\
\hline 29 & 0.87462 & 0.87321 & 0.87178 & 0.870 & & 0.86748 & & 60 \\
\hline 30 & 6603 & 0. & 10 & 63 & & 66 & 17 & 59 \\
\hline 31 & 5717 & 0. & 16 & 64 & & 59 & 4805 & 58 \\
\hline 32 & 0.84805 & 650 & 495 & 339 & 82 & 0.84025 & 0.83867 & 57 \\
\hline 33 & 3867 & 0.8 & 3549 & 389 & 28 & 0.83066 & 2904 & 56 \\
\hline 34 & 0.82904 & 0.82741 & 0.82577 & 0.82413 & 0.82248 & 0.82082 & 0.81915 & 55 \\
\hline 35 & 0. & 0. & 0 & 2 & 2 & 2 & 02 & 54 \\
\hline 36 & 02 & 0. & 58 & 86 & & 38 & 864 & 53 \\
\hline 37 & 9864 & 688 & 0.79512 & 35 & & 0.78980 & 0. & 52 \\
\hline 38 & 801 & 622 & 3442 & & & & 0.7 & 51 \\
\hline 39 & 0.77715 & 0.77531 & 0.77347 & 0.77162 & 0.76977 & 0.76791 & 0.76604 & 50 \\
\hline 40 & 0. & 0. & & & & & & 49 \\
\hline 41 & 75471 & 0.75280 & 0. & 96 & 0. & 0.74509 & 314 & 48 \\
\hline 42 & 0.74314 & 0.74120 & 0.73924 & 0.73728 & 0.73531 & 0.73333 & 0.73135 & 47 \\
\hline 43 & 0.73135 & 0.72937 & 0.72737 & 0.72537 & 0.72337 & 0.72136 & 0.71934 & 46 \\
\hline \multirow[t]{2}{*}{44} & 0.71934 & 0.71732 & 0.71529 & 0.71325 & 0.71121 & 0.70916 & 0.70711 & 45 \\
\hline & $60^{\prime}$ & $50^{\prime}$ & $40^{\prime}$ & $30^{\prime}$ & $20^{\prime}$ & $10^{\prime}$ & $0^{\prime}$ & \\
\hline
\end{tabular}


CORRUGATED BAR COMPANY, INC.

NATURAL TRIGONOMETRIC FUNCTIONS

\begin{tabular}{|c|c|c|c|c|c|c|c|c|}
\hline & & & & TANGENTS & & & & \\
\hline & $0^{\prime}$ & $10^{\prime}$ & $20^{\prime}$ & $30^{\prime}$ & $40^{\prime}$ & $50^{\prime}$ & $60^{\prime}$ & \\
\hline 0 & 0.00000 & 0.00291 & 0.00582 & 0.00873 & 0.01164 & 0.01455 & 0.01746 & 89 \\
\hline 1 & 0.01746 & 0.02036 & 0.02328 & 0.02619 & 0.02910 & 0.03201 & 0.03492 & 88 \\
\hline 2 & 0.03492 & 0.03783 & 0.04075 & 0.04366 & 0.04658 & 0.04949 & 0.05241 & 87 \\
\hline 3 & 0.05241 & 0.05533 & 0.05824 & 0.06116 & 0.06408 & 0.06700 & 0.06993 & 86 \\
\hline 4 & 0.06993 & 0.07285 & 0.07578 & 0.07870 & 0.08163 & 0.08456 & 0.08749 & 85 \\
\hline 5 & 0.08749 & 0.09042 & 0.09335 & 0.09629 & 0.09923 & 0.10216 & 0.10510 & 84 \\
\hline 6 & 0.10510 & 0.10805 & 0.11099 & 0.11394 & 0.11688 & 0.11983 & 0.12278 & 83 \\
\hline 7 & 0.12278 & 0.12574 & 0.12869 & 0.13165 & 0.13461 & 0.13758 & 0.14054 & 82 \\
\hline 8 & 0.14054 & 0.14351 & 0.14648 & 0.14945 & 0.15243 & 0.15540 & 0.15838 & 81 \\
\hline 9 & 0.15838 & 0.16137 & 0.16435 & 0.16734 & 0.17033 & 0.17333 & 0.17633 & 80 \\
\hline 10 & 0.17633 & 0.17933 & 0.18233 & 18534 & 18835 & 136 & 438 & 79 \\
\hline 11 & 0.19 & 0.1 & 0.2 & 0.2 & 0.2 & & 0. & 78 \\
\hline 12 & 0.21256 & 0.21560 & 0.21864 & 0.2 & 0.2 & 0. & 0.2 & 77 \\
\hline 13 & 0.23087 & 0.23393 & 0.23700 & 0.240 & 0.2 & 0. & 0.2 & 76 \\
\hline 14 & 0.24933 & 0.25242 & 0.25552 & 0.25862 & 0.26172 & 0.26483 & 0.26795 & 75 \\
\hline 15 & 0.26795 & 0.2 & 0 . & 0.2 & 0 & 0 & 0.28675 & 74 \\
\hline 16 & $0.2 S 675$ & 0.28990 & 0.2 & 0.29621 & 0.2 & 0.30255 & 0.30 & 73 \\
\hline 17 & 0.30573 & 0.30891 & 210 & 0.31530 & 0.31850 & 0.32171 & 0.32492 & 72 \\
\hline 18 & 0.32492 & 0.32814 & 136 & 0.33460 & 0.33783 & 0.34108 & 0.34433 & 71 \\
\hline 19 & 0.34433 & 0.34758 & 0.35085 & 0.35412 & 0.35740 & 0.36068 & 0.36397 & 70 \\
\hline 20 & 0 . & 27 & 0 & & 20 & & & 69 \\
\hline 21 & 0.38386 & 0.38721 & 0.39055 & 0.39391 & 727 & 065 & 0. & 68 \\
\hline 22 & 0.40403 & 0.40741 & 0.41081 & 0.41421 & 0.41763 & 0.4 & 0.42447 & 67 \\
\hline 23 & 0.42447 & 0.42791 & 0.43136 & 0.43481 & 828 & 0.4 & 0.44523 & 66 \\
\hline 24 & 0.44523 & 0.44872 & 0.45222 & 0.45573 & 0. & 0. & 0.4 & 65 \\
\hline 25 & 0.46631 & 0.46985 & & & & & 773 & 64 \\
\hline 26 & 0.48773 & 0.49134 & 0.49495 & 0.49858 & 0.50222 & 0.5 & 0.50953 & 63 \\
\hline 27 & 0.50953 & 0.51320 & 0.51688 & 0.52057 & 0.52427 & 0.52798 & 0.53171 & 62 \\
\hline 28 & 0.53171 & 0.53545 & 0.53920 & 0.54296 & 0.54674 & 0.55051 & 0.55431 & 61 \\
\hline 29 & 0.55431 & 0.55812 & 0.56194 & 0.56577 & 0.56962 & 0.57348 & 0.57735 & 60 \\
\hline 30 & 0.57735 & 0.58124 & 0.58513 & 0.58905 & 0.59297 & 0.59691 & 0.60086 & 59 \\
\hline 31 & 0.60086 & 0.60483 & 0.60881 & 0.61280 & 0.6 & 0.62083 & 0.6 & 58 \\
\hline 32 & 0.62487 & 0.62892 & 0.63299 & 0.63707 & 0.64117 & 0.64528 & 0.64941 & 57 \\
\hline 33 & 0.64941 & 0.65355 & & 0.66189 & 0.66608 & & 0.67451 & 56 \\
\hline 34 & 0.67451 & 0.67875 & 0.68301 & 0.68728 & 0.69157 & 0.69588 & 0.70021 & 55 \\
\hline 35 & & & & & & & & 54 \\
\hline 36 & 0.72654 & 0.73100 & 0.7 & 0.73996 & 0.7 & 0. & 0.75355 & 53 \\
\hline 37 & 0.75355 & 0.75812 & 0.76272 & 0.76733 & 0.77196 & 0.77661 & 0.78129 & 52 \\
\hline 38 & 129 & & & & & & & 51 \\
\hline 39 & 0.80978 & 0.81461 & 0.81946 & 0.82434 & 0.82923 & 0.83415 & 0.83910 & 50 \\
\hline 40 & 0.83910 & & & & & & & 49 \\
\hline 41 & 0.86929 & 0.87441 & & 0.884 & 0.88992 & 0.8 & 0.90040 & 48 \\
\hline 42 & 0.90040 & 0.90569 & 0.91099 & 0.91633 & 0.92170 & 0.92709 & 0.93252 & 47 \\
\hline 43 & 0.93252 & 0.9 & 0.9 & 0.94896 & 0.9 & 0.96008 & 0.96569 & 46 \\
\hline \multirow[t]{2}{*}{44} & 0.96569 & 0.97133 & 0.97700 & 0.98270 & 0.98843 & 0.99420 & 1.00000 & 45 \\
\hline & $60^{\prime}$ & $50^{\prime}$ & $40^{\prime}$ & $30^{\prime}$ & $20^{\prime}$ & $10^{\prime}$ & $0^{\prime}$ & \\
\hline
\end{tabular}


USEFUL DATA

NATURAL TRIGONOMETRIC FUNCTIONS

\begin{tabular}{|c|c|c|c|c|c|c|c|c|}
\hline & & & & NGENTS & & & & \\
\hline & $0^{\prime}$ & $10^{\prime}$ & $20^{\prime}$ & $30^{\prime}$ & $40^{\prime}$ & $50^{\prime}$ & $60^{\prime}$ & \\
\hline 0 & $\infty$ & 343.77371 & 171.88540 & 114.5 & 3979 & 68.75009 & 57.28996 & 00 \\
\hline 1 & 57.28996 & & 42.9 & & & & & \\
\hline 2 & 28.63625 & 26.43160 & 24.54176 & 22.90377 & & & & \\
\hline 3 & 19.08114 & 18.07498 & 17. 16934 & & & & 14.30067 & 00 \\
\hline 4 & 14.30067 & 14.72674 & 13.19688 & 12.70621 & 12.25051 & 11.82617 & 11.43005 & - \\
\hline 5 & 11.43005 & 11.05943 & 191 & 10 & 03 & 17 & 436 & . \\
\hline 6 & & 9.25530 & & & & & & \\
\hline 7 & 8.14435 & 7.95302 & & 7.59575 & 2871 & 73 & 7.11537 & 8 \\
\hline 8 & 7.11537 & 6.9 & & & & & 1375 & 81 \\
\hline 9 & 6.31375 & 6.19703 & 6.08444 & & & 5.76937 & 5.67128 & \\
\hline 10 & & & & & & & & 1 \\
\hline 1 & & & & & & & & 78 \\
\hline 2 & 4.7 & 5 & & & & & & 77 \\
\hline 13 & & 4.2 & & & & & & \\
\hline 14 & 4.01078 & 3.96165 & 3.91364 & & & & 3.73205 & 7 \\
\hline 15 & 05 & 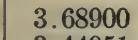 & 5 & & & & & 74 \\
\hline 6 & & & & & & & & $7 i$ \\
\hline 7 & & 4 & & & & & & $7:$ \\
\hline 18 & & 9 & & & & & & \\
\hline 19 & 2.9 & 00 & & & & & & 7 \\
\hline ח & 2 & & & & & & & \\
\hline & & & & & & & & \\
\hline & & & & & & & & 6 \\
\hline 23 & 2 . & & & & & & & \\
\hline 24 & 2.2 & 2857 & 2 & & & & & 6 \\
\hline 25 & & & & & & & & \\
\hline 6 & & & & & & & & \\
\hline 7 & 1 & 8 & & & & & & 62 \\
\hline 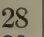 & & & & & & & & \\
\hline 29 & 1.8 & 4 & & & & & & \\
\hline 30 & 205 & & & & & & & \\
\hline 91 & & & & & & & & 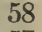 \\
\hline 32 & & & & & & & & 57 \\
\hline 33 & & & & & & & & 56 \\
\hline 34 & 1.48 & 330 & 1. & 1. & & & & \\
\hline 0 & & & & & & & & דר \\
\hline 36 & & & & & & & & 53 \\
\hline 37 & & & & & & & & 52 \\
\hline 38 & & & & & & & & 51 \\
\hline 39 & 1.2 & 1.22758 & 1.22031 & 1.2 & 1.20593 & 1. 19882 & 1.1 & \\
\hline 40 & & & & & & & & \\
\hline 41 & & & & & & & & \\
\hline 42 & & & & & & & & 1 \\
\hline 43 & & & & & & & & 10 \\
\hline \multirow[t]{2}{*}{44} & 1.03 & 1.02952 & 1.02355 & 1.01761 & 1.01170 & 1.00583 & 1.00000 & 45 \\
\hline & $60^{\prime}$ & $50^{\prime}$ & $40^{\prime}$ & $30^{\prime}$ & $20^{\prime}$ & $10^{\prime}$ & $0^{\prime}$ & \\
\hline
\end{tabular}


CORRUGATED BAR COMPANY, INC.

NATURAL TRIGONOMETRIC FUNCTIONS

\begin{tabular}{|c|c|c|c|c|c|c|c|c|}
\hline & \multicolumn{8}{|c|}{ SECANTS } \\
\hline & $0^{\prime}$ & $10^{\prime}$ & $20^{\prime}$ & $30^{\prime}$ & $40^{\prime}$ & $50^{\prime}$ & $60^{\prime}$ & \\
\hline 0 & 1.00000 & 1.00000 & 1.00002 & 1.00004 & 1.00007 & 1.00011 & 1.00015 & 89 \\
\hline 1 & 1.00015 & 1.00021 & 1.00027 & 1.00034 & 1.00042 & 1.000 & 1.00061 & 88 \\
\hline 2 & 1.00061 & 1.00072 & 1.00083 & 1.00095 & 1.00108 & 1.00122 & 1.00137 & 87 \\
\hline 3 & 1.00137 & 1.00153 & 1.00169 & 1.00187 & 1.00205 & 1.00224 & 1.00244 & 86 \\
\hline 4 & 1.00244 & 1.00265 & 1.00287 & 1.00309 & 1.00333 & 1.00357 & 1.00382 & 85 \\
\hline 5 & 1.00382 & 1.00408 & 1.00435 & 1.00463 & 1.00491 & 1.00521 & 1.00551 & 84 \\
\hline 6 & 1.00551 & 1.00582 & 1.00614 & 1.00647 & 1.00681 & 1.00715 & 1.00751 & 3 \\
\hline 7 & 1.00751 & 1.00787 & 1.00825 & 1.00863 & 1.00902 & 1.00942 & 1.00983 & 82 \\
\hline 8 & 1.00983 & 1.01024 & 1.01067 & 1.01111 & 1.01155 & 1.01200 & 1.01247 & 81 \\
\hline 9 & 1.01247 & 1.01294 & 1.01342 & 1.01391 & 1.01440 & 1.01491 & 1.01543 & 80 \\
\hline 10 & 1543 & 95 & 49 & 3 & 88 & 15 & 872 & 79 \\
\hline 11 & 1.01872 & 1.01930 & 1989 & 1.02049 & 1.0 & 1 . & 2234 & 78 \\
\hline 12 & 1.02234 & 1.02298 & 1.02362 & 1.02428 & 1.02494 & 1.02562 & 1.02630 & 77 \\
\hline 13 & 1.02630 & 1.02700 & 1.02770 & 1.02842 & 1.02914 & 1.02987 & 1.03061 & 76 \\
\hline 14 & 1.03061 & 1.03137 & 1.03213 & 1.03290 & 1.03 & 1. & 528 & 75 \\
\hline 15 & 1. & & 991 & 74 & 58 & 944 & 30 & 74 \\
\hline 16 & 1.04030 & 1.0 & 206 & 1. & $1 .($ & & 569 & 73 \\
\hline 17 & 1.04569 & 1.04663 & 1.04757 & 1.04853 & 950 & & 5146 & 72 \\
\hline 18 & 1.05146 & 1.05246 & 1.05347 & 1.0 & 1.0 & & 762 & 71 \\
\hline 19 & 1.0 & 869 & 1.0 & 1.0 & 1.0 & 3306 & 1.06418 & 70 \\
\hline 20 & 1.06418 & 1.06531 & 1.06645 & 1.06 & 1.06878 & 9995 & 1 & 69 \\
\hline 21 & 15 & 235 & 556 & & 1. & 27 & 853 & 68 \\
\hline 22 & 1.07853 & 1.07981 & 3109 & & & & 8636 & 67 \\
\hline 23 & 636 & 1. & & & & & & 66 \\
\hline 24 & 1.09464 & 1.09605 & 1.09750 & 1.09895 & 1.10041 & 1.10189 & 1.10338 & 65 \\
\hline 25 & 1.10 & 1. & 1. & 1. & 1. & & & 64 \\
\hline 26 & 1.11260 & & & & & & & 63 \\
\hline 27 & 233 & 400 & & & & & & 62 \\
\hline 28 & 257 & & & & & & & 61 \\
\hline 29 & 1.14335 & 1.14521 & 4707 & 1. & 1.15085 & 1.15277 & 1.15470 & 60 \\
\hline 30 & 1. & & & & & & & 59 \\
\hline 31 & 63 & 1.1 & & & & & & 58 \\
\hline 32 & 918 & 1.1 & 1. & & & & 36 & 57 \\
\hline 33 & 236 & 1. & & & & & 22 & 56 \\
\hline 0 & 622 & 1.2 & 1.2 & 1. & 1.2 & 30 & 77 & 55 \\
\hline 35 & 1.2 & & 1. & 1. & 1.2 & 1. & 1. & 54 \\
\hline 36 & 1.23607 & 1.2 & & 1.24400 & 1. 24669 & 1.24940 & 1.25214 & 53 \\
\hline 0 & 1.25214 & 489 & 1.25767 & 1.26047 & 1.26330 & 1.26615 & 1.26902 & 52 \\
\hline & 1.26902 & 1.27191 & 1.27483 & 1.27778 & 1.28075 & 1.28 & 1.28 & 51 \\
\hline 0 & 1.28676 & 1.2 & 1.29 & 1.29597 & 1.29909 & 1.30223 & 1.30 & 50 \\
\hline 40 & 1. & 1. & & & 1. & & & 49 \\
\hline 41 & 1.32501 & & 1.33177 & 1.33519 & 1.33864 & 1.34212 & 1.34563 & 48 \\
\hline 10 & 1.34563 & 1.34917 & 1.35274 & 1. 35634 & 1. 35997 & 1.36363 & 1.36733 & 47 \\
\hline & 1.36733 & 1.37105 & 1.37481 & 1.37860 & 1. 38242 & 1. 38628 & 1.39016 & 46 \\
\hline & 1.39016 & 1.39409 & 1.39804 & 1.40203 & 1.40606 & 1.41012 & 1.41421 & 45 \\
\hline & $60^{\prime}$ & $50^{\prime}$ & $40^{\prime}$ & $30^{\prime}$ & $20^{\prime}$ & $10^{\prime}$ & $0^{\prime}$ & \\
\hline & & & & & & & & \\
\hline
\end{tabular}


NATURAL TRIGONOMETRIC FUNCTIONS

\begin{tabular}{|c|c|c|c|c|c|c|c|c|}
\hline & & & & Cosecants & & & & \\
\hline & $0^{\prime}$ & 10 & $20^{\prime}$ & $30^{\prime}$ & $40^{\prime}$ & $50^{\prime}$ & $60^{\prime}$ & \\
\hline 0 & $\infty$ & 343.77516 & 171.88831 & 114.59301 & 85.94561 & 68.75736 & 57.29869 & \\
\hline 1 & 57.29869 & 49.11406 & 42.97571 & 38.20155 & 34.38232 & 31.25758 & 28.65371 & \\
\hline 2 & 28.65371 & 26.45051 & 24.56212 & 22.92559 & 21.49368 & 20.23028 & 19.10732 & \\
\hline 3 & 19.10732 & 18.10262 & 17.19843 & 16.38041 & 15. 63679 & 14.95788 & 14.33559 & \\
\hline 4 & 14.33559 & 13.76312 & 13.23472 & 12.74550 & 12.29125 & 11.86837 & 11.47371 & \\
\hline 5 & 11.47371 & 11.10455 & 10.75849 & 10.43343 & 10.12752 & 9.83912 & 9.56677 & \\
\hline 6 & 9.56677 & 9.30917 & 9.06515 & 8.83367 & 8.61379 & 8.40466 & 8.20551 & \\
\hline 7 & 8.20551 & 8.01565 & 7.83443 & 7.66130 & 7.49571 & 7.33719 & 7.18530 & \\
\hline 8 & 7.18530 & 3962 & 979 & 547 & 6.63633 & 6.51208 & 6.39245 & \\
\hline 9 & 6.39245 & 6.27719 & 6.16607 & 6.05886 & 5.95536 & 5.85539 & 5.75877 & \\
\hline 10 & 5.75877 & 66533 & 193 & 5.48740 & 5.40263 & 5.32049 & 084 & \\
\hline 11 & 5.24084 & 359 & 363 & 35 & 4.94517 & 4.87649 & 973 & \\
\hline 12 & 4.80973 & 1482 & 167 & 2023 & 4.56041 & 4.50216 & 4.44541 & \\
\hline 13 & 4.44541 & 012 & 622 & 8366 & 4.23239 & 4.18238 & 4.13357 & \\
\hline 14 & 4.13357 & 8591 & 4.03938 & 3.99393 & 3.94952 & 3.90613 & 3.86370 & \\
\hline 15 & 86370 & 223 & 66 & 98 & 15 & 15 & 96 & \\
\hline 16 & 796 & 154 & 37 & 94 & 3.4 & 3.45317 & 3.42030 & \\
\hline 17 & 3.42030 & 808 & 5649 & 2551 & 3.29512 & 3.26531 & 3.23607 & \\
\hline 18 & 607 & 0737 & 7920 & 5155 & 3.12440 & 3.09774 & 3.07155 & \\
\hline 19 & 155 & 84 & 3.02057 & 9574 & 2.97135 & 2.94737 & 2.92380 & \\
\hline 20 & 80 & & & & & 175 & 43 & \\
\hline 21 & 043 & 45 & 81 & 2850 & 2.70 & 884 & 47 & \\
\hline 22 & 947 & 5040 & 162 & 2.61313 & 2.59491 & 2.57698 & 2.55930 & \\
\hline 23 & 930 & 190 & 2474 & 0784 & 2.49119 & 2.47477 & 2.45959 & \\
\hline 24 & & 1264 & 2 & 142 & 2.39614 & 8107 & 20 & \\
\hline 25 & 620 & 54 & & 82 & 2.30875 & 2.29487 & 2.28117 & \\
\hline 26 & 8117 & 3766 & 132 & 16 & 2.22817 & 2.21535 & 2.2 & \\
\hline 27 & 2.20269 & 019 & 7786 & 2. & 2.15366 & 2.14178 & 2.13005 & \\
\hline 28 & 2.13005 & 2.11847 & & 74 & 2.08458 & 7356 & 2.06267 & \\
\hline 29 & 2.062 & 91 & .001 a & 2.03077 & 2.02039 & 2.01014 & 2.00000 & \\
\hline 30 & 00000 & 998 & 008 & 29 & 1.9 & 06 & 60 & \\
\hline & & 1.93226 & 302 & 38 & 1.90 & 1.89591 & 709 & \\
\hline 32 & 3708 & 1.87834 & 1.86970 & 1.86 & 1.85271 & 1.84435 & 1.83608 & \\
\hline 33 & 1.83608 & 1.82790 & 1.819 & & 1.80388 & 1.79604 & 1.78829 & \\
\hline 34 & 1.78829 & 1.78062 & 1.77303 & 1.76552 & 1.75808 & 1.75073 & 1.74345 & \\
\hline 35 & 45 & 4 & 1 & & 6 & & 30 & \\
\hline 36 & 1.70130 & 154 & 1.6 & & 1.67 & 09 & 1.66164 & \\
\hline 37 & 164 & 1.65526 & 1. & 1.6 & 1. 63648 & 1.63 & 1.62427 & \\
\hline 38 & 1. 62427 & & 229 & & 1.60054 & 1.59475 & 1.58902 & \\
\hline 39 & 1.58 & & 57771 & 1.57213 & 1.56661 & 1.56114 & 1.55572 & \\
\hline 40 & 1 & & & & 55 & & & 4 \\
\hline 41 & 1.52425 & 1.51918 & 1.514 & 1.50916 & 1.50422 & 1.49933 & 1.49448 & \\
\hline 42 & 1.49448 & 1.48967 & 1.48491 & 1.48019 & 1.47551 & 1.47087 & 1.46628 & \\
\hline 43 & 1.46628 & 1.46173 & 1.45721 & 1.45274 & 1.44831 & 1.44391 & 1.43856 & \\
\hline \multirow[t]{3}{*}{44} & 1.43956 & 1.43524 & 1.43096 & 1.42672 & 1.42251 & 1.41835 & 1.41421 & 4 \\
\hline & $60^{\prime}$ & $50^{\prime}$ & 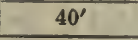 & $30^{\prime}$ & $20^{\prime}$ & $10^{\prime}$ & $0^{\prime}$ & \\
\hline & \multicolumn{8}{|c|}{ SECANTS } \\
\hline
\end{tabular}


FUNCTIONS OF NUMBERS 1 TO 49

\begin{tabular}{|c|c|c|c|c|c|c|c|c|}
\hline \multirow{2}{*}{ No. } & \multirow{2}{*}{ Square } & \multirow{2}{*}{ Cube } & \multirow{2}{*}{$\begin{array}{c}\text { Square } \\
\text { Root }\end{array}$} & \multirow{2}{*}{$\begin{array}{l}\text { Cube } \\
\text { Root }\end{array}$} & \multirow{2}{*}{ Logarithm } & \multirow{2}{*}{\begin{tabular}{|c|}
1,000 \\
$x$ \\
Reciprocal \\
\end{tabular}} & \multicolumn{2}{|c|}{ No. $=$ DIAMETER } \\
\hline & & & & & & & Circum. & Area \\
\hline 1 & 1 & 1 & 00 & 000 & 0000 & 1000.000 & 3.142 & 0.7854 \\
\hline 2 & 4 & 8 & & & & 500 . & & 416 \\
\hline 3 & 9 & 27 & 1.7321 & 1.4422 & 0.47712 & 333. & 9.425 & 7.0680 \\
\hline 4 & 16 & 64 & 2.0000 & 1.5874 & 0.60206 & 250.000 & 12.566 & 12.5664 \\
\hline 5 & 25 & 125 & 2.2361 & 1.7100 & 0.69897 & 200.000 & 15.708 & 19.6350 \\
\hline 6 & 36 & 216 & 2.4495 & 1.8171 & 0.77815 & 166.667 & 18.850 & 28.2743 \\
\hline 7 & 49 & 343 & 2.6458 & 1.9129 & 0.84510 & 142.857 & 21.991 & 38.4845 \\
\hline 8 & 64 & 512 & 2.8284 & 2.0000 & 0.90309 & 125.000 & 25.133 & 50.2655 \\
\hline 9 & 81 & 729 & & & 0.95424 & 111.111 & 28.274 & 63.6173 \\
\hline 10 & 100 & 1000 & 3.1623 & 2.1544 & 1.00000 & 100.000 & 31.416 & 78.5398 \\
\hline 11 & 121 & & & 2 & & 9 & & 33 \\
\hline 12 & 144 & 1728 & 3.4 & 2.2894 & & 83.3333 & 37.699 & 113.097 \\
\hline 13 & 169 & 2197 & 3.6 & 2.3513 & & 76.9231 & 40.841 & 132.732 \\
\hline 14 & 196 & 2744 & 3.7417 & 2.4101 & 13 & 71.4286 & 43.982 & 153.938 \\
\hline 15 & 225 & 3375 & 730 & 2.4662 & 609 & 66.6667 & 47.124 & 176.715 \\
\hline 16 & 256 & 4096 & 4.0000 & 2.5198 & 412 & 62.5000 & 50.265 & 201.062 \\
\hline 17 & 289 & 4913 & 4.1231 & 2. & 045 & 58.8235 & 53.407 & 226.980 \\
\hline 18 & 324 & 5832 & 4.2426 & 2.6207 & 527 & 55.5556 & 56.549 & 254.469 \\
\hline 19 & 361 & 6859 & 4.3589 & 2.6684 & 7875 & 52.6316 & 59.690 & 283.529 \\
\hline 20 & 400 & 8000 & 4.4 & 2.7144 & 1.30103 & 50. & 62.832 & 314.159 \\
\hline 21 & 4 & 1 & $x$ & & & & & \\
\hline 22 & & 18 & & & & & & 133 \\
\hline 23 & & & & & & 3 & 72.257 & .476 \\
\hline 24 & & & & & & 41.6 & 75.398 & 452.389 \\
\hline 25 & & & & & & & & 490.874 \\
\hline 26 & & & & & & & & 530.929 \\
\hline 27 & & & & & & & 4.823 & .555 \\
\hline 28 & & & & & & & 87.9 & .752 \\
\hline 29 & & & & & & & & 660.520 \\
\hline 30 & 900 & 27000 & 5.4772 & 3.1 & 1. & 33. & 48 & 706.858 \\
\hline 31 & & & & & & & & 768 \\
\hline 32 & 1024 & 768 & & & & 31.2500 & 100 & 248 \\
\hline 33 & 1089 & & & 3.2 & & 30.3030 & 10 & 855.299 \\
\hline 34 & & 39304 & & 3.2396 & & 29.4118 & 106.814 & 907.920 \\
\hline 35 & & & & & & 28.5714 & 109.956 & 962.113 \\
\hline 36 & & & & & & 27.7778 & 113.097 & 1017.88 \\
\hline 37 & & & & & & 27.0270 & 116.239 & 1075.21 \\
\hline 38 & & & & & & 26.31 & 119.381 & 1134.11 \\
\hline 39 & & & & & & & 122.522 & 1194.59 \\
\hline 40 & 1600 & & 6.32 & 3.42 & 1.602 & 0 & 12 & 1256. \\
\hline 41 & & & & & & & & \\
\hline 42 & 1764 & & & & & 23.8 & & 1385.44 \\
\hline 43 & 1849 & & & 3.50 & & 23.2558 & 135.09 & 1452.20 \\
\hline 44 & 1936 & 85184 & & 3.53 & & 22.7273 & 138.23 & 1520.53 \\
\hline 45 & 2025 & 91125 & 6.7082 & 3.55 & 1. 65321 & 22.2222 & 141.37 & 1590.43 \\
\hline 46 & 2116 & 97336 & & 3.58 & 1.66276 & 21.7391 & 144.51 & 1661.90 \\
\hline 47 & 2209 & 103823 & 6.8557 & 3.60 & 1.67210 & 21.2766 & 147.65 & 1734.04 \\
\hline 48 & 2304 & 110592 & 6.92 & 3.6 & & 20.8333 & 150.80 & 1809.56 \\
\hline 49 & 2401 & 117649 & 7.0000 & 3.6593 & 1. 69020 & 20.4082 & 153.94 & 1885.74 \\
\hline
\end{tabular}


USEFUL DATA

FUNCTIONS OF NUMBERS 50 TO 99

\begin{tabular}{|c|c|c|c|c|c|c|c|c|}
\hline \multirow{2}{*}{ No. } & \multirow{2}{*}{ Square } & \multirow{2}{*}{ Cube } & \multirow{2}{*}{$\begin{array}{c}\text { Square } \\
\text { Root }\end{array}$} & \multirow{2}{*}{$\begin{array}{l}\text { Cube } \\
\text { Root }\end{array}$} & \multirow{2}{*}{ Logarithm } & \multirow{2}{*}{$\begin{array}{c}1,000 \\
x \\
\text { Reciprocal }\end{array}$} & \multicolumn{2}{|c|}{ No. - DIAMEter } \\
\hline & & & & & & & Circum. & Area \\
\hline 50 & 2500 & 5000 & 7.0711 & 840 & 69897 & 20.0000 & 157.08 & 1963.50 \\
\hline & 2601 & & & & & 19.6078 & 160.22 & 2042.82 \\
\hline & 2704 & 140608 & 7.2111 & 3.7325 & 1.71600 & 19.2308 & 163.36 & 2123.72 \\
\hline & 2809 & 148877 & 7.2801 & 3.7563 & 1.72428 & 18.8679 & 166.50 & 2206.18 \\
\hline & 2916 & 157464 & 7.3485 & 3.7798 & 1. 73239 & 18.5185 & 169.65 & 2290.22 \\
\hline$\approx$ & 3025 & 166375 & 7.4162 & 3.8030 & 1.74036 & 18.1818 & 172.79 & 2375.83 \\
\hline 56 & & 175616 & 7.4 & 3.8259 & 1.74819 & 17.8571 & 175.93 & 2463.01 \\
\hline 57 & 3249 & & 7.5 & 3.8 & & 17.5439 & 179.07 & 2551.76 \\
\hline 58 & & & & & & 17.24 & & 2642.08 \\
\hline 59 & 3481 & 20 & & 30 & 085 & 16.9492 & 185.35 & 2733.97 \\
\hline 60 & 00 & 00 & 7 & & 15 & or & & .43 \\
\hline 61 & 3721 & 226981 & 7. & 3.9365 & 3533 & 16.3934 & 91.64 & 2922.47 \\
\hline 62 & 3844 & 239328 & 7.8740 & 3.9579 & 9239 & 16.1290 & 194.78 & 3019.07 \\
\hline 63 & & 250047 & 7.9373 & 3.9791 & 34 & 15.8730 & 197.92 & 7.25 \\
\hline 64 & 66 & 262144 & 8.0 & 0 & & 15.6250 & .06 & 0.99 \\
\hline 65 & 4225 & 625 & 8.0 & & & 15.3846 & .20 & 3.31 \\
\hline 66 & 4356 & 28 & 8. & & & 15.1515 & .35 & 1.19 \\
\hline 67 & 4489 & 30 & 8. & & & 140 & .49 & 5.65 \\
\hline 68 & 4624 & & 8.2 & & & 147 & 21 & .68 \\
\hline 69 & 4761 & & 8.3066 & 4.1 & 1.8 & 28 & 216 . & 3739.28 \\
\hline 70 & 4900 & 00 & & & & & & \\
\hline 71 & 5041 & & 8.4 & & 26 & 45 & 3.05 & .19 \\
\hline 72 & 5184 & 248 & 8.4853 & 4.1 & 1.85 & 13. 8889 & 6.19 & 1.50 \\
\hline 73 & 5329 & 017 & 8.5440 & 4.1 & 1.86332 & 13.6986 & 229.34 & 5.39 \\
\hline 74 & 5476 & 224 & 8.6023 & 4.19 & 1.86923 & 13.5135 & 232.48 & 0.84 \\
\hline 75 & 5625 & 75 & 8.6603 & 4.2172 & 1.87506 & 13.3333 & .62 & 7.86 \\
\hline 76 & 5776 & & 8.7178 & 4.2358 & 1. 88081 & 13.1579 & 238.76 & 4536.46 \\
\hline 77 & 5929 & & 8.7 & 4.2543 & 1.88 & 12.9870 & 241.90 & 4656.63 \\
\hline 78 & 6084 & & & 4.27 & & 12.8 & & 8.36 \\
\hline 79 & & 493039 & 8.8 & 4.29 & 1.89763 & 12.6582 & 2 & .67 \\
\hline 80 & 6400 & 512000 & 8.9 & 20 & 1.90309 & 12.5000 & & 55 \\
\hline 81 & 6561 & 41 & 9. & & 49 & 57 & .47 & .00 \\
\hline 82 & 6724 & 68 & 9.0 & 4. & 81 & 12.19 & 7.61 & 1.02 \\
\hline 83 & 6889 & 87 & 9.1 & 4.3 & 1. & 12.0482 & 260.75 & 0.61 \\
\hline 84 & 7056 & 04 & 9.1652 & 4.3 & 28 & 11.90 & 263.89 & 1.77 \\
\hline 85 & 7225 & 25 & 9.2195 & 4.3 & 1.9 & 11.7647 & 267.04 & 4.50 \\
\hline 86 & 7396 & & 9.2736 & 4.4 & 1.9 & 11. 6279 & 270.18 & 5808.80 \\
\hline 87 & 7569 & & 9.3274 & 4.4 & 1.9 & 11.4943 & 273.32 & 4.68 \\
\hline 88 & 7744 & & 9.38 & 4.44 & 1.9 & 11.36 & 276.46 & 2.12 \\
\hline 89 & & 704969 & 9.4340 & & 1.94939 & 11.23 & 279.60 & 6221.14 \\
\hline 90 & 8 & $7:$ & 9 & & $*$ & 11.1 & 4 & 73 \\
\hline 91 & 8281 & & & & & & & 3.88 \\
\hline 92 & 8464 & & 9.5 & & 79 & & & 6647.61 \\
\hline 93 & 8649 & 804357 & 9.6437 & & 848 & & & 6792.91 \\
\hline 94 & 8836 & 830584 & 9.6954 & & 313 & 10.6383 & 295.31 & 6939.78 \\
\hline 95 & 9025 & 857375 & 9.7468 & 4.5629 & 1. 97772 & 10.5263 & 298.45 & 7088.22 \\
\hline 96 & 9216 & $88 \pm 736$ & 9.7980 & 4.5789 & 1.98227 & 10.4167 & 301.59 & 7238.23 \\
\hline 97 & 9409 & 912673 & 9.8189 & 4.5947 & 1.98677 & 10.3093 & 304.73 & 7389.81 \\
\hline 98 & 9604 & 941192 & 9.8995 & 4.6104 & 1.99123 & 10.2041 & 307.88 & 7542.96 \\
\hline 99 & $9 S 01$ & 970299 & 9.9499 & 4. 6261 & 1.99564 & 10.1010 & 311.02 & 7697.69 \\
\hline
\end{tabular}


CORRUGATED BAR COMPANY, INC.

DECIMALS OF AN INCH FOR EACH $\frac{1}{64}$ th

\begin{tabular}{|c|c|c|c|c|c|c|c|c|c|}
\hline \multicolumn{4}{|c|}{ Fractions } & \multirow{2}{*}{ Decimals } & \multicolumn{4}{|c|}{ Fractions } & \multirow{2}{*}{ Decimals } \\
\hline 64ths & 32nds & 16 ths & 8ths & & 64ths & 32nds & 16 ths & 8ths & \\
\hline \multirow[t]{2}{*}{$\frac{1}{64}$} & & & & 0.015625 & $\frac{33}{64}$ & & & & 0.515625 \\
\hline & $\frac{1}{32}$ & & 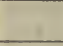 & 0.03125 & & $\frac{17}{32}$ & & & 0.53125 \\
\hline \multirow[t]{2}{*}{$\frac{3}{64}$} & & & & 0.046875 & $\frac{35}{64}$ & & & & 0.546875 \\
\hline & & $\frac{1}{16}$ & & 0.0625 & & & $\frac{9}{16}$ & & 0.5625 \\
\hline \multirow[t]{2}{*}{$\frac{5}{64}$} & & & 1 & 0.078125 & $\frac{37}{64}$ & & & & 0.578125 \\
\hline & $\frac{3}{32}$ & 4 & & 0.09375 & & $\frac{19}{32}$ & & & 0.59375 \\
\hline \multirow[t]{2}{*}{$\frac{7}{64}$} & & & & 0.109375 & $\frac{39}{64}$ & & & & 0.609375 \\
\hline & & & $1 / 8$ & 0.125 & & & & $5 / 8$ & 0.625 \\
\hline \multirow[t]{2}{*}{$\frac{9}{64}$} & & i & & 0.140625 & $\frac{41}{64}$ & & & & 0.640625 \\
\hline & $\frac{5}{32}$ & 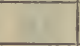 & 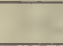 & 0.15625 & & $\frac{21}{32}$ & & & 0.65625 \\
\hline \multirow[t]{2}{*}{$\frac{11}{64}$} & & & & 0.171875 & $\frac{43}{64}$ & & & & 0.671875 \\
\hline & & $\frac{3}{16}$ & 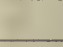 & 0.1875 & 4 & & $\frac{11}{16}$ & & 0.6875 \\
\hline \multirow[t]{2}{*}{$\frac{13}{64}$} & & 2 & & 0.203125 & $\frac{45}{64}$ & & & & 0.703125 \\
\hline & $\frac{7}{32}$ & 7 & - & 0.21875 & & $\frac{23}{32}$ & & & 0.71875 \\
\hline \multirow[t]{2}{*}{$\frac{15}{64}$} & & 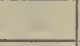 & & 0.234375 & $\frac{47}{64}$ & & & & 0.734375 \\
\hline & & E & $1 / 4$ & 0.25 & 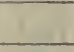 & & & $3 / 4$ & 0.75 \\
\hline \multirow[t]{2}{*}{$\frac{17}{64}$} & 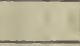 & & & 0.265625 & $\frac{49}{64}$ & & & & 0.765625 \\
\hline & $\frac{9}{32}$ & & & 0.28125 & 1 & $\frac{25}{32}$ & & & 0.78125 \\
\hline \multirow[t]{2}{*}{$\frac{19}{64}$} & & & . & 0.296875 & $\frac{51}{64}$ & & & & 0.796875 \\
\hline & & $\frac{5}{16}$ & & 0.3125 & & & $\frac{13}{16}$ & & 0.8125 \\
\hline \multirow[t]{2}{*}{$\frac{21}{64}$} & & & & 0.328125 & $\frac{53}{64}$ & & & & 0.828125 \\
\hline & $\frac{11}{32}$ & 18 & & 0.34375 & & $\frac{27}{32}$ & & & 0.84375 \\
\hline \multirow[t]{2}{*}{$\frac{33}{64}$} & 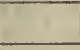 & & 7 & 0.359375 & $\frac{55}{64}$ & & & & 0.859375 \\
\hline & & & $3 / 8$ & 0.375 & 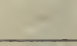 & & & $7 / 8$ & 0.875 \\
\hline \multirow[t]{2}{*}{$\frac{25}{64}$} & & & & 0.390625 & $\frac{87}{64}$ & & & & 0.890625 \\
\hline & $\frac{13}{32}$ & & & 0.40625 & & $\frac{29}{32}$ & & 1 & 0.90625 \\
\hline \multirow[t]{2}{*}{$\frac{27}{64}$} & & & & 0.421875 & $\frac{59}{64}$ & & & & 0.921875 \\
\hline & & $\frac{7}{16}$ & & 0.4375 & & & $\frac{15}{16}$ & & 0.9375 \\
\hline \multirow[t]{2}{*}{$\frac{29}{64}$} & 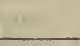 & 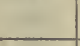 & 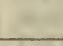 & 0.453125 & $\frac{61}{64}$ & & & & 0.953125 \\
\hline & $\frac{15}{32}$ & & & 0.46875 & & $\frac{81}{32}$ & & & 0.96875 \\
\hline \multirow[t]{2}{*}{$\frac{31}{64}$} & & & & 0.484375 & $\frac{63}{64}$ & & & & 0.984375 \\
\hline & & & $1 / 2$ & 0.5 & & & & 1 & 1.00 \\
\hline
\end{tabular}


DECIMALS OF A FOOT FOR EACH $\frac{1}{32} \mathrm{~d}$ OF AN INCH

\begin{tabular}{l|c|c|c|c|c|c|c|c|c|c|c|c}
\hline In. & $0^{\prime \prime}$ & $1^{\prime \prime}$ & $2^{\prime \prime}$ & $3^{\prime \prime}$ & $4^{\prime \prime}$ & $5^{\prime \prime}$ & $6^{\prime \prime}$ & $7^{\prime \prime}$ & $8^{\prime \prime}$ & $9^{\prime \prime}$ & $10^{\prime \prime}$ & $11^{\prime \prime}$ \\
\hline
\end{tabular}

\begin{tabular}{l|l|l|l|l|l|l|l|l|l|l|l|l|l}
0 & 0.0000 & 0.0833 & 0.1667 & 0.2500 & 0.3333 & 0.4167 & 0.5000 & 0.5833 & 0.6667 & 0.7500 & 0.8333 & 0.9167
\end{tabular} \begin{tabular}{ll|l|l|l|l|l|l|l|l|l|l|l|l|l} 
支 & 0.0026 & 0.0859 & 0.1693 & 0.2526 & 0.3359 & 0.4193 & 0.5026 & 0.5859 & 0.6693 & 0.7526 & 0.8359 & 0.9193
\end{tabular} \begin{tabular}{ll|l|l|l|l|l|l|l|l|l|l|l|l|l|l} 
18 & 0.0052 & 0.0885 & 0.1719 & 0.2552 & 0.3385 & 0.4219 & 0.5052 & 0.5885 & 0.6719 & 0.7552 & 0.8385 & 0.9219
\end{tabular}

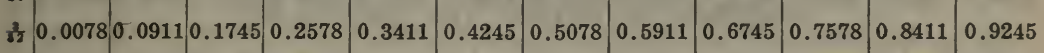
\begin{tabular}{ll|l|l|l|l|l|l|l|l|l|l|l|l|l|l}
$1 / 8$ & 0.0104 & 0.0937 & 0.1771 & 0.2604 & 0.3437 & 0.4271 & 0.5104 & 0.5937 & 0.6771 & 0.7604 & 0.8437 & 0.9271
\end{tabular} \begin{tabular}{l|l|l|l|l|l|l|l|l|l|l|l|l}
$\frac{8}{32}$ & 0.0130 & 0.0964 & 0.1797 & 0.2630 & 0.3464 & 0.4297 & 0.5130 & 0.5964 & 0.6797 & 0.7630 & 0.8464 & 0.9297
\end{tabular} \begin{tabular}{l|l|l|l|l|l|l|l|l|l|l|l|l|l|l|l} 
If & 0.0156 & 0.0990 & 0.1823 & 0.2656 & 0.3490 & 0.4323 & 0.5156 & 0.5990 & 0.6823 & 0.7656 & 0.8490 & 0.9323
\end{tabular} \begin{tabular}{l|l|l|l|l|l|l|l|l|l|l|l|l|l|l|l}
$\frac{7}{32}$ & 0.0182 & 0.1016 & 0.1849 & 0.2682 & 0.3516 & 0.4349 & 0.5182 & 0.6016 & 0.6849 & 0.7682 & 0.8516 & 0.9349
\end{tabular} \begin{tabular}{l|l|l|l|l|l|l|l|l|l|l|l|l|l|l|l|l}
$1 / 4$ & 0.0208 & 0.1042 & 0.1875 & 0.2708 & 0.3542 & 0.4375 & 0.5208 & 0.6042 & 0.6875 & 0.7708 & 0.8542 & 0.9375
\end{tabular} \begin{tabular}{ll|l|l|l|l|l|l|l|l|l|l|l|l}
3 & 0.0234 & 0.1068 & 0.1901 & 0.2734 & 0.3568 & 0.4401 & 0.5234 & 0.6068 & 0.6901 & 0.7734 & 0.8568 & 0.9401
\end{tabular} \begin{tabular}{l|l|l|l|l|l|l|l|l|l|l|l|l|l|l|l}
$\frac{3}{16}$ & 0.0260 & 0.1094 & 0.1927 & 0.2760 & 0.3594 & 0.4427 & 0.5260 & 0.6094 & 0.6927 & 0.7760 & 0.8594 & 0.9427
\end{tabular} \begin{tabular}{l|l|l|l|l|l|l|l|l|l|l|l|l|l|l|l|l}
$\frac{1}{2}$ & 0.0286 & 0.1120 & 0.1953 & 0.2786 & 0.3620 & 0.4453 & 0.5286 & 0.6120 & 0.6953 & 0.7786 & 0.8620 & 0.9453
\end{tabular} \begin{tabular}{l|ll|l|l|l|l|l|l|l|l|l|l|l|l|}
$3 / 8$ & 0.0312 & 0.1146 & 0.1979 & 0.2812 & 0.3646 & 0.4479 & 0.5312 & 0.6146 & 0.6979 & 0.7812 & 0.8646 & 0.9479
\end{tabular} \begin{tabular}{lllllllll|l|l|l|l|l|l}
$\frac{13}{3}$ & 0.0339 & 0.1172 & 0.2005 & 0.2839 & 0.3672 & 0.4505 & 0.5339 & 0.6172 & 0.7005 & 0.7839 & 0.8672 & 0.9505
\end{tabular} \begin{tabular}{l|l|l|l|l|l|l|l|l|l|l|l|l|l} 
I6 & 0.0365 & 0.1198 & 0.2031 & 0.2865 & 0.3698 & 0.4531 & 0.5365 & 0.6198 & 0.7031 & 0.7865 & 0.8698 & 0.9531
\end{tabular} \begin{tabular}{l|l|l|l|l|l|l|l|l|l|l|l|l|l|l}
$\frac{13}{3}$ & 0.0391 & 0.1224 & 0.2057 & 0.2891 & 0.3724 & 0.4557 & 0.5391 & 0.6224 & 0.7057 & 0.7891 & 0.8724 & 0.9557
\end{tabular} \begin{tabular}{l|lll|l|l|l|l|l|l|l|l|l|l|l}
$1 / 2$ & 0.0417 & 0.1250 & 0.2083 & 0.2917 & 0.3750 & 0.4583 & 0.5417 & 0.6250 & 0.7083 & 0.7917 & 0.8750 & 0.9583
\end{tabular}

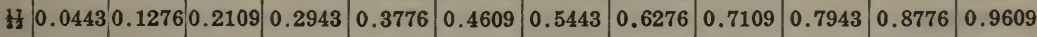
\begin{tabular}{lll|l|l|l|l|l|l|l|l|l|l|l|l|l} 
I6 & 0.0469 & 0.1302 & 0.2135 & 0.2969 & 0.3802 & 0.4635 & 0.5469 & 0.6302 & 0.7135 & 0.7969 & 0.8802 & 0.9635
\end{tabular} \begin{tabular}{l|l|l|l|l|l|l|l|l|l|l|l|l|l|l|l|}
12 & 0.0495 & 0.1328 & 0.2161 & 0.2995 & 0.3828 & 0.4661 & 0.5495 & 0.6328 & 0.7161 & 0.7995 & 0.8828 & 0.9661
\end{tabular} \begin{tabular}{l|l|l|l|l|l|l|l|l|l|l|l|l|l|l}
$5 / 8$ & 0.0521 & 0.1354 & 0.2188 & 0.3021 & 0.3854 & 0.4688 & 0.5521 & 0.6354 & 0.7188 & 0.8021 & 0.8854 & 0.9688
\end{tabular}

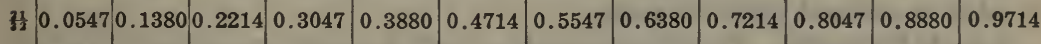

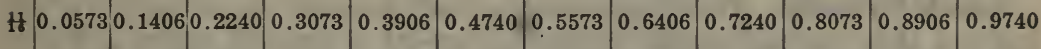
\begin{tabular}{l|l|l|l|l|l|l|l|l|l|l|l|l|l} 
3골 & 0.0599 & 0.1432 & 0.2266 & 0.3099 & 0.3932 & 0.4766 & 0.5599 & 0.6432 & 0.7266 & 0.8099 & 0.8932 & 0.9766
\end{tabular} \begin{tabular}{l|l|l|l|l|l|l|l|l|l|l|l|l|l|l|l}
$3 / 4$ & 0.0625 & 0.1458 & 0.2292 & 0.3125 & 0.3958 & 0.4792 & 0.5625 & 0.6458 & 0.7292 & 0.8125 & 0.8958 & 0.9792
\end{tabular} \begin{tabular}{ll|l|l|l|l|l|l|l|l|l|l|l}
$3 \frac{3}{2}$ & 0.0651 & 0.1484 & 0.2318 & 0.3151 & 0.3984 & 0.4818 & 0.5651 & 0.6484 & 0.7318 & 0.8151 & 0.8984 & 0.9818
\end{tabular} \begin{tabular}{l|l|l|l|l|l|l|l|l|l|l|l|l|l}
18 & 0.0677 & 0.1510 & 0.2344 & 0.3177 & 0.4010 & 0.4844 & 0.5677 & 0.6510 & 0.7344 & 0.8177 & 0.9010 & 0.9844
\end{tabular} \begin{tabular}{l|l|l|l|l|l|l|l|l|l|l|l|l|l|l|l} 
结 & 0.0703 & 0.1536 & 0.2370 & 0.3203 & 0.4036 & 0.4870 & 0.5703 & 0.6536 & 0.7370 & 0.8203 & 0.9036 & 0.9870
\end{tabular} \begin{tabular}{l|l|l|l|l|l|l|l|l|l|l|l|l|l|l|l}
$7 / 8$ & 0.0729 & 0.1562 & 0.2396 & 0.3229 & 0.4062 & 0.4896 & 0.5729 & 0.6562 & 0.7396 & 0.8229 & 0.9062 & 0.9896
\end{tabular} \begin{tabular}{ll|l|l|l|l|l|l|l|l|l|l|l}
32 & 0.0755 & 0.1589 & 0.2422 & 0.3255 & 0.4089 & 0.4922 & 0.5755 & 0.6589 & 0.7422 & 0.8255 & 0.9089 & 0.9922
\end{tabular} \begin{tabular}{l|l|l|l|l|l|l|l|l|l|l|l|l|l|l|l|l|} 
18 & 0.0781 & 0.1615 & 0.2448 & 0.3281 & 0.4115 & 0.4948 & 0.5781 & 0.6615 & 0.7448 & 0.8281 & 0.9115 & 0.9948
\end{tabular} \begin{tabular}{l|l|l|l|l|l|l|l|l|l|l|l|l|l|l|l|l|l} 
㨁 & 0.0807 & 0.1641 & 0.2474 & 0.3307 & 0.4141 & 0.4974 & 0.5807 & 0.6641 & 0.7474 & 0.8307 & 0.9141 & 0.9974
\end{tabular} 


\title{
AMERICAN SOCIETY FOR TESTING MATERIALS
}

\author{
Philadelphia, Pa., U. S. A.
}

AFFILIATED WITH THE

International Association For Testing Materials

\section{STANDARD SPECIFICATIONS FOR PORTLAND CEMENT}

ADOPTED, 1904; REVISED, 1908, 1909, 1916, 1917.

Portland cement is the product obtained by finely pulverizing clinker produced by calcining to ineipient fusion, an intimate and properly proportioned mixture of argillaceous and calcareous materials, with no additions subsequent to calcination excepting water and calcined or uncalcined gypsum.

\section{CHEMICAL PROPERTIES}

Chemical Limits. The following limits shall not be exceeded:

Loss on ignition, per cent . . . . . . . . . 4.00

Insoluble residue, per cent . . . . . . . . 0.85

Sulfuric anhydride $\left(\mathrm{SO}_{3}\right)$, per cent . . . . . . 2.00

Magnesia $(\mathrm{MgO})$, per cent . . . . . . . . . 5.00

\section{PHYSICAL PROPERTIES}

Specific Gravity. The specific gravity of cement shall be not less than 3.10 (3.07 for white Portland cement). Should the test of cement as received fall below this requirement a second test may be made upon an ignited sample. The specific gravity test will not be made unless specifically ordered.

Fineness. The residue on a standard No. 200 sieve shall not exeed 22 per cent by weight.

Soundness. A pat of neat cement shall remain firm and hard, and show no signs of distortion, cracking, checking, or disintegration in the steam test for soundness.

Time of Setting. The cement shall not develop initial set in less than 45 minutes when the Vicat needle is used, or 60 minutes when the Gillmore needle is used. Final set shall be attained within 10 hours.

Tensile Strength. The average tensile strength in pounds per square inch of not less than three standard mortar briquettes composed of one part cement and three parts standard sand, by weight, shall be equal to or higher than the following:

\begin{tabular}{c|c|c}
\hline $\begin{array}{c}\text { Age at Test } \\
\text { Days }\end{array}$ & Storage of Briquettes & $\begin{array}{c}\text { Tensile Strength } \\
\text { 1b. per sq. in. }\end{array}$ \\
\hline 7 & 1 day in moist air, 6 days in water & 200 \\
28 & 1 day in moist air, 27 days in water & 300 \\
\hline
\end{tabular}

The average tensile strength of standard mortar at 28 days shall be higher than the strength at 7 days.

\section{PACKAGES, MARKING AND STORAGE}

Packages and Marking. The cement shall be delivered in suitable bags or barrels with the brand and name of the manufacturer plainly marked thereon, unless shipped in bulk. A bag shall contain $94 \mathrm{lb}$. net. A barrel shall contain $376 \mathrm{lb}$. net. 
Storage. The cement shall be stored in such a manner as to permit easy access for proper inspection and identification of each shipment, and in a suitable weathertight building which will protect the cement from dampness.

\section{INSPECTION}

Inspection. Every facility shall be provided the purchaser for careful sampling and inspection at either the mill or at the site of the work, as may be specified by the purchaser. At least 10 days from the time of sampling shall be allowed for the completion of the 7-day test, and at least 31 days shall be allowed for the completion of the 28-day test. The cement shall be tested in accordance with the methods hereinafter prescribed. The 28-day test shall be waived only when specifically so ordered.

\section{REJECTION}

Rejection. The cement may be rejected if it fails to meet any of the requirements of these specifications.

Cement shall not be rejected on account of failure to meet the fineness requirement if upon retest after drying at 100 degrees $\mathrm{C}$. for one hour it meets this requirement.

Cement failing to meet the test for soundness in steam may be accepted if it passes a retest using a new sample at any time within 28 days thereafter.

Packages varying more than 5 per cent from the specified weight may be rejected; and if the average weight of packages in any shipment, as shown by weighing 50 packages taken at random, is less than that specified, the entire shipment may be rejected. 


\section{MANUFACTURERS' STANDARD SPECIFICATIONS FOR DEFORMED CONCRETE REINFORCEMENT BARS ROLLED FROM BILLETS \\ REVISED APRIL 21, 1914}

Manufacture. Steel may be made by either the open-hearth or Bessemer process. Bars shall be rolled from standard new billets.

Chemical and Physical Properties. The chemical and physical properties shall conform to the following limits:

\begin{tabular}{|c|c|c|c|}
\hline Properties Considered & $\begin{array}{l}\text { Structural } \\
\text { Grade }\end{array}$ & $\begin{array}{l}\text { Intermediate } \\
\text { Grade }\end{array}$ & $\begin{array}{l}\text { Hard } \\
\text { Grade }\end{array}$ \\
\hline 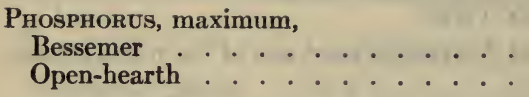 & $\begin{array}{l}0.10 \\
0.06\end{array}$ & $\begin{array}{l}0.10 \\
0.06\end{array}$ & $\begin{array}{l}0.10 \\
0.06\end{array}$ \\
\hline Ultimate tensile strength, lb. per sq. in. . & $55-70,000$ & $70-85,000$ & $80,000 \mathrm{~min}$. \\
\hline Yield point, minimum, lb. per sq. in. . . & 33,000 & 40,000 & 50,000 \\
\hline $\begin{array}{l}\text { Elongation, per cent in 8-in., minimum } \\
\text { CoLD BEND WITHOUT Fracture: }\end{array}$ & $\frac{1,250,000}{\text { Tens. Str. }}$ & $\frac{1,125,000}{\text { Tens. Str. }}$ & $\frac{1,000,000}{\text { Tens. Str. }}$ \\
\hline Bars under $3 / 4$-in. in diameter or thickness & $180^{\circ} d .=1 t$ & $180^{\circ} d=3 t$ & $180^{\circ} d=4 t$ \\
\hline $\begin{array}{l}\text { Bars } 3 / 4 \text {-in. in diameter or thickness and } \\
\text { over }\end{array}$ & $180^{\circ} d_{.}=2 t$ & $90^{\circ} \mathrm{d} .=3 \mathrm{t}$ & $90^{\circ} d .=4 t$. \\
\hline
\end{tabular}

The intermediate and hard grades will be used only when specified.

Chemical Determinations. In order to determine if the material conforms to the chemical limitations prescribed in the preceding tables, analysis shall be made by the manufacturer from a test ingot taken at the time of the pouring of each melt or blow of steel, and a correct copy of such analysis shall be furnished to the engineer or his inspector.

Yield Point. For the purposes of these specifications, the yield point shall be determined by careful observation of the drop of the beam of the testing machine, or by other equally accurate method.

Form of Specimens. Tensile and bending test specimens may be cut from the bars as rolled, but tensile and bending test specimens of deformed bars may be planed or turned for a length of at least nine inches if deemed necessary by the manufacturer in order to obtain uniform cross-section.

Number of Tests. (a)-At least one tensile and one bending test shall be made from each melt of open-hearth steel rolled, and from each blow or lot of ten tons of Bessemer steel rolled. In case bars differing $3 / 8$-inch and more in diameter or thickness are rolled from one melt or blow, a test shall be made from the thickest and thinnest material rolled. Should either of these test specimens develop flaws, or should the tensile test specimen break outside of the middle third of its gauged length, it may be discarded and another test specimen substituted therefor. In case a tensile test specimen does not meet the specifications, an additional test may be made.

(b)-The bending test may be made by pressure or by light blows. 
Modifications in Elongation for Thin and Thick Material. For bars less than $\frac{7}{16}$-inch and more than $3 / 4$-inch nominal diameter or thickness, the following modifcations shall be made in the requirements for elongation:

(a) - For each increase of $1 / 8$-inch in diameter or thickness above $3 / 4$-inch, a deduction of 1 shall be made from the specified percentage of elongation.

(b) - For each decrease of $\frac{1}{16}$-inch in diameter or thickness below $\frac{7}{16}$-inch, a deduction of 1 shall be made from the specified percentage of elongation.

Finish. Material must be free from injurious seams, flaws or cracks, and have a workmanlike finish.

Variation in Weight. Bars for reinforcement are subject to rejection if the actual weight of any lot varies more than 5 per cent over or under the theoretical weight of that lot.

NотE:-The chemical and physical properties are given for three different grades of steel. In using the specification care should be taken to indicate clearly the grade desired. 


\section{GENERAL SPECIFICATONS FOR MATERIALS AND LABOR USED IN REINFORCED CONCRETE CONSTRUCTION}

\section{MATERIALS}

Cement. The cement used for reinforced concrete construction shall be Portland cement which shall meet the requirements of the specifications and methods of tests last adopted by the American Society for Testing Materials.

Aggregates. (a) Fine.-Fine aggregates shall consist of uniformly graded sand or screenings by particles not exceeding $1 / 4$-inch in diameter; not more than 30 per cent by weight shall pass a sieve having 50 meshes per linear inch.

Particles shall be hard and clean; shall be free from coatings and soluble substances; shall contain no vegetable loam or other organic matter; and shall yield a 1:3 mortar of a strength at the age of seven days of not less than 70 per cent of that of 1:3 mortar of the same consistency and made with the same cement and standard Ottawa sand.

(b) Coarse.-Coarse aggregates shall consist of gravel or crushed stone which is retained on a screen having $1 / 4$-inch diameter holes and shall be graded from the smallest to the largest particles. The maximum size of particles shall be determined from the following table:

Nature of work

Maximum Sizes in Inches

Light slabs or partitions using mesh or expanded metal . . $1 / 2$

Flat Slab Floors, Beams and Slabs, Girders, Columns, Retaining Walls, Footings and other moderately heavy work

Heavy work .

$1 \frac{3 / 4}{11 / 2}$

Size and quality of stone for rubble concrete shall meet the approval of the Engineer.

All coarse aggregates shall be clean, hard, durable, free from coatings and all deleterious matter.

Water. The water used in mixing concrete shall be free from oil, acid, alkali or organic matter. Concrete shall not be mixed with sea water.

Metal Reinforcement. Bars used as reinforcement in reinforced concrete work shall be manufactured from new billet-steel and shall satisfy the specifications known as the Manufacturers' Standard Specifications for Billet-Steel Concrete Reinforcement Bars. All bars other than spiral wire shall be an approved deformed bar having a positive mechanical bond with the concrete equal to that of the Corrugated Bar, manufactured by the Corrugated Bar Company, Inc., Buffalo, N. Y.

\section{FORMS}

Character. Forms for reinforced concrete construction shall be substantial and unyielding, and shall conform to the design of the structure. They shall have a smooth surface in contact with the concrete, which shall be free from adhering material or from other defects which shall mar the finished surface. They shall be sufficiently tight to prevent the leakage of mortar.

The forms shall be so made that all interior angles caused by the junction of slabs and beams or other members shall be chamfered one inch. All exterior angles shall be made square.

Oiling and Inspection. Before placing the concrete in the forms, all debris in the space to be occupied by the concrete shall be removed. Handholes shall be provided at the base of the forms of all columns to render this space accessible for cleaning. 
The forms shall be thoroughly oiled (thin mineral oil) before the concrete is placed; or the surface shall be thoroughly wetted (excepting in freezing weather).

\section{PLACING OF REINFORCEMENT}

All reinforcement shall be placed in accordance with the plans furnished by the Engineer.

All loose rust or scale, all adhering material, and all oil or other substance which will tend to destroy bonding between the concrete and the reinforcement shall be removed before pouring begins.

\section{MIXING OF CONCRETE}

Mixing shall be done in a batch machine mixer of a type which will insure uniform distribution of the materials throughout the mass, and shall continue for the minimum time of one and one-half minutes after all ingredients are assembled in the mixer. For mixers of two or more cubic yards capacity the minimum time of mixing shall be two minutes. The drum of the machine shall be operated at a uniform speed of 200 feet per minute.

Unit of Measure. Measurements of fine and coarse aggregates and of cement, shall be by loose volume. The unit of measure shall be a bag of cement containing 94 pounds net, which should be considered the equivalent of one cubic foot.

Proportioning. The fine and coarse aggregates shall be so proportioned with the cement as to secure the ultimate compressive strength, in twenty-eight days, upon which the design of the structure was based.

The following table is recommended as the maximum ultimate compressive strength of different mixtures of concrete at twenty-eight days:

\section{Table of Compressive Strengths of Different Mixtures of Concrete}

(In Pounds per.Square Inch)

\begin{tabular}{l|c|r|r|r|r|r}
\hline \hline \multicolumn{1}{c|}{ Aggregate } & $1: 3^{*}$ & $1: 41 / 2 *$ & $1: 6 *$ & $1: 71 / 2 *$ & $1: 9 *$ \\
\hline Granite, trap rock . . . . . . . . . . . . . . & 3300 & 2800 & 2200 & 1800 & 1400 \\
Gravel, hard limestone and hard sandstone . . . . & 3000 & 2500 & 2000 & 1600 & 1300 \\
Soft limestone and sandstone . . . . . . . . . . . & 2200 & 1800 & 1500 & 1200 & 1000 \\
Cinders . . . . . . . . . . . . . . . . . . & 800 & 700 & 600 & 500 & 400 \\
\hline
\end{tabular}

Consistency. The materials shall be mixed wet enough to produce a concrete of such consistency as will flow sluggishly into the forms and about the metal reinforcement, and which at the same time can be conveyed from the mixer to the forms without separation of the coarse aggregate from the mortar. A properly mixed concrete is one which thoroughly sustains or supports the coarser aggregate throughout the mass and which when dumped from a barrel or buggy neither breaks nor flows readily over the edge.

Retempering. The remixing of mortar or concrete that has partly set shall not be permitted.

*The proportions here given are on the basis of separately measured aggregates. For instance; a 1:6 mixture refers to a mixture based on one part of cement and six parts of aggregates which were measured before being combined. A standard 1:2:4 mix, therefore, falls in the above class of 1:6 mix. 
Conveying and Placing. After the mixing of the concrete has been completed, it shall be conveyed as rapidly as possible to the place of deposit. No concrete shall be placed which has partly set. Where concrete is conveyed by spouting, the plant shall be of such size and design as to insure a practically continuous flow in the spout. The angle of the spout shall be such as to allow the concrete to flow without a separation of the ingredients. The spout shall be thoroughly cleaned by flushing before and after each run. Where it is impossible to deliver the concrete at the place of deposit without separation of the ingredients, the concrete shall be discharged upon a mixing board where it shall be mixed by turning until of uniform consistency before placing in the forms.

The concrete shall be deposited in the forms in such a manner as will permit the most thorough compacting obtained by working with a straight shovel or slicing tool kept moving up and down until all the ingredients are in their proper place.

Light horizontal reinforcement shall be raised from the bottom forms to allow the flow of the concrete under it. Where chairs are used, or where heavy reinforcement is definitely wired in place, the mass shall be thoroughly worked to insure contact of the mortar with the lower face of the reinforcing material.

Before concrete is placed upon previously poured concrete, care shall be taken to remove all debris from the concrete surface. All laitance shall be removed and the surface shall be thoroughly wetted and slushed with mortar consisting of one part Portland cement and two parts fine aggregate.

When it is necessary to stop pouring at a place where pouring will be resumed at a later date, all necessary grooves for joining future work shall be made before the concrete has set.

Construction Joints. Construction joints in columns shall be located at the base of the bell or flare occurring immediately below the floor slab in flat slab construction. In beam and girder construction the joint in the columns shall be located at the base of the lowest intersecting member at each floor.

Vertical joints formed by bulkheads which it may be necessary to construct in slab or beams shall be made at the center of the span of such slab or beam. In girders into which intersecting members are framed at the center of the span, the bulkhead shall be located within the middle third of the length of the span of such girder. On large beams or girders these bulkheads shall be placed inclined upward toward the nearest column.

Horizontal joints in large girders or other massive units shall be properly keyed by notching.

Lintel beams, whether above or below the slab, shall be poured monolithically with the slab.

No construction joints shall be allowed in footings, the pouring to be continued until the whole footing is completed to the base of the column.

All other joints in pouring shall be made only upon the approval of the Engineer.

Placing Concrete in Freezing Weather. No concrete shall be mixed or placed at a freezing temperature unless special precautions are taken to prevent the use of materials covered with ice crystals or containing frost, and to prevent the concrete from freezing before it has set and sufficiently hardened.

The material used shall be warmed well above the freezing point and the space of 
the structure in which pouring is taking place shall be maintained at a temperature well above the freczing point. Aggregates and water used shall not be heated to more than 70 degrces. The use of salt to lower the freezing point shall not be permitted.

Placing Concrete Under Water. Concrete may be placed only in still water, with the use of a tremie properly designed and operated. Concrete shall be mixed with more water than is ordinarily permissible so that it will flow readily through the tremie. Coarse aggregate used in concrete thus placed shall not be more than one inch in diameter.

In case the flow of concrete is interrupted, or in case it is necessary to provide a construction joint, care shall be taken to remove all laitance before proceeding with the work.

Protection of Exposed Surfaces. The surfaces of concrete exposed to premature drying shall be covered and kept wet for a period of at least seven days after pouring.

\section{REMOVAL OF FORMS}

Forms shall not be removed from the concrete until it is sufficiently hard to permit of this being done with safety.

In weather of a temperature above 60 degrees, the minimum time after pouring for the removal of forms shall be as follows:

Wall forms and forms for the sides of beams . . . . . . . 2 days

Golumn forms and forms under slabs of span less than 4 feet . 4 days

Slabs of span between supported girders or shoring between 4 and

10 feet . . . . . . . . . . . . . 6 days

Supports or shoring shall be maintained under horizontal members a minimum time after pouring in accordance with the following table:

Beams, Girders and Flat Slabs in ordinary building construction . 3 weeks

Spans over 30 feet . . . . . . . . . . . At least 1 month

Under all floors upon which building materials are being placed during construction, the supports or posts shall be left at least two weeks longer than specified in the above schedules.

In weather of a temperature below 60 degrees, the forms and supports shall be left in place a longer period, depending upon the weather encountered.

Especial care shall be taken in the removal of forms from any concrete that may have become frozen. Where it is likely that the concrete might have frozen, it may be tested by placing a piece of the concrete in warm water or upon a stove, after which, if the concrete is properly set, it shall not show any deterioration due to such treatment. A similar test may be made directly upon the structure by submitting it to the flame of a blow torch, which treatment will not produce any melting, if the concrete is properly set. If the concrete has frozen, the forms shall not be removed from it until it has had sufficient time to thoroughly thaw and set in warm weather. 


\section{RECOMMENDATIONS ON DESIGN AND WORKING STRESSES IN REINFORCED CON- CRETE CONSTRUCTION.}

(From the Final Report of the Joint Committee on Concrete and Reinforced Concrete, July 1, 1916)

Massive Concrete. In the design of massive or plain concrete, no account should be taken of the tensile strength of the material, and sections should usually be proportioned so as to avoid tensile stresses except in slight amounts to resist indirect stresses. This will generally be accomplished in the case of rectangular shapes if the line of pressure is kept within the middle third of the section, but in very large structures, such as high masonry dams, a more exact analysis may be required. Structures of massive concrete are able to resist unbalanced lateral forces by reason of their weight; hence the element of weight rather than strength often determines the design. A leaner and relatively cheap concrete, therefore, will often be suitable for massive concrete structures.

It is desirable generally to provide joints at intervals to localize the effect of contraction.

Massive concrete is suitable for dams, retaining walls, and piers in which the ratio of length to least width is relatively small. Under ordinary conditions this ratio should not exceed four. It is also suitable for arches of moderate span.

Reinforced Concrete. The use of metal reinforcement is particularly advantageous in members such as beams in which both tension and compression exist, and in columns where the principal stresses are compressive and where there also may be cross-bending. Therefore, the theory of design here presented relates mainly to the analysis of beams and columns.

General Assumptions. (a) Loads.-The forces to be resisted are those due to:

1. The dead load, which includes the weight of the structure and fixed loads and forces.

2. The live load, or the loads and forces which are variable. The dynamic effect of the live load will often require consideration. Allowance for the latter is preferably made by a proportionate increase in either the live load or the live load stresses. The working stresses hereinafter recommended are intended to apply to the equivalent static stresses thus determined.

In the case of high buildings the live load on columns may be reduced in accordance with the usual practice.

(b) Lengths of Beams and Columns.-The span length for beams and slabs simply supported should be taken as the distance from center to center of supports, but need not be taken to exceed the clear span plus the depth of beam or slab. For continuous or restrained beams built monolithically into supports the span length may be taken as the clear distance between faces of supports. Brackets should not be considered as reducing the clear span in the sense here intended, except that when brackets which make an angle of 45 degrees or more with the axis of a restrained beam are built monolithically with the beam, the span may be measured from the section where the combined depth of beam and bracket is at least one-third more than the depth of the beam. Maximum negative moments are to be considered as existing at the end of the span as here defined. 
When the depth of a restrained beam is greater at its ends than at midspan and the slope of the bottom of the beam at its ends makes an angle of not more than 15 degrees with the direction of the axis of the beam at midspan, the span length may be measured from face to face of supports.

The length of columns should be taken as the maximum unstayed length.

(c) Stresses. - The following assumptions are recommended as a basis for calculations:

1.-Calculations will be made with reference to working stresses and safe loads rather than with reference to ultimate strength and ultimate loads.

2.-A plane section before bending remains plane after bending.

3. - The modulus of elasticity of concrete in compression is constant within the usual limits of working stresses. The distribution of compressive stress in beams is, therefore, rectilinear.

4.-In calculating the moment of resistance of beams the tensile stresses in the concrete are neglected.

5.-The adhesion between the concrete and the reinforcement is perfect. Under compressive stress the two materials are, therefore, stressed in proportion to their moduli of elasticity.

6.-The ratio of the modulus of elasticity of steel to the modulus of elasticity of concrete is taken at 15 , except as modified in section on "Working Stresses."

7 -Initial stress in the reinforcement due to contraction or expansion of the concrete is neglected.

It is recognized that some of the assumptions given herein are not entirely borne out by experimental data. They are given in the interest of simplicity and uniformity, and variations from exact conditions are taken into account in the selection of formulas and working stresses.

The deflection of a beam depends upon the strength and stiffness developed throughout its length. For calculating deflection a value of 8 for the ratio of the moduli will give results corresponding approximately with the actual conditions.

T-Beams. In beam and slab construction an effective bond should be provided at the junction of the beam and slab. When the principal slab reinforcement is parallel to the beam, transverse reinforcement should be used extending over the beam and well into the slab.

The slab may be considered an integral part of the beam, when adequate bond and shearing resistance between slab and web of beam is provided, but its effective width shall be determined by the following rules:

(a) -It shall not exceed one-fourth of the span length of the beam;

(b) - Its overhanging width on either side of the web shall not exceed six times the thickness of the slab.

In the design of continuous T-beams, due consideration should be given to the compressive stress at the support.

Beams in which the T-form is used only for the purpose of providing additional compression area of concrete should preferably have a width of flange not more than three times the width of the stem and a thickness of flange not less than one-third of the depth of the beam. Both in this form and in the beam and slab form the web stresses and the limitations in placing and spacing the longitudinal reinforcement will probably be controlling factors in design. 
Floor Slabs Supported Along Four Sides. Floor slabs having the supports extending along the four sides should be designed and reinforced as continuous over the supports. If the length of the slab exceeds 1.5 times its width the entire load should be carried by transverse reinforcement.

For uniformity distributed loads on square slabs, one-half the live and dead load may be used in the calculations of moment to be resisted in cach direction. For oblong slabs, the length of which is not greater than one and one-half times their width, the moment to be resisted by the transverse reinforcement may be found by using a proportion of the live and dead load equal to that given by the formula $r=\frac{l}{b}-0.5$, where $l=$ length and $b=$ breadth of slab. The longitudinal reinforcement should then be proportioned to carry the remainder of the load.

In placing reinforcement in such slabs account may well be taken of the fact that the bending moment is greater near the center of the slab than near the edges. For this purpose two-thirds of the previously calculated moments may be assumed as carried by the center half of the slab and one-third by the outside quarters.

Loads carried to beams by slabs which are reinforced in two directions will not be uniformly distributed to the supporting beams and the distribution will depend on the relative stiffness of the slab and the supporting beams. The distribution which may be expected ordinarily is a variation of the load in the beam in accordance with the ordinates of a parabola, having its vertex at the middle of the span. For any given design, the probable distribution should be ascertained and the moments in the beam calculated accordingly.

Continuous Beams and Slabs. When the beam or slab is continuous over its supports, reinforcement should be fully provided at points of negative moment, and the stresses in concrete recommended in the section on "Working Stresses," should not be exceeded. In computing the positive and negative moments in beams and slabs continuous over several supports, due to uniformly distributed loads, the following rules are recommended:

(a) For floor slabs the bending moments at center and at support should be taken at $\frac{w l^{2}}{12}$ for both dead and live loads, where $w$ represents the load per linear unit and $l$ the span length.

(b) For beams the bending moment at center and at support for interior spans should be taken at $\frac{w l^{2}}{12}$ and for end spans it should be taken at $\frac{w l^{2}}{10}$ for center and interior support, for both dead and live loads.

(c) In the case of beams and slabs continuous for two spans only, with their ends restrained, the bending moment both at the central support and near the middle of the span should be taken at $\frac{w l^{2}}{10}$.

(d) At the ends of continuous beams the amount of negative moment which will be developed in the beam will depend on the condition of restraint or fixedness, and this will depend on the form of construction used. In the ordinary cases a moment of 
$\frac{w l^{2}}{16}$ may be taken; for small beams running into heavy columns this should be increased, but not to exced $\frac{w l^{2} \text {. }}{12}$

For spans of unusual length, or for spans of materially unequal length, more exact calculations should be made. Special consideration is also required in the case of concentrated loads.

Even if the center of the span is designed for a greater bending moment than is called for by $(a)$ or $(b)$, the negative moment at the support should not be taken as less than the values there given.

Where beams are reinforced on the compression side, the steel may be assumed to carry its proportion of stress in accordance with the ratio of moduli of elasticity, as given in the section on "Working Stresses." Reinforcing bars for compression in beams should be straight and should be two diameters in the clear from the surface of the concrete. For the positive bending moment, such reinforcement should not exceed one per cent of the area of the concrete. In the case of cantilever and continuous beams, tensile and compressive reinforcement over supports should extend sufficiently beyond the support and beyond the point of inflection to develop the requisite bond strength.

In construction made continuous over supports it is important that ample foundations should be provided; for unequal settlements are liable to produce unsightly, if not dangerous cracks. This effect is more likely to occur in low structures.

Girders, such as wall girders, which have beams framed into one side only, should be designed to resist torsional moment arising from the negative moment at the end of the beam.

Bond Strength and Spacing of Reinforcement. Adequate bond strength should be provided. The formula hereinafter given for bond stresses in beams is for straight longitudinal bars. In beams in which a portion of the reinforcement is bent up near the end, the bond stress at places, in both the straight bars and the bent bars, will be considerably greater than for all the bars straight, and the stress at some point may be several times as much as that found by considering the stress to be uniformly distributed along the bar. In restrained and cantilever beams full tensile stress exists in the reinforcing bars at the point of support and the bars should be anchored in the support sufficiently to develop this stress.

In case of anchorage of bars, an additional length of bar should be provided beyond that found on the assumption of uniform bond stress, for the reason that before the bond resistance at the end of the bar can be developed the bar may have begun to slip at another point and "running" resistance is less than the resistance before slip begins.

Where high bond resistance is required, the deformed bar is a suitable means of supplying the necessary strength. But it should be recognized that even with a deformed bar initial slip occurs at early loads, and that the ultimate loads obtained in the usual tests for bond resistance may be misleading. Adequate bond strength throughout the length of a bar is preferable to end anchorage, but, as an additional safeguard, such anchorage may properly be used in special cases. Anchorage furnished by short bends at a right angle is less effective than by hooks consisting of turns through 180 degrees. 
The lateral spacing of parallel bars should be not less than three diameters from center to center, nor should the distance from the side of the beam to the center of the nearest bar be less than two diameters. The clear spacing between two layers of bars should be not less than one inch. The use of more than two layers is not recommended, unless the layers are tied together by adequate metal connections, particularly at and near points where bars are bent up or bent down. Where more than one layer is used at least all bars above the lower layer should be bent up and anchored beyond the edge of the support.

Diagonal Tension and Shear. When a reinforced concrete beam is subjected to flexural action, diagonal tensile stresses are set up. A beam without web reinforcement will fail if these stresses exceed the tensile strength of the concrete. When web reinforcement, made up of stirrups or of diagonal bars secured to the longitudinal reinforcement, or of longitudinal reinforcing bars bent up at several points, is used, new conditions prevail, but even in this case at the beginning of loading the diagonal tension developed is taken principally by the concrete, the deformations which are developed in the concrete permitting but little stress to be taken by the web reinforcement. When the resistance of the concrete to the diagonal tension is overcome at any point in the depth of the beam, greater stress is at once set up in the web reinforcement.

For homogeneous beams the analytical treatment of diagonal tension is not very complex, the diagonal tensile stress is a function of the horizontal and vertical shearing stresses and of the horizontal tensile stress at the point considered, and as the intensity of these three stresses varies from the neutral axis to the remotest fibre, the intensity of the diagonal tension will be different at different points in the section, and will change with different proportionate dimensions of length to depth of beam. For the composite structure of reinforced concrete beams, an analysis of the web stresses, and particularly of the diagonal tensile stresses, is very complex; and when the variations due to a change from no horizontal tensile stress in the concrete at remotest fibre to the presence of horizontal tensile stress at some point below the neutral axis are considered, the problem becomes more complex and indefinite. Under these circumstances, in designing recourse is had to the use of the calculated vertical shearing stress as a means of comparing or measuring the diagonal tensile stresses developed, it being understood that the vertical shearing stress is not the numerical equivalent of the diagonal tensile stress, and that there is not even a constant ratio between them. It is here recommended that the maximum vertical shearing stress in a section be used as the means of comparison of the resistance to diagonal tensile stress developed in the concrete in beams not having web reinforcement.

Even after the concrete has reached its limit of resistance to diagonal tension, if the beam has web reinforcement, conditions of beam action will continue to prevail, at least through the compression area, and the web reinforcement will be called on to resist only a part of the web stresses. From experiments with beams it is concluded that it is safe practice to use only two-thirds of the external vertical shear in making calculations of the stresses that come on stirrups, diagonal web pieces, and bent-up bars, and it is here recommended for calculations in designing that two-thirds of the external vertical shear be taken as producing stresses in web reinforcement. 
It is well established that vertical members attached to or looped about horizontal members, inclined members secured to horizontal members in such a way as to insure against slip, and the bending of a part of the longitudinal reinforcement at an angle, will increase the strength of a beam against failure by diagonal tension, and that a welldesigned and well-distributed web reinforcement may under the best conditions increase the total vertical shear carried to a value as much as three times that obtained when the bars are all horizontal and no web reinforcement is used.

When web reinforcement comes into action as the principal tension web resistance, the bond stresses between the longitudinal bars and the concrete are not distributed as uniformly along the bars as they otherwise would be, but tend to be concentrated at and near stirrups, and at and near the points where bars are bent up. When stirrups are not rigidly attached to the longitudinal bars, and the proportioning of bars and stirrups spacing is such that local slip of bars occurs at stirrups, the effectiveness of the stirrups is impaired, though the presence of stirrups still gives an clement of toughness against diagonal tension failure.

Sufficient bond resistance between the concrete and the stirrups or diagonals must be provided in the compression area of the beam.

The longitudinal spacing of vertical stirrups should not exceed one-half the depth of beam, and that of inclined members should not exceed three-fourths of the depth of beam.

Bending of longitudinal reinforcing bars at an angle across the web of the beam may be considered as adding to diagonal tension resistance for a horizontal distance from the point of bending equal to three-fourths of the depth of beam. Where the bending is made at two or more points, the distance between points of bending should not exceed three-fourths of the depth of the beam. In the case of a restrained beam the effect of bending up a bar at the bottom of the beam in resisting diagonal tension may not be taken as extending beyond a section at the point of inflection, and the effect of bending down a bar in the region of negative moment may be taken as extending from the point of bending down of bar nearest the support to a section not more than three-fourths of the depth of beam beyond the point of bending down of bar farthest from the support but not beyond the point of inflection. In case stirrups are used in the beam away from the region in which the bent bars are condsidered effective, a stirrup should be placed not farther than a distance equal to one-fourth the depth of beam from the limiting sections defined above. In case the web resistance required through the region of bent bars is greater than that furnished by the bent bars, sufficient additional web reinforcement in the form of stirrups or attached diagonals should be provided. The higher resistance to diagonal tension stresses given by unit frames having the stirrups and bent-up bars securely connected together both longitudinally and laterally is worthy of recognition. It is necessary that a limit be placed on the amount of shear which may be allowed in a beam; for when web reinforcement sufficiently efficient to give very high web resistance is used, at the higher stresses the concrete in the beam becomes checked and cracked in such a way as to endanger its durability as well as its strength.

The section to be taken as the critical section in the calculation of shearing stresses will generally be the one having the maximum vertical shear, though experiments show that the section at which diagonal tension failures occur is not just at a support even though the shear at the latter point be much greater. 
In the case of restrained beams, the first stirrup or the point of bending down of bar should be placed not farther than one-half of the depth of beam away from the face of the support.

It is important that adequate bond strength or anchorage be provided to develop fully the assumed strength of all web reinforcement.

Low bond stresses in the longitudinal bars are helpful in giving resistance against diagonal tension failures and anchorage of longitudinal bars at the ends of the beams or in the supports is advantageous.

It should be noted that it is on the tension side of a beam that diagonal tension develops in a critical way, and that proper connection should always be made between stirrups or other web reinforcement and the longitudinal tension reinforcement, whether the latter is on the lower side of the beam or on its upper side. Where negative moment exists, as is the case near the supports in a continuous beam, web reinforcement to be effective must be looped over or wrapped around or be connected with the longitudinal tension reinforcing bars at the top of the beam in the same way as is necessary at the bottom of the beam at sections where the bending moment is positive.

Inasmuch as the smaller the longitudinal deformations in the horizontal reinforcement are, the less the tendency for the formation of diagonal cracks, a beam will be strengthened against diagonal tension failure by so arranging and proportioning the horizontal reinforcement that the unit stresses at points of large shear shall be relatively low.

It does not seem feasible to make a complete analysis of the action of web reinforcement, and more or less empirical methods of calculation are therefore employed. Limiting values of working stresses for different types of web reinforcement are given in the section on "Working Stresses." The conditions apply to cases commonly met in design. It is assumed that adequate bond resistance or anchorage of all web reinforcement will be provided.

When a flat slab rests on a column, or a column bears on a footing, the vertical shearing stresses in the slab or footing immediately adjacent to the column are termed punching shearing stresses. The element of diagonal tension, being a function of the bending moment as well as of shear, may be small in such cases, or may be otherwise provided for. For this reason the permissible limit of stress for punching shear may be higher than the allowable limit when the shearing stress is used as a means of comparing diagonal tensile stress. The working values recommended are given in the section on "Working Stresses."

Columns. By columns are meant compression members of which the ratio of unsupported length to least width exceeds about four, and which are provided with reinforcement of one of the forms hereafter described.

It is recommended that the ratio of unsupported length of column to its least width be limited to fifteen.

The effective area of hooped columns or columns reinforced with structural shapes shall be taken as the area within the circle enclosing the spiral or the polygon enclosing the structrual shapes.

Columns may be reinforced by longitudinal bars; by bands, hoops, or spirals, together with longitudinal bars; or by structural forms which are sufficiently rigid to 
have value in themselves as columns. The general effect of closely spaced hooping is to greatly increase the toughness of the column and to add to its ultimate strength, but hooping has little effect on its behavior within the limit of elasticity. It thus renders the concrete a safer and more reliable material, and should permit the use of a somewhat higher working stress. The beneficial effects of toughening are adequately provided by a moderate amount of hooping, a larger amount serving mainly to increase the ultimate strength and the deformation possible before ultimate failure.

Composite columns of structural steel and concrete in which the steel forms a column by itself should be designed with caution. To classify this type as a concrete column reinforced with structural steel is hardly permissible, as the steel, generally, will take the greater part of the load. When this type of column is used, the concrete should be adequately tied together by tie plates or lattice bars, which, together with other details, such as splices, etc., should be designed in conformity with standard practice for structural steel. The concrete may exert a beneficial effect in restraining the steel from lateral deflection and also in increasing the carrying capacity of the column. The proportion of load to be carried by the concrete will depend on the form of the column and the method of construction. Generally, for high percentages of steel, the concrete will develop relatively low unit stresses, and caution should be used in placing dependence on the concrete.

The following recommendations are made for the relative working stresses in the concrete for the several types of columns:

(a) Columns with longitudinal reinforcement to the extent of not less than 1 per cent and not more than 4 per cent, and with lateral ties of not less than $1 / 4$ inch in diameter 12 inches apart, nor more than 16 diameters of the longitudinal bar: the unit stress recommended for axial compression, on concrete piers having a length not more than four diameters, in section on "Working Stresses."

(b) Columns reinforced with not less than 1 per cent and not more than 4 per cent of longitudinal bars and with circular hoops or spirals not less than 1 per cent of the volume of the concrete and as hereinafter specified: a unit stress 55 per cent higher than given for $(a)$, provided the ratio of unsupported length of column to diameter of the hooped core is not more than 10 .

The foregoing recommendations are based on the following conditions:

It is recommended that the minimum size of columns to which the working stresses may be applied be 12 inches out to out.

In all cases longitudinal reinforcement is assumed to carry its proportion of stress in accordance with Section $(c) 6$, page 195. The hoops or bands are not to be counted on directly as adding to the strength of the column.

Longitudinal reinforcement bars should be maintained straight, and should have sufficient lateral support to be securely held in place until the concrete has set.

Where hooping is used, the total amount of such reinforcement shall be not less than one per cent of the volume of the column, enclosed. The clear spacing of such hooping shall not be greater than one-sixth the diameter of the enclosed column and preferably not greater than one-tenth, and in no case more than $2 \frac{1}{2}-\mathrm{in}$. Hooping is to be circular and the ends of bands must be united in such a way as to develop their full strèngth. Adequate means must be provided to hold bands or hoops in place so as to form a 
column, the core of which shall be straight and well centered. The strength of hooped columns depends very much upon the ratio of length to diameter of hooped core, and the strength due to hooping decreases rapidly as this ratio increases beyond five. The working stresses recommended are for hooped columns with a length of not more than ten diameters of the hooped core.

The Committee has no recommendation to make for a formula for working stresses for columns longer than ten diameters.

Bending stresses due to eccentric loads, such as unequal spans of beams, and to lateral forces, must be provided for by increasing the section until the maximum stress does not exceed the values above specified. Where tension is possible in the longitudinal bars of the columns, adequate connection between the ends of the bars must be provided to take this tension.

Reinforcing for Shrinkage and Temperature Stresses. When areas of concrete too large to expand and contract freely as a whole are exposed to atmospheric conditions, the changes of form due to shrinkage and to action of temperature are such that cracks may occur in the mass unless precautions are taken to distribute the stresses so as to prevent the cracks altogether or to render them very small. The distance apart of the cracks, and consequently their size, will be directly proportional to the diameter of the reinforcement and to the tensile strength of the concrete, and inversely proportional to the percentage of reinforcement and also to its bond resistance per unit of surface area. To be most effective, therefore, reinforcement (in amount generally not less than one-third of one per cent of the gross area) of a form which will develop a high bond resistance should be placed near the exposed surface and be well distributed. Where openings occur the area of cross-section of the reinforcement should not be reduced. The allowable size and spacing of cracks depends on various considerations, such as the necessity for water-tightness, the importance of appearance of the surface, and the atmospheric changes.

The tendency of concrete to shrink makes it necessary, except where expansion is provided for, to thoroughly connect the component parts of the frame of articulated structures, such as floor and wall members in buildings, by the use of suitable reinforcing material. The amount of reinforcement for such connection should bear some relation to the size of the members connected, larger and heavier members requiring stronger connections. The reinforcing bars should be extended beyond the critical section far enough, or should be sufficiently anchored to develop their full tensile strength.

Flat Slab. The continuous flat slab reinforced in two or more directions and built monolithically with the supporting columns (without beams or girders) is a type of construction which is now extensively used and which has recognized advantages for certain types of structures as, for example, warehouses in which large, open floor space is desired. In its construction, there is excellent opportunity for inspecting the position of the reinforcement. The conditions attending deposition and placing of concrete are favorable to securing uniformity and soundness in the concrete. The recommendations in the following paragraphs relate to flat slabs extending over several rows of panels in each direction. Necessarily the treatment is more or less empirical.

The co-efficients and moments given relate to uniformly distributed loads. 
(a) Column Capital.- - It is usual in flat slab construction to enlarge the supporting columns at their top, thus forming column capitals. The size and shape of the column capital affect the strength of the structure in several ways. The moment of the external forces which the slab is called upon to resist is dependent upon the size of the capital; the section of the slab immediately above the upper periphery of the capital carries the highest amount of punching shear; and the bending moment developed in the column by an eccentric or unbalanced loading of the slab is greatest at the under surface of the slab. Generally the horizontal section of the column capital should be round or square with rounded corners. In oblong panels the section may be oval or oblong, with dimensions proportional to the panel dimensions. For computation purposes, the diameter of the column capital will be considered to be measured where its vertical thickness is at least $1 \frac{1}{2}$ inches, provided the slope of the capital below this point nowhere makes an angle with the vertical of more than 45 degrees. In case a cap is placed above the column capital, the part of this cap within a cone made by extending the lines of the column capital upward at the slope of 45 degrees to the bottom of the slab or dropped panel may be considered as part of the column capital in determining the diameter for design purposes. Without attempting to limit the size of the column capital for special cases, it is recommended that the diameter of the column capital (or its dimension parallel to the edge of the panel) generally be made not less than one-fifth of the dimension of the panel from center to center of adjacent columns. A diameter equal to 0.225 of the panel length has been used quite widely and acceptably. For heavy loads or large panels especial attention should be given to designing and reinforcing the column capital with respect to compressive stresses and bending moments. In the case of heavy loads or large panels, and where the conditions of the panel loading or variations in panel length or other conditions cause high bending stresses in the column, and also for column capitals smaller than the size herein recommended, especial attention should be given to designing and reinforcing the column capital with respect to compression and to rigidity of connection to floor slab.

(b) Dropped Panel.-In one type of construction the slab is thickened throughout an area surrounding the column capital. The square or oblong of thickened slab thus formed is called a dropped panel or a drop. The thickness and the width of the dropped panel may be governed by the amount of resisting moment to be provided (the compressive stress in the concrete being dependent upon both thickness and width), or its thickness may be governed by the resistance to shear required at the edge of the column capital and its width by the allowable compressive stresses and shearing stresses in the thinner portion of the slab adjacent to the dropped panel. Generally however, it is recommended that the width of the dropped panel be at least fourtenths of the corresponding side of the panel as measured from center to center of columns, and that the offset in thickness be not more than five-tenths of the thickness of the slab outside the dropped panel.

(c) Slab Thickness.-In the design of a slab, the resistance to bending and to shearing forces will largely govern the thickness, and, in the case of large panels with light loads, resistance to deflection may be a controlling factor. The following formulas for minimum thicknesses are recommended as general rules of design when the diameter of the column capital is not less than one-fifth of the dimension of the panel from 
center to center of adjacent columns, the large dimension being used in the case of oblong panels. For notation, let

$$
\begin{aligned}
t & =\text { Total thickness of slab in inches. } \\
L & =\text { Panel length in feet. } \\
w & =\text { Sum of live load and dead load in pounds per square foot. }
\end{aligned}
$$

Then, for a slab without dropped panels, minimum $t=0.024 L \sqrt{w}+11 / 2$; for a slab with dropped panels, minimum $t=0.02 L \sqrt{w}+1$; for a dropped panel whose width is four-tenths of the panel length, minimum $t=0.03$ $L \sqrt{w}+11 / 2$.

In no case should the slab thickness be made less than six inches, nor should the thickness of a floor slab be made less than one-thirty-second of the panel length, nor the thickness of a roof slab less than one-fortieth of the panel length.

(d) Bending and Resisting Moments in Slabs.-If a vertical section of a slab be taken across a panel along a line midway between columns, and if another section be taken

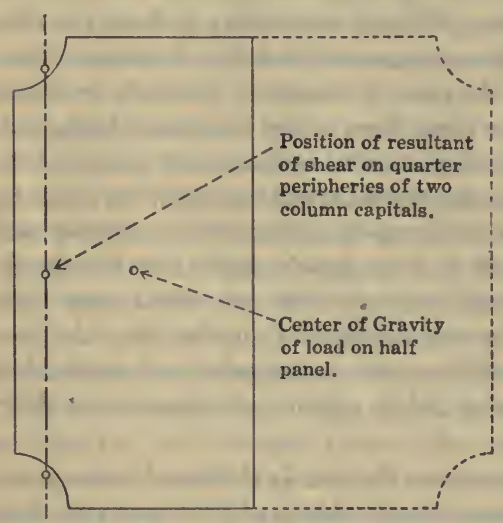

Fig. 10

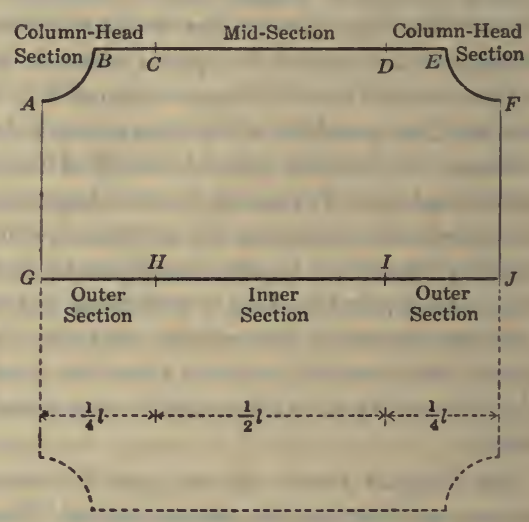

FIG. 11

along an edge of the panel parallel to the first section, but skirting the part of the periphery of the column capitals at the two corners of the panels, the moment of the couple formed by the external load on the half panel, exclusive of that over the column capital (sum of dead and live load) and the resultant of the external shear or reaction at the support at the two column capitals (see Fig. 10), may be found by ordinary static analysis. It will be noted that the edges of the area here considered are along lines of zero shear except around the column capitals. This moment of the external forces acting on the half panel will be resisted by the numerical sum of $(a)$ the moment of the internal stresses at the section of the panel midway between columns (positive resisting moment) and $(b)$ the moment of the internal stresses at the section referred to at the end of the panel (negative resisting moment). In the curved portion of the end section (that skirting the column), the stresses considered are the components which act parallel to the normal stresses on the straight portion of the section. Analysis shows that, for a uniformly distributed load, and round columns, and square 
panels, the numerical sum of the positive moment and the negative moment at the two sections named is given quite closely by the equation

$$
M_{\mathbf{x}}=\frac{1}{8} w l\left(l-\frac{2 \mathrm{c}}{3}\right)^{2}
$$

In this formula and in those which follow relating to oblong panels:

$w=$ sum of the live and dead load per unit of area;

$l=$ side of a square panel measured from center to center of columns;

$l_{1}=$ one side of the oblong panel measured from center to center of columns;

$l_{2}=$ other side of oblong panel measured in the same way;

$c=$ diameter of the column capital;

$M_{\mathrm{x}}=$ numerical sum of positive moment and negative moment in one direction.

$M_{y}=$ numerical sum of positive moment and negative moment in the other direction.

(See paper and closure, Statical Limitations upon the Steel Requirement in Reinforced Concrete Flat Slab Floors, by John R. Nichols, Jun. Am. Soc. C. E., Transactions Am. Soc. C. E. Vol. LXXVII.)

For oblong panels, the equations for the numerical sums of the positive moment and the negative moment at the two sections named become,

$$
\begin{aligned}
& M_{\mathrm{x}}=\frac{1}{8} w l_{2}\left(l_{1}-\frac{2 \mathrm{c}}{3}\right)^{2} \\
& M_{\mathrm{y}}=\frac{1}{8} w l_{1}\left(l_{2}-\frac{2 \mathrm{c}}{3}\right)^{2}
\end{aligned}
$$

Where $M_{\mathrm{x}}=$ is the numerical sum of the positive moment and the negative moment for the sections parallel to the dimensions $l_{2}$, and $M_{\mathrm{y}}$ is the numerical sum of the positive moment and the negative moment for the sections parallel to the dimensions $l_{1}$.

What proportion of the total resistance exists as positive moment and what as negative moment is not readily determined. The amount of the positive moment and that of the negative moment may be expected to vary somewhat with the design of the slab. It scems proper, however, to make the division of total resisting moment in the ratio of three-eighths for the positive moment to five-eighths for the negative moment.

With reference to variations in stress along the sections, it is evident from conditions of flexure that the resisting moment is not distributed uniformly along either the section of positive moment or that of negative moment. As the law of the distribution is not known definitely, it will be necessary to make an empirical apportionment along the sections; and it will be considered sufficiently accurate generally to divide the sections into two parts and to use an average value over each part of the panel section.

The relatively large breadth of structure in a flat slab makes the effect of local variations in the concrete less than would be the case for narrow members like beams. The tensile resistance of the concrete is less affected by cracks. Measurements of deformations in buildings under heavy load indicate the presence of considerable tensile resistance in the concrete, and the presence of this tensile resistance acts to decrease the intensity of the compressive stresses. It is believed that the use of moment co-efficients somewhat less than those given in a preceding paragraph as derived by analysis is warranted, the calculations of resisting moment and stresses in concrete 
and reinforcement being made according to the assumptions specified in this report and no change being made in the values of the working stresses ordinarily used. Accordingly, the values of the moments which are recommended for use are somewhat less than those derived by analysis. The values given may be used when the column capitals are round, oval, square or oblong.

(e) Names for Moment Sections.-For convenience, that portion of the section across a panel along a line midway between columns which lies within the middle two quarters of the width of the panel (HI, Fig. 11), will be called the inner section, and that portion in the two outer quarters of the width of the panel (GH and $I J$, Fig. 11) will be called the outer sections. Of the section which follows a panel edge from column capital to column capital and which includes the quarter peripheries of the edges of two column capitals, that portion within the middle two quarters of the panel width $(C D$, Fig. 11) will be called the mid-section, and the two remaining portions ( $A B C$ and $D E F$, Fig. 11), each having a projected width equal to one-fourth of the panel width, will be called the column-head sections.

(f) Positive Moment.-For a square interior panel, it is recommended that the positive moment for a section in the middle of a panel extending across its width be taken as $\frac{1}{25} w l\left(l-\frac{2 c}{3}\right)^{2}$. Of this moment, at least 25 per cent should be provided for in the inner section; in the two outer sections of the panel at least 55 per cent of the specified moment should be provided for in slabs not having dropped panels, and at least 60 per cent in slabs having dropped panels, except that in calculations to determine necessary thickness of slab away from the dropped panel at least 70 per cent of the positive moment should be considered as acting in the two outer sections.

(g) Negative Moment.-For a square interior panel, it is recommended that the negative moment for a section which follows a panel edge from column capital to column capital and which includes the quarter peripheries of the edges of the two column capitals (the section altogether forming the projected width of the panel) be taken as $\frac{1}{15} w l\left(l-\frac{2 c}{3}\right)^{2}$. Of this negative moment, at least 20 per cent should be provided for in the mid-section and at least 65 per cent in the two column-head sections of the panel, except that in slabs having dropped panels at least 80 per cent of the specified negative moment should be provided for in the two column-head sections of the panel.

(h) Moments for Oblong Panels. - When the length of a panel does not exceed the breadth by more than 5 per cent, computation may be made on the basis of a square panel with sides equal to the mean of the length and the breadth.

When the long side of an interior oblong panel exceeds the short side by more than one-twentieth and by not more than one-third of the short side, it is recommended that the positive moment be taken as $\frac{1}{25} w l_{2}\left(l_{1}-\frac{2 \mathrm{c}}{3}\right)^{2}$ on a section parallel to the dimension $l_{2}$, and $\frac{1}{25} w l_{1}\left(l_{2}-\frac{2 \mathrm{c}}{3}\right)^{2}$ on a section parallel to dimension $l_{1}$; and that the negative moment be taken as $\frac{1}{15} w l_{2}\left(l_{1}-\frac{2 c}{3}\right)^{2}$ on a section at the edge of the panel correspond- 
ing to the dimension $l_{2}$, and $\frac{1}{15} w l_{1}\left(l_{2}-\frac{2 \mathrm{c}}{3}\right)^{2}$ at a section in the other direction. The limitations of the apportionment of moment between inner section and outer section and between mid-section and column-head sections may be the same as for square panels.

(i) Wall Panels.-The co-efficient of negative moment at the first row of columns away from the wall should be increased 20 per cent over that required for interior panels, and likewise the co-efficient of positive moment at the section half way to the wall should be increased by 20 per cent. If girders are not provided along the wall or the slab does not project as a cantilever beyond the column line, the reinforcement parallel to the wall for the negative moment in the column-head section and for the positive moment in the outer section should be increased by 20 per cent. If the wall is carried by the slab this concentrated load should be provided for in the design of the slab. The co-efficient of negative moments at the wall to take bending in the direction perpendicular to the wall line may be determined by the conditions of restraint and fixedness as found from the relative stiffness of columns and slab, but in no case should it be taken as less than one-half of that for interior panels.

(j) Reinforcement.-In the calculation of moments all the reinforcing bars which cross the section under consideration and which fulfill the requirements given under paragraph $(l)$ of this chapter may be used. For a column-head section reinforcing bars parallel to the straight portion of the section do not contribute to the negative resisting moment for the column-head section in question. In the case of four-way reinforcement the sectional area of the diagonal bars multiplied by the sine of the angle between the diagonal of the panel and straight portion of the section under consideration may be taken to act as reinforcement in a rectangular direction.

(k) Point of Inflection.-For the purpose of making calculations of moments-at sections away from the sections of negative moment and positive moment already specified, the point of inflection on any line parallel to a panel edge may be taken as one-fifth of the clear distance on that line between the two sections of negative moment at the opposite ends of the panel indicated in paragraph $(e)$, of this chapter. For slabs having dropped panels the co-efficient of one-fourth should be used instead of one-fifth.

(l) Arrangement of Reinforcement.-The design should include adequate provision for securing the reinforcement in place so as to take not only the maximum moments, but the moments at intermediate sections. All bars in rectangular bands or diagonal bands should extend on each side of a section of maximum moment, either positive or negative, to points at least twenty diameters beyond the point of inflection as defined herein or be hooked or anchored at the point of inflection. In addition to this provision bars in diagonal bands used as reinforcement for negative moment should extend on each side of a line drawn through the column center at right angles to the direction of the band at least a distance equal to thirty-five one-hundredths of the panel length, and bars in diagonal bands used as reinforcement for positive moment should extend on each side of a diagonal through the center of the panel at least a distance equal to thirty-five one-hundredths of the panel length; and no splice by lapping should be permitted at or near regions of maximum stress except as just described. Continuity of reinforcing bars is considered to have advantages, and it is recommended that not more than one-third of the reinforcing bars in any direction be made of a length less 
than the distance center to center of columns in that direction. Continuous bars should not all be bent up at the same point of their length, but the zone in which this bending occurs should extend on each side of the assumed point of inflection, and should cover a width of at least one-fifteenth of the panel length. Mere draping of the bars should not be permitted. In four-way reinforcement the position of the bars in both diagonal and rectangular directions may be considered in determining whether the width of zone of bending is sufficient.

( $m$ ) Reinforcement at Construction Joints.-It is recommended that at construction joints extra reinforcing bars equal in section to 20 per cent of the amount necessary to meet the requirements for moments at the section where the joint is made be added to the reinforcement, these bars to extend not less than 50 diameters beyond the joint on each side.

(n) Tensile and Compressive Stresses.-The usual method of calculating the tensile and compressive stresses in the concrete and in the reinforcement, based on the assumptions for internal stresses given in this chapter, should be followed. In the case of the dropped panel the section of the slab and dropped panel may be considered to act integrally for a width equal to the width of the column-head section.

(o) Provision for Diagonal Tension and Shear.-In calculations for the shearing stress which is to be used as the means of measuring the resistance to diagonal tension stress, it is recommended that the total vertical shear on two column-head sections constituting a width equal to one-half the lateral dimensions of the panel, for use in the formula for determining critical shearing stresses, be considered to be one-fourth of the total dead and live load on a panel for a slab of uniform thickness, and to be three-tenths of the sum of the dead and live loads on a panel for a slab with dropped panels. The formula for shearing unit stress may then be written $v=\frac{0.25 W}{b j d}$ for slabs of uniform thickness, and $v=\frac{0.30 W}{b j d}$ for slabs with dropped panels, where $W$ is the sum of the dead and live load on a panel, $b$ is half the lateral dimension of the panel measured from center to center of columns, and $j d$ is the lever arm of the resisting couple at the section.

The calculation of what is commonly called punching shear may be made on the assumption of a uniform distribution over the section of the slab around the periphery of the column capital and also of a uniform distribution over the section of the slab around the periphery of the dropped panel, using in each case an amount of vertical shear greater by 25 per cent than the total vertical shear on the section under consideration.

The values of working stresses should be those recommended for diagonal tension and shear in the section on "Working Stresses."

( $p$ ) Walls and Openings.-Girders or beams should be constructed to carry walls and other concentrated loads which are in excess of the working capacity of the slab. Beams should also be provided in case openings in the floor reduce the working strength of the slab below the required carrying capacity.

(q) Unusual Panels.-The co-efficients, apportionments, and thicknesses recommended are for slabs which have several rows of panels in each direction, and in which the size of the panels is approximately the same. For structures having a width of one, 
two, or three panels, and also for slabs having panels of markedly different sizes, an analysis should be made of the moments developed in both slab and columns, and the values given herein modified accordingly. Slabs with paneled ceiling or with depressed paneling in the floor are to be considered as coming under the recommendations herein given.

(r) Bending Moments in Columns.-Provision should be made in both wall columns and interior columns for the bending moment which will be developed by unequally loaded panels, eccentric loading, or uneven spacing of columns. The amount of moment to be taken by a column will depend upon the relative stiffness of columns and slab, and computations may be made by rational methods, such as the principal of least work, or of slope and deflection. Generally, the larger part of the unequalized negative moment will be transmitted to the columns, and the column should be designed to resist this bending moment. Especial attention should be given to wall columns and corner columns.

\section{WORKING STRESSES}

General Assumptions. The following working stresses are recommended for static loads. Proper allowances for vibration and impact are to be added to live loads where necessary to produce an equivalent static load before applying the unit stresses in proportioning parts.

In selecting the permissible working stress on concrete, the designer should be guided by the working stresses usually allowed for other materials of construction, so that all structures of the same class composed of different materials may have approximately the same degree of safety.

The following recommendations as to allowable stresses are given in the form of percentages of the ultimate strength of the particular concrete which is to be used; this ultimate strength is that developed at an age of twenty-eight days, in cylinders 8 inches in diameter and 16 inches long, of proper consistency $\dagger$ made and stored under laboratory conditions. In the absence of definite knowledge in advance of construction as to just what strength may be expected, the committee submits the following values as those which should be obtained with materials and workmanship in accordance with the recommendations of this report.

Although occasional tests may show higher results than those here given, the Committee recommends that these values should be the maximum used in design.

Table of Compressive Strengths of Different Mixtures of Concrete (In Pounds per Square Inch)

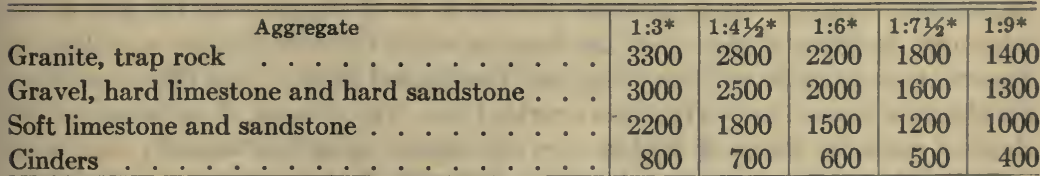

NOTE.-For variations in the moduli of elasticity see section on "Working Stresses."

Bearing. When compression is applied to a surface of concrete of at least twice the loaded area, a stress of 35 per cent of the compressive strength may be allowed in the area actually under load.

† See Consistency, page 191. 
Axial Compression. For concentric compression on a plain concrete pier, the length of which does not exceed four diameters, or on a column reinforced with longitudinal bars only, the length of which does not exceed 12 diameters, 22.5 per cent of the compressive strength may be allowed.

For other forms of columns the stresses obtained from the ratios given in the preceding section on "Design" may govern.

Compression in Extreme Fiber. The extreme fiber stress of a beam, calculated on the assumption of a constant modulus of elasticity for concrete under working stresses may be allowed to reach 32.5 per cent of the compressive strength. Adjacent to the support of continuous beams stresses 15 per cent higher may be used.

Sheer and Diagonal Tension. In calculations on beams in which the maximum shearing stress in a section is used as the means of measuring the resistance to diagonal tension stress, the following allowable values for the maximum vertical shearing stress in concrete, calculated by the method given in Formula 22 (see page 8) are recom. mended:

(a) - For beams with horizontal bars only and without web reinforcement, 2 per cent of the compressive strength.

(b) - For beams with web reinforcement consisting of vertical stirrups looped about the longitudinal reinforcing bars in the tension side of the beam and spaced horizontally not more than one-half the depth of the beam; or for beams in which longitudinal bars are bent up at an angle of not more than 45 degrees or less than 20 degrees with the axis of the beam, and the points of bending are spaced horizontally not more than three-quarters of the depth of the beam apart, not to exceed $41 / 2$ per cent of the compressive strength.

(c)-For a combination of bent bars and vertical stirrups looped about the reinforcing bars in the tension side of the beam and spaced horizontally not more than one-half of the depth of the beam, 5 per cent of the compressive strength.

(d) - For beams with web reinforcement (either vertical or inclined) securely attached to the longitudinal bars in the tension side of the beam in such a way as to prevent slipping of bar past the stirrup, and spaced horizontally not more than onehalf of the depth of the beam in case of vertical stirrups and not more than threefourths of the depth of the beam in the case of inclined members, either with longitudinal bars bent up or not, 6 per cent of the compressive strength.

The web reinforcement in case any is used should be proportioned by using twothirds of the external vertical shear in Formula 24 or 25 (see page 9). The effect of longitudinal bars bent up at an angle of from 20 to 45 degrees with the axis of the beam may be taken at sections of the beam in which the bent up bars contribute to diagonal tension resistance (see "Diagonal Tension and Shear," page 198) as reducing the shearing stresses to be otherwise provided for. The amount of reduction of the shearing stress by means of bent up bars will depend upon their capacity, but in no case should be taken as greater than $41 / 2$ per cent of the compressive strength of the concrete over the effective cross-section of the beam (Formula 22). The limit of tensile stress in the bent up portion of the bar calculated by Formula 25, using in this formula an amount of total shear corresponding to the reduction in shearing stress assumed for the bent up bars, may be taken as specified for the working stress of steel, but in the calculations the stress in the bar due to its part as longitudinal 
reinforcement of the beam should be considered. The stresses in stirrups and inclined members when combined with bent up bars are to be determined by finding the amount of the total shear which may be allowed by reason of the bent up bars, and subtracting this shear from the total external vertical shear. Two-thirds of the remainder will be the shear to be carried by the stirrups, using Formulas 24 or 25 (see page 9).

Where punching shear occurs, provided the diagonal tension requirements are met, a shearing stress of 6 per cent of the compressive strength may be allowed.

Bond. The bond stress between concrete and plain reinforcing bars may be assumed at 4 per cent of the compressive strength, or 2 per cent in the case of drawn wire. In the best types of deformed bar the bond stress may be increased, but not to exceed 5 per cent of the compressive strength of the concrete.

Reinforcement. The tensile or compressive stress in steel should not exceed 16,000 pounds per square inch.

In structural steel members the working stresses adopted by the American Railway Engineering Association are recommended.

Modulus of Elasticity. The value of the modulus of elasticity of concrete has a wide range, depending on the materials used, the age, the range of stresses between which it is considered, as well as other conditions. It is recommended that in computations for the position of the neutral axis, and for the resisting moment of beams and for compression of concrete in columns, it be assumed as:

(a)-One-fortieth that of steel, when the strength of the concrete is taken as not more than 800 pounds per square inch.

(b) - One-fifteenth that of steel, when the strength of the conerete is taken as greater than 800 pounds per square inch.

(c)-One-twelfth that of steel, when the strength of the concrete is taken as greater than 2,200 pounds per square inch, and less than 2,900 pounds per square inch, and

(d)-One-tenth that of steel, when the strength of the concrete is taken as greater than 2,900 pounds per square inch.

Although not rigorously accurate, these assumptions will give safe results. For the deflection of beams which are free to move longitudinally at the supports, in using formulas for deflection which do not take into account the tensile strength developed in the concrete, a modulus of one-eighth of that of steel is recommended. 


\section{SUBJECT INDEX}

AMrrican Society for Testing Materials, cement specifications

Areas-Circles

Circular segments.

Column sections

Corrugated Bars

PAGES

Reinforcement for slabs

$186-187$

Triangles

Various sections

Wire

182-183

174

$157-158$

164

148

175

168-171

167

BARS-Moments of inertia

160

Reinforcing . . .

164-166

Specifications

188-189

BEAMS-Continuous, moments and shears . . . . . . . . . . . . . . ${ }_{196-197}^{38-48}$

Quantities of concrete . . . . . . . . . . . 161

Rectangular, designing diagrams, explanation . . . . . . 10

Rectangular, diagrams for values of $j, k$ and $K \ldots \ldots \ldots$

Rectangular, formulas. . . . . . . . . . . 6

Rectangular, standard notation . . . . . . . . 5

Rectangular, tables of safe loads . . . . . . . . . 79-90

Rectangular, table of values of $p, k, j$ and $K \ldots \ldots$

Reinforced for compression, designing diagrams, explanation . . . 12-13

Reinforced for compression, diagram ......... 20

Reinforced for compression, formulas

Reinforced for compression, standard notation . . . . . . . 5

Span of . . . . . . . . . 194-195

Stirrup reinforcement, table . . . . . . . 107

Tee, continuous, explanation of tables . . . . . 59-60

Tee, designing diagrams, explanation . . . . . . . 11-12

Tee, diagrams for values of $K$ and $j \ldots \ldots$. . . . . . . 17-18

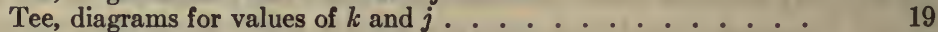

Tee, dimensions . . . . . . . . . . . . . . . 195

Tee, formulas ..................... 7

Tee, standard notation . . . . . . . . . . 5

Tee, tables of safe loads . . . . . . . . . . . . . 91-106

Wooden ......................... 173

BENdING and direct stress, combined . . . . . . . . . . . . 13-14

and direct stress, combined, diagrams . . . . . . . . 21-24

Bond-Formulas .

Standard notation . . . . . . . . . . 6

Strength . . . . . . . . . . . . . . 197-198

BRACKETS . . . . . . . . . . . . . . 194-195

BuILDING code requirements for live loads . . . . . . . . . . . . 51-52

BuILDING materials-weights of . . . . . . . . . . . 56

CAPs for reinforced concrete piles. . . . . . . . . . . . . . 141-142

CEMENT specifications. . . . . . . . . . . . . . . . 186-187

CrRCLes-Areas and circumferences . . . . . . . . . . . 182-183

Circtlar segments-Areas . . . . . . . . . . . . . . . 174

Circumferences of circles . . . . . . . . . . . . 182-183

Column-Heads, diagram for volume of concrete in . . . . . . . 162

Sections, areas, perimeters, etc. . . . . . . . 157-158

Spirals, pitch and percentage . . . . . . . . . 150

Spirals, standard wire and spacers . . . . . . . . . . 156

Spirals, weight per foot . . . . . . 151-155

Verticals, moments of inertia . . . . . . . . 159-160 
Columns-Bending moments

Designing diagrams, explanation .

Formulas .

Joint Committee recommendations

Length

200-202

Square tied, tables of safe loads

$122-132$

Standard notation

115-121

Tables, explanation.

Vertical steel for various percentages of core area

Wooden.

CoMPREssive reinforcement of beams, diagram

CONCRETE-General specifications

Massive

Quantities of materials

Construction-General specifications

Recommendations on design and working stresses, Joint Committee .

Corr-Plate floors-Explanation Tables

Corruaated Bars

194-211

108-109

110-113

CUBES and cube roots of numbers

Decimals of a foot.

182-183

of an inch

Deflection-General formulas $\ldots \ldots 26-37$

Desrgn - Final report of Joint Committee . . . . . . . . . . 194-211

Designing diagrams - Explanation

10-14

DIAGRAM-Compressive reinforcement of beams

Distribution of load for rectangular slabs ........................

Values of $K, j$ and $k$ for rectangular beams and slabs

Values of $k$ and $j$ for tee beams

Values of $K^{\prime}$ for combined bending and direct stress

Values of $k$ for combined bending and direct stress

Volume of concrete in column heads

Diagrams-Designing, explanation

Moment and shear coefficients for continuous beams, equal spans

Values of $F$ for combined bending and direct stress

Values of $K$ and $j$ for tee beams

Diagonal tension and shear.

198-200

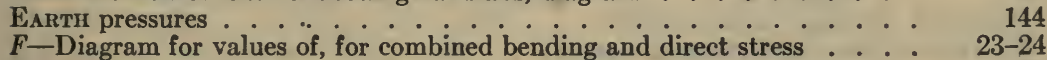

FireProofrng-Explanation of slab and beam tables . . . . . . 58

Flat Slab-Explanation of . . . . . . . . . . . 108-109

Column heads, diagram for volume of concrete in . . . . . 162

Joint Committee recommendations . . . . . . . . . 202-209

Floors, tables ................. 110-113

FLoors and roofs-Explanation of tables
Footings-Combined column, tables $\ldots \ldots$

Explanation of tables . . . . . . . . . 133-135

For reinforced concrete piles. . . . . . . . . . 141-142

Square column, table . . . . . . . . . . 140

Formulas-For columns . . . . . . . . . . . . . 114

For properties of sections. $\ldots$ 168-171

For reactions, bending moments, shears and deflections . . . 26-37

For reinforced concrete design ......... . . 5-9

For trigonometric solution of triangles . . . . . . 175 
Foundatrons-Bearing capacity of soils

Fractions and equivalent decimals .

Friction-Coefficients and angles

FunCtrons-Natural trigonometric

\section{of numbers}

176-181

Grration-Radius of, various sections

182-183

INERTIA-Moments of, bars

168-171

Moments of, column sections

Moments of, column verticals .

157-158

Moments of, various sections .

$j$-Diagram for values in rectangular beams and slabs.

168-171

$j$-Diagram for values in tee beams.

$j$-Table of values for rectangular beams and slabs

JorNT Committee-Final report on design and working stresses

$17-19$

$K$-Diagram for values of, rectangular beams and slabs

Diagram for values of, tee beams

Table of values, rectangular beams and slabs.

$k$-Diagram for values of, combined bending and direct stress

Diagram for values of, rectangular beams

Diagram for values of, tee beams

Table of values of, rectangular beams and slabs

LOAD-Building code requirements .

Distribution of, for rectangular slabs . . . . . . . . . . ${ }_{2} 25$

LoADs-Dead and live

Safe, for flat slab floors . . . . . . . . 110-113

Safe, for rectangular beams $\ldots \ldots$. . . . $79-90$

Safe, for spiral columns . . . . . . . . . . . . . . . 122-132

Safe, for square tied columns. . . . . . . . . . 115-121

Safe, superimposed for clay tile ribbed slabs . . . . . . . 67-72

Safe, superimposed for ribbed slabs . . . . . . . . 73-78

Safe, superimposed for solid concrete slabs . . . . . . . . . 61-66

Safe, for tee beams

In warehouses . . . . . . . . . . . $49-50$

For wooden beams and columns . . . . . . . . . 173

LogaritHMS of numbers . . . . . . . . . . . 182-183

MANUFACTURERs' standard specifications for deformed bars . . . . . . . 188-189

MATERIALS-Building, weight of .

Quantities for concrete Weights of

Modulus-Section, various sections

Moments-Bending, continuous beams

Bending, general formulas for

Of inertia, bars

Of inertia, column sections

Of inertia, column verticals

Of inertia, various sections

Theorem of three

188-189

168-171

$38-48$

28-37

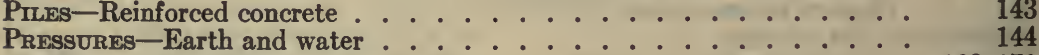

Propertres of sections . . . . . . . . . . . . 168-171

RADros of gyration of various sections . . . . . . . . . . . 168-171

REACTIONS-General formulas for . . . . . . . . . . 28-37

RECIPROCALS of numbers 
Reinforcement-Corrugated Bars .

REtaining walls-Cantilever

RiBBed slabs-Clay tile, safe load tables

Steel or wood forms, safe load tables

Roors-Floors and, explanation of tables

SECTION modulus-Various sections.

SHRINKAGE-Reinforcing for .

SLAB-Area of reinforcement per foot width

Flat, Joint Committee recommendation

SLABS-Clay tile ribbed, explanation of tables

Clay tile ribbed, tables of safe loads

Distribution of load for rectangular

Flat, explanation of

108-109

Flat, tables .

110-113

Ribbed, explanation of tables

Ribbed, table of safe loads

Solid concrete, explanation of tables

Solid concrete, tables of safe loads .

Sort values-Combined column footings Square column footings

SPECIFICATIONS for deformed bars

Squares and square roots of numbers.

STIRRUP reinforcement for uniformly loaded beams

STRESS-Combined bending and direct

Combined bending and direct, diagrams.

STresses-Joint Committee recommendations .

Caps for reinforced concrete piles $\ldots \ldots$. . . . 141-142

Cantilever retaining walls . . . . . . . . 145-146

Column spiral weights . . . . . . . . . 151-155

Column vertical steel. 
TABLES-Combined column footings

Corrugated Bars .

Decimals of a foot

136-139

Decimals of an inch

Earth and water pressures

184

Flat slab floors

Functions of numbers

110-113

Moments of inertia of bars

182-183

Moments of inertia of column verticals

Natural trigonometric functions

176-181

Properties of column sections

$157-158$

Properties of sections.

168-171

Quantities of materials for concrete . . . . . . . . 163

Reinforced concrete piles .

143

Safe loads, clay tile ribbed slabs

Safe loads, rectangular beams

$79-90$

Safe loads, ribbed slabs, steel or wood forms

$73-78$

Safe loads, solid concrete slabs

$61-66$

Safe loads, tee beams.

91-106

Spiral columns, safe loads .

122-132

Spiral wire and spacers .

Spirals, pitch and percentage

150

Square column footings .

140

Square tied columns, safe loads

$115-121$

Stirrup reinforcement for uniformly loaded beams

Timber .

$172-173$

Trigonometric solution of triangles

Values of $p, k, j$ and $K$

Volume of concrete in beams

Temperature-Reinforcing for

Triangles - Trigonometric solution of

Trigonometric - Natural, functions .

Volumes-Column sections

Of concrete in column heads

WEB reinforcement-Formulas for

WeIGHTS of building materials .

Column sections . . . . . .

Column spirals

Corrugated Bars . . . . . . . . . . . . . . . . . . . . . 164

Of materials $\ldots$

Of timber.

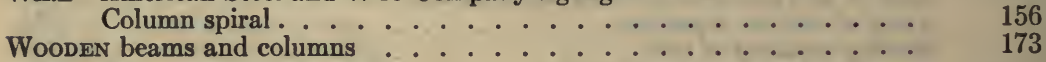




\title{
RICE LEADERS OF THE WORLD ASSOCIATION
}

\author{
Announces the Election To \\ MEMBERSHIP OF THE
}

CORRUGATED BAR COMPANY, INC.

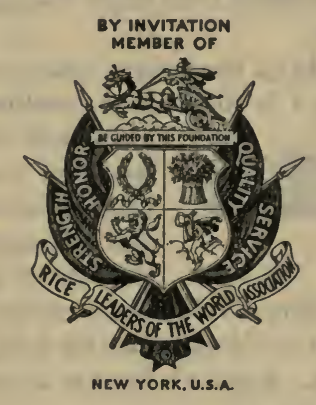

గHIs Association is a coöperative organization composed solely of 1 concerns which adhere to the highest standards in the conduct of their business. These are the

\section{QUALIFICATIONS FOR MEMBERSHIP}

HoNOR: A recognized reputation for fair and honorable business dealings.

QUALITY: An honest product, of quality truthfully represented.

Strength: A responsible and substantial financial standing.

Service: A recognized reputation for conducting business in prompt and efficient manner.

Membership in the Association is an evidence of the distinctive position which the Corrugated Bar Company, Inc. holds as a Specialist in Concrete Reinforcement and Design.

It testifies as to the quality of this company's products and service, and the character of the directing officials.

It is a further assurance that these products are made by a concern worthy of utmost confidence, as only manufacturers of the highest standing in name, product and policy are privileged to display the Association Emblem. 


\section{CORRUGATED BARS}

$7^{\mathrm{HE}}$ question of using plain or deformed bars for reinforced concrete construction has been answered in the United States where the volume of such construction is probably greater than in all the rest of the world. Fifteen years ago about $80 \%$ of the reinforcement used was plain bars, to-day $80 \%$ is deformed bars.

Nearly all building codes, architects' and engineers' specifications and committee recommendations of technical societies allow larger working values for the bond stress of deformed than for plain bars.

A review of available test data on bond shows clearly that American practice is on a very sound basis. Diagram 1 is the average resulting from the tabulation and

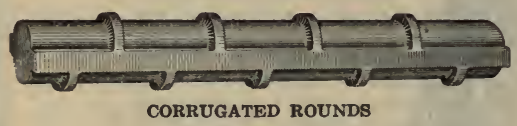
analysis of the thousands of bond tests made by recognized authorities in the United States and Europe on both plain and Corrugated Bars. The variation in the case of plain bars, from a very low value for a minimum to a maximum value which approximates only the average of Corrugated Bars, is due entirely to the fact that the bond of a plain bar is a function of accidental surface condition. A smooth bar has a very low bond value; a rusty, pitted, rough bar may have a high value.

An important difference in behavior between plain and Corrugated Bars is shown in Diagram 2. This diagram is a composite of load slip curves taken from University of Illinois tests where conditions were practically identical, and illustrates clearly that plain bars reach a maximum bond value at about 0.01 inch slip and then decrease while Corrugated Bars have much higher bond at the same slip and increase in resistance until a final value several times that of plain bars is reached.

It is not good engineering practice to leave the bond-the most important function of the reinforcement-to chance, while all the other physical properties of the material are required to conform to rigid specifications within narrow limits.

To realize the highest efficiency of bond value, a deformed bar of proper design is required; merely roughening the

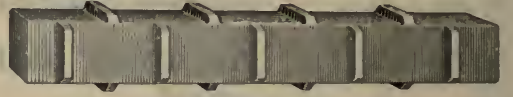

CORRUGATED SQUARES surface of a bar by haphazard projections may actually decrease its bond value. The twisted bar, for example, for years considered a standard deformed bar, has been found on careful examination to have lower bond resistance than a plain bar.

The conditions governing the design of a satisfactory deformed bar are clearly stated in Bulletin No. 71, University of Illinois, Engineering Experiment Station, as follows: "In a. deformed bar of good design the projections should present bearing faces as nearly as possible at right angles to the axis of the bar. The areas of the projections should be such as to preserve the proper ratio between the bearing stress against the concrete ahead of the projections and the shearing stress over the surrounding envelope."

Of all the deformed bars on the market the Corrugated Bar is the only one that substantially fulfills these requirements. 


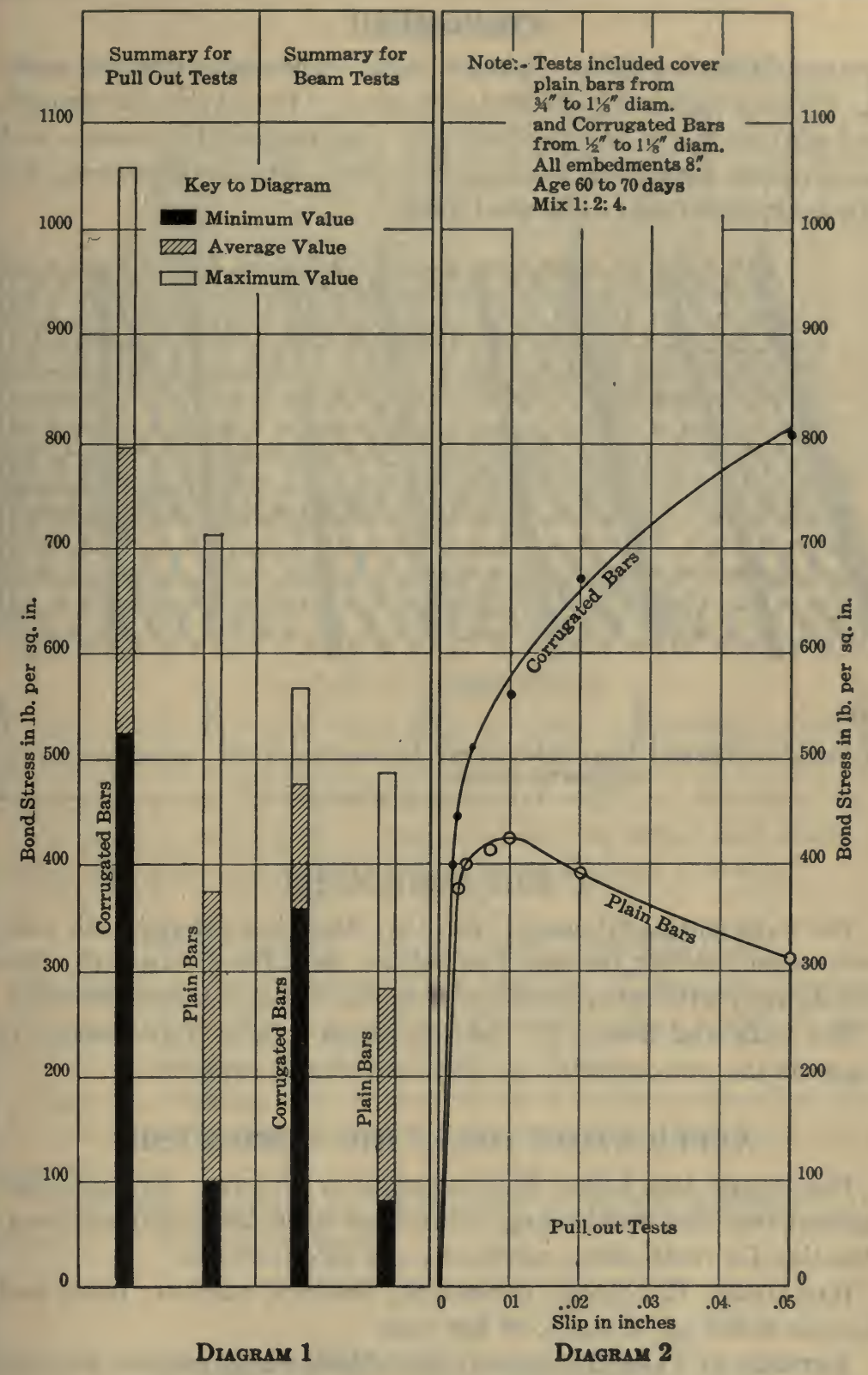




\section{CORR-MESH}

CORR-Mesh is a ribbed expanded metal-a one-piece product, made from a high-grade of rolled sheet steel. The ribs provide strength and stiffness to the sheets which give firm support to concrete and plaster both during construction and after. The metal between the ribs is expanded into a diamond mesh.

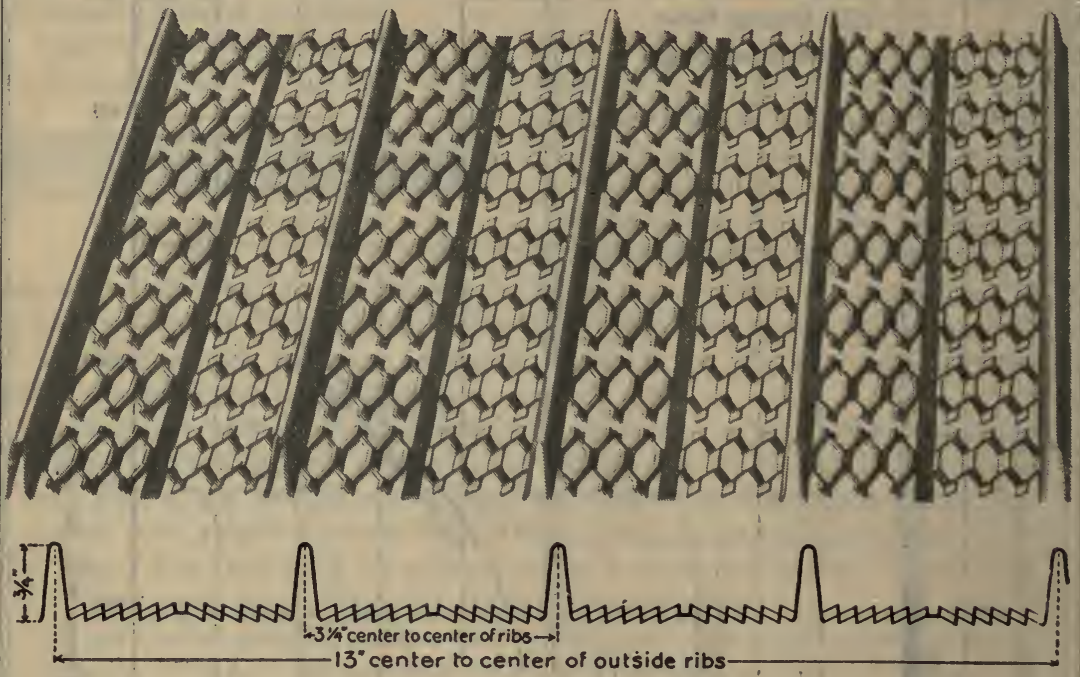

\section{3/4" RIB CORR-MESH}

For walls and partitions, $3 / 4^{\prime \prime}$ Rib Corr-Mesh is plastered both sides with cement mortar, forming a monolithic wall of great strength. The ribs do away with extra studding - a saving in material and labor cost.

For walls and floors, $3 / 4$ " Rib Corr-Mesh acts as form work. It supports the wet concrete; no deck centering is required.

\section{APPLICATION OF 3/4" RIB CORR-MESH}

Foundries and Light Manufacturing Plants: Replaces corrugated iron and wood siding. Corr-Mesh is the ideal method of construction for roofs, floors, partitions and exterior walls.

Rarlroads: Handsome, permanent, fireproof stations, sheds and wayside buildings in stucco at low cost.

Amusement Pakk Buildings: Corr-Mesh makes possible the only low cost construction on which insurance can be obtained. 


\section{CORR-MESH}

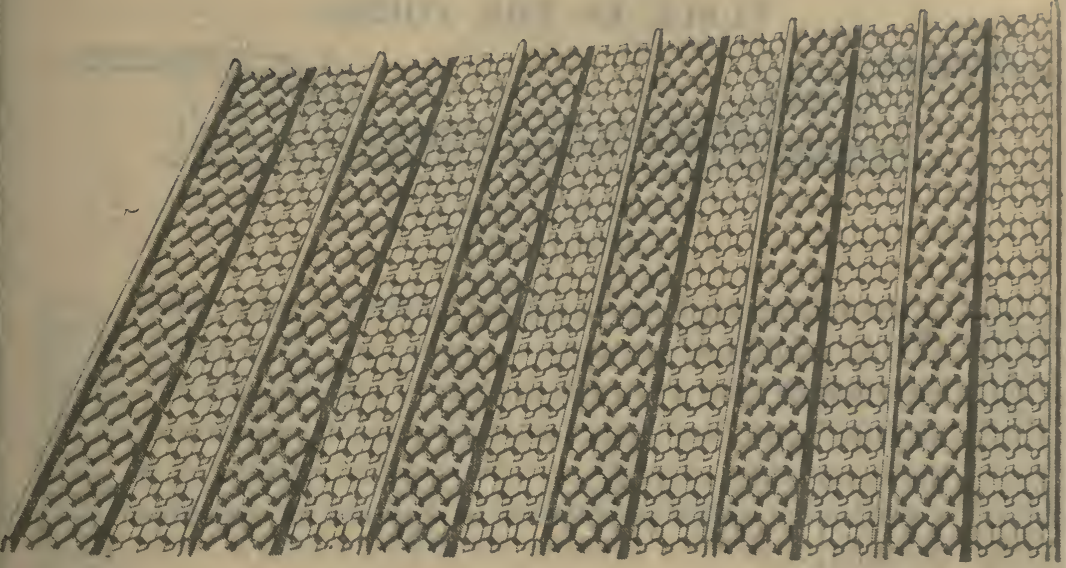

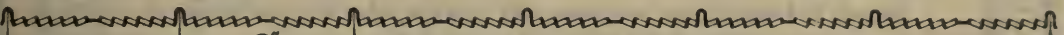
$\Vdash 3^{\prime \prime} \longrightarrow$ CENTER TO CENTER OF RIBS

18" CENTER TO CENTER OF OUTSIDE RIBS

\section{5/16" RIB CORR-MESH}

For ceilings, $5 / 10^{\prime \prime}$ Rib Corr-Mesh is used extensively, where it greatly reduces the material required in the supporting framework, and cuts down the cost of erection.

For stucco construction it eliminates furring strips and makes a strong and permanent reinforcement for the plaster covering.

\section{APPLICATION OF 5/16" RIB CORR-MESH}

Residences: Stucco walls are handsome, permanent and fireresisting. Old wooden houses may be transformed at small cost into attractive residences of greatly increased value.

Garages, Stables and Outbuildings of stucco construction with 5/1" Rib Corr-Mesh are low in cost, permanent, and free from repair expense.

Fences which present an artistic and substantial appearance are constructed with Corr-Mesh.

CORRUGATED BAR COMPANY, INC.

BUFFALO, N. Y. 


\section{FABRICATED REINFORCEMENT READY TO PLACE IN THE FORMS}

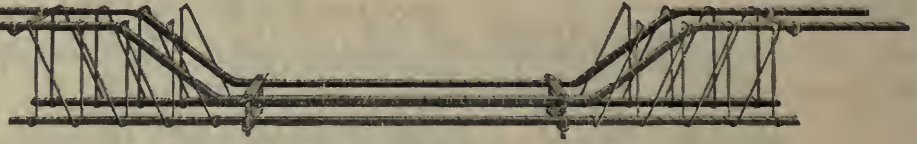

READY TO PLACE

CORR-BAR UNITS

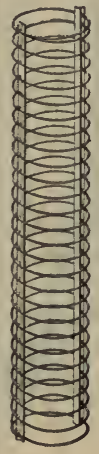

Мно fabricated reinforcement marks a turning point $N$ in reinforced concrete construction. It is only a question of time when the practice of organizing a fabrication crew, for each job, with local inexperienced common labor, must give way to well-established shop practice, in which hand labor is replaced by machine production.

Shop fabrication secures accuracy, thereby eliminating one important factor of uncertainty in construction. It secures economy and facilitates supervision and inspection. It saves storage and working space

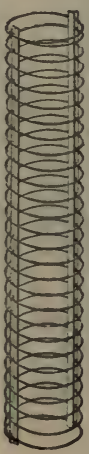
at the building site, often a matter of great importance, and by decreasing the amount of labor in the field removes some of the uncertainty and annoyance connected with the handling of labor.

Reinforcement should not only be fabricated in the shop, but assembled into units, as far as practicable, properly marked and made ready to place directly in the forms.

For beam reinforcement the Corr-Bar Unit is an ideal example of shop fabricated reinforcement. Instead of depending upon the skill of the individual workman to fabricate and assemble twenty to thirty pieces and place them properly in the forms, one unit is made in the shop by machine operators under the direction of an organization especially trained to do this work. The unit is inspected and marked and so equipped with supporting devices as to insure its proper place in the structure. 


\section{ENGINEERING SERVICE DEPARTMENT}

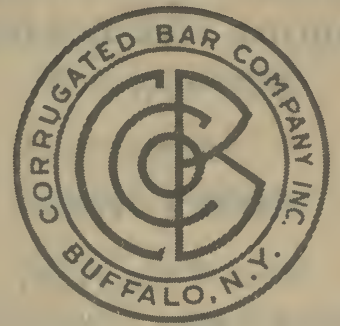

$T^{\mathrm{HE}}$ Corrugated Bar Company, Inc., since its inception, more than 1 twenty-five years ago, has been primarily an engineering organization. It has been a pioneer in the field of reinforced concrete not only in the development of scientifically correct reinforcing materials and systems of construction, but through investigation and presentation of rational methods of design.

Under the direction of its engineers elaborate and painstaking research and test programs have been carried out, earning for the company an enviable reputation and standing in professional circles.

The engineering department has developed under highly competitive conditions, making it of necessity efficient and economical in the execution of its designs and it is safe to say that for variety and wealth of experience it is unsurpassed by any organization of its kind. As a result of its experience it has been called upon frequently to act in a consulting capacity on numerous reinforced concrete structures in the United States and many foreign countries. The constantly increasing demand for this class of service resulted in the organization of an Engineering Service Department.

The service rendered by this department-divorced entirely from the company's products - is available to architects and engineers on a fee basis and consists of:

1. A study of conditions and selection of a type of construction best suited to the purpose of the building.

2. Preliminary and comparative sketches, estimates and cost data as a basis of negotiation between the architect or engineer and his client.

3. Preparation of detail plans and specifications including placing diagrams, fabrication details and bar lists.

By the use of this Service the owner obtains a low bid on the construction best suited to his building and it guarantees full patent protection on any materials or construction involved in the plans supplied. 


\section{CORRUGATED BAR COMPANY, INC. MUTUAL LIFE BUILDING BUFFALO, N. Y.}

\section{DISTRICT OFFICES}

New York, N. Y.

Whitehall Building, 17 Battery Place

Chicago, Ill.

Great Northern Building, 20 W. Jackson Street

Philadelphia, Pa.

Transportation Building, 26 S. Fifteenth Street

Boston, Mass.

- . 27 School Street

St. Louis, Mo.

Boatmen's Bank Building

- Detroit, Mich.

$\rightarrow \quad$ Penobscot Building

Milwaukee, Wis.

Wells Building

Kansas City, Mo.

Waldheim Building

St. Paul, Minn.

Pioneer Building

Atllanta, Ga.

Grant Building

Syracuse, N. Y.

Union Building

Houston, Texas

700 N. San Jacinto Street 


\section{ERRATA-“USEFUL DATA," FIRST EDITION}

Page 13, line 1, change 747 in denominator of fraction to 805 .

Page 13 , line 1 , change $7.76 \%$ to $7.2 \%$.

Page 13, line 2, change 7.76 to 7.2 .

Page 14, line 4, change 496 to 505.

Page 14, line 4, change 292 to 283.

Page 15 , in formula at top of page change $P$ to $p$.

Page 22, shift decimal point, in all figures at top of diagram, one place to the left, to read $0.17,0.18,0.20$, etc.

Page 26, line 3 from bottom, change $W$ to $w$. -

Page 26, bottom line, change $W_{\mathrm{m}}$ to $w_{\mathrm{m}}$. -

Page 27, line 4, change "foot" to "unit." -

Page 27, line 14, change $W_{\mathrm{m}}$ to $w_{\mathrm{m}}$.

Page 27, line 14, change "foot" to "unit."

Page 29, in formula 4 second equation, change $(l-x)$ to $(2 l-x)$.

Page 29, in formula 5 third equation, change $\frac{R_{1} x}{l}$ to $R_{1} x$.

Page 29, in formula 5 sixth equation, change " $d<b$ and on same side of load" to read " $a<b$ and $y$ on same side of load as $b . "$

Page 30 , in formula 9 , second equation from bottom, change $\frac{3 f S}{l}$ to $\frac{f S}{4 l}<$

Page 30, in formula 9, last equation, change $l^{3}$ to $L^{3}$.

Page 32 , in formula 13 , sixth equation, change $2 w$ to $w$.

Page 32 , in formula 15 , fourth equation, change $\frac{w l^{2}}{3}$ to $\frac{w l^{2}}{12}$.

Page 32 , in formula 15 , fifth equation, change $\frac{f S}{4 l^{2}}$ to $\frac{f S}{l^{2}}$.

Page 33 , in formula 18 , last equation, change $\frac{9 w l^{2}}{32}$ to $\frac{w l^{2}}{32}$.

Page 34, in formula 19, second equation, change $2 l$ to $4 l$. $\angle$

Page 34 , in formula 19, third equation, change $2 l$ to $4 l$.

Page 34, in formula 20, fourth equation, change to read $\frac{w_{2} x}{2}(l-x)+\frac{w_{1} x}{6 l}\left(l^{2}-x^{2}\right)$.

Page 35 , in formula 24 , fourth equation, change to read $M_{\mathbf{x}}=\frac{3 w l x}{20}-\frac{w l^{2}}{30}-\frac{w x^{3}}{6 l}$.

Page 36, in formula 26, last equation, change $l^{3}$ to $L^{3}$.

Page 37, change $M m=\frac{P x}{2 l}(2 l-a)$, to $M m=\frac{P}{8 l}(2 l-a)^{2}$.

Page 37, change $V m=R_{1}=\frac{(l-a)}{l} \Sigma P$, to $V m=R_{1}=P+p \frac{(l-a)}{l}$.

Page 37, change $V m=\frac{4 P}{l}(l-a)$, to $V m=R_{1}=\frac{4 P}{l}\left(l-\frac{3 a}{2}\right)$.

Page 37, change $x=\frac{1}{2 \Sigma P}(p b-P a)$, to $x=\frac{l}{2}+\frac{1}{2 \Sigma P}(p b-P a)$.

Page 141, in 7 pile cap, change 42.4 cu. yd. to 4.24 cu. yd.

Page 144, in nomenclature, change $P$ to $p$.

Page 149, in fourth column, change 0.57 to 0.75 under "Percent of Core Ares."

Page 175, Reverse the order of the first two formulas for "Area."

Page 175 in fourth line from the bottom, change 5 under the radical to $s$. 


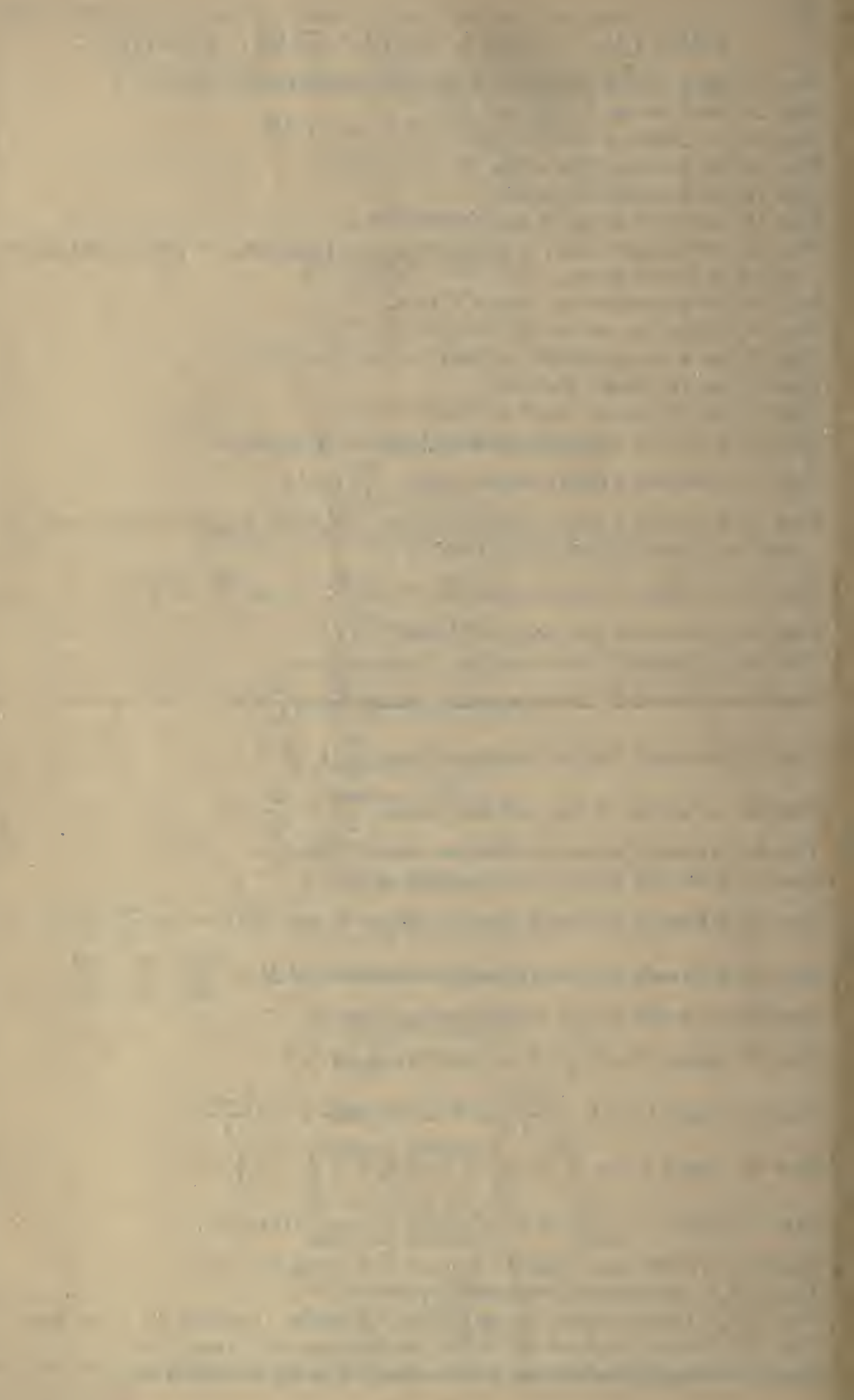


MATHHEWS-NORT THRUP

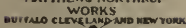

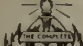

(4)

सhis? 




\section{PLEASE DO NOT REMOVE CARDS OR SLIPS FROM THIS POCKET}

\section{UNIVERSITY OF TORONTO LIBRARY}

S\&M

A

45 
